The copyright of this thesis vests in the author. No quotation from it or information derived from it is to be published without full acknowledgement of the source. The thesis is to be used for private study or noncommercial research purposes only.

Published by the University of Cape Town (UCT) in terms of the non-exclusive license granted to UCT by the author. 


\title{
Marine fish exploitation during the Middle and Later Stone Age of South Africa
}

\author{
by \\ Karen Loise van Niekerk
}

Thesis presented for the degree of DOCTOR OF PHILOSOPHY

In the Department of Archaeology UNIVERSITY OF CAPE TOWN

January 2011 


\begin{abstract}
Marine fish remains are not common in Middle Stone Age (MSA) sites in Africa. There are currently only two known MSA sites with good organic preservation in South Africa that contain marine fish remains in relatively high numbers: Blombos Cave (BBC) and Klasies River main site (KR). Marine fish exploitation is considered by some researchers as a marker of modern human behaviour, requiring cognitive and technological capacities thought to have only appeared after 50000 years ago, during the Later Stone Age (LSA). There is mounting artefactual evidence pointing towards an earlier date for modern human behaviour than previously thought, and most of the evidence currently comes from MSA assemblages in southern Africa. Both KR and BBC contain artefactual evidence for behavioural modernity during the MSA, at least as far back as 70 thousand years ago at BBC. The fish from these two sites, along with four LSA fish assemblages, were examined using a more systematic and comprehensive approach than has previously been applied to South African marine fish assemblages, and this has allowed a level of comparison between assemblages not previously possible. It has indicated that there is a large deficit of fish elements in the MSA relative to the LSA, and that age-related taphonomic processes have diminished the numbers of fish originally deposited. These analyses have revealed that people living at BBC and KR during the MSA could catch fish, and did so periodically, which is contrary to the model arguing for a late manifestation behavioural modernity.
\end{abstract}




\section{Acknowledgements}

I thank Judith Sealy for her guidance as supervisor and for access to the Hoffman's/Robberg Cave fish assemblage. Thanks to Christopher Henshilwood for access to the Blombos Cave and Blombosfontein 1 and 6 materials and to Hilary Deacon for access to the Klasies River main site fish remains. Sarah Wurz and Petro Keene were very helpful with regards to access of material curated at the IZIKO South African Museum, for which I am grateful, and it was a pleasure working with both of them. Thanks are due to Paul Cowley from the South African Institute of Aquatic Biodiversity (SAIAB) who very kindly and with considerable effort supplied me with a number of species that were lacking from my comparative collection. Special thanks to Nicholas Henshilwood and Morgan Wilson for their enthusiastic help in augmenting the comparative collection with their fishing expeditions. Drs P. and E. Heemstra, along with the publications department of the SAIAB, kindly gave me permission to use the illustrations of the fish species from their book (2004) Coastal Fishes of Southern Africa. Grahamstown: South African Institute for Aquatic Biodiversity and National Inquiry Service Centre.

A very special thank you to my mother, Laurette van Niekerk, for the inspirational artwork on the title page.

Funding for this project came from the National Research Foundation of South African and additional funding through Judith Sealy from the National Research Foundation, which I gratefully acknowledge. 


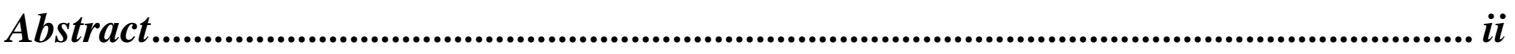

Acknowledgements.......................................................................................................... iii

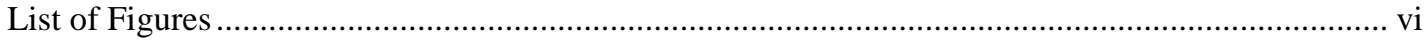

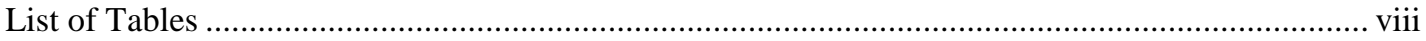

Chapter 1. Introduction ...........................................................................................

Chapter 2. Fish remains and archaeology ........................................................................... 8

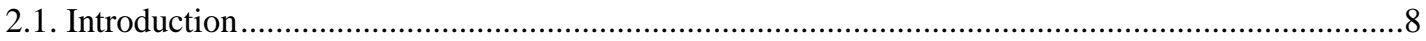

2.2. Archaeological fish analysis and information from anthropogenic fish remains ........................... 9

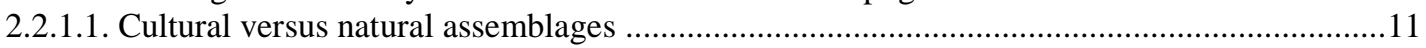

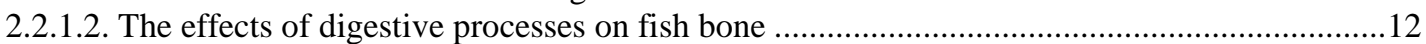

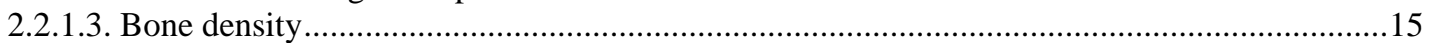

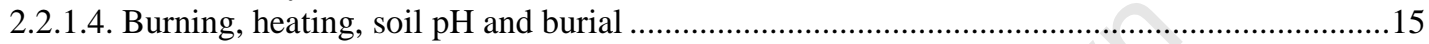

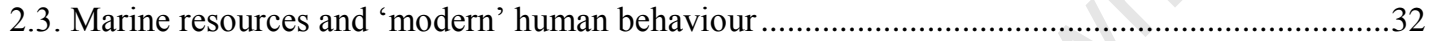

Chapter 3. Southern African archaeological fish analysis, species ecology and methods

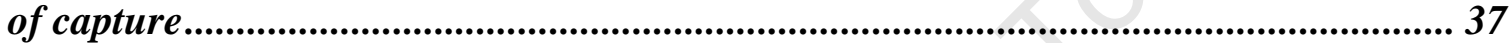

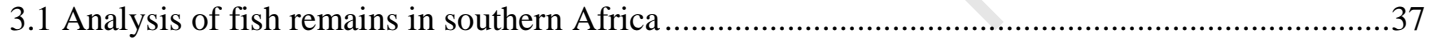

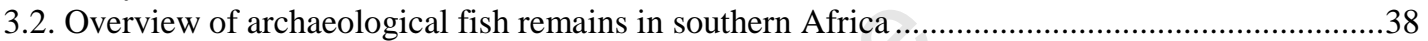

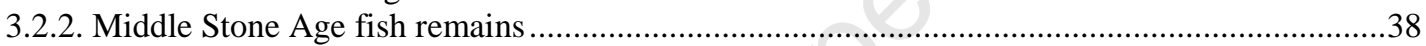

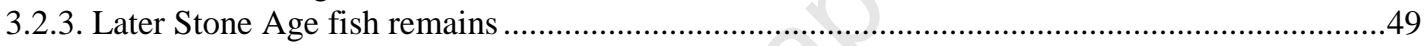

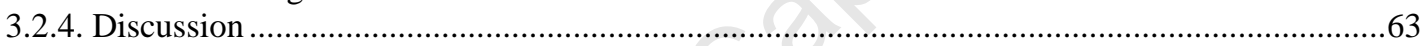

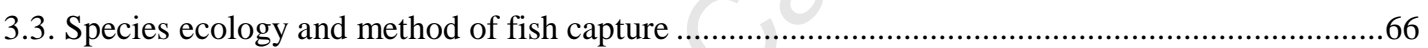

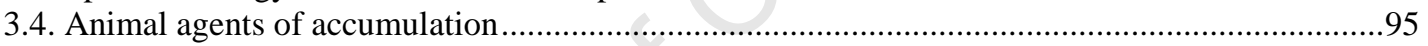

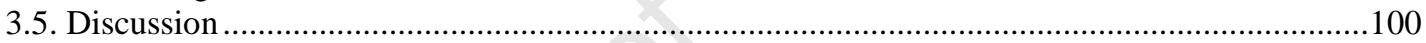

Chapter 4: Site Description and Background .................................................................. 104

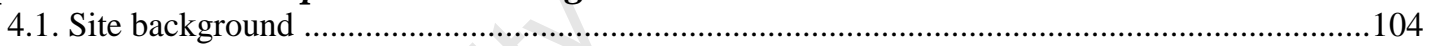

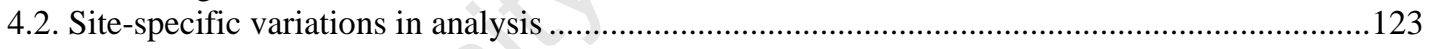

Chapter 5. Analytical methods.......................................................................................... 127

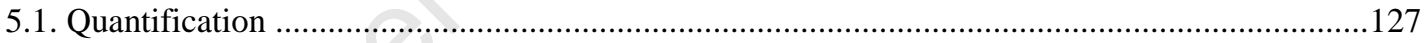

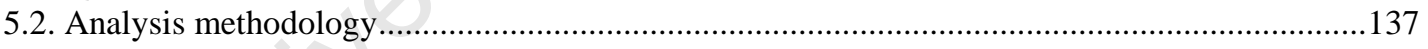

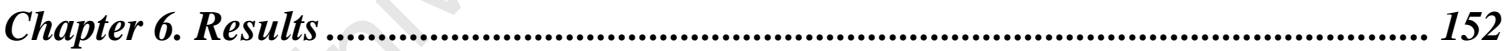

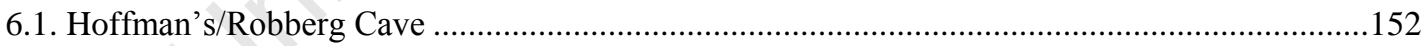

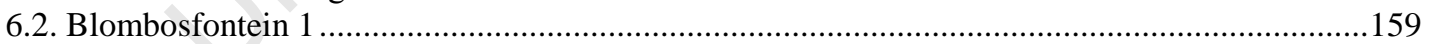

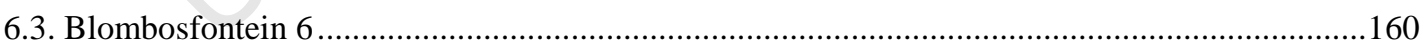

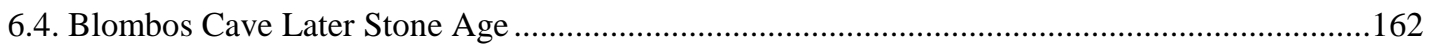

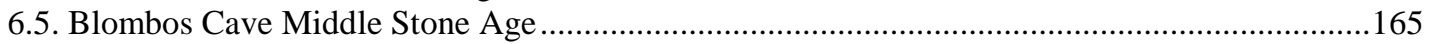

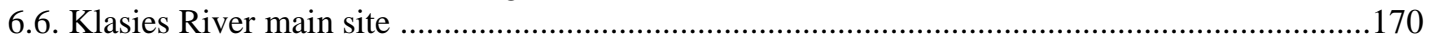

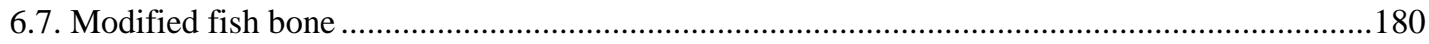

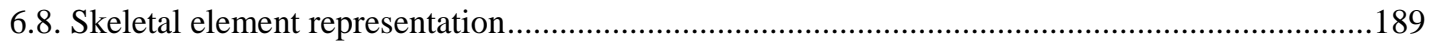

6.9. Skeletal element completeness based on percentage ..............................................................214

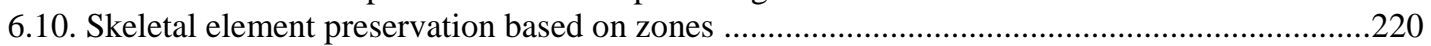

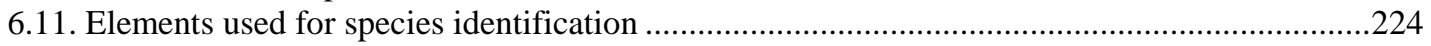

Chapter 7. Discussion ............................................................................................... 230

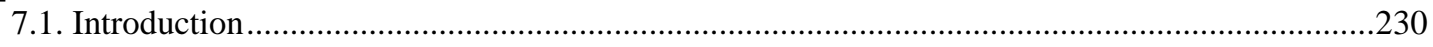

7.2. Agent of accumulation, method of capture, diachronic change and seasonality ........................231

7.3. The significance of fish in the diet of Stone Age people ...........................................................264

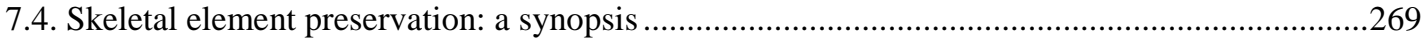

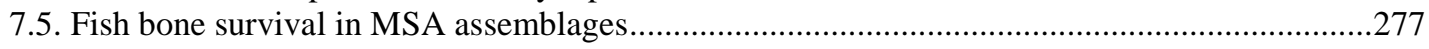




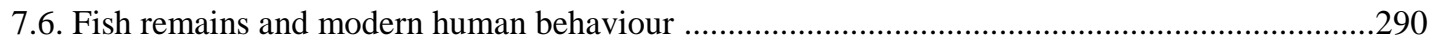

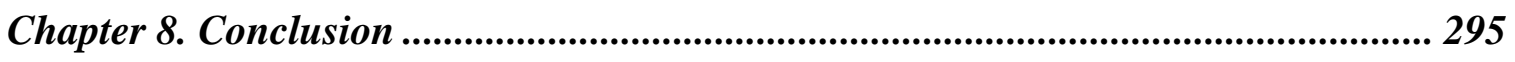

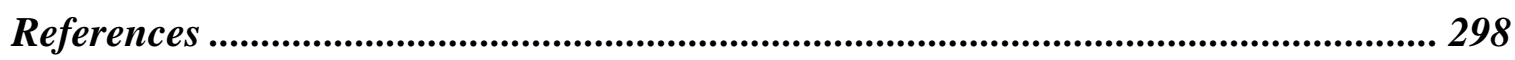

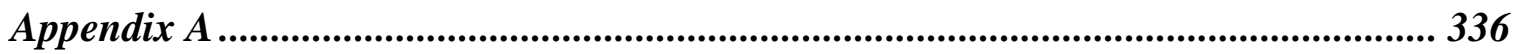




\section{List of Figures}

Figure 3.1. Distribution of archaeological sites in the western and eastern Cape that are referred to in the text........................................................................................... 39

Figure 3.2. Common near-shore fish species that occur in the southern Cape today....... 79 Figure 3.3. Location of sites that have bone hooks and illustration of a selection of hooks

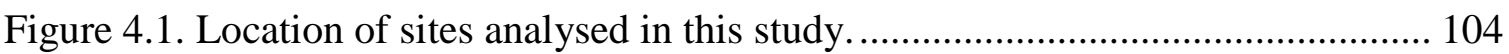

Figure 4.2. View of Hoffman's/Robberg Cave ........................................................... 105

Figure 4.3. Site plan of Hoffman's/Robberg Cave indicating the trench dug by Hoffman and the squares excavated by Sealy in 2007............................................................ 106

Figure 4.4. Stratigraphy of Hoffman's/Robberg Cave along the E/D section. ............... 107

Figure 4.5. View of Blombosfontein 1. Arrows indicate the deposit and the thick

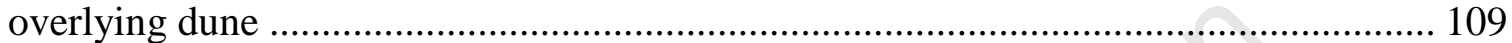

Figure 4.6. West section of Blombosfontein 1 and site layout ..................................... 110

Figure 4.7. Surface of Blombosfontein 6 showing shells and ash lenses ...................... 111

Figure 4.8. Blombosfontein 6 site layout indicating location of square excavated and

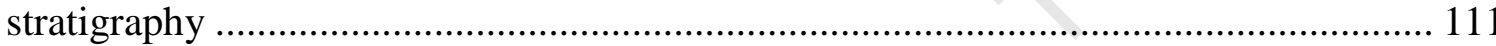

Figure 4.9. Location of Blombos Cave................................................................... 112

Figure 4.10. Site layout of Blombos Cave .................................................................. 113

Figure 4.11. Stratigraphy of the Blombos Cave Middle Stone Age layers along the D/E

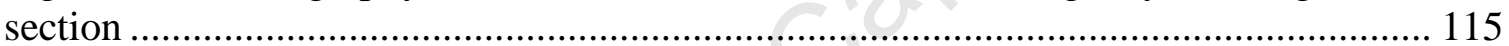

Figure 4.12. Blombos Cave Middle Stone Age artefacts from the M1/Still Bay phase. 116

Figure 4.13. Location of Klasies River main site. …………..................................... 118

Figure 4.14. Stratigraphy of Klasies River main site Cave 1A..................................... 119

Figure 4.15. Artefacts and hominid remains from Klasies River main site.................... 121

Figure 5.1. Location of the elements of the neurocranium........................................... 139

Figure 5.2. Location of elements from the lateral skull, pectoral girdle, pelvic girdle and

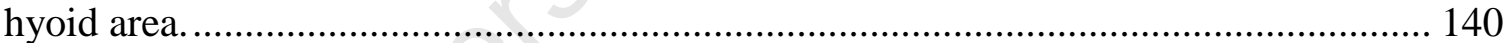

Figure 5.3. The modified York system data capture form used in this study ................. 142

Figure 5.4. Example of location of zones recorded. .................................................. 143

Figure 5.5. Examples of location of MNI feature ....................................................... 144

Figure 5.6. Location of measurements taken on palatine (a) and dentary (b) that differ from Morales and Rosenlund (1979)..................................................................... 146

Figure 6.1. MNI per $\mathrm{m}^{3}$ of the most common species at Hofffman's/Robberg Cave by

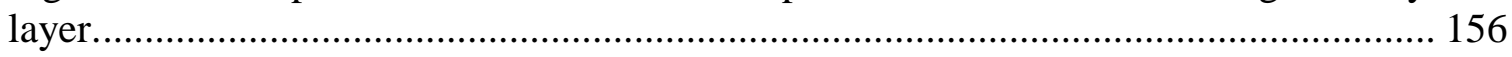

Figure 6.2. Reconstructed weights (in kg) based on MNI of Sarpa salpa and Seriola lalandi per layer at Hoffman's/Robberg Cave .............................................................. 156

Figure 6.3. Percentage per size class of the most common species found at Hoffman's/Robberg Cave based on NISP. 158

Figure 6.4. Comparison of percentages of size classes based on NISP of the most common species, all Sparidae combined and all species combined per Zostera and Shelly layers from Hoffman's/Robberg Cave... ..................................................................... 158 Figure 6.5. Density (MNI per $\mathrm{m}^{3}$ ) per layer of the most common species present in the Blombos Cave Later Stone Age assemblage ................................................................. 163 
Figure 6.6. Size category comparison between LSA material older and younger than 1600 $\mathrm{BP}$ at Blombos Cave based on NISP

Figure 6.7. Percentage of specimens per size class and phase of all species combined from the Blombos Cave Middle Stone Age assemblage.

Figure 6.8. Comparison of the percentage of specimens per size class and phase of all species combined from the interior (I) and exterior (E) of the Blombos Cave Middle Stone Age assemblage.

Figure 6.9. Percentage of specimens per size class of all species combined in the cultural (C) and sterile (S) units per member from the Klasies River main site Middle Stone Age assemblage..

Figure 6.10. Size categories of all Klasies River Middle Stone Age fish species combined per member based on NISP.

Figure 6.11. First vertebrae of Sparidae spp. showing damage on anterior side from Blombos Cave Later Stone Age layers

Figure 6.12. Percentage frequency of cut, possibly cut and unmodified Sparidae first vertebrae per size class from Blombos Cave Later Stone Age layers 186 Figure 6.13. An Epinephelus marginatus urohyal with a distinct cut mark on the anterior portion of the ventral side from Hoffman's/Robberg Cave layer TIM

Figure 6.14. Relative skeletal element frequency (\%MAU) of Dichistius capensis in the

Zostera and shelly layers at Hoffman's/Robberg Cave..

Figure 6.15. Relative skeletal element frequency (\%MAU) of Diplodus capensis at Blombos Cave LSA, Hoffman's/Robberg Cave Zostera and shelly layers and Blombosfontein 1

Figure 6.16. Relative skeletal element frequency (\%MAU) of Liza richardsonii at Blombos Cave MSA, Hoffman's/Robberg Cave Zostera and shelly layers, Klasies River main site cultural and sterile layers and Blombos Cave MSA interior 204 Figure 6.17. Relative skeletal element frequency (\%MAU) of Pomatomus saltatrix in the Zostera $(100 \% \mathrm{MAU}=2.5)$ and shelly $(100 \% \mathrm{MAU}=6)$ layers of Hoffman's/Robberg Cave 209

Figure 6.18. Location on skeleton of Pomatomus saltatrix elements found in the Zostera and shelly layers of Hoffman's/Robberg Cave. 209 Figure 6.19. Relative skeletal element frequency (\%MAU) of Seriola lalandi in the Zostera and shelly layers at Hoffman's/Robberg Cave.

Figure 6.20. Relative skeletal element frequency (\%MAU) of Sarpa salpa in the Zostera and shelly layers at Hoffman's/Robberg Cave 214

Figure 6.21. Distribution of element completeness scores of the most common Liza richardsonii elements from Blombos Cave Later Stone Age and Hoffman's Cave Zostera and shelly layers based on NISP..... 216

Figure 6.22. Distribution of element completeness scores of the most common Liza richardsonii elements from Klasies River main site sterile and cultural units and Blombos Cave Middle Stone Age based on NISP.. 218 Figure A-1. Illustration of the zones recorded per element and the 'MNI feature' of

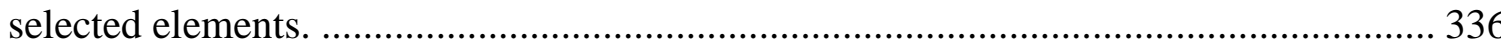
Figure A-2. Illustration of the premaxillae of species from various fish families. …... 337 Figure A-3. Illustration of the palatine bone of species from various fish families. ...... 338 Figure A-4. Illustration of the articular from species from various fish families. 339 
Figure A-5. Illustration of the dentary of species from various families.

Figure A-6. Illustration of the ceratohyal and epihyal bones from the hyoid region of fish from different families.

Figure A-7. Illustration of the lachrymal, jugal and and temporal bones of some Sparidae,

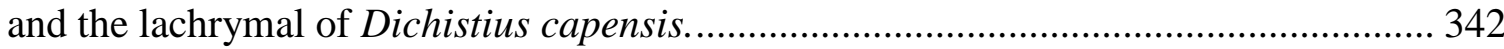

Figure A-8. Illustration of the parasphenoid bone of fish from different families. ........ 343

Figure A-9. Illustration of the supracleithrum and posttemporal bones of various fish

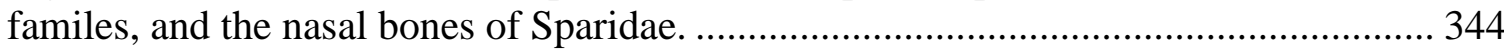

Figure A-10. Illustration of the pterygoid bone of various fish families. ..................... 345

Figure A-11. Illustration of the scapula, coracoid and symplectic bones of various fish

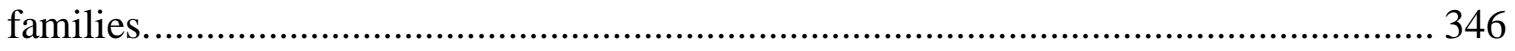

Figure A-12. Illustration of the urohyal bone from various fish families ..................... 347

Figure A-13. Illustration of the vomer of species from various fish families................. 348

Figure A-14. Illustration of the hymandibular bone of species from various families ... 349

Figure A-15. Illustration of the opercular of species from various fish families ........... 350

Figure A-16. Illustration of the preopercular of species from various fish families....... 351

Figure A-17. Illustration of the supraoccipital bone of species from various fish families

\section{List of Tables}

Table 3.1. Families and species identified from Klasies River main site by von den

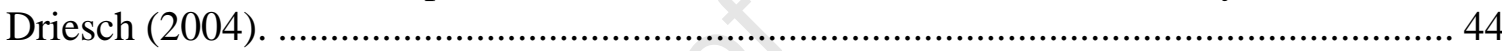

Table 3.2. Scores of the five most abundant fish families per layer and member from

Klasies River main site identified by von den Driesch (2004). .................................... 45

Table 3.3. Percentages per size class of bony fish per member from Klasies River main

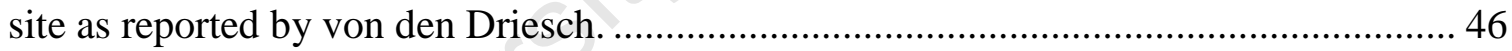

Table 3.4. MNI and NISP of fish identified from a sample of the Blombos Cave Later

Stone Age assemblage by van Niekerk (2005) ........................................................ 56

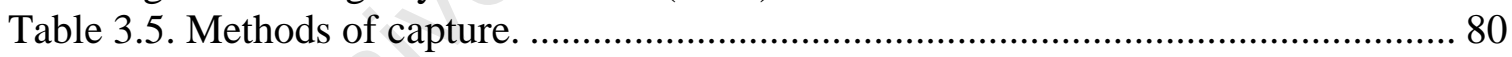

Table 3.6. Details of the fish remains contained in a sample of ten modern regurgitated cormorant pellets collected from the surface of Klasies River main site by von den

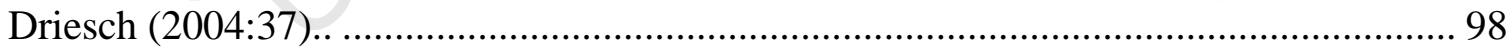

Table 4.1. Radiocarbon dates on charcoal for Hoffman's/Robberg Cave ...................... 107

Table 4.2. Excavated volumes $\left(\mathrm{m}^{3}\right)$ of squares D5c and D5d from Hoffman's/Robberg

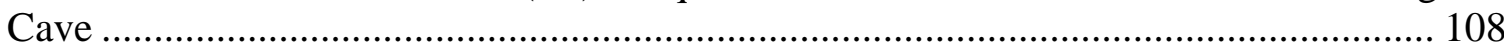

Table 4.3. Radiocarbon dates on charcoal for the Later Stone Age layers at Blombos

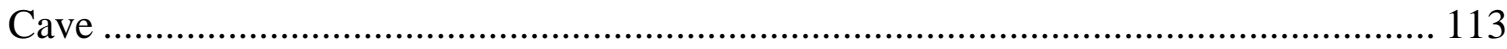

Table 4.4. Blombos Cave Middle Stone Age dates per phase based on optically

stimulated luminescence (OSL) and thermoluminescence (TL) dating techniques ....... 115

Table 4.5. Klasies River main site Middle Stone Age dates per phase based on amino acid racemization (AAR), Oxygen Isotope Stage (OIS), optically stimulated luminescence (OSL), thermoluminescence (TL), infra-red stimulated luminescence (IRSL), uranium series (US) and radiocarbon (C14) dating techniques .......................................... 120 
Table 4.6. Excavated volumes $\left(\mathrm{m}^{3}\right)$ of squares E5, F4, F5 and G5 from Blombos Cave

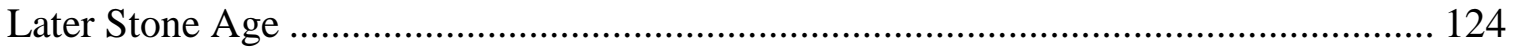

Table 5.1. Species in comparative collection............................................................. 138

Table 5.2. List of skeletal elements used for identification in this study....................... 150

Table 6.2. Percentage of NISP per size class and species for all layers combined at Hoffman's/Robberg Cave.......................................................................................... 157

Table 6.3. MNI and NISP of species at Blombosfontein 1........................................ 160

Table 6.4. Percentage of NISP per size class and species from Blombosfontein 1........ 160

Table 6.5. MNI and NISP of species at Blombosfontein 6......................................... 161

Table 6.6. Percentage of NISP per size class and species for all layers combined from

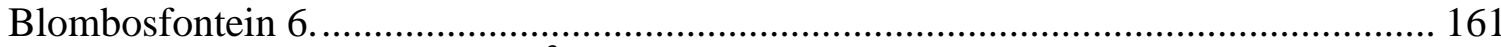

Table 6.7. MNI and NISP per $\mathrm{m}^{3}$ per layer of Blombos Cave Later Stone Age assemblage

Table 6.8. Percentage of NISP per size class and species for all layers combined from the Blombos Cave Later Stone Age assemblage. ............................................................ 165

Table 6.9. MNI and NISP per $10 \mathrm{~m}^{3}$ per phase in Blombos Cave Middle Stone Age.. .. 166 Table 6.10. Percentage of NISP per size class and phase from the Blombos Cave Middle

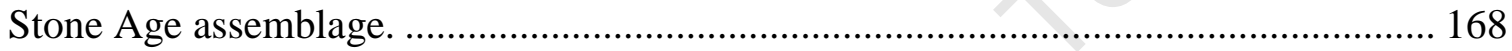

Table 6.11. Total NISP per member from Klasies River main site. ................................ 171 Table 6.12. Relative frequency (\%) of species in the cultural and sterile units per member from Klasies River main site based on NISP.

Table 6.13. Percentage of NISP per size class in the cultural (C) and sterile (S) units of the MSA III member of Klasies River main site. …………........................................ 175 Table 6.14. Percentage of NISP per size class in the cultural (C) and sterile (S) units of the HP member of Klasies River main site............................................................... 176 Table 6.15. Percentage of NISP per size class in the cultural (C) and sterile (S) units of the MSA II member of Klasies River main site......................................................... 177 Table 6.16. Percentage of NISP per size class in the cultural (C) and sterile (S) units of the MSA I member of Klasies River main site. ........................................................ 178 Table 6.17. Kolmogorov-Smirnov statistics from comparison of size classes between cultural and sterile units per member from Klasies River main site............................. 179 Table 6.18. Kolmogorov-Smirnov statistics from comparison of size classes of cultural and sterile units combined per member from Klasies River main site. 179 Table 6.19. Burnt (charred and calcined) specimens (NISP) from Hoffman's/Robberg Cave in the Zostera and shelly layers respectively.

Table 6.20. Number (NISP) of burnt bone and spines per Middle Stone Age phase at Blombos Cave.

Table 6.21. Number (NISP) of burnt specimens from Klasies River main site per cultural (C) and sterile (S) units per member, total per member and total of the whole assemblage

Table 6.22. Number of specimens (NISP) from Klasies River main site that showed evidence of gastric etching.

Table 6.23. Relative skeletal element frequency (\%MAU) of Argyrozona argyrozona 191 Table 6.24. Relative skeletal element frequency (\%MAU) of Argyrosomus inodorus/japonicus. 
Table 6.25. Relative skeletal element frequency (\%MAU) of Acanthistius sebastoides.

Table 6.26. Relative skeletal element frequency (\%MAU) of Cheilodactylus brachydactylus.

Table 6.27. Relative skeletal element frequency (\%MAU) of Chrysoblephus cristiceps

Table 6.28. Relative skeletal element frequency (\%MAU) of Chrysoblephus gibbiceps.

Table 6.29. Relative skeletal element frequency (\%MAU) of Chrysoblephus laticeps. 194 Table 6.30. Relative skeletal element frequency (\%MAU) of Cymatoceps nasutus..... 195 Table 6.31. Relative skeletal element frequency (\%MAU) of Dichistius capensis........ 196 Table 6.32. Relative skeletal element frequency (\%MAU) of Diplodus hottentotus..... 197 Table 6.33. Relative skeletal element frequency (\%MAU) of Diplodus capensis......... 198 Table 6.34. Relative skeletal element frequency (\%MAU) of Epinephelus marginatus.200 Table 6.35. Relative skeletal element frequency (\%MAU) of Gymnocrotaphus curvidens.

Table 6.36. Relative skeletal element frequency (\%MAU) of Galeichthys feliceps..... 202 Table 6.37. Relative skeletal element frequency (\%MAU) of Lithognathus lithognathus.

Table 6.38. Relative skeletal element frequency (\%MAU) of Liza richardsonii........... 203 Table 6.39. Relative skeletal element frequency (\%MAU) of Pachymetopon blochii... 206 Table 6.40. Relative skeletal element frequency (\%MAU) of Pomadasys olivaceum... 207 Table 6.41. Relative skeletal element frequency (\%MAU) of Petrus rupestris............. 207 Table 6.42. Relative skeletal element frequency (\%MAU) of Pomatomus saltatrix. .... 208 Table 6.43. Relative skeletal element frequency (\%MAU) of Rhabdosargus globiceps.

Table 6.44. Relative skeletal element frequency (\%MAU) of Spondyliosoma

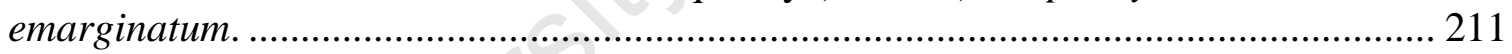

Table 6.45. Relative skeletal element frequency (\%MAU) of Seriola lalandi............... 212

Table 6.46. Relative skeletal element frequency (\%MAU) of Sarpa salpa. .................. 213

Table 6.47. Percentage per bone completeness score of the most common Liza richardsonii elements from Blombos Cave Later Stone Age and Hoffman's/Robberg Cave Zostera and shelly layers.

Table 6.48. Percentage per element completeness score of the most common Liza richardsonii elements from the Klasies River main site cultural and sterile units and Blombos Cave Middle Stone Age.

Table 6.49. Percentage per completeness score of all elements and species combined from the interior and exterior of the Blombos Cave Middle Stone Age excavations.... 218 Table 6.50. Percentage per completeness score of all elements and species for the cultural and sterile units respectively per member from Klasies River.

Table 6.51. Percentage per completeness score of all elements and species combined per assemblage.

Table 6.52. Comparison of the zones present of the most common elements of Liza richardsonii between all assemblages. 
Table 6.53. Comparison of zones present of the most common elements of Galeichthys feliceps between the cultural and sterile layers at Klasies River main site and Blombos Cave MSA 222

Table 6.54. Comparison of zones present of the most common elements of Sarpa salpa, Seriola lalandi and Dichistius capensis between the Zostera and shelly layers at Hoffman's/Robberg Cave.

Table 6.55. Frequency (\%) of elements per Sparidae from all sites combined (based on MAU) used for identification.

Table 6.55. Frequency (\%) of elements per non-Sparid species from all sites combined (based on MAU) used for identification.

Table 6.56. Elements that were not identifiable to species, and elements that consistently provided less than 5\% of species identifications for Sparidae and non-Sparidae respectively 229

Table 7.1. Percentage of NISP and MNI per size class and phase from the Blombos Cave Middle Stone Age and Later Stone Age assemblages. 248 Table 7.2. Percentage per completeness score of all elements and species combined of the Klasies River main site cultural and sterile units for specimens from fish smaller and larger than $30 \mathrm{~cm}$ respectively. 255 


\section{Chapter 1. Introduction}

The study of fish remains can contribute significantly to our understanding of how prehistoric societies utilised aquatic resources through space and time. Fish bones can contain a wealth of information on a number of issues ranging from changes in environmental conditions, people's adaptive flexibility and technological expertise, and the timing of human adaptations to coastal environments. Yet the study of archaeological fish remains was often neglected in favour of that of mammalian fauna, with the result that there was limited research into and evidence for the potentially considerable role fish played in the diet of early coastal inhabitants.

The ability to intensively exploit marine coastal resouces, including through fishing, is considered by some researchers to be a marker of modern human behaviour, which they argue only started after 50000 years ago, possibly as a result of a genetic mutation resulting in increased cognitive abilities (Avery et al. 2008; Halkett et al. 2003; Klein 1974, 1975, 1976, 1977a, b, 2008; Klein et al. 2004; Klein and Cruz-Uribe 1996, 2000a; Klein and Steele 2008). There is mounting evidence that behavioural modernity and marine resource exploitation in Africa extends further back in time, and the availability of these resources could have sustained populations during periods when interior regions were uninhabitable due to extreme climatic fluctuations. This adaptation to coastal environments possibly facilitated expansion of modern $H$. sapiens out of Africa, at an earlier time than formerly supposed (Marean et al. 2007; Walter et al. 2000). The significance of the role of fish as one of these aquatic resources has not yet been established.

The southern African coast is an ideal region for studying the exploitation of coastal and marine resources over a long period. It is rich in marine resources and has a very stable coastline, which has allowed the preservation of elevated coastal sites despite fluctuating sea levels, a situation which is found in few other parts of the world.

Marine fish remains are relatively common at many Later Stone Age (LSA) archaeological sites situated along the South African coast dating to the last ten thousand years. A few of these sites have tentative evidence of fishing gear but most do not contain any artefacts that can be directly associated with fishing. Bone 'fish' hooks are 
geographically limited to Later Stone Ages sites to the north and northeast of the foothills of the Drakensberg mountain range and are not associated with marine fish remains (van Niekerk 2002). Despite the lack of obvious fishing gear at many LSA sites researchers have not doubted that these people were actively engaged in fishing. This is partly due to the large numbers of fish remains at some archaeological sites.

In contrast, fish remains are rare in most Middle Stone Age (MSA) assemblages from South Africa. In the few instances where fish do occur in MSA contexts, their presence has mostly been attributed to fish-eating birds (Deacon 1989, 1995, 2001; Deacon and Geleijnse 1988) or alternately as a result of hominids collecting dead fish from the shore (Klein 2000; Klein and Cruz-Uribe 2000). It is generally thought that MSA people were not actively engaged in fishing. Some researchers interpret the absence or low numbers of fish in the MSA as an indication that people were not behaviourally modern and lacked the cognitive ability and technology to fully exploit coastal resources prior to the LSA (Avery et al. 2008; Halkett et al. 2003; Klein 1974, 1975, 1976, 1977a, b, 2000, 2008; Klein et al. 2004; Klein and Cruz-Uribe 1996, 2000; Klein and Steele 2008; Volman 1978, 1981). Others have argued that, rather than suffering from a cognitive deficiency, MSA people did not fish because they ranked fish lower than other easily available protein sources such as shellfish, and that the diminished return from the increased effort needed to catch fish as opposed to other available food resources, was not worth it (Deacon 1989; 1995, 2001; Henshilwood and Marean 2003, 2006).

There is increasing artefactual evidence from African MSA assemblages that the origins of modern human behaviour lie in the MSA, and not in the LSA, as previously thought, and these origins date at least as far back as 78k years ago (d'Errico et al. 2005; Henshilwood et al. 2009; Henshilwood and Marean 2003, 2006; Lombard and Phillipson 2010; Parkington et al. 2005; Rigaud et al. 2006; Texier et al. 2010; Wadley 2010; Wadley et al. 2009; Wurz 1999, 2000, 2008), and potentially as far back as 164k years ago (Brown et al. 2009).

Two South African MSA assemblages, Klasies River main site (KR) on the Tsitsikamma coast, and Blombos Cave (BBC) on the southern Cape coast, contain marine fish remains in fairly substantial numbers, and a third, Sibudu Cave (SBC), contains small quantities 
of marine and freshwater fish remains. Preliminary analysis of the BBC fish (Henshilwood et al. 2001), and analysis of the KR (von den Driesch 2004) and SBC (Plug 2006) fish remains have indicated that at least some of the fish were brought into these sites by humans, and in some instances MSA people were possibly engaged in active fishing. The possibility that MSA people were purposefully fishing is contrary to the model that places fishing as a modern human trait that was practised only in the LSA, after 50k years ago (Avery et al. 2008; Halkett et al. 2003; Klein 1974, 1975, 1976, 1977a, b, 2000, 2008; Klein et al. 2004; Klein and Cruz-Uribe 1996, 2000; Klein and Steele 2008; Volman 1978, 1981).

Fish remains can yield far more information than has traditionally been reported in analyses of South African fish remains. Previous analytical methods applied to South African archaeological fish assemblages were mostly limited to species identification and quantification, typically based on a few elements from the jaw apparatus that were considered to be robust and good species indicators (Poggenpoel 1987, 1996). Studies have shown that elements other than jaw bones can occur more frequently in assemblages (Poggenpoel 1984, 1996), but despite this evidence, most identification and quantification of marine fish species continued to be based on a limited set of elements (see Poggenpoel 1996, which contains the majority of reports on South African coastal fish assemblages). Some elements of species from the same family can be morphologically indistinguishable from one another, particularly species with similar ecology (Day 2002). This includes the jaw bones of some of the Sparidae which are most common in South African assemblages. Jaw elements alone are therefore not always the most suitable for identification and other, more suitable elements need to be identified for those species. No other studies have been published to demonstrate that jaw bones of the most common marine species in South Africa are consistently the best elements for identification or the best surviving elements.

Using a limited number of elements for identification and quantification is desirable in terms of the analyst's time limitations as assemblages can contain vast quantities of fish bone. A fish skeleton has a very large number of potentially identifiable bones: over sixty excluding vertebrae. Analysing so many bones per species is time-consuming and impractical. Some doubt has been expressed over whether the extra effort required to 
identify all elements is worth what may be a relatively small amount of additional information (Leach 1986; Wheeler and Jones 1989). Narrowing down the number of elements to those that are useful for differentiating between species and that are consistently preserved in archaeological sites can greatly reduce the amount of time spent on analysis. Using a restricted number of elements for identification of assemblages is practical, provided that it has been demonstrated that these elements are consistently the best surviving bones for each particular species (e.g. Barrett 1995; Leach 1986; Jones 1999). This has not yet been done for the large variety of South African fish species. Recording all elements that are identifiable to species can be very informative if the goal of the analyst is to produce more than a quantified species list. Skeletal element representation can be used to infer cultural practises such as processing and transport of fish, to differentiate between natural and cultural accumulations, and to determine the degree of attrition at an assemblage (Barrett 1997; Barrett et al. 1999; Butler 1993; Carvajal-Contreras et al. 2008; Jones 1984, 1986, 1999; Lubinski 1996; Nicholson 1992, 1993, 1996, 1998; Stewart 1989, 1991; van Neer and Pieters 1997; Zohar et al. 2001). The skeletal element composition of South African fish assemblages has seldom or never been reported on a species level, and element completeness has never been quantified. Cedric Poggenpoel, from the University of Cape Town, has done the vast majority of identification and interpretation of marine fish remains from southern African archaeological assemblages. He has subsequently retired and is no longer involved in fish analysis. There are currently no other marine fish specialists working on South African archaeological material.

In the light of all the potential information that can be extracted from fish remains, I felt that a more comprehensive and systematic method of analysis of South African fish assemblages was needed, which would allow comparisons between assemblages. This is particularly important for the KR and BBC assemblages, as these are the only South African MSA assemblages recovered to date that contain relatively substantial numbers of fish remains, and the rarity of fish in the MSA has frequently been used to infer cognitive deficiencies in MSA populations, despite increasing evidence from the artefactual record to the contrary. Taphonomic processes could have been instrumental in 
reducing the original MSA fish numbers, and a detailed approach was needed to determine to what degree these processes might have compromised the assemblages. Therefore, to get a better understanding of the taphonomic history of the MSA assemblages, fish remains from the two MSA sites of KR and BBC as well as four LSA sites were examined and a host of attributes were recorded for each specimen. The LSA assemblages are Hoffman's/Robberg Cave (H/RC), located on the Robberg Peninsula, the LSA fish from BBC, the open site of Blombosfontein 1 (BBF 1), and the shell midden Blombosfontein 6 (BBF 6). Both BBF 1 and BBF 6 are located close to BBC. These were chosen for analysis due to their close proximity to the MSA sites, and with the expectation that they contained similar species to the MSA assemblages, which would allow comparison between assemblages. Additionally, KR, BBF1 and 6 are open sites, whereas $\mathrm{BBC}$ and $\mathrm{H} / \mathrm{RC}$ are cave sites. There may be differences in element preservation between caves and open sites which can be detected through comparison of skeletal element representation and completeness. The KR fish assemblage has been re-analysed by separating the cultural and sterile units, and the BBC MSA assemblage has been enlarged since the preliminary report.

The key issues to be investigated in this thesis can be divided into two broad categories, although both are intertwined in terms of interpretation of the data.

One is to compare the species present in the different assemblages and to explore what this can tell us about how people acquired fish and whether this changed through time. An important aspect of the MSA assemblages studied, BBC and KR, is to establish the agent of accumulation of the fish. The first step will be to ascertain species composition, fish size, cultural and natural modification, skeletal element representation and element fragmentation. These patterns will be compared with those expected for non-human predators of fish. The fish remains from the open Klasies River site in particular have previously been attributed to fish eating birds that roost on the cliff above the deposit (Deacon 1995). Determining whether humans or other animals were responsible for the fish remains at these sites, and, if humans were the agents, how they acquired fish, can contribute to the debate about whether MSA hominids were capable of modern human behaviour. 
The second aspect investigated relates to the preservation of different skeletal elements in different contexts. This thesis will explore whether skeletal element preservation within each species is consistent at open and cave sites, as well as in assemblages of different ages. There is an implicit assumption that elements in the MSA assemblages have survived the taphonomic processes over many tens of millennia due to their greater robustness. Testing this assumption requires that the elements present in the MSA assemblages are also present in the LSA. If the same elements are consistently preserved in both MSA assemblages studied then these are likely to be very robust elements. This type of information can also provide an indication of the size of the original MSA fish assemblages. If only the most robust elements survived, the initial assemblages must have contained a much greater quantity of fish remains. The general condition of the elements from the different sites will be assessed in terms of completeness to further compare preservation between sites. In addition, the elements that allow identification to species will be identified to speed up future analyses.

The layout of the thesis is as follows:

Chapter 2 gives on overview of the taphonomic, actualistic and ethnoarchaeological work that has been done on archaeological fish remains in order to better understand the different processes that can affect fish remains. The significance of marine resources in the debate on the origins of modern human behaviour is assessed.

Chapter 3 gives background information on the history of archaeological fish analysis in South Africa and includes an overview of researchers who have worked with South African fish remains and their methods of analysis applied. It also reviews the current knowledge on fish remains from Middle and Later Stone Age sites in South Africa. In addition, details the ecology of the fish species that occur in the study area today are listed. The possible agents of accumulation of archaeological fish remains other than humans in the study area are considered.

Chapter 4 describes the ages and contexts of the assemblages that have been studied for this thesis. It includes notes on site-specific variations in analysis where applicable. Chapter 5 reviews the most common methods of quantification used in faunal analysis and how these measures are calculated, and covers the analysis methodology applied to 
the fish remains in this study.

Chapter 6 presents the results of the analysis in terms of species present, fish size, cultural modification and skeletal element representation and completeness per site.

Chapter 7 discusses and contextualises the results, and Chapter 8 summarises the findings of this study and makes some recommendations for future research. 


\section{Chapter 2. Fish remains and archaeology}

\subsection{Introduction}

The quantification and publishing of faunal remains in archaeological reports is a fairly recent development, and prior to the 1970s most animal remains received scant attention and the majority of reports included little more than species lists (Aldenderfer 1998; Grayson 1973; Landon 2005; Lyman 1994a; O'Connor 1996; Olsen 1971; Uerpmann 1973). In the early 1900s, while archaeologists were primarily concerned with chronology and typology, zoologists had begun to recognise the potential of faunal remains in terms of information on distribution, morphological changes and environmental reconstruction (Reitz and Wing 1999).

By the 1950s (with some earlier exceptions, see Reitz and Wing 1999) faunal analysis started to move beyond simple qualitative data, as archaeologists sought to extract more information from animal remains (Dart 1957; Uerpmann 1973; White 1953, 1956). By the 1970s a great many papers dealing with issues of quantification methodology and the information that can be derived from it were being published and the sub-disciplines of zooarchaeology or archaeozoology became a recognised field (O'Connor 1996; Reitz and Wing 1999).

\subsubsection{Zooarchaeology and archaeozoology}

Zooarchaeology and archaeozoology are two terms applied to the analysis of faunal remains from archaeological sites. The term zooarchaeology is typically used by American researchers and implies a cultural, rather than ecological or zoological, perspective of faunal remains (Reitz and Wing 1999, 2008). African and Eurasian researchers tend to use the term archaeozoology, which is thought to refer to faunal analysis with an emphasis on biology rather than anthropology (Reitz and Wing 1999, 2008). These terms are, however, not mutually exclusive, and not all archaeologists agree on the definitions and differentiation of the two. Alternative terms have also been suggested, particularly for sub-specialties within faunal analysis (Bartosiewicz 2001; Bobrowsky 1982; Olsen and Olsen 1981; Schramm 1982). Many African and Eurasian 'archaeozoologists' study faunal remains from an anthropological perspective so that in 
some instances the use of the term archaeozoology is based more on issues of nomenclature and tradition than a strictly zoological/ecological approach (Bartosiewicz 2001, 2008; Lyman 1982; Reitz and Wing 1999, 2008).

\subsection{Archaeological fish analysis and information from anthropogenic fish remains}

The analysis and reporting of archaeological fish remains has a long tradition in Europe and America and, as with other vertebrate fauna, publications on fish remains and methodology had increased significantly by the 1970s (Casteel 1976; Clason 1986; Colley 1990; Heinrich 1983; Lepiksaar and Heinrich 1977). Species lists on their own are still informative in situations where presence and absence of species could indicate environmental change, fishing technology employed or cultural choices, and exotic species could indicate possible trade (Colley 1990; Lyman 1986), but the information to be gleaned from fish bone data has moved well beyond simple species lists and has been applied to a wide range of research questions.

\subsubsection{Taphonomy, Experimental Archaeology and Ethnographic Studies}

Taphonomy is literally translated as the 'laws of burial' (Efremov 1940). Initially a discipline of palaeontology, it is now widely applied to archaeology. It entails the study of the processes that have affected living organisms from death until they form part of an archaeological assemblage or fossilizes. Excavation and subsequent analysis is also considered to form part of the taphonomic process (Gifford 1981; Lyman 1994a; Reitz and Wing 2008).

There are many processes that can affect what is ultimately excavated and analysed from a site, often starting from before the specimens are discarded and buried. These processes all contribute to a loss of information to the extent that the resulting assemblage (often a sample) is a small and potentially biased reflection of the original material deposited (Reitz and Wing 1999, 2008). Studying these processes and the signatures that they leave helps the archaeologist be aware of potential biases when quantifying and interpreting assemblages. Apart from identifying potential biases in an assemblage, taphonomic 
studies can also provide valuable information about cultural practices such as butchery and processing (Brain 1981; Bunn 1991; Lyman 1994a).

Taphonomy plays an important role in determining whether the agent of accumulation and modification of an assemblage or part thereof is cultural or natural. Prior to deposition the human activities that will subsequently affect the archaeological assemblage include butchery practices, processing methods, selective body part exploitation, cooking methods and digestion. These activities can leave some evidence in the form of cut marks, differential burning patterns, signs of heating, skeletal element representation and digestive etching on bones (Lyman 1994a). Non-cultural activities include animals contributing or removing material; modification of bones through scavenging, leaving gnaw marks or crushing; fat-rich specimens passing through the digestive system; burrowing animals disturbing the deposit or dying within the burrows; trampling (by humans or other animals); soil $\mathrm{pH}$; and site aspect and location (Brain 1981; Bunn 1991). Differential bone density affects the survival of elements (Butler and Chatters 1994; Lam and Pearson 2005). Excavation and sampling methodology and sieve sizes are further factors that can bias assemblages (Gifford 1981; Gobalet 2005; Vale and Gargett 2002; Zohar and Belmaker 2005). It is not always possible to distinguish between cultural and natural processes as both can leave similar traces (Andrews and Cook 1985; Behrensmeyer et al. 1986; Broughton et al. 2006; Butler 1993).

Studies of taphonomy rely heavily on experimental archaeology (Andrews 1995; Briggs 1995; Lyman 1994a; Steffen and Mackie 2005). Many researchers are engaged in actualistic or ethnographic studies of taphonomic processes in order to better understand and identify taphonomic agents, and interpret archaeological assemblages (Andrews 1995; Bartram and Marean 1999; Bunn et al. 1988; Capaldo 1997; Capaldo and Blumenschine 1994; Faith et al. 2007; Lam 1992; Lupo and O'Connell 2002; Lyman et al. 2003; Olsen and Shipman 1988; Pickering and Egeland 2006).

There are a number of actualistic and ethnographic studies that relate specifically to fish remains. Observations of skeletal element patterning after butchery and processing (from ethnographical or experimental work) offer valuable tools for interpreting the presence and absence of elements in archaeological assemblages. Methods of determining whether 
assemblages were accumulated by humans or other predators are of particular interest to this study. Examples of experimental archaeology relating to the processing and taphonomy of fish bones are listed below.

\subsubsection{Cultural versus natural assemblages}

Patterns of survival of fish elements from modern campsites have been compared to natural accumulations at Lake Turkana to aid in differentiating between cultural and natural assemblages (Gifford-Gonzalez et al. 1999; Stewart 1991; Stewart and GiffordGonzalez 1994). In addition to analyzing the fish remains from the modern campsites, observations of fish processing practices and interviews with the fishers provided additional information on bone alterations which could be visible in the archaeological record. Natural accumulations were found to differ from culturally derived assemblages in terms of skeletal element composition and taxonomic abundance. Cultural assemblages were found to be less diverse in terms of species present and fish size. The skeletal elements present in cultural assemblages differed from the natural assemblages as a result of processing and varied between species. The element composition of natural assemblages reflected the anatomical proportions of elements in the skeleton. In addition, some elements from the cultural assemblages displayed evidence of processing in the form of cut marks and burning.

Similar comparisons were made between natural and archaeological salmonid fish assemblages in Oregon, North America (Butler 1993). It was found that it is possible to distinguish between cultural and natural salmonid assemblages on the basis of skeletal element representation and completeness. Natural assemblages had more complete skeletons with an even representation of cranial and axial elements, whereas cultural collections showed uneven ratios of cranial and axial elements, and skeletons were less complete.

Zohar and Cooke (1997) observed patterns of element discard and survival of different sized fish from modern day salting and drying practices in Panama. They found that small and large fish were processed differently, resulting in characteristic skeletal element representation and damage to specific elements depending on the size of the specimen. The observed patterning was recognised in fish data from Atlit-Yama, a Holocene 
assemblage in Israel, suggesting that similar size-dependent processing of fish occurred at this site (Zohar et al. 2001).

\subsubsection{The effects of digestive processes on fish bone}

Evidence of digestive damage to fish bones and the skeletal elements present can indicate the agents of accumulation if the signals left by the respective agents are recognizable as such. The effects of the digestive processes on fish bone by mammals (including humans) (Butler 1996; Butler and Schroeder 1998; Jones 1984, 1986; Nicholson 1993b) and birds (Broughton et al. 2006; Erlandson et al. 2007; Stewart et al. 1999; von den Driesch 2004) have been studied to differentiate between cultural and biological agents of accumulation. Jones $(1984 ; 1986)$ recorded element survival and condition of a variety of fish species recovered after being fed to dogs, pigs, rats and humans. Nicholson (1993b) did a similar study investigating the effects of human digestive processes on fish elements, and whether these effects could be recognised in archaeological assemblages. Both studies found that only a small percentage of the originally ingested elements survived in the faeces of the different consumers. Many of the surviving elements were so damaged by the digestive processes that they could not be identified to species (Jones 1984, 1986; Nicholson 1993b).

Nicholson (1993b) found that head bones were particularly badly preserved and damaged after consumption by humans. The observed damage could not be related to the effects of chewing only, as elements of tiny specimens were completely absent. Thus element size might also affect survival. The most commonly preserved elements were eye lenses, which are not likely to survive in most archaeological assemblages as they do not calcify. Most vertebrae from this study were fragmented, with the centra separated due to damage to the struts connecting them. Several vertebrae showed dark brown or black staining, and most of these were badly preserved. The observed damage was not consistent, and some vertebrae displayed no staining or digestive damage at all. In addition, the study found that the extent of digestion on fish elements that have passed through the human gut varies depending on the efficiency of an individual's digestive system.

Butler's (1996) study of fish bones from a prehistoric human coprolite from California found that none of the elements recovered showed traces of digestive etching, and less 
than half were stained a darker colour. Elements from all areas of the skeleton were preserved, indicating that digestion in this instance did not discriminate between elements. The species present are Cyprinidae and Catostomidae, both Cypriniformes. Butler (1996) believes that this contrast with the results of Jones $(1984 ; 1986)$ and Nicholson's (1993b) studies suggests that the effects of digestion could be species specific, as the latter studies did not include Cypriniformes. She concludes that the absence of staining or digestive damage cannot be used as an indication that fish bones were not ingested by humans and therefore not of cultural origin.

Butler and Schroeder (1998) investigated the effects of digestive processes of humans and coyotes on tui chub (Cyprinidae) elements. They sampled modern human and coyote faeces as well as ancient human coprolites. As with Butler's (1996) previous study, they found that elements from the human coprolites were fairly well preserved with most elements represented, but in contrast to the latter study, many, but not all, elements showed evidence of digestion in the form of erosional pitting and deformation (Butler and Schroeder 1998). The condition and skeletal element representation of bones recovered from the modern coyote scats were the same as those from the human coprolites. Thus it is not possible to distinguish between humans or coyotes as agents of fish bone accumulation based on acid etching and element representation. Considerable bone loss was recorded in the modern human sample, but elements from all areas of the skeleton were present. Several skeletal elements that were found in the coyote scats and human coprolites were absent from the modern human sample. This most likely relates to the small modern human sample (3 fish were ingested). The original number ingested by the coyotes and ancient humans is unknown and it was therefore not possible to quantify element loss from these samples. A proffered explanation for the discrepancy between this study and those of Jones $(1984 ; 1986)$ and Nicholson (1993b) is that the species used in this study (Cyprinidae) has a higher bone density than those used in the latter. In addition, processing techniques prior to consumption (cooking, chewing etc.) could diversely affect the end product (Butler and Schroeder 1998).

Thus the effects of mammalian digestive processes on fish bone are not consistent and are contingent upon a number of factors. It seems conceivable that elements heavily eroded by digestive processes are less likely to survive in the archaeological record as they 
would be more fragile due to reduced structural integrity as well as more prone to disintegration due to an increased surface area exposed to microbial activities once deposited.

Broughton et al.'s (2006) study on differentiating between fish that had been through human digestive systems or fish regurgitated by barn owls was inconclusive. The study was based on skeletal element representation and digestive damage. The fish remains from modern owl pellets from a barn in Nevada and human coprolites from Homestead Cave, Utah, North America, showed the same (minimal) amount of digestive damage, whereas those from modern human faeces showed intensive damage, similar to that seen in coyote scats.

The composition and condition of fish bones from regurgitated bird pellets have been studied to determine whether fish eating birds are responsible for remains found at sites. Stewart et al. (1999) analysed the remains from African fish eagle pellets at Lake Turkana and found the assemblage to be very characteristic in terms of size of specimens recovered, element survivorship and completeness, prey choice and habitat and concluded that assemblages created by these birds should be recognizable as such. Von den Driesch (2004) analysed a sample of ten modern regurgitated pellets produced by cormorants roosting on the cliff above Klasies River main site. The fish from these pellets were analysed to species, skeletal element composition, condition and size. All of the bones from the pellets showed signs of digestion and most were heavily digested, which made her question whether such damaged bones would survive in the archaeological record. The results of Stewart et al. (1999) and von den Driesch's (2004) studies are pertinent to this thesis and are discussed further in Chapter 3.

Erlandson et al. (2007) analysed the faunal remains from a historic bald eagle (Haliaeetus leucocephalus) nest on the southwestern coast of California to determine whether one can differentiate between natural and cultural accumulations of fauna at archaeological sites where this bird species occurs. The fish species present, element composition and lack of evidence of digestive processes were virtually identical to remains from archaeological sites in the vicinity. Thus these criteria are not sufficient to distinguish between fish remains deposited by bald eagles and humans where both are possible contributors. Other 
factors such as evidence for burning, fish size and the presence or absence of fishing gear need to be taken into account to determine the agent of accumulation (Rick et al. 2001).

The results from these taphonomic studies of fish bone illustrate the importance of taphonomy in understanding the formation of assemblages, but also emphasize the need for actualistic studies of the species in question. Data obtained for one species cannot be applied to other species unless actualistic studies have shown that they are inclined to the same degrees of attrition as a result of digestion or other taphonomic processes.

\subsubsection{Bone density}

Bone density of salmon skull elements have been measured to determine whether skeletal element patterning observed in archaeological sites could be density-mediated (Butler and Chatters 1994). Some correlation between element survival and density were found but the study also showed that cultural practices can influence element representation and need to be considered when assessing the cause of selective element representation. Nicholson (1992a) assessed the relationship of the bone density of cod elements with these elements' survival in archaeological sites. She found that elements with higher densities did not always survive better than those with lower densities, and that the relationship between density and skeletal abundance was not predictable. Several elements from the archaeological samples with high bone densities such as the scapula, hypohyal and basipterygium were either absent or only present at very low frequencies, whereas those with relatively low densities such as the hyomandibular and parasphenoid occurred at higher frequencies. Bone shape appeared to be a more determining factor for element survival.

\subsubsection{Burning, heating, soil pH and burial}

The effects of burning on Sebastes (rockfish) element identifiability and size as well as otoliths were studied by Steffen and Mackie (2005). The authors examined an early Holocene fish assemblage from Haida Gwaii on Richardson Island, British Columbia. All fish remains in this assemblage were calcined, and initial analysis indicated that most species were relatively small in size. This smaller size contrasted with a nearby contemporaneous site, where specimens were larger, which prompted the authors to 
investigate the effect of heating on element size. Experimental burning of selected Sebastes elements at laboratory controlled temperatures of $900^{\circ} \mathrm{C}$ for 35 minutes resulted in an average of $9 \%$ shrinkage in size of elements. All elements apart from otoliths showed some shrinkage, and vertebrae reduced the most in size, by $10.9 \%$. Recalculation of the Haida Gwaii fish size taking shrinkage into account resulted in an increase of 20$31 \%$ in live weight estimation. Thus reconstructing live weight from calcined bones can underrepresent size if shrinkage is not taken into account. Otoliths were found to increase in size directly after burning, but subsequently, and in a matter of days, degraded to such a degree that they turned into ash. A lack of otoliths from burnt assemblages could therefore be due to their disintegration after excessive heating.

Nicholson (1993a) did experimental burning of modern fish vertebrae to detect whether changes in colour and surface morphology of heated fish bone could be used as indicators of the amount of heat archaeological bones were exposed to. She found that colour change was variable between species and is only useful for crude estimates of temperatures achieved. Post-depositional staining can discolour bones and mimic the effect of burning at low temperatures. Examination of surface morphology revealed that the effects of heating are also variable, and temperature determinations are unlikely to be more precise than to the nearest $100^{\circ} \mathrm{C}$. The surface morphology of bones heated to above $700^{\circ} \mathrm{C}$ was more distinct. Nicholson (1993a) questions the value of these determinations, as both colour and surface morphology are indications of the temperatures that the bone itself attained, and not of the heat source. Elements from a fish that lies partly on the periphery and partly in the centre of a fire will exhibit a wide range of colours. This variability makes it difficult to determine what kind of fire caused the burning (Nicholson 1993a).

Jousse et al. (2008) investigated whether there was any relationship between the location of bones in the skeleton (external and internal) and the degree of burning that could be discerned on these bones from a Late Holocene assemblage in Mali. They found no correlation between burnt elements and their degree of exposure to fire, with many of the internal bones such as vertebrae displaying the same amount of burning as those that would have been directly exposed to heat. This absence of patterning was interpreted to 
indicate post-depositional burning from a subsequent fire built on top of the bare bones, or to the bones having been thrown into the fire.

Richter (1986) developed a method for detecting whether fish bone has been heated through electron microscope examination of morphological changes in collagen before and after being subjected to either dry heating or boiling. It was found that increased temperatures resulted in increased changes in collagen morphology, which can start at temperatures as low as $60^{\circ} \mathrm{C}$. This method can be applied to archaeological fish bones to indicate heating which would aid in distinguishing between culturally and naturally accumulated assemblages.

Lubinski (1996) investigated the effects of heating and soil $\mathrm{pH}$ on Lake Whitefish (Salmonidae) bone survival. The results showed that cranial elements of this species are more susceptible to degradation in both acidic and alkaline soils than vertebrae, but degradation was most pronounced in acidic soils. Similar results were recorded for heated bone, both boiled and burned, and increased heating resulted in increased element destruction, which was also more evident under acidic conditions.

To determine the effect of soil type ( $\mathrm{pH}$ and drainage) and different heating methods on skeletal element preservation, Nicholson (1996) either boiled or baked several fish species (as well as a selection of birds and mammals) in various states (whole, gutted or filleted) prior to burial at four locations with different soil profiles. Fresh specimens of the same species were also buried. These were subsequently excavated after seven years. Considerable variation was found in element preservation between sites with similar soil $\mathrm{pH}$ and drainage. Less than $3 \%$ of the original skeletons interred were preserved at one location with acidic soil, and only otoliths and vertebrae survived. Bones were considerably better preserved at another acidic location with comparable drainage. The degree of element preservation of boiled and baked specimens varied between species. At the site with neutral soil, element preservation of uncooked fish also varied between species. Elements of species that had been baked were as well preserved as their uncooked counterparts. Bones were less well preserved in alkaline than in neutral soil, but skeletons were more complete than those from the acidic sites. The baked specimens from the alkaline soil were in better condition than those that were buried fresh. Elements 
from the boiled specimen were badly preserved in all contexts. In all soil types scales were absent although otoliths, which are calcium carbonate concretions, were preserved. There was no difference between the element preservation of small and large fish at most sites apart from at one acid context where herring bones were absent and large fish bones present in very low numbers.

No predictable relationship between bone survival and soil $\mathrm{pH}$ could be established, and the main factor influencing initial bone degradation is thought to be microbiological. Elements from fish buried whole were better preserved than those from defleshed uncooked fish, presumably due to the skin and scales affording some measure of protection from microbial agents. The location where the lowest numbers of elements were recovered was also where the carcasses were buried at the least depth, which could have contributed to increased microbial activity. The boiled specimens showed the highest rate of decomposition, and elements from fish deposited raw and intact were best preserved. This has implications for studies of archaeological fish assemblages as most fish were presumably cooked before consumption. The method of cooking applied affects element survival, and boiled fish elements are less likely to survive. If different species were subjected to different cooking methods, those that were boiled will be underrepresented.

Nicholson (1998) investigated the survival of fish (and other animals') bone that were buried in a compost heap for seven years in neutral to slightly alkaline soil. The results were surprising in that most of the elements were recovered, in very good condition, and mostly intact. Only the boiled fish lacked several cranial elements, showed extensive fragmentation, and was represented mostly by vertebrae which were missing their spines and processes. Fish scales were not found, but some might have been missed in the recovery process. No channeling or pitting was seen on any of the fish bone, and most post-depositional damage was on the articular facets of vertebral centra and along the edges of flat bones, where bits of bone were missing. Nicholson argues that the good state of preservation is due to processes of humification retarding decomposition within the compost heap and that, once the inclusion of organic matter stops, the natural degradation of the bone will resume, unless the bone has become mineralized. Thus although compost heaps can be considered analogous to archaeological living sites during occupation, with 
large amounts of organic matter deposited over relatively short periods of time, once the influx of organic matter ceases, bone will start and continue to degrade with time, despite the organic-rich surroundings.

\subsubsection{Season of capture}

Season of capture and scheduling of resources has been inferred from growth rings on various elements including otoliths, pectoral and dorsal spines, scales and vertebrae (Brewer 1987; Casteel 1972, 1974, 1976; Desse and Desse-Berset 1992; Higham and Horn 2000; van Neer et al. 1999; Walters 1992), although the method's success varies depending on the species involved and its growth rate (Andrews et al. 2003; van Neer 1993; van Neer et al. 2004a).

Andrews et al. (2003) have demonstrated some of the problems with inferring seasonality from otolith margin analysis. These include difficulty in reading growth rings as there is considerable variability in their formation, even within species, and the inherent subjectivity of the method if suitable comparative material is not available. Growth rings from archaeological otoliths can be particularly troublesome to read, as taphonomic processes tend to make the rings even less clear or totally obscured. They have shown that inferring season of capture from otoliths is most accurate when applied in conjunction with a large modern comparative collection of the same species of different ages from the same location, and with known date of capture.

Van Neer et al. (2004a) reported findings similar to those of Andrews et al. (2003) based on the incremental growth rings of otoliths from modern Pleuronectes platessa (plaice) and Melanogrammus aeglefinus (haddock) from the North Sea. They first analysed the margin types in terms of timing and appearance (opaque or hyaline) of a very large sample of modern otoliths from these species (4595 P. platessa and 1297 M. aeglefinus). They also measured the margin width increases per month on a subsample (387) of $P$. platessa, a method that had seldom been used before. They then compared the modern otoliths with those from a variety of contexts at Raversijde, a $15^{\text {th }}$ century AD fishing village on the Flemish coast. They found that, contrary to previous assumptions, the margins of modern $P$. platessa otoliths were not consistently either opaque or hyaline during specific seasons. Geographical location, sea temperature, fish age and growth rate 
are all variables that affect the timing and formation of the growth rings on otoliths, which can differ annually. Thus otolith edge type alone cannot be used to infer seasonality, particularly with small samples or samples from different contexts, as both types have been shown to occur throughout the year. Only if assemblages have close to $100 \%$ frequency of either hyaline or opaque margins can seasonality be assumed. The edge types of $M$. aeglefinus were even harder to interpret as the edges of some specimens displayed hyaline and opaque zones simultaneously. Marginal increment formation is affected by the same variables as other growth increments. The authors compared the size distribution of the marginal increments to those of full increments of otoliths formed during the same year of life from the same archaeological assemblage containing fish of different ages. The sizes of marginal rings were plotted against full rings from the same year of growth of fish of different ages. This method was shown to be most useful for identifying the beginning of the growth season when increment growth is rapid, but increment size was consistently smaller than that of full growth rings. Using this method, they determined that $P$. platessa were consistently caught during early spring at Raversijde. Oxygen isotope analysis of a random sample of otoliths from the site confirmed this finding. Inferring season of capture from the marginal increments of $M$. aeglefinus otoliths was less straightforward as there was considerable variation in the increment distribution. This suggests that this species was caught during more than one season, but determining the exact time of year is problematic. Once the initial growth spurt has passed, it is difficult to determine whether the growth margin reflects the end of the growing season or the phase when growth has stopped, which can encompass a period of half a year.

Higham and Horn (2000) analysed growth rings on prehistoric Pseudophycis bachus (red cod) otoliths from Shag River Mouth, New Zealand, in conjunction with a large number (>500) of modern $P$. bachus otoliths to reconstruct the season of capture. Otoliths of $P$. bachus have two discernible and seasonally specific growth rings that form annually. Growth rings in archaeological specimens were found to be more clearly distinguishable than the modern comparative specimens. Their results validated previous seasonal estimations based on oxygen isotope analysis of shellfish, which indicated winter 
occupation in the earlier levels, which also matches the current season of abundance of $P$. bachus in the area.

Analysis of growth increments in pectoral spines of the catfish Synodontis schall enables reconstruction of the animals' ages and growth rates, and has been used to investigate changes in levels of human predation and environmental conditions (Luff and Bailey 2000). Fish continue to grow throughout their life spans, and larger fish are usually assumed to be older individuals. Decreased fish size in assemblages is often interpreted as an indication of increased predation. Factors other than age also influence fish growth rates, in particular population densities and predation pressure. Dense populations of fish have increased competition for food, which results in slower growth rates, whereas lower numbers of fish have access to larger amounts of food and have increased growth rates. One possible cause of reduced fish density is increased predation by humans. Even though the average size of fish in an exploited population might decrease, due to older individuals not being replaced, younger fish from exploited populations can be bigger in size than their older counterparts in unexploited populations. The authors predicted that a size decrease due to increased predation will be visible in the archaeological record as fish with increased growth rates, younger age, and their age and size distribution will be more positively skewed. Conversely, if a size decrease was due to low predation, they expected to see reduced growth rates and increased age, and the size and age distribution would be broader and less positively skewed. If environmental deterioration was responsible for size reductions, they expected growth rates to decrease, and age distribution could be negatively skewed under severe conditions, but the overall age composition would not necessarily change.

Luff and Bailey (2000) used growth rings on pectoral spines to reconstruct the age of 167 modern S. schall specimens to compare with those of 115 archaeological specimens from Egyptian sites dating to the Pharaonic $\left(14^{\text {th }} \mathrm{C}\right.$. BC) and Late Roman $\left(5-6^{\text {th }} \mathrm{C}\right.$. AD) period. The modern sample showed growth rings can be clearly read from pectoral spines and that a certain amount of bone resorption tends to occur during the fourth year, which can be recognised and corrected for before age calculation. The growth rings were also clearly visible on the archaeological specimens. The combined size and age data of the modern and archaeological assemblages were compared to determine whether there were 
changes in predation pressure or environment. The modern sample indicated the highest predation pressure based on the criteria listed above. Fish from the Pharaonic period were mostly from the smaller size classes but larger than those from the Roman period, and sizes were strongly positively skewed, suggesting relatively intensive fishing during this period. The size reconstruction of the sample from the Roman period suggested that fish were less intensively exploited, but the age data was not in agreement with this hypothesis, as it indicated younger average ages than expected. The authors interpreted this to indicate a combination of extensive fishing and climatic deterioration during the Roman period, and that the processes were possibly linked. Climatic deterioration could have induced crop failure, which would have necessitated other means of subsistence, which could account for an increased reliance on fish during that period.

\subsubsection{Reconstructing fish size}

Reconstructed fish size is used to determine the contribution of fish to people's diet, what technology was employed to catch the fish and the environmental conditions at the time of capture (Colley 1990). Growth ring analysis of vertebrae has been used to reconstruct fish size and by extension palaeoclimates, where wider growth rings indicate larger fish that are indicative of warmer conditions (Brewer 1991; Casteel 1976). Fish size can be reconstructed using regression formulae calculated from measurements of modern comparative samples (Butler 1993, 1996; Capriles et al. 2008; Desse 1984; Desse and Desse-Berset 1996; Höglund 1972; Leach and Boocock 1995; Leach et al. 1997; Leach and Davidson 2001; Olson et al. 2008; Orchard 2003; Plug 2008; Zohar et al. 1997). Published regression analysis data and formulae for specific species can be useful resources for others working with the same species. However, as these formulae are often published by non-archaeologists who are working with fresh and intact elements, it is almost always necessary for people working with archaeological material to determine their own points of measurement and regression formulae due to the fragmented nature of most fish bone assemblages (Leach and Boocock 1995; Leach et al. 1997; Wheeler and Jones 1989; Zohar et al. 1997). The publication by Morales and Rosenlund (1979) which intended to standardize measurements of archaeological fish remains has been followed widely, but it is limited to a set number of elements and sometimes requires that the 
original measurement points are tweaked to compensate for specific species and fragmentation (Colley 1990; Zohar et al. 1997). Establishing regression formulae is prohibitive due to the cost and time of acquiring and processing sufficient modern samples and are therefore rarely carried out (Colley 1990). Caution should also be applied if using regression equations for the same species from different geographical locations as it has been shown that these can differ significantly (Granadeiro and Silva 2000).

\subsubsection{Species identification}

Elements of closely related fish species can look very similar morphologically, sometimes making it impossible to differentiate between them based on the elements more commonly used for identification. Descriptive and illustrative publications on the osteology and morphological differences between fish species are useful tools, particularly if an adequate comparative collection is not available. Compiling a comprehensive comparative fish collection requires considerable investment in terms of time, cost, and space. These factors can be prohibitive to researchers who only occasionally work with fish remains. In such instances the use of illustrations alone can be sufficient to identify fish bones to family if species specific identifications are not required. In addition, they can be used when dealing with assemblages containing species that are all very distinct morphologically. Illustrations are useful as references to use in the field for initial identification. They cannot, however, completely replace physical comparative specimens, as many of the subtle differences that allow identification are difficult to capture in two-dimensional formats.

Most publications on fish remains do not explain which elements or characteristics of elements species identifications were based on, although some do include useful descriptions and illustrations to help differentiate between morphologically similar species. A limited number of publications are dedicated solely to illustrating fish bones to aid identification, although not all are necessarily intended for use by archaeologists.

Lepiksaar (1983) illustrated many elements of northern European marine and freshwater fish in a privately distributed document named "Osteologica I Pisces". This document also provided his proposal for a standard nomenclature of fish elements and their 
particular features in Latin. Lepiksaar and Heinrich (1977) included photographs of certain fish elements in their publication on the fish remains from the Viking Age town Haithabu. Olsen (1968) illustrated a generalized fish skeleton, along with those of amphibians and reptiles, to enable archaeologists to distinguish between these classes during primary analysis of bone assemblages.

Van Neer's (1995) report on the fish remains from three Iron Age settlement mounds in Mali, Jenne-Jeno, Hambarketolo and Kaniana includes illustrations of some of the diagnostic elements that he used for identification of species. The illustrations are of the actual archaeological specimens that were found, and show what certain elements look like when fragmented. These can be useful for archaeologists who are not familiar with fish remains but work with similar assemblages.

Robinson et al. (2009) published images of Salmo salar (Atlantic salmon) and Salvelinus spp. (trout) vertebrae to illustrate the morphological characteristics that allow differentiation between trout and salmon. Based on initial analysis of archaeological assemblages in Northeast North America, S. salar appeared to be almost non-existent, and it was hypothesized that this species only colonized the area once the climate became more favourable during the Little Ice Age. Most identifications of possible archaeological salmon bones were lumped under a general "large salmonid" category, which includes both salmon and trout, as it was thought that it was not possible to differentiate between the species unless the few elements that do allow identification were present. Subsequently, more salmon have been identified at a number of sites in the area, and identifications were usually based on large intact vertebrae. Robinson et al. (2009) determined that the key differences between $S$. salar and trout were in the bone structure of vertebrae, particularly the vertebral rim, which they believe is sufficiently dissimilar to allow identification of even incomplete fragments. The differences were less clear on young specimens, as young salmon have less distinctive vertebral rims, and the cell pattern along the centrum of young trout closely resemble that of salmon. Using these criteria, they re-analysed bone previously identified as "large salmonid" from seven archaeological sites in the area, and concluded that all were in fact $S$. salar. These results indicate that salmon occurred in Northeast North America considerably earlier than was previously believed, as early as $7000-6500 \mathrm{BP}$. 
Gayet and van Neer (1990) published detailed illustrations of the diagnostic characteristics of the dorsal and pectoral spines of a number of African siluroids (catfish). Many species of catfish occur across Africa, and their spines are common in archaeological and palaeontological sites. These illustrations are very clear and useful for distinguishing between species and include descriptions of several characteristics of the spines per species.

Radu (2005) published a volume of illustrations of the elements of the 26 taxa of Central European freshwater fish which most commonly occur in archaeological sites in the area. The publication is specifically intended for archaeologists working with assemblages containing the same species.

Cannon (1987) published an illustrated manual for the identification of all fish elements that occur in the skeletons of four northern hemisphere fish families. She expressed concern about the fact that, while many archaeologists have some familiarity with mammal bone, few are able to recognise or identify disarticulated or fragmented fish bones. Failing to identify all fish elements while mammal elements are readily identified would result in fish remains being underrepresented in faunal reports. The manual is intended to help archaeologists familiarize themselves with all elements that occur in the fish skeleton. All elements, including those not often used for identification, are illustrated for one species per family. The families are Gadidae, Salmonidae, Scorpionidae and Pleuronectidae. The manual is a useful guide to understanding fish skeletal morphology and for identifying less familiar elements.

Publications on fish osteology that are intended for a wider audience, but which are useful for identifying specific elements, include the 'Dictionary of Evolutionary Fish Osteology' by Rojo (1991), and 'Fish Skulls: a study of the evolution of natural mechanisms' by Gregory (1933). The former contains descriptions of each element in terms of its location and function in the skeleton, and illustrations of several elements of a number of northern hemisphere fish families, including Cyprinidae, Gadidae and Lotidae. Gregory's (1933) volume includes illustrations of articulated fish skulls of a large number of fish families, including some that occur in southern African waters. The illustrations are useful indicators of the general differences in morphology between families. 
A relatively new development entails the publishing on the World Wide Web of resources to aid identification of fish remains. The North Atlantic Biocultural Organization Zooarchaeology Working Group (NABO) has an online interactive identification manual (NABONE) displaying images of the elements of a number of North Atlantic Gadid species. Several of the elements can be viewed from the lateral, mesial and ventral aspects. The Hawaii Biological Survey (HBS) from the Bishop Museum, Hawaii, has an online interactive key system with images of elements, including otoliths and scales, that allow identification of the more common Hawaiian fish species. It includes the names of the various features and processes that each element possesses.

\subsubsection{Culturally modified fish bone}

Intentionally modified fish bones are rare, but there are a few reported instances. Jousse et al. (2008) recovered two polished catfish (Arius gigas) spines from Kobadi, a Late Holocene site in the Niger Inland Delta in Mali. They are thought to have been used as tools, but are fragmented and their exact function is unknown.

Polished catfish spine spatulates were recovered from the Terminal Late Archaic (1.6 2.4 kya) layers of Arenosa Shelter in southwestern Texas. These are informal bone tools described as specimens "that have projecting narrow working areas with an oval cross section" (Jurgens 2005:202; 2008). Use-wear analysis indicated that most of the spine spatulates were used on silica-rich plant material, possibly as weaving tools. Some were used in hide working, including one that showed traces of having been used to pierce dry hide.

At Niah Cave, Borneo, stingray spine points that have been sharpened at both ends were found in layers dating to the early Holocene (Barton et al. 2009). Some of the spines retained traces of hafting residue and plant fibers which is consistent with historic examples of composite armatures using stingray barbs. The points are thought to have formed parts of barbed projectile spears or arrows. Based on the faunal remains associated with these points, the authors believe that they were used for hunting terrestrial fauna, but do not exclude the possibility that they could have been used for fishing. 
Drilled triggerfish (Balistidae) teeth and a drilled Naso (unicorn fish) caudal tang, dating to the $7^{\text {th }}-11^{\text {th }}$ century AD, were found at Maka Bay, a site on Rotuma Island in Polynesia (Allen et al. 2001). It is thought that these were drilled for use as ornaments. Although drilled fish teeth are common in Polynesia, this is the first report of drilled Balistid teeth. The drilled caudal tang is also the first reported instance of this kind of modification.

In South Africa, a fish spine from a shark species that appears to have been used as an awl was found at Gordon's Bay Midden in a layer dating to $3220 \pm 55$ BP. (van Noten 1974). Rudner and Rudner (1973:96) reported that "an artificially pointed fish bone with signs of red paint on it" had been excavated at a cave on the Robberg Peninsula which might be Hoffman's/Robberg Cave. There is some confusion about the exact location of the site due to the excavators' poor records. These are currently the only culturally modified fish bones reported from prehistoric contexts in South Africa.

Deliberate breaking of sharp or poisonous fish spines to avoid risk of injury has been recorded from archaeological assemblages. Spines of several species including catfish, weeverfishes and stingrays are serrated and covered with toxic mucous that can cause painful wounds, and removing them shortly after capture would reduce some of the risk of injury (Wheeler and Jones 1989). Von den Driesch (1983) recorded deliberately broken catfish (Synodontis sp.) spines from Karnak-North, temple of New Kingdom, near Luxor. Wall paintings from the Tomb of Akhouhotep and Tomb Ti confirm that fishermen regularly broke off pectoral spines from Synodontis species.

Evidence for the disarming of the locking mechanism of catfish spines by cutting the muscles at the base of the spines, was recorded at the Arenosa Rock Shelter by Jurgens (2008). This practice was identified from cutmarks located at the proximal ends of the spines, where the locking mechanism is located.

\subsubsection{Stable isotopes}

Stable isotope ratios are usually measured to trace processes in biogeochemical cycles, such as the flow of nutrients through food webs. In archaeology, applications include analysis of human remains to determine the contribution of aquatic foods to diet (Corr $e t$ al. 2009; Corr et al. 2005; Hu et al. 2009; Milner et al. 2004; Sealy 1989, 2006; Sealy 
and Cox 2003; Sealy and Pfeiffer 2000). Stable isotope ratios of human remains have also been used to identify differences between or displacement of populations based on the contribution of marine foods to their diet (Barrett and Beukens 2001; Barrett and Richards 2004; Sealy 2006).

A number of new studies measuring the isotope ratios of archaeological fish bone have been published. Miller et al. (2010) compared carbon and nitrogen isotope ratios of modern and archaeological fish from Lake Titicaca, Bolivia, to aid in determining the role of these species in the diet of inhabitants. They found that the carbon isotope signatures differed significantly between the archaeological and modern fish of the same species. The reasons for this change in values over time are not yet understood. The isotope values of fish overlapped with those of maize, so this approach cannot distinguish the relative contributions of these food items to peoples' diets.

Hufthammer et al. (2010) analysed oxygen isotopes of cod otoliths from two Mesolithic sites in Norway, Skoklefald and Skipshelleren, to determine season of capture. The technique entails fine scale sampling of the growth zones, and requires that the fish under study experienced seasonal temperature fluctuation. They found that both sites were occupied during late winter or early spring, and these determinations are tentatively substantiated by the presence of species that are more common during this time of year. As part of the study mentioned above, in which Robinson et al. (2009) developed a method of differentiating between salmon and trout bone, they also used strontium $(\mathrm{Sr})$ and calcium $(\mathrm{Ca})$ isotope ratios of $S$. salar bone to successfully distinguish between Atlantic and landlocked salmon from Sharrow, a Holocene site in Northeast North America. The increased $\mathrm{Sr} / \mathrm{Ca}$ ratios of the salmon samples relative to those of freshwater species at Sharrow indicated that all were Atlantic salmon, derived from marine rather than fresh water.

Recently, a novel approach of calculating oxygen and strontium isotope ratios of fish tooth enamel to determine the provenance of riverine and lacustrine archaeological fish remains was attempted, but with limited success. The method requires that in future studies more variables need to be taken into account (Dufour et al. 2007). These include 
determining the spatial heterogeneity in strontium ratios that can occur within a lake as well as possible changes of strontium isotope signals in teeth after burial.

Barrett et al. (2008) used a similarly novel approach using carbon and nitrogen isotope ratios of fish bone collagen to determine the geographical origin of dried cod. Peliminary results show that this method does indicate different locations and can be a useful tool in reconstructing medieval long distance trade.

\subsubsection{Sea surface temperatures}

Holocene sea surface temperatures have been reconstructed using oxygen isotopes from sea catfish otoliths (Andrus et al. 2002), although the validity of the method and the materials employed are contested (Andrus et al. 2003; Bearez et al. 2003). Other applications of otolith oxygen isotopes in climate reconstruction have been less controversial (Patterson 1998; Patterson et al. 1993) and new techniques are being developed to overcome some of the methodological difficulties (Andersson et al. 2008; Surge and Walker 2005; Walker and Surge 2006; Wurster and Patterson 2001).

\subsubsection{Fish origins, trade and processing}

The use of ancient fish DNA to identify and distinguish between morphologically similar species has increased considerably over the last decade as DNA extraction and sequencing techniques have improved (Butler and Bowers 1998; Ciesielski and Brzuzan 2003; Ciesielski et al. 2002; Nicholls et al. 2003; Speller 1999; Speller et al. 2005; Yang et al. 2004).

Arndt et al. (2003) used DNA to determine where catfish found in Roman and early Byzantine contexts in Turkey were procured to inform on past trade relations and origins. From the DNA they determined the species (Clarias gariepinus), and that it originated from the lower Nile, indicating that trade in fish occurred between the areas at these times. These findings are supported by other evidence of trade between these regions during the Roman and early Byzantine periods.

Although promising, the use of DNA for species identification is not always successful as bone is sensitive to taphonomic influences which can affect the preservation and location 
of DNA, even in fairly young material (Hlinka et al. 2002; Matisoo-Smith 2009). The method is also costly (van Neer et al. 2004b).

A large body of work from northern Europe and the Mediterranean centers around evidence for the origins and development of trade and exchange based on fish remains (Arndt et al. 2003; Barrett 1995, 1997; Barrett et al. 2008; Barrett et al. 2004b; Barrett et al. 2004a; Barrett et al. 1999; Sampson 1998; van Neer et al. 2004b; Zohar et al. 2001). Evidence for transport and processing of fish has been obtained through species identification, skeletal element representation and cut marks (Barrett 1997; Barrett et al. 1999; Carvajal-Contreras et al. 2008; van Neer and Pieters 1997; Zohar et al. 2001). Species found at locations where they do not occur naturally can indicate trade with the areas of origin, or long-distance travel by members of a single group. Before assuming trade activities, it needs to be ascertained whether the species could have occurred over larger areas in the past due to environmental changes.

Processing and transport of fish can be inferred from the relative skeletal element distribution of a species at a particular location. The processing of fish prior to drying for long term storage or trade often results in distinctive skeletal element patterning at the processing as well as the final destination site. Processing methods can vary according to the shape and size of the species, which will determine the ultimate relative skeletal element distribution (Norton et al. 1999; van Neer and Pieters 1997; Zohar and Cooke 1997). Fish heads spoil more rapidly than the body (Butler 1993) and their removal prior to processing for immediate consumption or discard has been recorded from ethnographic, historical and archaeological studies (Barrett 1997; Gifford-Gonzalez et al. 1999; Stewart and Gifford-Gonzalez 1994; van Neer and Pieters 1997). Decapitation would result in most of the skull elements being discarded at the processing site, and depending on the method employed, parts of the appendicular skeleton and vertebral column could either be removed with the head and left at the butchery site, or left attached to the fleshy body to be cured, and ultimately end up at the destination site, if intended for trade or transportation. Processing sites typically contain more cranial elements, and assemblages at receiving sites might have vertebrae from only the trunk area, but this is very variable and depends on the method of processing employed. Small 
fish might be processed whole, which would mask their economic importance at the processing location if they were removed intact (Norton et al. 1999). If the same anatomically skewed distribution of elements is consistently present at a site, it could indicate that the species was subjected to some form of processing.

On the Western Cape coast of South Africa various species of mullet (Mugilidae) are traditionally gutted, salted and wind dried whole. These are referred to locally as bokkoms. The antiquity of the practice is not known. Poggenpoel (1996) has suggested several prehistoric (Later Stone Age) sites with fish assemblages in the Western Cape may have been processing sites, because of the large discrepancies between quantities of non-vertebral elements relative to vertebrae. The processing method differed from that of bokkoms in that heads were removed prior to transportation and possible drying of the fleshy trunk area. Both Mugilidae and Sparidae were treated in this way. Further details are provided in Chapter Three.

Cut marks are much rarer on fish than on mammal bone, but where they occur with consistent patterning they are seen as the result of systematic fish processing (Barrett 1997; Colley 1990). Less consistent patterning of cut marks has been attributed to a range of activities including hook removal, filleting, gutting or altering jaws with sharp teeth to minimize danger of injury (Colley 1990; Jurgens 2005, 2008; Leach et al. 1984). Willis et al.'s (2008) experimental work has shown that most of the cut marks made during filleting occur on elements that are not diagnostic and thus seldom analysed; this could account for the rarity of cut marks reported on fish.

Fish remains from Neolithic sites in the Middle East have been interpreted to indicate trade (Beech 2002, 2003). The presence of non-local fish species (tuna) at an early Neolithic site in Mesopotamia suggests that these people were engaged in the trade of dried fish with people from the Arabian Gulf.

Evidence for fish processing by smoke drying was found in the form of charcoal pits and postholes associated with large amounts of fish from Late Palaeolithic sites in Egypt (van Neer et al. 2000). The first identification of a clear high quality fermented fish sauce, garum, from fish remains, previously known only from classical texts, inscriptions on amphorae and architectural structures, has been reported from an early Roman ceramic 
jar in Jordan (van Neer and Parker 2008). Other evidence for fish sauces that typically include fish bones and that are more visible archaeologically have been found in early and late Roman contexts (Desse-Berset and Desse 2000; Lepiksaar 1986; van Neer and Ervynck 1998, 2004; van Neer et al. 2006). Evidence for pickled fish has also been found in an amphora from Egypt, dating to the $7^{\text {th }}$ century AD (van Neer et al. 2007). Because of an absence of fish bones or scales inside ancient ceramic vessels from northern Europe, stable carbon and nitrogen isotope analysis of adsorbed residues was carried out to determine if these vessels were used to store marine products. Tentative results indicate this was the case (Craig et al. 2007).

\subsection{Marine resources and 'modern' human behaviour}

It has been argued that the systematic exploitation of aquatic resources, particularly those of marine origin, is one of the traits of modern human behaviour (Deacon 1989; Klein 1995; Milo 1998; Thackeray 1992). For some archaeologists, it is the conceptual and technological ability to fish and the recognition and exploitation of seasonally available resources (Klein 1995, 2008; Klein and Cruz-Uribe 1996, 2000b; Klein et al. 1999a). Some archaeologists believe that seasonal scheduling of food resources was not practiced by Middle Stone Age people, whose foraging strategies are compared to those of hyenas, where they opportunistically patrol the coast year-round (Klein et al. 1999b). Others have argued that seasonal resources are recognised by other animals with considerably smaller brains than hominins, and therefore the concept of seasonal mobility as an indication of human modernity is untenable (Henshilwood and Marean 2003). Attempts to reconstruct seasonal occupation of southern African Middle and Later Stone Age sites have not been very successful, and earlier hypotheses for or against seasonal movement have been challenged (e.g. Cable 1984; Hall 1990; Klein 2001, Klein and Cruz-Uribe 1996; Marean and Assefa 1999; Mazel 1989; Parkington 1976, 1991, 2001; Sealy et al., 2000; Sealy and van der Merwe 1985, 1986, 1992; Shackleton 1973).

Others consider that the recognition and consumption of shellfish indicates an awareness of the available resources no different to that of their Holocene successors (Deacon 1989). Baboons (Chacma ursinus) are also reported to collect shellfish, especially limpets, at low tide (Avery and Siegfried 1980). Joordens et al. (2009) argue that 
advanced technology such as fish hooks and boats must be present before modernity can be linked with aquatic resource consumption.

The earliest evidence of anatomically modern Homo sapiens exploiting marine resources (shellfish) occurs at Pinnacle Point 13B in the southern Cape at about $164 \mathrm{ka}$, a time when the African interior was very arid and inhospitable (Marean et al. 2007). The authors argue that this is evidence for broadening of dietary resources, perhaps in response to these environmental conditions, and, as the terrestrial food component of coastal environments tend to be less rich than inland, expansion of diets to include marine resources would have been essential in order to survive in this habitat (Marean et al. 2007). Shellfish is a nutritious and reliable food resource that is easily harvested. Once it was recognised as such, living in a coastal habitat would have been more attractive as adequate nutrition would have been available. It is hypothesized that once coastal habitats became more inhabitable, human expansion along coastal routes out of Africa became possible (Stringer 2000; Walter et al. 2000). The Pinnacle Point 13B shellfish from 164 - 120 ka was found in conjunction with other, less contested markers of modern human behaviour, namely pigment use and a bladelet technology suggesting the use of composite tools (Marean et al. 2007).

In southern Africa, marine shellfish exploitation predates the appearance of fish in MSA assemblages. Most coastal southern African MSA sites that have early evidence for shellfish exploitation have no fish bones recorded. These include Boegoeberg 2 (Klein et al. 1999a), Die Kelders (Avery et al. 1997; Schweitzer 1970), Diepkloof (Steele, pers. comm.), Herold's Bay (Brink and Deacon 1982), Hoedjies Punt (Volman 1978), Pinnacle Point caves 13A and 13B (Marean et al. 2007; Marean et al. 2004b) Sea Harvest (with the exception of one fish vertebra) (Volman 1978) and Ysterfontein 1 (Avery et al. 2008; Halkett et al. 2003; Klein et al. 2004).

Lower volumes and an increase in average size of shellfish, a lack of evidence for seasonal scheduling of coastal resources and a general lack of fish or fishing gear in the MSA relative to the LSA has led some researchers to conclude that MSA people were less efficient at utilizing coastal resources than their LSA counterparts (Avery et al. 2008; 
Klein 1988, 1989a, b, 1992, 1995, 1998, 2000, 2001, 2008; Klein et al. 2004; Volman 1978, 1981).

There is increasing evidence that Neanderthals were utilizing freshwater and marine resources, perhaps as far back as $115 \mathrm{ka}$ (Stiner 1994; Stringer et al. 2008; Zilhão et al. 2010). Even older evidence for aquatic resource utilization including freshwater fish, crabs and turtles has been discovered in Israel at Gesher Benot Ya'aqov, dating back to the Acheulean, at 790ka (Alperson-Afil et al. 2009). Tentative evidence of shellfish utilization by $H$. erectus at Trinil in Java 1.5 mya has also been published recently (Joordens et al. 2009). Erlandson (2001:306, Table 1) lists all the sites with tentative and confirmed incidences of aquatic species utilization associated with hominins. Whether aquatic resource exploitation can be considered an exclusively modern human trait associated with $H$. sapiens when it is shared with Neanderthals and other hominins is subject to debate (Joordens et al. 2009; Milliken 2007; Shipman 2008; Stringer et al. 2008). It has been pointed out that although there is increased evidence for aquatic resource use by Neanderthals and other hominins, the quantities reported are small compared with those reported from southern African sites associated with $H$. sapiens (d'Errico 2003), and the argument for systematic exploitation might not be tenable. Bailey (2009) believes that the smaller quantities of marine foods associated with Mousterian sites are simply a reflection of the lower productivity of the Mediterranean intertidal habitat, and that the antiquity of collecting and consumption of marine resources predates any cognitive expansion of $H$. sapiens and the appearance of modern human behaviour.

Fish bones have been found in association with a number of early hominid sites at Olduvai Gorge, and some tentative evidence suggests that early hominids (Homo habilis and possibly Homo erectus) were responsible for collecting some of these, and possibly even scheduling their seasonal rounds to exploit spawning or stranded fish (Stewart 1994). At these inland locations freshwater fish could be easily procured without the need for technology during spawning runs, when they congegrate in large numbers, or from drying lake and river pools during the dry season. Oppertunities for collecting stranded or spawning fish are more limited at coastal locations. Some fish undertake annual spawning migrations but these generally do not entail mass congregations in shallow waters where they could be collected by hand, and more advanced technology would be needed to take 
advantage of these situations. Sporadic cold water upwelling events resulting in washed up fish are generally limited to the summer months in southern Africa (Bower 1981; Hanekom 1989; Lutjeharms 2000). Due to the unpredictable nature of these events, it is unlikely that people would have scheduled their visits to the coast based on such an unreliable resource. Such events would, however, have presented people with several meals, and could have encouraged people to pursue fish as a food source.

It has been suggested that an adaptation to an aquatic diet rich in docosahexaenoic acid (DHA) played a significant role in hominin brain development, and that it was in fact this change in diet that allowed sufficient encephalization to lead to the evolution of Homo sapiens and ultimately the ability to become behaviourally modern (Broadhurst et al. 1998; 2002; Crawford et al. 1999a). The hypothesis is partially based on the location of early Homo spp. finds, which tend to centre around lacustrine environments such as the East African Rift Valley, as opposed to Australopithecines that inhabited forested areas, and modern $H$. sapiens fossils that occur at coastal sites in southern Africa (Broadhurst $e t$ al. 1998; 2002; Crawford et al. 1999a). The resources would initially have consisted of sessile foods, the consumption of which over time would have improved brain function leading to improved tool use and hand-eye coordination to allow more active methods of acquiring food such as fishing (Broadhurst et al. 2002; Cunnane and Crawford 2003).

Aquatic foods have the highest amounts of DHA and sessile organisms would have been easy to collect and a reliable and constant source of this essential fatty acid. They are, however, not the only source, and it has been argued that it is possible to synthesize sufficient DHA and other fatty acids needed for brain development from a completely terrestrial diet (Carlson and Kingston 2007b; Langdon 2006), although this is contested (Carlson and Kingston 2007a; Crawford 2006; Cunnane 2007; Cunnane et al. 2007; Joordens et al. 2007).

The increasing evidence for aquatic resource exploitation by hominins other than Homo sapiens makes it clear that this in itself cannot be considered to be an exclusively modern human trait. Compared to the Later Stone Age which has consistent evidence for fishing, it seems that active fishing during the Middle Stone age was less purposeful. Recent data shows that MSA people in southern Africa were capable of sourcing exotic raw materials, 
making complex tools, heat treating raw material prior to knapping and using advanced techniques to shape weapons and tools in stone and bone. These activities indicate an ability for forward planning, creating mental templates and for carrying out complex tasks (Brown et al. 2009; Lombard and Phillipson 2010; Mourre et al. 2010; Villa et al. 2009). It is also likely that some of these tools, together with marine beads used as personal ornaments, functioned in a symbolic way within a society whose behaviour was mediated by symbols (Henshilwood et al. 2004, d'Errico et al. 2005).

Obtaining marine fish generally requires more planning and technological innovation than procuring of freshwater fish, as there are fewer seasonally mediated opportunities for easy collecting of fish in a the coastal environment. Intentional targeting of marine fish indicates an awareness of tides, seasonal movement of fish and fish feeding ecology and a knowledge of attractant baits. Targeting marine fish would require forward planning and technological abilities that extend beyond the opportunistic gathering of stranded fish. Some fish can be caught using relatively simple technology such as wooden spears, but other species can only be caught with more advanced equipment such as hooks, which are composite tools, or through the erection of stone structures. As such, active fishing of species that cannot be caught by simple methods indicates complex strategies and perhaps advanced technology. These act as clear markers of cognitively modern human behaviour.

Currently there are only two Middle Stone Age assemblages in Africa, Klasies River and Blombos Cave, both in South Africa, that contain reasonable amounts of marine fish remains, some of which might have been caught by humans (Henshilwood et al. 2001b; von den Driesch 2004). 


\section{Chapter 3. Southern African archaeological fish analysis, species ecology and methods of capture}

\subsection{Analysis of fish remains in southern Africa}

\subsubsection{Background of archaeological fish remains analysis and analysts in southern Africa}

Fish remains from southern African archaeological sites received little attention in publications until around the 1940s. Early identifications of archaeological fish remains were made by ichthyologists and reports typically consist of a species list and occasional mention of the elements identified (e.g. Deacon 1970; Goodwin 1946). There are a few exceptions, such as the ichthyologist M-L. Penrith's report on the fish remains from Gordon's Bay Midden that includes fragment counts as well as a list of elements present (van Noten 1974).

Cedric Poggenpoel was the first archaeologist to systematically identify and quantify marine fish remains from sites in the southwestern Cape. He has been responsible for the majority of fish identifications at sites in this area since the early 1970's (Henshilwood 1995; Inskeep 1987; Klein 1972a; Parkington 1976; Poggenpoel 1984, 1987, 1996; Poggenpoel and Robertshaw 1981; Robey 1984; Schweitzer 1979; Schweitzer and Wilson 1982; Singer and Wymer 1982). Poggenpoel's method of identification is usually based on a predetermined set of robust elements, but he has also shown that the inclusion of other elements for certain species can increase both specimen count and number of taxa (Poggenpoel 1984, 1996).

Angela von den Driesch, well known for her contributions to faunal analysis in many parts of the world, including South Africa, analysed the MSA fish remains from Klasies River main site (2004). She identified a wide range of elements in order to obtain a species list and compared skeletal element representation between vertebral and nonvertebral elements.

Ina Plug has been responsible for most freshwater fish analysis in southern Africa (Plug $1989,2005,2006 a, b, 2008 ; 2003)$. She is the first archaeozoologist to publish prediction ratios for determining fish size for southern African freshwater fish species (Plug 2008). 
Plug uses a wide range of elements for identification and has in addition identified and published descriptions and illustrations of elements of morphologically similar species that can and cannot be reliably used for identification (2005).

Simon Hall analysed freshwater fish from a number of sites in the eastern Cape and Kwazulu-Natal but is no longer involved in fish analysis (1990; 1997). Poggenpoel, Plug and von den Driesch are now retired from their institutions, although Plug is still active in faunal analysis in South Africa and Lesotho (Plug 2005, 2006a, b, 2008; Plug et al. 2010).

\subsection{Overview of archaeological fish remains in southern Africa}

\subsubsection{Introduction}

There are relatively few intact MSA deposits along the southern Cape coast as many caves and open sites were inundated during the mid-Holocene high sea stand. Caves at an elevation above $10-15 \mathrm{~m}$ above present sea levels are most likely to preserve evidence of MSA occupation (van Andel 1989a; van Andel 1989b, 1990). The same applies to Later Stone Age deposits dating to the terminal Pleistocene and early and middle Holocene. During colder intervals of past glacial/interglacial cycles, the currently submerged Agulhas plain was dry land, at times extending as much as $100 \mathrm{~km}$ south of the present Cape Agulhas. There were undoubtedly many MSA and LSA sites there, all of which are now underwater. A great deal of information on the exploitation of fish and other marine resources has therefore been lost (Klein 1974).

\subsubsection{Middle Stone Age fish remains}

Middle Stone Age (MSA) sites with preserved fish remains are rare in southern Africa.

The coastal or near-coastal MSA sites with preserved fauna but little or no fish remains include Pinnacle Point caves 13A and 13B (Marean et al. 2004b), Boegoeberg 2 (Klein et al. 1999a), Diepkloof (Parkington et al. 2005; Texier et al. 2010), Herold's Bay (Brink and Deacon 1982), Hoedjies Punt (Volman 1978), Ysterfontein (Avery et al. 2008; Halkett et al. 2003; Klein et al. 2004) and Die Kelders (Avery et al. 1997; Schweitzer 1970) (Figure 3.1.). 


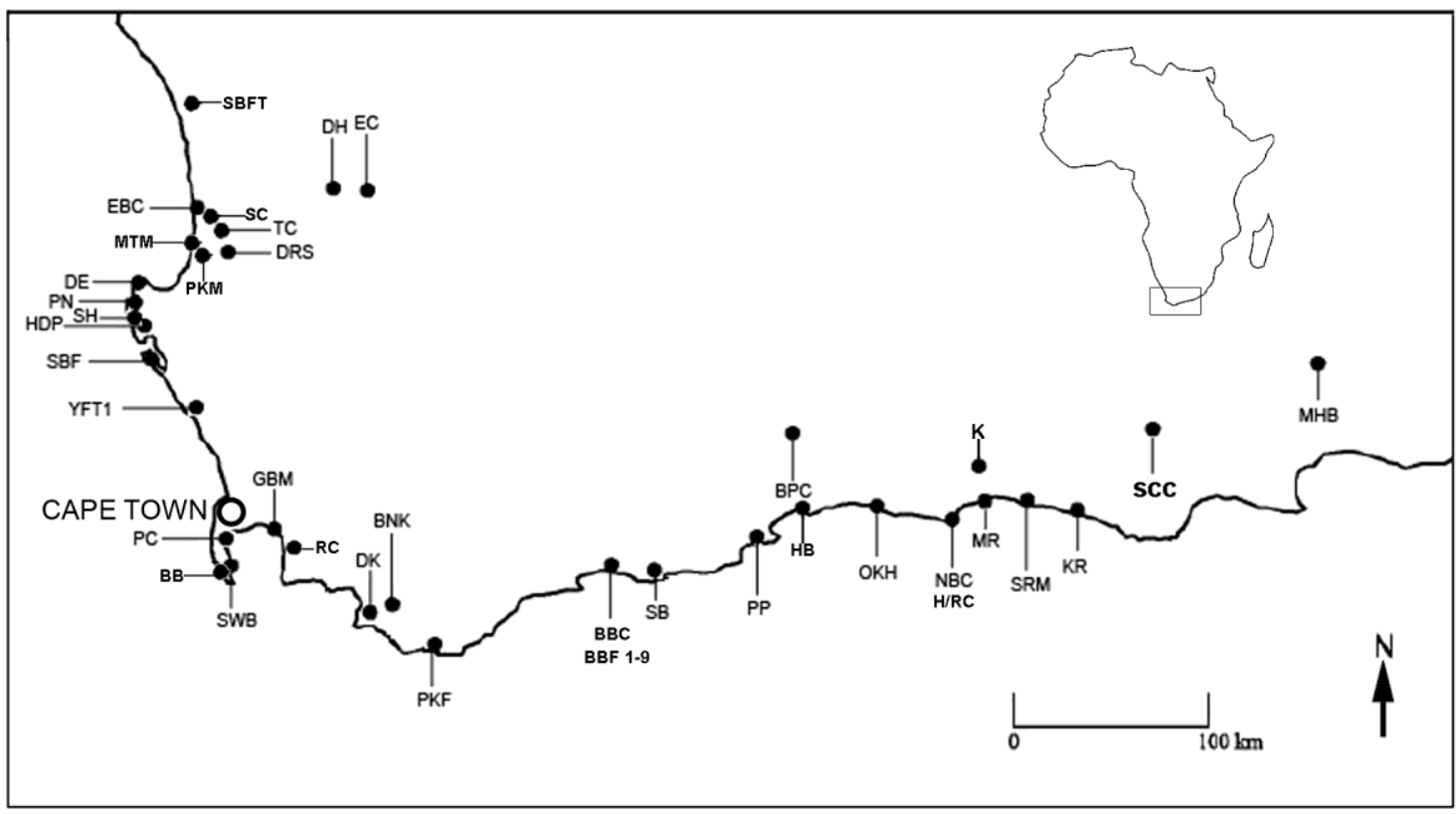

Figure 3.1. Distribution of archaeological sites in the western and eastern Cape that are referred to in the text.

BB - Bonteberg; BBC - Blombos Cave; BBF 1 \& 6 -Blombosfontein 1 and 6; BPC - Boomplaas Cave; BNK - Byneskranskop; DE - Duiker Eiland; DH - DeHangen; DRS - Diepkloof Rock Shelter; DK - Die Kelders; EBC - Elands Bay Cave; EC - Eland Cave, GBM - Gordon's Bay Midden; HB Herolds Bay; HDP - Hoedjiespunt; H/RC - Hoffman's/Robberg Cave; K - Kouga; KR - Klasies River; MR - Matjes River; MHB - Melkhoutboom; MTM - Mike Taylor's Midden; NBC - Nelson Bay Cave; OKH - Oakhurst Shelter; PC - Peers cave; PKF - Paapkuilfontein; PKM - Pancho's Kitchen Midden; PN - Paternoster; PP - Pinnacle Point; RC - Rooiels Cave; SB - Still Bay middens; SBF - Stofbergfontein; SBFT-Steenbokfontein; SH - Sea Harvest; SC - Spring Cave; SCC - Scott's Cave; SRM - Storms River Middens; SWB - Smitswinkelbaai Cave; TC - Tortoise Cave; YFT1 Ysterfontein 1

Boegoeberg 2 (BOG2) is a stratified MSA shell midden located in a shelter along the Atlantic coast of the Northern Cape Province, some 850km north of Cape Town (Klein et al. 1999a). The site was discovered and unfortunately mostly destroyed by bulldozing activities (Klein and Cruz-Uribe 1996). It contains abundant shellfish, some MSA lithic artefacts, and little bone, most of which are from cormorants (Klein 1999a). The site has not yet been fully described and no fish has been reported. During a brief assessment of this assemblage which is curated at the IZIKO South African Museum, I noted at least two large fish vertebrae in the unsorted material.

Diepkloof Rock Shelter (DRS) is a quartzite overhang located close to the Verlorenvlei approximately $18 \mathrm{~km}$ from the coast and $180 \mathrm{~km}$ north from Cape Town (Parkington and Poggenpoel 1987; Parkington et al. 1988; Parkington et al. 2005) (Figure 3.1). It contains 
a shallow LSA occupation overlaying a series of MSA assemblages. The MSA assemblage dates from before $130 \mathrm{ka}$ to $\sim 45 \mathrm{ka}$. It is one of the most continuous and complete MSA sequences in southern Africa and includes occupations from the PreStillbay, Stillbay, Howiesons Poort and Post-Howiesons Poort periods (Texier et al. 2010). The MSA bone is highly fragmented and friable, and is currently under study (Parkington and Poggenpoel 1987; Texier et al. 2010; Steele, pers. comm.). Apart from a single bone from an unprovenienced context, fish bones have not been found in the MSA assemblages (Steele, pers. comm.), but the presence of a limited amount of marine shellfish and some seal remains indicates that the inhabitants were accessing coastal resources (Texier et al. 2010). The conditions contributing to the poor preservation of the mammalian fauna would also affect fish bone if these had been present.

Sea Harvest (SH) and Hoedjies Punt (HDP) are two open MSA shell middens located $1.3 \mathrm{~km}$ apart on the west coast, approximately $120 \mathrm{~km}$ north from Cape Town (Volman 1978) (Figure 3.1). Both assemblages were capped by cemented deposits and the matrix surrounding the occupation residues were also cemented, which made conventional excavation procedures unpractical. Material was therefore collected and sieved from the base of the deposits where it had eroded out on repeated visits. SH dates between 40 and $130 \mathrm{ka}$, and contains well preserved shellfish and a small amount of bone including one cormorant and a single fish vertebra (Grine and Klein 1993; Volman 1978). The lithic assemblage and geomorphic setting is very similar to that of Ysterfontein 1, and it has been suggested that the sites may be contemporaneous (Avery et al. 2008). Details of the faunal component of HDP were not given apart from noting that shellfish is less well preserved than that of SH. Road-building activities has subsequently destroyed the remaining SH assemblage (Volman 1981).

Ysterfontein 1 (YFT1) is a stratified MSA rock shelter located on the coast $\sim 70 \mathrm{~km}$ northnorth-west of Cape Town (Klein et al. 2004) (Figure 3.1). The age of the deposits lies beyond the effective range of the radiocarbon method and the authors estimate an age between 110 and 71 kya (Avery et al. 2008). The presence of coprolites and distinctive damage to ostrich eggshell fragments indicate a degree of hyaena activity at the site, particularly in the upper layers where signs of human occupation are scarce. The deposits contain terrestrial fauna as well as marine birds, mammals (seals), and considerable 
amounts of shellfish, indicating the site's proximity to the coast during occupation. A few bones from fish smaller than $10 \mathrm{~cm}$ occur in the disturbed upper layers and are attributed to either small carnivores or regurgitated cormorant pellets (Avery et al. 2008; Halkett et al. 2003; Klein et al. 2004). Micromammals appear to be totally absent from the assemblage.

Die Kelders Cave 1 (DK1) is situated on the coast approximately $160 \mathrm{~km}$ east of Cape Town and contains both Later and Middle Stone Age deposits (Schweitzer 1970) (Figure 3.1). It is one of a complex of caves formed at the contact of Table Mountain Sandstone (TMS) (the base of the cave) and Bredasdorp limestone (roof). The MSA assemblage dates to between 70 - 60 ka (Avery et al. 1997; Feathers and Bush 2000; Schwarcz and Rink 2000). Fish, shellfish and seal are well preserved in the LSA layers (Schweitzer 1979). Shellfish and seals are present in the MSA layers, but preservation of the shellfish is very poor (Avery et al. 1997; Marean et al. 2000a). Micromammals and tortoise occur throughout the sequence. The preservation of large mammal bone varies from weathered at the top of the sequence to excellent at the bottom (Avery et al. 1997; Marean et al. 2000b). The presence of shellfish and seals indicates that the MSA inhabitants were exploiting marine resources. Due to the very steep downwards slope of the rocky sea floor in front of the cave, the coast was never further than a kilometer away except during the last glacial maximum, during which the cave was not occupied (van Andel 1989a). The degraded condition of the shellfish in the MSA relative to the LSA suggests that more extreme taphonomic processes affected the older material. These might relate to increased acidity due to the underlying TMS bedrock, and could be responsible for the absence of fish in the MSA layers. Other bone, including those of small micromammals, was preserved in the MSA layers.

Herold's Bay (HB) is an MSA midden situated in a sea-cut cave approximately $430 \mathrm{~km}$ northeast from Cape Town near the town of George (Brink and Deacon 1982) (Figure 3.1). It has not been dated directly, but is thought to be younger than $115 \mathrm{ka}$ (Volman 1984). Much of the site was destroyed by building operations before excavation commenced, and only a small portion of the original assemblage was preserved. The midden contained shellfish, some mammalian fauna and lithic artefacts. A considerably larger amount of mammalian fauna was recovered from the layers above the midden, 
which was accumulated by carnivores and porcupines. The human-occupied layers contain no marine fauna other than shellfish.

Pinnacle Point Site 13A (PP13A) is a dune deposit that lies below a quartzite overhang near Mossel Bay on the south coast of the Western Cape (Marean et al. 2004b) (Figure 3.1). Pinnacle Point Site 13B (PP13B) is a quartzite cave located close to PP13A.

Shellfish is present at PP13A in very low numbers (total minimum number of individuals $[\mathrm{MNI}]=60$ ) but are reportedly reasonably well preserved, although the most common species, Perna perna, was very fragmented and friable (Marean et al. 2004b). Some of the shellfish present in PP13B currently represent the oldest evidence of shellfish exploitation by Homo sapiens at $\sim 164 \mathrm{ka}$. These occur in low numbers (MNI = 89), with conditions of preservation within the site varying from burnt and chalky to relatively well preserved (Marean et al. 2007; Marean et al. 2004b). The condition of bone at these sites is reportedly excellent, due to the calcium carbonate rich water percolating through the walls and roof of the cave and overhang. Fish, bird and snake bone are absent from PP13A. A small number of fish bones were recovered from PP13B $(n=19)$ which have not yet been studied (Thompson 2008), a single bird bone occurred in the MSA roof spall facies, and no snake bones were found. Micromammals are rare in both assemblages, and most of the mammalian fauna are from larger mammals. Tortoise is abundant in both PP13A and PP13B, but at much lower frequencies than at most other MSA sites such as BBC, DK and YFT (Halkett et al. 2003; Henshilwood et al. 2001b; Klein and Cruz-Uribe 2000a; Marean et al. 2004b). The same is true for small mammals and micromammals, which are common in other MSA assemblages but rare at the Pinnacle Point sites. The authors interpret the lack of tortoises, micromammals, and small mammals as an indication that owls and raptors, common collectors of these species, did not play a significant role in the accumulation of the assemblage. The generally low number and often degraded condition of shells and lack of smaller faunal remains could instead be a function of differential preservation at these sites, which might affect smaller faunal remains, including those of fish, to a greater extent than those of larger animals.

The MSA levels at Eland's Bay Cave (Volman 1981) and Nelson Bay Cave (Klein 1972a, b) do not have preserved fauna (Figure 3.1). 
Sibudu Cave is a large rock shelter in northern Kwa-Zulu Natal. It is situated in a sandstone and shale cliff $15 \mathrm{~km}$ inland from the Indian Ocean and overlooking the Tongati River (Wadley 2001). The deposit contains a deep sequence of MSA occupation dating from >70 - $30 \mathrm{ka}$, overlain by Iron Age deposits (Wadley 2007). The site has exceptionally good organic preservation, which includes the preservation of small seeds. A small amount of freshwater (NISP=40) and marine (NISP=6) fish and shellfish occur in the MSA layers. The marine fish bones were too fragmented to be identified to genus, but most were from "fairly large" fish (Plug 2004; 2006a: 295). The site is too far from the coast for birds to have carried fish of this size (Plug 2006a), so it appears that the fish and shellfish were brought in by humans (Plug 2004, 2006a).

At Klasies River main site (KR) on the Tsitsikamma coast, the MSA sub-stages from the Deacon excavations contain a substantial number of fish bones relative to other MSA sites (Deacon and Geleijnse 1988) (Figure 3.1). The MSA deposits date to between 120 000 and >50 000 years ago. The highest mass of fish bone from a sample of the deposit was recorded in the sterile units, which the authors believe substantiates their theory that the fish remains are not anthropological, but originates from the regurgitated pellets of cormorants roosting above the site (Deacon 1989; Deacon and Geleijnse 1988). Very few fish remains are reported from the earlier Singer and Wymer (1982) excavations at KR, and those that are mentioned are from large specimens. A review of these remains by the author confirms this bias. The absence of smaller fish bones is partly due to the larger sieve sizes used in the Singer and Wymer excavations ( $1 / 4$ inch for Howieson's Poort material and $1 / 2$ inch screens for the rest, as opposed to the 2 and $3 \mathrm{~mm}$ sieves used in Deacon's excavation) (Singer and Wymer 1982). A large sieve size can result in considerable loss of material as fish and other small faunal remains pass through the sieve (Erlandson 2001; Gargett and Vale 2005; Gobalet 2005; Vale and Gargett 2002). In several instances only samples of finds were retained (Voigt 1982), and vertebrate fauna that were considered taxonomically unidentifiable were discarded during preliminary sorting in the field (Klein 1976). This selective sampling introduced an additional bias to the fish remains from the Wymer excavations.

Von den Driesch (2004) analysed a sample of the fish from KR main site from the Deacon excavation. At least 61 species from 35 families of bony fish, and 17 species 
from twelve families of cartilaginous fish were identified (Table 3.1). All but one of the species identified are marine inhabitants. The frequencies of the most common families were compared per square and member. Species of Clinidae, Sparidae and Gobiesocidae families occurred most frequently, but there was some variation in the most abundant species between squares of the same members (Table 3.2). The most significant difference in the families present was found in the LBS member, where Carangidae, not present in the five most abundant families of the other members, was the most frequently occurring fish family in two of the three squares.

Table 3.1. Families and species identified from Klasies River main site by von den Driesch (2004). Note that the names of some of the species and families have been changed to the taxonomic nomenclature used in this study which follows Heemstra and Heemstra (2004). This publication uses more up to date valid scientific names than was used by von den Driesch (2004)

\begin{tabular}{|c|c|c|c|}
\hline CHONDRICHTHYES & & OSTEICHTH & 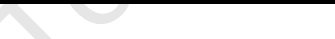 \\
\hline Hexanchidae & Clupeidae & Sciaenidae & Gobiidae \\
\hline Notorynchus cepedianus & Sardinops ocellatus & Argyrosornus hololep & Caffrogobius caffer \\
\hline Squalidae & Engraulidae & Atractoscion aequide & Blenniidae \\
\hline Squalus megalops & Engraulis cf. japonicus & Mullidae & Parablennius pilicornis \\
\hline Lamnidae & Plotosidae & Parupeneus spp. & Chalaroderma capito \\
\hline Carcharodon carcharias & Plotosus cf. nkunga & Dichistiidae & Clinidae \\
\hline Odontaspididae & Ariidae & Dichistius cap & Clinus spp. \\
\hline Carcharias taurus & Galeichthys feliceps & Haemulidae & Pavoclinus spp. \\
\hline Scyliorhinidae & Belonidae & Pomadasys spp. & Bythitidae \\
\hline Halaelurus natalensis & Ablennes hians & Sparidae & Bidenichthys capensis \\
\hline Haploblepharus spp. & Tylosurus crocodilus & Acanthopagrus berda & Cheilodactylidae \\
\hline Poroderma pantherinum & Merlucciidae & Boops salpa & Cheilodactylus spp. \\
\hline Carcharhinidae & Merluccius capensis & Chrysoblephus cristiceps & Chirodactylus brachydactylus \\
\hline Galeorhinus galeus & Teraponidae & Chrysoblephus gibbiceps & Gobiesocidae \\
\hline Mustelus spp. & Terapon jarbua & Cymatoceps nasutus & Chorisochismus dentex \\
\hline Carcharhinus spp. & Malacanthidae & Diplodus cervinus & Muraenidae \\
\hline Triakidae & Branchiostegus doliatus & Diplodus sargus & Echidna spp. \\
\hline Triakus megalopterus & Serranidae & Gymnocrotaphus curvidens & Lycodontis undulatus \\
\hline Sphyrnidae & Acanthistius sebastoides & Lithognathus lithognatus & Congridae \\
\hline Sphyrna zygaena & Epinephelus spp. & Pachymetopon aeneum & Conger spp. \\
\hline Rhinobatidae & Serranus spp. & Pagellus natalensis & Anguillidae \\
\hline Rhinobatos annulatus & Scorpaenidae & Polysteganus spp. & Anguilla spp. \\
\hline Rhynchobatus djiddensis & Helicolenus dactylop & Pterogymnus lanarius & Muraenesocidae \\
\hline Rajidae & Oplegnathidae & Rhabdosargus holubi & Muraenesox cinereus \\
\hline Raja alba & Oplegnathus conwayi & Sparodon durbanensis & Ostraciidae \\
\hline Dasyatidae & Carangidae & Spondyliosoma emarginatum & Ostracion spp. \\
\hline Dasyatis spp. & Caranx sexfasciatus & Scombridae & Diodontidae \\
\hline Myliobatidae & Lichia amia & Scomber japonicus & Diodon holocanthus \\
\hline Myliobatis aquila & Seriola lalandi & Gempylidae & Tetraodontidae \\
\hline Aetobatus narinari & Decapterus spp. & Thyrsites atun & Amblyrhynchotes honckenii \\
\hline $\begin{array}{l}\text { FRESH WATER FISH } \\
\text { Cichlidae } \\
\text { Oreochromis mossambicus }\end{array}$ & $\begin{array}{l}\text { Trachurus trachurus } \\
\text { Pomatomidae } \\
\text { Pomatomus saltatrix }\end{array}$ & $\begin{array}{l}\text { Mugilidae } \\
\text { Liza richardsoni } \\
\text { Mugil cf. cephalus }\end{array}$ & Arothron hispidus \\
\hline
\end{tabular}


Table 3.2. Scores of the five most abundant fish families per layer and member from Klasies River main site identified by von den Driesch (2004). A score of $1=$ most abundant, 5 = least abundant. Adapted from Figures 7, 8 and 9 in von den Driesch (2004:48-49)

\begin{tabular}{|c|c|c|c|c|c|c|c|c|c|c|c|c|c|}
\hline \multirow[b]{2}{*}{ SQUARE } & \multicolumn{3}{|c|}{$\begin{array}{l}\text { Upper member } \\
\text { (HP + MSA III) }\end{array}$} & \multicolumn{7}{|c|}{ SAS (MSA II) } & \multicolumn{3}{|c|}{ LBS (MSA I) } \\
\hline & E50 & H51 & J51 & M50 & N51 & Q52 & 050 & T51 & Y45 & PP38 & AA43 & $\mathrm{Z44}$ & PP38 \\
\hline Clinidae & 2 & 1 & 1 & 1 & 1 & 5 & 1 & 1 & 1 & 1 & 3 & 5 & 1 \\
\hline Sparidae & 4 & 4 & 3 & 3 & 2 & 4 & 2 & 2 & 2 & 2 & 2 & 2 & 2 \\
\hline Gobiesocidae & 3 & 2 & 2 & 4 & & & & & 4 & 3 & & 3 & 3 \\
\hline \begin{tabular}{|l|} 
Mugilidae \\
\end{tabular} & 5 & 3 & 4 & 2 & 3 & 3 & 3 & 4 & 3 & 4 & 4 & & 4 \\
\hline Ariidae & 1 & 5 & & 5 & 4 & 1 & 5 & & & & & & \\
\hline Gobiidae & & & 5 & & & & & & & & & & \\
\hline Serranidae & & & & & 5 & 2 & & 3 & 5 & & & & \\
\hline Cheilodactylidae & & & & & & & 4 & 5 & & 5 & & & \\
\hline Carangidae & & & & & & & & & & & 1 & 1 & 5 \\
\hline Teraponidae & & & & & & & & & & & 5 & & \\
\hline Engraulidae & & & & & & & & & & & & 4 & \\
\hline
\end{tabular}

Frequencies of vertebrae and other elements were compared by square and member and it was found that these were variable, but no specific pattern of element preservation between squares or members could be discerned. The majority of squares contained more than $80 \%$ vertebrae. Based on previous studies on fish element representation, von den Driesch (2004) believes that element preservation could be dependant on archaeological age, and that vertebrae survive better than other elements in older assemblages.

Fish size was reconstructed by direct comparison with comparative specimens and through measurement of the elements (von den Driesch 2004) (Table 3.3). The majority of fish in all members were in the $15-20 \mathrm{~cm}$ size class. The LBS member contained considerably more fish smaller than $15 \mathrm{~cm}$ than the other members. Von den Driesch (2004) found that most of the archaeological specimens were of the same size of those found in cormorant pellets, but all chronological units also contained specimens that are considerably larger than those cormorants can ingest. The skeletal element composition of fish bones in cormorant pellets differed from the archaeological assemblage, and vertebral centra were more damaged in the pellets than in the archaeological specimens. After considering other potential agents of accumulation of the fish bone and the generally good condition of the bone, she concluded that humans were responsible for at least some of the fish remains recovered from this site. 
Table 3.3. Percentages per size class of bony fish per member from Klasies River main site as reported by von den Driesch. Adapted from von den Driesch (2004:50, Fig. 11)

\begin{tabular}{|c|r|r|r|r|}
\hline Size class & $\begin{array}{c}\text { Upper member } \\
\text { (HP + MSA III) }\end{array}$ & $\begin{array}{c}\text { SAS Upper } \\
\text { (MSA II) }\end{array}$ & $\begin{array}{c}\text { SAS Lower } \\
\text { (MSA II) }\end{array}$ & LBS (MSA I) \\
\hline $\mathbf{c m}$ & $\%$ & $\%$ & $\%$ & $\%$ \\
\hline $5-10$ & $0-5$ & - & $15-20$ & $0-5$ \\
\hline $10-15$ & $10-15$ & $0-5$ & $15-20$ & $15-20$ \\
\hline $15-20$ & $40-45$ & $40-45$ & $35-40$ & $40-45$ \\
\hline $20-25$ & $20-25$ & $35-40$ & $10-15$ & 25 \\
\hline $25-30$ & $10-15$ & $10-15$ & $5-10$ & $5-10$ \\
\hline $30-40$ & $0-5$ & $5-10$ & 5 & $0-5$ \\
\hline $40-50$ & $0-5$ & $0-5$ & $0-5$ & $0-5$ \\
\hline$>50$ & $0-5$ & $0-5$ & $0-5$ & $0-5$ \\
\hline
\end{tabular}

Von den Driesch (2004) reasoned that fish remains from the culturally sterile layers at KR must have been accumulated by agents other than humans. There are some methodological problems with this analysis of the KR fish remains. The KR sequence contains alternating sterile and cultural (occupation) layers. The majority of fish remains by weight occur in the sterile layers that are thought to derive from cormorants that periodically roost on the cliff above the site (Deacon and Geleijnse 1988). Von den Driesch 2004 did not differentiate between fish remains from the cultural and sterile layers, thus potentially masking any anthropogenic signatures that could help identify whether humans were instrumental in collecting some of the fish. While the report contained much new and useful information, the data was presentated in a way that made it difficult to compare with other assemblages. For example, although a species list was supplied, quantification was presented either for all bony fishes combined, all cartilaginous fishes combined, or all fish per family, without distinguishing between the frequencies of individual species. As there can be major differences in the ecology of species of the same family, combining all species masks potentially useful information such as which are the most frequently occurring species, and therefore the habitats most frequently exploited. Different species may be acquired in different ways, some of which require more sophisticated technology than others, so this information is central to the key question of whether humans or other animals were responsible for the presence of fish in the site. In addition, previous work grouped fish elements into vertebral and nonvertebral elements, without detailing or quantifying the non-vertebral elements. Each fish skeleton contains a large number of non-vertebral elements, and knowing which elements 
were most common is important for comparison with other human and non-human accumulated assemblages to determine the agent of accumulation and the extent of attrition within an assemblage. Size estimates were also based on combined species per family data, as the number of size estimates per species was too low to allow statistical comparisons. The problem here is that the size estimates were for fish from the cultural and sterile layers combined, thereby averaging fish sizes that might in fact differ between the cultural and sterile layers, and which could potentially be used to identify accumulators other than cormorants in the cultural units. For this thesis, therefore, the assemblage from KR studied by von den Driesch was analysed again using the methods described in Chapter Five.

I would like to emphasize that this is not at all intended as a criticism of von den Driesch's work. On the contrary, hers is a pioneering study of fish from a southern African MSA assemblage, taking into account many factors that have not been considered before. Her work has inspired me to explore just how much information can be gleaned from fish bones if one moves beyond species lists.

Cedric Poggenpoel carried out a preliminary examination of the MSA fish remains recovered from the 1998 - 1999 excavations of Blombos Cave in the southern Cape (Henshilwood et al. 2001b) (Figure 3.1). Fish occurred in low frequencies in all MSA sub-stages, and ten species from seven families were identified from 664 bones (Table 3.4). The data was presented as NISP per phase. The fish remains from the interior of the cave and the exterior test trench were presented separately and compared. Black musselcracker, Cymatoceps nasutus, was the most common species in the interior, whereas sea catfish, Galeichthys feliceps, was the most frequently occurring species in the exterior. The largest number of specimens was recorded in the interior of the M1 phase and the exterior test trench of the M3 phase. 
Table 3.4. NISP of fish species per Middle Stone Age phase identified from the 1998 and 1999 excavation seasons at Blombos Cave. Note that the names of some of the species have been changed to the taxonomic nomenclature used in this study which follows Heemstra and Heemstra (2004). This publication uses more up to date valid scientific names than those used by von den Driesch (2004)

\begin{tabular}{|c|c|c|c|c|c|c|c|c|c|}
\hline \multirow[b]{2}{*}{ Taxa } & \multicolumn{4}{|c|}{ BBC Interior } & \multicolumn{4}{|c|}{ BBC Exterior } & \multirow{2}{*}{$\begin{array}{c}\text { Site } \\
\text { total }\end{array}$} \\
\hline & M1 & M2 & M3 & Total & M1 & M2 & M3 & Total & \\
\hline \multicolumn{10}{|l|}{ Chondrichthyes } \\
\hline Shark spp. & & 2 & & 2 & & & & & 2 \\
\hline \multicolumn{10}{|l|}{ Osteichthyes } \\
\hline Ariidae - Galeichthys feliceps & 6 & 3 & 5 & 14 & 8 & 7 & 68 & 83 & 97 \\
\hline Serranidae - Epinephelus andersoni & & & & & & & 1 & 1 & 1 \\
\hline Sparidae - Chrysoblephus gibbiceps & 3 & 1 & & 4 & & & & & 4 \\
\hline Sparidae - Cymatoceps nasutus & 26 & 31 & 4 & 61 & 1 & & 1 & 2 & 63 \\
\hline Sparidae - Spondyliosoma emarginatum & 2 & & 2 & 4 & & & 1 & 1 & 5 \\
\hline Dischistiidae - Dichistius capensis & & 1 & & 1 & & & 3 & 3 & 4 \\
\hline Sciaenidae - Argyrosomus japonicus & & & 1 & 1 & & & & & 1 \\
\hline Oplegnathidae - Oplegnathus conwayi & 1 & & & 1 & & & 5 & 5 & 6 \\
\hline Mugilidae - Liza richardsonii & 1 & & 1 & 2 & & $\infty$ & & & 2 \\
\hline Species indeterminate & 167 & 120 & 71 & 358 & 6 & 20 & 95 & 121 & 479 \\
\hline Total NISP & 206 & 158 & 84 & 448 & 15 & 27 & 174 & 216 & 664 \\
\hline
\end{tabular}

Some of the elements identified were from fish larger than $10 \mathrm{~kg}$, and most fish present were too large to represent roost litter from birds. Humans, rather than other agents of accumulation, are thought to have collected the fish. Poggenpoel (pers. comm.) noted that a number of the Galeichthys feliceps spines had green-stick fractures, indicating that they were broken while fresh, probably to avoid injury to those handling them. Most elements of this species were found in the excavations immediately outside the cave, where the catfish were presumably either cooked or their remains discarded. The majority of species identified can be caught with baited hooks but other methods such as trapping or spearing could also have been employed. Because a wide range of species was present, including types of fish that are not usually washed up on the shore, the authors argued that the fish were unlikely to have been acquired through scavenging wash-ups.

Poggenpoel based his identification on a wide range of elements, including teeth and spines (pers. comm.), but did not provide details of his analytical methodology in the brief preliminary report. Subsequent excavation seasons have increased the fish sample, and the whole assemblage has been examined for this thesis. 


\subsubsection{Later Stone Age fish remains}

Fish remains appear in numbers at coastal Later Stone Age (LSA) sites from the Terminal Pleistocene/Early Holocene period, around 12000 BP (Klein 1972b; Poggenpoel 1996). In the southern Cape, stone artefact assemblages dating to this period are known as 'Albany', and this term is sometimes used as a chronostratigraphic indicator. The absence of fish and other marine resources prior to this period is due to the fact that sea levels were lower, resulting in greater distances between currently visible sites and the sea. Sites that were close to the coastline at that time are now underwater (Klein 1972b, 1974; van Andel 1989a).

The earliest recorded LSA marine fish remains occur at Elands Bay Cave (EBC) on the west coast in very low frequencies at $13260 \pm 120 \mathrm{BP}$, and the most abundant fish and other marine animal remains are found in the 9600 year old layers that contain artefacts of the Albany industry (Parkington 2006; Poggenpoel 1996). At a number of other coastal sites there is a large increase in fish remains relative to mammals at the start of the Wilton period around $8000 \mathrm{BP}$, a trend that was first recognised by Goodwin at Oakhurst Shelter (Goodwin 1946; Klein 1974).

Elands Bay Cave is located on the west coast of the Western Cape and contained both Pleistocene and Holocene deposits (Parkington 2006) (Figure 3.1). The fish remains from the terminal Pleistocene layers at EBC consist predominantly of estuarine species, and the number of taxa increased as rising sea levels during the mid-Holocene brought the shore closer to the site (Poggenpoel 1996). More than 55000 fish bones were analysed from this assemblage (Poggenpoel 1996). Bone gorges, possibly used as fish hooks, are most common in the 9600 year old layers and are associated with the highest number of fish in the site $(\mathrm{MNI}=1107)$. The bone gorges will be discussed further later in this chapter in section 3.3 (Methods of Fish Capture). After an occupational hiatus between 8000 and $4300 \mathrm{BP}$, the site was reoccupied, by which time the fish species composition had changed significantly and included several species that do not enter estuaries, which Poggenpoel interprets as signifying further encroachment of the shore. Offshore species were identified and their presence attributed to scavenging of washed up specimens after cold water upwelling events which are common on the west coast. A significant decrease 
in taxa occurred approximately 1000 years ago, and only two species, Lithognathus lithognathus and Mugil cephalus, both of which are tolerant of low salinity and dependent on estuaries as nursery grounds, were present in very low numbers. Poggenpoel (1996) interprets this as an indication that the previously open estuary had closed, forming a coastal lake, which resulted in limited fishing opportunities for the inhabitants of EBC.

Identification and quantification of the EBC fish was based on a limited number of elements, selected for their robusticity (Poggenpoel 1996). These were premaxillae, maxillae and dentaries to identify Sparidae, basioccipitals and first vertebrae for Mugilidae and otoliths for Aridae. A total of 21 elements were selected for identification and their frequency per layer listed for all species combined. Maxillae of modern Lithognathus lithognathus were measured and the relationship between maxilla length and fork length was determined and the equation applied to the archaeological specimens. Small L. lithognathus frequent estuaries whereas larger ones seldom do. The reconstructed sizes of the archaeological specimens substantiated Poggenpoel's earlier interpretation that the older layers contained more estuarine fish. Barium/strontium ratios of modern specimens were compared with samples from EBC and confirmed that fishing occurred at the estuary mouth during the terminal Pleistocene.

Poggenpoel (1996) compared MNI counts based on the most frequently occurring elements with the numbers of vertebrae from EBC. The majority of layers younger than $4300 \mathrm{BP}$ showed a deficit of vertebrae, and most dating to the terminal Pleistocene displayed a surplus. The surplus could indicate that fish were decapitated and perhaps dried prior to being transported to the cave, which was 10 to $15 \mathrm{~km}$ from the shore at that time. A large deficit of vertebrae at $9600 \mathrm{BP}$, when the coast was nearer, has been interpreted as an indication of fish processing at the site, entailing beheading and drying, after which the trunks with the vertebrae intact were exported from the cave.

Tortoise Cave (TC) is a small shelter located on the bank of the Verlorenvlei, four kilometres inland from the coast (Poggenpoel 1996) (Figure 3.1). It was intermittently occupied between 7700 and 1800 BP (Jerardino 1993). It contains a large quantity of fish $(\mathrm{NISP}=>37000)$ and changes in species composition after $3400 \mathrm{BP}$ suggests that the 
Verlorenvlei had changed from estuarine conditions to a coastal lake. The largest number and variety of fish occur in the layers dated to between 3400 and 4200 BP. Vertebrae are under-represented throughout the sequence, and Poggenpoel (1996) believes that this was a processing site where the trunk region including vertebrae was transported elsewhere. Species identification was based on maxillae, premaxillae, dentaries, basioccipitals, first vertebrae and otoliths. Quantification was based on jaw bones for Sparidae, basioccipitals for Mugilidae and otoliths for Ariidae. A total MNI of 986 fish from nine species were identified.

Spring Cave (SC), located slightly inland from EBC, contains deposits dating between $\sim 460$ and 3890 BP (Poggenpoel 1996) (Figure 3.1). The fish remains from two squares were analysed, yielding eight species with a MNI of 69, among which Lithognathus lithognathus and Mugil cephalus occurred most frequently. The species composition indicates a change through time from mixed marine and estuarine fishing in the older layers to the utilization of closed estuarine systems in the younger layers. These findings match those observed at EBC and TC during the same time period and supports Poggenpoel's argument that the Verlorenvlei estuary was closed at that time. As in the late Holocene deposits of EBC and TC, a large deficit of vertebrae was noted, suggesting that the same processing methods were employed at all three sites (Poggenpoel 1996).

The LSA layers of Diepkloof Rock Shelter date between 390 and 1590 BP and contains preserved shellfish, seal and fish along with terrestrial vertebrates. Only two fish species were identified, Lithognathus lithognathus and Mugil cephalus, both in very low numbers (total MNI=14) (Poggenpoel 1996). The volume of material from which these 14 fish were identified is not known. Identification of the former was based on maxillae and dentaries, and the latter on supraoccipitals and parasphenoids. The bones were very fragmented and measurement was not possible. Both species can tolerate very low salinities and Poggenpoel (1996) believes that they were caught in the upper reaches of the Verlorenvlei in front of the cave.

A small quantity of fish $(\mathrm{MNI}=18)$ was recovered at Pancho's Kitchen Midden (PKM), located under a small rocky overhang on the west coast close to EBC (Jerardino 1998) (Figure 3.1). The site consists of a series of superimposed shell middens dating to 
between $~ 3570$ and 570 BP. The majority of fish present (identified by C. Poggenpoel) were sub-adults of species that frequent estuaries, suggesting that the inhabitants were fishing in the middle reaches of the nearby Verlorenvlei. Fish size was reconstructed using ultimate vertebrae, but no information was given on which species were measured. Fish remains were most common in the 2940 BP layer, but were present in very low numbers throughout the sequence. The species identified, in order of decreasing abundance, were Lithognathus lithognathus, Mugil cephalus, Rhabdosargus globiceps and Galeichthys feliceps.

Duiker Eiland (DE) is one of a number of shell middens located on the Vredenburg Peninsula (Figure 3.1). It is a shallow $(12 \mathrm{~cm})$ midden that dates to between $1700 \pm 50$ and $1930 \pm 70$ BP (Robertshaw 1979). Two species of fish, Liza richardsonii, $(\mathrm{MNI}=9)$ and Pachymetopon blochii $(\mathrm{MNI}=37)$, were recovered from a $3 \mathrm{~m}^{2}$ area excavated to a depth of 12cm (Poggenpoel 1996). Fish bone was in poor condition and fragmented due to exposure to the elements. Otoliths were the best preserved elements of P. blochii and quantification was based on these. MNI calculation for L. richardsonii was based on parasphenoids. Vertebrae were underrepresented relative to cranial elements and interpreted as an indication that the trunk region was removed and taken elsewhere (Poggenpoel 1996).

Paternoster Midden (PM) is located on the Vredenburg Peninsula to the south of Duiker Eiland (Robertshaw 1977) (Figure 3.1). The material dates to between $\sim 3100$ and 855 BP. Only one species, Pachymetopon blochii, was identified throughout the sequence (Poggenpoel 1996). Species identification was based on mandibles (elements of the jaw apparatus was referred to as mandibles in Poggenpoel 1996) and quantification on the most common cranial element, the endopterygoid. Poggenpoel (1996) increased the original MNI derived from endopterygoid bones by pairing these elements according to size before recalculation. He listed the differences in MNI values obtained using various P. blochii elements of this assemblage, illustrating that quantification based on jaw bones produced an MNI of 14, whereas the endopterygoid bones produced a much higher MNI of 72. Poggenpoel (1996) calculated the average size and weight of P. blochii from the PM and DE middens based on measurements derived from 116 modern specimens. The elements used for calculation were basioccipitals, first vertebrae, endopterygoids and 
mandibles (sic). The Paternoster specimens were consistently smaller than those found at Duiker Eiland, which he believes relates to differences in the coastal topography between the sites as larger P. blochii frequent deeper water.

Stofbergsfontein (SBF) is a shell midden located on the shore of the Langebaan Lagoon with a date of $1550 \pm 55 \mathrm{BP}$ (Robertshaw 1978) (Figure 3.1). Most of the species identified occur in the lagoon today, but the presence of Pachymetopon blochii and marine shellfish indicates that the inhabitants also exploited coastal resources (Poggenpoel 1996). Liza richardsonii is the most frequently occurring species in the midden, and is present in large numbers relative to the other species (MNI=277). Poggenpoel (1996) believes that these were caught in the lagoon by mass trapping methods such as stone walled fish traps, as three of these have been located in the vicinity. Robertshaw (1978) suggested that they could have been caught with nets, as they are still caught in the area today. Poggenpoel used basioccipitals and first vertebrae to identify L. richardsonii and dentaries and premaxillae for identification of the other species.

Smitswinkelbaai Cave (SWB) is located on the western side of False Bay and contains multiple layers of superimposed shell middens that date to between $\sim 1420$ and $1175 \mathrm{BP}$ (Poggenpoel 1996; Poggenpoel and Robertshaw 1981) (Figure 3.1). Fish are present throughout the sequence and are most common in the upper layers. The most common species present is Pachymetopon blochii followed by Cymatoceps nasutus. The species present are seldom caught in traps or netted, and all occur in the natural habitat in front of the cave, which Poggenpoel believes is probably where they were caught using hook and line technology. A bone splinter that was pointed at both ends and which had been ground in one area was recovered and identified by the authors as a potential fish gorge. The majority of layers show a deficit of vertebrae, which could indicate that fish were processed at the site and then transported elsewhere. Poggenpoel (1996) demonstrated that by including elements other than premaxillae and dentaries for identification, MNI counts can be increased significantly. The MNI calculated for fish in layer 5, for example, was 84 based on premaxillae and dentaries, but 105 based on endopterygoid counts. The total MNI in the site calculated on multiple elements increased from 381 to 418. 
Rooiels Cave (RC) is situated on the eastern side of False Bay next to the Rooiels River and approximately $2 \mathrm{~km}$ from the coast (Poggenpoel 1996) (Figure 3.1). The oldest occupational layer dates to $6100 \pm 120 \mathrm{BP}$; no other dates are available. The cave contains a large quantity of fish, most of which occur in the upper undated layers. The species composition is similar to that at Smitswinkelbaai Cave with Pachymetopon blochii and Cymatoceps nasutus occurring most frequently. The majority of species are exclusively marine inhabitants, indicating that most fishing took place at the coast despite the close proximity of the river to the cave. A deficit of vertebrae was recorded in the lower occupation layers, and a surplus in the upper layers. It was suggested that the surplus of vertebrae indicates that many of the fish were processed elsewhere, possibly at the coast two kilometers distant, and only the trunk regions containing vertebrae were brought to the cave. Estimates of MNI based on jaw bones $(n=274)$ were much lower than those based on cranial bones $(n=418)$. Poggenpoel (1996; Table 7:2) did not specify what elements or species provided the increased MNI counts.

Gordon's Bay Midden (GBM) on the eastern side of False Bay on the Cape Peninsula, contains fish in layers dated to 2700 - 3220 BP (van Noten 1974) (Figure 3.1). A total of 12905 fragments of fish bone weighing $1081 \mathrm{~g}$ were recovered from an approximately $3.7 \mathrm{x} 1.2 \mathrm{~m}$ trench dug to $3.7 \mathrm{~m}$ depth. Fish occur throughout the sequence, but are more common in terms of fragment numbers and weight in the lower levels. The only fish identified to species was adult and juvenile Rhabdosargus globiceps. Unidentified shark and Sparidae were also recorded. The ichthyologist Penrith based her species identification on a wide range of elements, and listed the number of fragments per element per species, but did not calculate MNI.

The LSA sequence at Die Kelders 1 (DK) consists of a number of stratified shell midden layers that accumulated between 2000 and 1500 BP (Avery et al. 1997; Schweitzer 1979). Poggenpoel identified at least eight species of fish from 4761 specimens (NISP), but the assemblage is heavily dominated $(94.5 \%)$ by Pachymetopon blochii throughout the sequence (Schweitzer 1975; Schweitzer 1979). Pachymetopon blochii live in dense kelp beds that occur on the steep rocky coast in front of the cave. Alternately, this species might have been purposely targeted (Schweitzer 1979). The steepness of the offshore environment precludes the use of intertidal traps in the immediate vicinity of the cave, 
and the wide range of sizes present suggests that both line fishing and nets were employed. Dentaries and premaxillae were used for identification and quantification as these were the best preserved elements.

Byneskranskop Cave 1 (BNK1) is located approximately 10km southeast of Die Kelders 1 and 6km inland from the coast (Schweitzer and Wilson 1978, 1982) (Figure 3.1). The deposit consists of occupation layers separated by hiatus periods and dates to between $\sim 13000$ and 255 BP. Eight bone gorges were recovered from the >8500 year old layers which will be discussed below in section 3.3 (Methods of Fish Capture). Utilization of marine resources, including fish, increased significantly after 6500 - 6000 years ago, and almost all fish bone occurs in the Wilton layers. This increase probably relates to rising sea levels bringing marine resources closer to the cave at this time (van Andel 1989a). Poggenpoel analysed the fish remains. As at Die Kelders 1, Pachymetopon blochii is the dominant species present, and accounts for $34.7 \%$ of the total fish (MNI=265) in the assemblage, followed by Rhabdosargus globiceps and Lithognathus lithognathus. Nine other species were identified but at much lower frequencies. Poggenpoel reconstructed lengths of L. lithognathus from BNK1 using dentaries and compared the sizes with those from Elands Bay Cave and modern estuarine specimens. The BNK1 specimens were consistently larger, indicating that they were caught at sea rather than in estuaries. No details were given on which elements were used for identifications and quantification. A sample of the Blombos Cave LSA fish remains that date to younger than 2000 years was analysed by van Niekerk (2005). All elements were considered for identification to species, and those that were used for identification listed for each species. No significant changes in species composition over time were noted, but densities decreased in the younger layers. A few more species were present in the older layers, but in low numbers, and this could be a function of the larger sample size in those layers. The most frequently occurring species in the assemblage was Liza richardsonii, but the majority of species present were Sparidae (Table 3.5). The ratio of vertebrae to cranial elements indicated a consistent shortfall of vertebrae. This could indicate that fish were processed at the site and the vertebral column attached to the trunk taken elsewhere, but as the remains are from a sample only, this pattern could be a function of sampling or preservation (Table 3.4). The diverse range of elements used to calculate MNI differed between species. Fish 
densities per layer (MNI and NISP) were calculated by volume excavated to allow for comparison between units of different sizes. An additional sample of fish from the LSA layers has been studied for this thesis and combined with the results of this previous work.

Table 3.5. MNI and NISP of fish identified from a sample of the Blombos Cave Later Stone Age assemblage by van Niekerk (2005)

\begin{tabular}{|c|c|c|c|c|c|c|}
\hline LAYER & 1 & 2 & 3 & 4 & 5 & TOTAL \\
\hline Taxa & MNI/NISP & MNI/NISP & MNI/NISP & MNI/NISP & MNI/NISP & MNI/NISP \\
\hline \multicolumn{7}{|l|}{ Chondrichthyes } \\
\hline Shark spp. & - & - & $1 / 2$ & - & - & $1 / 2$ \\
\hline \multicolumn{7}{|l|}{ Osteichthyes } \\
\hline \multicolumn{7}{|l|}{ Serranidae } \\
\hline Epinephelus marginatus & $1 / 1$ & - & $2 / 2$ & $4 / 16$ & $2 / 2$ & $9 / 21$ \\
\hline \multicolumn{7}{|l|}{ Sparidae } \\
\hline Chrysoblephus cristiceps & $2 / 3$ & $5 / 6$ & $1 / 7$ & $15 / 108$ & $6 / 31$ & $29 / 155$ \\
\hline Chrysoblephus laticeps & $1 / 1$ & $2 / 3$ & $4 / 13$ & $13 / 72$ & $6 / 27$ & $26 / 116$ \\
\hline Cymatoceps nasutus & $2 / 3$ & $1 / 1$ & $3 / 5$ & $6 / 24$ & $2 / 9$ & $14 / 42$ \\
\hline Diplodus capensis & - & - & - & $2 / 3$ & $2 / 9$ & $4 / 12$ \\
\hline Gymnocrotaphus curvidens & - & - & - & $2 / 8$ & $1 / 3$ & $3 / 11$ \\
\hline Pachymetopon blochii & - & - & - & $4 / 9$ & $3 / 6$ & $7 / 15$ \\
\hline Petrus rupestris & - & - & $1 / 1$ & - & $1 / 1$ & $2 / 2$ \\
\hline Sparodon durbanensis & - & - & - & - & $2 / 3$ & $2 / 3$ \\
\hline Spondyliosoma emarginatum & $2 / 2$ & $2 / 2$ & $3 / 7$ & $12 / 68$ & $5 / 21$ & $24 / 100$ \\
\hline Sparidae spp. & $14 / 52$ & $16 / 48$ & $40 / 184$ & $106 / 1091$ & $30 / 384$ & $207 / 1775$ \\
\hline \multicolumn{7}{|l|}{ Mugilidae } \\
\hline Liza richardsonii & $2 / 13$ & $4 / 10$ & $7 / 55$ & $41 / 374$ & $10 / 96$ & $64 / 548$ \\
\hline Species Indeterminate & $2 / 6$ & $1 / 5$ & $11 / 24$ & $44 / 69$ & $18 / 44$ & $76 / 148$ \\
\hline Total excluding vertebrae & $27 / 79$ & $31 / 76$ & $73 / 300$ & $249 / 1856$ & $88 / 639$ & $468 / 2950$ \\
\hline No. unidentified vertebrae & 87 & 58 & 292 & 1454 & 435 & 2326 \\
\hline Total & $27 / 166$ & $31 / 134$ & $73 / 592$ & $249 / 3310$ & $88 / 1074$ & $468 / 5276$ \\
\hline
\end{tabular}

A number of small LSA sites in the vicinity of Blombos Cave have been excavated by Henshilwood (1995; 2008), and the fish remains analysed by Cedric Poggenpoel. These include Blombosfontein (BBF) 1, 2, 6 and 7. Blombosfontein 1 and 2 are open sites located slightly inland from the coast that have been radiocarbon dated on shell to $\sim 6960$ and 6181 BP respectively (Figure 3.1). Bone preservation was poor at both of these sites, but some fish were present. Most of the species present are Sparidae, and a total MNI of nine was recorded at $\mathrm{BBF} 1$ and four at BBF2. Diplodus capensis was the most frequently occurring species. Other species identified at BBF 1 are Dichistius capensis, Diplodus hottentotus, Lithognathus lithognathus and Sparodon durbanensis. The fish assemblage of BBF1 was reanalysed in this study to determine which elements were best preserved. The BBF6 midden is located close to the shore and has been radiocarbon dated on shell to between $~ 3630$ and 4070 BP. Twenty three fish were identified from a 50x50cm column 
sample. The majority of fish occurred in the younger levels. The most frequently occurring species throughout the sequence is Cymatoceps nasutus, followed by Diplodus capensis, Spondyliosoma emarginatum, $S$. durbanensis and Petrus rupestris. This assemblage has been reanalysed for this thesis. BBF7 is an open site dating to $\sim 3100 \mathrm{BP}$, approximately $500 \mathrm{~m}$ from the coast with very poor bone preservation. Three individual fish were identified, all Sparidae. Poggenpoel used all body parts for identification but did not specify which elements provided the MNI counts.

No fish remains were recovered from the Blombosfontein sites BBF3, ( 5960 - 4322 BP), BBF4 ( 5680 - 3985 BP), BBF5 ( 5520 - 3802 BP) and BBF9 ( 480 - 1443 BP). Bone preservation in general was very poor in all but BBF9, and the absence of fish at these sites could relate to this. At BBF9, a small shelter located close to the coast, the condition of the macromammal bone was sufficient for species identification but the preservation of the micromammalian fauna was too poor for analysis. Fish remains appear to be totally absent from this site.

Nelson Bay Cave (NBC) is a large cave situated on the Robberg Peninsula 550km east of Cape Town that was intermittently occupied from $~ 125000$ to $\sim 455$ years ago (Butzer 1973; Inskeep 1972, 1987; Inskeep and Vogel 1985; Klein 1972a) (Figure 3.1). It is situated close to Hoffman's/Robberg Cave, which is one of the assemblages examined for this study. The Pleistocene and early Holocene deposits were excavated by Klein, and the material dating from about 5900 years ago and younger by Inskeep. The first appearance of fish is in the layer dated to $11500 \mathrm{BP}$, associated with artefacts signifying the beginning of the Albany industry (Klein 1972b). Cedric Poggenpoel (Inskeep 1987; Poggenpoel 1996) analysed the fish remains from both the Klein and Inskeep excavations, and based his identification and quantification on premaxillae, dentaries, basioccipitals, first vertebrae and otoliths.

The absence of fish in the older layers is ascribed to the greater distance of the cave from the shore prior to 11500 years ago (Klein 1972a, b). The initial fish assemblage consisted of four species, Rhabdosargus globiceps, Diplodus capensis, Lithognathus lithognathus and Dichistius capensis, which remained unchanged until 9000 years ago. Rhabdosargus globiceps was the most common species, but all occurred at very low frequencies 
(Poggenpoel 1996). After 9000 BP, in layers associated with the Albany industry, there was a significant increase in species numbers and frequencies, with six additional species present. Large numbers of bone gorges were present, but disappeared after 8500 years ago (Klein 1974). After 8500 years ago a wider range of fish were exploited, including a number of smaller species, such as Diplodus hottentotus, Pachymetopon blochii, Argyrozona argyrozona and Lithognathus mormyrus. In addition, the size of $R$. globiceps decreased relative to those in the older layers. Some of these smaller species are seldom caught on hooks, and Poggenpoel (1996) suggested that this signified a change in fishing technique from line fishing to the use of nets or tidal traps. These smaller species do not replace the species found in the earlier layers, therefore they more likely indicate an expansion of methods employed for fishing, rather than a replacement of the previous techniques.

The fish data from Inskeep's (1987) excavations of material dating to 5900 years and younger were presented in terms of raw numbers per unit as well as in densities per unit, i.e. numbers per square foot, to allow for comparison of fluctuations in fish numbers between units of different sizes. He could not calculate density per volume, as volume data was not available for all units, and densities were therefore given per area (Inskeep 1987). Fish weight was calculated by multiplying the number of fish recorded (MNI) with the mean weight per species to determine the meat contribution of each species per layer, which was then converted to fish weight per square foot (Inskeep 1987; Poggenpoel 1984).

Fish numbers at NBC decreased gradually between 5900 to 3300 years ago, but a more diverse range of species were taken (Inskeep 1987; Inskeep and Vogel 1985). These include the introduction of additional small species such as Liza richardsonii, Sarpa salpa and Clinidae/Blenniidae. The latter occurred at high frequencies throughout the sequence once they appeared, and made a sizeable contribution in terms of meat weight, considering that these are very small fish.

The transition from the Wilton to the Post-Wilton occurred at $3300 \mathrm{BP}$ at NBC, characterised by substantial changes in technology in terms of artefact type, raw material use and some aspects of subsistence. After 3300 years ago, informal macrolithic artefacts, 
usually made of quartzite, replaced microlithic tools made on fine-grained raw materials. Marine foods became much more important, signifying a change in economy and increased focus on food sources in the direct vicinity of the cave (Inskeep 1987). Similar changes have been recorded at other coastal sites during this time period (Binneman 1996; Döckel 1998).

After 3300 BP fish species and frequency increased significantly, with eight times more fish in these layers relative to the early Holocene. The weight of fish per unit area in the post $3300 \mathrm{BP}$ layers is almost double that of the older layers (Inskeep 1987; Poggenpoel 1996). The most common species in these layers are Sarpa salpa, Seriola lalandi and Lithognathus lithognathus, and these occur at much higher numbers than in the preceding time period. The frequency of Diplodus capensis, Pomatomus saltatrix and Rhabdosargus globiceps declined significantly after 3300 BP.

Three possible bone gorges were found in layers dating to $\sim 3000 \mathrm{BP}$ at NBC. A few grooved shale sinkers were found in the layers dating to $\sim 4520$ and $3350 \mathrm{BP}$, but the vast majority (122 out of 138 ) occur in the $<3300$ year old layers, at the same time that the fish remains increase significantly (Inskeep and Vogel 1985; Ludwig 2005). A single quartzite sinker with an age between 6000 and 9000 BP was found during Klein's excavation (Deacon 1978; 1984b). Sinkers are discussed in further detail under methods of fish capture below.

Some evidence for possible processing of fish at NBC was found in the form of a few concentrated patches of fish scales. As these were not common, Inskeep (1987) considered that they could signify activities that did not form part of normal daily food processing, and some fish might have been scaled prior to drying or smoking.

The fish remains from two shell middens near Storms River Mouth in the Tsitsikamma National Park were first analysed by the ichthyologist J.L.B. Smith. The middens are referred to as Storms River 1 and 2 (SRM1 and SRM2), and SRM2 is also known as Swartrif midden (Figure 3.1). These middens are undated but believed to be older than 2000 years (Deacon 1970). The species composition differed between the sites, with the Swartrif assemblage dominated by one fish, Diplodus capensis, whereas SRM1 contained a range of species. Smith used dentaries and maxillae to calculate MNI at both sites. 
Poggenpoel (1996) reanalysed the remains of SRM1 after noticing a lack of estuarine species, which he expected in sites located close to river mouths. By including all elements in MNI calculations, both the number of taxa and the overall number of fish were increased significantly. The reanalysis identified seven additional species, of which Liza richardsonii was the most common, contributing more than double the number of the combined total of other species. Identification of L. richardsonii was based on basioccipital bones, as jaw elements of this species did not survive well. The total MNI of fish at SRM1 was increased from 26 to 83. Poggenpoel did not reanalyse the Swartrif midden fish, but it is likely that the species composition would be similarly increased if more elements were used for identification. Two grooved stones, possible line sinkers, were recovered from Swartrif midden, and are discussed in more detail under method of capture.

Several reports on excavated coastal LSA sites mention the presence of fish but carry out little or no detailed analysis, making only observations in passing on changes in densities, species present or numbers of bones. Earlier excavation and curation methods in southern Africa differed markedly from current standards in terms of sampling and retrieval.

Faunal remains from some of these assemblages were either not retained or only samples were kept, although the fate of the fauna is not always explicitly stated in the publications. Unfortunately, some of the fish remains from earlier excavations appear to have been misplaced or lost (Schrire 1962, own observation).

At Oakhurst Shelter (OKH) in the southern Cape, located 14km inland, Goodwin (1938; 1946) noticed a marked increase in fish remains in the Wilton layers relative to the layers below (Figure 3.1). He remarked that "they increase enormously in number and variety" (Goodwin 1946:3), and proposed that this increase signified a change in fishing technology, and could indicate the first use of stone walled tidal fish traps. The majority of fish occurred in the upper layers of deposit, which was referred to as the Final Wilton or Wilton + Pottery (Goodwin 1938, 1946; Schrire 1962). Oakhurst Shelter has not been dated directly, but the presence of pottery indicates that most fish occurred in layers postdating $2000 \mathrm{BP}$. The fish remains were analysed by a museum ichthyologist who provided a brief list of the elements and species identified. The majority of species appeared to be Sparidae. This collection seemed ideal for detailed analysis, and 
considerable effort was expended to try to find the material for inclusion in this study, but it could not be located.

Sites in the southern Cape with fish remains reported but not analysed in detail include Glentyre Shelter, where fish bone increased sharply in the upper Wilton layers, but "no fish remains have survived in the collection" (Fagan 1960:88). Some fish bones are reported from Forest Hall Shelter, dated to $\sim 9770$ BP, but only a "representative collection and unusual items" of the excavated material was retained: two otoliths of kob (Sciaenidae) and two unidentified fish ribs (Wilson 1988:53). It is not clear what quantity of fish the original assemblage contained. Goodwin noted shell middens containing fish and pottery located close to stone walled fish traps at the mouth of the Slang Rivier close to Humansdorp, but no other detail was given and these were presumably never excavated (Goodwin 1946).

Matjes River Rock Shelter (MR) is a large shell midden situated approximately $14 \mathrm{~km}$ east of Nelson Bay Cave, and was intensively occupied during the last 10000 years (Figure 3.1). Some fish was reported from the site in layers younger than 7000 BP, but initial excavations in the first half of the $20^{\text {th }}$ century were crude and most of the food waste was discarded (Dreyer 1934; Hoffman 1958; Louw 1960). A subsequent limited excavation by Deacon (Döckel 1998) recovered more fish bone, at a higher density in the layers older than 7000 years than those above. The samples are small due to the limited excavation, and the fish have not been analysed.

Hoffman's/Robberg Cave (H/RC), which is one of the assemblages studied for this thesis, was first excavated by Hoffman about 50 years ago (Figure 3.1). He never published his work, but it is clear that, as in the early excavations at Matjes River, most of the food remains were discarded. The collection of excavated material curated in the National Museum in Bloemfontein includes a few large fish bones (Kyriacou and Sealy 2009). Other publications referring to this site, which was previously known as Hoffman's Cave, East Guanogat/Ghwanogat or Robberg Cave, do not mention the abundant fish remains (Deacon 1972; Fairhall et al. 1976; Klein 1974; Rudner and Rudner 1973).

A number of rock shelters were excavated in the Tsitsikamma area during the 1920s but the results never published. A subsequent attempt to locate these sites was only partially 
successful, but does report fish bone from one location, a cave at the Elandsbos River mouth (Turner 1970). Coldstream Cave is also listed in the report but no fish remains are mentioned. This author has located some of the material from Coldstream Cave, and can confirm that fish bones were in fact present at this site. This highlights the potential difficulty in discerning the presence or absence of fish remains based on older literature. Archaeological sites located along the coast further westwards with reported but unpublished fish remains include Bonteberg Shelter (BB) on the Cape Peninsula, which contains material dated to between 4505 and 2050 BP (Phillipson 1970). A total of 754 fish bones were counted but not identified to species (Maggs and Speed 1967). Steenbokfontein (SBFT) is a large cave located $1.8 \mathrm{~km}$ inland on the west coast north of EBC (Jerardino and Yates 1996) (Figure 3.1). It was intermittently occupied between $\sim 6070$ and 2200 BP. Fish are said to occur infrequently, but have not yet been quantified. Mike Taylor's Midden (MTM) is a megamidden located on the west coast south of EBC (Jerardino and Yates 1997). The deposit dates to between 2270 and 985 BP. Fish occur in low numbers and the remains from three squares were quantified, but not identified to species (total MNI $=11$ ). A number of middens located along the sandy shore south of EBC that were occupied repeatedly during the last 3000 years have some fish present, but in very low quantities (Jerardino 2003).

Some coastal LSA sites with analysed fauna contain little or no fish at all. The Paapkuil Fontein (PKF) middens 4, 5, 7 and 11 near Cape Agulhas and Still Bay (SB) middens 1 and 2 near the town of Stilbaai, with ages between $\sim 4850$ and 1320 years, are dominated by shellfish with little (Still Bay) or no fish present (Paapkuilfontein) (Hine 2008; Hine et al. 2010) (Figure 3.1). The Pearly Beach and Hawston shell middens located in the vicinity of Hermanus and dating to the last 2000 years contain very low numbers of fish, with Sparidae occurring most frequently (Avery 1976).

Recent studies (Sealy 2006; Sealy and Pfeiffer 2000) on the isotopic signatures of LSA human remains in the southern Cape have shown marked differences in the long-term diets of people living on the Robberg Peninsula, which includes Hoffman's/Robberg Cave and Nelson Bay Cave, and those living at Matjes River Rock Shelter some 14km to the east. The results indicate that, between 4500 and 2000 BP, Robberg inhabitants were 
eating more nitrogen-15 enriched high trophic level seafood such as seals or carnivorous fish than their Matjes River Rock Shelter contemporaries. The implication is that there were socially distinct groups occupying the landscape on a long-term basis, a situation that contradicts the widely held belief that prehistoric hunter-gatherers in southern Africa moved in mobile bands, were not sedentary, and movement between populations was fluid (Sealy 2006; Sealy and Pfeiffer 2000). The distinctly different isotopic signals also suggest that there were territorial boundaries between these groups, most probably geographic landmarks such as the Keurbooms River estuary that flows between Matjes River and the Robberg Peninsula.

The argument for increased seal consumption leading to heightened nitrogen values in the Robberg population has been challenged by Parkington (2007), citing a lack of archaeological evidence for a shift in seal exploitation at this time. It is possible that carnivorous fish or fish eating birds were the source of high trophic level marine protein for the Robberg people, rather than seals. The results from this analysis of the Hoffman's/Robberg Cave fish assemblage will help to clarify whether high trophic level fish contributed to people's diets at this time.

\subsubsection{Discussion}

The low number or absence of fish remains in MSA sites has been interpreted as an indication that MSA people lacked the technology or neurological sophistication to catch fish or exploit coastal resources fully, and therefore were not behaviourally modern (Avery et al. 2008; Klein 1975, 2008; Klein and Cruz-Uribe 1996, 2000b; Volman 1978). The lack of fishing gear in the form of bone gorges and stone sinkers as seen in some LSA sites is often quoted as an indication that MSA people lacked the technology to fish (Avery et al. 2008; Klein 1974, 2001, 2008; Klein et al. 2004; Volman 1978). Others believe that MSA people did not fish because they ranked fish lower than other available protein sources, not because they lacked the cognitive capacity to recognise fish as a food source or the technological know-how to catch them (Deacon 1989, 1995).

Based on the current faunal data, there clearly is a paucity of fish remains in South African MSA archaeological sites. Much of this apparent absence could be due to poor preservation of organic material in these older assemblages as there are very few MSA 
sites along the coast with good organic preservation. An unknown number of sites that would have been inhabited on the coastal plain when sea levels were lower than the current position are now invisible.

Fish remains are considerably more common in LSA sites, and there is little doubt that these people were accomplished fishers. Fish bones first appear in small numbers in LSA assemblages around 13000 years ago, but their absence prior to this date does not imply that fishing was not practiced earlier. Older LSA sites were further from the shore, since they date from periods of lowered sea level.

In several instances the species compositions reflect the habitat that was immediately available to the fishers, as evidenced by the changes in exploitation of estuarine to marine species as the conditions at the Verlorenvlei on the west coast changed. The same appears to be true at sites such as BNK1, DK1, SWB, DE and PM where Pachymetopon blochii is the predominant species. At these caves and middens the morphology of the coast in the immediate vicinity of the sites creates deeper pools with kelp forests close to the shore, the preferred habitat of this species. At some sites there is evidence that people fished further afield, for example at Rooiels Cave where the inhabitants predominantly fished at the coast despite the site's location slightly inland and close to the river, where one could expect more estuarine species, and at SBF where people fished at both the lagoon in front of the site and at the coast. Fishing at locations beyond the immediate vicinity of a site could indicate a preference for certain species, or alternately the fishers were combining fishing with shellfish gathering expeditions to the coast. The relatively low numbers of estuarine species at NBC suggests that these species were not specifically targeted but perhaps caught during infrequent visits to a nearby estuary (Inskeep 1987).

Not all coastal LSA sites contain large numbers of fish, and some contain very little or no fish at all. The largest assemblages analysed to date are from cave sites, and in general the open sites contain considerably less fish remains. This can in some cases be attributed to poor sampling methods, lack of reporting or issues of preservation. As a result it is difficult to assess the relative importance of fish in the subsistence strategies of the people who created these assemblages. There are, however, several coastal LSA assemblages where fishing did not appear to feature strongly in the occupants' 
subsistence regime. These tend to be open sites, but include at least one large cave, Steenbokfontein, which reportedly contains very few fish remains.

Lack of chronological continuity at most LSA sites makes it difficult to pinpoint whether there was a universal temporal shift towards more intensified fishing. The apparent sudden increase of fish at the start of the Wilton period as recorded at Oakhurst and BNK might be a reflection of rising sea levels making coastal resources more accessible to the inhabitants of these sites, which are currently situated slightly inland. At NBC, which contains one of the most complete and continuous LSA sequences, fish start appearing earlier than in the aforementioned sites. Its closer location to the coast and the steep offshore coastal topography in this area means that rising sea levels would have brought coastal resources closer to the cave at an earlier date. As at Oakhurst and BNK, fish remains increased at NBC during the start of the Wilton period, after which numbers at NBC decreased gradually until 3300 years ago, when fish numbers once again increased significantly. This abrupt increase in fish exploitation after $3300 \mathrm{BP}$ at NBC is not as evident at other coastal sites. Sites on the west coast that were occupied during this time period with substantial fish remains such as EBC and TC do not show the same intensification in fish procurement relative to earlier periods. The absence of similar evidence for intensification in the southern Cape currently appears due mostly to either a lack of excavated sites that were occupied during this period, or to uncertainties relating to the dating or abundance of fish in sites that were occupied at this time.

A methodological concern which complicates interpretation and comparison of data within and between assemblages is the general absence of calculation of density values per layer. An apparent abundance of fish can be misleading if the volume of excavated material differs significantly between layers or sites. Data from two of the abovementioned assemblages were analysed using density values to allow inter-site comparison - in one instance density of fish per square foot (Inskeep 1987), and in the other density of fish per cubic meter (van Niekerk 2005). The data from all the other assemblages listed above were presented in terms of raw numbers. Without knowledge of the actual volumes excavated, it is difficult to accurately identify temporal changes in frequency and to evaluate the significance of such changes. It must be acknowledged that 
it is not always possible to obtain excavation volume data to calculate densities, usually because volumes were not recorded or due to the data not being made available.

Although some mention is made of which elements were used for identification in most of the abovementioned studies of fish assemblages, the elements identified to species are seldom listed. This is largely because skeletal element representation did not form part of the research questions being addressed. In the instances where skeletal elements are pertinent to an argument, they are referred to as vertebral or cranial, with all appendicular bones included with the latter, and no distinction made between these many elements. The omission of this data is unfortunate as it is not possible to compare the results with other studies such as this one.

The interpretation of deficits or surpluses of vertebrae as evidence for fish processing in some of the LSA assemblages does not take into account other potential causal factors such as carnivore or rodent activity. As taphonomic indicators of gnawing on fish bones were not reported, it is unclear whether these signs were absent or simply not recorded, and to what extent animals could have altered the assemblages. In addition, deficits in cranial or vertebral elements could be a function of sample size. Unless a large spatial area has been excavated, and all bones included in analyses, these deficits might simply be a reflection of differential discard of fish heads and bodies within a living area.

\subsection{Species ecology and method of fish capture}

\subsubsection{Species ecology}

In order to interpret the fish species composition at a site, one needs an understanding of the ecology of each species in terms of habitat preference, diet, seasonal migration and method of capture. Differences and changes in species present within and across sites could be an indication of changes in capture technology or season of occupation. As it is not possible to determine whether sea levels and climatic changes over the last 120000 years resulted in changes in fish species' behaviour and ecology, it is assumed in this study that the ecology and behaviour of species were the same as they are presently. Differences in species composition can also be a reflection of the distribution of specific subtidal habitat types within the vicinity of the archaeological sites. The relative 
abundance and diversity of species can differ markedly depending on the reef architecture of an area (Lechanteur 2004). Water depth, reef elevation and the availability of caves for shelter all influence whether an area will be inhabited by particular fish species, and high reefs with large caves will house a greater number of species in terms of variety and density, and fish will be of larger size than on flatter, less complex reefs (Lechanteur 2004). Changes in sea levels could also affect the availability of preferred habitats. It should therefore be kept in mind that species composition can also be a reflection of what is available in the area based on subtidal morphology.

The following is a list of the fish species that are most common along the southern Cape coast and that could be expected in the archaeological assemblages analysed here (Figure 3.2). Most offshore and deepwater dwelling marine species have been omitted as these could only be captured from boats and there is no evidence for prehistoric seafaring vessels in southern Africa. Rock art scenes depicting spearfishing from small boats or rafts only occur inland, and according to historical records this technique was not practised south of the Limpopo (Breuil 1945; Willcox 1963). Data on the maximum size, diet, habitat and method of capture is included for each species.

Chondrichthyes (sharks, skates, rays) have not been included in Figure 3.2 as there are a vast number of these species in southern African waters and their ecologies differ markedly. It would not be possible to adequately describe all their potential habitats and diets in the current format. In addition, shark bones could not be identified to species in this thesis due to a lack of comparative specimens.

The few species that occur in an area only during a particular time of the year have an added note reflecting the season that they are mostly caught in the southern Cape. In general species that are more abundant during certain seasons are not a very good indicator of the season of capture, as this does not preclude their being caught during other seasons (Lamberth et al. 1995). If a seasonally abundant species is very common in an assemblage, it might be warranted to infer a particular season of capture, but not if these species only occur in small numbers. Species that can be considered to be fairly good indicators of season are Seriola lalandi and Pomatomus saltatrix, both of which undertake seasonal migrations up the east coast and are most abundant in the study area 
in summer (Lamberth et al. 1995; van der Elst 1998). Dichistius capensis is not a migratory fish (Attwood and Bennett 1994; Attwood and Cowley 2005), but does spawn in deeper water during summer (Smith and Heemstra 1995), and is more commonly caught during winter in the southern Cape (Attwood and Bennett 1990; Pradervand and Hiseman 2006).

Note: unless stated otherwise, the data in Figure 3.2 are from Branch and Beckley (1999), Froese and Pauly (2009), Heemstra and Heemstra (2004), Smith (1953), Smith and Heemstra (1995) and van der Elst (1998). 


\section{OSTEICHTHYES}

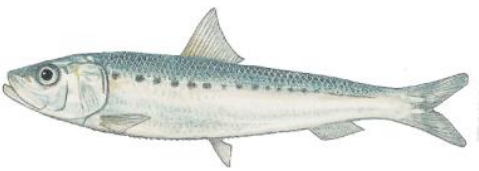

CLUPEIDAE (herrings and sardines)

Sardinops sagax (pilchard, sardine)

Maximum size: up to $30 \mathrm{~cm}$

Habitat: shoaling, offshore species

Diet: planktonic animals

Capture: killed by cold water upwelling, usually netted offshore

Notes: there are 13 species of Clupeidae in South African waters. Heavily preyed on by gamefish such as $S$. lalandi and $P$. saltatrix as well as birds, dolphins and seals

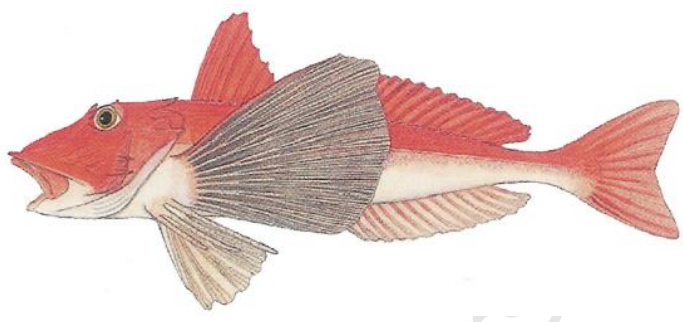

TRIGLIDAE (gurnards)

Maximum size: $70 \mathrm{~cm}$

Habitat: deep sandy bottoms

Diet: crustaceans

Capture: baited hooks, usually offshore

Notes: Seven species occur in southern Africa, they have distinctive scute-like scales and dermal plates

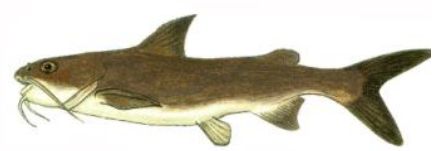

ARIIDAE

Galeichthys feliceps (white sea catfish)

Maximum size: $55 \mathrm{~cm}$

Habitat: mostly shallow but also deep coastal (up to $102 \mathrm{~m})$ water, estuaries. Juveniles $(<26 \mathrm{~cm})$ common in turbid estuaries, adult mouth-brooding males in estuaries from September to March

Diet: crustacea, fish

Capture: baited hooks, speared, stone walled fish traps (Avery 1975)

Notes: has no scales, toxic mucous coating on serrated pectoral and dorsal spines can cause painful wounds; more frequently caught at night

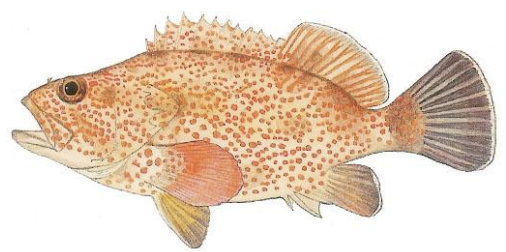

\section{SERRANIDAE (Rockcods/groupers) Acanthistius sebastoides (koester)}

Maximum size: $35 \mathrm{~cm}$

Habitat: Rocky reefs up to $25 \mathrm{~m}$

Diet: fish and crustaceans

Capture: baited hooks

Notes: solitary and sedentary species; common prey of great cormorant $P$. carbo (Randall et al. 2002)
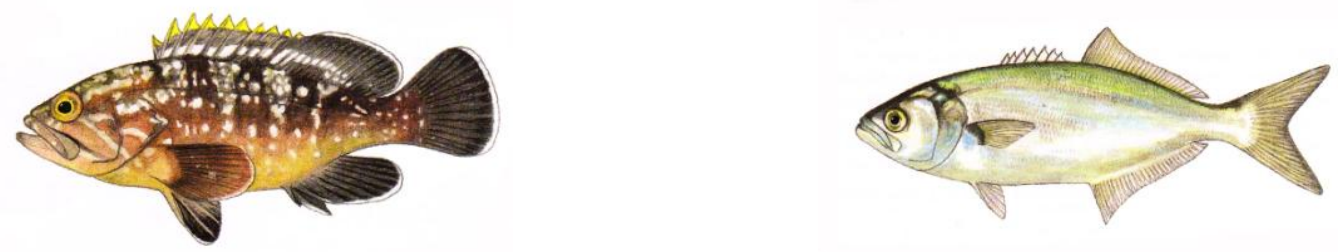
SERRANIDAE (Rockcods/groupers)

Epinephelus marginatus (yellowbelly rockcod)

Maximum size: $120 \mathrm{~cm}$

Habitat: rocky reefs up to $200 \mathrm{~m}$ depth, juveniles

$(<60 \mathrm{~cm})$ occur in tidal pools and gullies

Diet: fish, crustaceans, octopus

Capture: baited hooks and speared

Notes: some killed by cold water upwelling events

(Hanekom et al. 1989)

Season: most abundant in winter

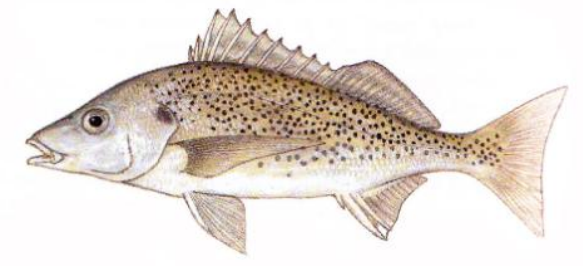

HAEMULIDAE (grunters)

Pomadasys commersonnii (spotted grunter)

Maximum size: $90 \mathrm{~cm}$

Habitat: estuaries, lagoons, shallow coastal areas,

tolerates fresh water

Diet: mussels and crustaceans

Capture: hooked or speared, usually near river

mouths in the surf

Notes: wary of noise; does not occur near rocks and

therefore unlikely to be caught by stone walled traps;

very sensitive to cold water upwelling

Season: most common in Cape during summer
POMATOMIDAE (bluefishes)

Pomatomus saltatrix (elf, shad)

Maximum size: $120 \mathrm{~cm}$

Habitat: shoaling, coastal waters including beaches and rocklands, estuaries. Juveniles $(<20 \mathrm{~cm})$ along shallow beaches in southern Cape

Diet: mostly fish, also cephalopods and crustaceans Capture: baited hooks, lures, stone walled fish traps (Avery 1975; Goodwin 1946; Kemp et al. 2009); good catches during frenzied inshore feeding Notes: usually caught during daytime with clear water; very sharp teeth can cut through (nylon) fishing line (Biden 1954); affected by cold water upwelling - large almost motionless shoals observed at river mouths (Hanekom et al. 1989);

Season: summer in southern Cape

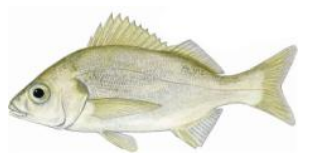

\section{HAEMULIDAE (grunters) Pomadasys olivaceum (piggy)}

Maximum size: $25 \mathrm{~cm}$, most inshore $<15 \mathrm{~cm}$

Habitat: Juveniles $(<13 \mathrm{~cm})$ in dense shoals around shallow reefs, adults occur deeper offshore reefs Diet: small shrimp, octopus, polychaete worms, hydrozoa

Capture: baited hooks, large numbers of juveniles killed by cold water upwelling 


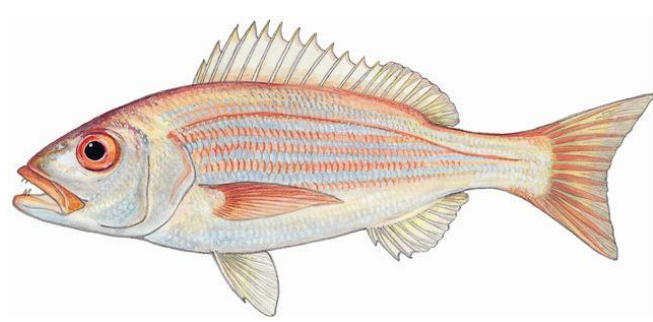

SPARIDAE (seabreams)

Argyrozona argyrozona (carpenter, silverfish)

Maximum size: $80 \mathrm{~cm}$

Habitat: adults deeper water offshore, juveniles

$(<30 \mathrm{~cm})$ nearshore in shallower water (Brouwer and

Griffiths 2005, 2006)

Diet: carnivorous: crabs, anchovies, worms, squid, amphipods

Capture: seldom landed from shore on baited hooks, mostly caught from boats

Notes: endemic, only occurs between Table Bay and northern Eastern Cape

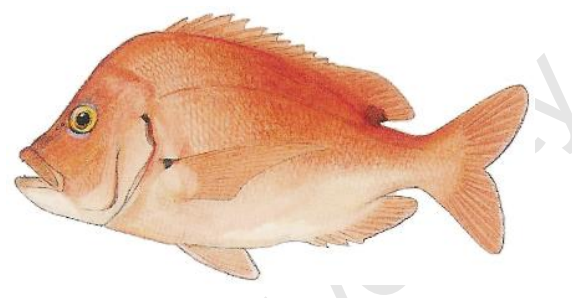

SPARIDAE (seabreams)

Chrysoblephus cristiceps (dageraad)

Maximum size: $70 \mathrm{~cm}$

Habitat: shoaling reef species, juveniles $(<37 \mathrm{~cm})$

closer inshore and sometimes in tidal pools

Diet: worms, crab, fish, sea urchins, starfish, squid

Capture: baited hooks

Notes: reportedly seldom caught from the shore

unless there are deep gullies

Season: most common during summer

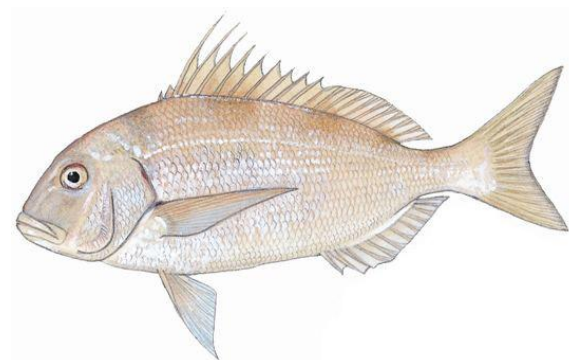

SPARIDAE (seabreams)

Cheimerius nufar (santer, soldier)

Maximum size: $75 \mathrm{~cm}$

Habitat: deep reefs and rocky bottoms, 7-130m depth

Diet: squid, fish, small crustaceans

Capture: seldom speared due to deep water habitat, baited hooks from shore in summer or when weather is stormy or during cold water upwelling events Notes: juveniles $(<34 \mathrm{~cm})$ in estuaries when storms approach, adults go inshore during cold water upwelling events

Season: summer

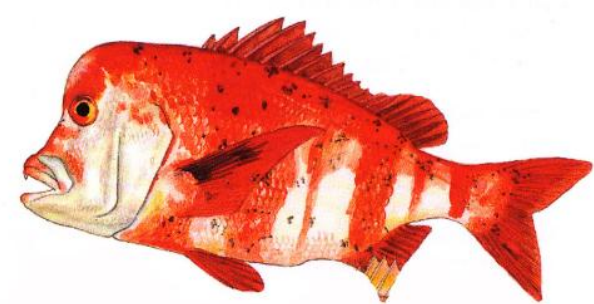

SPARIDAE (seabreams)

Chrysoblephus gibbiceps (red stumpnose)

Maximum size: $75 \mathrm{~cm}$

Habitat: adults (>30cm) over offshore reefs, 30 -

$150 \mathrm{~m}$ deep, juveniles over shallow reefs

Diet: crab, fish, molluscs, worms, octopus

Capture: difficult to hook, seldom caught from shore 


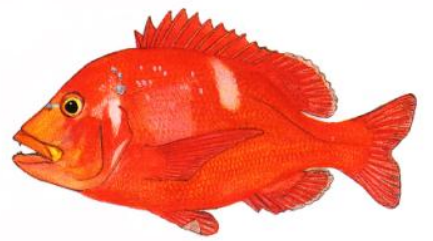

SPARIDAE (seabreams)

Chrysoblephus laticeps (roman, red roman)

Maximum size: $50 \mathrm{~cm}$

Habitat: rocky reefs up to $100 \mathrm{~m}$, adults $(>18 \mathrm{~cm})$

deeper water

Diet: fish, molluscs, sea urchins, crustaceans, worms

Capture: baited hooks, speared

Notes: reportedly very common and easily captured during the 1930's (Biden 1954); affected by cold water upwelling (Bower and Crawford 1981)

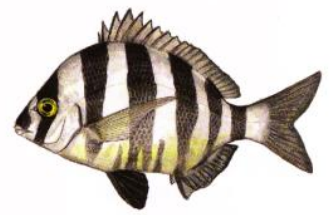

SPARIDAE (seabreams)

Diplodus hottentotus (zebra, wildeperd)

Maximum size: $60 \mathrm{~cm}$

Habitat: inshore, shallow water up to $120 \mathrm{~m}$, estuary mouths (Whitfield 2004), juveniles ( $<28 \mathrm{~cm})$ also in rock pools

Diet: omnivorous, seaweed, gastropods, fish, crustaceans

Capture: baited hooks, speared, most caught in deeper water (Pappe 1866), also found in stone walled fish traps (Avery 1975; Kemp et al. 2009) Notes: shelters in shallow water during cold water upwelling events (Hanekom et al. 1989)

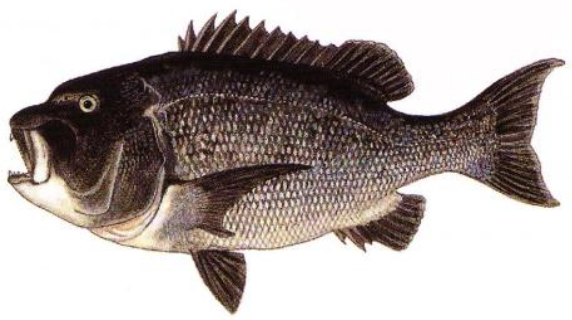

SPARIDAE (seabreams)

Cymatoceps nasutus (black musselcracker)

Maximum size: $130 \mathrm{~cm}$

Habitat: rocky reefs up to $100 \mathrm{~m}$ deep, juveniles $(<53 \mathrm{~cm})$ in shallower water

Diet: fish, urchins, molluscs, crustaceans

Capture: baited hooks, speared

Notes: very strong fighters once hooked; large specimens washed up after cold water upwelling events (Bower and Crawford 1981) and sheltering in shallow water (Hanekom et al. 1989). Fatty head was much sought after in the recent past (Biden 1954)

Season: most catches during winter

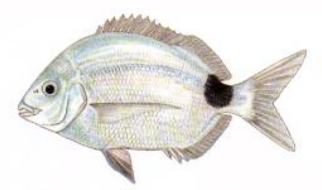

SPARIDAE (seabreams)

Diplodus capensis (dassie, blacktail) Maximum size: $45 \mathrm{~cm}$

Habitat: rocky inshore, turbulent water, juveniles $(<21 \mathrm{~cm})$ intertidal pools, estuary mouths

Diet: fish, crustaceans, gastropods, seaweed Capture: baited hooks, speared, stone tidal fish traps (Avery 1975; Goodwin 1946; Kemp et al. 2009) Notes: easily caught on light tackle especially in cloudy water; many juveniles killed by cold water upwelling events, adults shelter in shallow water (Bower and Crawford 1981; Hanekom et al. 1989) 


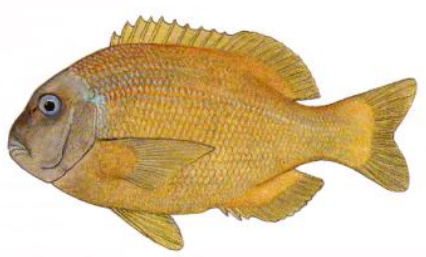

SPARIDAE (seabreams)

Gymnocrotaphus curvidens (john brown, janbruin)

Maximum size: $50 \mathrm{~cm}$

Habitat: shallow rocky areas, up to $80 \mathrm{~m}$ deep

Diet: ascidians

Capture: baited hooks, speared, not easily captured

Notes: mostly solitary

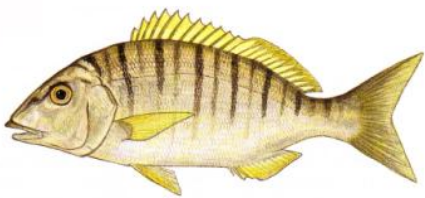

SPARIDAE (seabreams)

Lithognathus mormyrus (sand steenbras)

Maximum size: $55 \mathrm{~cm}$, most $<30 \mathrm{~cm}$ in southern

Africa

Habitat: shallow sandy areas, $10-50 \mathrm{~m}$ depth,

juveniles $(<13 \mathrm{~cm})$ shallower water near estuaries and bays

Diet: Invertebrates - shrimp, sea lice, bivalves

Capture: baited hooks

Notes: large numbers enter shallow water after cold water upwelling events (Hanekom et al. 1989)

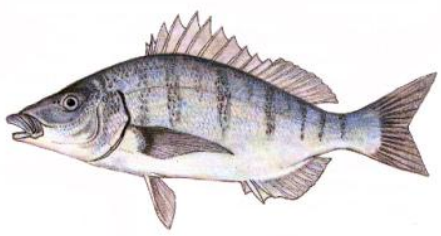

SPARIDAE (seabreams)

Lithognathus lithognathus (white steenbras)

Maximum size: $150 \mathrm{~cm}$

Habitat: adults sandy bottoms up to $25 \mathrm{~m}$, juveniles in estuaries, adolescents $(<65 \mathrm{~cm})$ surf-zones

Diet: crustaceans, molluscs and worms

Capture: baited hooks, speared, stone walled fish

traps (Avery 1975; Kemp et al. 2009)

Notes: affected by cold water upwelling, deaths as well as large numbers of 'stunned' fish lying

motionless below the water surface in estuaries reported (Hanekom et al. 1989), strong fighter once hooked. Historical records refer to large quantities being speared in an estuary in False Bay (Thompson 1913)

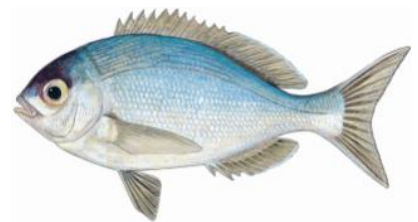

SPARIDAE (seabreams)

Pachymetopon aeneum (blue hottentot, copper bream)

Maximum size: $60 \mathrm{~cm}$

Habitat: deeper rocky reefs $20 m-75 m$

Diet: ascidians (sea squirts) and sponges

Capture: takes a variety of bait, but seldom caught or speared from shore due to preference for deeper water 


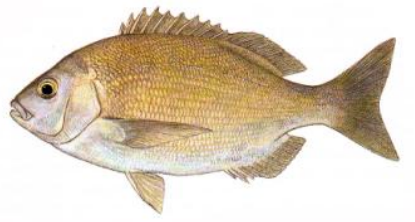

SPARIDAE (seabreams)

Pachymetopon blochii (hottentot)

Maximum size: $54 \mathrm{~cm}$

Habitat: kelp beds and rocky reefs up to $55 \mathrm{~m}$ depth

Diet: Omnivorous, adults mostly herbivorous but do

take bait

Capture: baited hooks, speared

Notes: Nieuhof 1654, (in Raven-Hart 1971:16), noted that "the Hottentots know how to catch it with a little hook", hence the common name.

A related species, $P$. grande, occurs eastwards from Knysna. A herbivore, it attains $70 \mathrm{~cm}$, inhabits shallow, rocky areas, is seldom hooked, but caught in stone walled fish traps

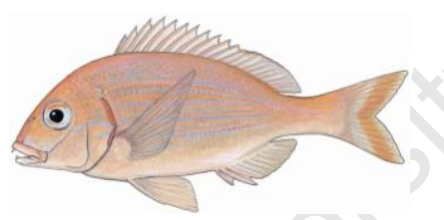

SPARIDAE (seabreams)

Pterogymnus laniarius (panga)

Maximum size: $45 \mathrm{~cm}$

Habitat: adults and juveniles shoaling in deep water between 50 and $120 \mathrm{~m}$

Diet: crabs, polychaete worms, mollusc, brittlestars

Capture: caught with baited hooks from offshore vessels

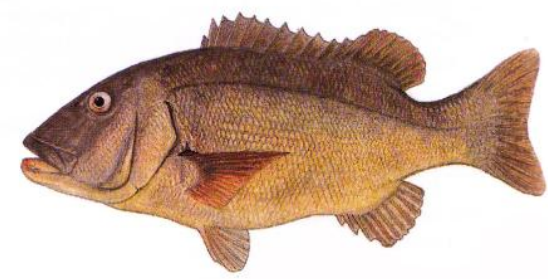

SPARIDAE (seabreams)

Petrus rupestris (red steenbras)

Maximum size: $200 \mathrm{~cm}$

Habitat: adults $(>57 \mathrm{~cm})$ deeper water over rocky

reefs, juveniles shallower reefs

Diet: fish, squid, octopus

Capture: baited hooks from shore over deep gullies, stone walled fish traps (Avery 1975; Goodwin 1946; Kemp et al. 2009)

Notes: high concentrations of vitamin A in the liver makes it toxic if eaten in large quantities

Season: most are caught during summer

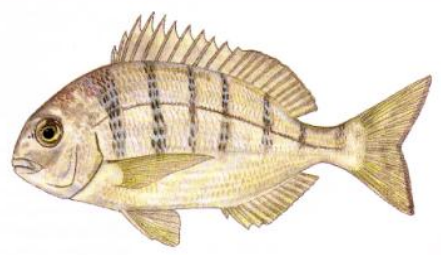

SPARIDAE (seabreams)

Rhabdosargus globiceps (white stumpnose)

Maximum size: $50 \mathrm{~cm}$

Habitat: sandy sea-beds, rocky outcrops up to $120 \mathrm{~m}$ depth. Juveniles $(<23 \mathrm{~cm})$ in estuaries and bays, surfzone

Diet: molluscs, crustaceans, worms

Capture: baited hooks from shore

Notes: most commonly caught at night

Season: most common in summer 


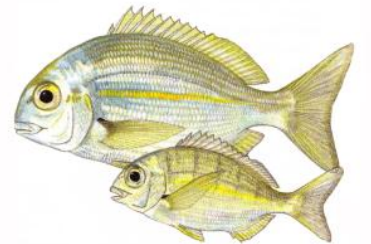

SPARIDAE (seabreams)

Rhabdosargus holubi (Cape stumpnose)

Maximum size: $45 \mathrm{~cm}$

Habitat: adults inhabit mostly marine, shallow water over rocky or sandy bottoms. Juveniles $(<19 \mathrm{~cm})$

dependent on estuaries as nurseries

Diet: crustaceans, molluscs, worms

Capture: baited hooks, stone walled fish traps (Avery

1975; Goodwin 1946; Kemp et al. 2009)

Notes: reported killed and sheltering after cold water upwelling events

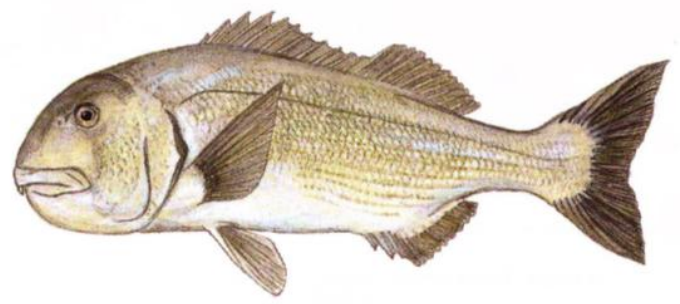

SPARIDAE (seabreams)

Sparodon durbanensis (white musselcracker)

Maximum size: $120 \mathrm{~cm}$

Habitat: rocky shores up to $30 \mathrm{~m}$ depth, juveniles $(<35 \mathrm{~cm})$ in gullies and tidal pools

Diet: mainly bivalves, also fish, worms, redbait, crabs

Capture: baited hooks or speared

Notes: the very strong jaws can straighten metal hooks, therefore strong tackle needed; affected by cold water upwellings - large almost motionless shoals observed at river mouths (Hanekom et al. 1989)

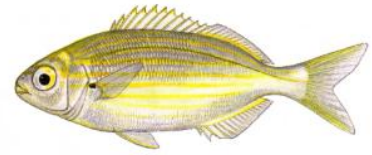

\section{SPARIDAE (seabreams) \\ Sarpa salpa (strepie, karanteen) Maximum size: $30 \mathrm{~cm}$}

Habitat: adults mostly in surf-zone, inshore reefs, juveniles $(<15 \mathrm{~cm})$ also in rock pools and estuaries

Diet: red algae

Capture: small baited hooks

Notes: shoaling species, juveniles in particular are affected by cold water upwelling events - both killed and sheltering in shallow waters/estuaries (Hanekom et al. 1989)

Season: most common in summer (Pradervand and Hiseman 2006)

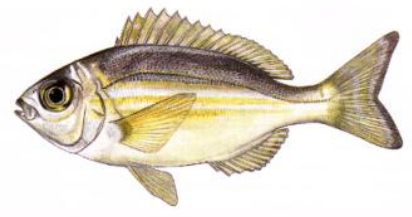

SPARIDAE (seabreams) Spondyliosoma emarginatum (steentjie)

Maximum size: $45 \mathrm{~cm}$

Habitat: rocky reefs, juveniles $(<22 \mathrm{~cm})$ sometimes in estuaries, shallow reefs

Diet: crabs, worms, amphipods, redbait

Capture: baited hooks

Notes: shoaling species; strongly affected by cold water upwelling events leading to mass mortalities 


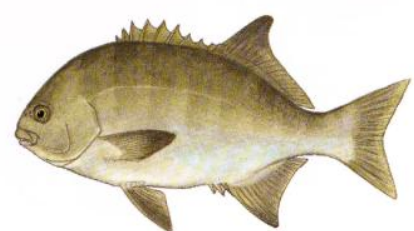

DICHISTIIDAE

Dichistius capensis (galjoen)

Maximum size: $80 \mathrm{~cm}$

Habitat: turbulent water at kelp beds, gullies and shallow rocky and sandy locations

Diet: shellfish, redbait, worms, algae, isopods

Capture: speared, baited hooks, stone walled fish traps (Avery 1975; Kemp et al. 2009)

Notes: Endemic, affected by cold water upwelling killed and sheltering (Hanekom et al. 1989); strong fighter; cooked flesh has black veins if fish is not bled straight after capture. Flesh fattiest during winter months.

Season: Most abundant in winter (Attwood and Bennett 1990; Pradervand and Hiseman 2006)

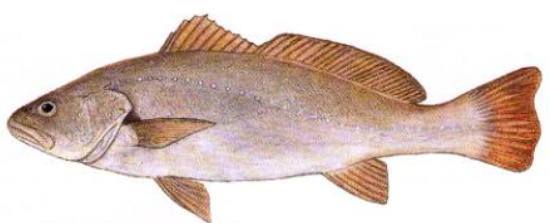

SCIAENIDAE (croakers/drums) Argyrosomus japonicus (dusky kob) Maximum size: $180 \mathrm{~cm}$

Habitat: estuaries, surf zone, deeper water up to $120 \mathrm{~m}$

Diet: fish, shrimp, squid

Capture: baited hooks from shore

Notes: A. inodorus is a closely related species that is usually only found offshore (Griffiths and Heemstra 1995). The differences between these two species are mostly internal and the exact species of kob in the comparative collection is not known. Therefore any bones identified as kob were recorded as $A$.

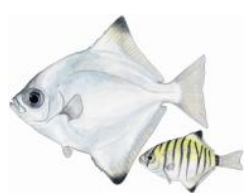

\section{MONODACTYLIDAE (moonies) Monodactylus falciformis (Cape moony, oval moony, kitefish)}

Maximum size: $25 \mathrm{~cm}$

Habitat: shallow reefs, estuaries, rivers

Diet: zooplankton, fish

Capture: can be caught on very light tackle

Notes: affected by cold water upwelling events

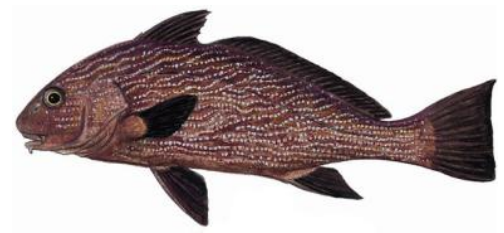

\section{SCIAENIDAE (croakers/drums) \\ Umbrina canariensis (baardman)}

Maximum size: $42 \mathrm{~cm}$

Habitat: primarily offshore, adults (>30 $\mathrm{cm}$ )

sometimes in shallow water, juveniles have been observed in tidal rock pools

Diet: benthic invertebrates

Capture: sometimes caught with baited hooks; speared in shallow water

Notes: Image is of related species, U. robinsoni 


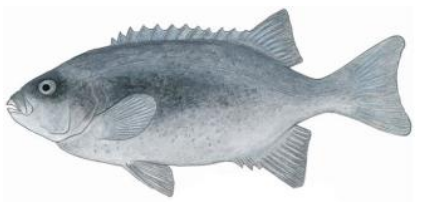

OPLEGNATHIDAE

Oplegnathus conwayi (Cape knifejaw)

Maximum size: $90 \mathrm{~cm}$

Habitat: deeper (>10m) inshore reefs, juveniles

$(<44 \mathrm{~cm})$ occur in tidal pools

Diet: omnivorous, mostly seaweed, sponges and redbait

Capture: commonly speared, seldom caught on hooks Notes: deaths from this, or closely related species, have been recorded from cold water upwelling events (Hanekom et al. 1989)

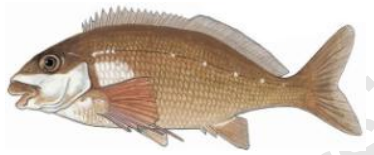

CHEILODACTYLIDAE (fingerfins) Chirodactylus brachydactylus (two-tone fingerfin)

Maximum size: $40 \mathrm{~cm}$

Habitat: shallow coastal reefs, never occurs in estuaries; juveniles common in rock pools $(<25 \mathrm{~cm})$

Diet: bivalves, crabs and amphipods extracted by sifting through sand

Capture: speared, seldom hooked

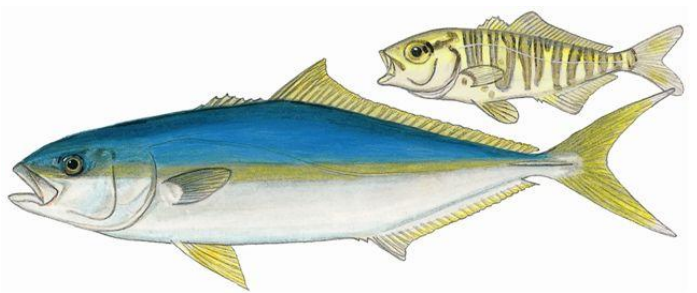

CARANGIDAE

Seriola lalandi (giant yellowtail) Maximum size: $150 \mathrm{~cm}$

Habitat: offshore, pelagic, coastal, adults mainly in warmer surface waters

Diet: fish, crustaceans, squid Capture: baited hooks and lures from deep rocky gullies, speared, netted. Occasionally caught in stone walled fish traps (Kemp et al. 2009)

Notes: shoaling predator, often associated with reef pinnacles. Adults $(>61.5 \mathrm{~cm})$ closer to shore, young in large shoals offshore

Season: most common in summer; episodes of frenzied feeding on small shoaling fish close to shore resulting in good catches are reported

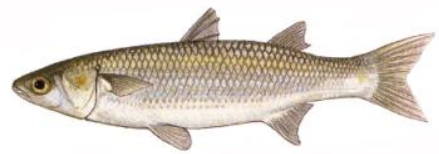

\section{MUGILIDAE}

Liza richardsonii (southern mullet, haarder)

Maximum size: $41 \mathrm{~cm}$

Habitat: dense shoals on rocky points, sandy beaches, estuaries. Young $(<20 \mathrm{~cm})$ use estuaries as nurseries Diet: diatoms, algae, zooplankton, detritus Capture: nets, large numbers in stone walled fish traps (Kemp et al. 2009)

Notes: abundant, shoaling species; seldom caught by hook, endemic, common gamefish prey 


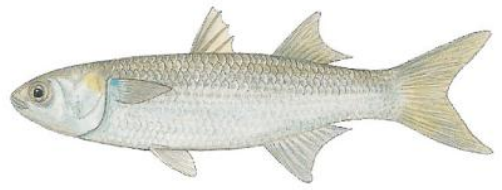

MUGILIDAE

Mugil cephalus (flathead mullet)

Maximum size: $80 \mathrm{~cm}$

Habitat: mostly estuaries, also rivers and sea, usually over muddy or sandy substrate. Juveniles $(<45 \mathrm{~cm})$

inhabit estuaries

Diet: detritus and diatoms

Capture: nets, stone walled tidal fish traps (Avery 1975; Goodwin 1946; Kemp et al. 2009), seldom hooked. Notes: abundant, in large schools of similarly sized fish; preyed on by great cormorant, $P$. carbo (Randall et al. 2002).

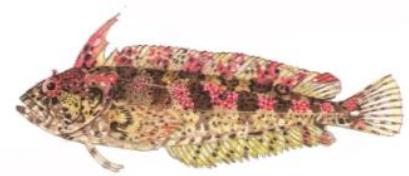

\section{CLINIDAE (klipfish)}

Maximum size: $30 \mathrm{~cm}$

Habitat: Rock pools and estuaries

Diet: carnivorous

Capture: Can be caught with small baited hooks

Notes: small species; common; at least 41 species in southern Africa; difficult to distinguish between species

\section{BLENNIIDAE (blennies)}

Maximum size: Most $<12 \mathrm{~cm}$

Habitat: rock pools, reef bottoms, shallow sand

Diet: seaweeds, crustaceans, hydrozoa, mussels

Capture: Small baited hooks

Notes: scaleless; around 45 species in southern Africa, difficult to distinguish between species; small size makes targeting by humans for food unlikely, preyed on by fish and cormorants

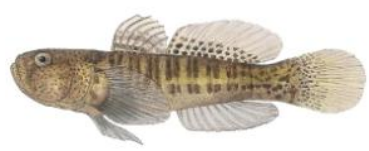

GOBIIDAE (gobies)

Maximum size: Most $<8 \mathrm{~cm}$

Habitat: rock pools, estuaries, rocky reefs, sandy or muddy bottoms, some pelagic species

Diet: crustaceans, worms, molluscs and sea urchins Capture: small nets

Notes: at least 111 species in southern Africa; difficult to distinguish between species; small size makes targeting by humans for food unlikely 


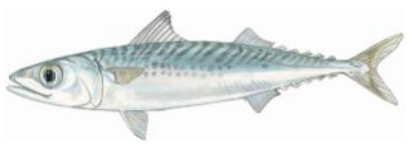

SCOMBRIDAE (mackerel)

Scomber japonicus (chub mackerel)

Maximum size: $50 \mathrm{~cm}$

Habitat: pelagic, surface waters close to shore up to

$300 \mathrm{~m}$ depth

Diet: plankton feeder

Capture: baited hooks

Notes: shoaling species

Season: winter in South

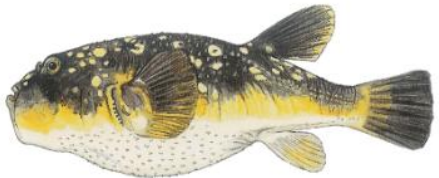

TETRAODONTIDAE (pufferfish)

Maximum size: up to $30 \mathrm{~cm}$

Habitat: bottom-dweller in shallow, sandy areas, also in estuaries and deeper reefs

Diet: small crabs, fish, invertebrates

Capture: baited hooks

Notes: the skin and viscera of pufferfish are highly toxic to humans and other mammals, but the flesh is edible, and considered a delicacy in Japan (known there as Fugu). Some predatory fish such as tuna feed on these species with no ill effect. There are over 30 species in Southern Africa

Figure 3.2. Common near-shore fish species that occur in the southern Cape today. The figure should be read from left to right. Note that Chondrichthyes have been excluded from this figure as they could not be identified to species due to a lack of comparative specimens.

\subsubsection{Methods of fish capture}

The species composition of an assemblage can provide insights as to how the fish were obtained. Fish, as with other fauna, inhabit specific ecological niches, have particular diets and behaviour, and are not all caught in the same manner. Determining how people acquired fish helps us to understand the mental and physical capabilities of hominins in the past, what technology they used and how this changed through time. Most species can be caught by a variety of means. Some fish species do however provide clearer evidence for the capture method than others. It cannot be assumed that only one method was employed at a particular time as several methods could have been used simultaneously, as witnessed by early travellers to the Cape (Lichtenstein 1930; Schapera 1930; Stow 1905). Information on the species that are caught by specific methods listed in Table 3.6 and Figure 3.2 is based on current data taken from the literature. This does not take into account that the numbers of fish were considerably higher in southern African waters prior to large scale commercial fishing, that average sizes of fish were bigger (Bennett 1991; Bennett and Attwood 1991), and that many species were probably easier to catch due to 
their abundance. Good evidence for these points is available in historical records from the late 1600s onwards (Lichtenstein 1930; Noble 1875; Thompson 1913).

Table 3.6. Methods of capture.

Fish caught in tidal stone traps within the last 60 years. $\mathrm{x}=$ reported; $\mathrm{xx}=$ large numbers; * $=$ rare; ? = exact species not certain (From Goodwin 1946; Avery 1975; Haddad 2003; Kemp 2006, 2008). Species that occur in the vicinity of BBC that were washed up after cold water upwelling events (From Hanekom et al. 1989; Bower \& Crawford 1981; * from Heemstra \& Heemstra 2004) $\downarrow=\langle 25 ; \uparrow=26$ -

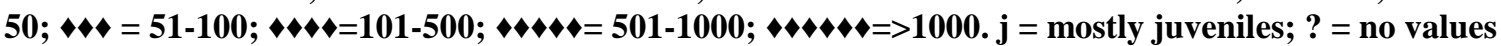
available.

\begin{tabular}{|c|c|c|c|c|c|c|c|}
\hline \multirow{2}{*}{\multicolumn{2}{|c|}{$\begin{array}{l}\text { Method of capture } \\
\text { Taxa }\end{array}$}} & \multirow[t]{2}{*}{ tidal traps } & \multicolumn{2}{|c|}{ upwelling } & \multirow[t]{2}{*}{ baited hook } & \multirow[t]{2}{*}{ speared } & \multirow[t]{2}{*}{ netted } \\
\hline & & & killed & shelter & & & \\
\hline \multicolumn{8}{|l|}{ Chondrichthyes } \\
\hline & Shark spp. & $\mathrm{x} ?$ & & & + & & \\
\hline Osteichthyes & & & & & $\infty$ & & \\
\hline Clupeidae & Sardinops sagax & & 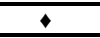 & & 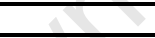 & & + \\
\hline Ariidae & Galeichthys feliceps & $\mathbf{x}$ & & & + & + & \\
\hline Serranidae & Acanthistius sebastoides & & & & + & & \\
\hline Serranidae & Epinephelus marginatus & & 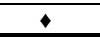 & 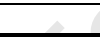 & $\mathrm{z}$ & + & \\
\hline Pomatomidae & Pomatomus saltatrix & $\mathbf{x}$ & & $\leftrightarrow \bullet \bullet$ & 4 & & \\
\hline Haemulidae & Pomadysas commersonni & $\mathbf{x}$ & $\downarrow ?$ & 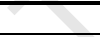 & + & + & \\
\hline Haemulidae & Pomadysas olivaceum & & $\bullet \bullet \bullet \bullet j$ & & + & & \\
\hline Sparidae & Argyrozona argyrozona & & & 2 & + & & \\
\hline Sparidae & Boopsoidea inornata & $\mathrm{x}^{*}$ & & $\overline{0}$ & + & & \\
\hline Sparidae & Cheimerius nufar & & + & $\leftrightarrow \bullet$ & + & & \\
\hline Sparidae & Chrysoblephus cristiceps & & 2 & & + & & \\
\hline Sparidae & Chrysoblephus gibbiceps & & 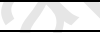 & & + & & \\
\hline Sparidae & Chrysoblephus laticeps & & $\checkmark$ & & + & + & \\
\hline Sparidae & Cymatoceps nasutus & $\mathbf{x} ?$ & $\bullet$ & & + & + & \\
\hline Sparidae & Diplodus hottentotus & $\mathbf{x}$ & & $\leftrightarrow \leftrightarrow \bullet$ & + & + & \\
\hline Sparidae & Diplodus capensis & $\mathbf{x x}$ & $\leftrightarrow \leftrightarrow \bullet \bullet j$ & $\leftrightarrow \leftrightarrow \bullet \bullet$ & + & + & \\
\hline Sparidae & Gymnocrotaphus curvidens & +2 & & & + & + & \\
\hline Sparidae & Lithognathus lithognathus & $\mathbf{x}$ & & $\leftrightarrow \leftrightarrow$ & + & + & \\
\hline Sparidae & Lithognathus mormyrus & & & $\leftrightarrow \bullet \bullet$ & + & & \\
\hline Sparidae & Pachymetopon aeneum & $\mathrm{x}^{*} ?$ & $\downarrow ?$ & & & & \\
\hline Sparidae & Pachymetopon blochii & $\mathrm{x}^{*} ?$ & & & + & + & \\
\hline Sparidae & Pachymetopon grande & $\mathbf{x}$ & & $\leftrightarrow \leftrightarrow \bullet$ & & & \\
\hline Sparidae & Pagellus natalensis & & $\leftrightarrow$ & & + & & \\
\hline Sparidae & Petrus rupestris & & & & + & & \\
\hline Sparidae & Pterogymnus laniarius & & & & & & \\
\hline Sparidae & Rhabdosargus globiceps & $\mathbf{x} ?$ & & & + & & \\
\hline Sparidae & Rhabdosargus holubi & $\mathbf{x} ?$ & + & $\leftrightarrow$ & + & & \\
\hline Sparidae & Sarpa salpa & $\mathbf{x x}$ & $\leftrightarrow \leftrightarrow \bullet$ j & $\bullet \bullet ৬ \bullet \bullet \bullet$ & + & & \\
\hline Sparidae & Sparodon durbanensis & $\mathbf{x}$ & & $\leftrightarrow$ & + & + & \\
\hline Sparidae & Spondyliosoma emarginatum & & $\leftrightarrow \leftrightarrow \leftrightarrow$ & & + & & \\
\hline Dichistiidae & Dichistius capensis & $\mathbf{x}$ & 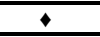 & $\leftrightarrow 4$ & + & + & \\
\hline Monodactylidae & Monodactylus falciformis & & 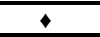 & $\leftrightarrow 4$ & + & & \\
\hline Sciaenidae & Argyrosomus inodorus/japonicus & $\mathbf{x}$ & & $\leftrightarrow 4$ & + & & \\
\hline Sciaenidae & Umbrina canariensis & $\mathbf{x} ?$ & & & + & + & \\
\hline Scombridae & Scomber japonicus & & $?$ & & + & & \\
\hline Oplegnathidae & Oplegnathus conwayi & & $\downarrow ?$ & & & + & \\
\hline Carangidae & Seriola lalandi & $\mathrm{x}^{*}$ & & & + & + & \\
\hline Carangidae & Trachurus trachurus & & + & 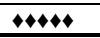 & + & & \\
\hline Cheilodactylidae & Chirodactylus brachydactylus & & & & & + & \\
\hline Mugilidae & Liza richardsonii & $\mathbf{x x}$ & & & & & + \\
\hline Mugilidae & Mugil cephalus & $\mathbf{x}$ & & & & & + \\
\hline Mugilidae & Mugil spp. & $\mathbf{x x}$ & & $\leftrightarrow \leftrightarrow \leftrightarrow \bullet \bullet$ & & & + \\
\hline Tetraodontidae & Lagocephalus lagocephalus & & 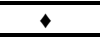 & & + & & \\
\hline
\end{tabular}




\subsubsection{Scavenging for fish}

The collection and consumption of dead fish and other marine animals that have washed ashore was recorded in the Cape during historical times (Raven-Hart 1967, 1971). It is quite likely that wash-ups were collected by people prehistorically. In this context it has been suggested that the low numbers of fish (and flying birds) in MSA contexts relative to those from the LSA signify that MSA people lacked the sophisticated technology to effectively exploit these resources, or exploited coastal resources more selectively, and that fish found in MSA contexts were most likely as a result of scavenging, or brought in by other animals during hiatus periods (Avery et al. 1997; Avery et al. 2008; Deacon and Geleijnse 1988; Halkett et al. 2003; Klein 1977a, 2001; Klein et al. 2004; Klein and CruzUribe 1996, 2000b; Singer and Wymer 1982).

There is no data available on the species or quantities of fish that get cast ashore due to random natural causes. An exception is when fish are stunned and killed after rapid cold water upwelling events. These are caused by sharp and rapid declines in water temperature due to easterly winds which occur almost exclusively during summer in the southern Cape (Hanekom et al. 1989; Schumann 1999). A sudden change in water temperature affects some species' metabolism and they are unable to adapt to the colder water sufficiently rapidly (Kerwath 2006). Such upwelling events can cause mass mortalities of certain species. The washed up dead fish presented opportunities for large scale food scavenging. Other species respond to the colder water by sheltering in warmer and shallower water, often estuaries, where they are reported to be stunned and sluggish (Bower and Crawford 1981; Hanekom et al. 1989). This sluggishness made them easy prey for humans using either spears or nets. Not all species move into the shallows, for example Chrysoblephus laticeps responds by sheltering in sea caves and crevices for up to three days after a cold water event (Kerwath 2006; Kerwath et al. 2007).

Cold water upwellings are restricted to certain parts of the coastline and have been recorded approximately $70 \mathrm{~km}$ east of Blombos Cave and the BBF sites (Schumann et al. 1982). Smaller upwelling plumes have been recorded near Still Bay, some $20 \mathrm{~km}$ east of Blombos Cave and the BBF sites (Swart and Largier 1987). Upwellings also occur in the coastal Storms River region approximately $55 \mathrm{~km}$ east of Hoffman's/Robberg Cave and are most pronounced at Cape St Francis, 80km further east of Storms River and 
approximately 50km east of Klasies River (Hanekom et al. 1989; Swart and Largier 1987). Not all upwelling events result in deaths. According to Hanekom et al. (1989) fish deaths related to cold water upwellings are restricted to the Tsitsikamma coast. Klasies River is situated within the Tsitsikamma region and Hoffman's/Robberg Cave is located just to the west of it.

The species affected by cold water upwelling events are listed in Table 3.6. The largest numbers killed are juvenile Sarpa salpa, Diplodus capensis and Pomadasys olivaceum, but several large specimens of other species, for example a $26 \mathrm{~kg}$ Cymatoceps nasutus, have also been reported (Bower and Crawford 1981).

If the species composition of an assemblage indicates that the majority of fish were obtained through scavenging of washed up species, it would imply summer occupation as these events occur almost exclusively during summer, although winter occupation cannot be excluded.

\subsubsection{Spearing of fish}

The use of spears for fishing is likely one of the oldest methods employed. The first spears were probably made out of wood or bamboo and would not have preserved well in most archaeological sites. Three examples of ancient wooden spears have been recovered from Lower Palaeolithic assemblages in Europe. At Schöningen, Germany, eight wooden spears, thought to have been used for hunting horses, but also found associated with fish, date back to $400 \mathrm{ka}$ (Thieme 1997, 1999), and a single wooden spear from Clacton-onSea, England, dates to roughly the same time period (Warren 1951; Wymer and Singer 1970). At Lehringen, Germany, a wooden spear dating to around $125 \mathrm{ka}$ is reported (Movius 1950).

Four wooden implements were found at Florisbad, South Africa, with an age of at least $120 \mathrm{ka}$. One of these was possibly part of a throwing stick (Bamford and Henderson 2003). Unmodified wood has been preserved at Diepkloof, with an age greater than $55 \mathrm{ka}$ (Parkington et al. 2005). Evidence of woodworking, mostly in the form of preserved wood shavings but also a few artefacts, has been found at some LSA sites in southern Africa (Clark 1958; Deacon 1976; Deacon and Deacon 1963; Mazel and Parkington 1981; Parkington 1978; Sealy et al. 2000; Wells 1965). Parkington and Poggenpoel (1971) 
reported two wooden 'points' preserved at the inland LSA cave De Hangen, one of which might be a projectile point. Seven wooden and three bone points that might have been hafted to arrow shafts were found in the LSA layers at Diepkloof (Parkington and Poggenpoel 1987).

Spearfishing with sharpened wooden sticks has been recorded historically (Burchell 1824; Raven-Hart 1967; Schapera 1930). Stow (1905) reports barbed bone points attached to bamboo shafts being used as harpoons used for fishing in the Gariep River and its tributaries, and the use of a bow and arrow for fishing. A line attached to the arrow was used for retrieval of the fish. According to Schapera (1930), Khoi pastoralists used sharpened wooden sticks to spear fish in deeper parts of rivers.

Rock art scenes depicting spearfishing are recorded at several sites, mostly inland, and the majority of the fish depicted appear to be freshwater species (Breuil 1945; Lee and Woodhouse 1970; Smits 1967; Vinnicombe 1976). In Walvis Bay, Namibia, it was reported that in 1835 "Fish were speared with a stick on to which a gemsbok horn had been mounted. Even today a place at the coast north of the harbour is known to the Hottentots as the place where fish were speared" (Sydow 1973). Stow (1905) also refers to arrows tipped with horn. Spear or arrow tips made of animal horn, which consists of keratin, are not likely to preserve well in the archaeological record (Davis 1987).

Barbed and smooth bone spear points associated with large quantities of freshwater fish have been found at the $\sim 90 \mathrm{ka}$ MSA sites of Katanda - Kt2, Kt9 and Kt16 - situated in the Semliki River, Zaire (Brooks et al. 1995; Feathers and Migliorini 2001; Yellen 1996, 1998; Yellen et al. 1995). Barbed points were also found at Ishango, $6 \mathrm{~km}$ from Katanda, with an age of $\sim 25 \mathrm{ka}$ (Brooks and Smith 1987). At White Paintings Shelter in Botswana barbed bone points associated with freshwater fish occur in layers dating between $4-21$ ka as well as in lower layers with an age of between 33 and 45 ka (Feathers 1997; Robbins et al. 2000; Robbins et al. 1994). Barbed bone points have also been found at the archaeological mound, Kobadi, in Mali, with an age of 4000 BP (Jousse et al. 2008). Barbed bone spear points have not been found in South African archaeological sites. Smooth bone points are common in LSA sites (Deacon 1984a). Bone points have been found in MSA contexts at Peers' Cave (d'Errico and Henshilwood 2007), Blombos Cave 
(d'Errico and Henshilwood 2007; Henshilwood and Sealy 1997; Henshilwood et al. 2001a; Henshilwood et al. 2001b), Klasies River (d'Errico and Henshilwood 2007; Singer and Wymer 1982) and Sibudu (Backwell et al. 2008). At least some of these were hafted to form arrow or spear tips (Backwell et al. 2008; d'Errico and Henshilwood 2007; Henshilwood and Sealy 1997).

Spears can also be fashioned by hafting lithics onto wooden or bamboo shafts, and evidence for spear armatures is found in the southern African MSA (Lombard 2006a, 2007b; Minichillo 2005; Villa in press; Villa et al. 2005; Villa et al. 2009; Wadley et al. 2009). Spear damage was noted on fish bones at the inland LSA site of Likoaeng, Lesotho (Plug, pers. comm.).

Spearing fish in marine contexts is most likely to take place in rock pools and over shallow reefs which many of the species expected inhabit. If stone walled tidal traps were employed, the trapped fish could have been speared. Alternatively, they could simply have been herded into a corner and collected by hand once trapped, and the use of spears would not have been essential. Species that inhabit shallow sandy surf zones or estuaries, such as Lithognathus lithognathus, can be speared in the shallows. One account reports that when a large shoal of all sizes of this species fed on sand mussel, Donax serra, they did not pay any heed to a person standing still in their midst, even brushing against the person without becoming alarmed (Biden 1954). In such instances they would be easy targets for spearing. Biden (1954) recounts that L. lithognathus used to be caught from the rocks at Walvis Bay, Namibia, by throwing spears fashioned from sticks, straightened hooks and line. Stunned or sluggish fish sheltering in shallow water or estuaries due to cold water upwelling events would be easy targets for spearfishing (3.5). Based on the evidence for possible spears and harpoons at archaeological sites in southern Africa reviewed above, it is clear that people in both the MSA and LSA possessed the technology for spearfishing. Whether this technology was employed will be determined through assessment of the species present in the studied assemblages.

\subsubsection{Stone walled tidal fish traps}

Stone walled tidal fish traps are constructions of rocks and cobbles laid in a specific way in the intertidal zone to form an enclosure. They are designed to entice fish to enter the 
traps during high tide and the fish then become trapped when the tide recedes. The fish can then easily be 'herded' into the corners of these enclosures using branches, splashing etc. and then collected by hand, in baskets or by spearing (Avery 1975; Goodwin 1946; Haddad 2003; Hine 2008; Hine et al. 2010; Kemp 2006; Kemp et al. 2009). The success of the traps is dependent on regular maintenance of the rock walls as well as favourable conditions at the time of capture. These include tides, wind direction, turbulence, moon phase and season. Coastal topography is a determining factor for the location of fish traps. Construction of stone walled traps are dependant on suitable raw material being available in the vicinity, and their function requires a gradual slope of the sea bed (Avery 1975). Fish traps are therefore unlikely to be built along sandy beaches lacking rocks or in areas where the rocky coast is steeply shelved. The species that are most commonly caught in stone walled fish traps are listed in Table 3.6.

A survey by the South African Heritage Resources Agency, reported by Kemp (2006) recorded 43 sets of fish traps along a 300km stretch of the southern Cape coast, from Cape Agulhas in the west to just east of Still Bay. More are located east of Still Bay to Mossel Bay. The currently visible fish traps closest to Blombos Cave and BBF 1 and 6 are about $20 \mathrm{~km}$ to the east at Still Bay, and $18 \mathrm{~km}$ to the west at Steenkoolfontein. The coastal topography in the vicinity of BBC and BBF is not suited to building traps. There are no records of stone fish traps in the immediate vicinity of Hoffman's/Robberg Cave, and this absence most likely relates to the steep coastal topography in this area. Fish traps have been recorded at Slang Rivier, located close to the Klasies River sites (Goodwin 1946).

The age of the traps is unknown but Goodwin (1946) proposed that they might date back to Wilton times (now known to begin $\sim 8000$ years ago), on the basis of the observation that numbers of fish increase in Wilton era deposits. Subsequently, Avery (1975) suggested an age of 3000 - 1700 years, which was the time when sea levels returned to approximately their present levels after the mid-Holocene high stand. Recent research based on both archival material and excavations suggests that these structures, at least those visible on the landscape today, were constructed and used by local farmers and likely date to the early 1900s (Hine 2008; Hine et al. 2010). A large number of existing fish traps were demolished around that time and the current distribution of traps does not reflect the previous, much wider, distribution. The majority of currently visible fish traps 
are located along the southern coast of the Western Cape, but they have also been recorded along the west coast (Hine 2008).

In order to determine whether there was any association between shell middens and fish traps, Hine (2008) excavated and analysed material from the Paapkuil Fontein and Still Bay middens, which are in close proximity to existing fish traps near Cape Agulhas and Still Bay. The middens did not produce fish remains in the quantities expected if the occupants were operating the traps, and no relationship between these middens and the fish traps could be established. From the available data on the catch composition of stone walled fish traps, based on observations and anecdotal evidence gathered during the past 65 years, mullet (Mugilidae) species are most frequently caught in large numbers in these traps, followed by Diplodus capensis in lesser quantities (Avery 1975; Goodwin 1946; Kemp 2006; Kemp et al. 2009). Several other species have also been recorded, but usually in smaller numbers (Table 3.6). Based on this data, Hine (2008) expects assemblages created by people operating fish traps to be heavily dominated by Liza richardsonii. Additionally, large hauls of mullet would require some sort of processing, most likely drying, which would take a number of days during which he expects that some of the catch would be consumed and the bones discarded at the processing site. If the fish were transported after processing, this should also be visible in the archaeological record. Based on his midden data and a review of the published fish data from the Western Cape, Hine (2008) concluded that there are no LSA sites with fish assemblages that appear to have been acquired by trapping. This supports his theory that the age of fish traps does not extend into prehistoric times. Possible exceptions are SBF, SRM1 and the LSA layers of $\mathrm{BBC}$, which are currently the only assemblages where the southern mullet, $L$. richardsonii, is the most frequently occurring species. Hine (2008) believes that the SBF and BBC mullet could have been caught with nets. The SBF mullet could have been caught in the lagoon directly in front of the site with baskets or nets, and drift nets are still used to catch this species in the lagoon today (Sunde and Isaacs 2008).

The recent age of currently visible fish traps and the absence of any relationship between these and the middens excavated by Hine (2008) does not preclude the possible antiquity of stone walled traps. Even if the extant and known historical stone walled fish traps are not prehistoric, it seems likely that once people recognised fish as a food source they 
would have been aware of the concept of trapping. Fish are known to occasionally become trapped in natural rockpools with receding tides and it would have been easy for hunter gatherers to take advantage on these occasions. Some small adjustments to natural rockpools could easily have made for more efficient trapping, although such alterations would no longer be visible. Such ad hoc adjustments to natural pools would have resulted in less predictable and smaller scale fishing than would be expected from specially constructed fish traps. Large numbers of shoaling species such as mullet are not likely to be caught in small traps as they would soon become aware of the enclosure's restrictions due to their large numbers.

\subsubsection{Hooks, line and sinkers}

\section{Hooks}

Bone fashioned into curved hooks has been found in association with freshwater fish remains at LSA sites in South Africa with ages from 4500 to $70 \pm 40 \mathrm{BP}$ but none have been found in assemblages with marine fish remains (van Niekerk 2002). These LSA bone hooks were not necessarily used for catching only fish and could also have been used for some other purposes, such as hooking frogs. The distribution of bone hooks appears to be geographically restricted to the north and northeast of the foothills of the Drakensberg, and none have been recorded further south or southwest (Figure 3.3).

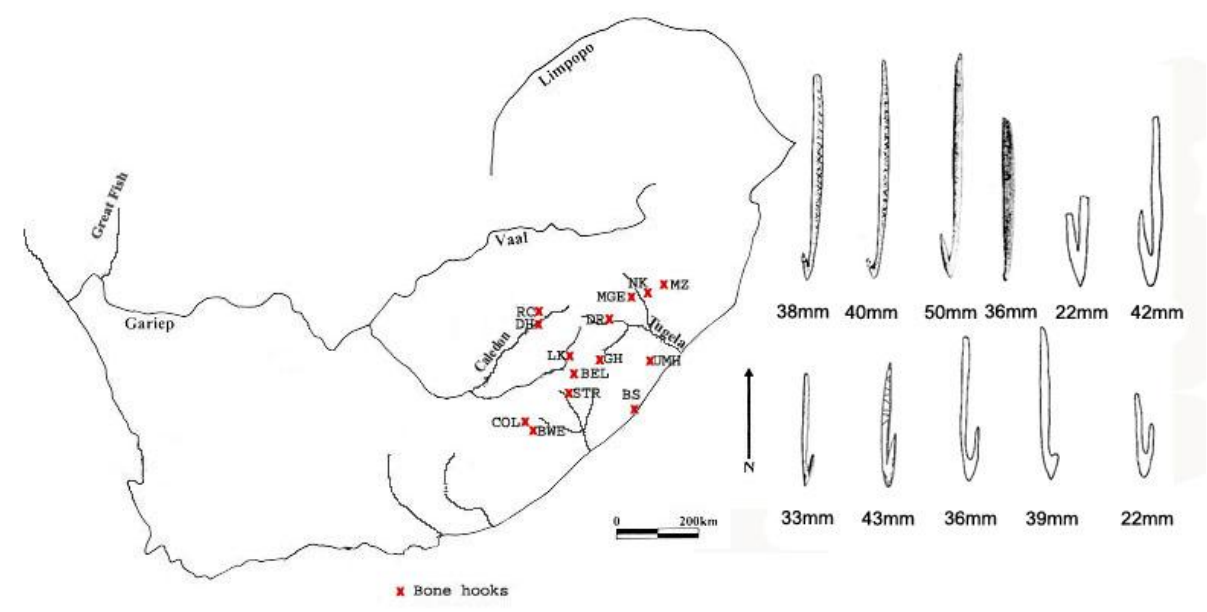

Figure 3.3. Location of sites that have bone hooks and illustration of a selection of hooks (not to scale length is indicated below each hook). $B E L=$ Bellvue, $B S=$ Borchers, $B W E=B o n a w e, C O L=C o l w i n t o n$, DH= De Hoop, $\mathbf{D R}=$ Driel, $\mathbf{G H}=$ Good Hope, $\mathbf{L K}=$ Likoaeeng, $\mathrm{MGE}=$ Mgede, $\mathrm{MZ}=$ Mzinyashana, NK= Nkupe, UMH= Umhlatuzana 
Fish gorges, also referred to as bone bi-points or straight hooks (Rick et al. 2001; Walters 1988), are symmetrical bone fragments that have been polished and sharpened at both ends. They have been found at just a few LSA coastal sites, namely Nelson Bay Cave (Deacon 1978; Klein 1972a), Byneskranskop 1 (Schweitzer and Wilson 1978; 1982), Elands Bay Cave (Poggenpoel 1996) and Matjes River (Döckel 1998). All occur in layers with ages of between 10000 and 7800 years ago. A single potential bone gorge has been found at Smitswinkelbaai Cave with an age of around 1175 BP (Poggenpoel and Robertshaw 1981). This 'gorge' is unlike those found in the early Holocene assemblages listed above, and looks more like a bone splinter that is pointed at both ends, but with very little evidence for being sharpened, and it is not polished 1981). Inskeep (1987) found one bone gorge during his excavations at NBC that is similar to that described by Deacon (1978) from Klein's (1972a) excavation at NBC, with an age of about 3000 years ago. He preferred to describe it as an 'awl' due to the low number of these artefacts from his excavations (only two others which he very tentatively identified as gorges were found) and the apparent lack of correlation between the number of fish and the number of gorges within layers from the Klein excavation. As with the SWB specimen, these 'gorges' or awls showed minimal shaping and polish, unlike the older specimens from the early Holocene.

Poggenpoel (1996) compared the sizes of the bone gorges from EBC and NBC in relation to the sizes of the contemporary fish. The NBC gorges were considerably bigger, and were associated with larger fish caught in coastal water, whereas the smaller EBC gorges were associated with smaller, juvenile fish, presumably caught in the estuary. Thus he believes that gorge size could be related to the size of fish targeted, which in turn depends on the habitat exploited.

Goodwin (1946) was informed about a fisherman in the Gouritz area, close to Still Bay, who had found a bone that was sharpened on both ends and tied in the middle to a line made of a shredded fibrous vine. This sounds much like the bone gorges found prehistorically. Gorges made from wood or sturdy thorns might have replaced those made of bone but are unlikely to survive in the archaeological record. M. Smith (Inskeep 1987) recounts seeing children in northern Mozambique using mimosa thorns and handmade twine for catching fish. In South Africa, rock art depicting fishermen using rods, line and 
apparent gorges has been reported by Willcox (1963) as well as Lee and Woodhouse (1970) but the locations of these images were not specified.

Fish gorges made from bone and other material such as shell, wood and stone are known from archaeological sites elsewhere in the world including Europe (Morris 1938; Olson et al. 2008; Sampson 1998; Straus 2001), United Kingdom (Ritchie 1977), United Arab Emirates (Beech 2003; Mery et al. 2008), North America (Erlandson et al. 2005; Erlandson 2001; Erlandson et al. 2008; Heizer 1949; Noah 1998; Rick and Erlandson 2000; Rick et al. 2005; Rick et al. 2001; Vellanoweth et al. 2003), Asia (Kuang-Ti 2001), North Africa (van Neer et al. 2000) and Oceania (Reinman 1967) - although there is some uncertainty whether those in Australia predate the arrival of Europeans (Dyall 2004; Walters 1988). Historical records also refer to the use of a variety of materials to make fish gorges elsewhere (Herdrich and Armstrong 2008).

Some doubt has been expressed about whether the fish gorges found in southern and western Cape archaeological sites were actually used to catch fish. This observation is based on the absence of bone gorges in many layers that contain large amounts of fish (Inskeep 1987; Poggenpoel 1996; Schweitzer and Wilson 1982). At Byneskranskop 1 (BNK) in particular, only four fish were present in the layers containing the eight gorges found at the site, and no gorges were reportedly found in the layers with the highest densities of fish (Schweitzer and Wilson 1982). Two of the bone artefacts from BNK illustrated and categorized as awls (Schweitzer and Wilson 1982: 78 Fig. 29, F \& K) could potentially be gorges if the criteria for describing gorges is applied - a bone fragment or sliver that has been sharpened at both ends (Deacon 1978). Specimen F is found in a layer with only one fish present, but specimen K occurs in the layer with the highest number of fish in the site. It has been suggested that the classification of certain bone artefacts that are sharpened at one end, but broken at the other, should be reconsidered as these might have been gorges, particularly in sites where a lot of fish remains are present (Noah 1998). At Nelson Bay Cave and Elands Bay Cave, it is worth noting that the appearance of bone gorges coincides with the appearance of fish at these sites. The same could apply at Matjes River Rock Shelter, but needs further investigation. 
The possibility that these bone gorges might have been used to catch birds has been raised (Klein 1983; Poggenpoel 1996). Ethnographic records of bone gorges used to catch birds are fairly common (De Laguna 1964; Gabriel et al. 2005; Gilmore 1953; Pyle 2009), but I have not found any records from southern Africa. Data on the quantities of birds found at the sites with bone gorges is not available and it is therefore not possible to determine whether there is any relationship between frequency of bone gorges and birds at this stage, although bird bones tend to be more common after the introduction of gorges, from the appearance of the Wilton to the late Holocene (Klein 1974).

Bone gorges from South African sites have thus far not been examined or described in detail. If these artefacts were used repeatedly, some traces could be expected close to the gorges' midsections, the area where they were presumably attached to some kind of cord or thong. A possible function of gorges as part of composite spearpoints as described by Barton et al. (2009), where the sharpened base of the point forms a barb to secure the fish once speared, has not been considered. Future microscopic analysis of these gorges could help determine their application, particularly if traces of usewear or residue are present.

\section{Line}

Both sinkers and gorges would need to be attached to string or cord, presumably made from plant fibres or sinew. Preserved string or twine is fairly rare, but has been recovered from a few sites with exceptional preservation such as at De Hangen (Parkington and Poggenpoel 1971), Scott's Cave (Deacon and Deacon 1963; Wells 1965), Dikbosch Shelter 1 (Humphreys 1974), Melkhoutboom (Deacon 1974, 1993), Eland Cave (Sealy et al. 2000), Kouga (Steyn et al. 2007) and a cave near Bredasdorp (Grobbelaar and Goodwin 1952). These are all very dry caves, and string or twine is unlikely to be preserved in the damper conditions that are typical of coastal sites.

\section{Sinkers}

In addition to those already mentioned from NBC and Swartrif midden, small grooved pebbles of shale, silcrete, sandstone and quartzite, interpreted as sinkers, have been found at Matjes River Rock Shelter ( 7700 - 5400 BP and younger than 3550 BP) (Döckel 1998; Louw 1960; Ludwig 2005), Klasies River Mouth Lower Midden ( 4800 BP) 
(Singer and Wymer 1982), Noetzie, near Knysna (Orton and Halkett 2007) and in the recent excavations at Hoffman's/Robberg Cave ( 4000 - 3000 BP) (Sealy pers. comm.).

Sinkers have not been found at sites along the west coast. Where both sinkers and gorges occur at the same site, none of the sinkers occur in the same layers as the bone gorges apart from the single quartzite sinker from Klein's excavation at NBC (Deacon 1978; 1984b). Based on the average weight of these pebbles - maximum $36.3 \mathrm{~g}$ at NBC, but most below 3g (Inskeep 1987) - Poggenpoel (1996) believes they were most likely used as line sinkers rather than net sinkers. Despite their generally small size, a brief experiment by Inskeep (1987) showed that they were adequate as line sinkers. Similarly sized and shaped sinkers from the United Arab Emirates are thought to have been used primarily as net sinkers, although their possible use as line sinkers was also considered (Beech 2003). The light weight of these grooved stones suggests that, if they functioned as line sinkers, they were used for fishing in relatively shallow waters (Beech 2003).

The large increase in sinkers after $3300 \mathrm{BP}$ at NBC coincides with a substantial increase in the number of fish remains. The species composition is essentially the same as in the lower layers, but differences in frequencies of some species were noted. Species that occur in higher frequencies in these layers such as Seriola lalandi and Sarpa salpa are most commonly caught on hooks, which could signify an association between sinkers and line fishing. Such an association is somewhat tenuous, as these species were also present in the older layers lacking sinkers, and the frequency of other species that are only caught on hooks (e.g. Pachymetopon blochii, Petrus rupestris) decreased after 3300 BP. The latter species do, however, occur in relatively low numbers throughout the sequence.

At Swartrif midden, the dominant species identified by Smith and associated with grooved sinkers is Diplodus capensis. This species is susceptible to cold water upwelling which is a common occurrence in the Tsitsikamma area, and such events might have been the source of this species (Deacon 1970). Alternately, it was caught on hooks and the sinkers were part of line fishing gear. Unfortunately, as Poggenpoel (1996) has demonstrated at the nearby SRM1, the method used for identification was based on only two elements which, at SRM1, resulted in a large number of species not being identified. The likelihood that species such as Liza richardsonii were present and possibly the dominant species at 
Swartrif midden, but missed due to methodological issues, as at SRM1, is high. This assemblage will have to be reanalysed to determine whether the initial species identification was correct before any further assumptions can be made on the function of the grooved stones from this site.

Pyle (2009) reports that in the Hawaiian Islands grooved pebbles, called Kolea stones, are used as weights attached with string to baited bone or wooden gorges to catch birds.

Grooved pebbles found in inland archaeological assemblages from this area thought to have been used for this purpose weigh between 46.3 - 114.3g (Pyle 2009). This is somewhat heavier than the heaviest grooved stone reported from NBC and it seems unlikely that the South African examples were used to catch birds.

\subsubsection{Nets and baskets/woven fish traps}

The use of nets or baskets to trap fish could account for the presence of certain species that are seldom hooked or speared and that are not usually caught in stone walled traps or rockpools. Fragments of well made woven nets have been found in LSA contexts at a few sites including Diepkloof (Parkington and Poggenpoel 1987), Melkhoutboom (Deacon 1974), and a cave near Bredasdorp (Grobbelaar and Goodwin 1952). Likewise fragments of pierced reeds that could be remnants of woven baskets or mats have been recovered from sites including De Hangen (Parkington and Poggenpoel 1971), Diepkloof (Parkington and Poggenpoel 1987) and Faraoskop (Manhire 1993). These sites are all located inland where dry conditions contributed to their preservation.

Depictions of fish and fishing scenes feature occasionally in rock art in southern Africa (Eastwood 1999; Ouzman 1995; Skelton 2000; Vinnicombe 1960; 1961). Several recorded rock art scenes depict nets associated with antelope (Manhire et al. 1985), and a few have been reported that show fish being caught in conical or other traps (Smits 1967; Vinnicombe 1961; Woodhouse 1979).

Historical records refer to fishing baskets made of twigs and reeds (Hambly 1937;

Schapera 1930; Stow 1905; Willcox 1963) and these are still used today by many societies (Ankei 1989; Skelton 2001). The baskets are placed near the banks of the river or in areas where spawning runs are anticipated, and the area around the baskets blocked by vegetation and sometimes stone weirs to force the fish into the basket opening (Stow 
1905). Driving of fish towards funnel-shaped reed traps placed across shallow streams, and the construction of reed fences and stone dams over dry river courses prior to floods have been recorded along the Orange River (Schapera 1930). Once the river subsides, fish that have been trapped are easily collected. The historical records refer to freshwater fishing although it seems probable that similar methods could have been used in estuaries. Historical accounts also refer to fishing with nets, for example Kolbe (1738, in Thompson 1913) stated that indigenous people at the Cape were very skilled at casting and drawing nets. If nets were employed one would expect a larger range of sizes per species to be represented in an assemblage because nets are less size-selective than other methods. Factors influencing characteristics of net-caught assemblages include the size of the mesh used and whether the species in question shoal in mixed size groups.

The grooved sinkers found at the sites listed above could also have functioned as net sinkers. The increased frequency of Liza richardsonii and Clinidae in the $<3300$ year old layers at NBC could indicate the use of nets, and the sinkers might have been used as net weights. The coastal topography in the area is unsuitable for fish traps, and the use of nets to catch $L$. richardsonii in particular, seems likely. Whether the nets were weighted down with stone sinkers is difficult to establish but seems likely, particularly if they were used as throw-nets, as such nets need to be weighted in order to sink rapidly and trap the fish (Gabriel et al. 2005). Net sinker weights and numbers used depend on several variables such as the weight of the net, turbidity of the water, and whether the net is used for fishing in bottom or surface waters (Lucas 1998).

\subsubsection{Piscicides}

Fishing with poison has been practiced by cultures across the world and is still employed today (Alvarez Arias 2000; Béarez 1998; De Smet 1998; Hambly 1937; Hornell 1941; Obomanu et al. 2007; Quigley 1956). Plants containing rotenoids or saponins, as well as certain Euphorbiaceae are most commonly used to kill or stun fish (Bearez 1998). The method entails crushing of the roots, leaves, bark or seeds before releasing it into a body of water (Neuwinger 2004). The chemical substances in these plants are toxic to fish, but are harmless to humans in small doses, and fish killed in this manner can be eaten by humans mostly without ill effects. Plants containing saponins give off a soapy substance 
when crushed and mixed with water, and it is thought that the traditional use of these plants for domestic washing inadvertently lead to the discovery of its potential for catching fish (Bearez 1998).

Plant piscicides are mostly associated with freshwater fishing in slow moving rivers or lakes but in the Indo Pacific they are also used in rock pools and during diving (Bearez 1998). There is at least one recorded incidence of possible piscicide use associated with marine fish from an archaeological site in Ecuador, dating to 900 - 350 BC (Béarez 1998). The fishes in question were very small $(31-79 \mathrm{~mm})$ species that frequent rock pools and are not preyed upon by the larger fish found in the assemblage, thus did not represent stomach contents of the bigger fish. The species composition made the use of nets unlikely, and the use of very small fishes in certain dishes as well as fishing with plant poisons was recorded in the area historically. It was concluded that, based on the above evidence, piscicides were most likely employed to catch these small fish (Bearez 1998). The plant species most commonly used as piscicides in South Africa, Burkea africana (wild syringa), Euphorbia ingens (common tree euphorbia), Mundulea sericea (visgif, corkbush), Adenium multiflorum (impala lily), Apodytes dimidiata (white pear), Millettia grandis (umzimbeet) and Tephrosia macropoda (lozane) do not occur in the southern Cape, and it is not clear whether they or others serving the same function ever occurred in this area (Brackenbury and Appleton 1997; Howes 1930; van Wyk and Gericke 2000). Rotenone, a plant based piscicide produced commercially, is frequently used to eradicate alien fishes from waterways or to sample natural fish assemblages (Enviro-Fish-Africa 2008). It is most effective when used in relatively calm or enclosed waters as the toxins are not dissipated as rapidly as in more turbulent water. Rotenone is said to be less effective on species with high mobility such as Sparidae, as they are able to escape from the poison, but this does not apply to all Sparidae (Buxton and Smale 1984).

The use of piscicides is difficult to discern in the archaeological record as it is unlikely to leave any physical traces unless the plant material is well preserved. Carbonised seeds sometimes preserve well, even when other plant remains are lost (Sievers and Wadley 2008). Seeds could indicate the use of piscicides if identifiable to species. Pollen and starch analysis could also point to the presence of piscicides, although it seems unlikely 
that toxic materials would be processed within the domestic area; they are more likely to have been processed closer to the water. The evidence from historical records suggests that piscicides formed a part of southern Africa's repertoire of fishing, at least in freshwater contexts. This is supported by Vinnicombe's (1961: 115) observation that "The Bushmen of the Kalahari erected stone weirs in the few river pools, and drove fish into lines of baskets. They made poison from Euphorbia, but had no hooks."

\subsection{Animal agents of accumulation}

Fish remains excavated at a site may not be anthropogenic, particularly when the remains are not associated with cultural artefacts. Other possible agents of accumulation of fish at coastal sites in the southern Cape are considered here.

\subsubsection{Fish-eating birds}

Several species of fish-eating birds occur on the coast, a few of which regurgitate pellets of partially digested or undigested bone which could contribute to archaeological deposits. The African fish eagle, Haliaeetus vocifer, prefers nesting and perching in trees (Harper et al. 2002), but occasionally nest on cliffs (Maclean 1993) and could contribute fish to an open site below a cliff such as Klasies River main site or the exterior of Blombos Cave. They are territorial birds that nest in pairs (Harper et al. 2002). Most of their fish prey weigh less than $1 \mathrm{~kg}$ but can be as heavy as $3.7 \mathrm{~kg}$. Larger specimens $(>2.5 \mathrm{~kg})$ cannot be carried in flight, and are planed along the water surface to be consumed on shore (Maclean 1993).

Studies by Stewart et al. $(1999 ; 1997)$ of the contents of Haliaeetus vocifer pellets collected from a roost that had been used by this species for approximately ten years at Koobi Fora, Kenya, showed that the majority of the fish caught were from inshore waters less than $5 \mathrm{~m}$ in depth, most were $30-45 \mathrm{~cm}$ in length with a mass of 300 and $400 \mathrm{~g}$, and specimens below $20 \mathrm{~cm}$ were mostly juveniles. Overall their choice of fish was selective, with less common species being the preferred prey. Excavation of two square meters of deposit below the roost to a depth of $5 \mathrm{~cm}$ in the one half and $25 \mathrm{~cm}$ in the other recovered 1551 fish bones (Stewart et al. 1999). Elements that best survived were cleithra and spines, whereas vertebrae and skull elements were poorly represented, and two thirds of the bones were fragmented. Most complete bones were from specimens smaller than 
$30 \mathrm{~cm}$, which the authors believe could be due to smaller specimens being swallowed whole, and therefore suffering less damage. No signs of digestion were noted on the fish bone, and most vertebrae consisted only of centra without processes (Stewart et al. 1999). Haliaeetus vocifer occasionally scavenge washed up fish and remains of two large fish $(\sim 14 \mathrm{~kg} / 105 \mathrm{~cm})$, thought to have been scavenged, were found in the roost (Stewart et al. 1997). An analysis by Whitfield and Blaber (1978) of $H$. vocifer pellets from Lake St. Lucia, South Africa, showed that the dominant prey species was Mugil cephalus. Most of the fish identified weighed between 400 and $500 \mathrm{~g}$ with a maximum of $3 \mathrm{~kg}$, and most occur inshore or within $30 \mathrm{~cm}$ from the surface. In addition, H. vocifer were observed taking prey from other birds. Skeletal element preservation and evidence of digestion on the prey elements were not recorded.

The cormorant species responsible for producing the modern cormorant pellets collected at Klasies River main site and studied by von den Driesch (2004) were not identified, but must have been either the Cape cormorant, Phalacrocorax capensis, or the larger whitebreasted cormorant, Phalocrocorax carbo. Most of the elements recovered from the pellets were from fish smaller than $15 \mathrm{~cm}$ and several were from fish less than $10 \mathrm{~cm}$ in length (Table 3.7). The largest fish represented in the pellets was $20 \mathrm{~cm}$. Non-vertebral elements were more common than vertebrae in all pellets analysed. Several near-shore and euryhaline (able to withstand a wide salinity range) species were identified. The species found were Galeichthys feliceps, Acanthistius sebastoides, Spondyliosoma emarginatum and undifferentiated Serranidae, Cheilodactylidae, Gobiesociedae, Clinidae and Mullidae (goatfishes). Although the study found that most prey species were small, some cormorants can take bigger prey, and $P$. carbo can swallow eels up to $60 \mathrm{~cm}$ (von den Driesch 2004). Eels, however, are long and thin and are easier to swallow than deeper bodied fish. The upper size limit of fish taken by $P$. carbo, other than eels, is $\sim 35 \mathrm{~cm}$ (Carss 1993; Opačak et al. 2004) with a preferred size of 15-20cm (Kirby et al. 1996). Phalacrocorax carbo has a diverse diet that includes species from a range of habitats, with its most common prey being the locally dominant species (Kirby et al. 1996; Linn and Campbell 1992; Suter 1995). This species does not prey on dead fish (Linn and Campbell 1992). On the southeastern coast of South Africa, the recorded prey of $P$. carbo includes reef and near-shore fish as well as juveniles of euryhaline species caught in estuaries 
(Randall et al. 2002). Apart from recently introduced freshwater species, the most common prey species recorded by Randall et al. (2002) were Myxus capensis, Mugil cephalus and Lisa dumerilii (Mugilidae, mullets) as well as Sarpa salpa and Diplodus capensis. Phalacrocorax capensis diets are less variable and their main prey consists of pelagic species such as Engraulis encrasicolus/japonicus (Engraulidae, anchovies), Sardinops sagax, Trachurus trachurus (Carangidae, horse mackerel) and Sufflogobius bibarbatus (Gobiidae, pelagic goby) (Crawford 2007; Crawford et al. 2007; Duffy et al. 1987). 
Table 3.7. Details of the fish remains contained in a sample of ten modern regurgitated cormorant pellets collected from the surface of Klasies River main site by von den Driesch (2004:37). Data recorded includes species present, percentage of vertebrae per sample, degree of digestion and fish size. Numbers of fish per sample were not specified in all instances.

\begin{tabular}{|c|c|c|c|c|c|}
\hline $\begin{array}{l}\text { Sample } \\
\text { number }\end{array}$ & Species & $\begin{array}{l}\text { Number } \\
\text { of fish }\end{array}$ & \%vertebrae & Comments & Fish length (cm) \\
\hline \multirow[t]{3}{*}{1} & Serranidae & & 60 & heavily digested & all $<15$ \\
\hline & Cheilodactylidae & & & & most $<10$ \\
\hline & Gobiesociedae & & & & \\
\hline \multirow[t]{2}{*}{2} & Galeichthys feliceps & 1 & 59.5 & digested & $1=20$ \\
\hline & Clinidae & several & & & rest $<12$ \\
\hline 3 & Acanthistius sebastoides & 4 & 49 & heavily digested & $<15$ \\
\hline \multirow[t]{2}{*}{4} & Serranidae & & & heavily digested & 15 \\
\hline & Clinidae & several & 52 & & $<10$ \\
\hline \multirow[t]{2}{*}{5} & Serranidae & 1 & 60 & & most $<10$ \\
\hline & Clinidae & & & & 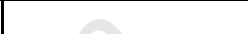 \\
\hline 6 & Clinidae & several & 62.5 & digested & $<12$, some $<5$ \\
\hline \multirow[t]{2}{*}{7} & Galeichthys feliceps & 1 & 65.8 & digested & 18 \\
\hline & Clinidae & & & & $<10$ \\
\hline \multirow[t]{3}{*}{8} & Serranidae & & 53.5 & heavily digested & $<15$ \\
\hline & Mullidae & & & +2 & most $<10$ \\
\hline & Gobiesociedae & & & 8 & \\
\hline 9 & Acanthistius sebastoides & 1 & 54.8 & digested & $<15$ \\
\hline \multirow[t]{2}{*}{10} & Serranidae & & 36.2 & heavily digested & 18, rest $<10$ \\
\hline & Spondyliosoma emarginatum & 1 & & & \\
\hline
\end{tabular}

The kelp gull, Larus dominicanus, sometimes nests on cliffs and regurgitates pellets from its diet that is dominated by bivalve mollusks, but also includes fish and a variety of scavenged foods (Bertellotti and Yorio 1999; Branch 1985; Maclean 1993). The smaller greyheaded gull, Larus cirrocephalus, also has a varied diet but does not nest on cliffs (Maclean 1993). It is not clear from the literature whether L. cirrocephalus casts pellets, and no data on the fish species composition of L. dominicanus pellets could be sourced. Based on the available data it would be difficult to infer whether either of these species could have contributed to an archaeological assemblage.

The jackass penguin, Spheniscus demersus, lives in burrows dug into banks or between boulders and occasionally inhabits caves (Simmons and Kemper 2003). Adults feed at sea and do not regurgitate pellets of hard undigested material like cormorants or owls (Beaune et al. 2009; Wilson 1985). They feed on pelagic species of Clupeidae and Gobiidae (Crawford et al. 1999b; Kemper et al. 2007; Wilson 1985). Remains of their prey could conceivably land up in archaeological sites as the stomach contents of these birds if they were being hunted for food by humans. Alternatively, if these birds made use of uninhabited archaeological sites for breeding, some might have died there, leaving their 
remains as well as their stomach contents behind. One could thus expect to see pelagic Gobiidae and Clupeidae bones in assemblages that contain penguin remains. This expectation has thus far not been met with South African archaeological assemblages. Only at Klasies River main site, where both penguin and fish remains occur, have Gobiidae and Clupeidae been identified (von den Driesch 2004).

One species of owl, Bubo africanus (spotted eagle owl), occasionally takes fish as prey in the southern Cape (Maclean 1993). It hunts nocturnally, roosts on ledges or in trees, and tends to move around rather than accumulate pellets in one place, although it sometimes returns to the same roost for several years (Andrews 1990; Avery 2001). Bubo africanus generally preys on small mammals weighing up to $140 \mathrm{~g}$ (Andrews 1990). No further information on the role of fish in this bird's diet could be obtained, and it seems unlikely that they regularly contributed fish remains to the archaeological assemblages studied here.

\subsubsection{Fish-eating mammals}

Cape clawless otters, Aonyx capensis, prey mainly on crabs but their diet also includes fish, fragments of which survive in their spraints in the form of otoliths, scales and bones (Nel and Somers 2007; Stuart and Stuart 2001; Verwoerd 1987). A variety of fish species will be taken depending on location (Emmerson and Philip 2004; Parker et al. 2005; Somers 2000). Most studies of $A$. capensis spraints do not specify whether the fragmented bones were identifiable, and recovered otoliths and scales are generally used for identification (Somers 2000; Watson and Lang 2004). Rowe-Rowe (1977) identified at least three fish elements from spraints, which suggests that they sometimes survive well enough to be identified. The size of fish taken by otters varies, but seems to be restricted mainly to relatively small specimens of 200mm and less (Rowe-Rowe 1977; Somers 2000). Some fish bones from otter spraints - or their stomach contents if they were killed by humans or natural causes - could conceivably end up in archaeological assemblages.

Bones and coprolites of brown hyaena, Hyaena brunnea, have been found at Klasies River main site (Deacon 2001a; Klein 1975), but not at Blombos Cave (Henshilwood et al. 2001b). Hyaena brunnea is primarily a scavenger and will take dead fish, as seen in scats from Namibia (Kuhn et al. 2008). Bones that have been through a hyaena's digestive 
system tend to be heavily eroded (Horwitz 1990; Klein et al. 1999a). If hyaenas were responsible for the presence of fish remains in archaeological sites then one would expect to see significant signs of digestion on these elements.

The Cape fur seal Arctocephalus pusillus pusillus, is the only endemic seal species in South Africa (Skinner and Chimimba 2005). It inhabits offshore islands as well as sandy or rocky shores in southern Africa, and does not inhabit dry caves on the mainland. They are therefore unlikely to be direct contributors of fish bone to locations inhabited by humans. The stomach contents of seals caught or scavenged by humans could potentially land up in archaeological assemblages. Cape fur seals' diet consists primarily of fish, as well as smaller quantities of cephalopods, crustaceans and occasional seabirds (Castley et al. 1991; Skinner and Chimimba 2005; Marks et al. 1997). The fish species most commonly preyed upon by seals in the southern cape are pelagic species such as Merluccius capensis (hake), Trachurus capensis (horse mackerel), Pterogymnus laniarius (panga), Sardinops occelata (sardine) and Engraulidae (anchovies) and bottom dwellers such as Cynoglossus zanzibarensis (sole) Austroglossus pectoralis (sole). Remains of these species could conceivably have entered an assemblage as the stomach contents of seals, particularly the pelagic species.

\subsection{Discussion}

The variety of possible means of acquiring specific species of fish can make interpretation of fishing methods employed somewhat vague. Species composition, size and ecology, local environmental determinants such as the suitability of coastal topography for specific methods, and artefacts associated with fishing are all factors that can help to reconstruct prehistoric fishing practices.

Fish that have washed up on shore after cold water upwelling events would almost certainly have been collected for consumption in prehistoric times. Based on the available data, several of the fish that are most often killed in large numbers by these events are juveniles of particular species. Assemblages dominated by these could indicate that the fish were acquired through scavenging of washed up specimens. Similarly, the presence of offshore species could indicate that these were collected after being washed up as these species cannot be captured from the shore. 
Spearing is probably the oldest method employed to catch fish, and primitive spears could have consisted of no more than a sharpened wooden stick, which would not survive in the archaeological record unless under exceptional circumstances of preservation. Artefacts other than wood that might have functioned as parts of fishing spears or harpoons have been found at a number of coastal MSA and LSA sites. Fish that congregate en masse in shallow water to feed, or those that have been stunned or shelter in bays after cold water upwelling events would make easy targets for spearing. Likewise, fish caught in tidal traps or reed enclosures could have been speared, although spears would not have been essential as the trapped fish could be collected by hand, with nets or baskets.

Hine's (2008; Hine et al. 2010) argument against the antiquity of fish traps if the majority of species expected are Mugilidae is persuasive. If correct, then the presence of Mugilidae in assemblages suggests that nets or baskets were used to catch these fish, as these species are seldom caught with hooks. This does not, however, exclude the possibility that less formal trapping methods using natural rock pools, perhaps needing only slight adjustment, were used. The species composition of fish caught in natural pools would not be as consistent as those recorded from specially built and maintained traps as these catches would be more dependent on chance than premeditated capture methods.

Whether bone gorges functioned as fish hooks is not yet clear. Their association with the first appearance of fish at at least two of the sites suggests a link between the two, but the presence of bone gorges in layers with very few fish remains casts some doubt on their function. At this stage it is not possible to determine whether there is any relationship between the presence of bone gorges and birds. It is possible that gorges were used for both fishing and fowling, as the method of employment is essentially the same. From the species compositions at the sites with marine fish remains, it is clear that fishing with hooks was practiced in prehistoric times, as some commonly occurring species are seldom caught in any other way.

In the absence of any hook-shaped hooks at sites containing marine fish, the most probable implement used to replace bone gorges would be similar implements fashioned out of wood or thorns. This practice has been recorded in the recent past in southern Africa, and the use of gorges made in a variety of materials to catch fish appears to be 
universal. If wood or thorns replaced bone, these are less likely to survive in the archaeological record, and would explain the lack of obvious fishing equipment. Gorges made out of durable material other than bone, such as stone or shell, have not been recorded in southern African sites.

The grooved stones that have been recovered from a few sites in southern Cape and interpreted as sinkers could have been used as either line or net sinkers. It is not currently possible to exclude either function based on the species associated with sinkers, and further analysis of the fish assemblages associated with these artefacts is needed.

Historical records refer to indigenous people using nets and basket traps to catch fish, and it is clear from the artefactual and rock art records that prehistoric people were adept at making baskets, string and nets. Although most historical accounts refer to the use of nets and particularly baskets for fishing in freshwater habitats, and most of the artefactual evidence has been found at inland locations, these methods could have been employed at coastal locations too. The lack of nets and baskets at coastal sites is most likely due to poor preservation. Shoaling species such as mullet and herrings are most commonly caught with nets, particularly in open waters. Nets or basket traps used in estuaries would capture a more diverse range of species.

Piscicides could quite feasibly have been used in rock pools or calm marine waters to kill or stun fish but their use would be difficult to determine in the absence of information on available piscicides in the study area and which species are most likely to be affected. Assemblages dominated by species not usually captured by the methods listed here might indicate the use of piscicides. The species affected would presumably be those that inhabit shallow water or rock pools where they could be collected easily as there is no evidence for the use of water craft during the Stone Ages.

At present only a very small amount of research has been conducted on the effects of digestive processes on fish of animals that are possible agents of accumulation at southern African archaeological sites, although such studies are quite common elsewhere. The pioneering work done by von den Driesch on the composition of cormorant pellets needs to be expanded, and extensive research on the signals left by other southern African fish predators needs to be conducted. Data from such studies would make important 
contributions to the study and understanding of the taphonomic history of fish assemblages. 


\section{Chapter 4: Site Description and Background}

\subsection{Site background}

The fish from the following sites have been analysed for this study: Hoffman's/Robberg Cave, Klasies River main site, Blombosfontein 1, Blombosfontein 6 and Blombos Cave (Figure 4.1). The sites were chosen in order to compare fish assemblages from both open and cave sites as well as from sites of different ages. In addition, due to the geographic proximity of these sites, it was expected that in general the same species would have been available at all locations, as they are today, barring the possible intrusion of some tropical species during periods of increased water temperature. Similarity in species availability allows more in depth investigation of the taphonomic processes that affect the survival of particular elements under different conditions and through time.

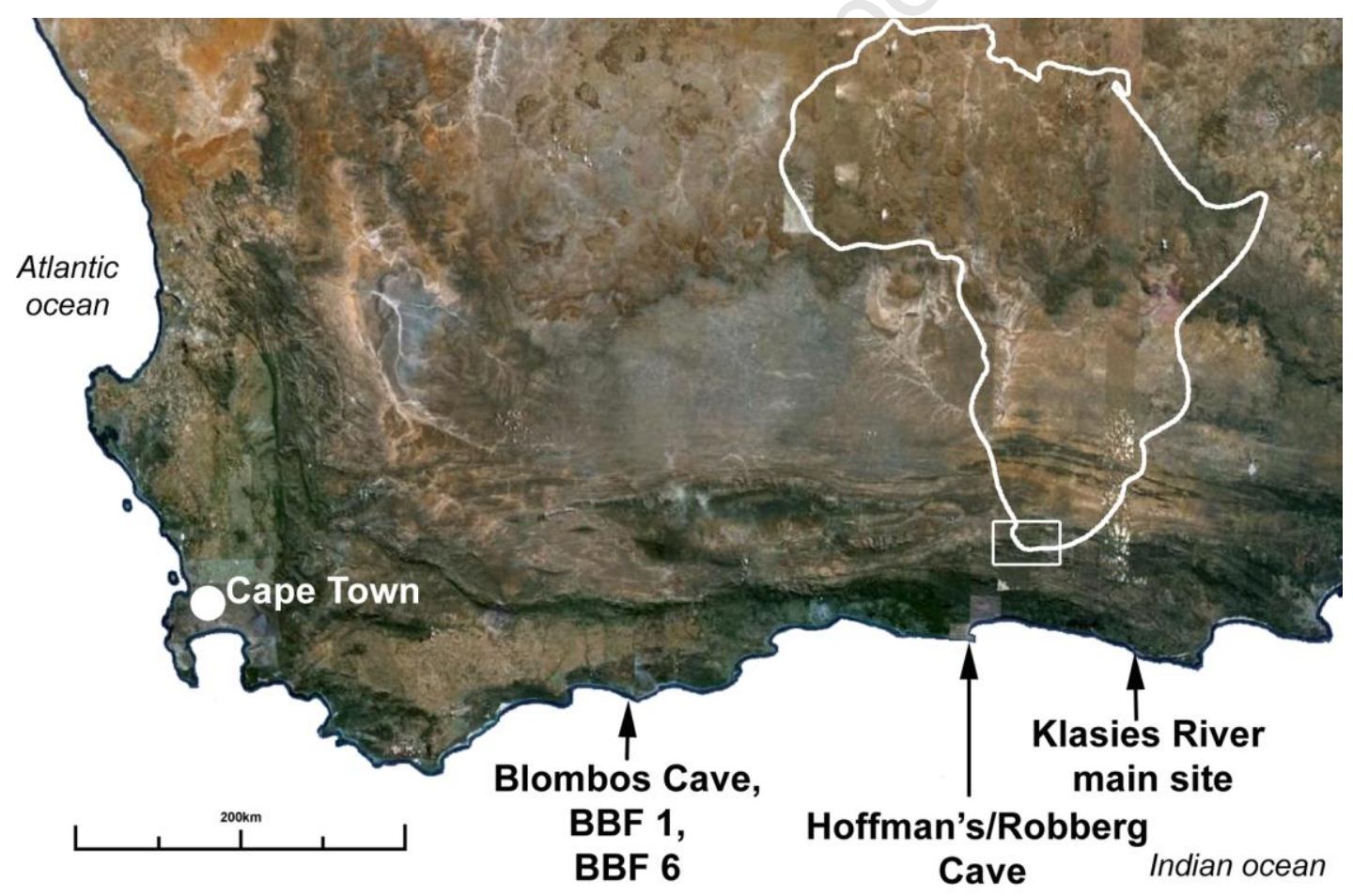

Figure 4.1. Location of sites analysed in this study. Image adapted from http://visibleearth.nasa.gov/

\subsubsection{Hoffman's/Robberg Cave}

Hoffman's/Robberg Cave (H/RC) is a large cave situated at $34^{\circ} 06^{\prime} 12.12^{\prime \prime} \mathrm{S}$, $23^{\circ} 23^{\prime} 09.58^{\prime \prime}$ E on the Robberg Peninsula on the southeastern coast of the Western Cape, 
South Africa (Kyriacou 2009) (Figure 4.1). The cave is one of several in the area formed through wave-cutting during a period of high sea levels into what is known as 'contact' breccia that is sandwiched between Silurian Table Mountain quartzite below and quartzitic sandstone of the Cretaceous Uitenhage Series above (Butzer 1973). The cave is situated next to the eastern Agulhas Bank (EAB), part of the southern African continental shelf, where the coastal shelf is narrow relative to the central Agulhas Bank (CAB) to the west (Brouwer and Griffiths 2006). It is located $1200 \mathrm{~m}$ WSW of Nelson Bay Cave (NBC) and the closest river, the Piesangs, is 4700m NNE (Figure 4.2).

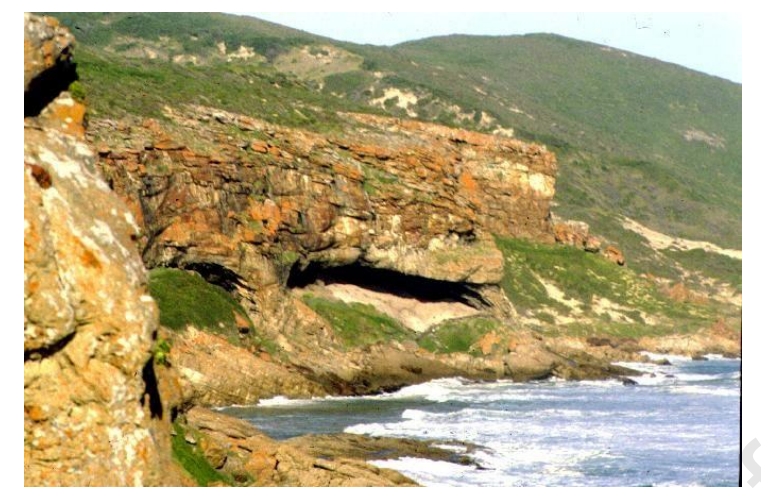

Figure 4.2. View of Hoffman's/Robberg Cave (Image courtesy of H. Deacon)

The cave, earlier known as East Guanogat/Ghwanogat, Hoffman's Cave or Robberg Cave, along with others in the area, was subject to several incidents of skeleton collecting and guano digging in the early 1900's although the literature on these early explorations is somewhat unclear with regards to which caves were involved (Rudner and Rudner 1973). In 1917, subcontractors of the South African Museum dug a test trench below stalactites at H/RC. During 1932 a team from Stellenbosch University excavated at three cave sites in the area and it is likely that one of them was H/RC (Rudner and Rudner 1973). Further excavations at $\mathrm{H} / \mathrm{RC}$ were initiated by A.C. Hoffman during the late 1950s. Only a selected sample of the material recovered was retained, and this is curated at the National Museum of Bloemfontein (Kyriacou 2009; Kyriacou and Sealy 2009). In order to obtain an unselected sample of artifacts and food remains, renewed excavations were carried out in 2007-2008 by a team led by Judith Sealy from the University of Cape Town (Figure 4.3). 


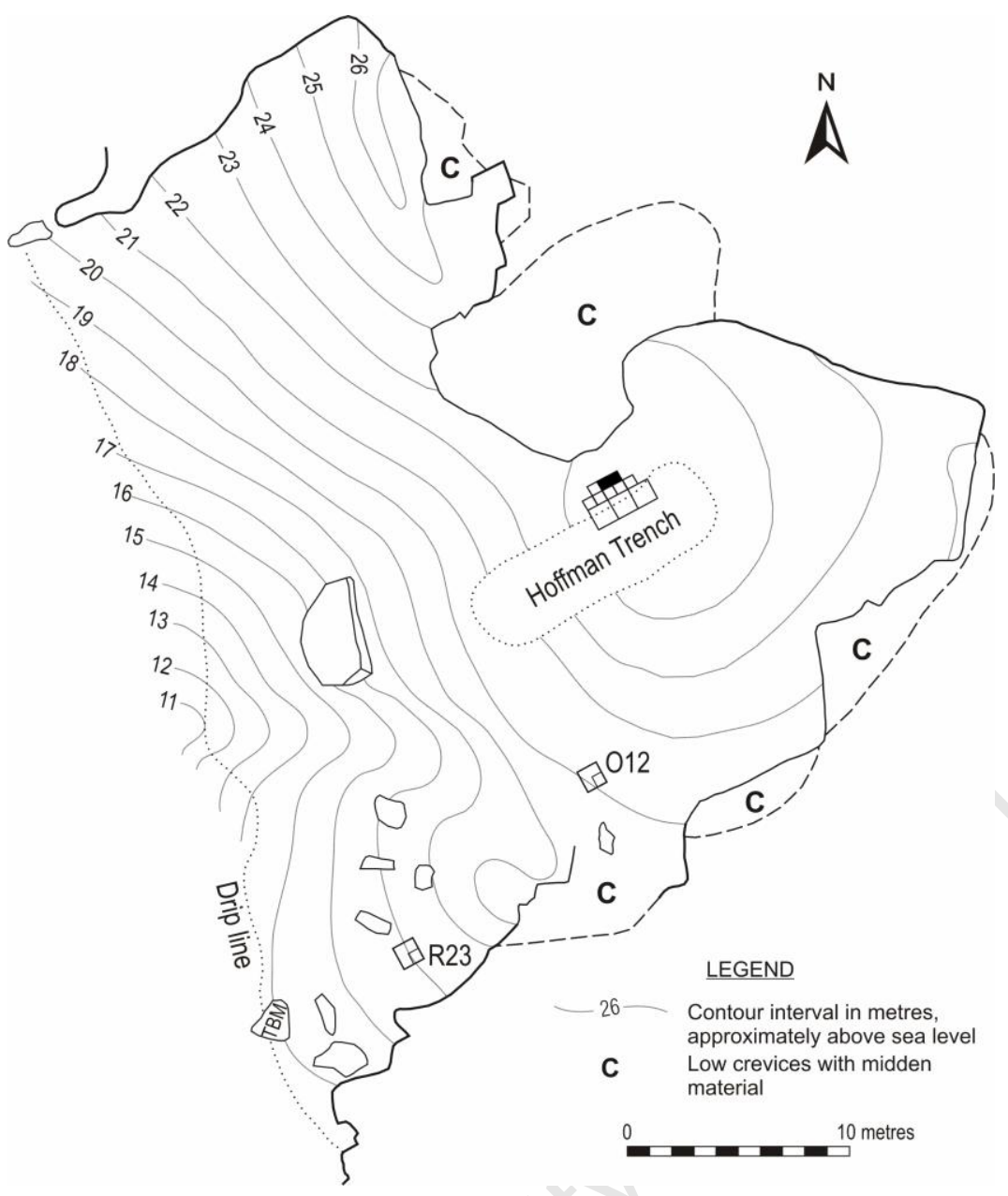

Figure 4.3. Site plan of Hoffman's/Robberg Cave indicating the trench dug by Hoffman and the squares excavated by Sealy in 2007 . The larger squares $(1 \mathrm{~m} \times 1 \mathrm{~m})$ are designated E6 (closest to drip line), E5 and E4. The fish analysed for this study comes from the two dark quadrats (D5c and D5d). (Image courtesy of J. Sealy)

The newly excavated material from H/RC dates to between 3000 and 4000 BP (Table 4.1); the latter date is somewhat older than dates obtained by Klein and Deacon for shell samples from Hoffman's trench (Fairhall et al. 1976). The deposit is approximately $1.6 \mathrm{~m}$ deep. The upper layers of deposit are characterised by dense mats of an estuarine eelgrass, Zostera capensis, that is thought to have been used by LSA people as bedding (Goodwin 1938). Some shellfish is present but their preservation is poor. A thin, heavily burnt and consolidated layer, IVAN, separates these Zostera-rich layers from those below (Figure 4.4). The layers below IVAN are mostly shell-rich with well preserved shellfish and little or no Zostera present. Layer IVAN represents an occupational hiatus of a few hundred 
years between the upper and lower layers. The Zostera-rich layers above and shell-rich layers below IVAN are referred to as 'Zostera' and 'shelly' respectively in this study.

Table 4.1. Radiocarbon dates on charcoal for Hoffman's/Robberg Cave

\begin{tabular}{|l|l|l|l|}
\hline LAYER & AGE B.P. & MATERIAL & REF. NO. \\
\hline Upper Zostera & $3370 \pm 40$ & charcoal & Beta-241142 \\
\hline Lower Zostera & $3310 \pm 40$ & charcoal & Beta-241149 \\
\hline Upper Shelly & $3760 \pm 40$ & charcoal & Beta-241144 \\
\hline Lower Shelly & $3920 \pm 40$ & charcoal & Beta-241147 \\
\hline Occupation base & $3990 \pm 50$ & charcoal & Beta-241146 \\
\hline
\end{tabular}

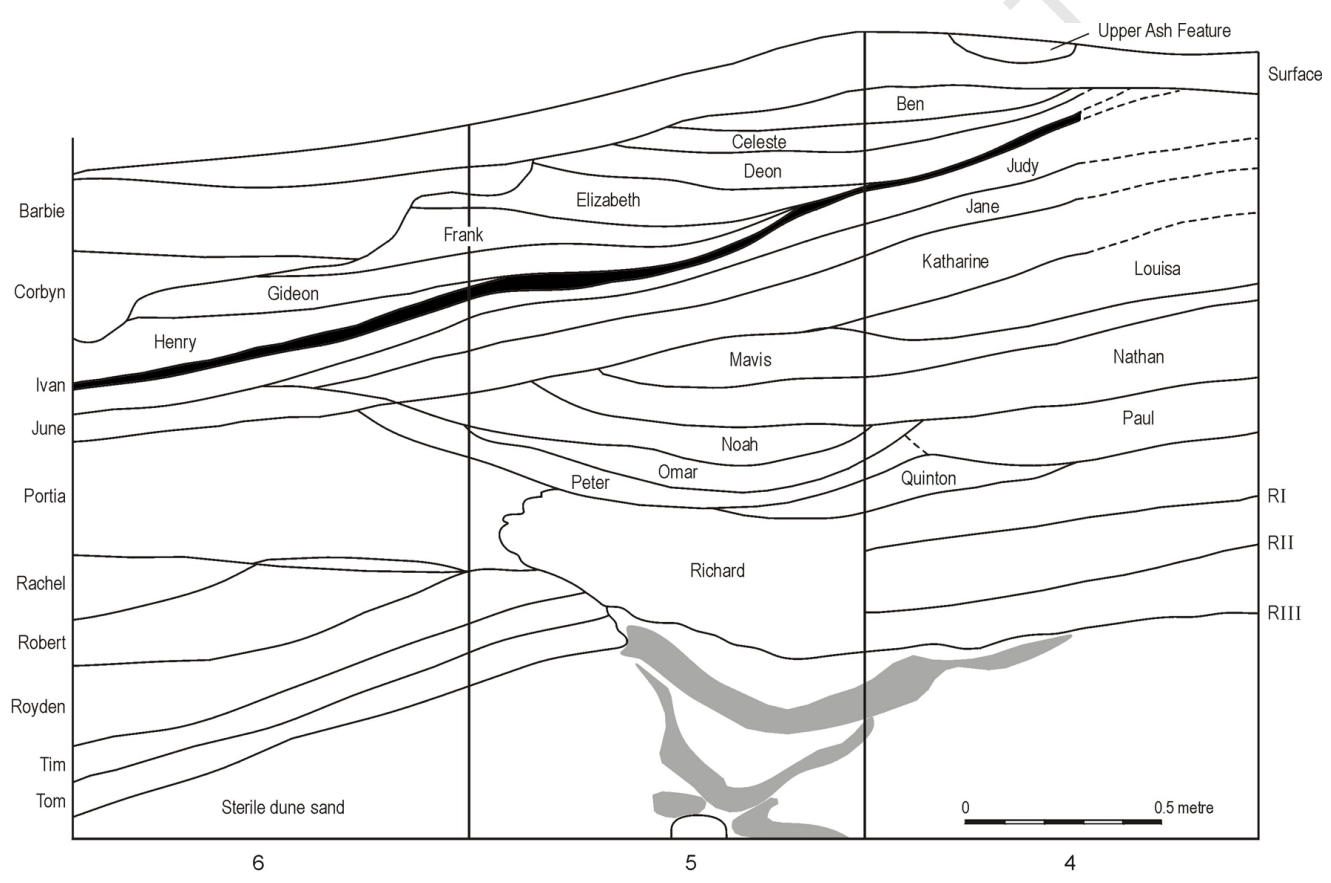

Figure 4.4. Stratigraphy of Hoffman's/Robberg Cave along the E/D section. The layer separating the Zostera-rich and shell-rich layers, IVAN, has been highlighted in black. (Image courtesy of J. Sealy)

The total excavated volume of the shelly layers is higher than that of the Zostera layers, partly due to the larger number of shelly layers (ten versus seven in the Zostera layers). The volumes per individual layer are however quite similar and range between 0.02 to $0.07 \mathrm{~m}^{3}$ in all but the three lowermost shelly layers, where excavated volumes are higher and range between 0.1 and $0.15 \mathrm{~m}^{3}$ (Table 4.2). 
Table 4.2. Excavated volumes $\left(\mathrm{m}^{3}\right)$ of squares D5c and D5d from Hoffman's/Robberg Cave

\begin{tabular}{|c|c|c|c|}
\hline Layer & Zostera $\left(\mathrm{m}^{3}\right)$ & Layer & Shelly $\left(\mathrm{m}^{3}\right)$ \\
\hline SURFACE & 0.05 & IVAN & 0.02 \\
\hline BEN & 0.02 & JANE & 0.05 \\
\hline CELESTE & 0.04 & JUDY & 0.02 \\
\hline DEON & 0.03 & KATHARINE & 0.07 \\
\hline ELIZABETH & 0.07 & MAVIS & 0.03 \\
\hline FRANK & 0.07 & NATHAN & 0.04 \\
\hline GIDEON & 0.04 & PETER & 0.06 \\
\hline \multirow[t]{3}{*}{ HENRY } & 0.04 & PORTIA & 0.10 \\
\hline & & RICHARD & 0.15 \\
\hline & & TOM & 0.10 \\
\hline total Zostera & 0.35 & to tal shelly & 0.63 \\
\hline
\end{tabular}

The study of the mammalian and other vertebrate fauna is in progress, but from personal observation during the separation of fish from other fauna it is clear that fish remains heavily dominate the assemblage. There are few mammalian bones and no tortoise bone present.

The Hoffman's/Robberg Cave lithic assemblage is characteristic of the post-Wilton period, and contains informal lithics predominantly made on locally available raw material (Deacon and Deacon 1999; Kyriacou 2009). Other artefacts include shale palettes, bone points, awls, tubes and rings, marine shell pendants and edge-ground marine shell artefacts, all similar to those found in the contemporary layers of nearby NBC (Inskeep 1987; Kyriacou 2009). Grooved pebble sinkers were recovered but these have not yet been studied. The transition from Wilton to post-Wilton at NBC occurred somewhat later, at $3300 \mathrm{BP}$.

The deposits at $\mathrm{H} / \mathrm{RC}$ derive from people living a hunter-gatherer lifestyle. Recent research based on stable isotope analysis of skeletons from a variety of sites in the vicinity of $\mathrm{H} / \mathrm{RC}$ suggests that there were geographically distinct groups with different diets occupying the landscape during the time of the occupation of Hoffman's/Robberg Cave (Sealy 2006; Sealy and Pfeiffer 2000). Burials from H/RC and other sites on the Robberg Peninsula have high $\delta^{15} \mathrm{~N}$ values, indicating diets including large quantities of high trophic level marine protein foods. 


\subsubsection{Blombosfontein 1}

Blombosfontein 1 (BBF 1) (previously named GSF 6, Henshilwood 1995) is a single occupation open site situated in the dune area approximately $90 \mathrm{~m}$ above sea level at $34^{\circ} 24^{\prime} 31.18 \mathrm{~S}$ and $21^{\circ} 12^{\prime} 48.02 \mathrm{E}$ (Henshilwood 1995, 2008) (Figure 4.1). The site spans approximately $22 \times 9 \mathrm{~m}$ and the deposit is heavily brecciated (Figure 4.5). Two square meters of $5-8 \mathrm{~cm}$ depth were excavated and the deposit sieved through $1.5 \mathrm{~mm}$ mesh (Figure 4.6). All the fish remains from these squares were analysed. BBF 1 is radiocarbon dated on shell to $6960 \pm 70$ BP. (Pta-6177) (Henshilwood 2008). The date has been corrected to compensate for the marine reservoir effect by subtracting 400 years from the uncalibrated shell date (Henshilwood 2008).

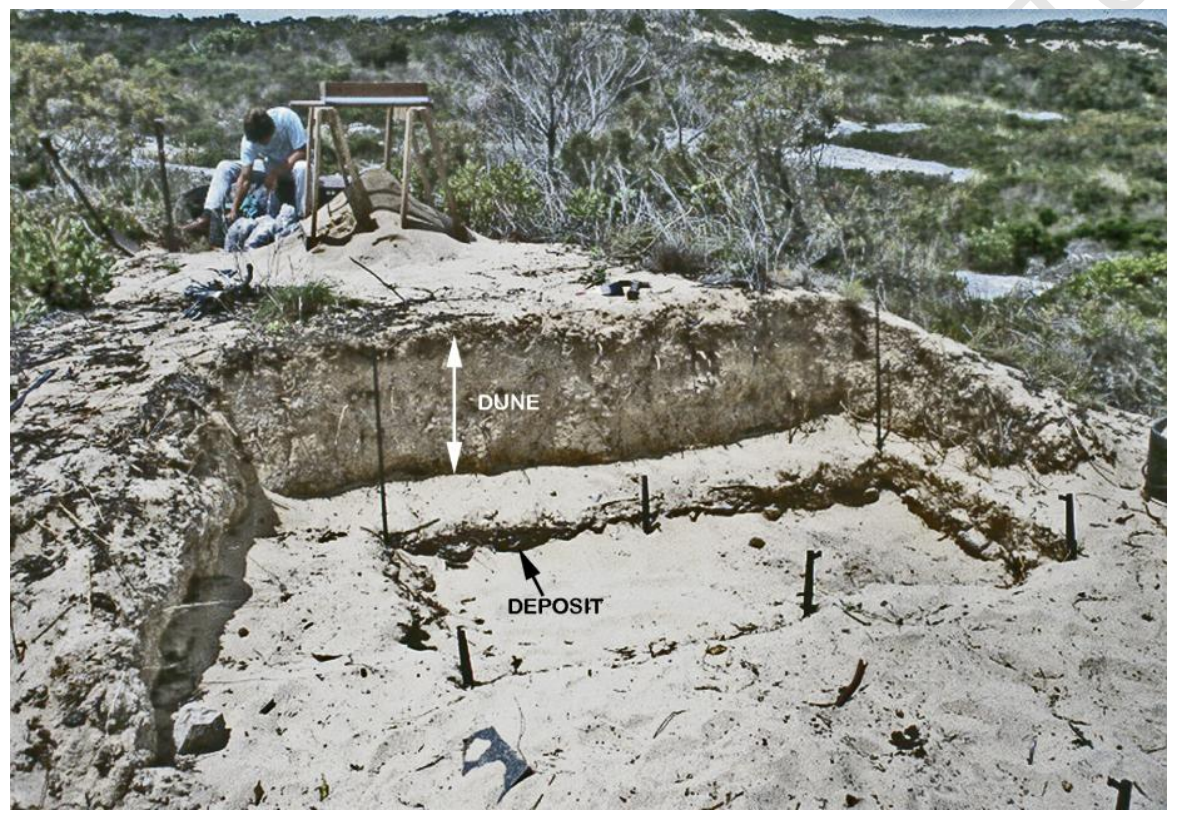

Figure 4.5. View of Blombosfontein 1. Arrows indicate the deposit and the thick overlying dune (Image courtesy of C. Henshilwood) 


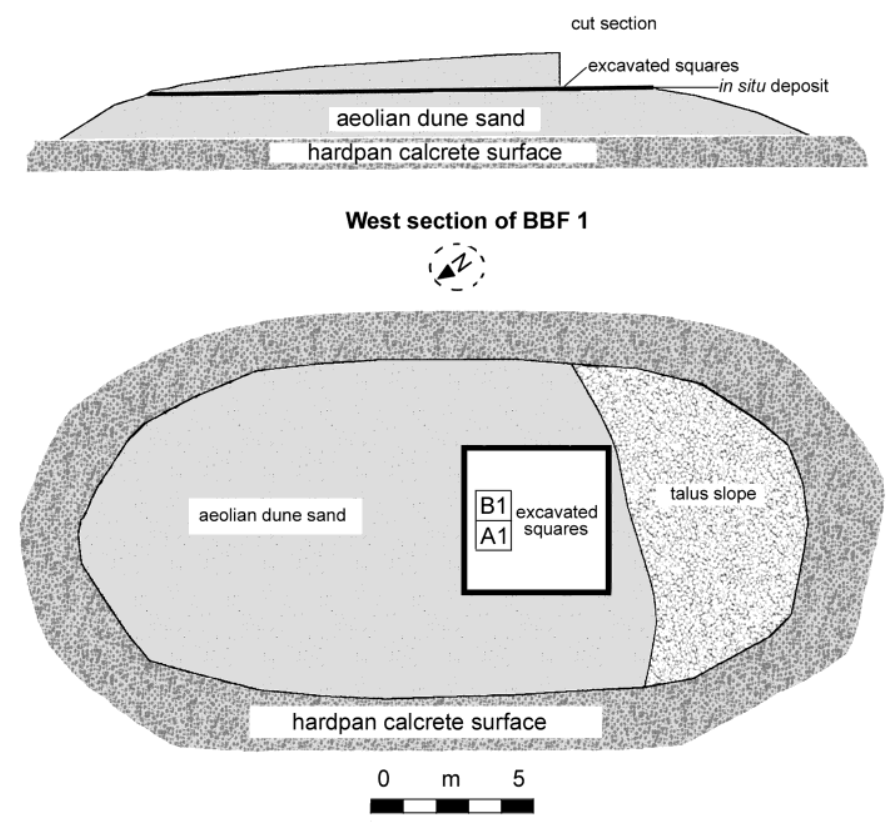

Figure 4.6. West section of Blombosfontein 1 and site layout (Image courtesy of C. Henshilwood)

No large mammal bone was recovered but tortoises were relatively abundant as were a variety of shellfish species. Oxygen isotope analysis of the edge increments of opercula of the shellfish Turbo sarmaticus, as well as the shell of Scutellastra tabularis, indicates winter occupation at this site (Henshilwood 2008). The site is currently exposed in an aeolian dunefield but during the time of its brief occupation conditions were warm and moist, with summer rainfall, and this area was forested (Henshilwood 2008).

\subsubsection{Blombosfontein 6}

Blombosfontein 6 (BBF 6), (previously named GSF 6, Henshilwood 1995), is a large $\left(>500 \mathrm{~m}^{2}\right)$ open midden situated $20 \mathrm{~m}$ from the ocean at $34^{\circ} 24^{\prime} 54.30 \mathrm{~S}$ and $21^{\circ} 13^{\prime} 12.13 \mathrm{E}$ (Henshilwood 2008) (Figures 4.1, 4.7). A 50 x 50cm column was excavated to a depth of $1.3 \mathrm{~m}$, revealing 12 discrete depositional events (Figure 4.8). The material analysed was recovered from $1.5 \mathrm{~mm}$ sieves. Two radiocarbon dates on shell, one each from the top and bottom of the sequence, date the deposit to between $3630 \pm 70$ BP. (Pta-6178) and 4070 \pm 60 BP. (Pta-6709) (Henshilwood 2008). 


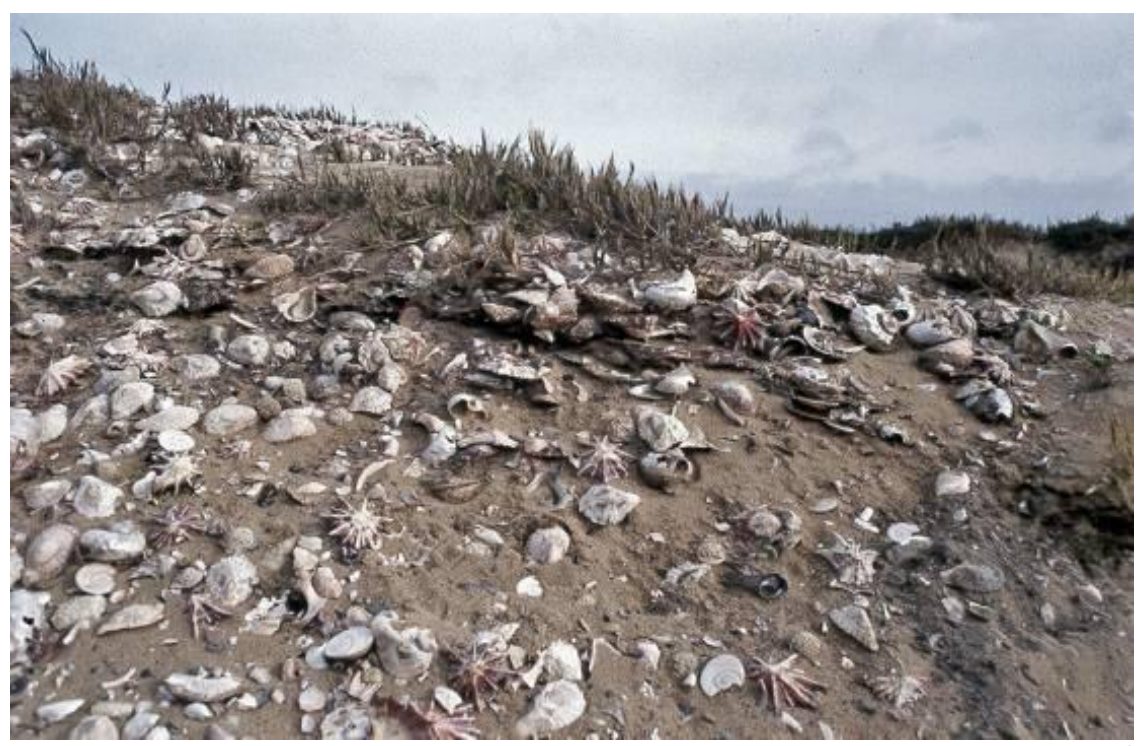

Figure 4.7. Surface of Blombosfontein 6 showing shells and ash lenses (Image courtesy of C. Henshilwood)

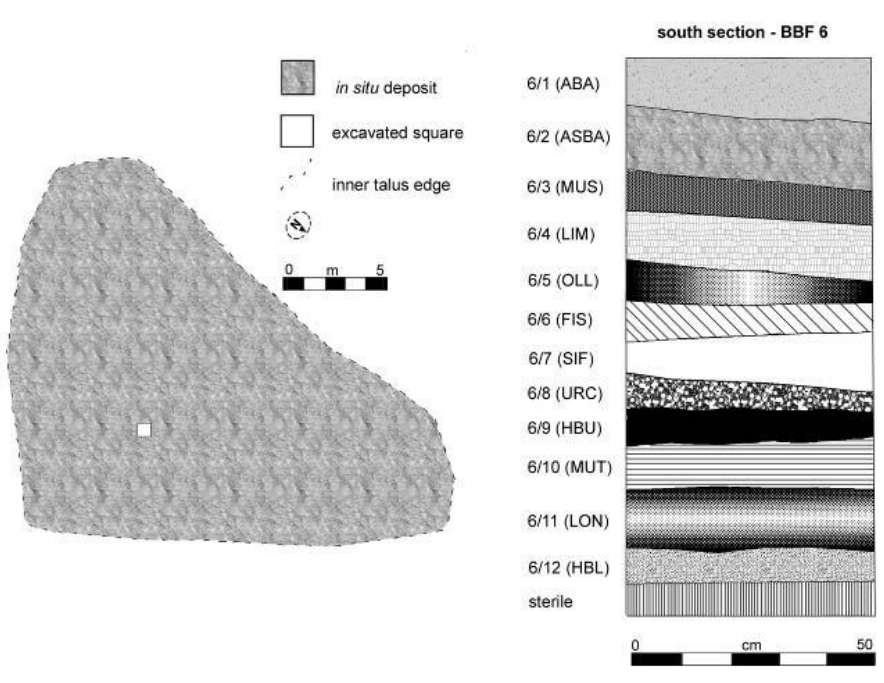

Figure 4.8. Blombosfontein 6 site layout indicating location of square excavated and stratigraphy (Image courtesy of C. Henshilwood)

The deposit is heavily dominated by shellfish with very few lithic or other cultural materials (Figure 4.7). No large mammal bone was found and smaller mammal bones were poorly preserved or fragmented and these could not be identified to species. There was no consistent patterning in the species of shellfish found through the sequence. The lack of cultural material and vast quantities of shellfish suggest that this was a shellfish 
processing or extraction site and not an occupation site (Henshilwood 2008). Oxygen isotope analysis of the edge increments of Turbo sarmaticus opercula indicates winter occupation for four of the layers, and a summer occupation for the youngest layer (Henshilwood 2008).

\subsubsection{Blombos Cave}

Blombos Cave (BBC) is a relatively small $\left(\sim 55 \mathrm{~m}^{2}\right)$ limestone cave situated at $34^{\circ} 24^{\prime} 54.58^{\prime \prime} \mathrm{S}, 20^{\circ} 13^{\prime} 31.21^{\prime \prime} \mathrm{E}$ (Figure 4.1). It is approximately $34 \mathrm{~m}$ above sea level and $100 \mathrm{~m}$ from the Indian Ocean on the southern Cape coast of the Western Cape Province, South Africa (Henshilwood et al. 2001b) (Figure 4.9). Chris Henshilwood commenced the excavations in 1991 and these are ongoing. The site contains both Middle (MSA) and Later Stone Age (LSA) deposits. In addition to excavations within the cave, a small test excavation was carried out directly in front of the dripline and this area on the talus contained MSA but no LSA deposits. The layers of this talus excavation cannot be directly correlated with those inside the cave, but it is possible to divide them into the three broad phases, M1, M2 and M3 based on their cultural content (Henshilwood et al. 2001b). All the recovered material was sieved through both $3 \mathrm{~mm}$ and $1.5 \mathrm{~mm}$ screens. Fish remains studied for this thesis comprise all the material from the MSA layers (all squares) retained in the $3 \mathrm{~mm}$ screens, and all the remains from the LSA layers in square G5 (Figure 4.10).

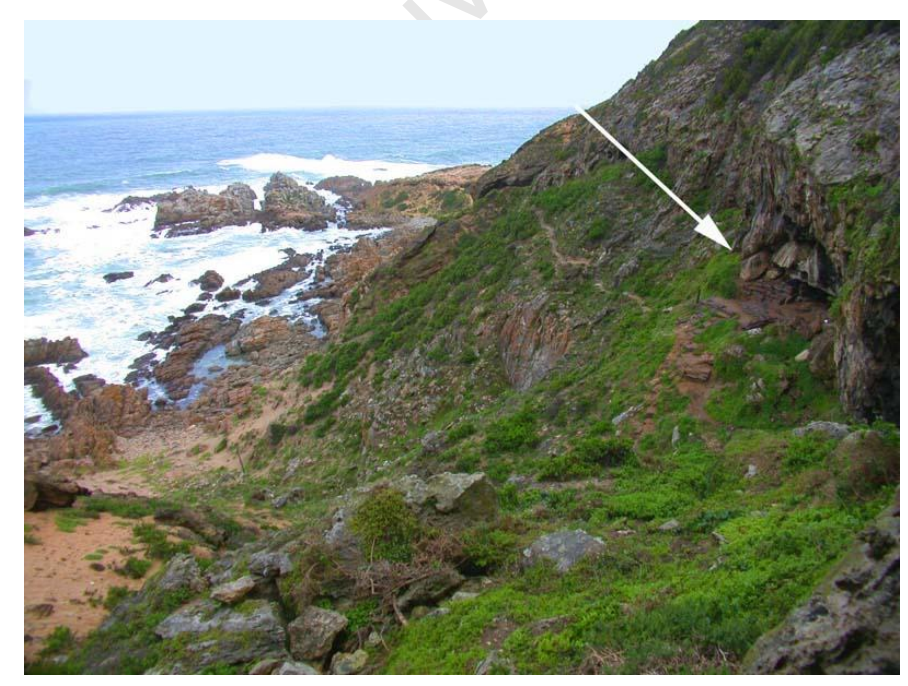

Figure 4.9. Location of Blombos Cave. Image courtesy of C. Henshilwood 


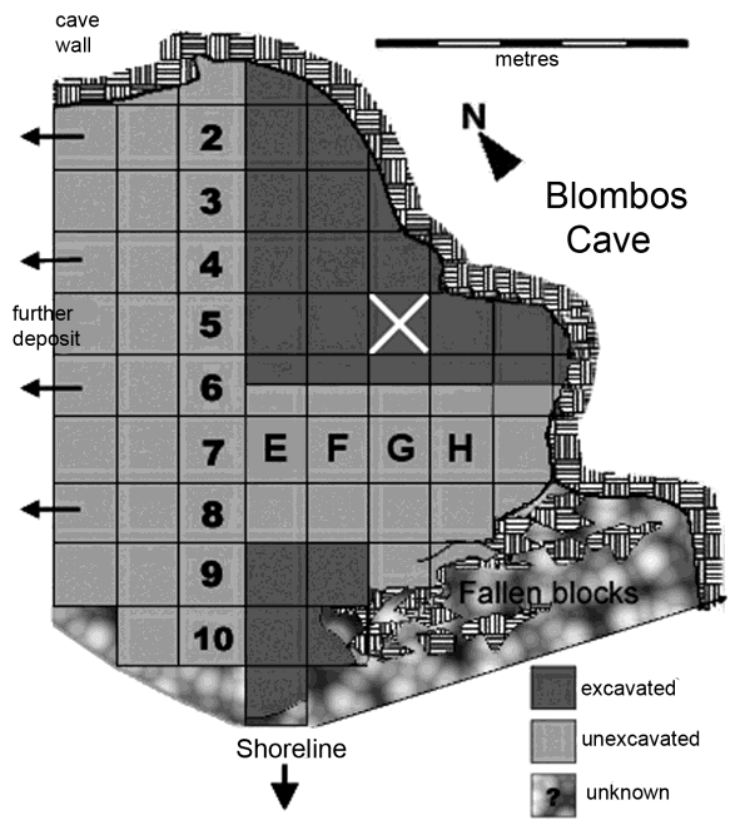

Figure 4.10. Site layout of Blombos Cave. The white cross indicates the location of the LSA sample analysed for this study

\subsubsection{Blombos Cave Later Stone Age}

The BBC LSA occupation is dated to between $290 \pm 20$ BP (Pta-6184) and $1995 \pm 43$

BP(Wk-25554) (Henshilwood 1995) (Table 4.3). Excavation units have been grouped into five layers based on stratigraphy and contents. Layer 6, also referred to as BBC HIATUS, a layer of mostly sterile yellow aeolian sand, is a hiatus unit separating the LSA from the MSA layers. It contains some archaeological material, probably trampled in from Layer 5 above, but does not contain any stratified separations or other features. Material from Layer 6 has not been included in this study as its provenance is unclear.

Table 4.3. Radiocarbon dates on charcoal for the Later Stone Age layers at Blombos Cave

\begin{tabular}{|l|l|l|l|}
\hline LAYER & AGE BP & MATERIAL & REF. NO \\
\hline Layer 1 & $290 \pm 20$ & charcoal & Pta-6184 \\
\hline \multirow{4}{*}{ Layer 2 } & $1230 \pm 65$ & charcoal & Wk-25553 \\
\cline { 2 - 4 } & $1557 \pm 59$ & charcoal & Wk-25552 \\
\hline Layer 3 & $1856 \pm 51$ & charcoal & Wk-25551 \\
\hline & $1964 \pm 53$ & charcoal & Wk-25550 \\
\cline { 2 - 4 } & $1994 \pm 47$ & charcoal & Wk-25548 \\
\cline { 2 - 4 } Layer 4 & $2019 \pm 50$ & charcoal & Wk-25555 \\
\hline & $1840 \pm 50$ & charcoal & Pta-6185 \\
\cline { 2 - 4 } Layer 5 & $1986 \pm 52$ & charcoal & Wk-25549 \\
\cline { 2 - 4 } & $1995 \pm 43$ & charcoal & Wk-25554 \\
\hline
\end{tabular}


The older LSA layers at BBC date to the period when herders first become archaeologically visible in southern Africa. Prior to 2000 BP the evidence indicates that only hunter-gatherers were present in the Western Cape (Klein 1986; Sadr 2008; Sadr et al. 2003; Smith et al. 1991). Sheep bones were found in Layers 5 and 6, and two were directly dated by accelerator mass spectrometry (AMS) to $1960 \pm 50 \mathrm{BP}$ (OxA-4543) and $1880 \pm 55$ (OxA-4544) BP. The physical location and size of the site would have made access difficult for herders with livestock, but the presence of small amounts of sheep bone and pottery in the oldest layers suggests that there was some contact between huntergatherers and herders in the area at that time (Henshilwood 1995; 2008). Oxygen isotope analysis of the edge increments of Turbo sarmaticus opercula indicated that layers 1 , and 3 were probably occupied during mid-winter, and layers 2, 4 and 5 were occupied during spring or autumn (Henshilwood 2008).

A wide range of mammals including seals were consumed on-site. The majority of bovids fall in the small to small-medium size classes, and the Cape dune mole-rat, Bathyergus suillus, is the most commonly occurring small mammal (Henshilwood 1995, 1997). Distinctive burn patterns on the mole-rat maxillae indicate that they were cooked by humans, and not brought in by another predator (Henshilwood 1997). Shellfish is common, particularly in the older layers.

\subsubsection{Blombos Cave Middle Stone Age}

The BBC MSA layers date between $\sim 72 \mathrm{ka}$ to $\sim 100 \mathrm{ka}$ (Jacobs et al. 2006; Tribolo 2006) (Table 4.4). As mentioned above, the MSA layers are separated from the LSA layers by a $5-60 \mathrm{~cm}$ thick layer of sterile aeolian dune sand (BBC Hiatus) with an age of $\sim 69-70 \mathrm{ka}$. The dune layer was laid down during a time of sea level regression, when an influx of aeolian sand covered the MSA levels and then sealed the cave entrance. The cave entrance reopened again during the mid- to late Holocene and was first reoccupied 2000 years ago (Henshilwood 2005; Henshilwood et al. 2001b). 
Table 4.4. Blombos Cave Middle Stone Age dates (in kya) per phase based on optically stimulated luminescence (OSL) and thermoluminescence (TL) dating techniques

\begin{tabular}{|l|l|l|l|l|l|}
\hline & \multicolumn{3}{|c|}{ Phase } & BBC M3 \\
\hline Dates in ka & Method & BBC HIATUS & BBC M1 (Still Bay) & BBC M2 & \\
\hline Henshilwood et al. 2002 & OSL & $69 \pm 5-70 \pm 5$ & & & \\
\hline Henshilwood et al. 2002 & TL & & $68.5 \pm 7.5-84.8 \pm 7.3$ & & \\
\hline Jacobs et al. 2003a & OSL (single grain) & $69.2 \pm 3.9$ & & & \\
\hline Jacobs et al. 2003b & OSL (multiple grain) & $67.3 \pm 3.8$ & & & \\
\hline Jacobs et al. 2006 & OSL & $67.8 \pm 4.2$ & $72.7 \pm 3.1$ & $76.8 \pm 3.1-84.6 \pm 5.8$ & $98.9 \pm 4.5$ \\
\hline Tribolo et al. 2006 & TL & & $74 \pm 5-78 \pm 6$ & & \\
\hline
\end{tabular}

The MSA layers have been grouped into three phases based on artefactual content and stratigraphy. These phases are, from oldest to youngest, M3, M2 and M1 (Still Bay) (Figure 4.11).

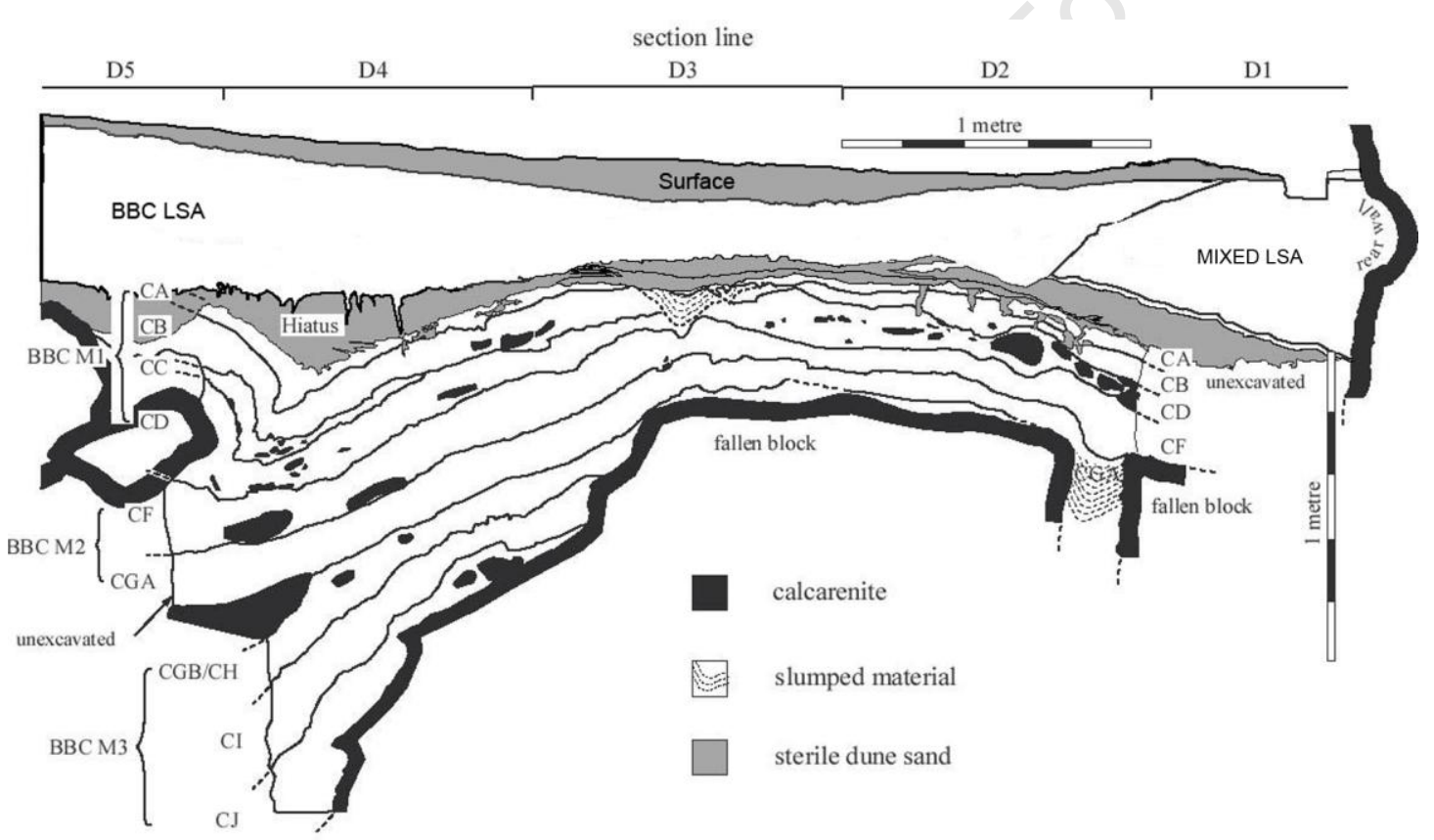

Figure 4.11. Stratigraphy of the Blombos Cave Middle Stone Age layers along the D/E section

The uppermost phase, M1, or the Still Bay phase, contains high densities of bifacial foliate points that are typical of the Still Bay techno-tradition (Henshilwood et al. 2001b; Villa et al. 2009) (Figure 4.12). Over 39 Nassarius kraussianus shell beads were found in this layer, and they provide the oldest known evidence for personal adornment in southern Africa (d'Errico et al. 2005; Henshilwood et al. 2004). Two slabs of ochre engraved with complex geometric patterns (Henshilwood et al. 2009; Henshilwood et al. 2002) and three 
deliberately engraved bone fragments (d'Errico and Henshilwood 2007; d'Errico et al. 2001; Henshilwood et al. 2001a) were also found in M1. The occurrence of shell beads as well as engraved ochre and bone suggests that the M1 inhabitants had the cognitive capacity for mediating their behaviour with symbols and this included the production of symbolic material culture (d'Errico and Henshilwood 2007; Henshilwood et al. 2001a; Henshilwood et al. 2009; Henshilwood et al. 2002; Henshilwood and Marean 2003).

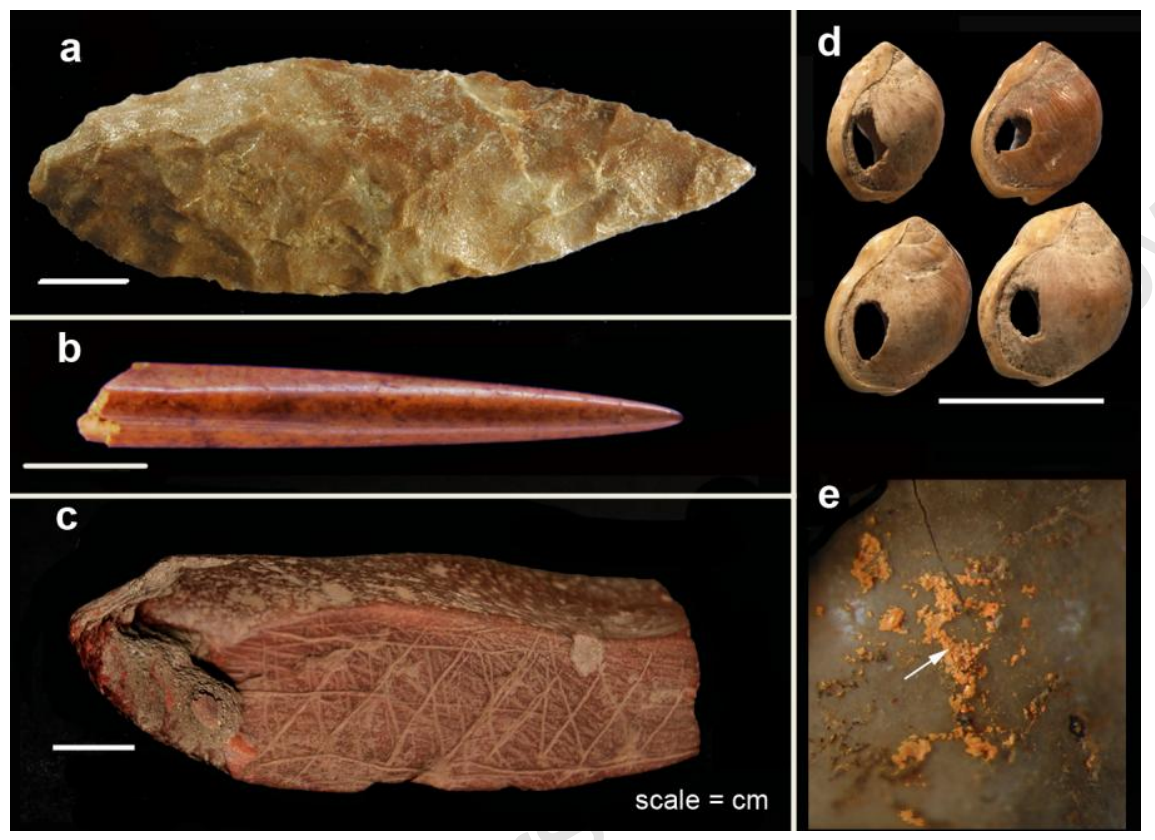

Figure 4.12. Blombos Cave Middle Stone Age artefacts from the M1/Still Bay phase. a: silcrete bifacial point ; b: polished bone point ; : engraved ochre ; d: perforated $N$. kraussianus beads; e: detail of ochre residue on $\mathbf{N}$. kraussianus bead (Images courtesy of C. Henshilwood and F. d'Errico)

Several bone tools were found in M1 but are more frequent in the M2 phase. They are mostly awls but there are also a number of points, some of which are highly polished. There is clear evidence that some of these points were hafted (d'Errico and Henshilwood 2007; Henshilwood and Sealy 1997; Henshilwood et al. 2001a). Bone tools are rare in the MSA and the finds at BBC represent the highest number known from a single MSA site in southern Africa. Some Still Bay bifacial points also occur in M2, and two Nassarius kraussianus beads were found in this phase. 
Shellfish occur in all phases but are most common in the M3 phase where dense shell middens associated with hearths occur (Henshilwood et al. 2001b). Bone and shell are generally well preserved across most of the cave but plant materials and charcoals are rare in the MSA levels.

The highest density of ochre is found in M3 and a high percentage of these pieces show evidence of utilization (Henshilwood et al. 2009; Henshilwood et al. 2001b). The lithics of M3 are still under study but preliminary results indicate that they differ from the typical MSA I and II patterns reported for Klasies River main site (Soressi 2005). A total of nine isolated human teeth or tooth fragments that conform morphologically to anatomically modern Homo sapiens have been found from all phases (Grine and Henshilwood 2002; Grine et al. 2000).

\subsubsection{Klasies River main site}

Klasies River (KR) main site is a complex of caverns and overhangs located at $34^{\circ} 6^{\prime} 29.17^{\prime \prime} \mathrm{S} 24^{\circ} 23^{\prime} 24.50^{\prime \prime} \mathrm{E}$ on the Tsitsikamma coast (Figure 4.13). The site is in the Eastern Cape Province and lies $43 \mathrm{~km} \mathrm{WNW}$ of Cape St Francis. It is approximately $0.5 \mathrm{~km}$ from the Klasies River itself, which is currently a narrow stream that enters the sea through a narrow gorge and the river mouth is blocked by a sandbar (von den Driesch 2004). The caverns and overhangs are cut into quartz arenite of the Silurian Cape Supergroup and a fossil dune above the site contributes lime-rich water which has helped to preserve the deposit. Preservation of the deposit is variable depending on location. Deposits closer to the cliff face are better preserved due to the presence here of alkaline water contributed from the overlying dune. Further away from the cliff face and within the caverns the organics are less well preserved due to the actions of acidic groundwater (Deacon 2001a). 


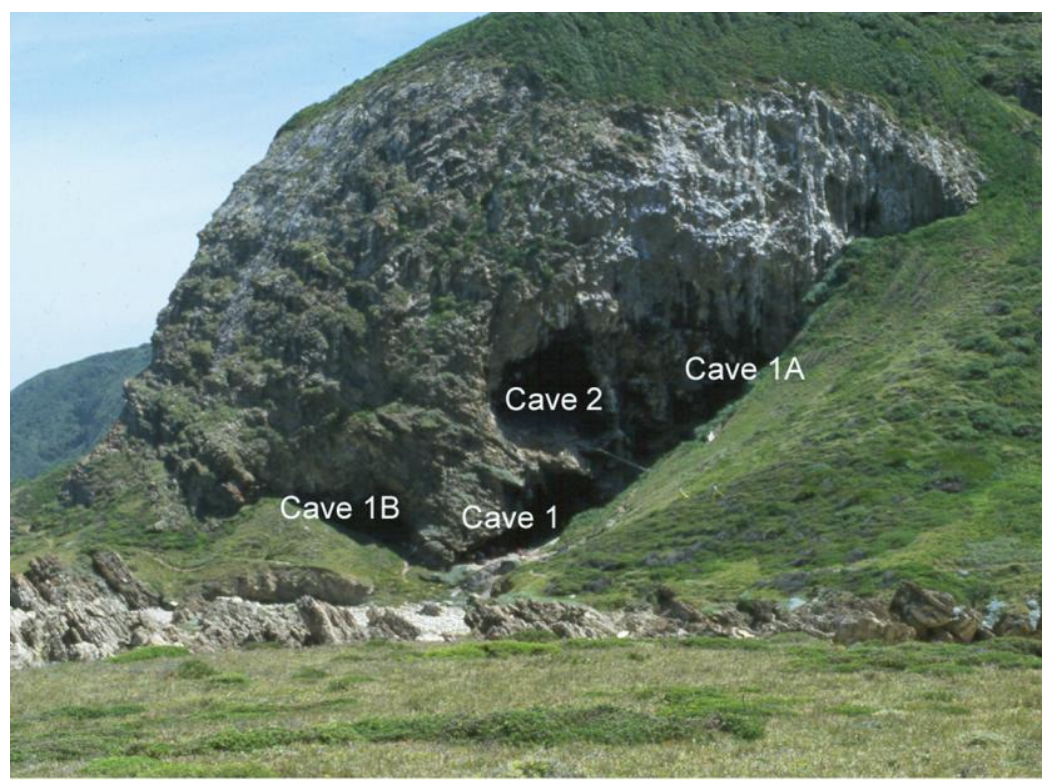

Figure 4.13. Location of Klasies River main site. Image courtesy of H. Deacon

KR main site is technically a single depository open site consisting of a number of caverns containing deposit with strata that can be correlated directly with each other (Deacon 1989, 2008; Singer and Wymer 1982) (Figure 4.14). As the sediment built up against the cliff face, the deeper caverns, cave 1 and 1C, were sealed off. Further accumulation of deposit subsequently filled cave 2 . The full extent of deposit reached $20 \mathrm{~m}$ against the cliff face. The deposits were undercut by water action during a higher sea stand around 6000 years ago, when between two thirds and three quarters of the material was removed (Deacon 2001a; Wurz 2000). The majority of the deposit dates to the MSA with a thin capping of Holocene material in some areas.

Initial excavations of LSA and MSA deposits were carried out by John Wymer in 1967 and 1968 (Singer and Wymer 1982). Large volumes were excavated and relatively large screens $(1 / 2$ and $1 / 4$ inch) were used to sieve the material. Based primarily on technological analysis of lithics, the MSA layers were grouped into four discrete chronological substages or phases (Singer and Wymer 1982). The cultural phases, from oldest to youngest, are MSA I, MSA II, Howieson's Poort (HP) and MSA III (Figure 4.14). An additional layer above MSA III, MSA IV, contains some MSA material but is thought to not be in situ (Deacon and Geleijnse 1988). 


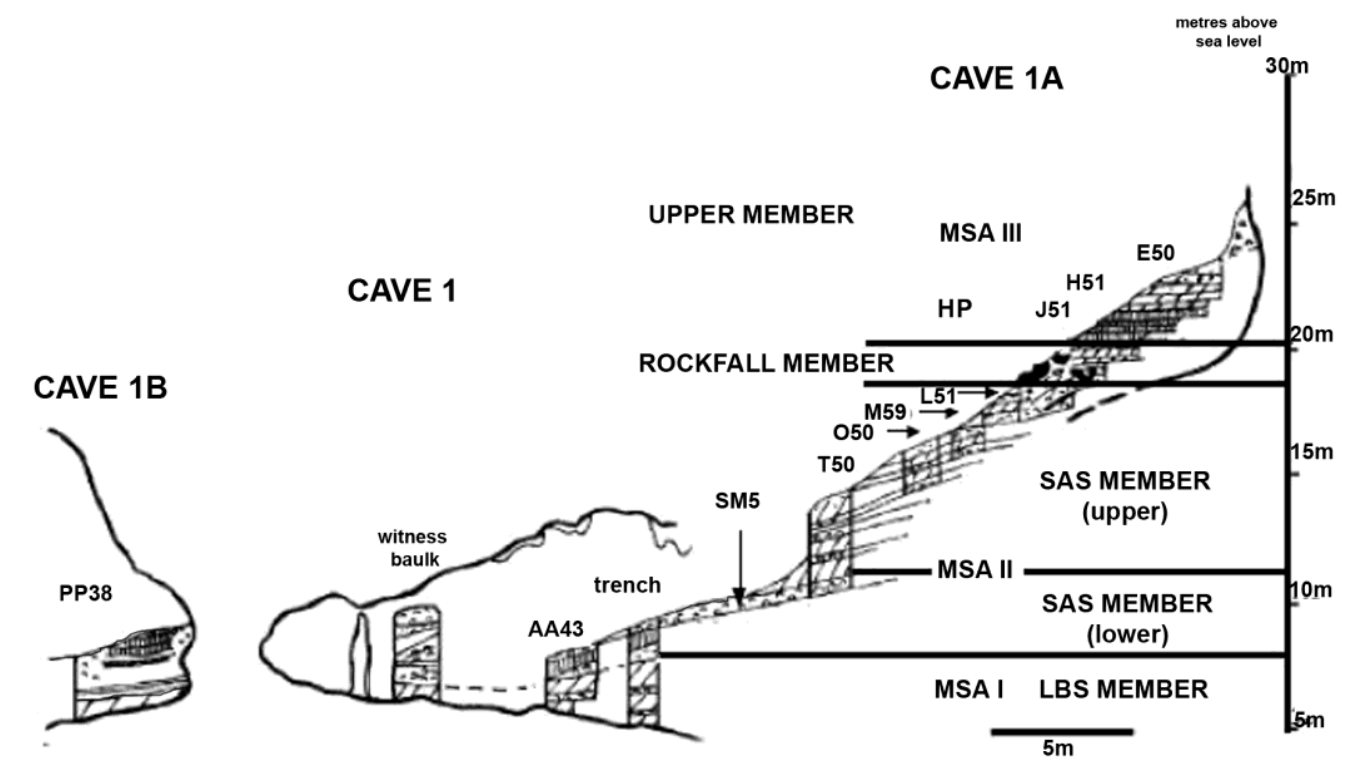

Figure 4.14. Stratigraphy of Klasies River main site Cave 1A (after Deacon and Geleijnse 1988 and Wurz 2000)

Limited scale further excavations of the MSA layers were subsequently conducted by Hilary Deacon, starting in 1984 (Deacon 1995; Deacon and Geleijnse 1988). Excavation was at a microstratigraphic level and smaller screens ( 2 and $3 \mathrm{~mm}$ ) were used to sieve the material (Deacon and Geleijnse 1988). These subsequent excavations showed that the MSA deposit is well stratified and consists of alternating occupational units containing artefacts, hearths, ash, faunal remains, and sandy non-occupational units (hereafter referred to as either cultural or sterile units) (Deacon 1995, 2008; Deacon and Geleijnse 1988). The sterile non-occupational units are thought to have accumulated over extensive periods of time based on their thickness relative to the thinner cultural occupational units, particularly in the MSA I and MSA III members (Deacon and Geleijnse 1988). Volume data are not available, although the section profiles indicate that the volume of the sterile units in the MSA I and III members could be up to double that of the cultural units. The sterile units of the HP occur less frequently and are thinner than the cultural units of this member (Deacon and Geleijnse 1988). Deacon grouped units with similar lithologies together to form members. This was done to achieve a finer resolution of the stratigraphy, which would allow distinction between culturally and naturally accumulated deposits, and thereby a better understanding of the site formation processes. 
The lowest member, associated with the technological sub-stage MSA I, was named the LBS member (light brown sand). Above this is the SAS (shell and sand) member, divided into a series of sub-members depending on their location and to conform to Singer and Wymer's stratigraphy (SAS RBS, SAS B, SASU, SAS W, SAS R). The SAS member is associated with MSA II and is sometimes split into SAS Upper (or MSA II Upper) and SAS Lower (MSA II Lower), based on technological differences in artefact manufacture (Wurz 2000, 2002). The RF (rockfall) member lies above SAS, and represents a period of very low frequency of human occupation. The RF member is usually included in the MSA II (Wurz 2002). The Upper member includes the sub-stages MSA III and HP. These members conform broadly to the sub-stages assigned by Singer and Wymer (1982), but the microstratigraphic approach enabled a better understanding of the very complex stratigraphy that is characterised by slumping, truncation and plastic flow of layers under conditions of water saturation and heavy overburdens.

Several dating techniques have been applied to the site. This has resulted in a spread of dates, partly due to variations in the geochemical and preservational conditions of the material dated (Bada and Deems 1975; Feathers 2002; Grün et al. 1990; Jacobs et al. 2008; Shackleton 1982; Tribolo 2003; Vogel 2001; Wurz 2002) (Table 4.5). The base of the sequence has a maximum age of $125 \mathrm{ka}$ and the uppermost layers lie beyond the limit of radiocarbon dating, with a minimum age of $50 \mathrm{ka}$ (Deacon and Geleijnse 1988).

Table 4.5. Klasies River main site Middle Stone Age dates per phase (in kya) based on amino acid racemization (AAR), Oxygen Isotope Stage (OIS), optically stimulated luminescence (OSL), thermoluminescence (TL), infra-red stimulated luminescence (IRSL), uranium series (US) and radiocarbon $(\mathrm{C} 14)$ dating techniques

\begin{tabular}{|c|c|c|c|c|c|c|}
\hline & & & & Sub & -stage & \\
\hline & Method & Sample materi & MSA III & HP & MSA II & $\overline{\text { MSA I }}$ \\
\hline Bada and Deems 1975 & AAR & bone & & & 89 & $90-110$ \\
\hline Shackelton 1982 & OIS & shell & & & OIS5c/5a & OIS5e \\
\hline Grun et al. 1990 & ESR & tooth & & $40-60$ & $88-93$ & \\
\hline Vogel 2001 & US & stalagmite & & & $85.2 \pm 2.1-101.7 \pm 7$ & $113 \pm 3.4$ \\
\hline Vogel 2001 & US & carbonate crust & & $70.9 \pm 5.3$ & $82.7 \pm 7.0$ & \\
\hline Vogel 2001 & US & shell & & & $28.5 \pm 2.2-37.4 \pm 3.2$ & \\
\hline Vogel 2001 & C14 & charcoal & $>45$ & $>40 ;>50$ & & \\
\hline Feathers 2002 & OSL/IRSL & sand & $\sim 50$ & $55-60$ & $75-85$ & $110-115$ \\
\hline \begin{tabular}{|l|} 
Tribolo 2003 \\
\end{tabular} & $\mathrm{TL}$ & stone & $60 \pm 5$ & $56 \pm 3$ & & \\
\hline Jacobs et al. 2008 & OSL & sand & $57.9 \pm 2.3$ & $63.4 \pm 2.6-65.5 \pm 2.6$ & $>71.6 \pm 2.9$ & \\
\hline Member & & & Upper & Upper & SAS & LBS \\
\hline
\end{tabular}


Throughout the KR MSA sequence and within each sub-stage there are stylistic changes in the production of typologically similar lithic artefacts that is not related to their function, and a similar pattern occurs at other MSA sites (Wurz 2000). This variability through time is interpreted by Wurz $(2000 ; 2002)$ as signifying that MSA people behaved, in terms of time-restricted patterning, in the same way as LSA people, suggesting no cognitive differences between the two groups.

The HP sub-stage is one of two MSA industries in southern Africa that is typologically very distinct (the other being the Still Bay techno-tradition) (Villa et al. 2010; Wurz 1999). The HP is characterised by standardised backed artefacts often made on non-local raw materials (Deacon 1989; Deacon and Wurz 2002; Wurz 1997, 1999, 2008) (but see Villa et al. 2010 for earlier examples of artefact standardisation) (Figure 4.15c).
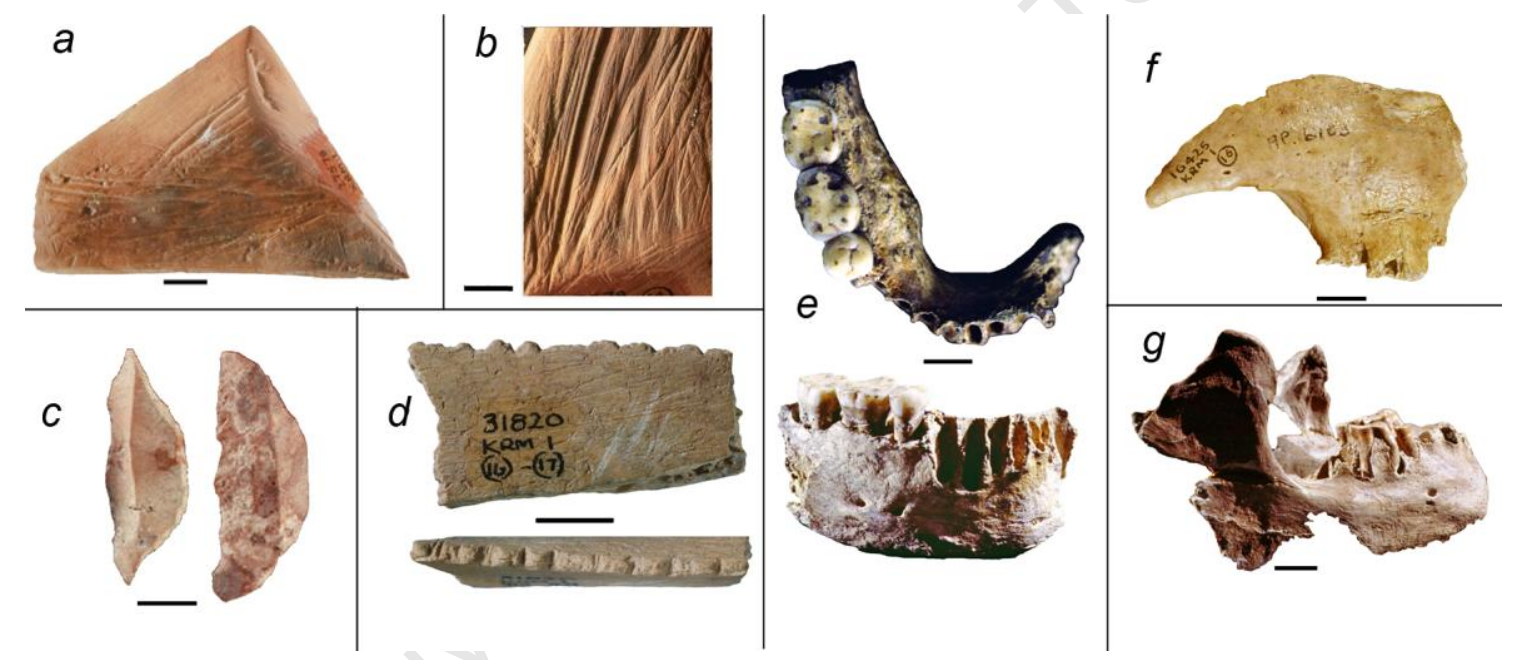

Figure 4.15. Artefacts and hominid remains from Klasies River main site. a: ochre crayon from HP; b: gouged ochre from MSA II; c: HP backed segments; d: notched bone from MSA II; e: specimen \# KRM 13400 mandible from MSA II; f: \# KRM 16425 frontal with cut marks and tearing from MSA II, g: \# KRM 41815 mandible from MSA II. Scale is in cm. (Images courtesy of H. Deacon, C. Henshilwood, F. d'Errico and S. Wurz)

Utilized and modified ochre, although not common, has been found in all sub-stages, including pieces that appear to have dendritic/covergent motifs engraved on them from the HP and MSA II, and several 'crayons' (Knight et al. 1995; Watts 1998; Wurz 2000) (Figure 4.15a, b; Table 4.5). Three notched/denticulated bone artefacts were recovered during the Wymer excavation of the MSA II levels (d'Errico and Henshilwood 2007; 
Wurz 2000) (Figure 4.15d). An additional bone fragment from the HP appears to have been deliberately engraved (d'Errico and Henshilwood 2007).

KR contains some remains of anatomically modern humans with an age of between 90 and 110 kya (Deacon 1995; Feathers 2002; Groves and Thorne 2000; Royer et al. 2009; Singer and Wymer 1982) (Figure 4.15e, f, g). Some of the human remains found are thought to fall on the edge of modern human morphological parameters, and are unusually variable by the standards of contemporary human populations (Rightmire et al. 2006; Wolpoff and Caspari 1996). Several of the human remains from the Wymer excavation are burnt and some show cut marks or impact scars, including one fragment that was damaged when the bone was still fresh (Deacon 2008; Deacon and Deacon 1999) (Figure $4.15 \mathrm{e}, \mathrm{f}, \mathrm{g})$. Because of the fragmentary state of the human remains, and the fact that these were found in the same context as other faunal remains, there was speculation that cannibalism was practised at the site (Deacon and Deacon 1999; White 1987). Subsequent re-evaluation of the contexts of these finds has led Deacon (2008) to conclude that they are all from the same provenance, and represent a minimum of five individuals who were butchered and killed in the same manner as other animals found at the site. A further two cannibalized individuals were found in layers $20 \mathrm{ka}$ older than those found during the Wymer excavations. Together, these finds suggest that incidents of cannibalism did take place at the site from $~ 90 \mathrm{ka}$ (Deacon 2008).

Until recently, KR produced the oldest evidence for the systematic exploitation of marine shellfish by Homo sapiens (Deacon 1995; Thackeray 1988). Recent excavations in cave 13B at Pinnacle Point, southern Cape, have revealed even older evidence dating to $164 \mathrm{ka}$ (Marean et al. 2007; Marean et al. 2004b). Shellfish occur in all sub-stages at Klasies but are least common in the HP and some of these layers contain no shellfish (Thackeray 1988). This low frequency or absence of shellfish in the HP has been attributed to lowered sea levels resulting in greater distances between the site and the coast (Thackeray 1988). Fish remains occur in all sub-stages but are more abundant, by weight, in the sterile layers of all the members above the MSA I (Deacon and Geleijnse 1988). The weight of fish in the MSA I member was not reported. 


\subsection{Site-specific variations in analysis}

Due to differences in the excavation methodology employed, and the unavailability of certain data from some sites, there is some variation in how the data from the various assemblages are presented in this thesis. This relates mostly to calculation of MNI and density values. Other site-specific variations in analysis of the different assemblages, mostly related to the particular characteristics of each, are listed below.

\section{Hoffman's/Robberg Cave}

Some of the layers as excavated are equivalent and the data for these has been combined here and the following names applied: Peter and Paul have been combined and named Peter; Portia and Below Peter named Portia; and Tom and Below Tim named Tom. MNI and NISP are presented per cubic metre per layer. Data from layer Ivan has been included with the shelly layers where these have been combined to compare with the Zostera layers as this unit, although essentially a hiatus layer, still contains material and clearly does not relate to the Zostera layers (Sealy, pers. comm). Fish remains retained in the $3 \mathrm{~mm}$ sieve were examined for this study.

\section{Blombosfontein 1}

The fish remains examined are from material that was retained in a $1.5 \mathrm{~mm}$ sieve. Due to the brecciated nature of the deposit the material had to be manually removed from consolidated blocks of calcareous matrix (Henshilwood 2008), and the fish remains studied here are only those that could be removed, and therefore not the full assemblage. Volume data was not available and therefore densities per volume were not calculated.

\section{Blombosfontein 6}

Henshilwood $(1995 ; 2008)$ reports fish present in six of the 12 layers excavated. I could find fish from only three layers; material from the other layers could not be located. The material examined is from deposit retained in a $1.5 \mathrm{~mm}$ sieve. Volume data was not available therefore all data are presented as raw counts.

Blombos Cave Later Stone Age

Fish from one square meter (G5 - NISP=2132) was examined for this study, and additional data (NISP=5185) from a previous study (van Niekerk 2005) from squares F4, 
F5 and E5 has been added to some of the results presented here to increase the sample size (Total NISP=7317). The method of counting specimens in the previous study was the same as in this study, and therefore the data for specimen counts (NISP) could be combined to calculate derivatives of this measure (MNI, MNE, MAU etc.). Details of the analysis methodology are given in Chapter 5. Specimen attributes such as element completeness, size estimation, bone modification and skeletal zone representation were not recorded in the previous study. Fish remains (NISP of 14) from the surface unit, SUR, have been excluded from Layer 1 as SUR consists mostly of a large volume $\left(0.80 \mathrm{~m}^{3}\right)$ of aeolian sand with small scattered pockets of deposit (Henshilwood 1995). It is not clear whether these small amounts of deposit are in situ, and including them would significantly reduce the apparent density of fish remains in Layer 1, biasing the results relative to other layers.

The total excavated volume of the squares from which fish data (MNI, NISP, MNE and MAU) are presented is $2.54 \mathrm{~m}^{3}$ (Table 4.6). The volume of the combined units of layer 4 is considerably higher than that of most of the other layers. The material retained in the $3 \mathrm{~mm}$ sieve was examined for this study.

Table 4.6. Excavated volumes $\left(\mathrm{m}^{3}\right)$ of squares E5, F4, F5 and G5 from Blombos Cave Later Stone Age

\begin{tabular}{|c|c|}
\hline & Volume $\left(\mathrm{m}^{3)}\right.$ \\
\hline \begin{tabular}{|l} 
LAYER 1 \\
\end{tabular} & 0.34 \\
\hline LAYER 2 & 0.48 \\
\hline LAYER 3 & 0.37 \\
\hline LAYER 4 & 0.86 \\
\hline LAYER 5 & 0.48 \\
\hline Total & 2.54 \\
\hline
\end{tabular}

Blombos Cave Middle Stone Age

Fish bones from all three phases of the MSA were examined from all excavations up to and including the 2008 season. The data from the exterior test excavation is presented separately except where all layers per phase are combined. The exterior material is located $2.5 \mathrm{~m}$ away from the interior excavation. Although this distance does not exclude interdependence of material between the two areas, I have nevertheless calculated the MNIs for the exterior trench as if they were independent, in order to give an indication of the minimum number of fish represented by the NISP in this area. 
The MNIs of the exterior material may be somewhat inflated relative to the interior. This is because the individual layers of the exterior trench could not be correlated with any other layers, and therefore each layer as excavated was taken as unique and the MNIs calculated per layer before being combined per phase. In the interior of the cave, several sub-layers known to form part of a bigger layer were combined before MNIs were calculated.

\section{Klasies River main site}

The fish remains from a sample of the KR assemblage from the 1994 and 1998 excavations by Deacon were examined. This is the same material that was analysed by von den Driesch (2004). The few fish bones that could be located from the earlier Wymer excavations (Singer and Wymer 1982) were included (NISP=5). The KR data are presented as NISPs for the cultural units and the sterile units, with each category grouped within each member. Thus the data for the MSA I (LBS member) is presented for all the MSA I cultural units combined, and separately for all the MSA I sterile units combined. The same applies to the other members. Data from the units that form the Upper member are presented separately for the HP and MSA III, and not combined into the single Upper member. Data from the SAS member have not been split into upper and lower SAS submembers as it was not possible to accurately assign individual units to their respective sub-members. As the members of the KR sequence named by Deacon conform to the substages of the MSA chronological sequence as proposed by Singer and Wymer (1982), and as the data from the Upper member have been split into its sub-stages here, I present the data per MSA sub-stage, i.e. MSA I, II, HP and MSA III.

MNIs have not been calculated for the KR assemblage because the deposit contains many small occupational units interspersed with sterile units of unknown depth or volume and there is little information on the correlation of units between squares. As the unit equivalents between squares were not available for this study, the data could not be combined and any attempt at calculating MNI per unit would result in artificially inflated counts. Information about the volumes of deposit excavated was not available, so it was not possible to calculate the average density of fish per unit or member as was done for $\mathrm{BBC}$ and $\mathrm{H} / \mathrm{RC}$. High numbers for some layers may therefore reflect larger volumes of 
deposit excavated, and are not directly comparable with data from sites for which densities are reported. As the material from the MSA IV phase at KR is thought to not be in situ (Deacon and Geleijnse 1988), it has been excluded from this analysis. 


\section{Chapter 5. Analytical methods}

\subsection{Quantification}

\subsubsection{Introduction}

Quantification is a key aspect of faunal analysis and most analyses entail using at least one method of determining taxonomic abundance. Very few reports now contain only species lists, although on their own these lists have their uses (Lyman 1986). The methods of quantification used in any study depend on the research questions to be addressed and the nature of the assemblages. All quantification systems have positive and negative attributes that influence their suitability for particular research questions and assemblages and the advantages and disadvantages of the various methods have been discussed extensively (Casteel 1976, 1977; Grayson 1973, 1984; Grayson and Frey 2004; Klein and Cruz-Uribe 1984; Lyman 1994a, b, 2008; Marshall and Pilgram 1993; O'Connor 2000, 2001; Plug and Plug 1990; Reitz and Wing 1999; Ringrose 1993). Although these discussions and debates frequently stem from the analysis of mammalian fauna, much of it relates to fish and other faunal remains too. The following is an overview of the most commonly used quantification methods in zooarchaeology.

\section{Note on terminology}

As suggested by others (Grayson 1984; Lyman 1994a, b, 2008; Reitz and Wing 1999), and for the sake of consistency, I use the terms element and specimen following Shotwell $(1955 ; 1958)$. An element refers to a whole, isolated bone or tooth, whereas a specimen can either be a whole bone or tooth, or just a part of it, as typically found in archaeological assemblages, where intact bones are rare. Therefore an element is always also a specimen, but a specimen is an element only if complete. A unit refers to material from a specific time period at a single site (Reitz and Wing 1999).

\subsubsection{Number of Identified Specimens (NISP)}

NISP usually refers to specimens that have been identified to element and taxon (Grayson 1984; Lyman 1994a, b, 2008; Reitz and Wing 1999), although there are instances when NISP has been calculated without identification to either element or taxon (Reitz and Wing 1999). Such instances are probably more appropriately named NSP, or number of 
specimens, a related term which encompasses all bone and teeth, fragmented or not, including those that have not been identified to taxon (Lyman 2008), or only to class (fish, mammal, etc.) (McKechnie 2005). NISP is usually derived from this, although NSP might not always be calculated.

The acronym TNF (total number of fragments) is sometimes used in place of NISP (Gilbert and Singer 1982; O'Connor and Barrett 2006). NISP values are considered to be primary data and the most basic unit by which faunal remains are counted (Grayson 1984; Horton 1984; Lyman 2008; Reitz and Wing 1999). NISP is one of the first and earliest methods of quantification employed by archaeologists (Lyman 2008). The most frequently cited advantages of NISP is that it is easily counted while identification is done, as well as being additive, i.e. subsequent NISP counts from the same assemblage can simply be added to the previous value without any need for recalculation (Klein and Cruz-Uribe 1984; Lyman 2008; O'Connor 2001).

Several possible problems with using NISP as an indication of species abundance have been raised. There is often an implicit understanding that with NISP, each identified fragment (specimen) represents a single individual (Grayson 1984; Lyman 2008; O'Connor and Barrett 2006; O'Connor 2001; Watson 1979). This assumption is seldom expressed (Grayson 1984), although a few have done so (Cornwall 1974: 242; Hesse and Perkins 1974: 151). It is also this assumption, which does not allow for interdependence between specimens, which is of most concern when using NISP, as it is difficult to prove that each specimen is independent of all others within a site, and the presence of articulated bones at some sites also disproves independence of all specimens, at least at those sites (Grayson 1984; Lyman 2008; Ringrose 1993).

It is now generally accepted that raw NISP values cannot be used to indicate taxonomic abundance, as they do not take into account taphonomic and other factors that could alter the number of specimens that are ultimately recovered. This has led to the development of several other techniques to estimate species abundance (Landon 2005; Lyman 1994b). The key problems with using NISP as a direct proxy for species abundance are listed below. Some animals have more elements than others, and the elements of some are easier to identify than others, leading to an overrepresentation of those species with more, or more 
readily identifiable elements (Daly 1969; Grayson 1984; Klein and Cruz-Uribe 1984; Reitz and Wing 1999; Shotwell 1955; Wheeler and Jones 1989). Fish bones in particular are quite different to most terrestrial fauna in that their skeletons contain considerably more unidentifiable to species elements, and many of the taxonomically distinct elements are fragile and more prone to damage, therefore more likely to be rendered unidentifiable (Vale and Gargett 2002; Wheeler and Jones 1989). Several methods of overcoming the problem of intertaxonomic differences in terms of varying numbers of elements have been proposed. These generally entail comparing the frequencies of only the elements that occur in all species in the same number, or weighting (or correcting) the NISP using different formulae to compensate for differences in element numbers or element identifiability (Daly 1969; de Ruiter 2004; Hesse and Perkins 1974; Lyman 2008; O'Connor and Barrett 2006; Plug 1988; Reitz and Wing 1999; Shotwell 1955, 1958). Using NISP as an indication of species richness (when it is not assumed that one specimen equals one animal) is based on the assumption that the whole carcass was deposited in the site. This does not take into account that several factors can contribute to less than the entire skeleton ending up in the site, and subsequently recovered for analysis. Cultural, biological and environmental activities including off-site processing of animals, refuse disposal, carnivore activity, rodent burrowing, rainfall, extremes in temperatures, soil acidity and erosion will all result in dispersal of elements of the original carcass within and beyond the site (Daly 1969; Gilbert and Singer 1982; Grayson 1984; Klein and CruzUribe 1984; Krantz 1968; Plug 1988; Reitz and Wing 1999; Uerpmann 1973; White 1953).

Sampling strategies will also affect NISP values, with sieved collections having varying numbers of fragments, depending on the mesh size used, and hand collected samples typically having the lowest number of specimens (O'Connor 2001), which further complicates comparisons between assemblages. In addition, most assemblages are, essentially, samples of samples (Plug 1988). Even if the entire site has been excavated, and all material recovered, the various factors leading to bone attrition will result in a smaller number of bones surviving than what was originally deposited (Plug 1988). 
Several factors can lead to single elements becoming fragmented on-site, and the resultant specimens would not be a true reflection of the original numbers of animals if each fragment was counted as being representative of one animal. Grayson (1984: 22) cites the example of differential butchery of different sized animals that would lead to higher NISP values of certain species, which is a reflection of butchery technique, rather than species abundance. Other processes such as trampling, as well as the weight of the overburden and sediment compaction, will also lead to inflated counts particularly if some bones of certain species are more prone to breakage (Darwent and Lyman 2002). Likewise, on-site butchery practices that differentiate between different, or different-sized animals, will result in some becoming more fragmented. NISP values do not take into account that some bones preserve better than others, that this varies between species, and that what is left in a site is not a direct reflection of what was originally deposited (Grayson 1984). NISP as an estimate of the relative abundance of a species in a site would be viable only if all animals underwent exactly the same taphonomic process and all bones were accounted for, a situation which seldom, if ever, occurs. If the entire skeleton of a single animal is preserved, then that species would be grossly overrepresented relative to those that, for one reason or another, are represented by only a few elements (Grayson 1984).

Grayson (1984) clearly demonstrates the effects that fragmentation, and therefore sample increase, can have on simple statistical tests of significant differences between species, even if the whole assemblage underwent the same degree of fragmentation. With increased fragmentation (and therefore increased NISP), the differences between units in an assemblage can become statistically highly significant, whereas the difference between the same units, without the theoretical fragmentation, is not statistically significant. The degree of fragmentation will affect the identifiability of specimens (Lyman 2008). Increased fragmentation will increase NISP values up to a certain point, after which further fragmentation will result in the loss of diagnostic marks which allow identification to element and taxon, and therefore a decrease in NISP (Lyman 2008; Marshall and Pilgram 1993).

NISP values as indicators of relative species abundance are still routinely listed and used in reports of fish remains, usually directly as 'raw' counts, without any weighting or 
correction for differences in numbers or identifiability of elements, and are often the only quantification method used or presented (Barrett 1997; Barrett et al. 2004a; Bazzanella et al. 2007; Jurgens 2008).

\subsubsection{Minimum Number of Individuals (MNI)}

As the name infers, MNI is a measure of the minimum number of animals (individuals) needed to account for all the elements present in an assemblage per species, and is not an absolute indication of the number of animals that were originally deposited (Reitz and Wing 1999). Theodore White (1953), a palaeontologist, is usually accredited with being the first to apply the method to archaeology in the English speaking world, although palaeontologists were already using MNI calculations much earlier (Howard 1930; Stock 1929). Casteel (1977) noted that the MNI method was applied to archaeological assemblages in Russia as early as the 1880s. MNI is secondary data, derived from primary data (Horton 1984; Reitz and Wing 1999, 2008). There are several ways in which MNI can be calculated, all based on the above premise, but with varying resultant values.

The most basic MNI calculation entails determining which element per species and unit occurs most frequently, treating each element as an individual bone, and the number of the most common element is then taken as the MNI of that particular species for that unit. With this technique it is impossible to count the same animal twice. This is the method suggested by White (1953) and is considered to be the traditional, or "Whitean" method of calculating MNI (Giovas 2009: 1558; Lyman 1994a; 2008: 122; Reitz and Wing 2008). The underlying assumption is that all left and right elements are matched, which is not necessarily true (Ringrose 1993; White 1953). It has been proposed that that this should perhaps be called the MMNI - the "Minimum Minimum Number of Individuals", as this is the lowest MNI value possible, and there are several ways that the analyst can increase this number (Ringrose 1993: 127). This suggestion, however, has not been acted upon. MNI is essentially calculated from MNE, taking sides and other splitting criteria such as size into account (Lyman 2008; Marean et al. 2001).

Binford (1978) originally calculated MNI by dividing the total number of bilaterally paired specimens by two (or more, depending on how many of a certain element occurs within the skeleton of a particular species), instead of separating symmetrical elements. 
Some researchers followed suit which created confusion about what was meant by MNI unless the method was explicitly stated (Reitz and Wing 1999). Although not as influential in large assemblages, in small assemblages this can result in a markedly different MNI to that which is calculated by counting the highest of either left or right elements (Klein and Cruz-Uribe 1984). Binford (1984) later revised his naming of this method and changed it to MAU (Minimum Animal Units). His intention was to calculate body-part representation rather than taxonomic abundance. Reitz and Wing (1999) advise against using this method as an MNI calculator, and add that, at least in 1999, some researchers were still using Binford's old MNI method. Their 2008 edition omits this sentence, and the impression is that it is no longer used to calculate MNI (Reitz and Wing 2008).

Pairing (or matching) of bones by size can sometimes significantly alter the resultant MNI values (Klein and Cruz-Uribe 1984; Lyman 2008; Ringrose 1993). For example, if a specific unit contains 4 left and 7 right dentaries of a certain species, the unpaired MNI would be the highest number of the two, namely 7 . If, however, an attempt was made to match the bones in terms of size, and none were found to be of equal size, in other words none were from the same animal, it could be said that the MNI of that unit is 11 . Thus pairing has the possibility to increase the MNI count of any symmetrical element. Conversely, even if all 4 of the left-sided bones matched 4 of the right-sided ones, the MNI would still remain 7. Matching bones in terms of age and sex works on the same principle (Bökönyi 1970).

Orchard (2005) devised a method of calculating the MNI of fish assemblages by reconstructing fish length using size regression formulae. The reconstructed fish sizes, based on a number of elements, were compared at 95\% confidence levels. Each fish that does not overlap with another at this confidence level can then be considered to be a discrete individual. This method increased the MNI calculated using White's (1953) method significantly. The advantage of this method is that more than one element can be included to calculate MNI (Orchard 2005), which might help to overcome some problems of differential preservation within a site.

Another method of calculating MNI entails estimating the fraction present of a fragment of a particular zone of an element, summing the fractions, and adding this sum to the 
number of complete elements (Klein and Cruz-Uribe 1984). The resultant number is rounded up to the next if not whole. This system is prone to under-representing MNIs (and MNEs) if the zones chosen are large and consists of several areas that could be identified individually if fragmented (Marean et al. 2001). A problem with this method is that it does not take the specific part of the element fraction present into account, hence there is a high possibility of counting the same element more than once.

The MNI of fragmented bone is often calculated by dividing each element into several diagnostic zones, which are recorded individually, and the most frequently occurring zone is then used (Dobney and Rielly 1988; Reitz and Wing 1999, 2008). Initially developed to record fragmentation (Dobney and Rielly 1988; Watson 1979), it is also used for calculating MNI and NISP. Watson (1979) emphasised that that each zone should only occur once per element and that more than $50 \%$ of it needs to be present to avoid counting the same bone twice. Fragments smaller than $50 \%$ of a zone are therefore excluded from analysis. The exclusion of these smaller fragments can be problematic as data can be lost, particularly in heavily fragmented assemblages, where there is an increased likelihood of fragments smaller than $50 \%$ of the whole (Marean et al. 2001). Likewise, Dobney and Rielly (1988) require more than $50 \%$ of a zone in order for it to be used for MNI calculation, but also record those smaller than $50 \%$ to document fragmentation. Using the zone method removes much of the subjectivity of estimating what proportion of an element is present, although not all (Dobney and Rielly 1988; Watson 1979).

The strengths of MNI are that it is less sensitive to fragmentation than NISP as the danger of counting the same animal twice is eliminated. Each animal can only be represented by one element or specimen. MNI is, however, affected by extensive fragmentation, where increasing numbers of fragments have lost their diagnostic features, thereby reducing both MNI and NISP (Lyman 2008). MNI is also not as affected by off-site butchery or other activities, cultural or biological, that result in less than the whole carcass being deposited in the site. Additionally, the problem of differential numbers of elements between species is not relevant (Klein and Cruz-Uribe 1984). MNI also addresses the not inconsiderable problem of interdependence of elements that plagues NISP calculations, as each animal can be represented by only one element or specimen (Grayson 1984; Klein and Cruz- 
Uribe 1984). This is, however, the case only if it is certain that the assemblages from which the fauna come are independent from one another (Grayson 1984).

One of the most frequently cited problems with MNI is that the totals cannot be summed, requiring recalculation if additional material is added from the same context, often resulting in decreased MNI values (Grayson 1984; Klein and Cruz-Uribe 1984; Lyman 2008; O'Connor 2001; Plug and Plug 1990; Ringrose 1993; Watson 1979). The relationships between MNI values are not always directly comparable between collections, particularly if the analysts are not explicit about their method of calculation (Klein and Cruz-Uribe 1984; O'Connor 2001; Ringrose 1993; Watson 1979).

When attempts are made to match pairs of bones within an assemblage, the resultant data will be problematic when compared with material that has not been analysed in this way, whether from the same or other sites. Such data are also not additive, i.e. all material from a particular site will have to be analysed by this method at the same time to ensure that all possible matches have been considered. Pairing can also be problematic as bilaterally paired bones are not totally symmetrical and can therefore lead to missing some pairs as well as misidentifying pairs (Klein and Cruz-Uribe 1984; Lyman 2006). Matching pairs in small assemblages can affect MNI much more than in large assemblages (Klein and CruzUribe 1984).

Another common problem is that MNI tends to exaggerate less abundant species as a single bone of a species in an assemblage can have the same MNI (of 1) as several bones of another (Klein and Cruz-Uribe 1984; Lyman 2008). Several analysts, particularly those working with fish, restrict the elements used to determine MNI to a predetermined selection of robust, diagnostic bones (Barrett 1995; Casteel 1976; Colley 1989; Poggenpoel 1984; Vale and Gargett 2002; Wheeler and Jones 1989). The choice of diagnostic elements differs between analysts, and is mostly a function of the type of material that is preserved and available for analysis as well as the range of species present (Casteel 1974, 1976; Wheeler and Jones 1989). Restricting the elements used for identification can result in losing data (and MNI counts) if the excluded elements are more numerous. For some, the amount of time that needs to be invested to identify less robust or 
distinctive elements is not worth the effort for the small returns in terms of increases in sample size and species present (Vale and Gargett 2002).

From the above it is clear that not all analysts calculate MNI in the same way, and the manner in which it is calculated in terms of symmetrical elements, pairing and whether fragmented elements are included, and if so, how, all affect the comparability of data, which contributes to its unpopularity with some researchers (Grayson 1984; O'Connor 2001).

\subsubsection{Minimum Number of Elements (MNE)}

MNE refers to the minimum number of skeletal elements per species that the specimens in an assemblage can account for. It is the total of each specific element per species and is mostly used to measure skeletal element abundance and element survivability rather than taxonomic abundance (Lyman 1994a; Ringrose 1993). The abundance of skeletal elements within an assemblage can inform on taphonomic issues such as on and off-site butchery patterns, carnivore damage and element survivability (Lyman 1994a; Marean and Spencer 1991; Reitz and Wing 1999, 2008). As with most quantification methods, various methods of calculating MNE are employed, with varying results (Lyman 1994a; Marean et al. 2001).

MNE is basically calculated in the same way as MNI, but gives a value per individual element, as opposed to per individual skeleton. Many of the same factors that can influence MNI values apply to MNE calculations, including whether age, sex and size classes are taken into account or not (Bunn and Kroll 1986; Lyman 1994a). The inclusion or exclusion of mammalian long-bone shaft fragments, for example, can result in considerable differences in the calculated MNE, that directly affect interpretation of taphonomic processes (Barba and Domínguez-Rodrigo 2005; Bunn and Kroll 1986; Dominguez-Rodrigo et al. 2007; Marean 1998; Marean et al. 2001; Marean et al. 2000a; Marean et al. 2004a; Marean and Frey 1997; Marean and Kim 1998; Marean and Spencer 1991; Pickering et al. 2003; Stiner 1991, 2002; Todd and Rapson 1988). Although fish do not possess shaft fragments, similar arguments can be made for the inclusion or exclusion of certain portions of fish elements when calculating MNE. 
Whether left and right elements are summed or counted as separate elements differs between analysts and the research questions asked. Separation of bilaterally paired elements can inform about differential treatment of various body parts, and bilaterally paired elements will usually be summed if the question is about element survivability (Binford 1978, 1984; Ringrose 1993; White 1952, 1955, 1956). If the latter is the case, then an additional calculation is typically performed by dividing the number of elements of a species (MNE) by the number of times that they occur in a complete skeleton to quantify the Minimal Animal Unit (MAU) (Binford 1978, 1984; Lyman 1994a, 2008). These normed values are usually standardised by assigning a value of 100 to the most common element, and scaling the other element values relative to that element and is usually referred to as percentage MAU (\%MAU) (Binford 1984; Lyman 2008). This standardising method is also often applied to MNI, MNE and NISP counts to allow graphic comparisons between different sample sizes (Cruz-Uribe and Klein 1998; de Ruiter et al. 2008; Grayson and Frey 2004; Lyman 2008).

The development and use of computer based programs for determining MNE (and other quantification units, as well as recording cutmark locations) has gained popularity. Methods differ, but generally entail digitally recording the location and size of a specimen on pre-formed templates for each element and the parts that overlap the most will be used for the MNE (Abe et al. 2002; Fischer 2007; Marean et al. 2001; Marean and Spencer 1991).

The weaknesses of MNE and, by extension, MAU and \%MAU, are similar to those of MNI. The methods used to calculate it differ widely, aggregation or splitting of data sets require that it be recalculated, and as it is a minimum estimate, it is not suitable for statistical comparisons with other minimum values as the upper end of the range is unknown (Lyman 2008). MAU as normed MNE specimen counts are widely used but can be problematic if there is a large discrepancy between counts of left and right elements as the MAU will then be much lower than the MNI (Grayson 1984; Lyman 2008). Standardization of any of the above methods (MNI, MNE, NISP and MAU) by scaling counts (elements or species) relative to the most abundant count results in a relationship between the counts that is very sensitive to changes in the value of the most common element or species (Lyman 2008; Ringrose 1993). Most of the varying methods of 
calculating MNI and MNE are attempts to increase the number, in order to get closer to the 'real' MNI or MNE (Orchard 2005). The 'real' MNI and MNE however, cannot ever be determined (Lyman 2008).

Any of the above methods used are useful in terms of species present (as a species list) at the sites, but as cultural, rather than natural assemblages, they are more a reflection of the inhabitants' skills and preferences in procuring fish than an indication of the immediate environment, with the exception of species that occupy very specific habitats or do not occur in the area today. The need to be clear and transparent about methodologies employed during analysis is constantly reiterated (Dobney and Rielly 1988; Lyman 2008; Morlan 1994; O'Connor and Barrett 2006; Reitz and Wing 1999, 2008; Ringrose 1993). Without transparency in method employed, there is no way of knowing whether data are comparable. Reports using Binford's (1978) earlier method of calculating MNI without explicitly stating that this particular method was employed are particularly problematic when compared with reports using White's (1953) method, or derivatives thereof (Klein and Cruz-Uribe 1984). The same applies to all other methods of quantification that have ambiguous methods lurking behind a single acronym.

\subsection{Analysis methodology}

Fish bones were initially separated from the mammalian and other fauna for each archaeological assemblage from the various sites analysed, then separated by skeletal element per square and unit before identification to species. Bones were washed and dried prior to identification where needed. All bones were identified by comparison with a modern comparative collection prepared by the author (Table 5.1). There were two exceptions where identifications were made of fish that were not in the comparative collection. Specimens listed as 'Shark spp.' include all elasmobranch (shark, ray, chimaera and skate) identifications and were based on mineralized vertebral centra. Mineralized vertebral centra, along with teeth and dermal denticles are often the only elements of cartilaginous fishes preserved in archaeological assemblages as most elements of these species are poorly calcified (Wheeler and Jones 1989). Shark vertebrae are distinctly different in shape to those of bony fishes as they lack haemal and neural spines, and are easily identified. Identifications of specimens of the Triglidae (as Triglidae spp.) were 
based on comparison of dermal plates with illustrations in Gregory (1933) and Smith and Heemstra (1995).

Table 5.1. Species in comparative collection ( $N=$ number)

\begin{tabular}{|c|c|c|c|c|c|c|c|}
\hline Family & Species & Common name & $\mathbf{N}$ & Family & Species & Common name & $\mathbf{N}$ \\
\hline Anguillidae & Anguillidae spp. & eel & 1 & Sparidae & Pterogymnus laniarius & panga & 2 \\
\hline Clupeidae & Sardinops sagax & herring, pilchard & 1 & Sparidae & Rhabdosargus globiceps & white stumpnose & 6 \\
\hline Ariidae & Galeichthys feliceps & white sea catfish & 5 & Sparidae & Rhabdosargus holubi & Cape stumpnose & 1 \\
\hline Serranidae & Acanthistius sebastoides & koester & 1 & Sparidae & Sarpa salpa & strepie & 2 \\
\hline Serranidae & Epinephelus marginatus & $\begin{array}{l}\text { yellowbelly } \\
\text { rockcod }\end{array}$ & 2 & Sparidae & Sparodon durbanensis & white musselcracker & 9 \\
\hline Pomatomidae & Pomatomus saltatrix & elf & 3 & Sparidae & Spondyliosoma emarginatum & steentjie & 1 \\
\hline Haemulidae & Pomadysas commersonni & spotted grunter & 2 & Dischistiidae & Dichistius capensis & galjoen & 3 \\
\hline Haemulidae & Pomadysas olivaceum & piggy & 1 & Monodactylidae & Monodactylus falciformis & Cape moony & 1 \\
\hline Sparidae & Argyrozona argyrozona & silver, carpenter & 1 & Sciaenidae & Argyrosomus inodorus/japonicus & kob & 6 \\
\hline Sparidae & Cheimerius nufar & santer & 1 & Sciaenidae & Atractoscion aequidens & $\begin{array}{l}\text { Cape salmon, } \\
\text { geelbek }\end{array}$ & 2 \\
\hline Sparidae & Chrysoblephus cristiceps & Dageraad & 2 & Sciaenidae & Umbrina capensis & baardman & 8 \\
\hline Sparidae & Chrysoblephus gibbiceps & red stumpnose & 5 & Oplegnathidae & Oplegnathus conwayi & Cape knifejaw & 1 \\
\hline Sparidae & Chrysoblephus laticeps & red roman & 7 & Carangidae & Seriola lalandi & giant yellowtail & 1 \\
\hline Sparidae & Cymatoceps nasutus & $\begin{array}{l}\text { black } \\
\text { musselcracker }\end{array}$ & 2 & Cheilodactylidae & Chirodactylus brachydactylus & two-tone fingerfin & 1 \\
\hline Sparidae & Diplodus cervinus hottentotus & zebra, wildeperd & 2 & Mugilidae & Liza richardsonii & $\begin{array}{l}\text { southern mullet, } \\
\text { haarder }\end{array}$ & 8 \\
\hline Sparidae & Diplodus sargus capensis & dassie, blacktail & 7 & Mugilidae & Mugil cephalus & flathead mullet & 1 \\
\hline Sparidae & Gymnocrotaphus curvidens & $\begin{array}{l}\text { john brown, } \\
\text { janbruin }\end{array}$ & 2 & Clinidae & Clinidae spp. & klipfish & 1 \\
\hline Sparidae & Lithognathus lithognathus & white steenbras & 1 & Gobiidae & Gobiidae spp. & gobies & 2 \\
\hline Sparidae & Pachymetopon blochii & hottentot & 2 & Scombridae & Scomber japonicus & mackerel & 1 \\
\hline Sparidae & Petrus rupestris & Red steenbras & 3 & Total & & & 108 \\
\hline
\end{tabular}

With the exclusion of teeth, scales, fins, rays, pterygiophores (other than the first anal) and most spines, which are considered undiagnostic in this study (although some teeth and scales can be diagnostic), each whole or fragmented piece of bone that could be identified as a whole or part of a specific element of fish was considered as an identifiable bone specimen and included in this study (Bunn 1982). Fragments that could be identified as fish, but not to element, were not included nor quantified in this study. The location of the elements referred to in this study is shown in Figure 5.1 and Figure 5.2. 


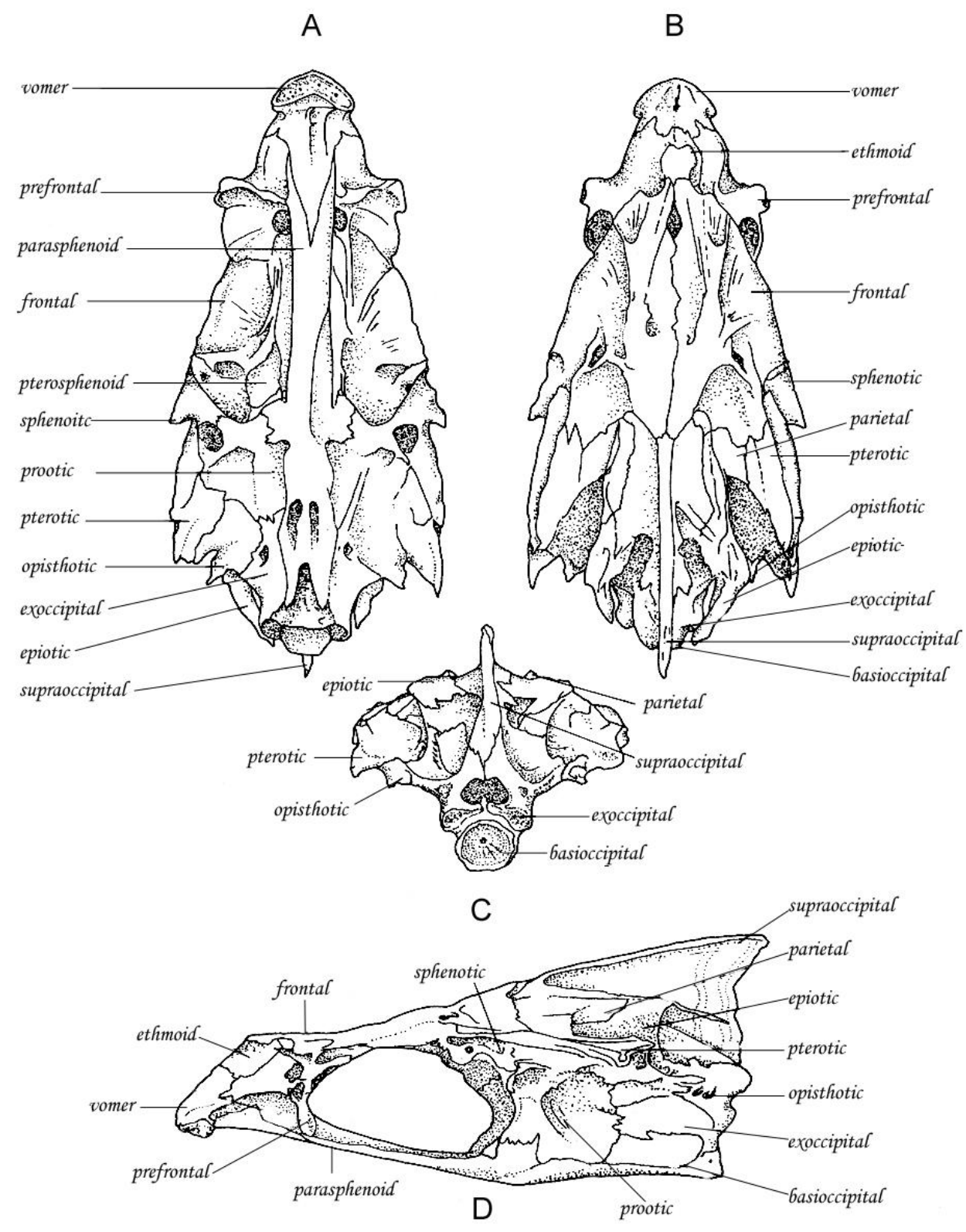

Figure 5.1. Location of the elements of the neurocranium. Adapted from Cannon 1987 


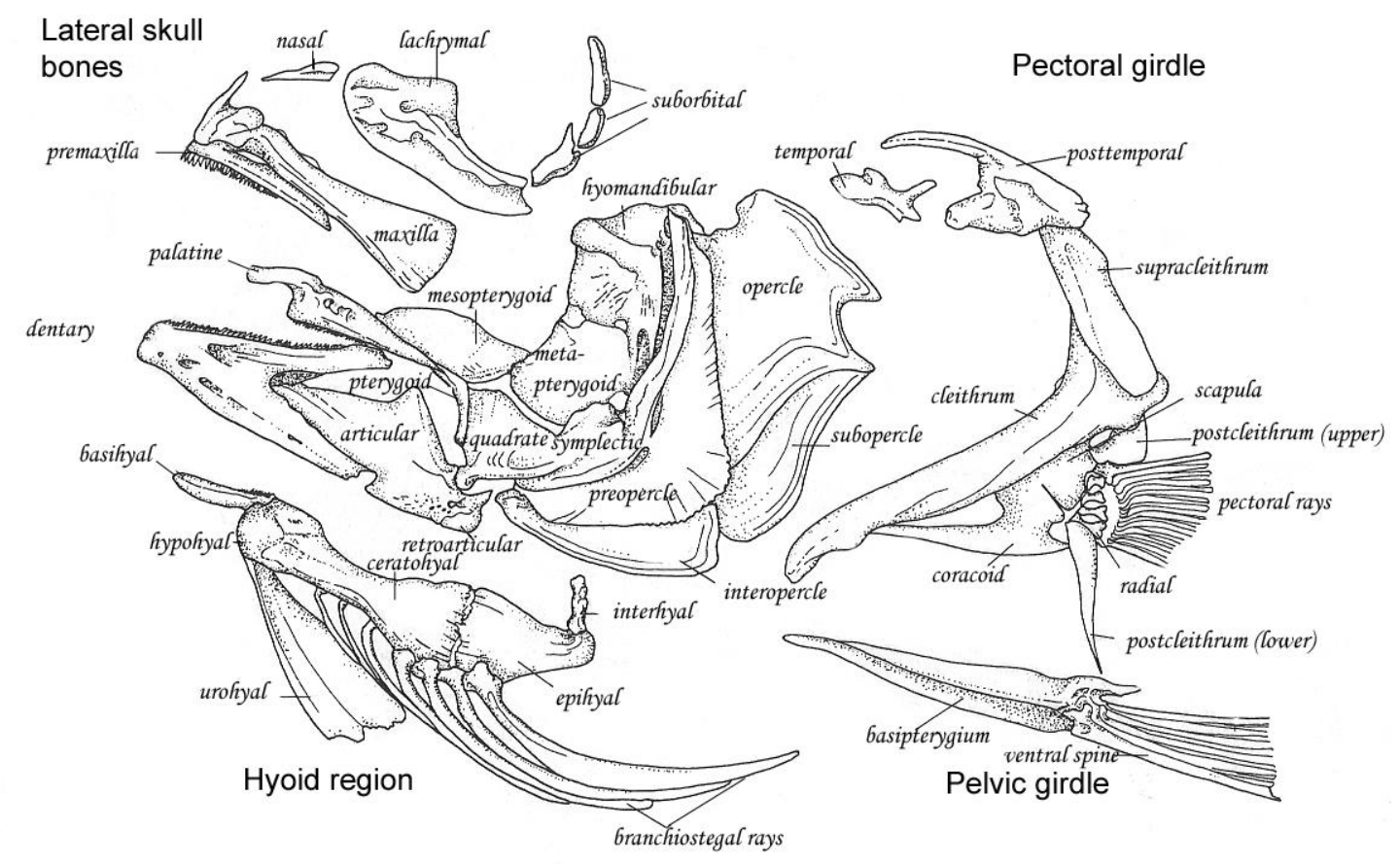

Figure 5.2. Location of elements from the lateral skull, pectoral girdle, pelvic girdle and hyoid area. Adapted from Cannon 1987

Apart from the first, ultimate and penultimate vertebrae, other vertebrae were counted but not identified to family or species, with the exception of some that were clearly identifiable to species, e.g. Galeichthys feliceps, and in the case of Chondrichthyes, to Class (cartilaginous fishes). Similarly, the spines of G. feliceps are serrated and easily identified and were included but unidentified spines were recorded only in the MSA assemblages and not in the LSA assemblages. Teeth and scales were quantified only in the MSA assemblages. These elements are very common in LSA sites and were recorded in the MSA assemblages only in order to have a full list of elements that survived in the much older sites. Pharyngeal bones were not identified to species as many of these elements are missing from the comparative collection due to the way in which commercially obtained specimens are cleaned prior to sale which entails removal of these bones. The number of skeletal elements studied after the exclusion of the abovementioned elements, as well as all vertebrae apart from the first, ultimate and penultimate vertebrae, varies across families but averages around 60 . 
Morphological characteristics that allow distinction between species of the same genus have been described and illustrated in van Niekerk (2004) and will not be repeated here. All bones of the same element per square and unit were grouped together and examined simultaneously for identification to species. It was found that identification was assisted when all the same elements could be compared with each other, as suggested by Bunn (1982), rather than in isolation This method is also less time-consuming than identifying mixed elements individually as the comparative specimens of a specific bone then need be unpacked only once per element per assemblage. Unlike Bunn's (1982) study, however, no attempt has been made here to match bones into pairs in terms of size, because the material studied here consists of samples from archaeological sites, rather than all the material from a site.

\subsubsection{Recording}

All identifiable specimens were directly entered into a modified version of the York system (Harland et al. 2003), a Microsoft Access database recording system developed at the University of York for faunal identification. It was adapted to include South African species. This system has a user-friendly interface that allows the recording of many different variables of a single specimen on a form with several drop-down menus (Figure 5.3). Only the options relevant to this study were recorded. 


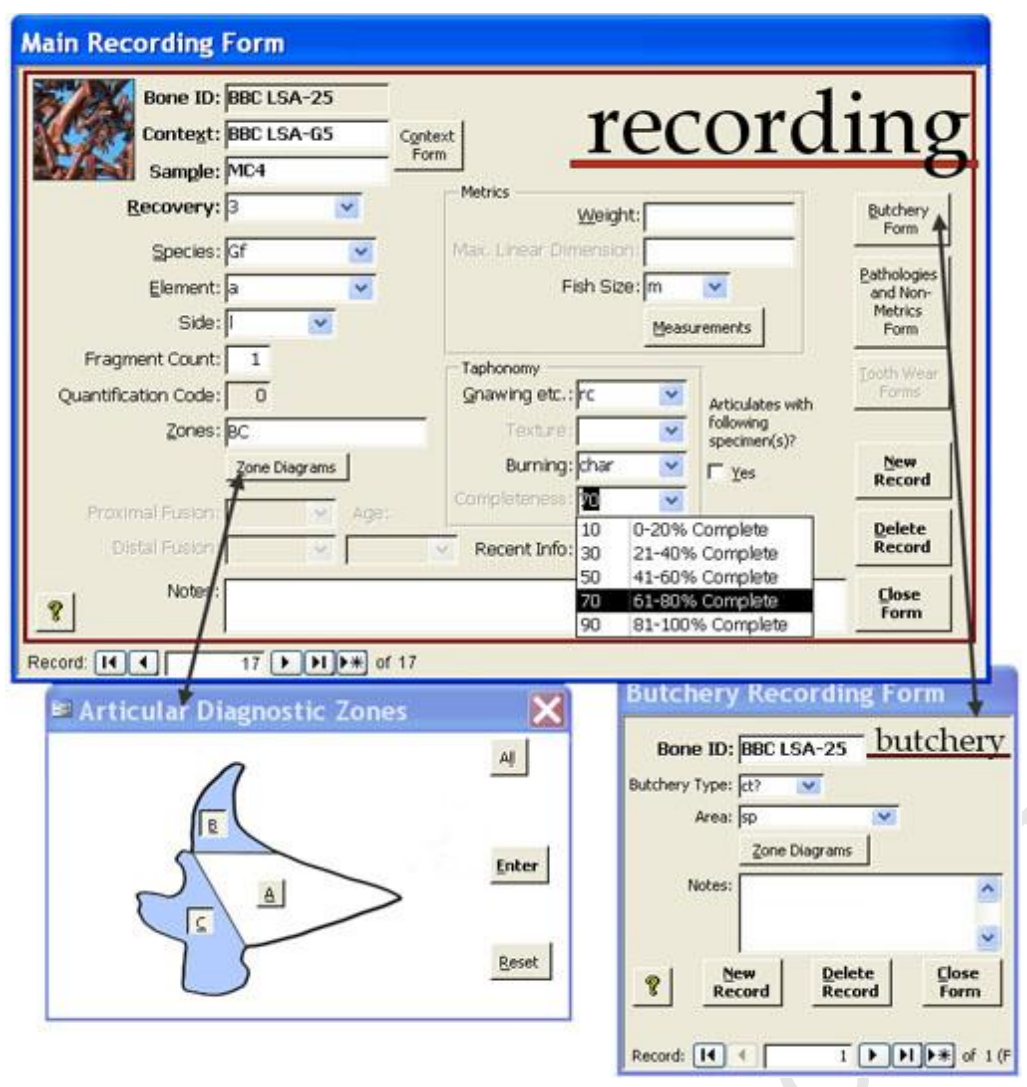

Figure 5.3. The modified York system data capture form used in this study

The system automatically generates a unique specimen number for each specimen, but the generally small size of fish bones precluded marking each with the full code. Bones were therefore marked with only the number portion of the code, or else the specimens were put into numbered packets. Each assemblage was entered into a new database. Data categories entered for each specimen are as follows: site, square, unit, recovery (sieve size), species, element, side, zone, fish size, gnawing etc., burning and completeness. The butchery form was used when applicable. The system allows the recording of several of the same elements from the same species at once (under fragment count), provided that none have any individual features that require recording (e.g. cutmarks, zones etc.), as the same specimen number is then ascribed to all, although separate measurements for each specimen are possible.

Zones were recorded to determine which parts of which elements survive, and whether there is a universal pattern in the preservation of specific parts (zones) of each element per 
species within and between sites. Zone recording also gives an indication of the degree of fragmentation of an assemblage.

Zone refers to what part or portion of the element is present, and was entered through the use of zone diagrams with different parts of each element having a corresponding letter (ABCD, etc.) (Figures 5.3, 5.4, Appendix Figure A-1). The original York system records zones on 18 elements, based on the morphology of Gadidae from the northern hemisphere (Harland et al. 2003). As it was intended to record all elements in this study, new zone diagrams were constructed and some of the existing ones altered to compensate for differences in species morphology. The number of zones per element varies, depending on the complexity of the element's shape and often on its breakage pattern, if characteristic, with some elements having only two zones to record (e.g. pterosphenoid), and others up to five (e.g. premaxillae). Harland et al. (2003) use these zones to calculate NISP and MNE and stipulate that more than $50 \%$ of a zone needs to be present for it to be recorded to prevent recording the same element twice. This was not followed here as sometimes the diagnostic parts were found to comprise less than $50 \%$ of a zone, and ignoring these would have resulted in the loss of identifiable specimens in the data as the less diagnostic parts of a zone are unlikely to be identified to element, even less to species.

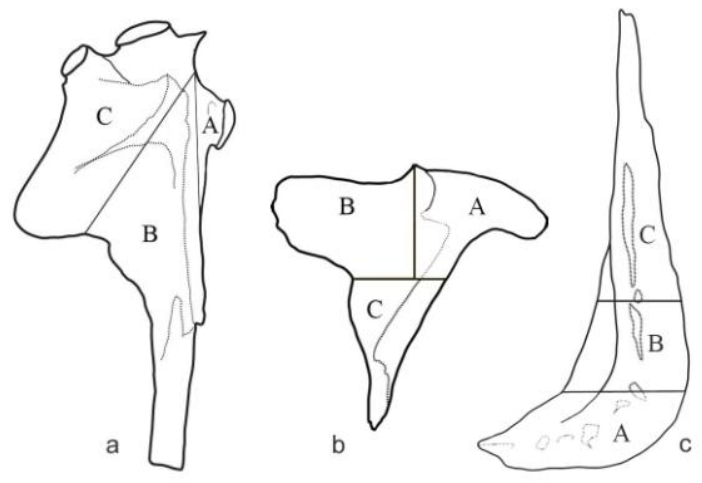

Figure 5.4. Example of location of zones recorded. (a) Hyomandibular; (b) palatine; (c) preopercular In order to avoid counting an element more than once, a single diagnostic feature was identified (where possible) for each zone (Morlan 1994). These will be referred to here as the MNI features. As the name suggests, these features are the basis for the calculation of MNI. If, for example, zone A is present in a palatine (Figures 5.5, A-1), but the MNI 
feature is lacking, then zone A will not be recorded for that specimen, even if it comprises more than $50 \%$ of the specimen (as there is a chance that the missing feature part will be found and recorded, thereby counting the same specimen twice). Due to the shapes and generally small sizes of the zones, this problem was seldom encountered, and the MNI feature usually comprised more than $50 \%$ of the zone.
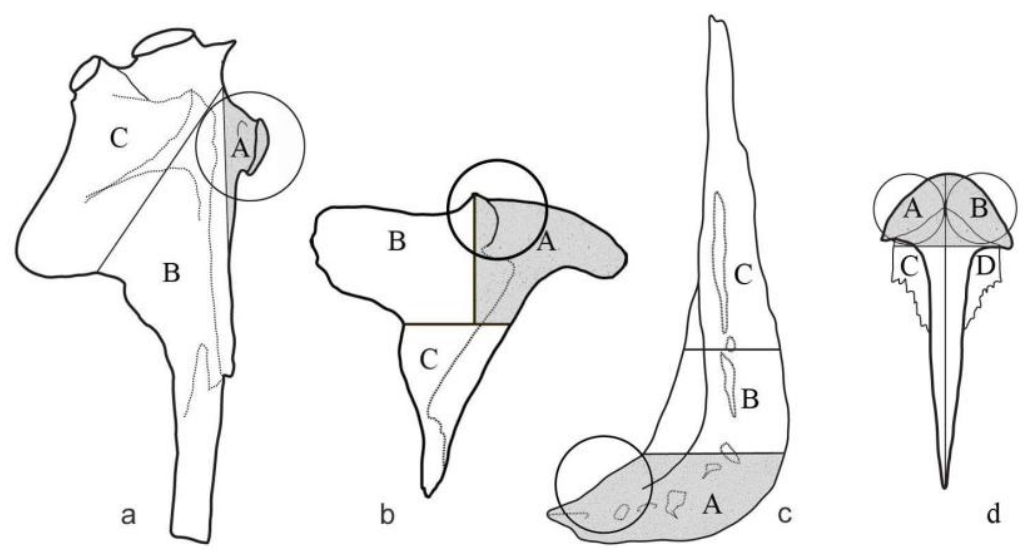

Figure 5.5. Examples of location of MNI feature. (a) hyomandibular; (b) palatine; (c) preopercular; (d) vomer. The shaded areas indicate the zone(s) containing the MNI features (circled) that need to be present in order for that bone to be included in MNI calculation

The York system refers to all these zones as diagnostic zones (Harland et al. 2003) (Figure 5.3). In this study most of the zones per element are considered to be diagnostic only to element and sometimes family, but not to species. It is likely that more zones are diagnostic to species in assemblages containing a range of species of families that are morphologically distinct, or assemblages dominated by a small number of species.

With mammalian fauna, it is common for analysts to assign several diagnostic zones to individual elements and to use the most frequently occurring zone as the MNI (Dobney and Rielly 1988; Marean et al. 2001; Moreno-Garcia et al. 1996; Morlan 1994; Outram et al. 2005; Reitz and Wing 1999; Watson 1979). The use of diagnostic zones for fish elements is uncommon, except by researchers who use the York protocol (Barrett 1995; Harland et al. 2003). The general morphology of fish bones is very different from that of terrestrial and marine mammals and other vertebrates, as well as quite variable between species (Butler 1994; Casteel 1976; Colley 1989; Nicholson 1998; Vale and Gargett 2002; Wheeler and Jones 1989). In the assemblages studied here, the majority of fish are from 
the same family (Sparidae), and because of the general similarity in morphology within this family, there is usually only one part (feature) of each element with characteristics that allow identification to species. Most other zones allow identification only to element, usually to family and sometimes to genus. That is not to imply that every MNI feature allows identification to species. Some elements are too similar morphologically to be of use in species identification, and determining which these are forms part of this study. The non-Sparid species encountered here tend to have more diagnostic zones. Species such as Galeicthyes feliceps have very distinctive bones and several parts of a single bone are diagnostic.

'Fish size' on the recording form refers to a set of predetermined size categories: tiny (0 150mm); small (151 - 300mm); medium (301 - 500mm); large (501 - 800); extra large $(801-1000 \mathrm{~mm})$ and extra extra large $(>1000 \mathrm{~mm})$. These are qualitative estimates of the total length (TL) of the fish by comparison with the modern comparative specimens. These size categories are those originally defined in the York system and seemed appropriate for the material studied here. Once analysis was in progress the subjectivity of size estimation became clear when dealing with specimens that were on the border between two size classes. This is, however, always a problem when using categories, regardless of how they are defined. Size estimates were not recorded for elements that could not be identified to family as there can be great variation in the TL of fish of different families with similarly shaped and sized elements (e.g. postcleithra). As the minimum number of individuals (MNI) was calculated without regard to size due to the subjectivity of the size estimates (see below), reconstructed fish sizes are presented based on the number of identified specimens (NISP) per size category. It is an indication of the overall number of specimens per size class per assemblage, rather than numbers of individuals.

Measurements were taken on selected specimens that could be identified to species (articular, basioccipital, dentary, first vertebra, maxilla, palatine, premaxilla and quadrate) following Morales and Rosenlund (1979) where possible. As the morphologies of the dentaries and palatines of local species differed substantially from those illustrated, and the preservation of certain landmarks was consistently poor, new measurement locations were created for these elements (Figure 5.6). The measurements have not been included in 
this thesis as the comparative collection does not contain enough specimens of any species to calculate regression formulae for size reconstruction, but they will be useful for future analysis if it can be established that there is a relationship between fish size and the measurements taken, as Poggenpoel (1996) and Plug (2008) have done for certain species.

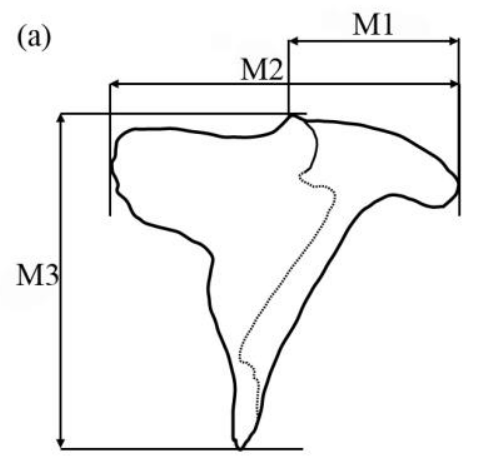

(b)

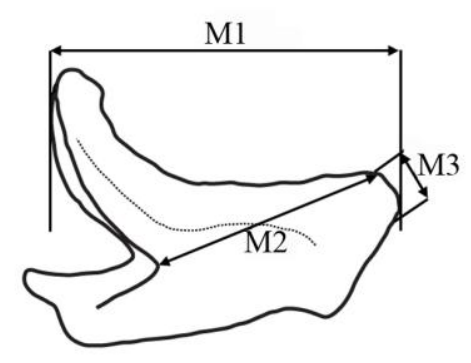

Figure 5.6. Location of measurements taken on palatine (a) and dentary (b) that differ from Morales and Rosenlund (1979). Bones illustrated are left elements from Chrysoblephus cristiceps

Entries under the field 'Taphonomy' include burning, which was recorded as either white (calcined) or black where appropriate. Options under 'Gnawing' include root etching, acid etching, carnivore gnawing, rodent gnawing and various permutations thereof. These modifications were identified with the naked eye, a hand lens and at times a 10-40x binocular microscope. 'Completeness' records the percentage of the entire element present per specimen in increments of 19 and 20\%, starting from 10 (0 to 20\% complete) to 90 (81 to $100 \%$ complete), similar to the method employed by Zohar et al. (2001).

Once all data had been captured the records were exported into Microsoft Excel for analysis.

\subsubsection{Quantification}

The methods of quantification applied to the data are NISP, MNI, MNE and its derivatives MAU and \%MAU. Where possible, the MNI and NISP for each assemblage were also calculated relative to the volume excavated and presented as $\mathrm{n} / 1 \mathrm{~m}^{3}$ (number per cubic meter), to give a more realistic representation of the density of fish per layer. This could not be done with the Klasies River and Blombosfontein 1 and 6 material as the excavated volume data was not available, therefore only the 'raw' numbers are presented. 
NISP was calculated by counting the number of identified specimens per element and species. Specimens that could be identified merely as 'fish' and not to element were not counted. Specimens that could be identified to element but not to species were recorded under the 'Species indeterminate' category. Interdependence of specimens is not guaranteed as they were recorded even if the part present comprised less than $50 \%$ of the zone in which it occurred. NISP in this study is intended to indicate the quantity of fish bone and the degree of fragmentation of elements within each site. It is not an indication of the actual number of individual species.

NISP was calculated for all species and all layers and is a direct tally of all the bones within each site that could be identified to element and species where appropriate. Although using NISP as determined here for certain calculations is less than ideal as some elements might be counted twice, it is the only fundamental measure - as opposed to derived measures like MNI, MNE etc. (Lyman 2008) - and is used to calculate frequencies of size classes, percentage completeness and other measures.

MNI was calculated following the traditional White (1953) method. The most frequently occurring element per species (based on the MNI feature) was taken as the MNI for that species per individual layer. In this calculation bilaterally paired elements are considered as individual elements, therefore left and right sides were counted separately. No attempt was made to match pairs of elements in terms of size due to the subjectivity of the size estimations. MNI was calculated for each species per individual layer. MNI was not calculated for the Species Indeterminate category, as the likelihood of these bones belonging to one of the already identified animals was high. Similarly, MNI was also not calculated for any of the grouped species categories (e.g. Sparidae spp.) as this would artificially inflate numbers if the elements are from the same fish that could be identified to species on other elements. MNI was not calculated in the instances where excavation layers were combined as interdependence of elements could not be guaranteed.

MNE, MAU and \%MAU were used to determine which elements of which species survive best and whether this survival is constant throughout time. The intention was to determine which of the elements per species are consistently well preserved under different environmental conditions. If the same elements are always preserved regardless of 
conditions, then it might be possible to compile a list of elements to use for future identification per species. Conversely, if there is a great deal of variation in which elements survive per species per site, then the list of elements used for identification will need to be expanded. It is currently not possible to determine the role of density-mediated attrition on elements as there is no data on the bone mineral densities and the effects of trampling, weathering and other taphonomic processes on specific elements of the species studied here. Previous studies have shown that the relationship between bone density and element survival can vary greatly between species, and it is not appropriate to use bone density data from other species in this study (Nicholson 1996). Good element survival does not always correlate with higher bone densities, and aspects such as element shape have been found to be a more determining factor in element preservation (Nicholson 1996).

NISP as calculated in this study is not an appropriate measure to use to determine skeletal element representation as it does not take fragmentation of a single element into account. There is therefore the danger of overestimating the survivability of a certain element if it tends to break into 2 or more identifiable fragments. MNE per element is calculated here in the same way as MNI but without regard to side, in other words lefts and rights of each bilateral element are combined, as it is assumed that there is unlikely to be any natural cause of differential preservation between lateral elements of symmetrical fish. The lateral elements of asymmetrically shaped species such as flatfish (Pleuronectidae etc), where both eyes are on one side of the 'face', would have to be considered individually, as it is likely that these morphologically different bilateral elements might preserve differently, but none were identified in the assemblages studied.

As MNE is the sum of each element, regardless of how many times each occurs in the skeleton, non-median elements will be overrepresented when compared to median elements. Therefore all MNE values were divided by the number of times that each element occurs in the skeleton to produce MAU. As most of the identifications in this study were on bilaterally paired and median elements, calculating MAU entailed dividing bilateral elements by two. Vertebrae were excluded from MAU calculations as very few were identified to species. Fish elements apart from vertebrae that occur more than twice 
in the skeleton (e.g. radials, pharyngeal bones, etc.) could not be identified to species in this study and were excluded from these calculations.

The problems that have been associated with MAU are deemed to be of little relevance here. They relate mostly to ignoring differences in the numbers of left and right elements which could inform on differential treatment of (mainly large mammal) carcasses (Lyman 2008; White 1953). It is unlikely that processing of a symmetrically shaped fish would result in a preference for one side over the other. It is also assumed that natural taphonomic processes will not affect one side more than the other. The intention of MAU here is to determine which elements from which species survive best under which circumstances, rather than skeletal completeness or element representation in terms of differential processing. Differential processing would be difficult to determine unless all vertebrae were identified to species, which is not the case here.

In order to compare MAU across different sample sizes, values have been standardized by calculating the percentages of all elements relative to the most common element (Binford 1984; Bunn and Kroll 1986; Lyman 1994b). This is referred to as the \%MAU and is useful for graphic representations of the frequencies of elements per species between sites and samples of different sizes. \%MAU is also a measure of the number of elements expected relative to the number observed (Binford 1984).

Pharyngeal, basibranchial and radial bones were not identified to species and were lumped in the pharyngiobranchial category. Elements that were not found in the assemblages have been excluded from some of the element lists to reduce empty spaces and facilitate reading of tables. The full list of elements that were considered for identification in this analysis is in Table 5.2. Where the data was available, all MNI and NISP values have been normalized per volume excavated to allow comparison between assemblages and layers and are presented as MNI/NISP per $\mathrm{m}^{3}$ or, in the case of the smaller assemblages, as MNI/NISP per $10 \mathrm{~m}^{3}$ (Cannon 1999). 
Table 5.2. List of skeletal elements used for identification in this study

\begin{tabular}{|llll|}
\hline \multicolumn{4}{c|}{ ELEMENT } \\
\hline Articular & Frontal & Palatine & Retroarticular \\
Basioccipital & Hyomandibular & Parasphenoid & Scapula \\
Basipterygium & Hypohyal low er & Parietal & Sphenotic \\
Basisphenoid & Hypohyal upper & Penultimate vertebrae & Spine \\
Ceratohyal & Infraorbital \#3 & Pharyngobranchial & Subopercle \\
Circumorbital \#1 & Infraorbital \#4 & Postcleithrum & Supracleithrum \\
Circumorbital \#2 & Interopercular & Posttemporal & Supraoccipital \\
Cleithrum & Jugal & Prefrontal & Symplectic \\
Coracoid & Lachrymal & Premaxilla & Temporal \\
Dentary & Maxilla & Preopercular & Upper postcleithrum \\
Epihyal & Mesopterygoid & Prootic & Urohyal \\
Epiotic & Metapterygoid & Pterosphenoid & Urostyle \\
Ethmoid & Nasal & Pterotic & Vertebra \\
Exoccipital & Opercular & Pterygoid & Vomer \\
First anal pterygiophore & Opisthotic & Quadrate & \\
First Vertebra & Otolith & Radial & \\
\hline
\end{tabular}

\subsubsection{Identifying skeletal elements that are useful for identification to species}

Fish contain a large number of skeletal elements that can potentially be used for species identification, and identifying every bone to skeletal element in large assemblages is very time consuming. It would therefore be very useful to narrow down the numbers of elements used for identification to expedite analysis, but not at the cost of potentially losing data. Poggenpoel $(1984,1996)$ considered dentaries and premaxillae to be the most reliable elements to use for species level identification of most species apart from Mugilidae, where basioccipitals and first vertebrae were better species indicators. Most of Poggenpoel's (1984; 1987; 1996; Poggenpoel and Robertshaw 1981) species identifications were based on these elements as well as maxillae and otoliths. His study of fish remains from Paternoster Midden, where only one species, Pachymetopon blochii, was present, has shown that other elements are often more numerous than dentaries or premaxilla. The most frequently occurring element of $P$. blochii was not the same between sites. At Paternoster, as well as Smitswinkelbaai, the endopterygoid was the most frequently occurring element, whereas at Duiker Eiland otoliths were most common.

Apart from these studies by Poggenpoel, there has been no systematic research on determining which elements are useful species indicators of South African marine fish. In this thesis all elements used for identification were listed per species to determine which elements can be used as species indicators. The results were compared between 
assemblages to determine whether the same elements were constantly the best preserved, or if there was variation in element survival frequency between sites. This was done in order to identify which elements can be excluded from future analyses of assemblages containing the same species.

The method used here differs from those used previously with South African fish assemblages in that all elements were initially considered as potentially identifiable to species, and all elements per species were recorded. In addition, cultural modification, element completeness, part of element preserved and size estimations were systematically recorded for each specimen, which has not been done previously.

This methodology has led to the findings that are presented in the following chapter. 


\section{Chapter 6. Results}

\subsection{Hoffman's/Robberg Cave}

\subsubsection{Species present}

A total of 19875 specimens were examined from 2 adjacent 50x50cm quadrats (D5c and D5d) spanning the complete stratigraphy from top to bottom. Layer Deon, which was present in D5b but not D5d, did not contain any fish bone. 12797 specimens could not be identified to family, of which 10756 (84\%) were vertebrae. These were recorded under 'species indeterminate'. The total number of individual fish (MNI) identified in the two quadrats is 605. There were 3631 specimens (NISP) in the combined Zostera layers, of which 1254 (34.5\%) were identified to family and/or species. In the shelly layers 5824 (35.9\%) out of 16244 specimens were identified to family and/or species.

Twenty nine species from at least 26 genera and twelve families were identified. In total there were at least 18 species present in the Zostera layers and 26 in the shelly layers. With the exception of Sparodon durbanensis, all species that were present in the Zostera layers occur in the shelly layers. The species that only occur in the shelly layers are present in low numbers (total NISP $=109$ ) and their presence could be a function of the larger sample sizes within the shelly layers.

The greatest species diversity was in the shelly layers Katherine $(n=22)$ and Tom $(n=19)$. The largest number of species in the Zostera layers was in layer Frank $(n=13)$. The highest density of fish in terms of MNI in the shelly layers was in layer Judy and in the Zostera layers in layer Ben. Overall, the density of fish per cubic metre was much higher in the shelly layers (Tables 6.1a,b). Note that the quantities listed in Tables 6.1a and b are MNI and NISP per cubic metre. 
Table 6.1a. MNI and NISP per $\mathrm{m} 3$ per species and layer of the Zostera layers from the Hoffman's/Robberg Cave Later Stone Age assemblage

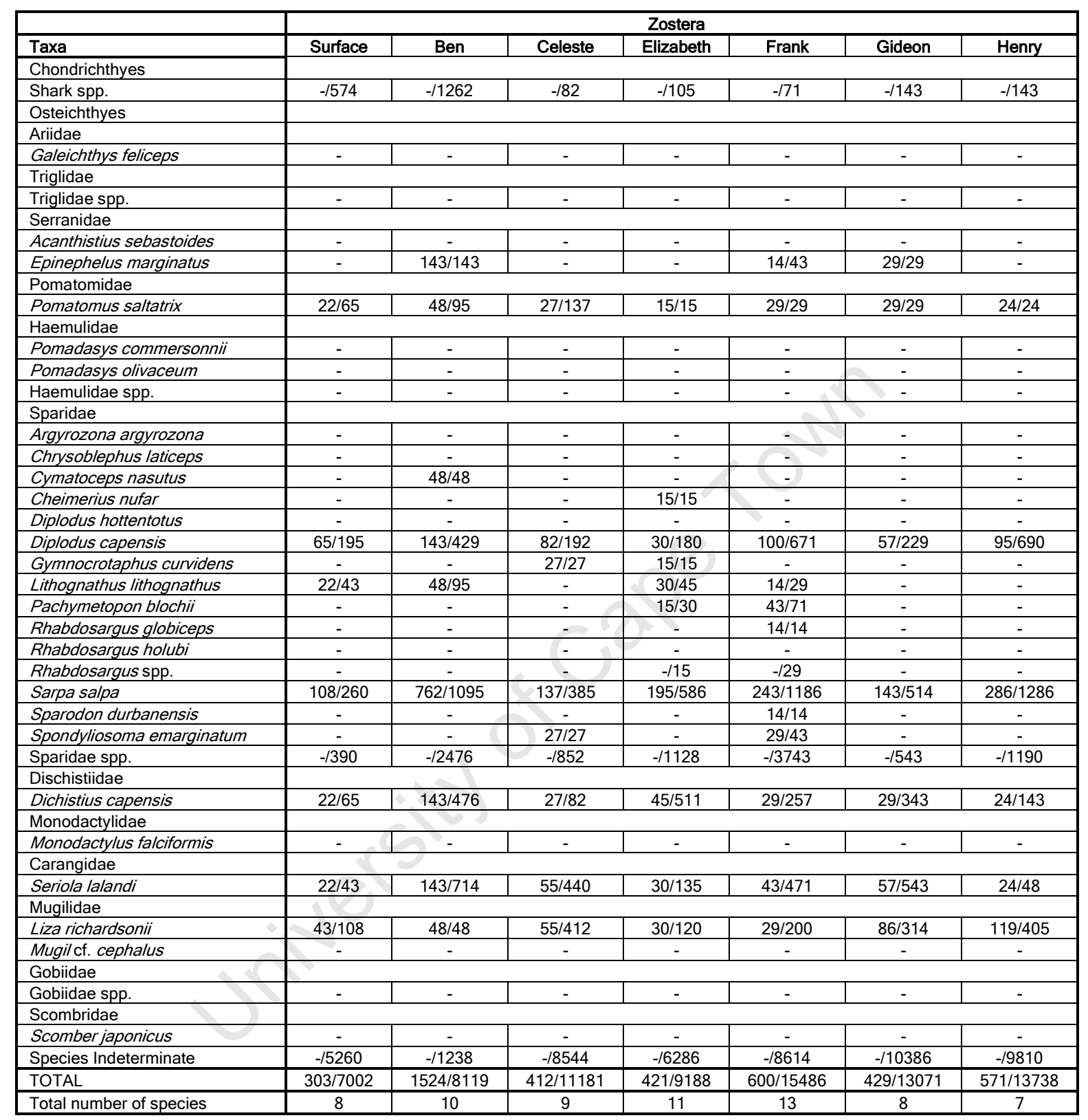


Table 6.1b. MNI and NISP per $\mathrm{m} 3$ per species and layer of the Shelly layers from the Hoffman's/Robberg Cave Later Stone Age assemblage. Note that the site total reflects the density of fish per $\mathrm{m} 3$ of the whole assemblage

\begin{tabular}{|c|c|c|c|c|c|c|c|c|c|c|c|}
\hline \multirow[b]{2}{*}{ Taxa } & \multicolumn{10}{|c|}{ Shelly } & \multirow[b]{2}{*}{$\begin{array}{l}\text { Site } \\
\text { total }\end{array}$} \\
\hline & Ivan & Jane & Judy & \begin{tabular}{|c|}
$\begin{array}{c}\text { Katherin } \\
\mathrm{e}\end{array}$ \\
\end{tabular} & Mavis & Nathan & Peter & Portia & Richard & Tom & \\
\hline \multicolumn{12}{|l|}{ Chondrichthyes } \\
\hline Shark spp. & $-/ 952$ & $-/ 398$ & $-/ 1476$ & $-/ 1300$ & $-/ 321$ & $-/ 457$ & $-/ 143$ & $-/ 142$ & $-/ 48$ & - & $-/ 300$ \\
\hline \multicolumn{12}{|l|}{ Osteichthyes } \\
\hline \multicolumn{12}{|l|}{ Ariidae } \\
\hline Galeichthys feliceps & - & - & - & $14 / 14$ & - & - & - & - & - & $20 / 51$ & $4 / 6$ \\
\hline \multicolumn{12}{|l|}{ Triglidae } \\
\hline Triglidae spp. & - & -120 & - & - & $-/ 36$ & - & - & - & $-/ 95$ & $-/ 173$ & $-/ 34$ \\
\hline \multicolumn{12}{|l|}{ Serranidae } \\
\hline Acanthistius sebastoides & $48 / 48$ & - & - & $14 / 29$ & - & - & - & - & - & - & $2 / 3$ \\
\hline Epinephelus marginatus & - & - & - & $14 / 14$ & - & $29 / 86$ & $18 / 18$ & - & - & $20 / 82$ & $10 / 20$ \\
\hline \multicolumn{12}{|l|}{ Pomatomidae } \\
\hline Pomatomus saltatrix & $48 / 48$ & $20 / 102$ & $48 / 48$ & $29 / 143$ & $71 / 143$ & $86 / 171$ & - & $10 / 49$ & $7 / 14$ & $20 / 102$ & $23 / 60$ \\
\hline \multicolumn{12}{|l|}{ Haemulidae } \\
\hline $\begin{array}{l}\text { Pomadasys } \\
\text { commersonnii }\end{array}$ & - & - & - & - & - & - & - & $10 / 20$ & - & - & $1 / 2$ \\
\hline Pomadasys olivaceum & - & - & - & $29 / 257$ & - & - & $18 / 18$ & - & - & $10 / 20$ & $4 / 21$ \\
\hline Haemulidae spp. & - & - & - & $-/ 14$ & - & - & - & - & - & $-/ 10$ & $-/ 2$ \\
\hline \multicolumn{12}{|l|}{ Sparidae } \\
\hline Argyrozona argyrozona & - & - & - & $29 / 29$ & $36 / 71$ & - & - & - & - & - & $3 / 4$ \\
\hline Chrysoblephus laticeps & - & $20 / 20$ & - & - & - & - & - & - & - & $10 / 10$ & $3 / 2$ \\
\hline Cymatoceps nasutus & $48 / 48$ & - & - & $14 / 29$ & - & - & - & - & - & $10 / 71$ & $6 / 11$ \\
\hline Cheimerius nufar & - & - & - & $14 / 14$ & - & - & - & - & - & - & $2 / 2$ \\
\hline Diplodus hottentotus & - & - & - & $14 / 57$ & $36 / 107$ & $29 / 29$ & - & $10 / 10$ & - & - & $4 / 9$ \\
\hline Diplodus capensis & $95 / 333$ & $41 / 429$ & $95 / 524$ & $200 / 1914$ & $71 / 571$ & $171 / 1771$ & $18 / 54$ & $68 / 391$ & $95 / 667$ & $173 / 1153$ & $111 / 639$ \\
\hline $\begin{array}{l}\text { Gymnocrotaphus } \\
\text { curvidens }\end{array}$ & - & - & - & $57 / 100$ & - & Or & - & $10 / 10$ & - & $20 / 51$ & $12 / 16$ \\
\hline Lithognathus lithognathus & - & - & - & $14 / 43$ & $36 / 36$ & $29 / 86$ & - & $29 / 49$ & $7 / 75$ & $20 / 61$ & $17 / 41$ \\
\hline Pachymetopon blochii & - & $20 / 20$ & $95 / 95$ & $43 / 43$ & - & $29 / 29$ & - & $10 / 10$ & $20 / 34$ & $51 / 92$ & $26 / 30$ \\
\hline Rhabdosargus globiceps & - & - & - & - & - & $29 / 29$ & - & $10 / 10$ & $7 / 7$ & $10 / 31$ & $6 / 7$ \\
\hline Rhabdosargus holubi & - & $20 / 41$ & $48 / 95$ & $14 / 29$ & - & - & - & - & $20 / 27$ & $41 / 102$ & $15 / 20$ \\
\hline Rhabdosargus spp. & - & $-/ 20$ & $-/ 95$ & $-/ 29$ & - & - & - & - & $-/ 82$ & $-/ 71$ & $-/ 28$ \\
\hline Sarpa salpa & $619 / 2095$ & $347 / 2694$ & $429 / 2571$ & $314 / 2671$ & $214 / 1071$ & $429 / 2457$ & $54 / 196$ & $68 / 421$ & $136 / 830$ & $378 / 1959$ & $264 / 1167$ \\
\hline Sparodon durbanensis & - & - & $-\quad$ & - & - & - & - & - & - & - & $1 / 1$ \\
\hline $\begin{array}{l}\text { Spondyliosoma } \\
\text { emarginatum }\end{array}$ & $48 / 48$ & - & - & $14 / 14$ & - & $57 / 114$ & - & - & $20 / 34$ & $41 / 92$ & $20 / 26$ \\
\hline Sparidae spp. & $-/ 2333$ & $-/ 5265$ & $-/ 5095$ & $-/ 7886$ & $-/ 2786$ & $-/ 6086$ & $-/ 411$ & $-/ 1928$ & $-/ 2619$ & $-/ 6949$ & $-/ 3113$ \\
\hline \multicolumn{12}{|l|}{ Dischistiidae } \\
\hline Dichistius capensis & $48 / 286$ & $41 / 245$ & $95 / 714$ & $57 / 1043$ & $36 / 214$ & $29 / 143$ & - & $29 / 411$ & $27 / 327$ & $41 / 898$ & $39 / 389$ \\
\hline \multicolumn{12}{|l|}{ Monodactylidae } \\
\hline Monodactylus falciformis & - & - & - & $14 / 14$ & - & - & - & $10 / 10$ & - & - & $2 / 2$ \\
\hline \multicolumn{12}{|l|}{ Carangidae } \\
\hline Seriola lalandi & $48 / 571$ & $82 / 755$ & $190 / 2095$ & $86 / 1557$ & $71 / 357$ & $114 / 514$ & $36 / 196$ & $20 / 245$ & $7 / 48$ & $10 / 20$ & $42 / 379$ \\
\hline Mugilidae & 0 & & & & & & & & & & \\
\hline Liza richardsonii & $143 / 857$ & $143 / 2041$ & $571 / 3571$ & $186 / 4657$ & $71 / 286$ & $29 / 143$ & - & $117 / 538$ & $20 / 231$ & $112 / 1786$ & $86 / 885$ \\
\hline Mugilcf. cephalus & - & - & - & $14 / 14$ & - & - & - & - & - & - & $1 / 1$ \\
\hline \multicolumn{12}{|l|}{ Gobiidae } \\
\hline Gobiidae spp. & - & - & - & - & - & - & - & - & - & $-/ 10$ & $-/ 1$ \\
\hline \multicolumn{12}{|l|}{ Scombridae } \\
\hline Scomber japonicus & - & - & - & - & - & - & - & - & $7 / 7$ & - & $1 / 1$ \\
\hline Species Indeterminate & $-/ 13905$ & $-/ 31122$ & $-/ 17667$ & $-/ 34000$ & $-/ 5821$ & $-/ 10657$ & $-/ 1321$ & $-/ 9271$ & $-/ 8459$ & $-/ 31133$ & $-/ 13058$ \\
\hline TOTAL & $\begin{array}{c}1143 / 2152 \\
4 \\
\end{array}$ & $\begin{array}{c}796 / 4325 \\
5 \\
\end{array}$ & $\begin{array}{c}1571 / 3404 \\
8 \\
\end{array}$ & \begin{tabular}{|c|}
$1186 / 5591$ \\
4 \\
\end{tabular} & \begin{tabular}{|c|}
$643 / 1182$ \\
1 \\
\end{tabular} & $\begin{array}{c}1057 / 2277 \\
1 \\
\end{array}$ & $\begin{array}{c}143 / 235 \\
7 \\
\end{array}$ & \begin{tabular}{|c|}
$401 / 1351$ \\
3 \\
\end{tabular} & \begin{tabular}{|c|}
$374 / 1360$ \\
2 \\
\end{tabular} & \begin{tabular}{|c|}
$990 / 4492$ \\
9 \\
\end{tabular} & $\begin{array}{c}707 / 2028 \\
0 \\
\end{array}$ \\
\hline Total number of species & 10 & 11 & 9 & 22 & 11 & 13 & 6 & 14 & 14 & 19 & 29 \\
\hline
\end{tabular}

The H/RC assemblage contains a diverse range of species. Just less than half (14) of the species identified at $\mathrm{H} / \mathrm{RC}$ are Sparidae. The remainder is from a varied number (9) of 
families. The most frequently occurring species, present in most layers, are Dichistius capensis, Diplodus capensis, Liza richardsonii, Seriola lalandi and Sarpa salpa.

All of the species identified in the assemblage occur in the area today. Most of the species inhabit near-shore areas. Several frequent estuaries but none are exclusively estuarine inhabitants. Two offshore species, Argyrozona argyrozona and Triglidae spp., were found in very small numbers in the shelly layers (Table 6.1b).

There is some variation in the frequencies and densities of species between layers. Liza richardsonii is most common in layer Judy, and quantities above and below this layer are considerably lower. The number of species present in layer Judy (9) is considerably lower than in layer Katherine (22) below, yet the density (in terms of MNI) of the most frequently occurring species is consistently higher in Judy, with the exception of Diplodus capensis, which is more frequent in Katherine. A similar trend is seen in layer Ben, where the overall number of species present is low (10), but the numbers of Seriola lalandi, Sarpa salpa and Dichistius capensis are more than double those of the preceding or following layers (Figure 6.1, Table 6.1b). The density of Diplodus capensis and Pomatomys saltatrix is also higher in layer Ben, but not to the same extent as the aforementioned species. Only one of the most frequently occurring species, $L$. richardsonii, shows little change in numbers between layer Ben and those layers above and below.

Sarpa salpa is the most frequently occurring species in terms of MNI and NISP at H/RC, but it is a rather small fish, and the meat contribution of one specimen is low relative to that of some of the other common species, particularly Seriola lalandi. Based on average species weight data from Poggenpoel (1984) and Inskeep (1987), one S. lalandi equals 26 S. salpa in weight. Reconstructed weights based on this data show that the meat contribution of $S$. salpa is lower in all but the oldest layer relative to that of S. lalandi (Figure 6.2). 


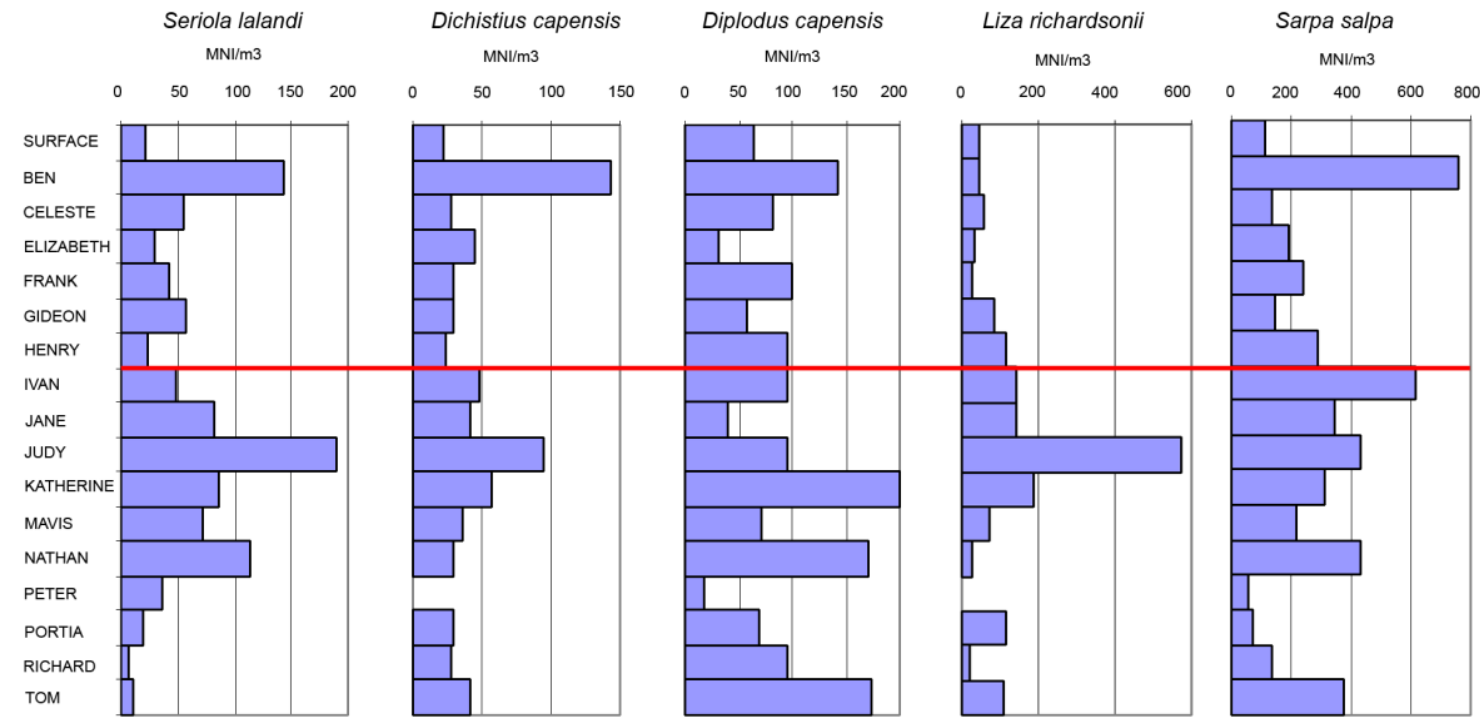

Figure 6.1. MNI per $\mathrm{m}^{3}$ of the most common species at Hofffman's/Robberg Cave by layer. The horizontal red line indicates the hiatus between the shelly and Zostera layers

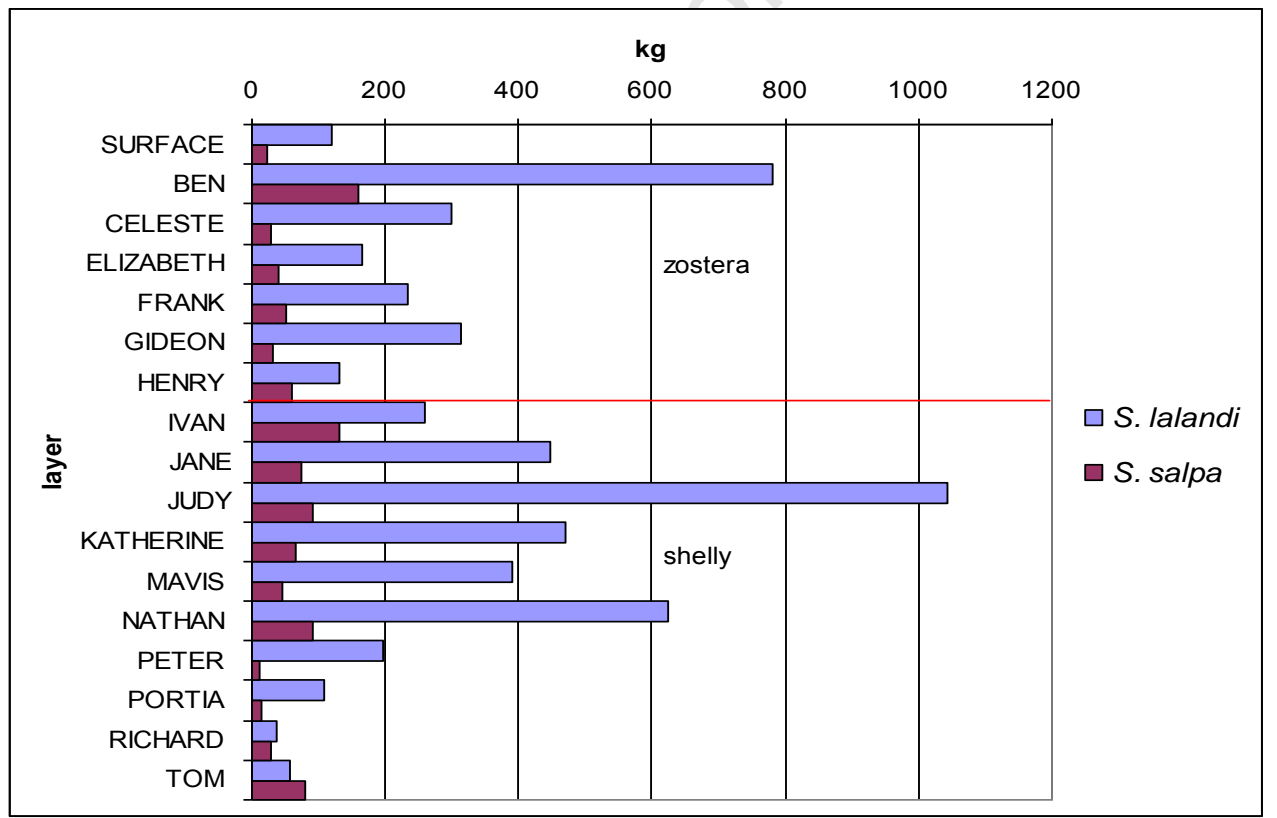

Figure 6.2. Reconstructed weights (in kg) based on MNI of Sarpa salpa and Seriola lalandi per layer at Hoffman's/Robberg Cave 


\subsubsection{Size reconstruction}

The size category data are based on NISP, but note that not all specimens could be assigned to a size category due to breakage or other factors and therefore the numbers are lower than the actual NISP per species (Table 6.2).

Table 6.2. Percentage of NISP per size class and species for all layers combined at Hoffman's/Robberg Cave. $n=$ NISP of elements that could be used for size estimations

\begin{tabular}{|c|c|c|c|c|c|c|c|}
\hline & & TINY & SMALL & MEDIUM & LARGE & XLARGE & XXLARGE \\
\hline Taxa & $\mathrm{n}$ & $\begin{array}{c}0- \\
150 \mathrm{~mm}\end{array}$ & $151-300 \mathrm{~mm}$ & $301-500 \mathrm{~mm}$ & $501-800 \mathrm{~mm}$ & $801-1000 \mathrm{~mm}$ & $>1000 \mathrm{~mm}$ \\
\hline \multicolumn{8}{|l|}{ Osteichthyes } \\
\hline \multicolumn{8}{|l|}{ Ariidae } \\
\hline G. feliceps & 6 & - & 50 & 50 & - & - & - \\
\hline Serranidae & & & & & & $\mathrm{B}$ & \\
\hline Acanthistius sebastoides & 3 & - & 100 & - & - & $-\quad$ & - \\
\hline Epinephelus marginatus & 20 & - & 10 & 65 & 25 & - & - \\
\hline \multicolumn{8}{|l|}{ Pomatomidae } \\
\hline Pomatomus saltatrix & 59 & - & - & 100 & - & - & - \\
\hline \multicolumn{8}{|l|}{ Haemulidae } \\
\hline Pomadasys commersonnii & 2 & - & - & 100 & - & - & - \\
\hline Pomadasys olivaceum & 21 & 4.8 & 95.2 & - & - & - & - \\
\hline Haemulidae spp. & 2 & - & 100 & - & - & - & - \\
\hline \multicolumn{8}{|l|}{ Sparidae } \\
\hline Argyrozona argyrozona & 4 & - & - & 75 & 25 & - & - \\
\hline Cheimerius nufar & 2 & - & - & - & 100 & - & - \\
\hline Chrysoblephus laticeps & 2 & - & - & 100 & - & - & - \\
\hline Cymatoceps nasutus & 11 & - & 9.1 & - & 90.9 & - & - \\
\hline Diplodus hottentotus & 9 & - & 22.2 & 77.8 & - & - & - \\
\hline Diplodus capensis & 623 & 0.8 & 82 & 17.2 & - & - & - \\
\hline Gymnocrotaphus curvidens & 16 & - & 68.8 & 31.3 & - & - & - \\
\hline Lithognathus lithognathus & 40 & - & 87.5 & 12.5 & - & - & - \\
\hline Pachymetopon blochii & 27 & - & 96.3 & 3.7 & - & - & - \\
\hline Rhabdosargus globiceps & 7 & - & 28.6 & 71.4 & - & - & - \\
\hline Rhabdosargus holubi & 20 & - & 85 & 15 & - & - & - \\
\hline Sarpa salpa & 1142 & 0.2 & 99.6 & 0.3 & - & - & - \\
\hline Sparodon durbanensis & 1 & - & - & 100 & - & - & - \\
\hline Spondyliosoma emarginatum & 25 & - & 100 & - & - & - & - \\
\hline Sparidae spp. & 3063 & 0.4 & 89.1 & 9.1 & 1.3 & 0.1 & - \\
\hline Dischistiidae & 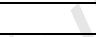 & 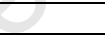 & & & & & \\
\hline Dichistius capensis & 379 & - & 10.3 & 87.1 & 2.6 & - & - \\
\hline \multicolumn{8}{|l|}{ Monodactylidae } \\
\hline Monodactylus falciformis & 2 & - & 100 & - & - & - & - \\
\hline \multicolumn{8}{|l|}{ Carangidae } \\
\hline Seriola lalandi & 365 & - & 0.8 & 1.4 & 68.5 & 29.3 & - \\
\hline \multicolumn{8}{|l|}{ Mugilidae } \\
\hline Liza richardsonii & 867 & - & 99.2 & 0.8 & - & - & - \\
\hline Mugil cf. cephalus & 1 & - & 100 & - & - & - & - \\
\hline \multicolumn{8}{|l|}{ Gobiidae } \\
\hline Gobiidae spp. & 1 & - & 100 & - & - & - & - \\
\hline \multicolumn{8}{|l|}{ Scombridae } \\
\hline S. japonicus & 1 & - & 100 & - & - & - & - \\
\hline Species indeterminate & 1852 & 0.5 & 89.7 & 9.1 & 0.7 & 0.1 & - \\
\hline Total & 8573 & 0.3 & 82.7 & 11.8 & 3.9 & 1.3 & - \\
\hline
\end{tabular}

The distributions of the sizes of the most common species are not significantly different between the Zostera-rich and the shell-rich layers of H/RC $(\mathrm{K}-\mathrm{S}$ statistic $=0.059, \mathrm{p}<0.5)$

(Figure 6.3). Overall, there is a slight decrease of larger fish in the shelly layers, which could be a function of the much larger sample size in those layers (Figure 6.4). Sarpa 
salpa and Liza richardsonii have been excluded from this figure as these are almost exclusively ( $>99 \%)$ in the small size category. As there were no major differences in size between the Zostera and shelly units, the data have been combined in Table 6.2.

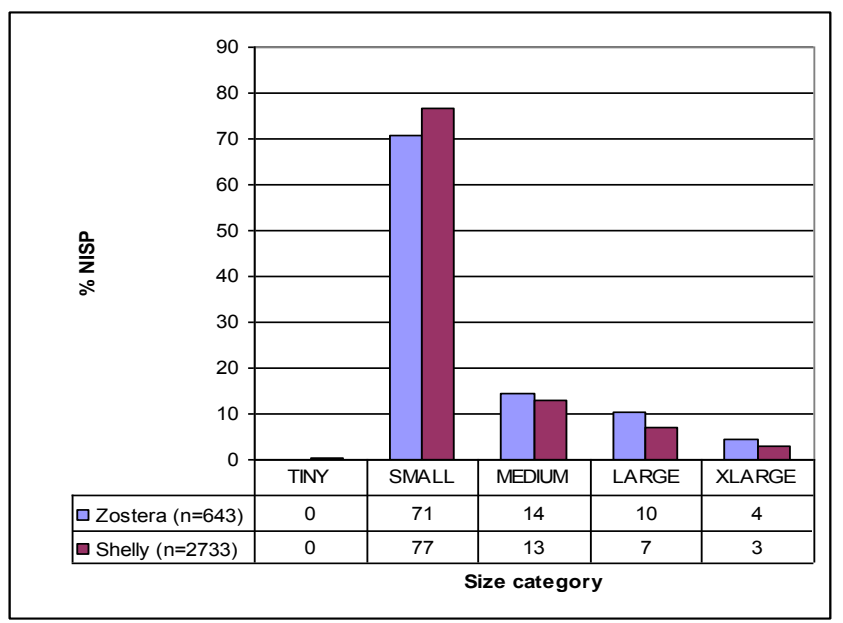

Figure 6.3. Percentage per size class of the most common species found at Hoffman's/Robberg Cave based on NISP. The species are Seriola lalandi, Sarpa salpa, Diplodus capensis, Dichistius capensis and Liza richardsonii

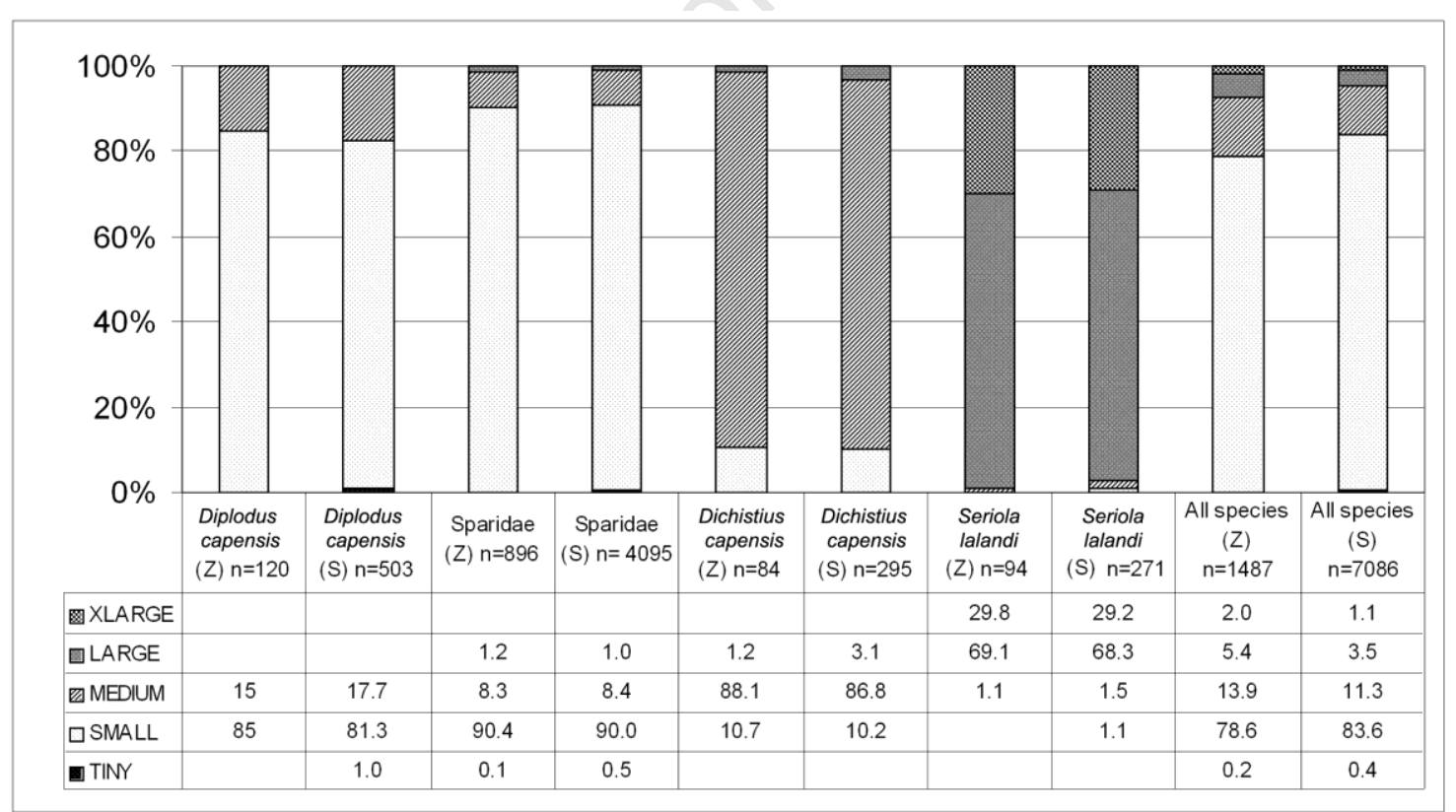

Figure 6.4. Comparison of percentages of size classes based on NISP of the most common species, all Sparidae combined and all species combined per Zostera and Shelly layers from Hoffman's/Robberg Cave. Note that Liza richardsonii and Sarpa salpa have been excluded from this chart and that the Sparidae category includes all Sparidae combined. Z=Zostera layers, $S=$ Shelly layers. 
The majority of specimens fall in the small category (82.7\%), followed by medium sized specimens (11.8\%) (Table 6.2). No bones from this assemblage were from fish larger than $1000 \mathrm{~mm}$. Most of the specimens in the assemblage are from mature individuals, with some smaller specimens of species such as Dichistius capensis and Epinephelus marginatus. There is a small number of specimens in the tiny category $(<150 \mathrm{~mm} ; \mathrm{n}=30)$.

Seriola lalandi can attain $1500 \mathrm{~mm}$, but none in the assemblage were larger than $1000 \mathrm{~mm}$, and the largest number fall into the large $(501-800 \mathrm{~mm})$ size category. The very low number of small and medium $S$. lalandi ( $\mathrm{n}=8$ out of 365 ) could be a reflection of this species' ecology as juvenile specimens tend to shoal further offshore (Froese and Pauly 2009; Lamberth et al. 1994). All of the Pomatomys saltatrix specimens fall in the medium size category (301 -500mm) and are from adult fish (Lamberth et al. 1994), although this species can grow considerably larger and can attain $1200 \mathrm{~mm}$ in length.

Most (85\%) of the Rhabdosargus holubi specimens are in the small (151-300mm) size category. This category can include both adults and juveniles of this species, and it is not possible to determine how many of these were either. Juvenile $(<19 \mathrm{~cm}) R$. holubi are estuarine dependant, whereas adults are primarily marine dwellers.

\subsection{Blombosfontein 1}

\subsubsection{Species present}

A total of 117 specimens derived from three families, four genera and four species were examined from BBF 1 (Table 6.3). Fifty seven specimens (49\%) could not be identified, 47 of which were vertebrae. Two articulated vertebrae were found brecciated together. The species list is very limited, with Diplodus capensis the most common species, followed by Dichistius capensis. The only other specimens identified were one Liza richardsonii and one specimen of an unidentified species of the genus Rhabdosargus. All of the species are near-shore inhabitants and occur in the area at present. 
Table 6.3. MNI and NISP of species at Blombosfontein 1

\begin{tabular}{|l|c|}
\hline Taxa & MNI/NISP \\
\hline Osteichthyes & \\
\hline Sparidae & \\
\hline Diplodus capensis & $3 / 19$ \\
\hline Rhabdosargus spp. & $1 / 1$ \\
\hline Sparidae spp. & $-/ 35$ \\
\hline Dischistiidae & \\
\hline Dichistius capensis & $2 / 4$ \\
\hline Mugilidae & \\
\hline Liza richardsonii & $1 / 1$ \\
\hline Species indeterminate & $-/ 57$ \\
\hline Total & $7 / 117$ \\
\hline
\end{tabular}

\subsubsection{Size reconstruction}

A limited range of sizes is represented at BBF 1, with the majority of specimens $(80.9 \%)$ in the medium size category, and the remainder in the small category (Table 6.4). The size ranges of those that could be identified to species are of adult specimens. Despite the small mesh size used during sieving $(1.5 \mathrm{~mm})$, no specimens were from individuals smaller than $15 \mathrm{~mm}$. The low number of specimens precludes any meaningful interpretation of the sizes of the species present.

Table 6.4. Percentage of NISP per size class and species from Blombosfontein 1. $n=$ NISP of elements that could be used for size estimations

\begin{tabular}{|c|c|c|c|c|c|c|c|}
\hline & $\mathbf{n}$ & TINY & SMALL & MEDIUM & LARGE & XLARGE & XXLARGE \\
\hline Taxa & & $0-150 \mathrm{~mm}$ & $151-300 \mathrm{~mm}$ & $301-500 \mathrm{~mm}$ & $501-800 \mathrm{~mm}$ & $801-1000 \mathrm{~mm}$ & $>1000 \mathrm{~mm}$ \\
\hline Osteichthyes & & & 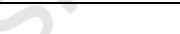 & & & & \\
\hline Sparidae & & & 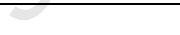 & & & & \\
\hline Diplodus capensis & 19 & -2 & - & 100.0 & - & - & - \\
\hline Sparidae spp. & 34 & - & 29.4 & 70.6 & - & - & - \\
\hline Dischistiidae & & + & & & & & \\
\hline Dichistius capensis & 4 & - & - & 100.0 & - & - & - \\
\hline Mugilidae & D & & & & & & \\
\hline Liza richardsonii & 1 & - & 100.0 & - & - & - & - \\
\hline Species indeterminate & 10 & - & 20.0 & 80.0 & - & - & - \\
\hline Total & 68 & - & 19.1 & 80.9 & - & - & - \\
\hline
\end{tabular}

\subsection{Blombosfontein 6}

\subsubsection{Species present}

A total of 225 specimens were examined from BBF 6, with fish from two families, three genera and three species identified (Table 6.5). Most of the identified specimens are Sparidae. Unidentified specimens constitute $81 \%$ of the assemblage, of which $91 \%$ $(n=166)$ are vertebrae. The species present occur in the area at present. Two of the species 
inhabit shallow near-shore waters, and the third, Chrysoblephus cristiceps, prefers deeper water, which can occur close to the shore if there are deep gullies present.

Table 6.5. MNI and NISP of species at Blombosfontein 6

\begin{tabular}{|l|c|c|c|c|}
\hline LAYER & ASBA & LIM & HBL & TOTAL \\
\hline Taxa & MNI/NISP & MNI/NISP & MNI/NISP & MNI/NISP \\
\hline Osteichthyes & & & & \\
\hline Sparidae & & & & \\
\hline Chrysoblephus cristiceps & - & - & $1 / 1$ & $1 / 1$ \\
\hline Diplodus capensis & $1 / 1$ & - & - & $1 / 1$ \\
\hline Sparidae spp. & $-/ 21$ & $-/ 5$ & $-/ 11$ & $-/ 37$ \\
\hline Mugilidae & & & & \\
\hline Liza richardsonii & $1 / 1$ & $1 / 2$ & - & $2 / 3$ \\
\hline Species indeterminate & $-/ 58$ & $-/ 111$ & $-/ 14$ & $-/ 183$ \\
\hline TOTAL & $2 / 81$ & $1 / 118$ & $1 / 26$ & $4 / 225$ \\
\hline
\end{tabular}

Fish teeth were not quantified, but it was noted that at this site most were smashed or broken. Most of the bones were also fragmented, and those in layer 6/4(LIM) were covered by a calcified layer that further hindered identification, particularly of surface modification. Data from all 3 layers were combined due to the low number of identified species and the generally low number of fish from this site (Table 6.5).

\subsubsection{Size reconstruction}

At BBF 6 the largest number of specimens are in the small and medium size category (37.5 and 33.9\% respectively), but large and tiny specimens are also present (Table 6.6).

All identified specimens fall within the adult size range for each species.

Table 6.6. Percentage of NISP per size class and species for all layers combined from Blombosfontein 6. $n=$ NISP of elements that could be used for size estimations

\begin{tabular}{|l|r|c|c|c|c|c|c|}
\hline & & TINY & SMALL & MEDIUM & LARGE & XLARGE & XXLARGE \\
\hline Taxa & $\mathbf{n}$ & $\mathbf{0 - 1 5 0} \mathbf{m m}$ & $\begin{array}{c}\mathbf{1 5 1 -} \\
\mathbf{3 0 0} \mathbf{m m}\end{array}$ & $\begin{array}{c}\mathbf{3 0 1 -} \\
\mathbf{5 0 0} \mathbf{m m}\end{array}$ & $\begin{array}{c}\mathbf{5 0 1 -} \\
\mathbf{8 0 0} \mathbf{m m}\end{array}$ & $\begin{array}{c}\mathbf{8 0 1 -} \\
\mathbf{1 0 0 0} \mathbf{m m}\end{array}$ & $\mathbf{> 1 0 0 0} \mathbf{m m}$ \\
\hline Osteichthyes & & & & & & & \\
\hline Sparidae & & & & & & & \\
\hline Chrysoblephus cristiceps & 1 & - & - & - & 100.0 & - & - \\
\hline Diplodus capensis & 1 & - & - & 100.0 & - & - & - \\
\hline Sparidae spp. & 37 & 10.8 & 24.3 & 37.8 & 27.0 & - & - \\
\hline Mugilidae & & & & & & & \\
\hline Liza richardsonii & 3 & - & 100.0 & - & - & - & - \\
\hline Species indeterminate & 14 & - & 64.3 & 28.6 & 7.1 & - & - \\
\hline Total & 56 & 7.1 & 37.5 & 33.9 & 21.4 & - & - \\
\hline
\end{tabular}




\subsection{Blombos Cave Later Stone Age}

\subsubsection{Species present}

A total of 2132 specimens from square G5 spanning the whole LSA sequence were examined for this study. Data from an additional 5185 specimens were added from a previous study (van Niekerk 2005) to increase the sample size. The additional data were used to calculate NISP, MNI and MNE. All other calculations were based only on the data collected for this study (from square G5). The data are presented in MNI and NISP per 10 cubic metres to allow comparison with the BBC MSA data (Table 6.7).

Table 6.7. MNI and NISP per $10 \mathrm{~m}^{3}$ per layer of Blombos Cave Later Stone Age assemblage. Note that the TOTAL column represents the MNI and NISP per $10 \mathrm{~m}^{3}$ of the whole assemblage (Layers 1 to 5 combined)

\begin{tabular}{|c|c|c|c|c|c|c|}
\hline & LAYER 1 & LAYER 2 & LAYER 3 & LAYER 4 & LAYER 5 & TOTAL \\
\hline Taxa & MNI/NISP/10m ${ }^{3}$ & MNI/NISP/10m ${ }^{3}$ & MNI/NISP/10m ${ }^{3}$ & MNI/NISP/10m ${ }^{3}$ & MNI/NISP/10m ${ }^{3}$ & MNI/NISP/10m ${ }^{3}$ \\
\hline Chondrichthyes & & & & 3 & & \\
\hline Shark spp. & - & - & $30 / 50$ & 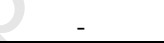 & - & $4 / 10$ \\
\hline \multicolumn{7}{|l|}{ Serranidae } \\
\hline Epinephelus marginatus & - & - & $50 / 50$ & $70 / 330$ & $40 / 100$ & $40 / 140$ \\
\hline \multicolumn{7}{|l|}{ Sparidae } \\
\hline Chrysoblephus cristiceps & $30 / 60$ & $100 / 190$ & $30 / 190$ & $200 / 1640$ & $120 / 780$ & $120 / 770$ \\
\hline Chrysoblephus laticeps & $30 / 30$ & $60 / 120$ & $110 / 370$ & $150 / 990$ & $120 / 580$ & $\mathbf{1 1 0 / 5 3 0}$ \\
\hline Cymatoceps nasutus & $60 / 90$ & $20 / 20$ & $80 / 130$ & $70 / 320$ & $40 / 230$ & $60 / 190$ \\
\hline Diplodus capensis & - & $20 / 40$ & - & $50 / 120$ & $40 / 330$ & 30/110 \\
\hline Gymnocrotaphus curvidens & - & $20 / 20$ & - & $40 / 130$ & $20 / 100$ & $20 / 70$ \\
\hline Pachymetopon blochii & $-8>$ & - & - & $200 / 270$ & $60 / 140$ & $80 / 120$ \\
\hline Petrus rupestris & - & - & $30 / 30$ & - & $20 / 40$ & $10 / 10$ \\
\hline Sparodon durbanensis & - & - & - & - & $40 / 80$ & $10 / 20$ \\
\hline Spondyliosoma emarginatum & $30 / 30$ & $20 / 20$ & $80 / 130$ & $150 / 700$ & $100 / 370$ & $90 / 330$ \\
\hline Sparidae spp. & $-/ 1310$ & $-/ 1790$ & $-/ 5580$ & $-/ 19970$ & $-/ 11000$ & $-/ 10160$ \\
\hline \multicolumn{7}{|l|}{ Dichistiidae } \\
\hline Dichistius capensis & - & - & - & $10 / 10$ & - & $4 / 4$ \\
\hline \multicolumn{7}{|l|}{ Mugilidae } \\
\hline Liza richardsonii & $60 / 380$ & $80 / 390$ & $210 / 2000$ & $580 / 7000$ & $290 / 2870$ & $310 / 3330$ \\
\hline Species indeterminate & $-/ 2630$ & $-/ 2520$ & $-/ 9710$ & $-/ 25330$ & $-/ 11640$ & $-/ 13020$ \\
\hline Total & $200 / 4520$ & $330 / 5120$ & $610 / 18260$ & $1520 / 56790$ & $910 / 28270$ & $870 / 28800$ \\
\hline Total number of species & 5 & 7 & 8 & 10 & 11 & 13 \\
\hline
\end{tabular}

Out of a total of 7317 specimens, 1425 (19.5\%) could be identified to genus and species, $2582(35 \%)$ could only be identified to family (Sparidae), two (vertebrae) were identified as unidentified shark species, and 3308 (45\%) specimens could not be identified to family, 
of which 3005 (91\%) were vertebrae. A total of 13 species, twelve genera and four families were identified. Most species (9) are Sparidae.

The number of species found increased with time, with the fewest (5) in the youngest layer, and up to eleven species identified in Layer 5. The additional species in the older layers occurred in low numbers, and their presence could be attributed to the much larger sample size in these layers. The overall density of fish was highest in Layer 4 and lowest in Layer 1 (Table 6.7). Five species were present in all layers, namely Chrysoblephus cristiceps, Chrysoblephus laticeps, Cymatoceps nasutus, Spondyliosoma emarginatum and Liza richardsonii. The latter was consistently the most common species in all layers (Table 6.7, Figure 6.5).

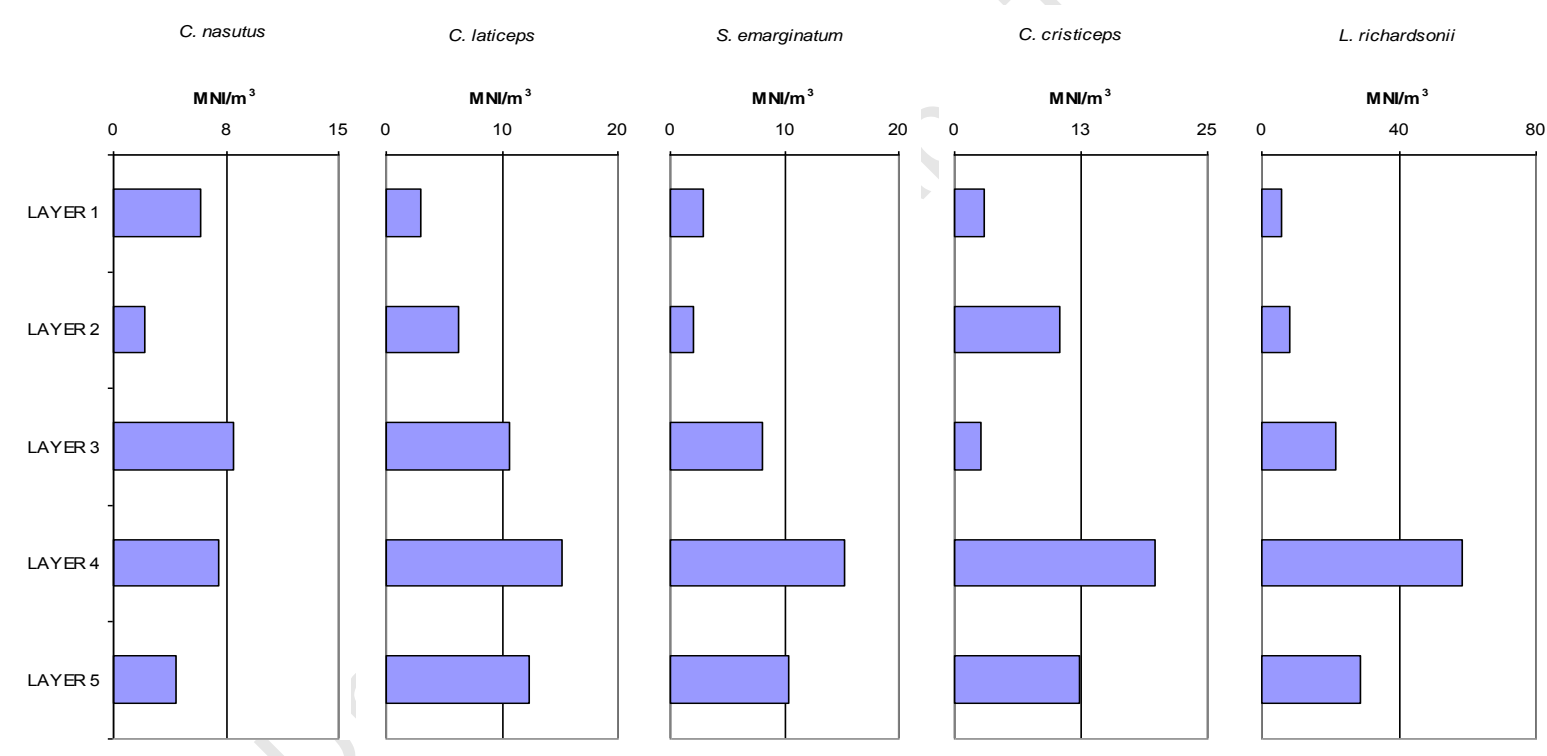

Figure 6.5. Density (MNI per $\mathrm{m}^{3}$ ) per layer of the most common species present in the Blombos Cave Later Stone Age assemblage

All of the species present are those that inhabit near shore habitats. One species, Liza richardsonii, frequents estuaries as well as coastal waters. Chrysoblephus cristiceps and Petrus rupestris inhabit deeper water but can occur close to shore if there are deep gullies, which are present near Blombos Cave. 


\subsubsection{Size reconstruction}

The BBC LSA material was initially separated into two groups based on the dates, namely pre $1600 \mathrm{BP}$ (Layers 3-5) and post $1600 \mathrm{BP}$ (Layers 1 and 2), to determine whether there were any significant differences in size categories between these two periods (Figure 6.6). Due to the relatively low numbers of species with reconstructed size categories, the data are presented for Liza richardsonii and all Sparidae combined. No significant differences were seen $(\mathrm{K}-\mathrm{S}$ statistic $=0.17, \mathrm{p}<0.5)$ which could in part be attributed to the low number of specimens that could be assigned to size categories in the post $1600 \mathrm{BP}$ material. The apparent increase in tiny and medium sized L. richardsonii in the post $1600 \mathrm{BP}$ assemblage is most likely due to the small sample size ( $\mathrm{n}=9$ ) (Figure 6.6).

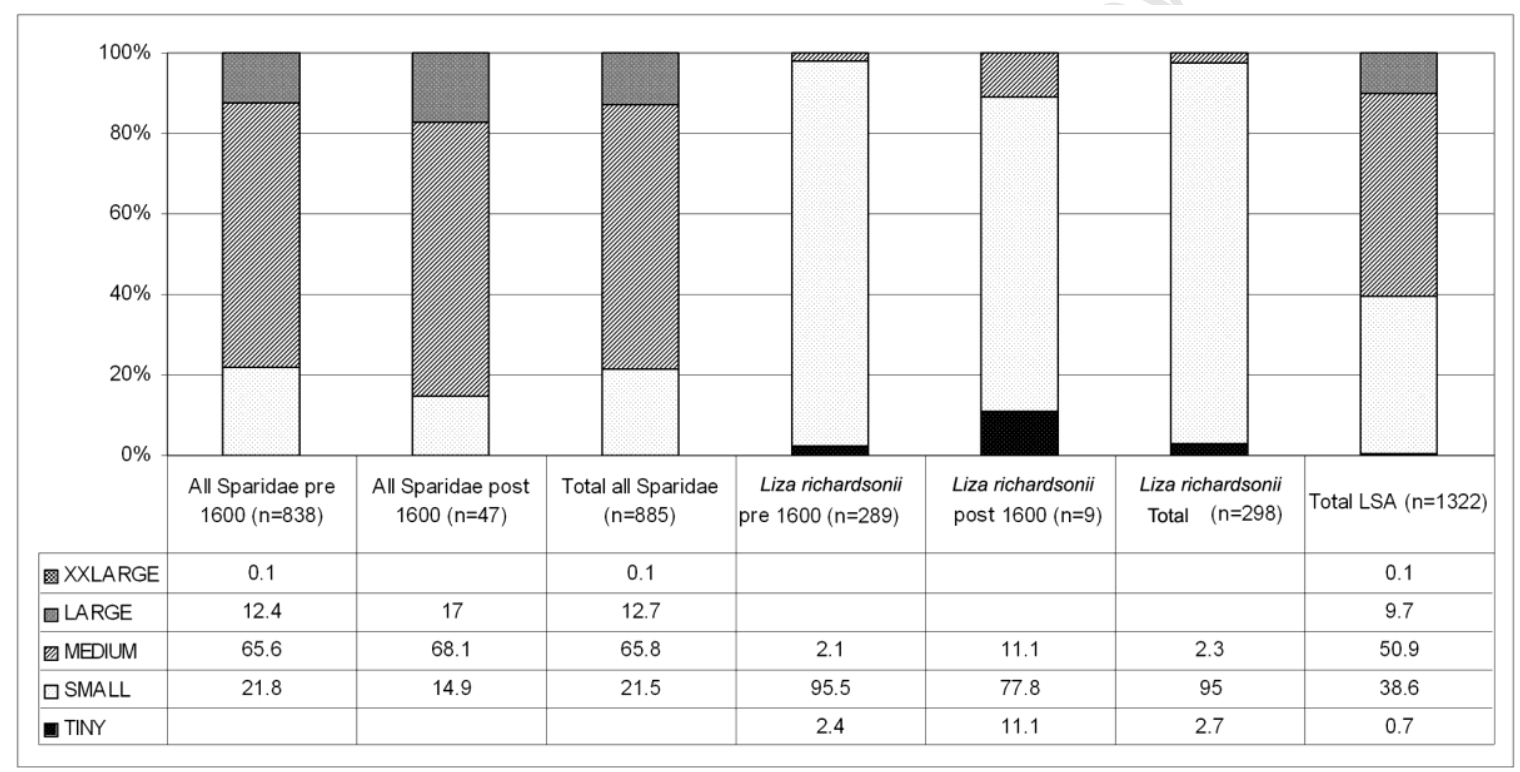

Figure 6.6. Size category comparison between LSA material older and younger than $1600 \mathrm{BP}$ at Blombos Cave based on NISP

As there are no significant differences in size between these two time periods, size categories per species are presented for all layers combined in Table 6.8. The majority of fish are in the medium (50.9\%) followed by small category (38.6\%). No specimens were recorded in the extra large category, and one specimen, a single Petrus rupestris dentary, is from a fish bigger than $1000 \mathrm{~mm}$ (XXlarge). Very few specimens are from fish smaller than $15 \mathrm{~cm}(\mathrm{n}=9)$. The sizes of Liza richardsonii specimens range from tiny to medium, although most (95\%) are small. 
Table 6.8. Percentage of NISP per size class and species for all layers combined from the Blombos Cave Later Stone Age assemblage. $n=$ NISP of elements that could be used for size estimations

\begin{tabular}{|c|c|c|c|c|c|c|c|}
\hline & & TINY & SMALL & MEDIUM & LARGE & XLARGE & XXLARGE \\
\hline Taxa & $\mathbf{n}$ & $0-150 \mathrm{~mm}$ & $151-300 \mathrm{~mm}$ & $301-500 \mathrm{~mm}$ & $501-800 \mathrm{~mm}$ & $801-1000 \mathrm{~mm}$ & $>1000 \mathrm{~mm}$ \\
\hline \multicolumn{8}{|l|}{ Osteichthyes } \\
\hline \multicolumn{8}{|l|}{ Serranidae } \\
\hline E. marginatus & 15 & - & - & 60 & 40 & - & - \\
\hline \multicolumn{8}{|l|}{ Sparidae } \\
\hline C. cristiceps & 44 & - & - & 56.8 & 43.2 & - & - \\
\hline C. laticeps & 18 & - & - & 100 & - & - & - \\
\hline C. nasutus & 5 & - & - & 20 & 80.0 & - & - \\
\hline D. sargus capensis & 16 & - & 37.5 & 62.5 & - & - & - \\
\hline G. curvidens & 7 & - & - & 100.0 & - & - & - \\
\hline P. blochii & 17 & - & 100 & - & - & - & - \\
\hline P. rupestris & 1 & - & - & - & - & - & 100 \\
\hline S. emarginatum & 13 & - & 100 & - & - & - & - \\
\hline Sparidae spp. & 763 & - & 20.2 & 68.2 & 11.7 & - & - \\
\hline \multicolumn{8}{|l|}{ Dischistiidae } \\
\hline D. capensis & 1 & - & - & 100 & - & - & - \\
\hline \multicolumn{8}{|l|}{ Mugilidae } \\
\hline L. richardsonii & 298 & 2.7 & 95 & 2.3 & - & - & - \\
\hline Species indeterminate & 124 & 0.8 & 29.8 & 61.3 & 8.1 & - & - \\
\hline Total & 1322 & 0.7 & 38.6 & 50.9 & 9.7 & - & 0.1 \\
\hline
\end{tabular}

\subsection{Blombos Cave Middle Stone Age}

\subsubsection{Species present}

A total of 860 specimens were examined from the Blombos Cave Middle Stone Age layers and at least 17 species from 12 genera and 11 families were identified (Table 6.9). Four hundred and seven (47\%) specimens could not be identified to family, of which 173 $(42.5 \%)$ were vertebrae and $143(35 \%)$ scales. The total MNI of the assemblage is 84 . Sixteen species are present in the interior layers, and 8 in the exterior. The quantity of fish from the exterior area is low (total NISP=156) but the average density is higher than in the interior. Most of the exterior fish are from the M3 phase where seven species are present. The M1 and M2 exterior layers each have two species present. Many of the vertebrae lacked neural and haemal spines. The data in Table 6.9 is presented as MNI/NISP per $10 \mathrm{~m}^{3}$ due to the low sample sizes. 
Table 6.9. MNI and NISP per $10 \mathrm{~m}^{3}$ per phase in Blombos Cave Middle Stone Age. Total columns show the MNI and NISP per $10 \mathrm{~m}^{3}$ for the combined volume of interior and exterior material excavated, per phase and for the entire assemblage. Note that the columns for the BBC MSA ALL PHASES represent the MNI and NISP per $10 \mathrm{~m}^{3}$ of the whole assemblage (M1, M2 and M3 combined)

\begin{tabular}{|c|c|c|c|c|c|c|c|c|c|c|c|c|}
\hline & \multicolumn{3}{|c|}{ M1 } & \multicolumn{3}{|c|}{ M2 } & \multicolumn{3}{|c|}{ M3 } & \multicolumn{3}{|c|}{ BBC MSA ALL PHASES } \\
\hline & $\begin{array}{l}\text { TOTAL } \\
{ }^{\prime} / 10 \mathrm{M}^{3}\end{array}$ & $\begin{array}{c}\text { INTERIO } \\
\mathrm{R} / 10 \mathrm{M}^{3} \\
\end{array}$ & $\begin{array}{c}\text { EXTERIO } \\
\mathrm{R} / 10 \mathrm{M}^{3} \\
\end{array}$ & $\begin{array}{l}\text { TOTAL } \\
{ }^{\prime 10 \mathrm{M}^{3}} \\
\end{array}$ & $\begin{array}{c}\text { INTERIO } \\
\mathrm{R} / 10 \mathrm{M}^{3} \\
\end{array}$ & $\begin{array}{c}\text { EXTERIO } \\
{\text { R } / 10 \mathrm{M}^{3}}\end{array}$ & $\begin{array}{l}\text { TOTAL } \\
/ 1 \mathbf{1 0 M}^{3}\end{array}$ & $\begin{array}{c}\text { INTERIO } \\
\mathrm{R} / 10 \mathrm{M}^{3} \\
\end{array}$ & \begin{tabular}{|c|} 
EXTERIO \\
R $/ 10 \mathrm{M}^{3}$ \\
\end{tabular} & $\begin{array}{l}\text { TOTAL } \\
/ \mathbf{1 0 M}^{3}\end{array}$ & $\begin{array}{c}\text { INTERIO } \\
\mathrm{R} / 10 \mathrm{M}^{3} \\
\end{array}$ & $\begin{array}{c}\text { EXTERIO } \\
{\text { R } / 10 M^{3}}^{3}\end{array}$ \\
\hline Taxa & $\begin{array}{c}\mathrm{MNI} / \mathrm{NIS} \\
\mathbf{P}\end{array}$ & MNI/NISP & MNI/NISP & $\begin{array}{c}\mathrm{MNI} / \mathrm{NIS} \\
\mathbf{P}\end{array}$ & MNI/NISP & MNI/NISP & $\begin{array}{c}\mathrm{MNI} / \mathrm{NIS} \\
\mathrm{P}\end{array}$ & $\mathrm{MNI} / \mathrm{NISP}$ & MNI/NISP & $\begin{array}{c}\mathrm{MNI} / \mathrm{NIS} \\
\mathrm{P}\end{array}$ & MNI/NISP & MNI/NISP \\
\hline \multicolumn{13}{|l|}{ Chondrichthyes } \\
\hline Shark spp. & $-/ 2$ & $-/ 2$ & - & $-/ 12$ & $-/ 13$ & - & $-/ 16$ & $-/ 19$ & - & $-/ 10$ & $-/ 11$ & - \\
\hline \multicolumn{13}{|l|}{ Osteichthyes } \\
\hline \multicolumn{13}{|l|}{ Ariidae } \\
\hline Galeichthys feliceps & $4 / 29$ & $2 / 29$ & $24 / 24$ & $16 / 29$ & $6 / 11$ & $142 / 249$ & $43 / 162$ & $12 / 35$ & $279 / 1115$ & $22 / 81$ & $7 / 27$ & $173 / 606$ \\
\hline \multicolumn{13}{|l|}{ Serranidae } \\
\hline Acanthistius sebastoides & - & - & - & - & - & - & $2 / 2$ & - & $15 / 15$ & $1 / 1$ & - & $7 / 7$ \\
\hline Epinephelus marginatus & - & - & - & $3 / 5$ & $3 / 6$ & - & $2 / 2$ & $2 / 2$ & - & $1 / 2$ & $1 / 2$ & - \\
\hline Serranidae spp. & - & - & - & - & - & - & $-/ 2$ & - & $-/ 15$ & $-/ 1$ & - & -17 \\
\hline \multicolumn{13}{|l|}{ Haemulidae } \\
\hline Haemulidae spp. & - & - & - & $-/ 5$ & $-/ 6$ & - & - & - & - & $-/ 1$ & $-/ 1$ & - \\
\hline \multicolumn{13}{|l|}{ Sparidae } \\
\hline Chrysoblephus cristiceps & $2 / 2$ & $2 / 2$ & - & $3 / 5$ & - & $36 / 71$ & $2 / 2$ & $2 / 2$ & - & $2 / 3$ & $1 / 1$ & $7 / 14$ \\
\hline Chrysoblephus gibbiceps & $10 / 19$ & $10 / 21$ & - & $3 / 13$ & $3 / 14$ & - & - & - & - & $4 / 10$ & $4 / 11$ & - \\
\hline Chrysoblephus laticeps & $2 / 2$ & $2 / 2$ & - & - & - & - & - & - & - & $1 / 1$ & $1 / 1$ & - \\
\hline Cymatoceps nasutus & $2 / 2$ & $2 / 2$ & - & - & - & - & - & - & - & $1 / 1$ & $1 / 1$ & - \\
\hline Pachymetopon blochii & $2 / 2$ & $2 / 2$ & - & $3 / 3$ & $3 / 3$ & - & $3 / 3$ & $2 / 2$ & $15 / 15$ & $3 / 3$ & $2 / 2$ & $7 / 7$ \\
\hline Pterogymnus laniarius & - & - & - & $3 / 3$ & $3 / 3$ & - & - & - & - & $1 / 1$ & $1 / 1$ & - \\
\hline Spondyliosoma emarginatum & $2 / 4$ & $2 / 4$ & - & - & - & - & $2 / 2$ & - & $15 / 15$ & $1 / 2$ & $1 / 1$ & $7 / 7$ \\
\hline Sparidae spp. & $-/ 207$ & $-/ 223$ & $-/ 24$ & $-/ 252$ & $-/ 261$ & $-/ 142$ & $-/ 48$ & $-/ 51$ & $-/ 29$ & $-/ 157$ & $-/ 168$ & $-/ 50$ \\
\hline Dischistiidae & & & & & & & 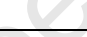 & & & & & \\
\hline Dichistius capensis & $2 / 2$ & $2 / 2$ & - & - & - & - & $2 / 2$ & $2 / 2$ & - & $1 / 1$ & $1 / 1$ & - \\
\hline Dichistiidae spp. & - & - & - & - & - & - & $-/ 10$ & $-/ 2$ & $-/ 73$ & $-/ 4$ & $-/ 1$ & $-/ 36$ \\
\hline Sciaenidae & . & & & & 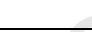 & 8 & 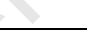 & & & & & \\
\hline $\begin{array}{l}\text { Argyrosomus } \\
\text { inodorus/japonicus }\end{array}$ & - & - & - & - & - & - & $2 / 3$ & $2 / 4$ & - & $1 / 1$ & $1 / 1$ & - \\
\hline Oplegnathidae & & & & & e & 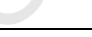 & & & & & & \\
\hline Oplegnathus conwayi & $2 / 2$ & $2 / 2$ & - & - & - & - & - & - & - & $1 / 1$ & $1 / 1$ & - \\
\hline Mugilidae & & & & & +8 & & & & & & & 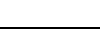 \\
\hline Liza richardsonii & $8 / 21$ & $8 / 23$ & - & $5 / 30$ & $6 / 33$ & - & $5 / 9$ & $6 / 10$ & - & $6 / 18$ & $7 / 20$ & - \\
\hline Mugilidae spp. & - & - & - & $-/ 3$ & $-/ 3$ & - & - & - & - & $-/ 1$ & $-/ 1$ & - \\
\hline \multicolumn{13}{|l|}{ Clinidae } \\
\hline Clinidae spp. & $-/ 2$ & $-/ 2$ & - & - & - & - & $-/ 3$ & $-/ 2$ & $-/ 15$ & $-/ 2$ & $-/ 1$ & $-/ 7$ \\
\hline \multicolumn{13}{|l|}{ Gobiidae } \\
\hline Gobiidae spp. & - & - & - & 18 & 18 & - & 17 & 12 & 144 & 15 & 13 & 122 \\
\hline Species Indeterminate & $-/ 344$ & $-/ 355$ & $-/ 224$ & $-/ 298$ & $-/ 316$ & $-/ 71$ & $-/ 193$ & $-/ 144$ & $-/ 565$ & $-/ 273$ & $-/ 264$ & $-/ 361$ \\
\hline TOTAL & $36 / 640$ & $34 / 671$ & $24 / 272$ & $36 / 666$ & $24 / 677$ & $178 / 533$ & $63 / 466$ & $28 / 277$ & $324 / 1901$ & $46 / 580$ & $29 / 520$ & $201 / 1124$ \\
\hline Total species & 12 & 12 & 2 & 10 & 9 & 2 & 12 & 10 & 7 & 17 & 16 & 8 \\
\hline
\end{tabular}

The majority of species (7) are Sparidae. Both the M1 and M3 phases have twelve species present, and ten species are in the M2. Four species occur in all phases, namely Chrysoblephus cristiceps, Galeichthys feliceps, Liza richardsonii and Pachymetopon blochii. Galeichthys feliceps and Liza richardsonii are the two most common species in the assemblage. One species, Acanthistius sebastoides, is present only in the exterior. Galeichthys feliceps is considerably more abundant in the exterior than interior in M2 and particularly in M3. The overall density of fish is low in all phases. The highest density $\left(\mathrm{MNI}\right.$ per $10 \mathrm{~m}^{3}$ ) of fish occurs in the M3 phase.

All the BBC MSA fish examined are marine species and all occur in the area today. The majority are near-shore species, with the exception of Pterogymnus laniarius, which 
occurs offshore. The unidentified Gobiidae specimens could be from either near-shore or pelagic species. Three of the species present, Liza richardsonii, Argyrosomus

inodorus/japonicus and Galeichthys feliceps, frequent both estuaries and coastal habitats.

\subsubsection{Size reconstruction}

The majority of BBC MSA fish fall in the small to large $(151-800 \mathrm{~mm})$ size categories with a few smaller and bigger exceptions. The proportions of size categories are very similar between the M1 and M2 phases (K-S statistic $=0.16, \mathrm{p}<0.5)$ (Figure 6.7). Most of the specimens in the M1 and M2 phases are from medium sized fish, which comprise $46.2 \%$ and $37.7 \%$ of the assemblages respectively. Small specimens are the second most common size category represented in both M1 and M2. The older M3 phase has considerably more specimens in the small category (59.7\%) and fewer large ones relative to M1 and M2 above (K-S statistic between M1 and M3 $=0.14, \mathrm{p}<0.5$, and between M2 and M3 $=0.15, \mathrm{p}<0.5)$. M3 is the only phase that contains specimens from fish larger than $1000 \mathrm{~mm}(\mathrm{n}=2)$. Tiny specimens are present in all three phases, at a constant ratio, albeit in low numbers (total $n=39$ ).

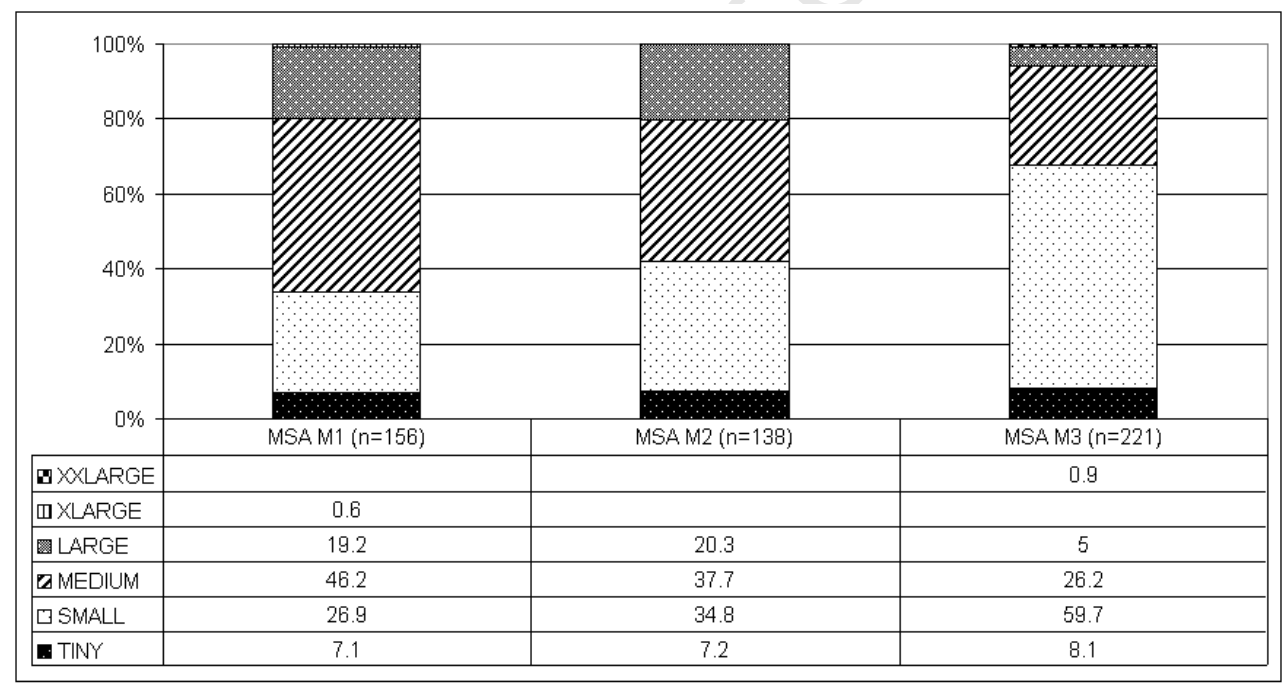

Figure 6.7. Percentage of specimens per size class and phase of all species combined from the Blombos Cave Middle Stone Age assemblage. Percentages are based on NISP of elements that could be used for size estimations

With the exception of one specimen of Acanthistius sebastoides, the small specimens in the M3 are from smaller fish of the same species that occur in larger sizes in the M1 and M2 (Tables 6.10a, b, c). The difference in size is most notable between the M3 and M1 phases. In the M3 the majority of Galeichthys feliceps and all Pachymetopon blochii and 
Dichistius capensis specimens are small, whereas all the specimens of these species are medium sized in the M1.

Table 6.10a. Percentage of NISP per size class in phase M1 from the Blombos Cave Middle Stone Age assemblage. $n=$ NISP of elements that could be used for size estimations

\begin{tabular}{|c|c|c|c|c|c|c|c|}
\hline \multicolumn{8}{|c|}{ M1 } \\
\hline Taxa & & TINY & SMALL & MEDIUM & LARGE & XLARGE & XXLARGE \\
\hline Total $n=156$ & $\mathrm{n}$ & $0-150 \mathrm{~mm}$ & $151-300 \mathrm{~mm}$ & $301-500 \mathrm{~mm}$ & $501-800 \mathrm{~mm}$ & $801-1000 \mathrm{~mm}$ & $>1000 \mathrm{~mm}$ \\
\hline \multicolumn{8}{|l|}{ Osteichthyes } \\
\hline \multicolumn{8}{|l|}{ Ariidae } \\
\hline Galeichthys feliceps & 14 & & & 100 & & & \\
\hline \multicolumn{8}{|l|}{ Sparidae } \\
\hline Chrysoblephus gibbiceps & 8 & & & 12.5 & 87.5 & & \\
\hline Chrysoblephus laticeps & 1 & & & 100 & & & \\
\hline Cymatoceps nasutus & 1 & & & & 100 & & \\
\hline Pachymetopon blochii & 1 & & & 100 & & & \\
\hline Spondyliosoma emarginatum & 2 & & 100 & & & & \\
\hline Sparidae spp. & 56 & & 12.5 & 62.5 & 23.2 & 1.8 & \\
\hline \multicolumn{8}{|l|}{ Dischistiidae } \\
\hline Dichistius capensis & 1 & & & 100 & & & \\
\hline \multicolumn{8}{|l|}{ Oplegnathidae } \\
\hline Oplegnathus conwayi & 1 & & 100 & & & & 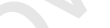 \\
\hline \multicolumn{8}{|l|}{ Mugilidae } \\
\hline Liza richardsonii & 8 & & 100 & & & 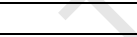 & \\
\hline \multicolumn{8}{|l|}{ Clinidae } \\
\hline Clinidae spp. & 1 & 100 & & & & 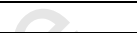 & \\
\hline \multicolumn{8}{|l|}{ Gobiidae } \\
\hline Gobiidae spp. & 1 & 100 & & & + & H & \\
\hline Species indeterminate & 61 & 14.8 & 39.3 & 31.1 & 14.8 & 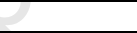 & \\
\hline Total \% & & 7.1 & 26.9 & 46.2 & 19.2 & 0.6 & \\
\hline
\end{tabular}

Table 6.10b. Percentage of NISP per size class in phase M2from the Blombos Cave Middle Stone Age assemblage. $n=$ NISP of elements that could be used for size estimations

\begin{tabular}{|c|c|c|c|c|c|c|c|}
\hline \multicolumn{8}{|c|}{ M2 } \\
\hline Taxa & & TINY & SMALL & MEDIUM & LARGE & XLARGE & XXLARGE \\
\hline Total $n=138$ & $\mathrm{n}$ & $0-150 \mathrm{~mm}$ & $151-300 \mathrm{~mm}$ & $301-500 \mathrm{~mm}$ & $501-800 \mathrm{~mm}$ & $801-1000 \mathrm{~mm}$ & $>1000 \mathrm{~mm}$ \\
\hline Osteichthyes & & & & 32 & & & \\
\hline Ariidae & & & 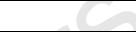 & 8 & & & \\
\hline Galeichthys feliceps & 11 & & 54.5 & 45.5 & & & \\
\hline Serranidae & & & 20 & & & & \\
\hline Epinephelus marginatus & 2 & & 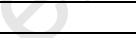 & 50 & 50 & & \\
\hline Haemulidae & & 8 & & & & & \\
\hline Haemulidae spp. & 2 & 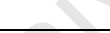 & 100 & & & & \\
\hline Sparidae & & +2 & & & & & \\
\hline Chrysoblephus gibbiceps & 5 & 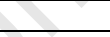 & & & 100 & & \\
\hline Pachymetopon blochii & 1 & & 100 & & & & \\
\hline Pterogymnus laniarius & 1 & - & 100 & & & & \\
\hline Sparidae spp. & 46 & 4.3 & 21.7 & 41.3 & 32.6 & & \\
\hline Mugilidae & & & & & & & \\
\hline Liza richardsonii & 11 & 9.1 & 90.9 & & & & \\
\hline Gobiidae & & & & & & & \\
\hline Gobiidae spp. & 3 & 100 & & & & & \\
\hline Species indeterminate & 56 & 7.1 & 32.1 & 48.2 & 12.5 & & \\
\hline Total \% & & 7.2 & 34.8 & 37.7 & 20.3 & & \\
\hline
\end{tabular}


Table 6.10c. Percentage of NISP per size class in phase M3 from the Blombos Cave Middle Stone Age assemblage. $n=$ NISP of elements that could be used for size estimations

\begin{tabular}{|c|c|c|c|c|c|c|c|}
\hline \multicolumn{8}{|c|}{ M3 } \\
\hline Taxa & & TINY & SMALL & MEDIUM & LARGE & XLARGE & XXLARGE \\
\hline Total $n=221$ & $\mathrm{n}$ & $0-150 \mathrm{~mm}$ & $151-300 \mathrm{~mm}$ & $301-500 \mathrm{~mm}$ & $501-800 \mathrm{~mm}$ & $801-1000 \mathrm{~mm}$ & $>1000 \mathrm{~mm}$ \\
\hline \multicolumn{8}{|l|}{ Osteichthyes } \\
\hline \multicolumn{8}{|l|}{ Ariidae } \\
\hline Galeichthys feliceps & 92 & & 64.1 & 35.9 & & & \\
\hline \multicolumn{8}{|l|}{ Serranidae } \\
\hline \multirow{2}{*}{$\begin{array}{l}\text { Acanthistius sebastoides } \\
\text { Epinephelus marginatus }\end{array}$} & 1 & & 100 & & & & \\
\hline & 1 & & & 100 & & & \\
\hline Serranidae spp. & 1 & & & 100 & & & \\
\hline \multicolumn{8}{|l|}{ Sparidae } \\
\hline \multirow{4}{*}{$\begin{array}{l}\text { Chrysoblephus cristiceps } \\
\text { Chrysoblephus gibbiceps } \\
\text { Pachymetopon blochii } \\
\text { Spondyliosoma emarginatum }\end{array}$} & 3 & & & 100 & & & \\
\hline & 2 & & & 50 & 50 & & \\
\hline & 1 & & 100 & & & & \\
\hline & 1 & & 100 & & & & \\
\hline Sparidae spp. & 19 & & 21.1 & 36.8 & 42.1 & & \\
\hline \multicolumn{8}{|l|}{ Dischistiidae } \\
\hline Dichistius capensis & 1 & & 100 & & & & \\
\hline Dichistiidae spp. & 6 & & 50 & 50 & & & 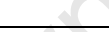 \\
\hline \multicolumn{8}{|l|}{ Sciaenidae } \\
\hline Argyrosomus inodorus/japonicus & 2 & & & & & & 100 \\
\hline \multicolumn{8}{|l|}{ Mugilidae } \\
\hline Liza richardsonii & 3 & & 100 & & & 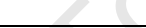 & \\
\hline \multicolumn{8}{|l|}{ Clinidae } \\
\hline Clinidae spp. & 2 & 100 & & & & 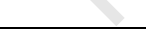 & \\
\hline \multicolumn{8}{|l|}{ Gobiidae } \\
\hline Gobiidae spp. & 3 & 100 & & & & 81 & \\
\hline Species indeterminate & 83 & 15.7 & 71.1 & 10.8 & 2.4 & + & \\
\hline Total \% & & 8.1 & 59.7 & 26.2 & 5.0 & & 0.9 \\
\hline
\end{tabular}

The low number of size estimations (eight each) on specimens from the exterior material of M1 and M2 phases precludes meaningful comparison with those from the interior of these two phases. A larger number of size estimations were made on the exterior M3 material $(n=124)$, which allows comparison of the size classes present between the interior and exterior of this phase (Figure 6.8). The majority of specimens from exterior M3 are small, followed by medium sized specimens, at slightly higher ratios than for the interior M3. The interior M3 has more large specimens relative to the exterior and includes 2 specimens from fish bigger than $1000 \mathrm{~mm}$. Tiny $(<150 \mathrm{~mm})$ specimens are somewhat better represented in the M3 interior. Overall, the differences between size class compositions of the interior and exterior material from the M3 phase are not statistically significant (K-S statistic $=0.18, \mathrm{p}<0.05)$. 


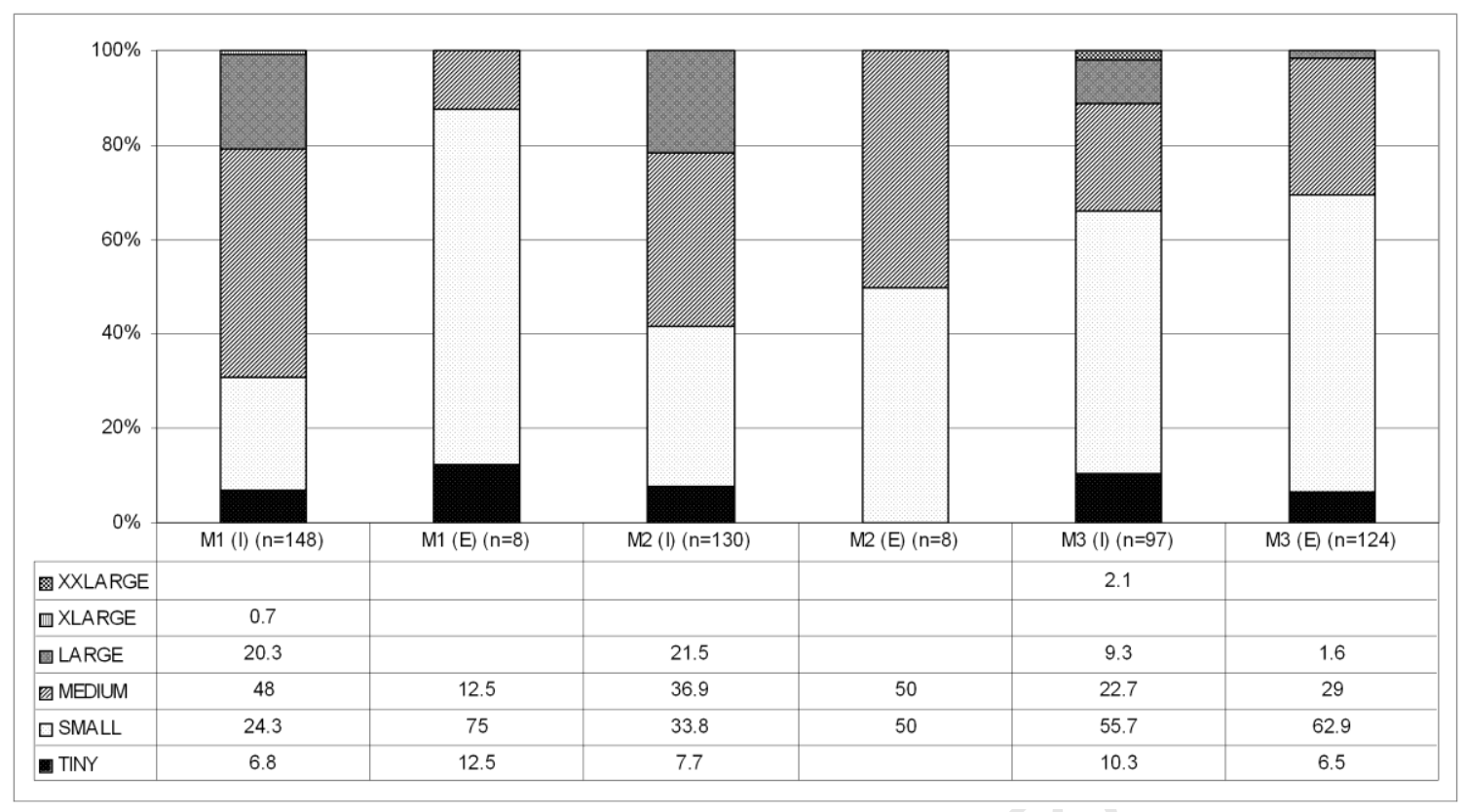

Figure 6.8. Comparison of the percentage of specimens per size class and phase of all species combined from the interior (I) and exterior (E) of the Blombos Cave Middle Stone Age assemblage. Percentages are based on the NISP of specimens that could be used for size estimations

\subsection{Klasies River main site}

\subsubsection{Species present}

A total of 12816 specimens were examined from KR main site. Of these, 5434 specimens from 17 families, at least 22 genera and 31 species were identified, 24 of which could be identified to species level (Table 6.11). The remaining 7382 (57.6\%) specimens could not be identified to family, and most of these (96\%) are unidentified vertebrae. Scales were not well preserved and only one was found. The total number of specimens (NISP) in the cultural units is 5562, and 7254 in sterile units. Vertebral elements constitute $89 \%$ of the specimens in the combined cultural units, $13 \%$ of which are from unidentified shark species, and $93 \%$ of the specimens in the sterile units are vertebral elements, of which $16 \%$ are from unidentified shark species. The MSA II member contains the largest number of specimens. Neural and haemal spines were missing from most vertebrae in both the cultural and sterile units. Note that, unlike the BBC and H/RC data, the KR data are presented as 'raw' NISP without any density calculations, for the reasons explained in Chapter Five. 
Table 6.11. Total NISP per member from Klasies River main site. $C=$ cultural units; $S=$ sterile units

\begin{tabular}{|c|c|c|c|c|c|c|c|c|c|c|c|c|c|c|c|}
\hline \multirow[b]{2}{*}{ Taxa } & \multicolumn{3}{|c|}{ MSA III } & \multicolumn{3}{|c|}{ HP } & \multicolumn{3}{|c|}{ MSA II } & \multicolumn{3}{|c|}{ MSA I } & \multirow{2}{*}{\begin{tabular}{|c|}
$\mathbf{C}$ \\
TOTAL \\
\end{tabular}} & \multirow{2}{*}{$\begin{array}{c}\mathbf{S} \\
\text { TOTAL }\end{array}$} & \multirow{2}{*}{$\begin{array}{c}\text { SITE } \\
\text { TOTAL }\end{array}$} \\
\hline & C & S & TOTAL & C & S & TOTAL & C & $\mathrm{s}$ & TOTAL & C & $\mathrm{S}$ & TOTAL & & & \\
\hline \multicolumn{16}{|l|}{ Chondrichthyes } \\
\hline Shark spp. & 97 & 357 & 454 & 233 & 241 & 473 & 154 & 258 & 412 & 152 & 189 & 341 & 635 & 1045 & 1680 \\
\hline \multicolumn{16}{|l|}{ Osteichthyes } \\
\hline \multicolumn{16}{|l|}{ Clupeidae } \\
\hline Clupeidae spp. & - & 16 & 16 & 11 & - & 11 & - & - & - & - & - & - & 11 & 16 & 27 \\
\hline \multicolumn{16}{|l|}{ Ariidae } \\
\hline Galeichthys feliceps & 198 & 336 & 534 & 98 & 88 & 186 & 128 & 141 & 269 & 31 & 44 & 75 & 455 & 609 & 1064 \\
\hline Triglidae & & & & & & & & & & & & & & & \\
\hline Triglidae spp. & - & - & - & - & - & 0 & - & 1 & 1 & 1 & 7 & 8 & 1 & 8 & 9 \\
\hline \begin{tabular}{|l|} 
Serranidae \\
\end{tabular} & & & & & & & & & & & & & & & \\
\hline Acanthistius sebastoides & - & - & - & 3 & 8 & 11 & 9 & 15 & 24 & 2 & 5 & 7 & 14 & 28 & 42 \\
\hline Epinephelus marginatus & - & - & - & - & - & - & - & - & - & 1 & - & 1 & 1 & - & 1 \\
\hline Pomatomidae & & & & & & & & & & & & & & & \\
\hline Pomatomus saltatrix & 1 & 1 & 2 & 4 & 4 & 8 & 2 & 6 & 8 & 3 & 1 & 4 & 10 & 12 & 22 \\
\hline Haemulidae & & & & & & & & & & & & & & & \\
\hline Pomadasys commersonnii & - & - & - & - & 1 & 1 & - & - & - & - & - & - & - & 1 & 1 \\
\hline Pomadasys olivaceum & - & - & - & - & - & - & - & - & - & 1 & 1 & 2 & 1 & 1 & 2 \\
\hline Sparidae & & & & & & & & & & & & 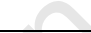 & & & \\
\hline Argyrozona argyrozona & - & - & - & 1 & - & 1 & - & - & - & - & - & - & 1 & - & 1 \\
\hline Diplodus capensis & - & - & - & - & - & - & 2 & 1 & 3 & - & - & - & 2 & 1 & 3 \\
\hline Gymnocrotaphus curvidens & - & - & - & - & 2 & 2 & 2 & 1 & 3 & - & - & - & 2 & 3 & 5 \\
\hline Lithognathus lithognathus & - & 1 & 1 & 2 & 6 & 8 & 5 & 2 & 7 & - & 2 & 2 & 7 & 11 & 18 \\
\hline Pachymetopon blochii & - & - & - & - & 1 & 1 & - & - & - & - & 1 & 1 & - & 2 & 2 \\
\hline Petrus rupestris & - & - & - & - & - & - & 2 & - & 2 & 2 & - & 2 & 4 & - & 4 \\
\hline Pterogymnus laniarius & - & - & - & - & - & - & - & 1 & 1 & - & 1 & 1 & - & 2 & 2 \\
\hline Rhabdosargus globiceps & - & - & - & - & 3 & 3 & - & 1 & 1 & - & - & - & - & 4 & 4 \\
\hline Rhabdosargus holubi & - & - & - & - & - & - & - & - & - & 1 & - & 1 & 1 & - & 1 \\
\hline Sarpa salpa & - & - & - & - & - & - & - & 7 & 7 & 7 & 3 & 10 & 7 & 10 & 17 \\
\hline Sparodon durbanensis & - & - & - & - & - & - & 2 & - & 2 & - & - & - & 2 & - & 2 \\
\hline Spondyliosoma emarginatum & - & - & - & - & - & - & - & - & - & - & 1 & 1 & - & 1 & 1 \\
\hline Sparidae spp. & 7 & 12 & 19 & 14 & 41 & 55 & 37 & 43 & 80 & 48 & 34 & 82 & 106 & 130 & 236 \\
\hline Dischistiidae & & & & & & 8 & + & & & & & & & & \\
\hline Dichistius capensis & 1 & - & 1 & 2 & 2 & 4 & 1 & 4 & 5 & 2 & 4 & 6 & 6 & 10 & 16 \\
\hline Dichistiidae spp. & - & - & - & - & - & - & - & 1 & 1 & - & - & - & - & 1 & 1 \\
\hline Sciaenidae & & & & & & 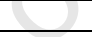 & . & & & & & & & & \\
\hline Argyrosomus inodorus/japonicus & 3 & 11 & 14 & 9 & 5 & 14 & 3 & 7 & 10 & 9 & 14 & 23 & 24 & 37 & 61 \\
\hline Sciaenidae spp. & - & - & - & 2 & - & 2 & 5 & 1 & 6 & 1 & 4 & 5 & 8 & 5 & 13 \\
\hline Carangidae & & & & 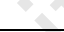 & 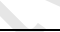 & & & & & & & & & & \\
\hline Seriola lalandi & - & - & - & - & - & - & 1 & - & 1 & - & - & - & 1 & - & 1 \\
\hline Carangidae spp. & - & - & - & 1 & - & 1 & - & 1 & 1 & 254 & 9 & 263 & 255 & 10 & 265 \\
\hline Cheilodactylidae & & & & & & & & & & & & & & & \\
\hline C. brachydactylus & - & 2 & 2 & 11 & 6 & 17 & 6 & 9 & 15 & 3 & 6 & 9 & 20 & 23 & 43 \\
\hline \begin{tabular}{|l|} 
Mugilidae \\
\end{tabular} & & & 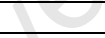 & & & & & & & & & & & & \\
\hline Liza richardsonii & 21 & 27 & 48 & 54 & 46 & 100 & 69 & 129 & 198 & 39 & 47 & 86 & 182 & 249 & 431 \\
\hline Clinidae & & 2 & & & & & & & & & & & & & \\
\hline Clinidae spp. & 20 & 22 & 42 & 123 & 239 & 362 & 169 & 329 & 498 & 155 & 217 & 372 & 467 & 807 & 1274 \\
\hline Gobiidae & 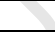 & 8 & & & & & & & & & & & & & \\
\hline Gobiidae spp. & 5 & 19 & 24 & 23 & 50 & 73 & 15 & 13 & 28 & 25 & 16 & 41 & 68 & 98 & 166 \\
\hline Scombridae & & & & & & & & & & & & & & & \\
\hline Scomber japonicus & - & - & - & - & - & - & - & - & - & 2 & 3 & 5 & 2 & 3 & 5 \\
\hline Scombridae spp. & - & - & - & - & - & - & - & 2 & 2 & - & - & - & - & 2 & 2 \\
\hline Tetraodontidae & & & & & & & & & & & & & & & \\
\hline Tetraodontidae spp. & 1 & - & 1 & - & - & 0 & - & 4 & 4 & 1 & 6 & 7 & 2 & 10 & 12 \\
\hline Species indeterminate & 370 & 738 & 1108 & 961 & 1155 & 2116 & 790 & 1430 & 2220 & 1147 & 793 & 1940 & 3267 & 4116 & 7382 \\
\hline Total NISP & 429 & 833 & 1262 & 1210 & 1569 & 2779 & 1119 & 2007 & 3126 & 1702 & 1168 & 2870 & 4460 & 5577 & 10036 \\
\hline Number of species & 9 & 10 & 6 & 14 & 10 & 18 & 17 & 16 & 15 & 19 & 19 & 23 & 25 & 25 & 31 \\
\hline
\end{tabular}

The majority of identified species are Sparidae $(n=12)$, but these occur in relatively low numbers (Table 6.11). Unidentified shark species are the most commonly occurring specimens, followed by unidentified Clinidae. These two categories make up $30.9 \%$ and $23.5 \%$ respectively of all specimens that could be identified beyond fish. The most common fish that could be identified to species in the whole assemblage is Galeichthys 
feliceps, which comprises $20 \%$ of the identified specimens, followed by Liza richardsonii $(8 \%)$. These are consistently the most common identified species in both the cultural and sterile units of all members.

In total, the sterile units contain $57 \%$ of the examined fish, and the cultural units $43 \%$. Total NISP is higher in the sterile units of all members apart from the MSA I, where more specimens occur in the cultural units. More than half of the additional specimens in the cultural units of the MSA I are scutes from unidentified Carangidae. Most of the specimens responsible for the higher NISP in the sterile units of the MSA II that could be identified to beyond fish are from unidentified shark and Clinidae. In the HP, Clinidae and Gobiidae account for most of the higher NISP in the sterile units. In the MSA III high numbers of shark and Galeichthys feliceps specimens account for almost half of the higher NISP in the sterile layers, whereas the rest are mainly from unidentified fish. The sterile units contain marginally more species than the cultural units in all members. The biggest difference in number of species between the cultural and sterile units occurs in the MSA II member which contains 16 and 20 species respectively. The number of species present decreases from the oldest to the youngest members in both the cultural and sterile units, with the lowest number of species present in the MSA III (Table 6.11).

Some differences in the species present in the cultural and sterile units are apparent. Six species are present in the cultural units only, namely Seriola lalandi, Sparodon durbanensis, Petrus rupestris, Rhabdosargus holubi, Epinephelus marginatus and Argyrozona argyrozona. The latter occurs only in the HP, whereas the others are present only in the MSA I and II assemblages. Species that are present in the sterile units only are Pachymetopon blochii, Rhabdosargus globiceps, Pterogymnus laniarius, Pomadasys commersonnii and Spondyliosoma emarginatum. All of these species, in both the cultural and sterile units, are present in very low numbers, and contribute a total NISP of 10 to each of the cultural and sterile assemblages. The rest of the species occur in both the cultural and sterile units, although not always simultaneously in the same member Specimens of Clinidae and Shark are consistently more common in the sterile units of all members. The NISP of Galeichthys feliceps, Liza richardsonii and Argyrosomus inodorus/japonicus is higher in the sterile units of all members apart from the HP, where these species are more common in the cultural units. 
The KR data are presented as NISP without correction for volumes excavated. It would therefore be inappropriate to interpret these numbers as actual indicators of fish numbers, and increases in NISP between members can be misleading. Relative changes in frequency of species within a member should give a better indication of changes in species frequency, assuming that there is not too much of a difference in fragmentation between the members. The relative changes in frequency of species that occur in the cultural and sterile units per member were compared (Table 6.12). Clinidae and shark species were the most common species in the MSA I in both the cultural and sterile units. The relative frequency of Clinidae decreased steadily from the older to younger members in the cultural units, but was highest in the sterile units of the HP. Shark species were the most common in the HP, but are fairly common in all members. The frequency of Galeichthys feliceps was highest in the MSA III (cultural and sterile units), and lowest in the MSA I. Changes in the frequencies of species per member are very similar, particularly in the MSA I and MSA II. Sharks occur more frequently in the sterile units of the MSA III, and G. feliceps in the cultural units.

The majority of species present inhabit near-shore regions, but at least two offshore species, Pterogymnus laniarius and Argyrozona argyrozona, are present in small numbers. One specimen of the former occurs in the cultural units of the HP, and one each of the latter occurs in the sterile units of the MSA II and MSA III members. 
Table 6.12. Relative frequency $(\%)$ of species in the cultural and sterile units per member from Klasies River main site based on NISP. Unidentified Sparidae, Carangidae, Scombridae and Sciaenidae have been excluded

\begin{tabular}{|c|c|c|c|c|c|c|c|c|}
\hline \multirow{3}{*}{$\begin{array}{l}\text { Taxa } \\
\text { Chondrichthyes }\end{array}$} & MSA III & HP & MSA II & MSA I & MSA III & HP & MSA II & MSA I \\
\hline & \multicolumn{4}{|c|}{ CULTURAL } & \multicolumn{4}{|c|}{ STERILE } \\
\hline & & & & & & & & \\
\hline Shark spp. & 27.8 & 40.5 & 27.0 & 34.8 & 45.1 & 5.6 & 27.7 & 33.3 \\
\hline \multicolumn{9}{|l|}{ Clupeidae } \\
\hline Clupeidae spp. & & 1.9 & & & 2.0 & & & \\
\hline \multicolumn{9}{|l|}{ Ariidae } \\
\hline Galeichthys feliceps & 57.1 & 17.1 & 22.5 & 7.1 & 42.4 & 20.0 & 15.2 & 7.7 \\
\hline \multicolumn{9}{|l|}{ Triglidae } \\
\hline Triglidae spp. & & & & 0.2 & & & 0.1 & 1.2 \\
\hline \multicolumn{9}{|l|}{ Serranidae } \\
\hline Acanthistius sebastoides & & 0.5 & 1.6 & 0.5 & & 1.8 & 1.6 & 0.9 \\
\hline Epinephelus marginatus & & & & 0.2 & & & & \\
\hline \multicolumn{9}{|l|}{ Pomatomidae } \\
\hline Pomatomus saltatrix & 0.3 & 0.7 & 0.4 & 0.7 & 0.1 & 0.9 & 0.6 & 0.2 \\
\hline \multicolumn{9}{|l|}{ Haemulidae } \\
\hline Pomadasys commersonnii & & & & & & 0.2 & & \\
\hline Pomadasys olivaceum & & & & 0.2 & & & & 0.2 \\
\hline \multicolumn{9}{|l|}{ Sparidae } \\
\hline Argyrozona argyrozona & & 0.2 & & & & & & \\
\hline Diplodus capensis & & & 0.4 & & & & 0.1 & \\
\hline Gymnocrotaphus curvidens & & & 0.4 & & & 0.5 & 0.1 & \\
\hline Lithognathus lithognathus & & 0.3 & 0.9 & & 0.1 & 1.4 & 0.2 & 0.4 \\
\hline Pachymetopon blochii & & & & & & 0.2 & 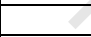 & 0.2 \\
\hline Pterogymnus laniarius & & & & & & & 0.1 & 0.2 \\
\hline Petrus rupestris & & & 0.4 & 0.5 & & & + & \\
\hline Rhabdosargus globiceps & & & & & & 0.7 & 0.1 & \\
\hline Rhabdosargus holubi & & & & 0.2 & & & 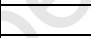 & \\
\hline Sarpa salpa & & & & 1.6 & & 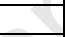 & 0.8 & 0.5 \\
\hline Sparodon durbanensis & & & 0.4 & & & + & & \\
\hline Spondyliosoma emarginatum & & & & & & 急 & & 0.2 \\
\hline \multicolumn{9}{|l|}{ Dischistiidae } \\
\hline Dichistius capensis & 0.3 & 0.3 & 0.2 & 0.5 & & 0.5 & 0.4 & 0.7 \\
\hline Dichistiidae spp. & & & & & & & 0.1 & \\
\hline \multicolumn{9}{|l|}{ Sciaenidae } \\
\hline Argyrosomus inodorus/japonicus & 0.9 & 1.6 & 0.5 & 2.1 & 1.4 & 1.1 & 0.8 & 2.5 \\
\hline \multicolumn{9}{|l|}{ Carangidae } \\
\hline Seriola lalandi & & & 0.2 & & & & & \\
\hline \multicolumn{9}{|l|}{ Cheilodactylidae } \\
\hline Chirodactylus brachydactylus & & 1.9 & 1.1 & 0.7 & 0.3 & 1.4 & 1.0 & 1.1 \\
\hline Mugilidae & & & 20 & & & & & \\
\hline Liza richardsonii & 6.1 & 9.4 & 12.0 & 8.8 & 3.4 & 10.4 & 13.9 & 8.3 \\
\hline \multicolumn{9}{|l|}{ Clinidae } \\
\hline Clinidae spp. & 5.8 & 21.4 & 29.7 & 35.5 & 2.8 & 54.3 & 35.4 & 38.2 \\
\hline \multicolumn{9}{|l|}{ Gobiidae } \\
\hline Gobiidae spp. & 1.4 & 4.0 & 2.6 & 5.7 & 2.4 & 1.1 & 1.4 & 2.8 \\
\hline Scombridae & +2 & & & & & & & \\
\hline Scomber japonicus & 8 & & & 0.5 & & & & 0.5 \\
\hline \multicolumn{9}{|l|}{ Tetraodontidae } \\
\hline Tetraodontidae spp. & 0.3 & & & 0.2 & & & 0.4 & 1.1 \\
\hline Total \% & 100 & 100 & 100 & 100 & 100 & 100 & 100 & 100 \\
\hline Total NISP & 347 & 574 & 570 & 437 & 792 & 441 & 930 & 568 \\
\hline
\end{tabular}

\subsubsection{Size reconstruction}

Size classes for the KR material from the cultural and sterile units were calculated separately per member (Figure 6.9). The majority of specimens are vertebrae, and as size estimates were not made on these except for the first, penultimate and ultimate vertebrae, the number of specimens for which size can be estimated is low. All of the groups are dominated by specimens in the small size category, followed by medium sized specimens (Figure 6.9). 


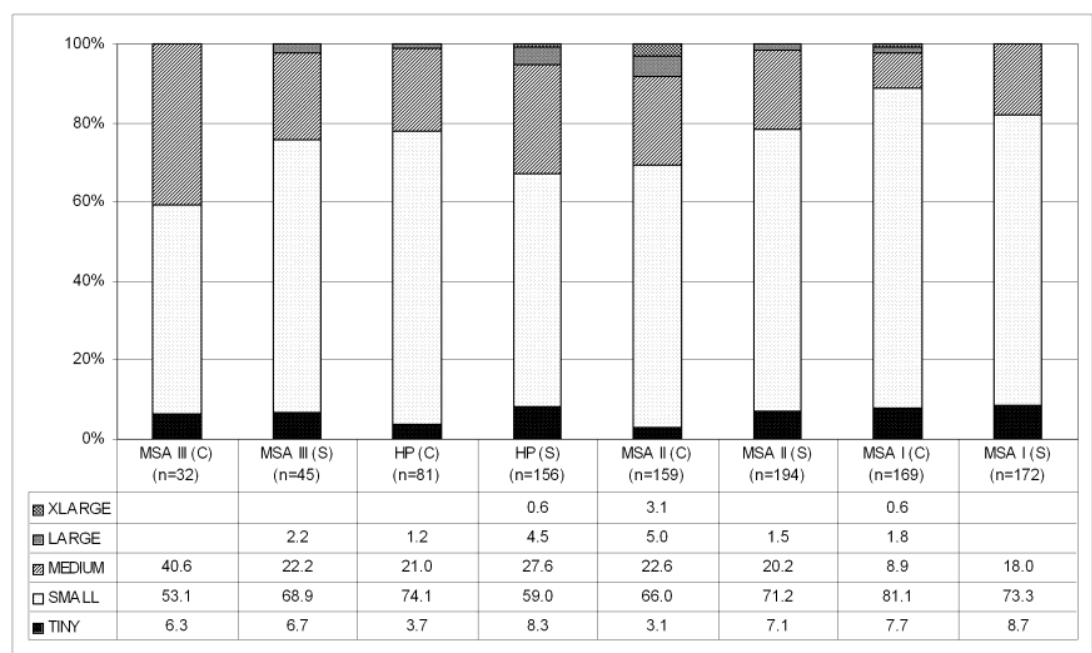

Figure 6.9. Percentage of specimens per size class of all species combined in the cultural $(C)$ and sterile (S) units per member from the Klasies River main site Middle Stone Age assemblage. Percentages are based on the NISP of elements that could be used for size estimations.

All units have tiny fish present, and the MSA I and II cultural units and HP sterile unit yield the largest number of size categories: from tiny to extra extra large (Figure 6.0,

Tables 6.12, 6.13, 6.14, and 6.15).

Table 6.13. Percentage of NISP per size class in the cultural (C) and sterile (S) units of the MSA III member of Klasies River main site. $n=$ NISP of elements that could be used for size estimations

\begin{tabular}{|c|c|c|c|c|c|c|c|c|c|c|}
\hline \multirow{2}{*}{\multicolumn{3}{|c|}{$\begin{array}{l}\text { SIZE } \\
\text { Total: Cultural }(n=32) \text { Sterile }(n=45)\end{array}$}} & \multirow{2}{*}{\multicolumn{2}{|c|}{$\begin{array}{c}\text { TINY } \\
0-150 \mathrm{~mm}\end{array}$}} & \multirow{2}{*}{\multicolumn{2}{|c|}{$\begin{array}{c}\text { SMALL } \\
151-300 \mathrm{~mm}\end{array}$}} & \multirow{2}{*}{\multicolumn{2}{|c|}{$\begin{array}{c}\text { MEDIUM } \\
301-500 \mathrm{~mm}\end{array}$}} & \multirow{2}{*}{\multicolumn{2}{|c|}{$\begin{array}{c}\text { LARGE } \\
501-800 \mathrm{~mm}\end{array}$}} \\
\hline & & & & & & & & & & \\
\hline Taxa & $\mathrm{n}(\mathrm{C})$ & $\mathbf{n}(\mathbf{S})$ & $\begin{array}{l}\text { MSA III } \\
\text { (C) }\end{array}$ & $\begin{array}{l}\text { MSA III } \\
\text { (S) }\end{array}$ & $\begin{array}{l}\text { MSA III } \\
\text { (C) }\end{array}$ & $\begin{array}{l}\text { MSA III } \\
\text { (S) }\end{array}$ & $\begin{array}{l}\text { MSA III } \\
\text { (C) }\end{array}$ & $\begin{array}{l}\text { MSA III } \\
\text { (S) }\end{array}$ & $\begin{array}{l}\text { MSA III } \\
\text { (C) }\end{array}$ & $\begin{array}{c}\text { MSA III } \\
\text { (S) }\end{array}$ \\
\hline Osteichthyes & & & 2 & & & & & & & \\
\hline Clupeidae & & 8 & 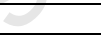 & & & & & & & \\
\hline Clupeidae spp. & & 6 & - & - & - & 100.0 & - & - & - & - \\
\hline Ariidae & 1 & 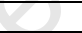 & & & & & & & & \\
\hline Galeichthys feliceps & 4 & 9 & - & - & 50.0 & 44.4 & 50.0 & 55.6 & - & - \\
\hline Pomatomidae & \begin{tabular}{|l|l|} 
\\
\end{tabular} & 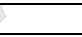 & & & & & & & & \\
\hline Pomatomus saltatrix & 1 & 1 & - & - & 100.0 & - & - & 100.0 & - & - \\
\hline Sparidae & & & & & & & & & & \\
\hline Lithognathus lithognathus & 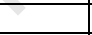 & 1 & - & - & - & 100.0 & - & - & - & - \\
\hline Sparidae spp. & 6 & 9 & - & - & 33.3 & 88.9 & 66.7 & 11.1 & - & - \\
\hline Dischistiidae & & & & & & & & & & \\
\hline Dichistius capensis & 1 & & - & - & - & - & 100.0 & - & - & - \\
\hline Sciaenidae & & & & & & & & & & \\
\hline $\begin{array}{l}\text { Argyrosomus } \\
\text { inodorus/japonicus }\end{array}$ & 1 & 4 & & & 100.0 & 75.0 & - & 25.0 & - & - \\
\hline Mugilidae & & & & & & & & & & \\
\hline L. richardsonii & 2 & & - & - & 50.0 & - & 50.0 & - & - & - \\
\hline Clinidae & & & & & & & & & & \\
\hline Clinidae spp. & 2 & 2 & 50.0 & - & 50.0 & 100.0 & - & - & - & - \\
\hline Gobiidae & & & & & & & & & & \\
\hline Gobiidae spp. & 2 & 5 & - & 60.0 & 100.0 & 40.0 & - & - & - & - \\
\hline Tetraodontidae & & & & & & & & & & \\
\hline Tetraodontidae spp. & 1 & & - & - & 100.0 & - & - & - & - & - \\
\hline Species indeterminate & 12 & 8 & 8.3 & - & 50.0 & 62.5 & 41.7 & 25.0 & - & 12.5 \\
\hline Total $\%$ & & & 6.3 & 6.7 & 53.1 & 68.9 & 40.6 & 22.2 & - & 2.2 \\
\hline
\end{tabular}


Table 6.14. Percentage of NISP per size class in the cultural (C) and sterile (S) units of the HP member of Klasies River main site. $n=$ NISP of elements that could be used for size estimations

\begin{tabular}{|c|c|c|c|c|c|c|c|c|c|c|c|c|}
\hline \multirow{2}{*}{\multicolumn{3}{|c|}{$\begin{array}{l}\text { SIZE } \\
\text { Total: Cultural }(n=81) \text { Sterile }(n=156)\end{array}$}} & \multirow{2}{*}{\multicolumn{2}{|c|}{$\begin{array}{c}\text { TINY } \\
0-150 \mathrm{~mm}\end{array}$}} & \multicolumn{2}{|c|}{ SMALL } & \multicolumn{2}{|c|}{ MEDIUM } & \multicolumn{2}{|c|}{ LARGE } & \multicolumn{2}{|c|}{ XLARGE } \\
\hline & & & & & \multicolumn{2}{|c|}{$151-300 \mathrm{~mm}$} & \multicolumn{2}{|c|}{$301-500 \mathrm{~mm}$} & \multicolumn{2}{|c|}{$501-800 \mathrm{~mm}$} & \multicolumn{2}{|c|}{$801-1000 \mathrm{~mm}$} \\
\hline Taxa & $n(C)$ & $n(S)$ & HP (C) & HP (S) & HP (C) & HP (S) & $\mathrm{HP}(\mathrm{C})$ & HP (S) & HP (C) & HP (S) & $\mathrm{HP}(\mathrm{C})$ & HP (S) \\
\hline \multicolumn{13}{|c|}{ 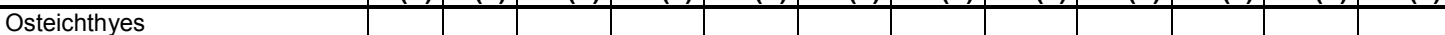 } \\
\hline \multicolumn{13}{|l|}{ Clupeidae } \\
\hline Clupeidae spp. & 1 & & - & - & 100.0 & - & - & - & - & - & - & - \\
\hline \multicolumn{13}{|l|}{ Ariidae } \\
\hline Galeichthys feliceps & 3 & 9 & - & - & 100.0 & 66.7 & - & 33.3 & - & - & - & - \\
\hline \multicolumn{13}{|l|}{ Serranidae } \\
\hline Acanthistius sebastoides & 4 & 3 & 25.0 & - & 75.0 & 100.0 & - & - & - & - & - & - \\
\hline \multicolumn{13}{|l|}{ Pomatomidae } \\
\hline Pomatomus saltatrix & 3 & 3 & - & - & 66.7 & 66.7 & - & 33.3 & 33.3 & - & - & - \\
\hline \multicolumn{13}{|l|}{ Haemulidae } \\
\hline Pomadasys commersonnii & & 1 & - & - & - & - & - & 100.0 & - & - & - & - \\
\hline Sparidae & & & & & & & & & & 2 & & \\
\hline Argyrozona argyrozona & 1 & & - & - & 100.0 & - & - & - & - & - & - & - \\
\hline Gymnocrotaphus curvidens & & 2 & - & - & - & 100.0 & - & - & - & - & - & - \\
\hline Pachymetopon blochii & & 1 & - & - & - & - & - & 100.0 & - & - & - & - \\
\hline Lithognathus lithognathus & 2 & 6 & - & - & 50.0 & 50.0 & 50.0 & 33.3 & - & - & - & 16.7 \\
\hline Rhabdosargus globiceps & & 3 & - & - & - & 100.0 & - & - & - & - & - & - \\
\hline Sparidae spp. & 10 & 40 & - & 2.5 & 60.0 & 47.5 & 40.0 & 42.5 & - & 7.5 & - & - \\
\hline \multicolumn{13}{|l|}{ Dischistiidae } \\
\hline Dichistius capensis & 2 & 2 & - & - & - & 50.0 & 100.0 & - & - & 50.0 & - & - \\
\hline \multicolumn{13}{|l|}{ Sciaenidae } \\
\hline Argyrosomus inodorus/japonicus & 2 & 5 & - & - & - & 20.0 & 100.0 & 40.0 & - & 40.0 & - & - \\
\hline Sciaenidae spp. & 2 & & - & - & 50.0 & - & 50.0 & - & - & - & - & - \\
\hline \multicolumn{13}{|l|}{ Cheilodactylidae } \\
\hline Chirodactylus brachydactylus & 6 & 6 & - & - & 100.0 & 100.0 & - & - & - & - & - & - \\
\hline \multicolumn{13}{|l|}{ Mugilidae } \\
\hline Liza richardsonii & 7 & 12 & - & - & 100.0 & 91.7 & - & 8.3 & - & - & - & - \\
\hline \multicolumn{13}{|l|}{ Clinidae } \\
\hline Clinidae spp. & 7 & 11 & - & 18.2 & 100.0 & 81.8 & - & - & - & - & - & - \\
\hline \multicolumn{13}{|l|}{ Gobiidae } \\
\hline Gobiidae spp. & 4 & 16 & 50.0 & 50.0 & 50.0 & 50.0 & - & - & - & - & - & - \\
\hline Tetraodontidae & & & & 20 & & & & & & & & \\
\hline Tetraodontidae spp. & 1 & & - & - & 100.0 & - & - & - & - & - & - & - \\
\hline Species indeterminate & 26 & 36 & - & 5.6 & 73.1 & 50.0 & 26.9 & 41.7 & - & 2.8 & - & - \\
\hline Total \% & & & 3.7 & 8.3 & 74.1 & 59.0 & 21.0 & 27.6 & 1.2 & 4.5 & - & 0.6 \\
\hline
\end{tabular}


Table 6.15. Percentage of NISP per size class in the cultural (C) and sterile (S) units of the MSA II member of Klasies River main site. $n=$ NISP of elements that could be used for size estimations

\begin{tabular}{|c|c|c|c|c|c|c|c|c|c|c|c|c|}
\hline \multirow{2}{*}{\multicolumn{3}{|c|}{$\begin{array}{l}\text { SIZE } \\
\text { Total: Cultural }(n=159) \text { Sterile }(n=194)\end{array}$}} & \multirow{2}{*}{\multicolumn{2}{|c|}{$\begin{array}{c}\text { TINY } \\
0-150 \mathrm{~mm}\end{array}$}} & \multirow{2}{*}{\multicolumn{2}{|c|}{$\begin{array}{c}\text { SMALL } \\
151-300 \mathrm{~mm}\end{array}$}} & \multirow{2}{*}{\multicolumn{2}{|c|}{$\begin{array}{c}\text { MEDIUM } \\
301-500 \mathrm{~mm}\end{array}$}} & \multirow{2}{*}{\multicolumn{2}{|c|}{$\begin{array}{c}\text { LARGE } \\
501-800 \mathrm{~mm}\end{array}$}} & \multirow{2}{*}{\multicolumn{2}{|c|}{$\begin{array}{c}\text { XLARGE } \\
801-1000 \mathrm{~mm}\end{array}$}} \\
\hline & & & & & & & & & & & & \\
\hline ( & $\mathrm{n}(\mathbf{C})$ & $\mathbf{n}(\mathbf{S})$ & $\begin{array}{c}\text { MSA II } \\
\text { (C) }\end{array}$ & $\begin{array}{c}\text { MSA II } \\
\text { (S) }\end{array}$ & $\begin{array}{c}\text { MSA II } \\
\text { (C) }\end{array}$ & $\begin{array}{c}\text { MSA II } \\
\text { (S) }\end{array}$ & $\begin{array}{c}\text { MSA II } \\
\text { (C) }\end{array}$ & $\begin{array}{c}\text { MSA II } \\
(\mathrm{S})\end{array}$ & $\begin{array}{c}\text { MSA II } \\
\text { (C) }\end{array}$ & $\begin{array}{c}\text { MSA II } \\
\text { (S) }\end{array}$ & $\begin{array}{c}\text { MSA II } \\
\text { (C) }\end{array}$ & $\begin{array}{l}\text { MSA II } \\
\text { (S) }\end{array}$ \\
\hline \multicolumn{13}{|l|}{ Osteichthyes } \\
\hline \multicolumn{13}{|l|}{ Ariidae } \\
\hline Galeichthys feliceps & 33 & 14 & - & - & 72.7 & 35.7 & 27.3 & 64.3 & - & - & - & - \\
\hline \multicolumn{13}{|l|}{ Serranidae } \\
\hline Acanthistius sebastoides & 9 & 7 & - & - & 100.0 & 100.0 & - & - & - & - & - & - \\
\hline \multicolumn{13}{|l|}{ Pomatomidae } \\
\hline Pomatomus saltatrix & 1 & & - & - & - & - & 100.0 & - & - & - & - & - \\
\hline \multicolumn{13}{|l|}{ Sparidae } \\
\hline Diplodus capensis & 2 & 1 & - & - & 100.0 & 100.0 & - & - & - & - & - & - \\
\hline Gymnocrotaphus curvidens & 2 & 1 & - & - & - & - & 100.0 & 100.0 & - & - & - & - \\
\hline Lithognathus lithognathus & 5 & 2 & - & 50.0 & 20.0 & 50.0 & 20.0 & - & 20.0 & - & 40.0 & - \\
\hline Petrus rupestris & 2 & & - & - & - & - & - & - & - & - & 100.0 & - \\
\hline Pterogymnus laniarius & & 1 & - & - & - & 100.0 & - & - & - & - & - & - \\
\hline Rhabdosargus globiceps & & 1 & - & - & - & 100.0 & - & - & - & - & - & - \\
\hline Sarpa salpa & & 7 & - & 14.3 & - & 85.7 & - & - & - & - & - & - \\
\hline Sparodon durbanensis & 2 & & - & - & - & - & - & - & 100.0 & - & - & - \\
\hline Sparidae spp. & 30 & 38 & 3.3 & - & 66.7 & 71.1 & 23.3 & 23.7 & 6.7 & 5.3 & - & - \\
\hline \multicolumn{13}{|l|}{ Dischistiidae } \\
\hline Dichistius capensis & 1 & 4 & - & - & - & - & 100.0 & 75.0 & - & 25.0 & - & - \\
\hline \multicolumn{13}{|l|}{ Sciaenidae } \\
\hline Argyrosomus inodorus/japonicus & 4 & & - & - & 25.0 & - & 50.0 & - & 25.0 & - & - & - \\
\hline Sciaenidae spp. & 5 & 1 & - & - & - & 100.0 & 80.0 & $-\quad$ & 20.0 & - & - & - \\
\hline \multicolumn{13}{|l|}{ Carangidae } \\
\hline Seriola lalandi & 1 & & - & - & - & - & - & - & - & - & 100.0 & - \\
\hline \multicolumn{13}{|l|}{ Cheilodactylidae } \\
\hline Chirodactylus brachydactylus & 6 & 7 & 16.7 & - & 83.3 & 85.7 & - & 14.3 & - & - & - & - \\
\hline \multicolumn{13}{|l|}{ Mugilidae } \\
\hline Liza richardsonii & 7 & 22 & - & - & 100.0 & 95.5 & - & 4.5 & - & - & - & - \\
\hline \multicolumn{13}{|l|}{ Clinidae } \\
\hline Clinidae spp. & 12 & 29 & 8.3 & 20.7 & 91.7 & 79.3 & - & - & - & - & - & - \\
\hline \multicolumn{13}{|l|}{ Gobiidae } \\
\hline Gobiidae spp. & 4 & 2 & 25.0 & 100.0 & 50.0 & - & 25.0 & - & - & - & - & - \\
\hline Tetraodontidae & & & & & 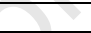 & & & & & & & \\
\hline Tetraodontidae spp. & & 3 & - & 33.3 & - & 66.7 & - & - & - & - & - & - \\
\hline Species indeterminate & 33 & 54 & 3.0 & 5.6 & 69.7 & 70.4 & 24.2 & 24.1 & 3.0 & - & - & - \\
\hline Total $\%$ & & & 3.1 & 7.2 & 66.0 & 72.2 & 22.6 & 19.1 & 5.0 & 1.5 & 3.1 & - \\
\hline
\end{tabular}


Table 6.16. Percentage of NISP per size class in the cultural (C) and sterile (S) units of the MSA I member of Klasies River main site. $n=$ NISP of elements that could be used for size estimations

\begin{tabular}{|c|c|c|c|c|c|c|c|c|c|c|c|c|}
\hline \multirow{2}{*}{\multicolumn{3}{|c|}{$\begin{array}{l}\text { SIZE } \\
\text { Total: Cultural }(n=169) \text { Sterile }(n=172)\end{array}$}} & \multirow{2}{*}{\multicolumn{2}{|c|}{$\begin{array}{c}\text { TINY } \\
0-150 \mathrm{~mm}\end{array}$}} & \multirow{2}{*}{\multicolumn{2}{|c|}{\begin{tabular}{c|} 
SMALL \\
$151-300 \mathrm{~mm}$
\end{tabular}}} & \multirow{2}{*}{\multicolumn{2}{|c|}{$\begin{array}{c}\text { MEDIUM } \\
301-500 \mathrm{~mm}\end{array}$}} & \multirow{2}{*}{\multicolumn{2}{|c|}{$\begin{array}{c}\text { LARGE } \\
501-800 \mathrm{~mm} \\
\end{array}$}} & \multirow{2}{*}{\multicolumn{2}{|c|}{$\begin{array}{c}\text { XLARGE } \\
801-1000 \mathrm{~mm}\end{array}$}} \\
\hline & & & & & & & & & & & & \\
\hline Taxa & $\mathrm{n}(\mathrm{C})$ & $\mathrm{n}(\mathrm{S})$ & $\begin{array}{c}\text { MSA I } \\
\text { (C) }\end{array}$ & $\begin{array}{c}\text { MSA I } \\
\text { (S) }\end{array}$ & $\begin{array}{l}\text { MSA I } \\
\text { (C) }\end{array}$ & $\begin{array}{c}\text { MSA I } \\
\text { (S) }\end{array}$ & $\begin{array}{c}\text { MSA I } \\
\text { (C) }\end{array}$ & $\begin{array}{l}\text { MSA I } \\
\text { (S) }\end{array}$ & $\begin{array}{c}\text { MSA I } \\
\text { (C) }\end{array}$ & $\begin{array}{l}\text { MSA I } \\
\text { (S) }\end{array}$ & $\begin{array}{l}\text { MSA I } \\
\text { (C) }\end{array}$ & $\begin{array}{l}\text { MSA I } \\
\text { (S) }\end{array}$ \\
\hline \multicolumn{13}{|l|}{ Osteichthyes } \\
\hline \multicolumn{13}{|l|}{ Ariidae } \\
\hline Galeichthys feliceps & 3 & 4 & - & - & 66.7 & 75.0 & 33.3 & 25.0 & - & - & - & - \\
\hline \multicolumn{13}{|l|}{ Serranidae } \\
\hline Acanthistius sebastoides & 2 & 2 & - & - & 100.0 & 100.0 & - & - & - & - & - & - \\
\hline Epinephelus marginatus & 1 & & - & - & - & - & 100.0 & - & - & - & - & - \\
\hline \multicolumn{13}{|l|}{ Pomatomidae } \\
\hline Pomatomus saltatrix & 1 & & - & - & - & - & 100.0 & - & - & - & - & - \\
\hline \multicolumn{13}{|l|}{ Haemulidae } \\
\hline Pomadasys olivaceum & 1 & 1 & - & - & 100.0 & 100.0 & - & - & - & - & - & - \\
\hline \multicolumn{13}{|l|}{ Sparidae } \\
\hline Lithognathus lithognathus & & 2 & - & - & - & - & - & 100.0 & - & - & - & - \\
\hline Pachymetopon blochii & & 1 & - & - & - & - & - & 100.0 & - & - & - & - \\
\hline Petrus rupestris & 2 & & - & - & - & - & 50.0 & - & - & - & 50.0 & - \\
\hline Pterogymnus laniarius & & 1 & - & - & - & 100.0 & - & - & - & - & - & - \\
\hline Rhabdosargus holubi & 1 & & - & - & 100.0 & - & - & - & - & - & - & - \\
\hline Sarpa salpa & 7 & 3 & - & - & 100.0 & 33.3 & - & 66.7 & - & - & - & - \\
\hline Spondyliosoma emarginatum & & 1 & - & - & - & 100.0 & - & - & - & - & - & - \\
\hline Sparidae spp. & 40 & 34 & - & 8.8 & 87.5 & 70.6 & 12.5 & 20.6 & - & - & - & - \\
\hline \multicolumn{13}{|l|}{ Dischistiidae } \\
\hline Dischistius capensis & 2 & 4 & - & - & 50.0 & - & 50.0 & 100.0 & - & - & - & - \\
\hline \multicolumn{13}{|l|}{ Sciaenidae } \\
\hline Argyrosomus inodorus/japonicus & 3 & 8 & - & - & 33.3 & 50.0 & - & 50.0 & 66.7 & - & - & - \\
\hline Sciaenidae spp. & 1 & 4 & - & - & - & 75.0 & - & 25.0 & 100.0 & - & - & - \\
\hline Cheilodactylidae & & & & & & & - & 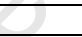 & & & & \\
\hline Chirodactylus brachydactylus & 2 & 4 & - & - & 100.0 & 100.0 & - & - & - & - & - & - \\
\hline \multicolumn{13}{|l|}{ Mugilidae } \\
\hline Liza richardsonii & 4 & 16 & - & - & 100.0 & 93.8 & -8 & 6.3 & - & - & - & - \\
\hline \multicolumn{13}{|l|}{ Clinidae } \\
\hline Clinidae spp. & 20 & 34 & 35.0 & 14.7 & 65.0 & 82.4 & - & 2.9 & - & - & - & - \\
\hline \multicolumn{13}{|l|}{ Gobiidae } \\
\hline Gobiidae spp. & 6 & 11 & 16.7 & 27.3 & 83.3 & 72.7 & - & - & - & - & - & - \\
\hline \multicolumn{13}{|l|}{ Scombridae } \\
\hline Scomber japonicus & 1 & 2 & - & - & 100.0 & 100.0 & - & - & - & - & - & - \\
\hline \multicolumn{13}{|l|}{ Tetraodontidae } \\
\hline Tetraodontidae spp. & 1 & 6 & - & 33.3 & 100.0 & 66.7 & - & - & - & - & - & - \\
\hline Species indeterminate & 71 & 34 & 7.0 & 5.9 & 85.9 & 73.5 & 7.0 & 20.6 & - & - & - & - \\
\hline Total \% & & & 7.7 & 8.7 & 81.1 & 73.3 & 8.9 & 18.0 & 1.8 & - & 0.6 & - \\
\hline
\end{tabular}

There is no clear patterning in terms of size overall between cultural and sterile units. In the MSA III and II members there are more small specimens in the sterile units relative to cultural, whereas in the MSA I and HP members small specimens are more common in the cultural units. These differences are not very large, and the differences between sizes in the cultural and sterile units are not statistically significant in any of the members (Table 6.17). Note that the sample size of the MSA III cultural units consists of only 32 specimens, which is lower than the ideal minimum of 40 when comparing samples using the Kolmogorov-Smirnov test. 
Table 6.17. Kolmogorov-Smirnov statistics from comparison of size classes between cultural and sterile units per member from Klasies River main site. No differences are statistically significant $(\mathbf{p}<\mathbf{0 . 0 5})$

\begin{tabular}{|l|r|r|r|r|}
\hline Member & MSA III C & HP C & MSA II C & MSA I C \\
\hline MSA III S & 0.16 & & & \\
\hline HP S & & 0.10 & & \\
\hline MSA II S & & & 0.10 & \\
\hline MSA I S & & & & 0.07 \\
\hline
\end{tabular}

No major differences in size of individual species between the cultural and sterile layers were noted. There are a few more medium sized specimens of Liza richardsonii in the sterile layers of the MSA I, II and HP members, whereas those in the cultural layers are all small sized. The numbers of individual species are too low to infer statistically significant differences in size between the cultural and sterile units.

Some difference in size was noted between the species that only occur in either the cultural or sterile units. Those that occur only in the cultural units are generally larger than those found only in the sterile units. Six of the specimens in the sterile units are from small fish, and the other four are from medium sized fish. In the cultural units, there are two specimens each from small, medium and large fish, and four from extra large fish. When the data for the cultural and sterile units are combined, some patterning in size change becomes apparent (Figure 6.10). There is a gradual decrease in smaller and increase in medium sized fish from the oldest to youngest members. The MSA II and HP members contain the most fish that are bigger than medium sized. The size differences between the MSA I and the MSA II and HP as seen in Figure 6.10 are statistically significant (K-S statistic $=0.11$ and $0.14, \mathrm{p}<0.05$, respectively). The fish sizes in the MSA III member are not statistically different to those of the other members (Table 6.18).

Table 6.18. Kolmogorov-Smirnov statistics from comparison of size classes of cultural and sterile units combined per member from Klasies River main site. * denotes that the differences are statistically significant $(\mathbf{P}<0.05)$

\begin{tabular}{|l|r|r|r|l|}
\hline & \multicolumn{1}{|l|}{ MSA I } & \multicolumn{1}{|l|}{ MSA II } & HP & MSA III \\
\hline MSA III & 0.17 & 0.06 & 0.02 & \\
\hline HP & ${ }^{*} 0.14$ & 0.04 & & \\
\hline MSA II & ${ }^{*} 0.11$ & & & \\
\hline MSA I & & & & \\
\hline
\end{tabular}




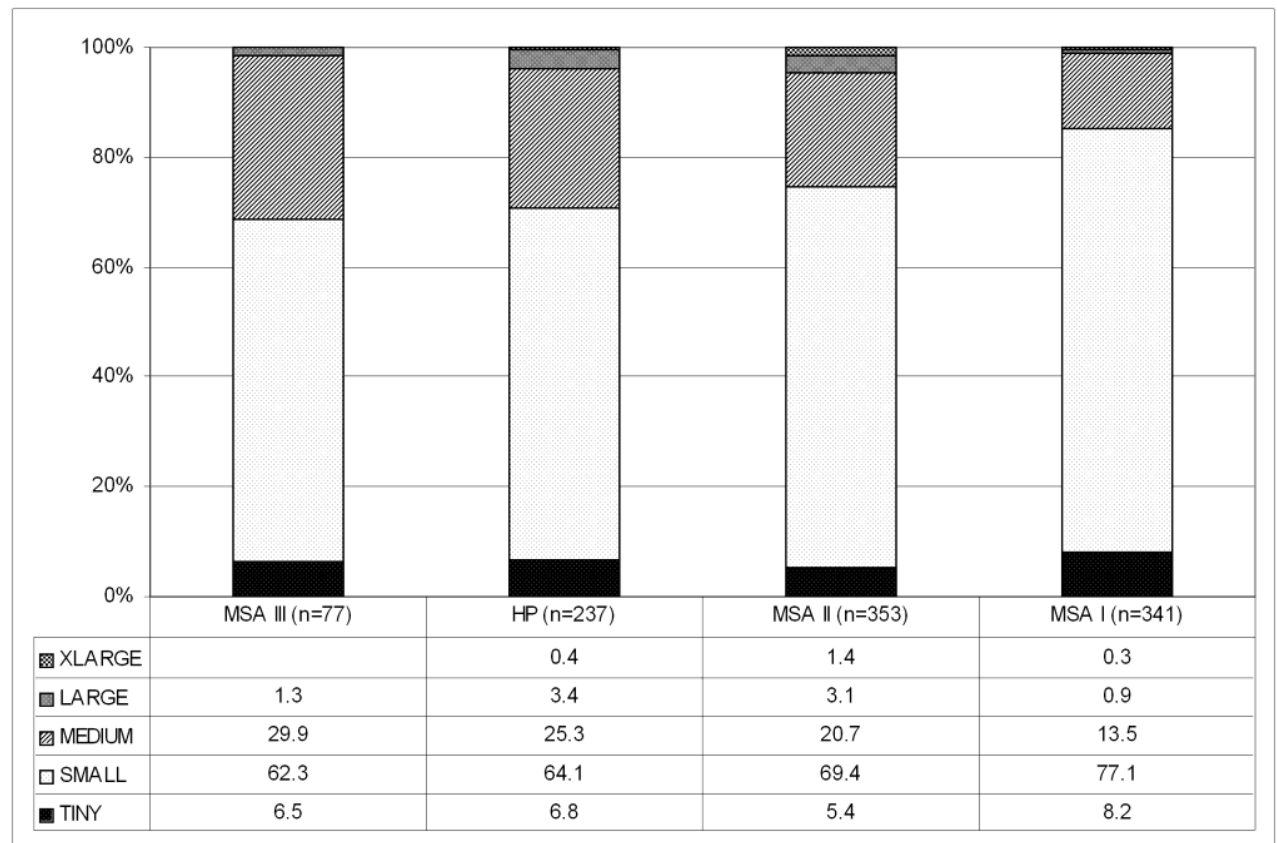

Figure 6.10. Size categories of all Klasies River Middle Stone Age fish species combined per member based on NISP. Size data from the cultural and sterile layers per member have been combined

\subsection{Modified fish bone}

\subsubsection{Culturally modified fish bone}

All specimens were examined for evidence of cultural modification in the form of cut marks, polish or intentional modification. All burnt specimens were recorded.

Examination of fracture patterns on elements can be informative in terms of pre and postdepositional processes affecting the assemblage. Bones that were broken while fresh (green) can be recognised by green-bone fractures, which are characterised by smooth fracture surfaces at acute or obtuse angles to the cortical surface (Outram 2001). Fracture patterns on dry, more brittle bones show different patterns of breakage. Unfortunately I did not have sufficient experience in recognizing bone fracture patterning on fish bone to record these attributes. Other cultural bone modifications such as chop and scrape marks, bone flakes, flake scars and percussion pitting, often recorded on long bone shaft fragments and associated with marrow removal, and gouge marks, associated with disarticulation (Fisher 1995), are not usually associated with fish remains, as fish bones do not contain marrow or other substances worth extracting. As with fracture patterns, I lacked experience in identifying these kinds of modifications, and none were recorded. Chewing of bones typically results in a crushed or distorted appearance, and is most often 
recorded on vertebrae (Nicholson 1993b; Wheeler and Jones 1989). Other digestive processes can also cause deformation of elements (Butler and Schroeder 1998). Some deformation of elements was noted during analysis, but these attributes were not recorded for this study.

It was not feasible to compare the frequencies of vertebrae versus cranial elements to determine whether any form of processing that would separate the head and body such as drying was practiced. The assemblages contained species other than Sparidae and Mugilidae that have more diverse numbers of vertebrae present and, as most vertebrae were not identified to species, calculations of deficit or surplus based on an average number of vertebrae would be inappropriate.

\subsubsection{Burning}

Experimental studies of burn patterning relative to element location on different species in various conditions (fleshed/filleted etc.) can be very informative on how fish was handled or prepared as well as inform on element survival (Nicholson 1993a, 1995; Steffen and Mackie 2005). Defleshed bones are usually calcined when exposed to direct heat, and charred when located between 1 and $15 \mathrm{~cm}$ below a hearthfire (Asmussen 2009). No burning experiments were conducted for this study, and currently no data on burn patterning are available for southern African species. Further investigation of burn patterning from the detailed data is a subject for future research.

No burnt specimens were recorded in the BBF1 assemblage. In the BBF6 assemblage three specimens were burnt, two from unidentified Sparidae and one Diplodus capensis specimen. Only six of the BBC LSA specimens showed evidence of burning, and these all occur in layer four. Four specimens are from unidentified Sparidae, and two are from Liza richardsonii. Burnt vertebrae were not recorded in the BBC LSA assemblage.

The H/RC assemblage contains several burnt specimens from a variety of species and elements including 3 that are calcined (Table 6.19). Burnt vertebrae were not recorded for this study due to their sheer quantity and because this was not the focus of the study. The data presented here is from all other elements apart from vertebrae. Five species with recorded burnt elements occur in both the Zostera and shelly layers. An additional two species in the Zostera layers show evidence of burning (Diplodus capensis and Spondyliosoma emarginatum). There are more burnt specimens in the Zostera than shelly 
layers $-5.2 \%$ of all non-vertebral specimens are burnt compared to $0.5 \%$ of the shelly material Table 6.19.

Table 6.19. Burnt (charred and calcined) specimens (NISP) from Hoffman's/Robberg Cave in the Zostera and shelly layers respectively. Note that burnt vertebrae were not quantified and are excluded from this table

\begin{tabular}{|l|l|l|c|c|}
\hline & SPECIES & BURNING & ZOSTERA & SHELLY \\
\hline Osteichthyes & & & & \\
\hline Sparidae & Diplodus capensis & charred & 4 & - \\
\hline & Pachymetopon blochii & charred & - & 1 \\
\hline & Sarpa salpa & charred & 5 & 1 \\
\hline & Sarpa salpa & calcined & - & 1 \\
\hline & Spondyliosoma emarginatum & charred & 1 & - \\
\hline & Sparidae spp. & charred & 27 & 18 \\
\hline & Dichistius capensis & charred & 11 & 2 \\
\hline Carangidae & Dichistius capensis & calcined & 1 & - \\
\hline Mugilidae & Seriola lalandi & charred & 3 & 1 \\
\hline & Liza richardsonii & charred & 10 & 4 \\
\hline \multicolumn{2}{l}{ Species indeterminate } & charred & 21 & 13 \\
\hline Total NISP & Species indeterminate & 1 & - \\
\hline Total \% of all specined & 84 & 41 \\
\hline Total \% excluding vertebrae & & 2.3 & 0.3 \\
\hline
\end{tabular}

In the BBC MSA assemblage a low number (NISP 27, 3.1\% of total) of specimens show traces of burning (Table 6.20). Only one specimen from the exterior trench is burnt, a single Galeichthys feliceps vertebra. All other burnt elements are from inside the cave, most from unidentified species and some from Sparidae that could not be identified to species. Two specimens in the M2 are calcined while all others are burnt black. All of the burnt spines from unidentified species (in all phases) are burnt only at the distal ends. This constitutes $20 \%$ of the total number of unidentified spines. The burn pattern on the spines suggests that the fish were roasted whole, with the proximal ends of the spines still embedded in the flesh and thus protected from direct heat. None of the G. feliceps spines were burnt. 
Table 6.20. Number (NISP) of burnt bone and spines per Middle Stone Age phase at Blombos Cave. Unburnt spines have been included in this table for comparison and are shaded in grey

\begin{tabular}{|c|c|c|c|c|c|c|c|}
\hline PHASE & FAMILY & SPECIES & \begin{tabular}{|l|} 
ELEMENT \\
\end{tabular} & BURNING & NISP & $\%$ Burnt spines & $\%$ Burnt total \\
\hline \multirow{9}{*}{ M1 } & Ariidae & Galeichthys feliceps & Spine & none & 1 & - & - \\
\hline & Sparidae & Sparidae spp. & Nasal & charred & 1 & - & - \\
\hline & Sparidae & Sparidae spp. & Tooth & charred & 2 & - & - \\
\hline & Sparidae & Sparidae spp. & Vertebra & charred & 1 & - & - \\
\hline & & Species indeterminate & Coracoid & charred & 1 & - & - \\
\hline & & Species indeterminate & Spine & charred & 5 & 26.3 & - \\
\hline & & Species indeterminate & Spine & none & 14 & - & - \\
\hline & & Species indeterminate & Vertebra & charred & 3 & - & - \\
\hline & & \multicolumn{3}{|l|}{ Total burnt } & 13 & - & 3.9 \\
\hline \multirow{6}{*}{ M2 } & Ariidae & Galeichthys feliceps & Spine & none & 1 & - & - \\
\hline & & Species indeterminate & Spine & charred & 3 & 17.6 & - \\
\hline & & Species indeterminate & Spine & none & 14 & - & - \\
\hline & & Species indeterminate & Vertebra & calcined & 2 & - & - \\
\hline & & Species indeterminate & Vertebra & charred & 4 & - & - \\
\hline & & \multicolumn{3}{|l|}{ Total burnt } & 9 & - & 3.5 \\
\hline \multirow{5}{*}{ M3 } & Ariidae & Galeichthys feliceps & spine & none & 1 & - & - \\
\hline & Sparidae & Sparidae & Tooth & charred & 1 & - & - \\
\hline & & Species indeterminate & Spine & charred & 2 & 22.2 & - \\
\hline & & Species indeterminate & Spine & none & 7 & - & - \\
\hline & & Species indeterminate & Vertebra & charred & 1 & - & - \\
\hline \multirow{3}{*}{ M3 exterior } & Ariidae & Galeichthys feliceps & Spine & none & 5 & - & - \\
\hline & Ariidae & Galeichthys feliceps & Vertebra & charred & 1 & - & - \\
\hline & & \multicolumn{3}{|l|}{ Total burnt } & 5 & - & 1.8 \\
\hline \multicolumn{5}{|c|}{ TOTAL burnt all specimens and phases } & 27 & 22.2 & 3.1 \\
\hline
\end{tabular}

At KR, the percentage of burnt bone is higher in the cultural units of all members, but the overall number of burnt bone is low ( $n=449,3.5 \%$ of site total) (Table 6.21). The highest number of burnt specimens is in the cultural units of the HP (NISP $=234,15.1 \%$ ), which is not unexpected as these units are known to contain many discrete hearths and carbonized material (Deacon and Geleijnse 1988). Out of the 449 burnt bones, 24 (5.3\%) are calcined, 19 of which occur in the cultural units, with the majority $(n=15)$ in the HP. Four calcined specimens occur in the HP sterile units, and one was found in the MSA I sterile units. The majority $(n=434)$ of burnt elements are vertebrae. All of the calcined specimens, apart from one otolith, are vertebrae. None of the spines $(n=56)$ in the KR assemblage show any evidence of burning. 
Table 6.21. Number (NISP) of burnt specimens from Klasies River main site per cultural (C) and sterile (S) units per member, total per member and total of the whole assemblage

\begin{tabular}{|c|c|c|c|c|c|c|c|c|c|c|c|c|c|c|c|}
\hline \multirow[b]{2}{*}{ TAXA } & \multirow[b]{2}{*}{ ELEMENT } & \multirow[b]{2}{*}{ BURNING } & \multicolumn{3}{|c|}{ MSA III } & \multicolumn{3}{|c|}{ HP } & \multicolumn{3}{|c|}{ MSA II } & \multicolumn{3}{|c|}{ MSA I } & \multirow{2}{*}{\begin{tabular}{|c|} 
TOTAL \\
NISP \\
\end{tabular}} \\
\hline & & & C & $\mathrm{s}$ & TOTAL & C & $\mathbf{s}$ & TOTAL & C & $\mathrm{s}$ & TOTAL & C & $\mathrm{s}$ & TOTAL & \\
\hline \multicolumn{16}{|l|}{ Chondrichthyes } \\
\hline Shark spp. & Vertebra & calcined & 1 & - & 1 & 5 & 2 & 7 & - & - & - & - & - & - & 8 \\
\hline Shark spp. & Vertebra & charred & 5 & 1 & 6 & 29 & 8 & 37 & 2 & 2 & 4 & 4 & 4 & 8 & 55 \\
\hline \multicolumn{16}{|l|}{ Clupeidae } \\
\hline Clupeidae spp. & Vertebra & calcined & - & - & - & 1 & - & 1 & - & - & - & - & - & - & 1 \\
\hline Clupeidae spp. & Vertebra & charred & - & 1 & 1 & 2 & - & 2 & - & - & - & - & - & - & 3 \\
\hline \multicolumn{16}{|l|}{ Ariidae } \\
\hline Galeichthys feliceps & articular & charred & 1 & - & 1 & - & - & - & - & - & - & - & - & - & 1 \\
\hline Galeichthys feliceps & Vertebra & calcined & 1 & - & 1 & - & - & - & - & - & - & - & - & - & 1 \\
\hline Galeichthys feliceps & Vertebra & charred & 10 & 6 & 16 & 26 & - & 26 & 2 & 3 & 5 & 1 & 1 & 2 & 49 \\
\hline \multicolumn{16}{|l|}{ Sparidae } \\
\hline Sparidae spp. & First Vertebra & charred & - & - & - & 2 & - & 2 & - & 1 & 1 & - & - & - & 3 \\
\hline Sparidae spp. & Tooth & charred & - & - & - & - & - & - & 1 & 1 & 2 & - & - & - & 2 \\
\hline Sparidae spp. & Posttemporal & charred & - & - & - & - & 1 & 1 & - & - & - & - & - & - & 1 \\
\hline Sparidae spp. & Pterotic & charred & - & - & - & 1 & - & 1 & - & - & - & 1 & - & 1 & 2 \\
\hline \multicolumn{16}{|l|}{ Dischistiidae } \\
\hline Dichistius capensis & Ceratohyal & charred & - & - & - & 1 & - & 1 & - & - & - & - & - & - & 1 \\
\hline \multicolumn{16}{|l|}{ Sciaenidae } \\
\hline Argyrosomus inodorus/japonicus & Vertebra & charred & 1 & - & 1 & - & - & - & - & - & - & - & - & - & 1 \\
\hline \multicolumn{16}{|l|}{ Cheilodactylidae } \\
\hline Chirodactylus brachydactylus & Vertebra & charred & - & - & - & 2 & - & 2 & - & - & - & - & - & - & 2 \\
\hline Chirodactylus brachydactylus & Vertebra & calcined & - & - & - & - & 1 & 1 & - & - & - & - & - & - & 1 \\
\hline \multicolumn{16}{|l|}{ Mugilidae } \\
\hline Liza richardsonii & First Vertebra & charred & - & - & - & 1 & - & 1 & - & - & - & - & - & - & 1 \\
\hline Liza richardsonii & Penultimate Vertebra & charred & - & - & - & 1 & 1 & 2 & - & - & - & - & - & - & 2 \\
\hline Liza richardsonii & Ultimate vertebra & charred & - & - & - & - & 1 & 1 & - & - & - & - & - & - & 1 \\
\hline Liza richardsonii & Vertebra & calcined & - & - & - & 1 & - & 1 & - & - & - & - & - & - & 1 \\
\hline Liza richardsonii & Vertebra & charred & - & - & - & 9 & - & 9 & 2 & - & 2 & - & - & - & 11 \\
\hline Cliniidae & & & & 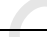 & 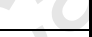 & & & & & & & & & . & \\
\hline Cliniidae spp. & First Vertebra & charred & - & - & - & - & - & - & 1 & - & 1 & - & - & - & 1 \\
\hline Cliniidae spp. & Premaxilla & charred & - & - & - & - & - & - & 1 & - & 1 & - & - & - & 1 \\
\hline Cliniidae spp. & Vertebra & calcined & - & - & - & 1 & - & 1 & - & - & - & - & - & - & 1 \\
\hline Cliniidae spp. & Vertebra & charred & - & - & - & 24 & 3 & 27 & 6 & 3 & 9 & 10 & 6 & 16 & 52 \\
\hline Gobiidae & & & 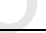 & & & & & & & & & & & & \\
\hline Gobiidae spp. & First Vertebra & calcined & 1 & - & 1 & - & - & - & - & - & - & - & - & - & 1 \\
\hline Species indeterminate & Articular & charred & - & - & - & - & - & - & - & 1 & 1 & - & - & - & 1 \\
\hline Species indeterminate & Basioccipital & charred & - & - & - & - & 1 & 1 & - & - & - & - & - & - & 1 \\
\hline Species indeterminate & First Vertebra & charred & - & - & - & 2 & - & 2 & - & - & - & - & - & - & $\underline{2}$ \\
\hline Species indeterminate & Otolith & calcined & - & - & - & 1 & - & 1 & - & - & - & - & - & - & 1 \\
\hline Species indeterminate & Penultimate Vertebra & charred & - & - & - & 1 & - & 1 & - & - & - & - & - & - & 1 \\
\hline Species indeterminate & Pharyngobranchial & charred & - & - & - & - & - & - & 1 & - & 1 & 1 & 1 & 2 & 3 \\
\hline Species indeterminate & Symplectic & charred & - & - & - & - & - & - & - & - & - & - & 1 & 1 & 1 \\
\hline Species indeterminate & Ultimate Vertebra & calcined & - & - & - & 1 & - & 1 & - & - & - & - & - & - & 1 \\
\hline Species indeterminate & Ultimate Vertebra & charred & - & - & - & 1 & - & 1 & - & - & - & 1 & 1 & 2 & 3 \\
\hline Species indeterminate & Vertebra & calcined & 1 & - & 1 & 5 & 1 & 6 & - & - & - & - & 1 & 1 & 8 \\
\hline Species indeterminate & Vertebra & charred & 14 & 2 & 16 & 117 & 32 & 149 & 15 & 14 & 29 & 23 & 7 & 30 & 224 \\
\hline Total (NISP) & & & 35 & 10 & 45 & 234 & 51 & 285 & 31 & 25 & 56 & 41 & 22 & 63 & 449 \\
\hline Total $\%$ burnt & & & 4.8 & 0.6 & 2.0 & 15.1 & 2.7 & 8.3 & 2.2 & 1.0 & 1.5 & 2.2 & 1.6 & 1.9 & 3.5 \\
\hline
\end{tabular}

\subsubsection{Cut marks}

During analysis it was noted that several of the first vertebrae of Sparidae from the BBC

LSA assemblage were damaged on the anterior faces of the centra (Figure 6.11). The damage was suggestive of having been caused by a cutting or shearing action. All the first vertebrae from the entire BBC LSA excavation were extracted and examined for the same damage. Some of the BBF 6 first vertebrae showed the same damage on the anterior side of the centra. One second vertebra - sometimes referred to as the axis, although this term is not considered appropriate for fish (Rojo 1991) - from BBF 6 also showed damage on 
the anterior centrum. Some first vertebrae from BBC LSA had the same damage on the posterior portion of the centra, with none on the anterior side. This type of cutting/shearing damage was not recorded at any of the other assemblages studied.

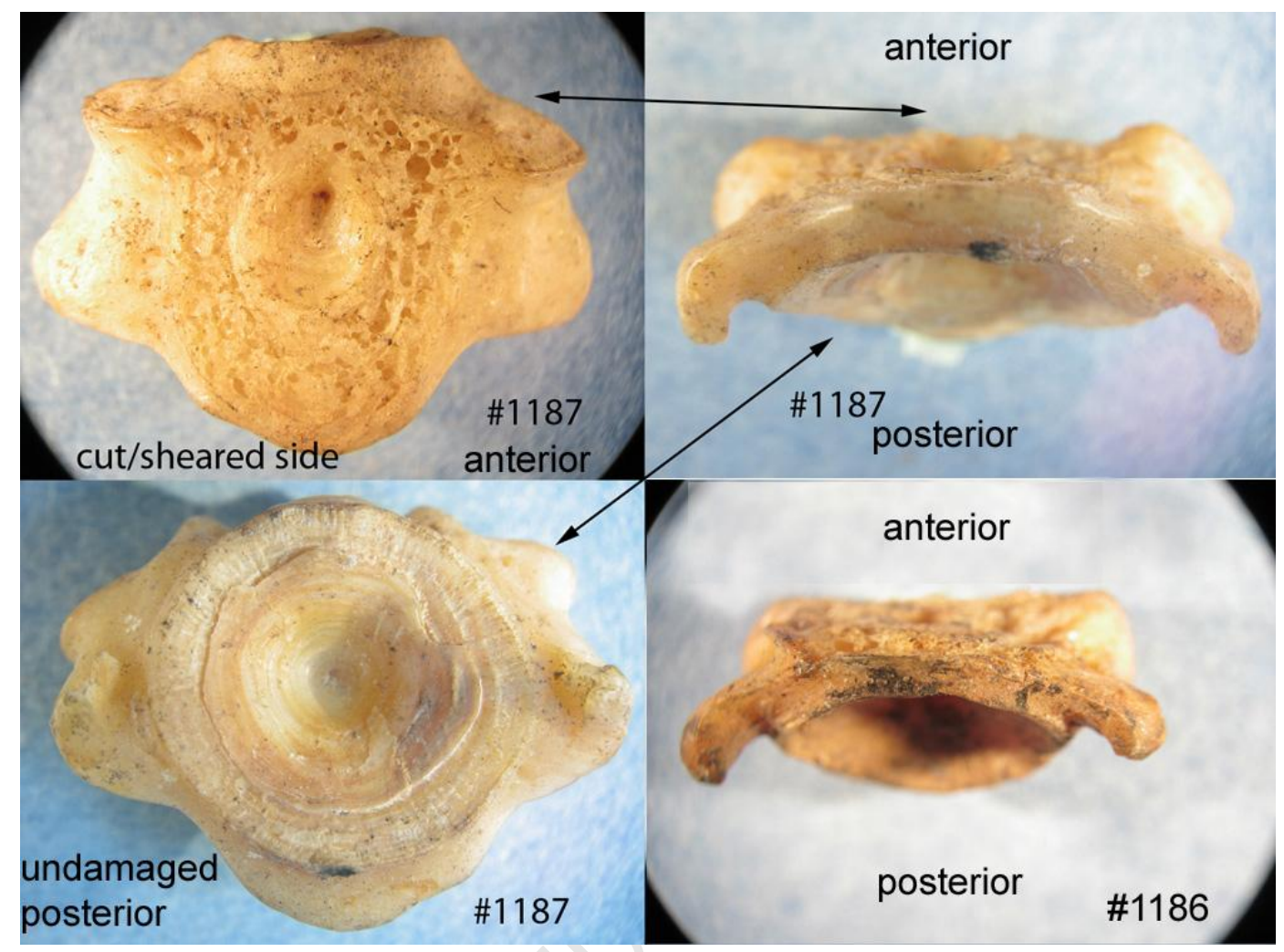

Figure 6.11. First vertebrae of Sparidae spp. showing damage on anterior side from Blombos Cave Later Stone Age layers

Basioccipitals, which articulate with the anterior centra of the first vertebra, were also inspected for damage. The majority of basiocciptals were fragmented and the centra distorted, therefore it was difficult to ascertain whether any damage on the posterior relates to the damage seen on several of the first vertebrae. One basioccipital from F5 COK showed some damage on the right ventral edge of the posterior centra, which might be the result of some sort of cutting action, but the damage was not as clear as that recorded on the first vertebrae.

The damage was restricted to Sparidae, with none noticed in other families. It was not possible to distinguish between Sparidae based on the first vertebra and all were recorded as Sparidae spp. Of 96 first vertebrae from BBC LSA, 11 (11\%) appeared to be definitely cut, all on the anterior side. Seventeen (18\%) were possibly cut, 13 on the anterior side 
and 4 on the posterior side. Sixty-eight (71\%) first vertebrae showed no damage at all. The majority of cut and possibly cut specimens were from medium sized fish (Figure 6.12).

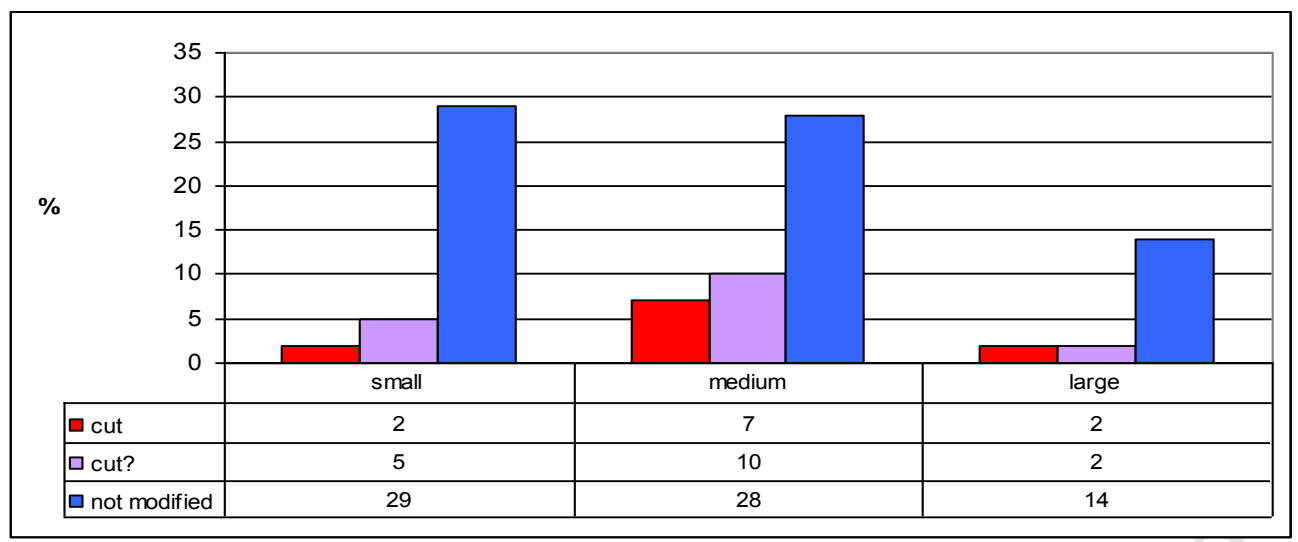

Figure 6.12. Percentage frequency of cut, possibly cut and unmodified Sparidae first vertebrae per size class from Blombos Cave Later Stone Age layers

The damage could indicate that BBC LSA people were processing some of their fish by removal of the head. Actualistic studies are needed to confirm these observations, but these were beyond the scope of this project.

At $\mathrm{H} / \mathrm{RC}$ in one of the shelly layers a single Epinephelus marginatus urohyal from a large specimen has a clear cutmark ventrally near the anterior edge (Layer Tim, H/RC\#1848) (Figure 6.13). The urohyal is a median bone located at the throat region of the fish (Figure 5.2). Van Neer (1995) noted cutmarks on the external side of an interhyal from a large Nile perch from Jenné-Jeno in Mali. Cutting the fish's head in this area would allow simultaneous removal of the branchial arch and hyoid bones (van Neer 1995). Removal of the branchial arch, which contains the gills, would be preferable as the gills spoil quickly. This is common practice among commercial and recreational fishers today to prevent spoilage. Although the urohyal does not belong to the hyoid arch (Rojo 1991), it is located directly below it. Removal of the hyoid and branchial arch could result in damage to the urohyal and might account for the cutmark seen on the H/RC specimen. Alternatively, the fish's throat might have been cut with a sharp stone blade. There is currently no information available on whether this kind of damage results from gill removal or throat cutting. Further experimental work is needed to determine which actions would result in the cutmark seen. 


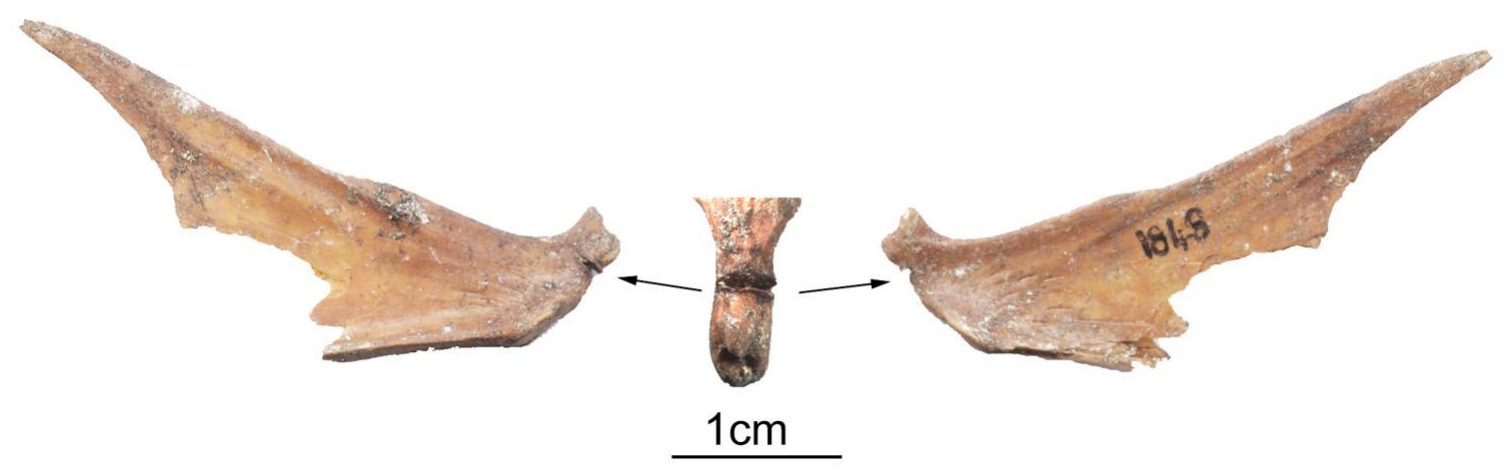

Figure 6.13. An Epinephelus marginatus urohyal with a distinct cut mark on the anterior portion of the ventral side from Hoffman's/Robberg Cave layer TIM

A few other elements from $\mathrm{H} / \mathrm{RC}$ showed marks that might be cut marks or the result of trampling, and once again actualistic studies are needed to investigate this damage, which was beyond the scope of this project. It is nevertheless significant to see clear evidence of cutmarks on fish. These are rarely observed (Willis et al. 2008), and this is the first time that cutmarks on fish have been reported from prehistoric archaeological sites in South Africa. No cut marks were recorded on elements from the KR or BBC MSA assemblages. During Poggenpoel's initial analysis of the BBC MSA fish, he noted (pers. comm.) that some of the Galeichthys feliceps spines from BBC MSA appeared to have green-bone fractures, signifying that these bones were broken while still fresh. Although I was not able to verify this, four of the six spines recorded from the BBC MSA were broken, and none of the breaks were modern. Modern breakage is identifiable through the colour contrast between the fresh break and the surrounding bone, whereas on ancient breaks the fracture site tends to be the same colour as the undamaged parts of the bone. The fracture planes of all four broken spines were the same colour as the rest of the bone, signifying that these elements were broken a long time ago. The proximal ends of all G. feliceps spines were inspected for evidence of disabling of the spines' locking mechanism (Jurgens 2008), but none were found.

\subsubsection{Non-cultural modification}

Natural alterations such as root etching, acid etching and carnivore or rodent gnawing were recorded for each element. Evidence of gnawing was very scarce. One subopercular bone from a medium unidentified Sparidae in the BBC LSA assemblage (BBCLSA\#771, square G5, layer 4) appeared to have been punctured by a tooth. 
Extensive root etching can obscure other surface modifications such as cut marks or gnaw marks on bones (Willis et al. 2008). Root etching on bones exposes additional surfaces of the bone which can make it more vulnerable to degradation (Fisher 1995). Only the presence or absence of root etching, and not the extent thereof, was recorded in this study. Root etching was recorded on 427 specimens from square G5 of the BBC LSA. This constitutes $29 \%$ of the specimens examined for this study, excluding vertebrae, as root etching was not recorded on these elements in the LSA assemblages. In the BBC MSA 27 specimens in the M1 were root etched, eight in M2 and six in the M3. Three elements from BBF 1 were root etched, and five from BBF 6 . None of the specimens from H/RC showed signs of root etching. Root etching was recorded on nine specimens from the cultural units of KR and 16 from the sterile units. The number of specimens with root etching in the BBC LSA is quite high, and this damage could potentially have obscured additional evidence for processing and or gnawing.

Signs of gastric etching were recorded on some specimens from KR in the cultural and sterile units, and none was recorded from the other assemblages. Nineteen specimens from the cultural units showed gastric etching, and fifteen in the sterile units (Table 6.22). Elements with gastric etching that could be identified to species belonged to Acanthistius sebastoides, Galeichthys feliceps and Diplodus capensis. Gastric etching was also recorded on some elements from unidentified Clinidae and Sparidae, as well as on unidentified vertebrae. All of the specimens are from small sized fish $(15-30 \mathrm{~cm})$, apart from the unidentified vertebrae, which did not have size data recorded. The most likely source of the etched bones are regurgitated cormorant pellets, as these birds are known to roost above the site, and the damage is identical to that recorded and illustrated from modern cormorant pellets by von den Driesch (2004: 53, Figure 12). In addition, three of the species were recorded in modern cormorant pellets by von den Driesch (2004). The possibility that the gastric etching was due to human digestive processes cannot be ruled out, but it cannot currently be determined due to the lack of data on the effects of the human digestive system on bones from these species. 
Table 6.22. Number of specimens (NISP) from Klasies River main site that showed evidence of gastric etching. Tick marks indicate specimens that appeared to be 'fresher' than their counterparts from the same contexts. $\mathrm{C}=$ cultural, $\mathrm{S}=$ sterile

\begin{tabular}{|c|c|c|c|c|}
\hline TAXA & ELEMENT & KR C & KR S & $\begin{array}{l}\text { APPEARS } \\
\text { FRESH? }\end{array}$ \\
\hline \multirow{2}{*}{$\begin{array}{l}\text { Ariidae } \\
\text { Galeichthys feliceps }\end{array}$} & Ceratohyal & 1 & & \\
\hline & Vomer & 1 & & \\
\hline \multirow{8}{*}{$\begin{array}{l}\text { Serranidae } \\
\text { Acanthistius sebastoides }\end{array}$} & articular & 1 & & $\checkmark$ \\
\hline & Ceratohyal & 1 & & \\
\hline & Dentary & 1 & & $\checkmark$ \\
\hline & Frontal & 1 & & $\checkmark$ \\
\hline & Maxilla & 1 & & $\checkmark$ \\
\hline & Palatine & 1 & & $\checkmark$ \\
\hline & Parasphenoid & 1 & & $\checkmark$ \\
\hline & Premaxilla & & 1 & $\checkmark$ \\
\hline \multirow{2}{*}{$\begin{array}{l}\text { Sparidae } \\
\text { Sparidae spp. }\end{array}$} & articular & & 4 & \\
\hline & Premaxilla & 1 & & $\checkmark$ \\
\hline \multirow{2}{*}{$\begin{array}{l}\text { Dischistidae } \\
\text { Diplodus capensis }\end{array}$} & & & & \\
\hline & Palatine & 1 & & $\checkmark$ \\
\hline \multirow{2}{*}{$\begin{array}{l}\text { Clinidae } \\
\text { Clinidae spp. }\end{array}$} & articular & 1 & & $\checkmark$ \\
\hline & Dentary & & 2 & $\checkmark$ \\
\hline Species indeterminate & Vertebra & 7 & 8 & $\checkmark$ \\
\hline \multicolumn{2}{|l|}{ TOTAL (NISP) } & 17 & 15 & \\
\hline
\end{tabular}

It was noted that almost all of these bones had a 'fresh' appearance, and were lighter in colour than others from the same contexts (Table 6.22). A number of other specimens, 39 in the cultural and 30 in the sterile units, displayed the same 'fresh' appearance as those listed in Table 6.22, but without evidence for gastric etching. These bones were not modern intrusions, and their appearance was significantly different to the few clearly very fresh looking specimens found in the assemblage, which were excluded from the database. As half of the 'fresher' looking elements showed signs of digestion, these could conceivably have been deposited by cormorants or other animal agents in between episodes of more intensive natural and cultural deposition, which might explain the different colouring of these bones. With the exception of Diplodus capensis, the 'fresher' looking elements that lack signs of digestion are from the same species listed in Table 6.22, and also include 2 elements of Liza richardsonii.

\subsection{Skeletal element representation}

The skeletal element representation per species was compared between sites to determine whether element survivorship differed under specific conditions (cave versus open sites) and with age. These data are based MAU, which is the MNE divided by the number of times each element occurs in a species' skeleton. In order to compare MAU across different sample sizes, it has been standardized by calculating the frequencies of all elements relative to the most common element. This is referred to as the \%MAU (element 
survivorship). It was derived by scaling all values relative to the highest MAU value, which is set to 100 .

Since very few vertebrae were identified to species they were excluded from these calculations except for the first, ultimate and penultimate vertebrae. Including those vertebrae that are easily identifiable will give a biased impression of the skeletal element composition of certain species which could result in misinterpretation of the data. The elements in the element survivorshop (\%MAU) tables are arranged into broad categories based on their location in the skeleton as assessed visually. Only a few species' ultimate and penultimate vertebrae were identifiable to species. These have been included in the tables but it should be kept in mind that the absence of terminal vertebrae of most species cannot be interpreted as a real absence, as it could simply be a function of identifiability.

\subsubsection{Argyrozona argyrozona}

Four different elements of this species were identified (Table 6.23). Most $(n=3)$ are from the shelly layers of $\mathrm{H} / \mathrm{RC}$, and one element (palatine) is present in the cultural units of $\mathrm{KR}$. Palatines were not recovered from H/RC. Premaxillae are the best surviving elements at $\mathrm{H} / \mathrm{RC}$ and palatines at $\mathrm{KR}$. 
Table 6.23. Relative skeletal element frequency (\% MAU) of Argyrozona argyrozona. $100 \% \mathrm{MAU}=$ highest MAU for species which is set at 100. MAU=MNE/number of times the element occurs in an individual animal's skeleton. In this table, the MAU of 0.5 under $\mathrm{KR}(\mathrm{C})$ is derived from dividing the MNE of the most frequently occurring element by the number of times that that element occurs in this species' skeleton. In this instance, there is only one element in this unit, the palatine, which is a bilaterally paired element, in other words there are two per skeleton. The palatine MNE in the KR (C) was 1 (not shown). Therefore the MAU is 1 (MNE) divided by 2 (as there are 2 per skeleton), which results in 0.5. Likewise, the highest MAU of 1 under $\mathrm{H} / \mathrm{RC}$ (S) is derived from MNE, which in this instance is from the premaxilla (MNE is not shown, but the \% MAU of 100 for the premaxilla indicates that this was the most frequently occurring element). Premaxillae are bilaterally paired elements, therefore the MNE was divided by two to derive the MAU. As the MAU is 1, it follows that the MNE was 2. A MAU of one (which in this instance equals $100 \% \mathrm{MAU}$ ) indicates that the minimum number of individuals of this species represented at the site was one. If all the bones from this species were present, all elements would have a $\% \mathrm{MAU}$ of 100 . The \% MAU of the other elements that are below $100 \%$ indicates how many of these elements were observed, relative to how many are expected (the expected number being based on the highest number (MAU) that constitutes100\% MAU). Note that MAU is not the same as MNI: it is not a measure of taxonomic abundance, but of element survivorship

(H/RC: $\mathrm{Z}=$ Zostera, $\mathrm{S}=$ shelly) (KR: $\mathrm{C}=$ cultural, $\mathrm{S}=$ sterile $)$

\begin{tabular}{|c|c|c|c|c|c|}
\hline A. argyrozona & Site type & \multicolumn{2}{|c|}{ CAVE } & \multicolumn{2}{|c|}{ OPEN } \\
\hline \multirow[b]{3}{*}{ Element location } & \multirow[b]{2}{*}{ Site } & \multicolumn{2}{|c|}{$\mathrm{H} / \mathrm{RC}$} & \multicolumn{2}{|c|}{$\mathrm{KR}$} \\
\hline & & $\mathrm{Z}$ & $S$ & $\mathrm{C}$ & $\mathrm{S}$ \\
\hline & $100 \% \mathrm{MAU}=(\mathrm{n})$ & 0 & 1 & 0.5 & 0 \\
\hline \multirow{3}{*}{ lateral skull } & Premaxilla & & 100 & 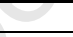 & \\
\hline & Palatine & & + & 100 & \\
\hline & Quadrate & & 50 & & \\
\hline \multirow[t]{2}{*}{ skull } & Prefrontal & & 50 & & \\
\hline & Number of elements & 0 & 3 & 1 & 0 \\
\hline
\end{tabular}

\subsubsection{Argyrosomus inodorus/japonicus}

Nine elements of this species were found, six of which only occur in the sterile layers at KR. The most common elements of this species at KR are basioccipitals in the cultural and first vertebrae in the sterile units. Both elements are from the skull region (Table 6.24). One element of this species, the articular, is present in the interior of BBC MSA; this element also occurs in the sterile layers of KR. 
Table 6.24. Relative skeletal element frequency (\% MAU) of Argyrosomus inodorus/japonicus. $100 \% \mathrm{MAU}=$ highest MAU for this species is set at 100 and all other values are scaled to this. (KR: $\mathrm{C}$ = cultural, $\mathbf{S}=$ sterile) (BBC MSA: $\mathbf{I}=$ interior, $\mathbf{E}=$ exterior)

\begin{tabular}{|c|c|c|c|c|}
\hline A. inodorus/japonicus & Site type & \multirow{2}{*}{\multicolumn{2}{|c|}{$\frac{\text { OPEN }}{\mathrm{KR}}$}} & CAVE \\
\hline \multirow[b]{3}{*}{ Element location } & & & & BBC MSA \\
\hline & Site & $\mathrm{C}$ & $\mathrm{S}$ & 1 \\
\hline & $100 \% \mathrm{MAU}=(\mathrm{n})$ & 3 & 6 & 0.5 \\
\hline \multirow{4}{*}{ lateral skull } & Articular & & 17 & 100 \\
\hline & Premaxilla & & 8 & \\
\hline & Palatine & 17 & 8 & \\
\hline & Quadrate & & 8 & \\
\hline hyoid (throat region) & Epihyal & & 8 & \\
\hline \multirow{5}{*}{ skull } & Basioccipital & 100 & 67 & \\
\hline & First Vertebra & 67 & 100 & \\
\hline & Otolith & & 8 & \\
\hline & Vomer & & 17 & \\
\hline & Number of elements & 3 & 9 & 1 \\
\hline
\end{tabular}

\subsubsection{Acanthistius sebastoides}

A total of 14 elements of this species were identified (Table 6.25). Most elements $(n=10)$ are in KR cultural units, followed by eight in the sterile units. Four out of the eight elements in the sterile units are not present in the cultural units. The best surviving elements in the KR cultural units are parasphenoid and vomer bones, both from the skull region. The most common element in the KR sterile units is the first vertebrae, which did not occur in the cultural units. Articular bones are the best preserved elements at both $\mathrm{H} / \mathrm{RC}$ and BBC MSA (exterior).

Table 6.25. Relative skeletal element frequency (\%MAU) of Acanthistius sebastoides. 100\% MAU = highest MAU for this species is set at 100 and all other values are scaled to this. (H/RC: $Z=Z$ Zostera, $S$ = shelly) (KR: $\mathbf{C}=$ cultural, $\mathbf{S}=$ sterile) (BBC MSA: $\mathbf{I}=$ interior, $\mathbf{E}=$ exterior $)$

\begin{tabular}{|c|c|c|c|c|c|}
\hline \multirow[t]{3}{*}{ A. sebastoides } & Site type & CAVE & \multicolumn{2}{|c|}{ OPEN } & \multirow{2}{*}{\begin{tabular}{|c|} 
OPEN \\
BBC MSA
\end{tabular}} \\
\hline & \multirow[b]{2}{*}{ Site } & $\mathrm{H} / \mathrm{RC}$ & \multicolumn{2}{|c|}{$\mathrm{KR}$} & \\
\hline & & \begin{tabular}{|l|l}
$Z$ & $S$ \\
\end{tabular} & $C$ & $\mathrm{~S}$ & $E$ \\
\hline Element location & $100 \% \mathrm{MAU}=(\mathrm{n})$ & 0 & 2 & 2 & 0.5 \\
\hline \multirow{7}{*}{$\begin{array}{l}\overline{\bar{a}} \\
\frac{v}{\omega} \\
\frac{\pi}{0} \\
\frac{\omega}{\omega}\end{array}$} & Articular & 100 & 25 & & 100 \\
\hline & Dentary & 50 & 25 & 17 & \\
\hline & Maxilla & & 50 & & \\
\hline & Premaxilla & & & 33 & \\
\hline & Palatine & & 25 & & \\
\hline & Quadrate & & 25 & & \\
\hline & Opercular & & 25 & & \\
\hline hyoid (throat region) & Ceratohyal & & 25 & 17 & \\
\hline \multirow{5}{*}{$\begin{array}{l}\overline{\bar{z}} \\
\frac{v}{\omega}\end{array}$} & Basioccipital & & & 33 & \\
\hline & First Vertebra & & & 100 & \\
\hline & Frontal & & 25 & 17 & \\
\hline & Parasphenoid & & 100 & & \\
\hline & Vomer & & 100 & 67 & \\
\hline \multirow[t]{2}{*}{ pectoral girdle } & Posttemporal & & & 17 & \\
\hline & Number of elements & 0 & 10 & 8 & $\overline{1}$ \\
\hline
\end{tabular}

\subsubsection{Chirodactylus brachydactylus}

The most common element of this species is the first vertebrae in both the sterile and cultural layers of KR, followed by the frontals (Table 6.26). The quadrate is the only 
element that occurs exclusively in the sterile layers, and ceratohyal and epihyal bones from the hyoid region occur only in cultural layers.

Table 6.26. Relative skeletal element frequency (\%MAU) of Chirodactylus brachydactylus. 100\% MAU = highest MAU for that species is set at 100. (KR: $\mathrm{C}=$ cultural, $\mathrm{S}=$ sterile)

\begin{tabular}{|l|l|r|r|}
\hline C. brachydactylus & Site type & \multicolumn{2}{|c|}{ OPEN } \\
\hline \multirow{4}{*}{ Element location } & \multirow{2}{*}{ Site } & \multicolumn{2}{|c|}{ KR } \\
\cline { 2 - 4 } & $100 \% \mathrm{MAU}=(\mathrm{n})$ & 9 & \multicolumn{1}{c|}{$\mathrm{S}$} \\
\hline \multirow{2}{*}{ lateral skull } & Dentary & 6 & 9 \\
\cline { 2 - 4 } & Quadrate & & 5 \\
\hline \multirow{2}{*}{ hyoid (throat region) } & Ceratohyal & 6 & \\
\cline { 2 - 4 } & Epihyal & 6 & \\
\hline \multirow{2}{*}{ skull } & First vertebra & 100 & 100 \\
\cline { 2 - 4 } & Frontal & 11 & 9 \\
\hline & Number of elements & 5 & 4 \\
\hline
\end{tabular}

\subsubsection{Chrysoblephus cristiceps}

This species occurred only at sites in the vicinity of Blombos Cave. Elements from the lateral skull region are most frequently preserved (Table 6.27). Nasal bones are the most common elements in both the BBC LSA and BBC MSA exterior. The two elements preserved in the BBC MSA interior differ from the two found in the exterior. All the elements found in the older sites are also present in the BBC LSA assemblage.

Table 6.27. Relative skeletal element frequency (\%MAU) of Chrysoblephus cristiceps. 100\%MAU = highest MAU for that species is set at 100. (BBC MSA: $\mathrm{I}=$ interior, $E=$ exterior)

\begin{tabular}{|c|c|c|c|c|c|}
\hline C. cristiceps & Site type & CAVE & OPEN & CAVE & OPEN \\
\hline \multirow[b]{3}{*}{ Element location } & & $\mathrm{BBC}$ & BBF 6 & \multicolumn{2}{|c|}{ BBC MSA } \\
\hline & Site & LSA & BBF 6 & 1 & $E$ \\
\hline & $100 \% \mathrm{MAU}=(\mathrm{n})$ & 17 & 0.5 & 0.5 & 0.5 \\
\hline \multirow{7}{*}{ 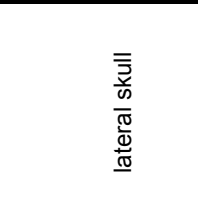 } & 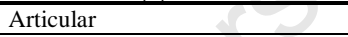 & 73 & 100 & & \\
\hline & Dentary & 39 & & & \\
\hline & Premaxilla & 79 & & & \\
\hline & Retroarticular & 36 & & & \\
\hline & Lacrimal & 58 & & 100 & \\
\hline & Nasal & 100 & & & 100 \\
\hline & Preopercular & 88 & & 100 & \\
\hline \multirow{4}{*}{$\frac{\overline{\bar{z}}}{\frac{v}{\omega}}$} & Frontal & 9 & & & \\
\hline & Infraorbital \#3 & 15 & & & \\
\hline & Prefrontal & 24 & & & 100 \\
\hline & Supraoccipital & 42 & & & \\
\hline \multirow[t]{2}{*}{ pectoral girdle } & Supracleithrum & 36 & & & \\
\hline & Number of elements & 12 & 1 & 2 & 2 \\
\hline
\end{tabular}

\subsubsection{Chrysoblephus gibbiceps}

Elements of this species occurred only in the interior MSA layers of Blombos Cave (Table 6.28). Ten elements were identified, of which most are lateral skull bones apart from one skull element, the prefrontal. Premaxillae are the most frequently occurring elements. 
Table 6.28. Relative skeletal element frequency (\% MAU) of Chrysoblephus gibbiceps. 100\% MAU = highest MAU for that species is set at 100. ( $\mathrm{I}=$ interior $)$

\begin{tabular}{|c|c|c|}
\hline C. gibbiceps & Site type & CAVE \\
\hline \multirow[b]{3}{*}{ Element location } & \multirow[b]{2}{*}{ Site } & BBC MSA \\
\hline & & 1 \\
\hline & $100 \% \mathrm{MAU}=(\mathrm{n})$ & 1.5 \\
\hline \multirow{9}{*}{ 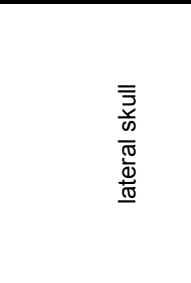 } & Articular & 33 \\
\hline & Dentary & 67 \\
\hline & Maxilla & 33 \\
\hline & Premaxilla & 100 \\
\hline & Retroarticular & 33 \\
\hline & Palatine & 67 \\
\hline & Hyomandibular & 67 \\
\hline & Opercular & 33 \\
\hline & Preopercular & 33 \\
\hline \multirow[t]{2}{*}{ skull } & Prefrontal & 33 \\
\hline & Number of elements & 10 \\
\hline
\end{tabular}

\subsubsection{Chrysoblephus laticeps}

Elements of this species were found only at BBC and H/RC. Eleven elements were found in BBC LSA, and two in H/RC shelly (Table 6.29). The nasal bone is the most common in the LSA assemblages. The supraoccipital was the only element found in the MSA, and is also present in the LSA.

Table 6.29. Relative skeletal element frequency (\%MAU) of Chrysoblephus laticeps. 100\% MAU = highest MAU for that species is set at 100. (H/RC: $Z=$ Zostera, $S=$ shelly) (BBC MSA: $I=$ interior, $E$ $=$ exterior)

\begin{tabular}{|c|c|c|c|c|c|}
\hline C. laticeps & Site type & CAVE & \multicolumn{2}{|c|}{ CAVE } & CAVE \\
\hline \multirow[b]{3}{*}{ Element location } & \multirow[b]{2}{*}{ Site } & BBC & \multicolumn{2}{|c|}{$\mathrm{H} / \mathrm{RC}$} & BBC MSA \\
\hline & & LSA & $Z$ & $\mathrm{~S}$ & 1 \\
\hline & $100 \% \mathrm{MAU}=(\mathrm{n})$ & 14 & 0 & 0.5 & $\overline{1}$ \\
\hline \multirow{7}{*}{ 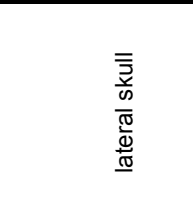 } & Articular & 82 & & & \\
\hline & 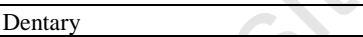 & 25 & & & \\
\hline & 900 & 64 & & & \\
\hline & Retroarticular & 36 & & & \\
\hline & Lacrimal & 46 & & 100 & \\
\hline & Nasal & 100 & & 100 & \\
\hline & Preopercular & 54 & & & \\
\hline \multirow{5}{*}{$\frac{\overline{\bar{z}}}{\frac{\bar{v}}{\omega}}$} & Frontal & 14 & & & \\
\hline & Infraorbital \#3 & 29 & & & \\
\hline & Prefrontal & 21 & & & \\
\hline & Supraoccipital & 14 & & & 100 \\
\hline & Number of elements & 11 & 0 & 2 & 1 \\
\hline
\end{tabular}

\subsubsection{Cymatoceps nasutus}

This species occurred only in $\mathrm{H} / \mathrm{RC}$ and the BBC LSA and MSA assemblages. The greatest variety of elements of this species were found in BBC LSA $(n=16)$ (Table 6.30). Three elements occur in $\mathrm{H} / \mathrm{RC}$ shelly that do not occur in BBC LSA. The palatine occurs in all three assemblages, and is the only element present in the MSA in the interior of BBC. The most common element differs between sites. 
Table 6.30. Relative skeletal element frequency (\% MAU) of Cymatoceps nasutus. 100\% MAU = highest MAU for that species is set at 100. $(\mathrm{H} / \mathrm{RC}: \mathrm{Z}=\mathrm{Zostera}, \mathrm{S}=$ shelly $)(\mathrm{BBC}$ MSA: $\mathrm{I}=$ interior, $\mathrm{E}=$ exterior)

\begin{tabular}{|c|c|c|c|c|c|}
\hline C. nasutus & Site type & CAVE & \multirow{2}{*}{\multicolumn{2}{|c|}{$\begin{array}{l}\text { CAVE } \\
\text { H/RC }\end{array}$}} & \multirow{3}{*}{$\begin{array}{c}\text { CAVE } \\
\text { BBC MSA } \\
\text { I }\end{array}$} \\
\hline \multirow[b]{3}{*}{ Element location } & \multirow[b]{2}{*}{ Site } & $\mathrm{BBC}$ & & & \\
\hline & & LSA & $Z$ & $S$ & \\
\hline & $100 \% \mathrm{MAU}=(\mathrm{n})$ & 6 & 0.5 & 11 & 0.5 \\
\hline \multirow{10}{*}{$\begin{array}{l}\overline{\bar{a}} \\
\frac{\vec{v}}{\omega} \\
\frac{\sqrt[\pi]{0}}{\frac{d}{\omega}} \\
\frac{\omega}{0}\end{array}$} & Articular & 8 & & & \\
\hline & Dentary & 33 & & & \\
\hline & Maxilla & 8 & & 9 & \\
\hline & Premaxilla & 100 & & 5 & \\
\hline & Palatine & 17 & & 5 & 100 \\
\hline & Pterygoid & & & 5 & \\
\hline & Lacrimal & 17 & & 5 & \\
\hline & Nasal & 42 & & 5 & \\
\hline & Hyomandibular & 33 & & & \\
\hline & Preopercular & 42 & & & \\
\hline \multirow{2}{*}{ hyoid (throat region) } & Epihyal & 8 & 100 & & \\
\hline & Urohyal & & & 18 & \\
\hline \multirow{6}{*}{$\frac{\overline{\bar{z}}}{\frac{\bar{v}}{\omega}}$} & Epiotic & 17 & & & \\
\hline & Ethmoid & 17 & & & \\
\hline & Infraorbital \#3 & 17 & & & \\
\hline & Parasphenoid & 33 & & & \\
\hline & Prefrontal & 8 & & & 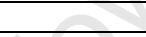 \\
\hline & Vomer & & & 100 & 8 \\
\hline \multirow[t]{2}{*}{ pectoral girdle } & Supracleithrum & 8 & & & 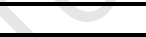 \\
\hline & Number of elements & 16 & 1 & 8 & 1 \\
\hline
\end{tabular}

\subsubsection{Cheimerius nufar}

Only two elements of this species were found, a dentary in the Zostera layers and a maxilla in the shelly layers at $\mathrm{H} / \mathrm{RC}$.

\subsubsection{Dichistius capensis}

The widest range of identifiable elements from this species is at $\mathrm{H} / \mathrm{RC}$, where 42 different elements were identified in the shelly layers, and 30 in the Zostera layers (Table 6.31). All the elements that occur at other sites are also present in both the Zostera and shelly layers at $\mathrm{H} / \mathrm{RC}$. In total, there are more elements preserved in the sterile layers at $\mathrm{KR}(\mathrm{n}=8)$ than in the cultural layers $(n=5)$. Most elements from the sterile layers are from the lateral skull, whereas those from the cultural layers are from the skull and hyoid region. Only lateral skull elements of Dichistius capensis are present at BBC MSA, whereas elements from all parts of the skeleton are present at KR. The elements preserved in BBC MSA interior also occur at KR in both the sterile and cultural layers. 
Table 6.31. Relative skeletal element frequency (\% MAU) of Dichistius capensis. 100\% MAU = highest MAU for that species is set at 100. $(\mathrm{H} / \mathrm{RC}: \mathrm{Z}=\mathrm{Zostera}, \mathrm{S}=$ shelly $)(\mathrm{KR}: \mathrm{C}=$ cultural, $\mathrm{S}=$ sterile) (BBC MSA: I = interior, $\mathrm{E}=$ exterior)

\begin{tabular}{|c|c|c|c|c|c|c|c|c|}
\hline \multirow{4}{*}{$\begin{array}{l}\text { D. capensis } \\
\text { Element location }\end{array}$} & \multirow{3}{*}{$\begin{array}{l}\text { Site type } \\
\text { Site }\end{array}$} & \multirow{3}{*}{$\frac{\frac{\text { CAVE }}{\text { BBC }}}{\text { LSA }}$} & \multirow{2}{*}{\multicolumn{2}{|c|}{$\begin{array}{l}\text { CAVE } \\
\text { H/RC }\end{array}$}} & \multirow{3}{*}{$\begin{array}{l}\text { OPEN } \\
\text { BBF } 1 \\
\text { BBF } 1\end{array}$} & \multirow{2}{*}{\multicolumn{2}{|c|}{$\frac{\text { OPEN }}{\text { KR }}$}} & \multirow{3}{*}{$\begin{array}{c}\frac{\text { CAVE }}{\text { BBC MSA }} \\
1\end{array}$} \\
\hline & & & & & & & & \\
\hline & & & $\bar{Z}$ & $\bar{S}$ & & C & $\mathrm{S}$ & \\
\hline & $100 \% \mathrm{MAU}=(\mathrm{n})$ & 1 & 5 & 12 & 1 & 1 & 1 & 1 \\
\hline \multirow{15}{*}{ 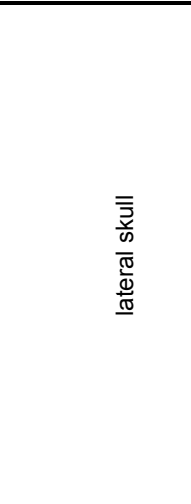 } & Dentary & & 22 & 63 & & & 50 & \\
\hline & Maxilla & & 89 & 75 & & & & \\
\hline & Premaxilla & & 56 & 100 & & & 100 & 100 \\
\hline & Retroarticular & & & 13 & & & & \\
\hline & Mesopterygoid & & & 13 & & & & \\
\hline & Palatine & & 22 & 54 & 50 & & 100 & \\
\hline & Pterygoid & & 22 & 38 & & & & \\
\hline & Quadrate & & 33 & 58 & & & & \\
\hline & Lacrimal & & 22 & 17 & & & & \\
\hline & Hyomandibular & 100 & 100 & 46 & & & 50 & \\
\hline & Interopercular & & 44 & 33 & & & & \\
\hline & Opercular & & 11 & 21 & & & 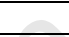 & \\
\hline & Preopercular & & 22 & 46 & & & & \\
\hline & Suboperclular & & & 8 & & & 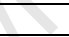 & \\
\hline & Upper postcleithrum & & 11 & 29 & & $\Delta$ & 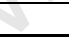 & \\
\hline \multirow{5}{*}{ 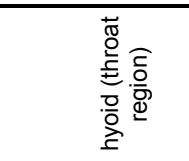 } & Ceratohyal & & 22 & 46 & & 100 & & \\
\hline & Epihyal & & 22 & 17 & 50 & 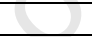 & & \\
\hline & Hypohyal lower & & 22 & 29 & 8 & 50 & 50 & \\
\hline & Hypohyal upper & & & 13 & & 8 & & \\
\hline & Urohyal & & 67 & 33 & 8 & & & \\
\hline \multirow{13}{*}{$\begin{array}{l}\overline{\overline{2}} \\
\frac{v}{\omega}\end{array}$} & Basioccipital & & 22 & 17 & 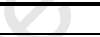 & 100 & & \\
\hline & Epiotic & & 11 & 4 & 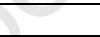 & & & \\
\hline & Ethmoid & & & 8 & 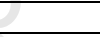 & & & \\
\hline & Exoccipital & & 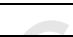 & 17 & 8 & & & \\
\hline & First vertebra & & 67 & 25 & & 100 & 100 & \\
\hline & Frontal & & 11 & 13 & & & & \\
\hline & Parasphenoid & & & 33 & & & & \\
\hline & Prootic & & 11 & 8 & & & & \\
\hline & Pterosphenoid & & & 4 & & & & \\
\hline & Pterotic & 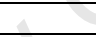 & 22 & 17 & & & & \\
\hline & Sphenotic & 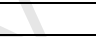 & & 8 & & & & \\
\hline & Supraoccipital & +2 & 44 & 50 & & & & \\
\hline & Vomer & 9 & 22 & 8 & & & & \\
\hline \multirow{7}{*}{$\begin{array}{l}\frac{0}{\overline{0}} \\
\cdot \overline{0} \\
\frac{\bar{\sigma}}{0} \\
\frac{0}{0} \\
\frac{0}{0}\end{array}$} & Basipterygium & & 22 & 13 & & & & \\
\hline & Cleithrum & & 44 & 54 & & & & \\
\hline & 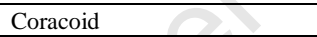 & & & 33 & & & & \\
\hline & Postcleithrum & & 22 & 21 & & & & \\
\hline & Posttemporal & & & 58 & & & & \\
\hline & Scapula & & 78 & 29 & & & 50 & \\
\hline & Supracleithrum & & & 4 & & & & \\
\hline \multirow[t]{2}{*}{ tail } & Urostyle & & 22 & 25 & & & & \\
\hline & Number of elements & 1 & 30 & 42 & 3 & 5 & 8 & 2 \\
\hline
\end{tabular}

At $\mathrm{H} / \mathrm{RC}$ elements from all regions of the skeleton are present (Table 6.31, Figure 6.14). The frequency of elements that only occur in the shelly layers is generally low (17\% or less), with the exception of posttemporal (58\%), parasphenoid and coracoid bones (33\% each). This low frequency of elements that are absent in the Zostera layers probably relates to the larger sample size in the shelly layers. Skull elements are less common in the Zostera layers. Premaxillae and maxillae are the most common in the shelly layers, and hyomandibular bones followed by maxillae are the most frequently occurring elements in the Zostera layers. 


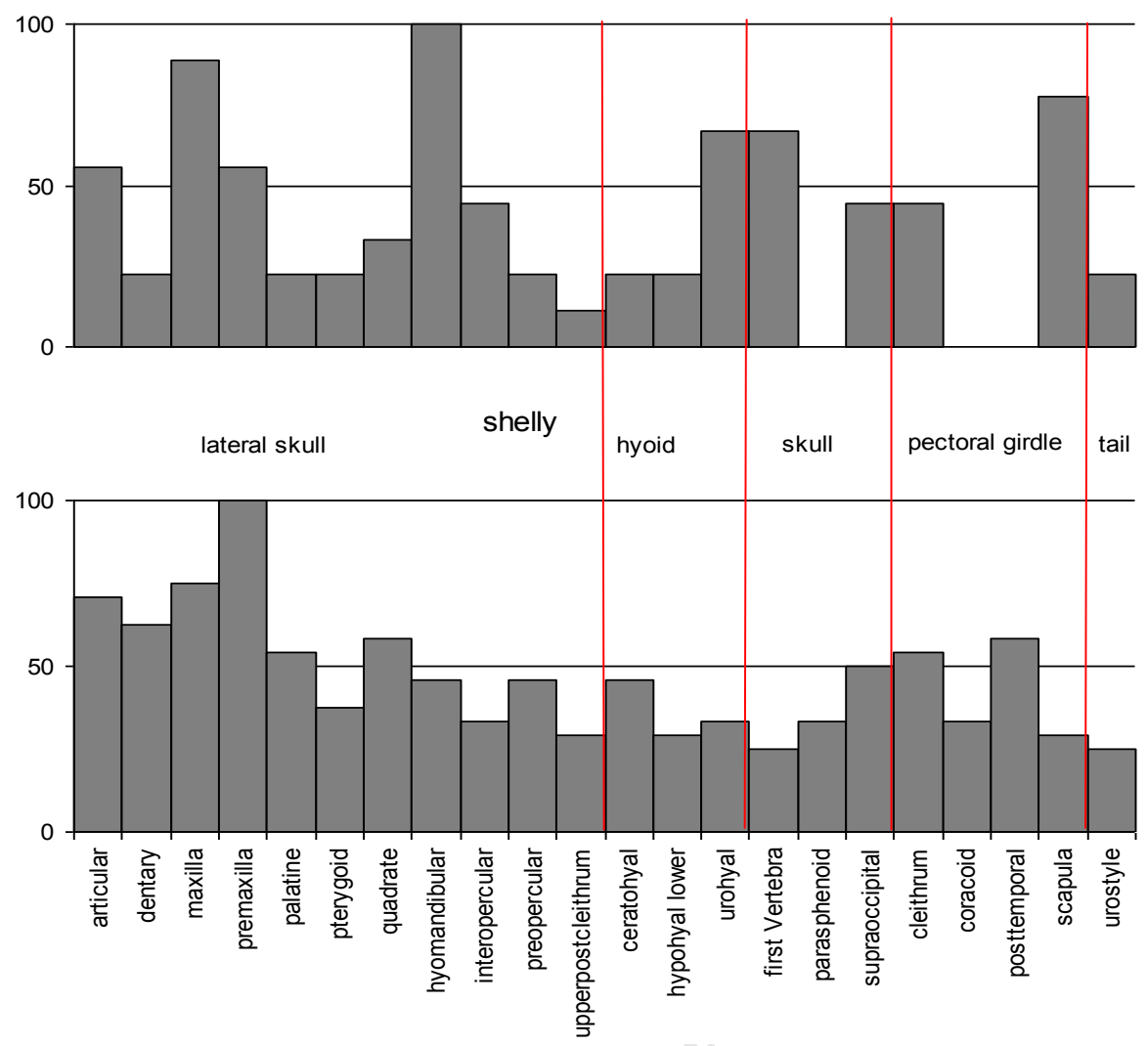

Figure 6.14. Relative skeletal element frequency (\% MAU) of Dichistius capensis in the Zostera and shelly layers at Hoffman's/Robberg Cave. Note that elements with frequencies below $25 \%$ in both the Zostera and shelly layers have been omitted.

The wide range of elements present is at least partly due to the bones of this species being relatively easily identifiable.

\subsubsection{Diplodus hottentotus}

Elements of this species only occurred in the shelly layers of H/RC (Table 6.32).

Premaxillae are the most common element, followed by ceratohyal, epihyal and basioccipital bones in equal ratios.

Table 6.32. Relative skeletal element frequency (\% MAU) of Diplodus hottentotus. 100\% MAU = highest MAU $\mathrm{f}$ or that species is set at 100. $(\mathrm{H} / \mathrm{RC}: \mathrm{Z}=\mathrm{Zostera}, \mathrm{S}=$ shelly $)$

\begin{tabular}{|l|l|r|r|}
\hline D. cervinus hottentotus & Site type & \multicolumn{2}{|c|}{ CAVE } \\
\hline \multirow{5}{*}{ Element location } & \multirow{2}{*}{ H/RC } \\
\cline { 2 - 4 } & Site & \multicolumn{2}{|c|}{ S } \\
\cline { 2 - 4 } lateral skull & $100 \% \mathrm{MAU}=(\mathrm{n})$ & & 1.5 \\
\hline \multirow{2}{*}{ hyoid (throat region) } & Maxilla & & 33 \\
\cline { 2 - 4 } & Premaxilla & & 60 \\
\hline skull & Ceratohyal & & 67 \\
\hline & Epihyal & 0 & 67 \\
\hline & Basioccipital & & 5 \\
\hline
\end{tabular}




\subsubsection{Diplodus capensis}

The highest number of elements was found in the H/RC shelly layers $(n=30)$ (Table 6.33).

The majority of elements in the open sites are from the lateral skull bones. Dentaries, premaxillae and palatines are the best represented elements in the open sites, and premaxillae and articulars are the most common elements in the cave sites. Elements preserved in MSA assemblages are restricted to dentaries, palatines and premaxillae.

Elements of the pectoral girdle are underrepresented at all sites, with only the supracleithrum present in BBC LSA and H/RC shelly. Most of the skull elements are in the $\mathrm{H} / \mathrm{RC}$ shelly layers.

Table 6.33. Relative skeletal element frequency (\%MAU) of Diplodus capensis. 100\%MAU = highest MAU for that species is set at 100. (H/RC: $Z=$ Zostera, $S=$ shelly $)(K R: C=$ cultural, $S=$ sterile $)$

\begin{tabular}{|c|c|c|c|c|c|c|c|c|}
\hline Diplodus capensis & Site type & CAVE & \multicolumn{2}{|c|}{ CAVE } & OPEN & OPEN & \multicolumn{2}{|c|}{ OPEN } \\
\hline \multirow[b]{3}{*}{ Element location } & \multirow[b]{2}{*}{ Site } & $\mathrm{BBC}$ & \multicolumn{2}{|c|}{ H/RC } & $\overline{B B F} 6$ & BBF 1 & \multicolumn{2}{|c|}{$\overline{K R}$} \\
\hline & & LSA & Z & $\mathrm{S}$ & BBF 6 & BBF 1 & $\mathrm{C}$ & $\mathrm{S}$ \\
\hline & $100 \% \mathrm{MAU}=(\mathrm{n})$ & 2 & 17 & 48 & 1 & 1 & & $\overline{1}$ \\
\hline \multirow{14}{*}{$\begin{array}{l}\overline{\bar{a}} \\
\frac{1}{\omega} \\
\frac{\pi}{\frac{\pi}{2}} \\
\frac{\Phi}{\omega}\end{array}$} & Articular & 100 & 24 & 34 & 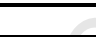 & 20 & & \\
\hline & Dentary & 67 & 67 & 78 & 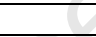 & 1 & & 100 \\
\hline & Maxilla & 33 & 15 & 63 & 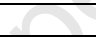 & 40 & & \\
\hline & Premaxilla & 67 & 100 & 100 & 100 & 80 & & 100 \\
\hline & Retroarticular & 33 & 3 & 9 & +8 & 40 & & \\
\hline & Mesopterygoid & & 6 & 25 & 8 & & & \\
\hline & Metapterygoid & & & 1 & & & & \\
\hline & Hyomandibular & 33 & 3 & 8 & & & & \\
\hline & Palatine & 67 & 64 & 51 & & 100 & & 100 \\
\hline & Pterygoid & 67 & 6 & 17 & & & & \\
\hline & Quadrate & 33 & 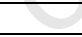 & 1 & & & & \\
\hline & Lacrimal & 33 & 3 & 2 & & & & \\
\hline & Opercular & 33 & 9 & 7 & & & & \\
\hline & Preopercular & 67 & 8 & 5 & & & & \\
\hline \multirow{5}{*}{ 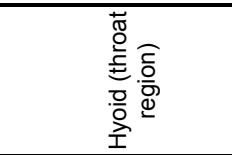 } & Ceratohyal & 33 & 15 & 23 & & 80 & & \\
\hline & Epihyal & 67 & 3 & 10 & & & & \\
\hline & Hypohyal lower & & 6 & 4 & & & & \\
\hline & Hypohyal upper & & 3 & 4 & & & & \\
\hline & Urohyal & & & 23 & & & & \\
\hline \multirow{10}{*}{$\frac{\overline{\overline{2}}}{\frac{\bar{v}}{\omega}}$} & Epiotic & 33 & 9 & 10 & & & & \\
\hline & Exoccipital & & & 1 & & & & \\
\hline & First vertebra & & 6 & & & & & \\
\hline & Frontal & & & 3 & & & & \\
\hline & Parasphenoid & 67 & 12 & 2 & & 80 & & \\
\hline & Parietal & & & 7 & & & & \\
\hline & Prefrontal & & 9 & 10 & & & & \\
\hline & Pterosphenoid & 67 & & 16 & & & & \\
\hline & Supraoccipital & 67 & 12 & 27 & & & & \\
\hline & Vomer & & 6 & 6 & & & & \\
\hline \multirow[t]{2}{*}{ pectoral girdle } & Supracleithrum & 33 & & 1 & & & & \\
\hline & Number of elements & 19 & 21 & 29 & 1 & 7 & 0 & 3 \\
\hline
\end{tabular}

A wide range of elements from the hyoid region are present at $\mathrm{H} / \mathrm{RC}$, but these occur at relatively lower frequencies than the few hyoid elements found in BBC LSA and BBF 1 (Table 6.33, Figure 6.15). There are eight more elements in the H/RC shelly layers than in the Zostera layers. Seven of these occur at very low frequencies ( $<10 \% \mathrm{MAU})$ and their presence can be attributed to the larger sample size from the shelly layers. One element 
from the Zostera layers (first vertebrae) does not occur in any of the other assemblages.

The urohyal occurs only in $\mathrm{H} / \mathrm{RC}$ shelly. All of the elements found at the open sites also occur in both LSA cave sites. The lack of bones from the tail region is due to those elements not being identifiable to species rather than an indication of an actual absence of these elements.

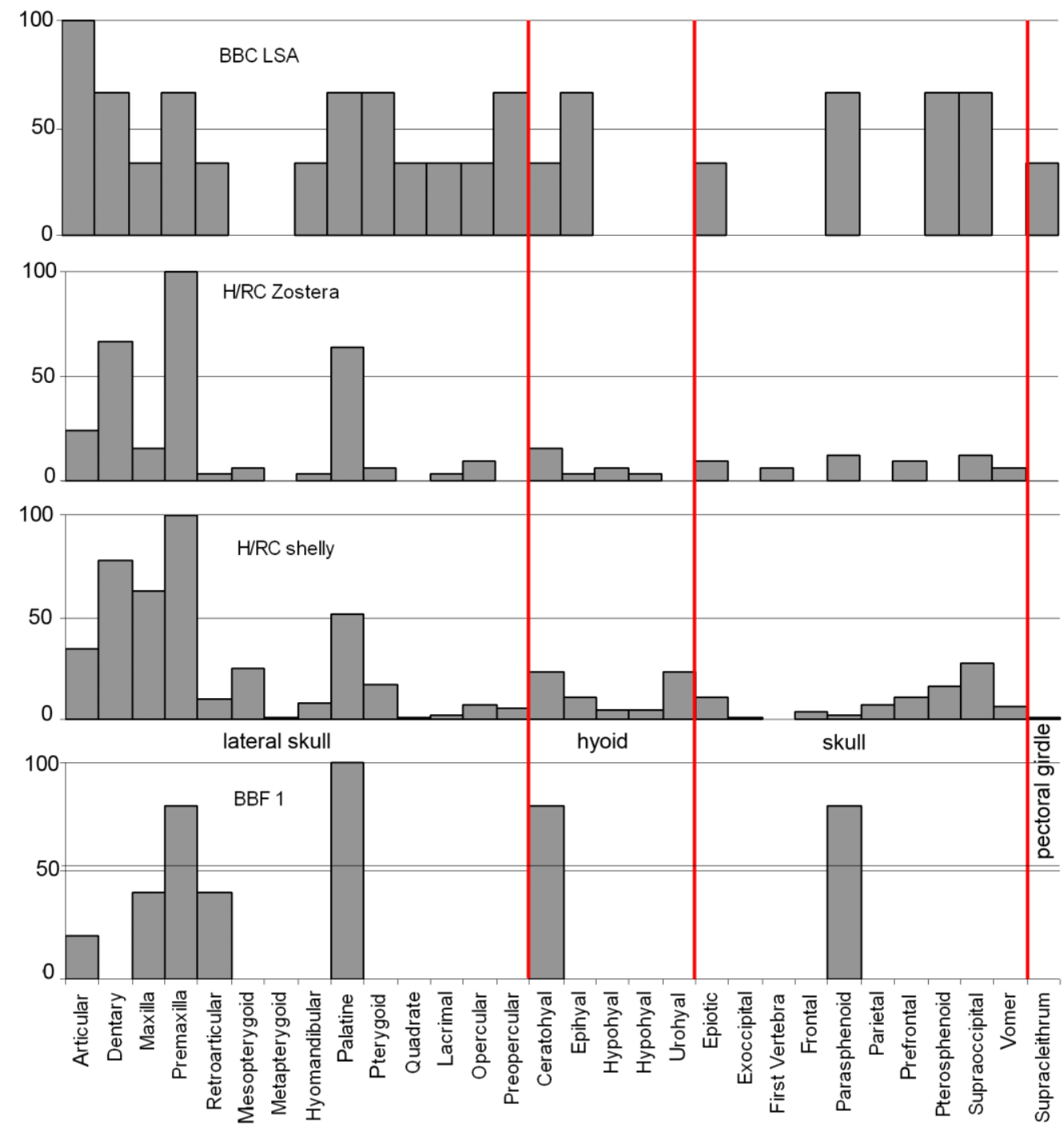

Figure 6.15. Relative skeletal element frequency (\% MAU) of Diplodus capensis at Blombos Cave LSA, Hoffman's/Robberg Cave Zostera and shelly layers and Blombosfontein 1

\subsubsection{Epinephelus marginatus}

In total, 34 elements of this species were identified (Table 6.34). The biggest variety of elements are in BBC LSA ( $n=26)$. Five elements not present in BBC LSA are found in $\mathrm{H} / \mathrm{RC}$. One of the four elements found in the Zostera layers of $\mathrm{H} / \mathrm{RC}$ is also present in the 
shelly layers. The shelly layers contain five elements that were not found in BBC LSA, and two of the four elements found in the Zostera layers do not occur in the BBC LSA.

The most common elements, present in three of the assemblages, are basipterygiums and scapulae, both from the pectoral girdle. All three elements preserved in BBC MSA are from the pectoral girdle. The coracoid occurs only in BBC MSA. Only one element, a hyomandibular, was found in an open context at KR.

Table 6.34. Relative skeletal element frequency (\% MAU) of Epinephelus marginatus. 100\% MAU = highest MAU for that species is set at 100. (H/RC: $Z=$ Zostera, $S=$ shelly) (KR: $C=$ cultural, $S=$ sterile) (BBC MSA: $\mathrm{I}=$ interior, $\mathrm{E}=$ exterior)

\begin{tabular}{|c|c|c|c|c|c|c|c|}
\hline E. marginatus & Site type & CAVE & \multicolumn{2}{|c|}{ CAVE } & \multicolumn{2}{|c|}{ OPEN } & CAVE \\
\hline \multirow[b]{3}{*}{ Element location } & \multirow[b]{2}{*}{ Site } & $\mathrm{BBC}$ & \multicolumn{2}{|c|}{$\mathrm{H} / \mathrm{RC}$} & \multicolumn{2}{|c|}{$\mathrm{KR}$} & BBC MSA \\
\hline & & LSA & Z & $\mathrm{S}$ & C & $S$ & 1 \\
\hline & $100 \% \mathrm{MAU}=(\mathrm{n})$ & 2 & 1.5 & 1 & 0.5 & 0 & 0.5 \\
\hline \multirow{16}{*}{ 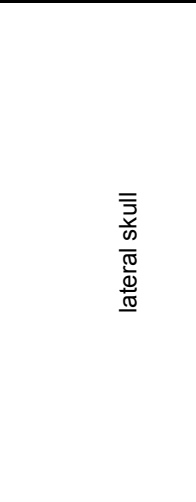 } & Articular & 25 & & & & & $\mathrm{C}$ \\
\hline & Dentary & & 67 & & & & 8 \\
\hline & Maxilla & 25 & 33 & & & & $\overline{l n}$ \\
\hline & Premaxilla & 25 & & & & & +3 \\
\hline & Retroarticular & & & 50 & & & +2 \\
\hline & Mesopterygoid & 25 & & & & & \\
\hline & Metapterygoid & 100 & & & & 8 & \\
\hline & Pterygoid & 50 & & & $\angle$ & 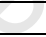 & \\
\hline & Quadrate & 50 & 100 & & 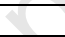 & & \\
\hline & Lacrimal & 25 & & & 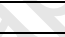 & & \\
\hline & Hyomandibular & & & 50 & 100 & & \\
\hline & Interopercular & 25 & & & & & \\
\hline & Opercular & 50 & 8 & 50 & & & \\
\hline & Preopercular & 25 & 2 & & & & \\
\hline & Subopercular & 50 & $\bar{z}$ & & & & \\
\hline & Upper postcleithrum & 25 & & & & & \\
\hline \multirow{3}{*}{ 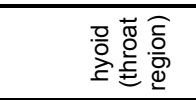 } & Epihyal & 25 & & & & & \\
\hline & Hypohyal lower & 8 & & 50 & & & \\
\hline & Urohyal & 50 & & 100 & & & \\
\hline \multirow{8}{*}{$\frac{\overline{\bar{z}}}{\frac{\vec{v}}{\omega}}$} & Basioccipital & 50 & & & & & \\
\hline & First Vertebra & 50 & & & & & \\
\hline & Parasphenoid & 50 & & & & & \\
\hline & Parietal & 25 & & & & & \\
\hline & Prefrontal & 25 & & & & & \\
\hline & Pterosphenoid & & & 100 & & & \\
\hline & Pterotic & 50 & & & & & \\
\hline & Vomer & 50 & & & & & \\
\hline \multirow{7}{*}{ 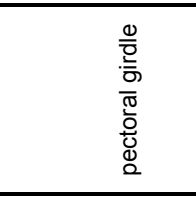 } & Basipterygium & 25 & & 50 & & & 100 \\
\hline & Cleithrum & 25 & & 100 & & & \\
\hline & Coracoid & & & & & & 100 \\
\hline & Posttemporal & & 33 & 50 & & & \\
\hline & Scapula & 50 & & 50 & & & 100 \\
\hline & Supracleithrum & 25 & & 50 & & & \\
\hline & Number of elements & 26 & 4 & 11 & 1 & 0 & 3 \\
\hline
\end{tabular}

\subsubsection{Gymnocrotaphus curvidens}

The greatest number of elements are in the BBC LSA $(n=13)$, followed by the H/RC shelly layers $(n=6)$ (Table 6.35). Dentaries and premaxillae are the only elements preserved in the MSA at KR in both cultural and sterile layers. Dentaries are present at low frequencies in H/RC shelly, but were not found in H/RC Zostera or BBC LSA.

Premaxillae are the only elements present in the H/RC Zostera layers, and this element 
occurred in all the assemblages. Articular bones are most common in the shelly layers of $\mathrm{H} / \mathrm{RC}$. In the BBC LSA layers a number of skull elements are most common.

Table 6.35. Relative skeletal element frequency (\%MAU) of Gymnocrotaphus curvidens. $100 \% \mathrm{MAU}=$ highest MAU for that species is set at 100. (H/RC: $Z=$ Zostera, $S=$ shelly) (KR: $C=$ cultural, $S=$ sterile)

\begin{tabular}{|c|c|c|c|c|c|c|}
\hline G. curvidens & Site type & CAVE & \multicolumn{2}{|c|}{ CAVE } & \multicolumn{2}{|c|}{ OPEN } \\
\hline \multirow[b]{3}{*}{ Element location } & \multirow[b]{2}{*}{ Site } & \multirow{2}{*}{$\begin{array}{l}\text { BBC } \\
\text { LSA }\end{array}$} & \multicolumn{2}{|c|}{$\mathrm{H} / \mathrm{RC}$} & \multicolumn{2}{|c|}{$\mathrm{KR}$} \\
\hline & & & $\bar{Z}$ & $\mathrm{~S}$ & $\mathrm{C}$ & $\mathrm{S}$ \\
\hline & $100 \% \mathrm{MAU}=(\mathrm{n})$ & 1 & 1 & 6 & 0.5 & 1 \\
\hline & Articular & 50 & & 100 & & \\
\hline & Dentary & & & 9 & 100 & 100 \\
\hline$\overline{\bar{z}}$ & Maxilla & 50 & & 9 & & \\
\hline 辛 & Premaxilla & 50 & 100 & 55 & 100 & 50 \\
\hline$\overline{\frac{\pi}{0}}$ & Palatine & 50 & & & & \\
\hline 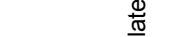 & Nasal & 50 & & & & \\
\hline & Hyomandibular & 50 & & & & \\
\hline & Symplectic & 50 & & & & \\
\hline & Epiotic & 100 & & 36 & & \\
\hline & Parietal & 100 & & & & 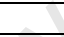 \\
\hline$\overline{\bar{z}}$ & Prootic & 50 & & & & P \\
\hline & Pterosphenoid & 100 & & & & D \\
\hline & Supraoccipital & 100 & & & 8 & \\
\hline pectoral girdle & Posttemporal & 100 & & 9 & 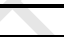 & \\
\hline & Number of elements & 13 & 1 & 6 & 2 & 2 \\
\hline
\end{tabular}

\subsubsection{Galeichthys feliceps}

In total, 19 elements of this species were identified (Table 6.36). The majority $(\mathrm{n}=12)$ occurred in the sterile units of KR. Six of the seven elements found in the cultural units also occur in the sterile units. Otoliths are the most frequently occurring elements at KR in both cultural and sterile units. Otoliths are present in the BBC MSA exterior at relatively high frequencies, but are absent from the interior and H/RC. Dorsal spines are most frequent in the BBC MSA exterior, and occur at low frequencies in the KR cultural units. Spines were not found in the other assemblages. Two elements found in the interior (prefrontal and cleithrum) of BBC MSA were not found at any of the other sites. Nasal bones are present only in H/RC. Prefrontals, cleithra, pectoral spines and symplectics were present only in the MSA at BBC. 
Table 6.36. Relative skeletal element frequency (\% MAU) of Galeichthys feliceps. $100 \% \mathrm{MAU}=$ highest MAU for that species is set at 100. $(\mathrm{H} / \mathrm{RC}: \mathrm{Z}=\mathrm{Zostera}, \mathrm{S}=$ shelly $)(\mathrm{KR}: \mathrm{C}=$ cultural, $\mathrm{S}=$ sterile)

(BBC MSA: I = interior, $\mathrm{E}=$ exterior)

\begin{tabular}{|c|c|c|c|c|c|c|c|}
\hline \multirow[b]{4}{*}{ Element location } & Site type & \multirow{2}{*}{\multicolumn{2}{|c|}{$\begin{array}{l}\text { CAVE } \\
\text { H/RC } \\
\end{array}$}} & \multirow{2}{*}{\multicolumn{2}{|c|}{$\begin{array}{c}\text { OPEN } \\
\text { KR } \\
\end{array}$}} & \multirow{2}{*}{\multicolumn{2}{|c|}{$\begin{array}{c}\text { CAVE } \\
\text { BBC MSA }\end{array}$}} \\
\hline & \multirow[b]{2}{*}{ Site } & & & & & & \\
\hline & & $Z$ & $S$ & $\mathrm{C}$ & $\mathrm{S}$ & 1 & $E$ \\
\hline & $100 \% \mathrm{MAU}=(\mathrm{n})$ & 0 & 1.5 & 19 & 7.5 & 1 & 4 \\
\hline \multirow{6}{*}{ 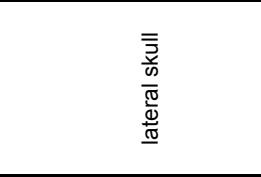 } & Articular & & & 5 & 7 & 50 & 63 \\
\hline & Dentary & & & & 7 & & 13 \\
\hline & Nasal & & 33 & & & & \\
\hline & Hyomandibular & & & & 7 & & \\
\hline & Symplectic & & & & & & 13 \\
\hline & Opercular & & & 5 & 20 & & \\
\hline hyoid (throat region) & Ceratohyal & & & 5 & 20 & & \\
\hline \multirow{8}{*}{$\frac{\overline{\bar{z}}}{\omega}$} & Basioccipital & & & 5 & & & 25 \\
\hline & Epiotic & & & & 7 & & \\
\hline & First Vertebra & & 100 & & 13 & 100 & 25 \\
\hline & Frontal & & 33 & & 13 & & \\
\hline & Otolith & & & 100 & 100 & & 75 \\
\hline & Prefrontal & & & & & 50 & \\
\hline & Sphenotic & & & & 7 & & \\
\hline & Vomer & & & 11 & 13 & & 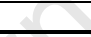 \\
\hline \multirow{3}{*}{ pectoral girdle } & Cleithrum & & & & & 50 & 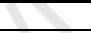 \\
\hline & Spine dorsal & & & 5 & & 100 & 100 \\
\hline & Spine pectoral & & & & & 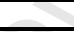 & 50 \\
\hline \multirow[t]{2}{*}{ tail } & Urostyle & & & 11 & 80 & 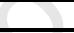 & \\
\hline & Number of elements & 0 & 3 & 8 & 12 & 5 & 8 \\
\hline
\end{tabular}

\subsubsection{Lithognathus lithognathus}

Most elements occur in the shelly layers of $\mathrm{H} / \mathrm{RC}(\mathrm{n}=8)$ (Table 6.37). Premaxillae are the most frequently occurring elements at $\mathrm{H} / \mathrm{RC}$. Maxillae are the best preserved elements in the cultural units of KR. A number of elements are equally well represented in KR sterile. First vertebrae occurred only at KR in both cultural and sterile layers. Palatine, pterygoid and lachrymal bones occur only at H/RC. All identified elements were from the skull and lateral skull region.

Table 6.37. Relative skeletal element frequency (\%MAU) of Lithognathus lithognathus. 100\% MAU = highest MAU for that species is set at 100. (H/RC: $Z=$ Zostera, $S=$ shelly) (KR: $C=$ cultural, $S=$ sterile)

\begin{tabular}{|c|c|c|c|c|c|}
\hline L. lithognathus & Site type & \multicolumn{2}{|c|}{ CAVE } & \multicolumn{2}{|c|}{ OPEN } \\
\hline \multirow[b]{3}{*}{ Element location } & \multirow[b]{2}{*}{ Site } & \multicolumn{2}{|c|}{$\mathrm{H} / \mathrm{RC}$} & \multicolumn{2}{|c|}{$\mathrm{KR}$} \\
\hline & & Z & $\mathrm{S}$ & $\mathrm{C}$ & $S$ \\
\hline & $100 \% \mathrm{MAU}=(\mathrm{n})$ & 2 & 5.5 & 1.5 & 1 \\
\hline \multirow{7}{*}{ 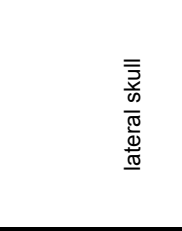 } & Articular & 25 & 18 & & 100 \\
\hline & Dentary & & 36 & 33 & 50 \\
\hline & Maxilla & 50 & & 100 & 100 \\
\hline & Premaxilla & 100 & 100 & & 100 \\
\hline & Palatine & 25 & 73 & & \\
\hline & Pterygoid & 25 & 18 & & \\
\hline & Lacrimal & & 9 & & \\
\hline \multirow{4}{*}{$\begin{array}{l}\overline{\overline{2}} \\
\frac{v}{\omega}\end{array}$} & First vertebra & & & 67 & 100 \\
\hline & Frontal & & 18 & 67 & \\
\hline & Vomer & & 18 & & 100 \\
\hline & Number of elements & 5 & 8 & 4 & 6 \\
\hline
\end{tabular}




\subsubsection{Liza richardsonii}

A large number of elements of this species were identified, particularly from the LSA cave sites. The largest number of elements are present in BBC LSA ( $n=39)$, followed by H/RC shelly $(n=37)$ (Table 6.38). Overall, urostyles and first vertebrae are the most common.

These, along with hyomandibular and basioccipital bones, occur in the highest number of assemblages. The frequency of the most common elements varies between assemblages.

The elements present in the BBF 1 and BBF 6 open sites also occur at the other open and cave sites.

Table 6.38. Relative skeletal element frequency (\% MAU) of Liza richardsonii. 100\% MAU = highest MAU for that species is set at 100. (H/RC: $Z=$ Zostera, $S=$ shelly) (KR: $C=$ cultural, $S=$ sterile) (BBC MSA: $\mathrm{I}$ = interior, $\mathrm{E}=$ exterior)

\begin{tabular}{|c|c|c|c|c|c|c|c|c|c|}
\hline L. richardsonii & Site type & CAVE & \multicolumn{2}{|c|}{ CAVE } & \multirow{3}{*}{$\begin{array}{l}\text { OPEN } \\
\text { BBF } 6 \\
\text { BBF } 6\end{array}$} & \multirow{3}{*}{$\begin{array}{c}\text { OPEN } \\
\text { BBF } 1 \\
\text { BBF } 1\end{array}$} & \multicolumn{2}{|c|}{ OPEN } & \multirow{2}{*}{$\begin{array}{c}\text { CAVE } \\
\text { BBC MSA }\end{array}$} \\
\hline \multirow[b]{3}{*}{ Element location } & \multirow[b]{2}{*}{ Site } & BBC & $\mathrm{H} / \mathrm{F}$ & & & & + & & \\
\hline & & LSA & $Z$ & $\mathrm{~S}$ & & & $\mathrm{C}$ & $\mathrm{S}$ & 1 \\
\hline & $100 \% \mathrm{MAU}=(\mathrm{n})$ & 54 & 8 & 49 & 1 & 1 & 9 & 7 & 5 \\
\hline \multirow{11}{*}{ 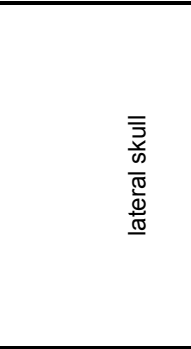 } & Articular & 16 & & 11 & & 8 & & & \\
\hline & Dentary & 18 & 13 & 9 & 2 & & & 14 & 10 \\
\hline & Maxilla & 7 & 6 & 11 & 8 & & & & \\
\hline & Premaxilla & 3 & & 7 & $\omega_{n}$ & & 6 & 7 & \\
\hline & Palatine & & & 4 & +2 & & & 7 & \\
\hline & Quadrate & 17 & & 10 & 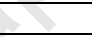 & & & & \\
\hline & Hyomandibular & 51 & 31 & 54 & & & & 43 & 30 \\
\hline & Interopercular & 9 & 6 & 12 & & & & & \\
\hline & Opercular & 26 & 13 & 26 & & & 11 & 7 & \\
\hline & Preopercular & 10 & $\bar{x}$ & 8 & & & & & \\
\hline & Subopercular & 10 & 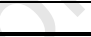 & 1 & & & & & \\
\hline \multirow{4}{*}{ 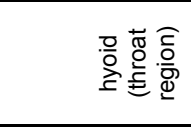 } & Ceratohyal & 37 & 25 & 37 & & & & & 20 \\
\hline & Epihyal & 6 & 6 & 4 & & & & & 10 \\
\hline & Hypohyal upper & 1 & & & & & & & \\
\hline & Urohyal & 100 & 13 & 29 & & & & 14 & \\
\hline \multirow{18}{*}{$\frac{\overline{\bar{z}}}{\mathrm{v}}$} & Basioccipital & 96 & 50 & 53 & 40 & & 22 & 43 & 20 \\
\hline & Epiotic & 16 & 25 & 47 & & & & & \\
\hline & Epiotic 2 & 3 & 6 & 9 & & & & & \\
\hline & Ethmoid & & & 4 & & & & & \\
\hline & Exoccipital & 46 & 31 & 47 & & & & 7 & 10 \\
\hline & First Vertebra & 31 & 13 & 55 & & & 100 & 100 & \\
\hline & Frontal & 19 & 13 & 39 & & & 6 & 79 & \\
\hline & Opisthotic & 2 & & & & & & & \\
\hline & Otolith & 2 & & & & & & & \\
\hline & Parasphenoid & 44 & 13 & 14 & & & & 14 & 20 \\
\hline & Parietal & & 19 & & & & & & \\
\hline & prefrontal & 6 & & 1 & & & & & \\
\hline & Prootic & 33 & & 19 & & & & & \\
\hline & Pterosphenoid & 1 & & & & & & & \\
\hline & Pterotic & 24 & 19 & 63 & 20 & & & 14 & \\
\hline & Sphenotic & 19 & 25 & 20 & & & & & \\
\hline & Supraoccipital & 22 & 13 & 37 & & & & 14 & \\
\hline & vomer & & & 10 & & & & & \\
\hline \multirow{7}{*}{ 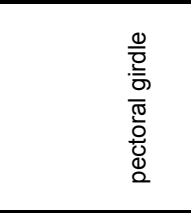 } & Basipterygium & 10 & 25 & 33 & & & & 14 & \\
\hline & Cleithrum & 67 & 31 & 56 & & & & & 20 \\
\hline & Coracoid & 28 & & 5 & & & & & \\
\hline & Postcleithrum & 43 & & 26 & & & & & 10 \\
\hline & Posttemporal & 1 & & 4 & & & & & 10 \\
\hline & Scapula & 22 & & 20 & & & & & 10 \\
\hline & Supracleithrum & 11 & & 1 & & & & & \\
\hline \multirow{4}{*}{$\overline{\bar{\pi}}$} & 1st anal pterygiophore & 2 & & & & & & & \\
\hline & Penultimate vertebrae & 30 & 75 & 84 & & & 44 & 57 & \\
\hline & Urostyle & 74 & 100 & 100 & 40 & 100 & 22 & 86 & 100 \\
\hline & Number of elements & 39 & 23 & 37 & 3 & 1 & 7 & 16 & 12 \\
\hline
\end{tabular}


Figure 6.16 provides a graphic representation of the data in Table 6.38. Elements that constituted less than $10 \%$ frequency in all assemblages have been omitted from this figure, as well as the BBF 1 and BBF 6 data, due to the small numbers in those assemblages.

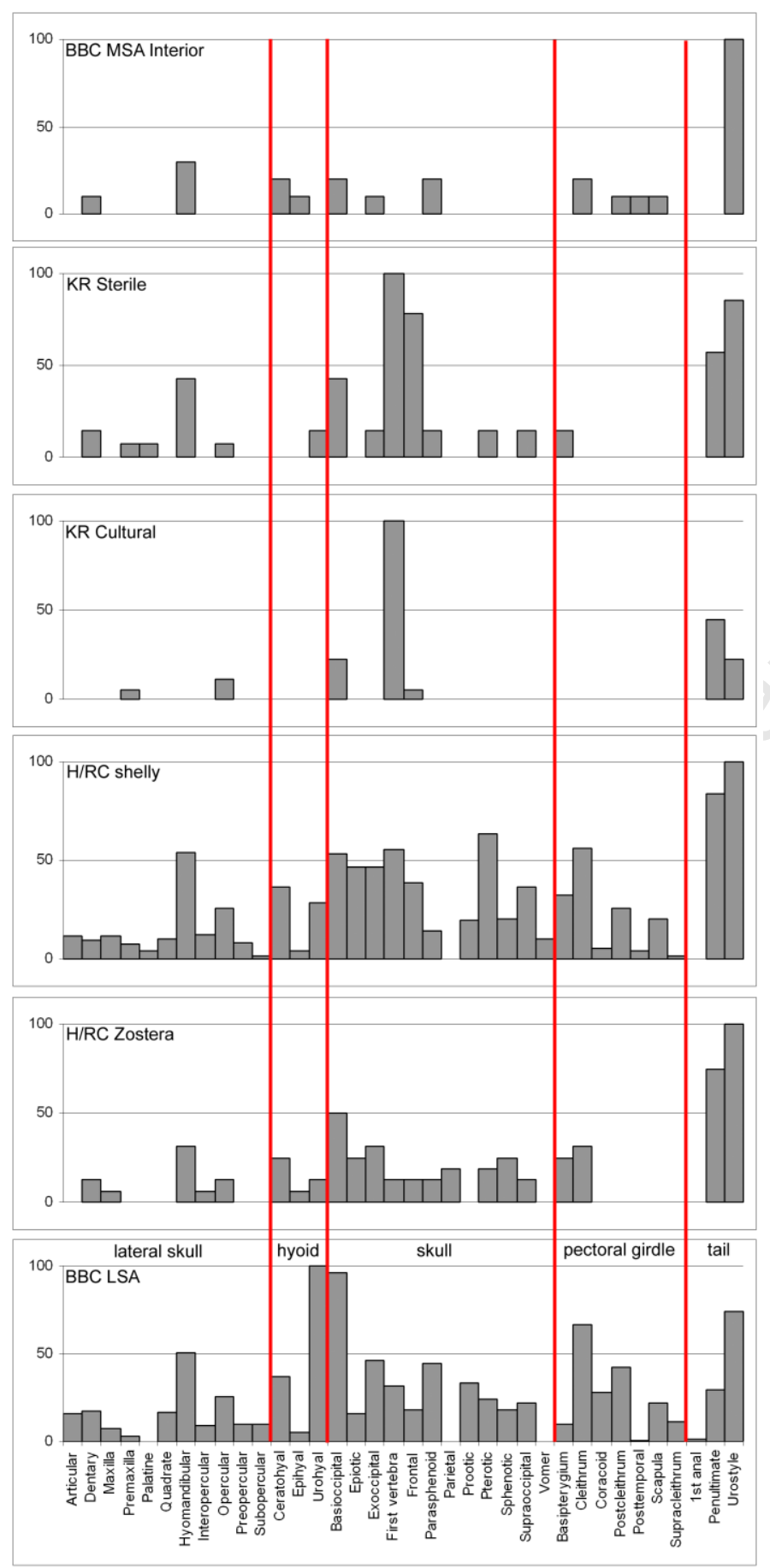

Figure 6.16. Relative skeletal element frequency (\%MAU) of Liza richardsonii at Blombos Cave MSA, Hoffman's/Robberg Cave Zostera and shelly layers, Klasies River main site cultural and sterile layers and Blombos Cave MSA interior 
The BBC LSA element composition is very similar to that of the H/RC shelly layers, but the individual element frequencies differ. H/RC Zostera layers have fewer elements than the shelly layers, but all the elements in the Zostera layers also occur in the shelly layers, with the exception of the parietal.

The combined KR cultural layers contain the lowest number of elements present $(n=7)$, all of which are also present in the KR sterile layers. Only two of the KR cultural elements occur in the BBC MSA (urostyle and basioccipital), whereas 6 of the KR sterile elements are present in the BBC MSA interior layers. The pattern of element survival is not consistent between the MSA assemblages. For example, the first vertebra is the most frequently occurring element in both the cultural and sterile units of $\mathrm{KR}$, yet none were preserved in BBC MSA. Pectoral girdle elements are underrepresented in the open assemblages.

\subsubsection{Monodactylus falciformis}

Only two elements of this species occurred, one maxillae and one cleithra, both in the Zostera layers of $\mathrm{H} / \mathrm{RC}$.

\subsubsection{Mugil cf. cephalus}

One element was identified, a basioccipital at $\mathrm{H} / \mathrm{RC}$ in the shelly layers. As there are several Mugil species in southern African waters, and the comparative collection only contains Mugil cephalus, the term Mugil cf. cephalus is used here, as this single element could belong to another Mugil species not present in the collection.

\subsubsection{Oplegnathus conwayi}

This species is represented by one premaxilla in the BBC MSA (interior).

\subsubsection{Pachymetopon blochii}

The largest number of elements representing this species is in the LSA at BBC $(n=6)$ (Table 6.39). Maxillae are the most common elements at the MSA open contexts of KR and $\mathrm{BBC}$ exterior. Overall, the supracleithrum is the most frequently occurring element of this species and is found in four of the cave assemblages. Maxillae, lachrymal and supraoccipital bones occur only in the MSA assemblages. 
Table 6.39. Relative skeletal element frequency (\%MAU) of Pachymetopon blochii. $100 \% \mathrm{MAU}=$ highest MAU for that species is set at 100. (H/RC: $\mathrm{Z}=$ Zostera, $\mathrm{S}=$ shelly) $(\mathrm{KR}$ : $\mathrm{C}=$ cultural, $\mathrm{S}=$ sterile) (BBC MSA: I = interior, $\mathrm{E}=$ exterior)

\begin{tabular}{|c|c|c|c|c|c|c|c|c|}
\hline P. blochii & Site type & \multirow{2}{*}{$\begin{array}{c}\text { CAVE } \\
\text { BBC }\end{array}$} & \multicolumn{2}{|c|}{ CAVE } & \multicolumn{2}{|c|}{ OPEN } & CAVE & OPEN \\
\hline \multirow[b]{3}{*}{ Element location } & \multirow[b]{2}{*}{ Site } & & \multicolumn{2}{|c|}{$\mathrm{H} / \mathrm{RC}$} & \multicolumn{2}{|c|}{$\mathrm{KR}$} & \multicolumn{2}{|c|}{ BBC } \\
\hline & & LSA & Z & $S$ & $\mathrm{C}$ & $S$ & I & $E$ \\
\hline & $100 \% \mathrm{MAU}=(\mathrm{n})$ & 11 & 3 & 10.5 & 0 & 0.5 & 1 & 0.5 \\
\hline$\overline{\bar{z}}$ & Maxilla & & & & & 100 & & 100 \\
\hline$\frac{\vec{v}}{\omega}$ & Premaxilla & 10 & 17 & & & & 50 & \\
\hline $\bar{\pi}$ & Pterygoid & & & 5 & & & & \\
\hline$\stackrel{0}{\mathbb{0}}$ & Lacrimal & & & & & 100 & & \\
\hline & Ethmoid & 29 & & & & & & \\
\hline$\overline{\overline{5}}$ & Pterosphenoid & 5 & & & & & & \\
\hline$\frac{\vec{v}}{\omega}$ & Supraoccipital & & & & & & 100 & \\
\hline & Vomer & 10 & & & & & & \\
\hline pectoral girdle & Supracleithrum & 100 & 100 & 100 & & & 50 & \\
\hline tail & Urostyle & 10 & & & & & & \\
\hline & Number of elements & 6 & 2 & 2 & 0 & 2 & 3 & 1 \\
\hline
\end{tabular}

\subsubsection{Pomadasys commersonnii}

Only 2 elements represent this species, dentaries in H/RC shelly, and palatines in KR HP sterile.

\subsubsection{Pterogymnus laniarius}

Very few elements represent this species, the most common is the articular in both BBC MSA interior and KR sterile units. The only other element identified is the dentary in the KR sterile units.

\subsubsection{Pomadasys olivaceum}

This species is represented by 16 elements in the $\mathrm{H} / \mathrm{RC}$ shelly layers, with the basioccipital and supraoccipital being the most common (Table 6.40). Several elements from all regions apart from the tail are present at $\mathrm{H} / \mathrm{RC}$. The premaxilla is the only element preserved in the KR open assemblage (cultural and sterile units), and this element is not present in the $\mathrm{H} / \mathrm{RC}$ assemblage. 
Table 6.40. Relative skeletal element frequency (\%MAU) of Pomadasys olivaceum. 100\% MAU = highest MAU for that species is set at 100. $(\mathrm{H} / \mathrm{RC}: \mathrm{Z}=\mathrm{Zostera}, \mathrm{S}=$ shelly $)(\mathrm{KR}$ : $\mathrm{C}=$ cultural, $\mathrm{S}=$ sterile)

\begin{tabular}{|c|c|c|c|c|c|}
\hline P. olivaceum & Site type & \multirow{2}{*}{\multicolumn{2}{|c|}{$\begin{array}{l}\text { CAVE } \\
\text { H/RC }\end{array}$}} & \multirow{2}{*}{\multicolumn{2}{|c|}{$\frac{\text { OPEN }}{\mathrm{KR}}$}} \\
\hline \multirow[b]{3}{*}{ Element location } & \multirow[b]{2}{*}{ Site } & & & & \\
\hline & & Z & $\mathrm{S}$ & C & $\mathrm{S}$ \\
\hline & $100 \% \mathrm{MAU}=(\mathrm{n})$ & 0 & 2 & 0.5 & 0.5 \\
\hline \multirow{6}{*}{ 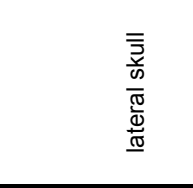 } & Premaxilla & & & 100 & 100 \\
\hline & Retroarticular & & 25 & & \\
\hline & Palatine & & 25 & & \\
\hline & Hyomandibular & & 25 & & \\
\hline & Opercular & & 25 & & \\
\hline & Subopercular & & 25 & & \\
\hline \multirow{3}{*}{$\begin{array}{l}\text { 흥 동 } \\
\text { 을을 }\end{array}$} & Ceratohyal & & 25 & & \\
\hline & Epihyal & & 25 & & \\
\hline & Urohyal & & 50 & & \\
\hline \multirow{6}{*}{$\frac{\overline{\bar{z}}}{\frac{\vec{v}}{\omega}}$} & Basioccipital & & 100 & & \\
\hline & Otolith & & 50 & & \\
\hline & Parietal & & 25 & & \\
\hline & Pterotic & & 25 & & \\
\hline & Sphenotic & & 50 & & \\
\hline & Supraoccipital & & 100 & & \\
\hline \multirow{3}{*}{ pectoral girdle } & Cleithrum & & 50 & & \\
\hline & Posttemporal & & 25 & & \\
\hline & Number of elements & 0 & 16 & 1 & 1 \\
\hline
\end{tabular}

\subsubsection{Petrus rupestris}

Most of the elements representing this species are from the lateral skull region. One skull element, the basioccipital, was present in the cultural units of KR (Table 6.41). Dentaries and retroarticular bones occur in both cave (BBC LSA) and open (KR MSA) contexts.

Table 6.41. Relative skeletal element frequency (\%MAU) of Petrus rupestris. 100\% MAU = highest MAU for that species is set at 100. (KR: $C=$ cultural, $S=$ sterile)

\begin{tabular}{|c|c|c|c|c|}
\hline P. rupestris & Site type & CAVE & \multicolumn{2}{|c|}{ OPEN } \\
\hline \multirow[b]{3}{*}{ Element location } & \multirow[b]{2}{*}{ Site } & $\mathrm{BBC}$ & \multicolumn{2}{|c|}{$\mathrm{KR}$} \\
\hline & & LSA & C & $\mathrm{S}$ \\
\hline & $100 \% \mathrm{MAU}=(\mathrm{n})$ & 1 & 1 & \\
\hline \multirow{4}{*}{$\begin{array}{l}\overline{\bar{\pi}} \\
\frac{\bar{d}}{\bar{d}} \overline{\overline{2}} \\
\underline{\underline{\sigma}}\end{array}$} & Dentary & 100 & 50 & \\
\hline & Premaxilla & & 50 & \\
\hline & Retroarticular & 100 & 50 & \\
\hline & Opercular & 100 & & \\
\hline \multirow[t]{2}{*}{ skull } & Basioccipital & & 100 & \\
\hline & Number of elements & 3 & 4 & 0 \\
\hline
\end{tabular}

\subsubsection{Pomatomus saltatrix}

The largest number of elements are in the H/RC shelly layers $(n=16)$ (Table 6.42).

Dentaries, followed by premaxillae, are the most common elements in both the Zostera and shelly layers of H/RC. First vertebrae are the most common in the open KR assemblage in cultural and sterile units. This element is also present in H/RC shelly, at a low frequency relative to others from this assemblage. The vomer occurred only in the cultural units of KR. 
Table 6.42. Relative skeletal element frequency (\% MAU) of Pomatomus saltatrix. $100 \% \mathrm{MAU}=$ highest MAU for that species is set at 100. (H/RC: $Z=$ Zostera, $S=$ shelly $)(K R: C=$ cultural, $S=$ sterile)

\begin{tabular}{|c|c|c|c|c|c|}
\hline P. saltatrix & Site type & \multirow{2}{*}{\multicolumn{2}{|c|}{$\frac{\text { CAVE }}{\text { H/RC }}$}} & \multicolumn{2}{|c|}{ OPEN } \\
\hline \multirow[b]{3}{*}{ Element location } & \multirow[b]{2}{*}{ Site } & & & & \\
\hline & & Z & $\mathrm{S}$ & $\mathrm{C}$ & $\mathrm{S}$ \\
\hline & $100 \% \mathrm{MAU}=(\mathrm{n})$ & 2.5 & 6 & 2 & 2 \\
\hline \multirow{7}{*}{ 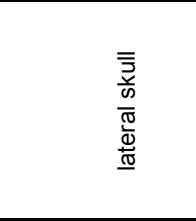 } & Articular & & 25 & & \\
\hline & Dentary & 100 & 100 & & \\
\hline & Premaxilla & 100 & 33 & & \\
\hline & Palatine & 20 & 8 & & \\
\hline & Pterygoid & 40 & 25 & & \\
\hline & Quadrate & & 25 & 25 & \\
\hline & Hyomandibular & 20 & 8 & & \\
\hline \multirow{3}{*}{ 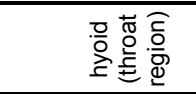 } & Ceratohyal & & 17 & & \\
\hline & Epihyal & & 17 & & \\
\hline & Hypohyal upper & & 8 & & \\
\hline \multirow{5}{*}{$\begin{array}{l}\overline{\overline{2}} \\
\frac{\vec{v}}{\omega}\end{array}$} & Basioccipital & & 17 & & \\
\hline & Ethmoid & & 17 & & \\
\hline & First Vertebra & & 17 & 100 & 100 \\
\hline & Pterotic & & 17 & & \\
\hline & Vomer & & & 50 & \\
\hline \multirow{3}{*}{ pectoral girdle } & Coracoid & & 8 & & \\
\hline & Scapula & & 17 & & \\
\hline & Number of elements & 5 & 16 & 3 & 1 \\
\hline
\end{tabular}

All five elements that occur in the H/RC Zostera layers are limited to the lateral skull region, whereas elements from all regions apart from the tail are present in the shelly layers (Figure 6.17). The lack of tail elements of this species is due to these elements not being identifiable to species. Most of the elements that are only in the shelly layers occur at relatively low frequencies. This could relate to the larger sample size in these layers. Alternately, the distinct lack of elements from areas other than the lateral skull region in the Zostera layers could indicate differential preservation of elements of this species between these assemblages. 


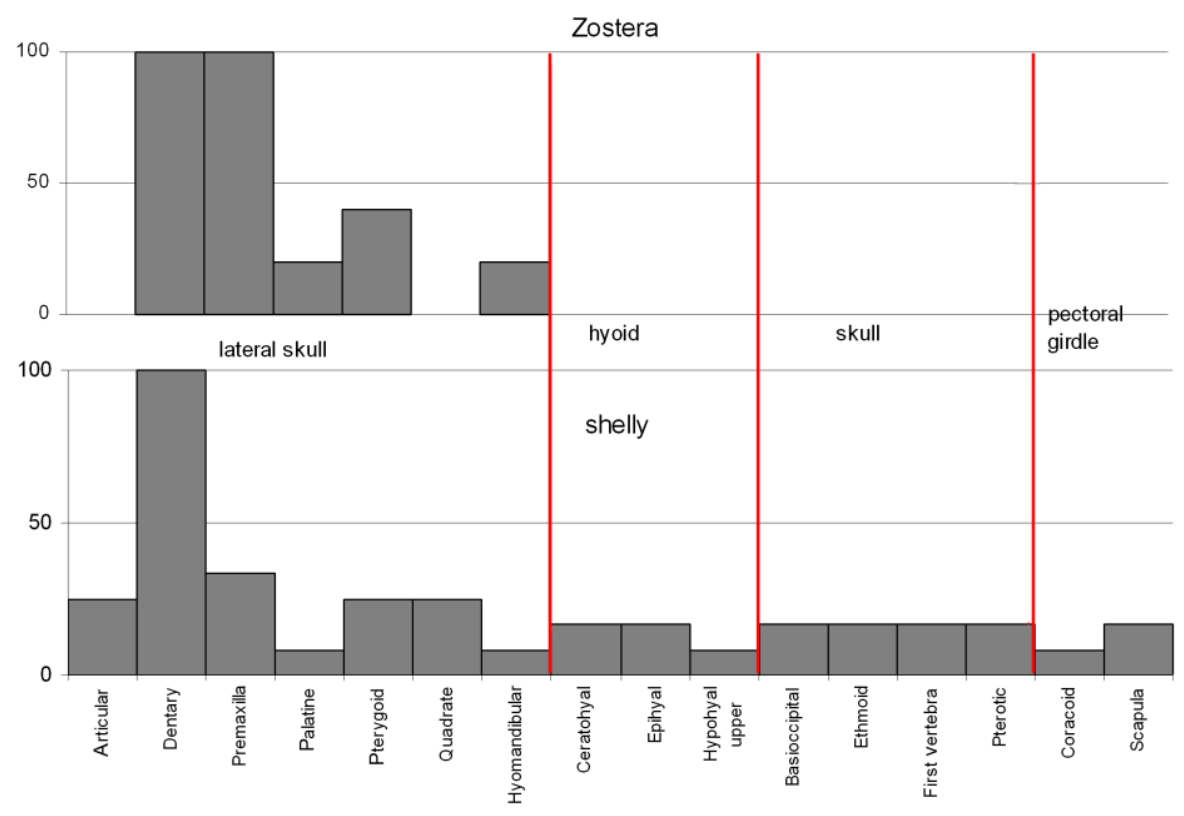

Figure 6.17. Relative skeletal element frequency (\% MAU) of Pomatomus saltatrix in the Zostera $(100 \%$ MAU $=2.5)$ and shelly $(100 \%$ MAU = 6) layers of Hoffman's/Robberg Cave

Figure 6.18 provides a graphic illustration of the differences in location of Pomatomus saltatrix elements between the Zostera and shelly layers.

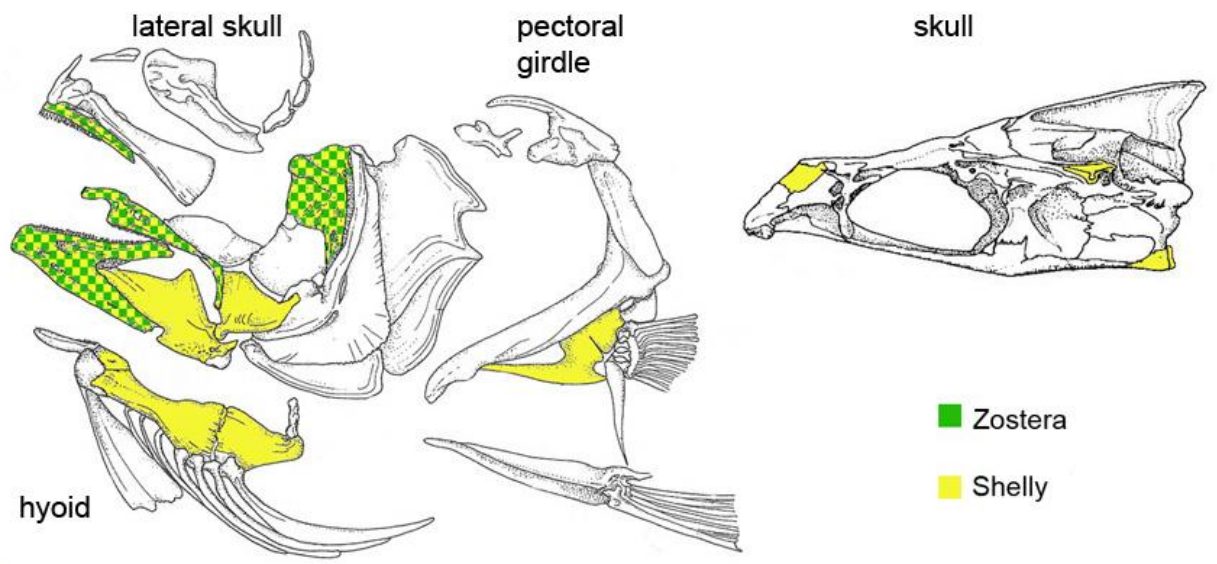

Figure 6.18. Location on skeleton of Pomatomus saltatrix elements found in the Zostera and shelly layers of Hoffman's/Robberg Cave

\subsubsection{Rhabdosargus globiceps}

This species is represented by a low number of elements, most of which occur in the H/RC shelly layers ( $n=6$ ) (Table 6.43). Only one element, the pterygoid, occurs in both the Zostera and shelly layers of $\mathrm{H} / \mathrm{RC}$, and the two elements found in the sterile $\mathrm{KR}$ layers (frontal and nasal bones) were not found in the other assemblages. 
Table 6.43. Relative skeletal element frequency (\%MAU) of Rhabdosargus globiceps. 100\% MAU = highest MAU for that species is set at 100. (H/RC: $Z=$ Zostera, $S=$ shelly) (KR: $C=$ cultural, $S=$ sterile)

\begin{tabular}{|c|c|c|c|c|c|}
\hline R. globiceps & Site type & \multicolumn{2}{|c|}{ CAVE } & \multicolumn{2}{|c|}{ OPEN } \\
\hline \multirow[b]{3}{*}{ Element location } & \multirow[b]{2}{*}{ Site } & \multicolumn{2}{|c|}{$\mathrm{H} / \mathrm{RC}$} & \multicolumn{2}{|c|}{$\mathrm{KR}$} \\
\hline & & $Z$ & $\mathrm{~S}$ & $\mathrm{C}$ & $\mathrm{S}$ \\
\hline & $100 \% \mathrm{MAU}=(\mathrm{n})$ & 0.5 & 0.5 & 0 & 1.5 \\
\hline \multirow{5}{*}{ 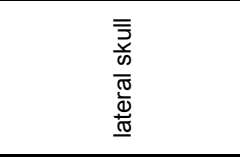 } & Dentary & & 100 & & \\
\hline & Pterygoid & 100 & 100 & & \\
\hline & Lacrimal & & 100 & & \\
\hline & Nasal & & & & 33 \\
\hline & Symplectic & & 100 & & \\
\hline \multirow{2}{*}{ hyoid (throat region) } & Ceratohyal & & 100 & & \\
\hline & Epihyal & & 100 & & \\
\hline \multirow[t]{2}{*}{ skull } & Frontal & & & & 100 \\
\hline & Number of elements & 1 & 6 & 0 & 2 \\
\hline
\end{tabular}

\subsubsection{Rhabdosargus holubi}

Four elements represent this species in all the assemblages studied. Three are in the shelly layers of $\mathrm{H} / \mathrm{RC}$, with the frontal the most common element, the other two being maxillae and parasphenoid bones which occur at low frequencies (12\% MAU each). Dentaries are present only in the cultural units of KR.

\subsubsection{Sparodon durbanensis}

This species is represented by five elements. Three occurred in the BBC LSA, where the ceratohyal was the most frequently occurring element, followed by the quadrate and epiotic. The palatine was the only element of this species present at $\mathrm{H} / \mathrm{RC}$ in the Zostera layers, and the premaxilla in the KR cultural units.

\subsubsection{Spondyliosoma emarginatum}

The largest number of elements are in BBC LSA $(n=14)$ (Table 6.44). The most common element at BBC LSA is the ceratohyal, followed by opercular and pterygoid. At H/RC the pterygoid is the most common element in both shelly and Zostera layers. The supracleithrum is the only element found at KR and was in the sterile layers. At BBC MSA interior only the articular and retroarticular were present, and only dentaries were found in the exterior. 
Table 6.44. Relative skeletal element frequency (\% MAU) of Spondyliosoma emarginatum. 100\% MAU $=$ highest MAU for that species is set at 100. $(\mathrm{H} / \mathrm{RC}: \mathrm{Z}=\mathrm{Zostera}, \mathrm{S}=$ shelly) $(\mathrm{KR}: \mathrm{C}=$ cultural, $\mathrm{S}=$ sterile) (BBC MSA: I = interior, $\mathrm{E}=$ exterior)

\begin{tabular}{|c|c|c|c|c|c|c|c|c|}
\hline S. emarginatum & Site type & CAVE & \multicolumn{2}{|c|}{ CAVE } & \multicolumn{2}{|c|}{ OPEN } & \multicolumn{2}{|c|}{ CAVE } \\
\hline \multirow[b]{3}{*}{ Element location } & \multirow[b]{2}{*}{ Site } & $\mathrm{BBC}$ & \multicolumn{2}{|c|}{$\mathrm{H} / \mathrm{RC}$} & \multicolumn{2}{|c|}{$\mathrm{KR}$} & \multicolumn{2}{|c|}{ BBC MSA } \\
\hline & & LSA & \multicolumn{2}{|l|}{$Z$} & \multirow[t]{2}{*}{$\mathrm{C}$} & $\mathrm{S}$ & $\frac{1}{05}$ & $\mathrm{E}$ \\
\hline & $100 \% \mathrm{MAU}=(\mathrm{n})$ & 7 & 1.5 & 5 & & 0.5 & 0.5 & 0.5 \\
\hline \multirow{7}{*}{ 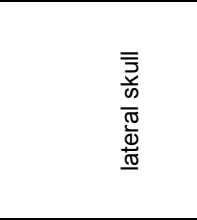 } & Articular & 21 & & & & & 100 & \\
\hline & Dentary & & & & & & & 100 \\
\hline & Premaxilla & 43 & & & & & & \\
\hline & Retroarticular & & & 10 & & & 100 & \\
\hline & Pterygoid & 86 & 100 & 100 & & & & \\
\hline & Opercular & 86 & & & & & & \\
\hline & Preopercular & 7 & & & & & & \\
\hline \multirow{3}{*}{ 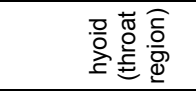 } & Ceratohyal & 100 & & & & & & \\
\hline & Epihyal & 71 & & & & & & \\
\hline & Urohyal & 71 & & & & & & \\
\hline \multirow{7}{*}{$\frac{\overline{\bar{z}}}{\frac{\vec{v}}{\omega}}$} & Epiotic & 7 & & & & & & \\
\hline & Ethmoid & & 67 & & & & & \\
\hline & Frontal & 21 & & & & & & \\
\hline & Parasphenoid & 57 & & & & & & \\
\hline & Prefrontal & 71 & & & & & & \\
\hline & Pterosphenoid & 21 & & & & & 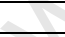 & \\
\hline & Vomer & 14 & & & & & + & \\
\hline \multirow[t]{2}{*}{ pectoral girdle } & Supracleithrum & & & & & 100 & 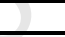 & \\
\hline & Number of elements & 14 & 2 & 2 & 0 & 1 & 2 & 1 \\
\hline
\end{tabular}

\subsubsection{Seriola lalandi}

Apart from $\mathrm{H} / \mathrm{RC}$, this species is represented by only one element, the basioccipital in the cultural layers of KR (Table 6.45). At H/RC, S. lalandi is represented by a large number of elements, most of which occur in the shelly layers $(n=39)$. The ceratohyal is the most frequently occurring element in the Zostera layers, and the parasphenoid in the shelly layers. With the exception of the postclethrum, all elements that occur in the Zostera layers are also present in the shelly layers. The majority of elements that are present only in the shelly layers occur at low frequencies ( $<15 \% \mathrm{MAU})$ and their presence could be attributed to the larger sample size. Two elements, the basisphenoid and supraoccipital, are however present at relatively high frequencies (45\%MAU and 55\% MAU respectively).

Elements from the skull region are more common and occur in higher frequencies in the shelly layers (Figure 6.19). Hyoid and tail elements generally occur at higher frequencies in the Zostera layers. There is considerable variation in the frequencies of element survival between the Zostera and shelly layers. 
Table 6.45. Relative skeletal element frequency (\%MAU) of Seriola lalandi. $100 \% \mathrm{MAU}=$ highest MAU for that species is set at 100. $(\mathrm{H} / \mathrm{RC}: \mathrm{Z}=\mathrm{Zostera}, \mathrm{S}=$ shelly $)(\mathrm{KR}: \mathrm{C}=$ cultural, $\mathrm{S}=$ sterile $)$

\begin{tabular}{|c|c|c|c|c|c|c|c|c|c|}
\hline S. lalandi & Site type & \multirow{2}{*}{\multicolumn{2}{|c|}{$\begin{array}{l}\text { CAVE } \\
\text { H/RC }\end{array}$}} & & & \multirow{2}{*}{\multicolumn{2}{|c|}{$\begin{array}{l}\text { CAVE } \\
\text { H/RC }\end{array}$}} & \multirow{2}{*}{\multicolumn{2}{|c|}{$\begin{array}{c}\text { OPEN } \\
K R\end{array}$}} \\
\hline \multirow[b]{3}{*}{ Element location } & \multirow[b]{2}{*}{ Site } & & & & & & & & \\
\hline & & Z & $\mathrm{S}$ & & & Z & $S$ & C & $\mathrm{S}$ \\
\hline & $100 \% \mathrm{MAU}=(\mathrm{n})$ & 5 & 11 & & & & & 1 & 0 \\
\hline \multirow{14}{*}{ 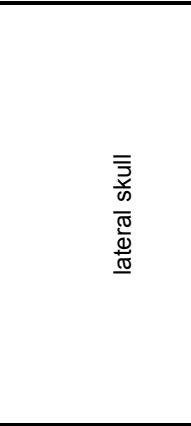 } & Articular & 60 & 55 & \multirow{11}{*}{$\begin{array}{l}\overline{\bar{z}} \\
\frac{v}{\omega}\end{array}$} & Basioccipital & 20 & 82 & 100 & \\
\hline & Dentary & 70 & 68 & & Basispenoid & & 45 & & \\
\hline & Maxilla & 30 & 50 & & First Vertebra & 20 & 36 & & \\
\hline & Premaxilla & 80 & 82 & & Frontal & & 9 & & \\
\hline & Retroarticular & 30 & 14 & & Opisthotic & 10 & 36 & & \\
\hline & Palatine & 10 & 41 & & Parasphenoid & 40 & 100 & & \\
\hline & Pterygoid & 10 & 45 & & Parietal & & 9 & & \\
\hline & Quadrate & 50 & 82 & & Prootic & 20 & 32 & & \\
\hline & Jugal & & 5 & & Pterotic & 10 & 41 & & \\
\hline & Hyomandibular & 40 & 32 & & Supraoccipital & & 55 & & \\
\hline & Interopercular & 10 & 18 & & Vomer & 40 & 45 & & \\
\hline & Opercular & & 5 & \multirow{7}{*}{ 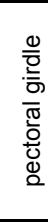 } & Basipterygium & 10 & 18 & & \\
\hline & Preopercular & & 5 & & Cleithrum & 30 & 36 & & \\
\hline & Upperpostcleithrum & & 9 & & Coracoid & 10 & 27 & & \\
\hline \multirow{4}{*}{ 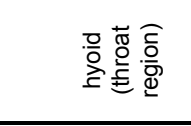 } & Ceratohyal & 100 & 18 & & Postcleithrum & 10 & 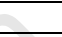 & & \\
\hline & Epihyal & 20 & 5 & & Posttemporal & & 14 & & \\
\hline & Hypohyal lower & 10 & 18 & & Scapula & 50 & 82 & & \\
\hline & Hypohyal upper & 20 & 14 & & Supracleithrum & 40 & 18 & & \\
\hline \multirow{3}{*}{ skull } & Epiotic & & 9 & \multirow{2}{*}{ tail } & Penultimate vertebrae & 20 & 18 & & \\
\hline & Exoccipital & & 14 & & Urostyle & 60 & 27 & & \\
\hline & \multicolumn{5}{|l|}{ Number of elements } & 29 & 39 & 1 & 0 \\
\hline
\end{tabular}

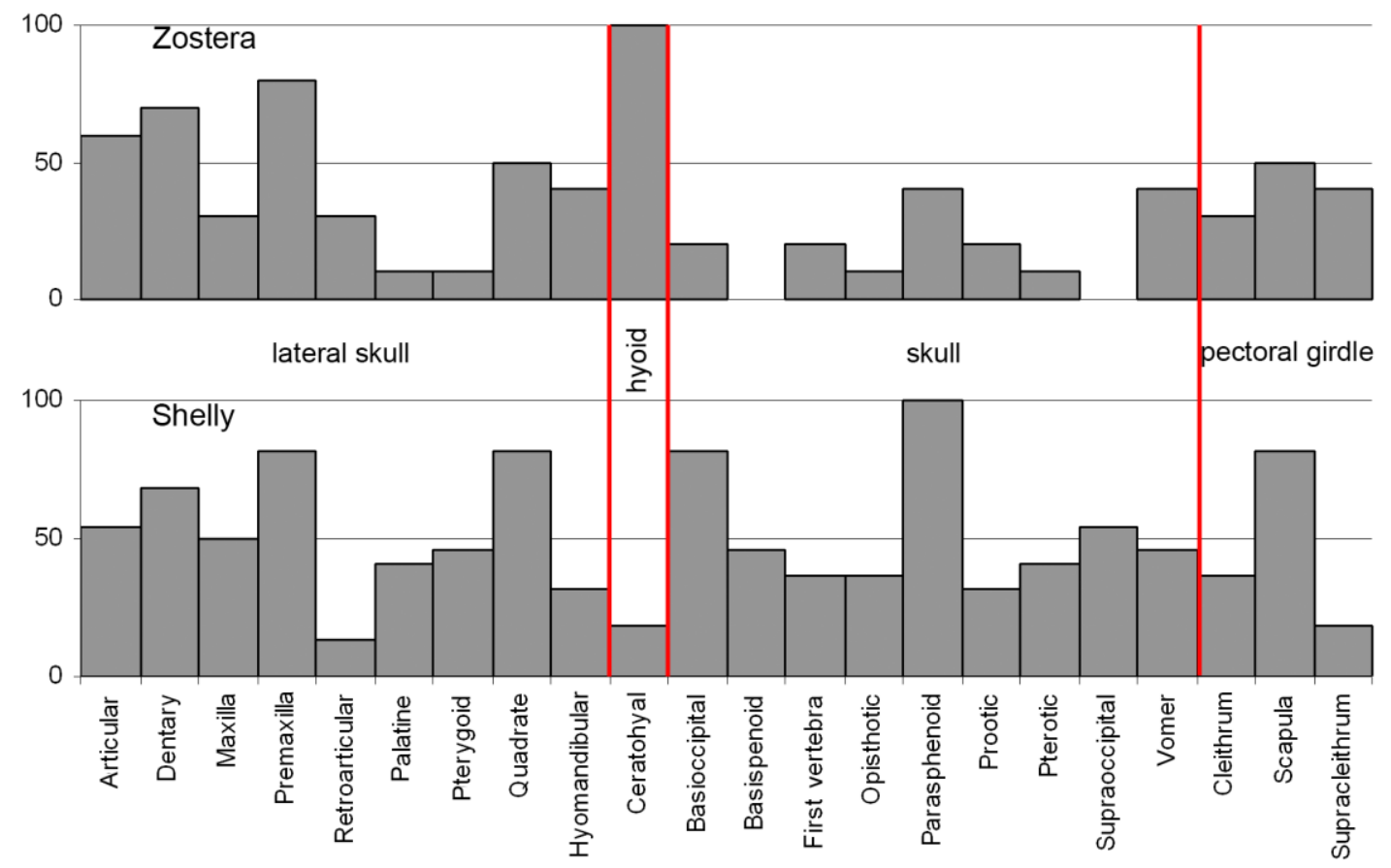

Figure 6.19. Relative skeletal element frequency (\%MAU) of Seriola lalandi in the Zostera and shelly layers at Hoffman's/Robberg Cave. Note that elements with frequencies below $25 \%$ in both the Zostera and shelly layers have been omitted. 
The large number of identifiable elements for this species is due in part to the fact that it is morphologically quite distinct from other species. The presence of tail elements indicates that whole fish were brought into the cave.

\subsubsection{Sarpa salpa}

The largest number of elements are present in the H/RC shelly layers $(n=18)$, followed by H/RC Zostera $(n=10)$ (Table 6.46). A total of 5 elements were found at KR. The three elements that occur in the cultural units of KR are also present in the sterile units. All elements that are preserved in the KR assemblage are also present in $\mathrm{H} / \mathrm{RC}$ in both the shelly and Zostera layers. At KR the best preserved elements are first vertebrae in the cultural and dentaries in the sterile units.

Table 6.46. Relative skeletal element frequency (\%MAU) of Sarpa salpa. $100 \% \mathrm{MAU}=$ highest MAU for that species is set at 100. (H/RC: $Z=$ Zostera, $S=$ shelly) (KR: $C=$ cultural, $S=$ sterile)

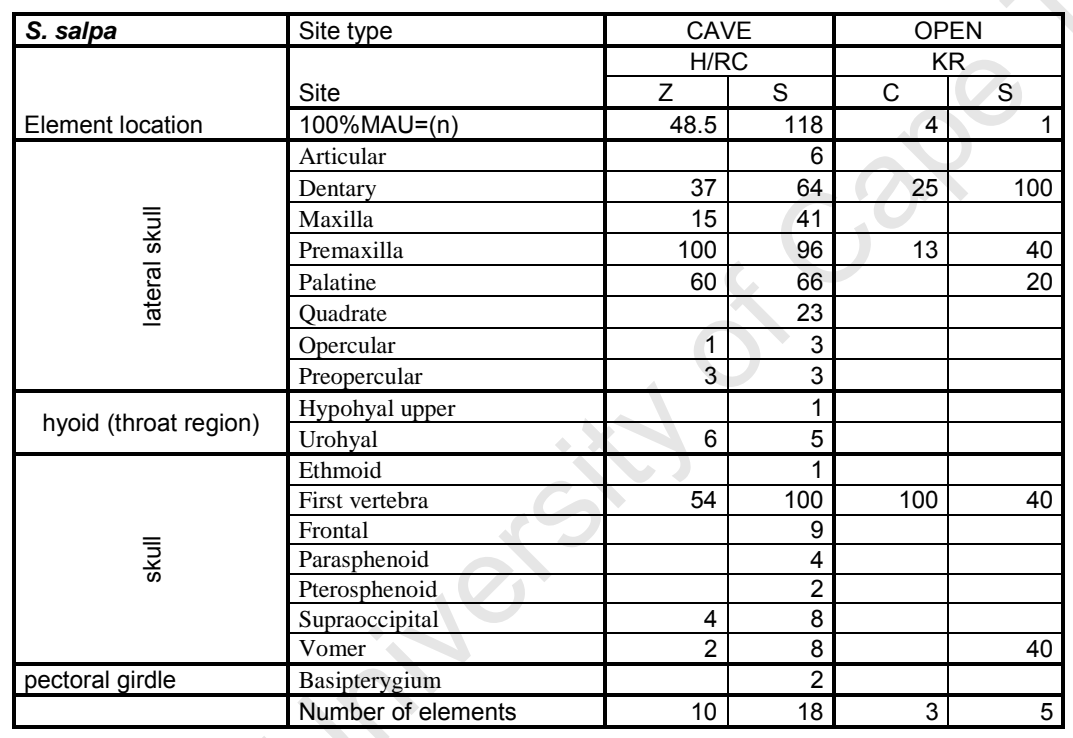

First vertebrae are also the best preserved elements in the shelly layers of $\mathrm{H} / \mathrm{RC}$, followed closely by premaxillae with $96 \% \mathrm{MAU}$. In the Zostera layers of $\mathrm{H} / \mathrm{RC}$ premaxillae are the most common. With the exception of the quadrate, which is not present in the Zostera layers, the same five elements are best preserved in both the Zostera and shelly layers (Figure 6.20). Hyoid and skull elements occur in low frequencies apart from first vertebrae, and the basipterygium is the only pectoral girdle element present, restricted to the shelly layers. 


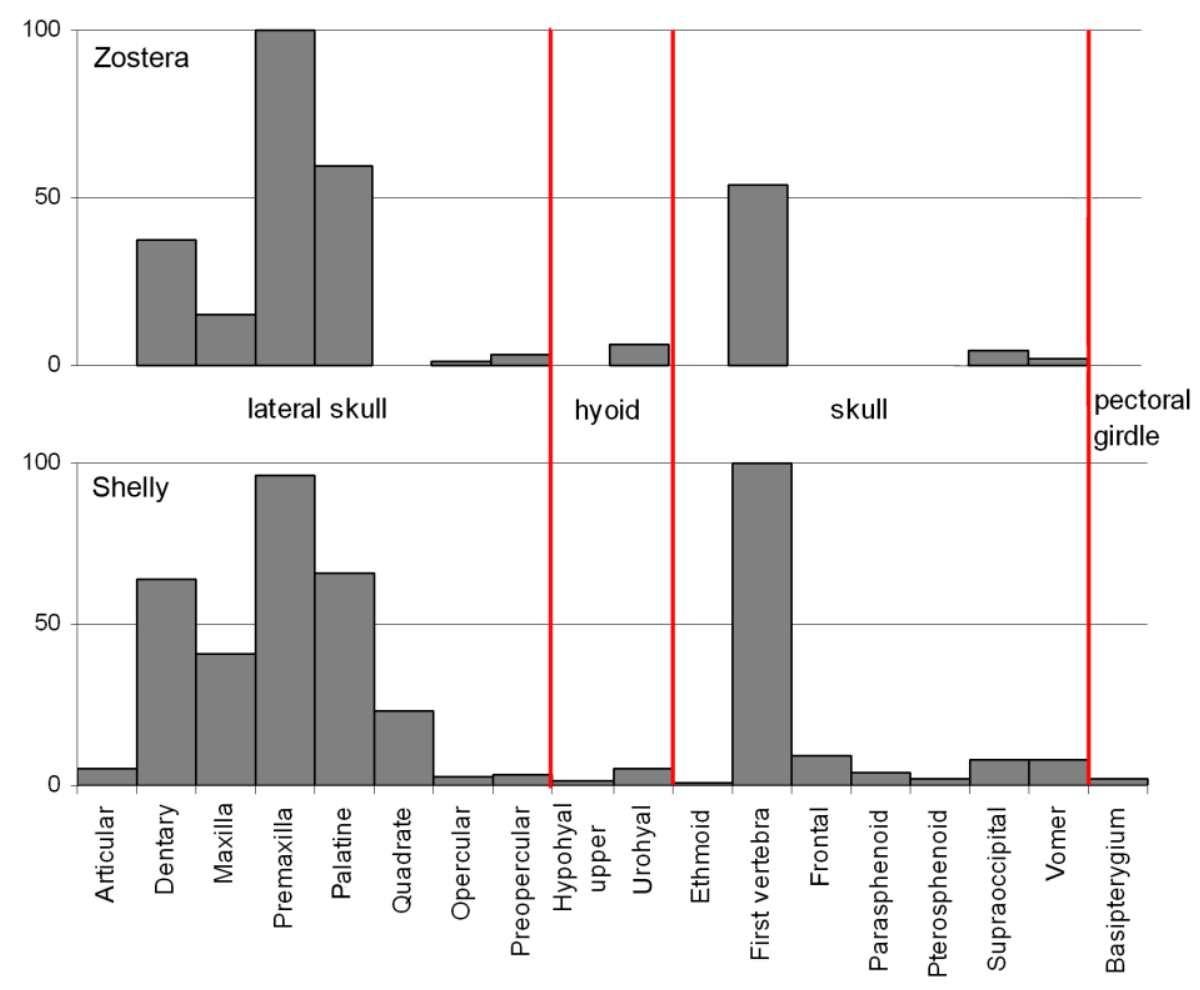

Figure 6.20. Relative skeletal element frequency (\% MAU) of Sarpa salpa in the Zostera and shelly layers at Hoffman's/Robberg Cave

\subsubsection{Scomber japonicus}

Very few elements of this species were recorded $(n=4)$. In the open KR MSA sterile layers it is represented by dentaries and premaxillae, and by first vertebrae in the cultural layers. The pterotic is the only element present in the shelly layers of H/RC.

\subsection{Skeletal element completeness based on percentage}

The degree of completeness, which can also be seen as the degree of fragmentation, of each specimen was recorded using five percentage categories (bone completeness scores) starting from $0-20 \%$ (very incomplete or fragmented) to $80-100 \%$ (very complete or mostly intact). These qualitative measures give an indication of what proportion of the total element is left, and therefore the amount of physical damage to each element. The wide range of species that are not present at all sites and the generally small number of specimens in the MSA and open LSA assemblages made it difficult to assess the completeness of elements across sites per individual species. In addition, the considerable variation in elements present per species between sites did not allow comparison and meaningful interpretation of the completeness of elements of most species based on the 
current data. The many species in the assemblages studied here are represented by different elements at varying and generally low frequencies between sites.

The levels of completeness of Liza richardsonii elements were compared as this species occurs at all sites and in relatively high numbers compared to other species. The BBF 1 and 6 data were excluded due to the low numbers. In order to compare the completeness of $L$. richardsonii elements across sites a large number of elements had to be excluded as they often only occurred in one or two of the assemblages.

Only elements that occurred in all three of the LSA cave assemblages (BBC LSA, H/RC Zostera and H/RC shelly) were included in Figure 6.21 and Table 6.47. The BBC LSA elements are generally more complete than those at H/RC. The difference in element completeness between the BBC LSA and H/RC shelly as well as Zostera and shelly combined, is statistically significant ( $\mathrm{K}-\mathrm{S}$ statistic $=0.22, \mathrm{p}<0.05$, in both instances). There is no significant difference in element completeness between the BBC LSA and $\mathrm{H} / \mathrm{RC}$ Zostera elements $(\mathrm{K}-\mathrm{S}$ statistic $=0.15, \mathrm{p}<0.05)$. Element completeness scores are very similar between the Zostera and shelly layers at $\mathrm{H} / \mathrm{RC}$, with the exception of ultimate vertebrae and epiotic bones that are more complete in the Zostera layers, and hyomandibular bones are more complete in the shelly layers. The differences between the Zostera and shelly layers are not statistically significant ( $\mathrm{K}-\mathrm{S}$ statistic $=0.09, \mathrm{p}<0.05)$. The numbers of individual elements across sites were too low to allow for significance testing at this scale.

Table 6.47. Percentage per bone completeness score of the most common Liza richardsonii elements from Blombos Cave Later Stone Age and Hoffman's/Robberg Cave Zostera and shelly layers. Calculations are based on NISP. The \% per completeness score at the base of the table refers to the percentage of the total elements that fall into each of the five completeness score categories

\begin{tabular}{|c|c|c|c|c|c|c|c|c|c|c|c|c|c|c|c|c|c|c|}
\hline \multirow[b]{3}{*}{ ELEMENT } & \multicolumn{6}{|c|}{ BBC LSA } & \multicolumn{6}{|c|}{ H/RC ZOSTERA } & \multicolumn{6}{|c|}{ H/RC SHELLY } \\
\hline & \multirow[b]{2}{*}{ NISP } & \multicolumn{5}{|c|}{$\%$ COMPLETE } & \multirow[b]{2}{*}{ NISP } & \multicolumn{5}{|c|}{$\%$ COMPLETE } & \multirow[b]{2}{*}{ NISP } & \multicolumn{5}{|c|}{$\%$ COMPLETE } \\
\hline & & $0-20$ & $20-40$ & $40-60$ & $60-80$ & $80-100$ & & $0-20$ & $20-40$ & $40-60$ & $60-80$ & $80-100$ & & $0-20$ & $20-40$ & $40-60$ & $60-80$ & $80-100$ \\
\hline Basioccipital & 16 & & & 19 & 19 & 63 & 4 & & & 25 & 50 & 25 & 26 & & 19 & 19 & 31 & 31 \\
\hline Ceratohyal & 13 & & & & 23 & 77 & 4 & & & & 25 & 75 & 36 & & & 6 & 22 & 72 \\
\hline Cleithrum & 36 & 3 & 22 & 14 & 22 & 39 & 5 & & 40 & 40 & 20 & & 77 & 3 & 45 & 35 & 9 & 8 \\
\hline Epiotic & 6 & & & & & 100 & 4 & & & & 25 & 75 & 46 & & & 7 & 65 & 28 \\
\hline Exoccipital & 8 & & & & 50 & 50 & 5 & & & 20 & 20 & 60 & 49 & & 4 & 20 & 14 & 61 \\
\hline Frontal & 5 & & & 20 & 40 & 40 & 2 & & 50 & & & 50 & 39 & 3 & 10 & 15 & 15 & 56 \\
\hline Hyomandibular & 13 & & & & 23 & 77 & 5 & & & 40 & 40 & 20 & 56 & 7 & 18 & 16 & 18 & 41 \\
\hline Penultimate vertebra & 9 & & & & & 100 & 6 & & & & & 100 & 41 & & & & & 100 \\
\hline Pterotic & 8 & & & 13 & & 88 & 3 & & 33 & & 33 & 33 & 63 & 2 & 3 & 27 & 46 & 22 \\
\hline Ultimate vertebra & 15 & & & & 20 & 80 & 8 & & & & 50 & 50 & 49 & & & 4 & 49 & 47 \\
\hline$\%$ per completeness s & & 1 & 6 & 8 & 20 & 65 & & & 9 & 13 & 28 & 50 & & 2 & 12 & 17 & 27 & 43 \\
\hline
\end{tabular}




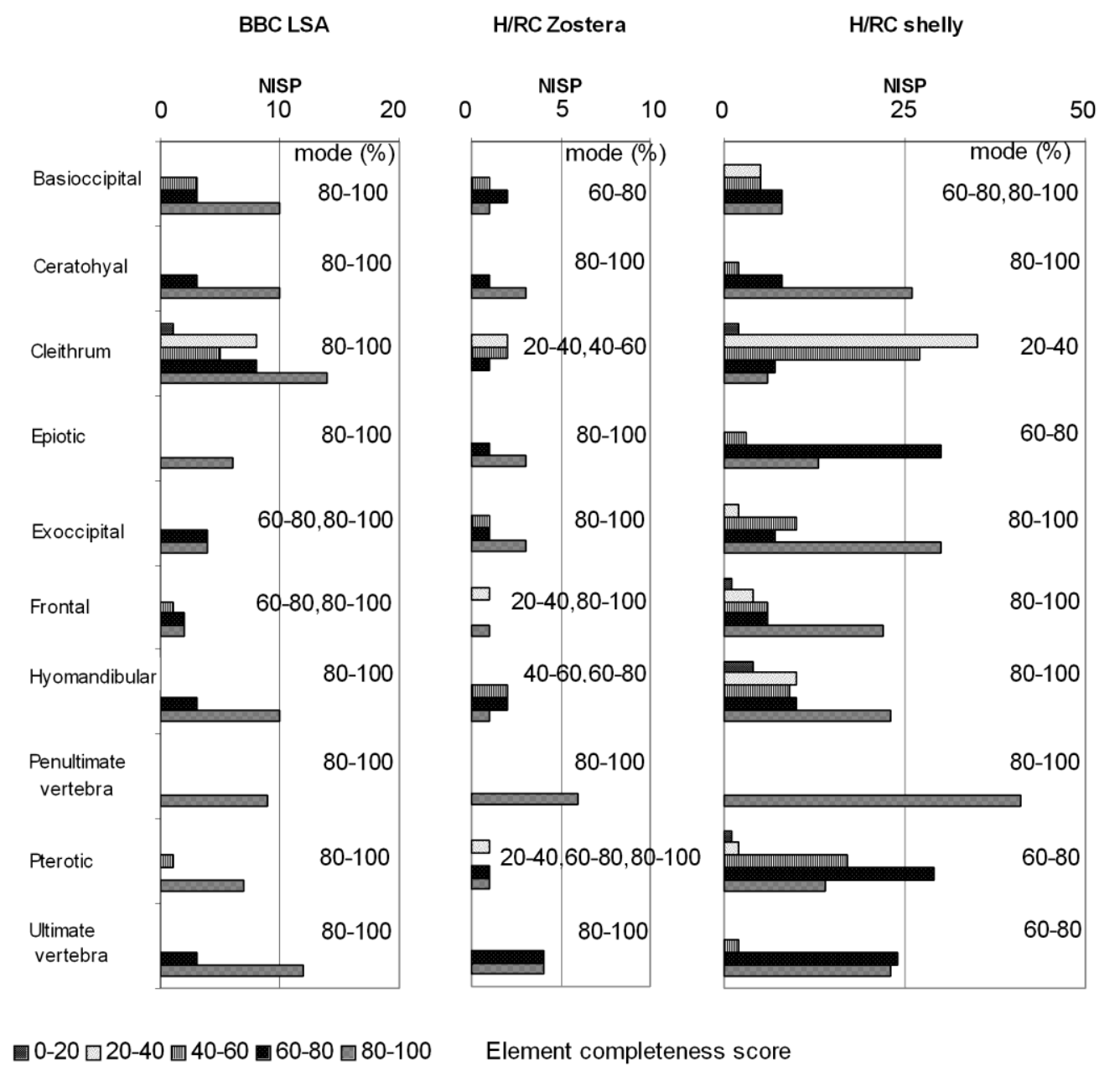

Figure 6.21. Distribution of element completeness scores of the most common Liza richardsonii elements from Blombos Cave Later Stone Age and Hoffman's Cave Zostera and shelly layers based on NISP. Stacked bars on the left indicate the completeness scores. Scores are $0-20 \%, 20-40 \%, 40-60 \%$, $60-80 \%$ and $80-100 \%$ complete (see legend at bottom of chart). The most frequent score (mode) per element is indicated to the right of that element per assemblage.

Due to the low number of Liza richardsonii elements in the BBC MSA the data from the three phases have been combined (Table 6.48, Figure 6.22). Likewise, the KR data for cultural and sterile units have been combined respectively per member. First vertebrae and frontal bones are more complete in the cultural units at $\mathrm{KR}$, and premaxillae are more complete in the sterile units. Basioccipital, epihyal and hyomandibular bones are better preserved at BBC MSA than at KR. Dentaries and parasphenoid bones are less well preserved at BBC MSA compared to the KR units. All of the BBC MSA specimens are $>40 \%$ complete, whereas in the KR assemblages element completeness scores range from very fragmented to mostly intact. Combined element completeness scores indicate that the 
majority of specimens in the BBC MSA and KR cultural units fall into the $80-100 \%$ score category, followed by the $60-80 \%$ category (Table 6.48). Most of the specimens in the KR sterile units fall in the $40-60 \%$ score category, followed by the $80-100 \%$ category.

Table 6.48. Percentage per element completeness score of the most common Liza richardsonii elements from the Klasies River main site cultural and sterile units and Blombos Cave Middle Stone Age. Calculations are based on NISP. The \% per completeness score at the base of the table refers to the percentage of the total elements that fall into each of the five completeness score categories

\begin{tabular}{|c|c|c|c|c|c|c|c|c|c|c|c|c|c|c|c|c|c|c|}
\hline \multirow[b]{3}{*}{ ELEMENT } & \multicolumn{6}{|c|}{ KR STERILE } & \multicolumn{6}{|c|}{ KR CULTURAL } & \multicolumn{6}{|c|}{ BBC MSA } \\
\hline & \multirow[b]{2}{*}{$\mathrm{NISP}$} & \multicolumn{5}{|c|}{$\%$ COMPLETE } & \multirow[b]{2}{*}{ NISP } & \multicolumn{5}{|c|}{$\%$ COMPLETE } & \multirow[b]{2}{*}{ NISP } & \multicolumn{5}{|c|}{$\%$ COMPLETE } \\
\hline & & $0-20$ & $20-40$ & $40-60$ & $60-80$ & $80-100$ & & $0-20$ & $20-40$ & $40-60$ & $60-80$ & $80-100$ & & $0-20$ & $20-40$ & $40-60$ & $60-80$ & $80-100$ \\
\hline Basioccipital & 3 & & & 67 & 33 & & 2 & & 50 & 50 & & & 1 & & & & & 100 \\
\hline Dentary & 2 & & & 50 & 50 & & & & & & & & 1 & & & & 100 & \\
\hline Epihyal & 1 & & & & 100 & & & & & & & & 1 & & & & & 100 \\
\hline Exoccipital & 7 & & & & 29 & 71 & & & & & & & 1 & & & & 100 & \\
\hline First Vertebra & 11 & & & 82 & 9 & 9 & 9 & & & 11 & 56 & 33 & & & & & & \\
\hline Frontal & 6 & 33 & 17 & 33 & 17 & & 1 & & & & 100 & & & & & & & \\
\hline Hyomandibular & 1 & 100 & & & & & & & & & & & 3 & & & & 33 & 67 \\
\hline Opercular & 1 & & 100 & & & & 2 & 50 & 50 & & & & s & 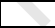 & & & & \\
\hline Parasphenoid & 4 & & & & & 100 & & & & & & & 1 & & & 100 & & \\
\hline Penultimate vertebra & 1 & & & & & 100 & 4 & & & & & 100 & 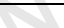 & & & & & \\
\hline Premaxilla & 6 & & & 17 & 50 & 33 & 1 & & & 100 & & & & & & & & \\
\hline Ultimate vertebra & & & & & & & 2 & & & & 50 & 50 & 5 & & & & 20 & 80 \\
\hline $\begin{array}{l}\text { \% per completeness } \\
\text { score }\end{array}$ & & 7 & 5 & 35 & 23 & 30 & & 5 & 10 & 14 & 33 & 38 & & & & 8 & 31 & 62 \\
\hline
\end{tabular}

The majority of specimens from both the interior and exterior excavations of BBC MSA fall in the mostly complete (80-100\%) category (Table 6.49). The interior and exterior contain a similar frequency of very fragmented specimens (0-20\% complete). The second most common completeness category in the exterior is 40-60\%, whereas the interior specimens are slightly more intact, with $19 \%$ of specimens falling in the $60-80 \%$ category. As there were too few specimens in the exterior for which completeness scores could be recorded, it was not possible to determine whether these differences were significantly different. 


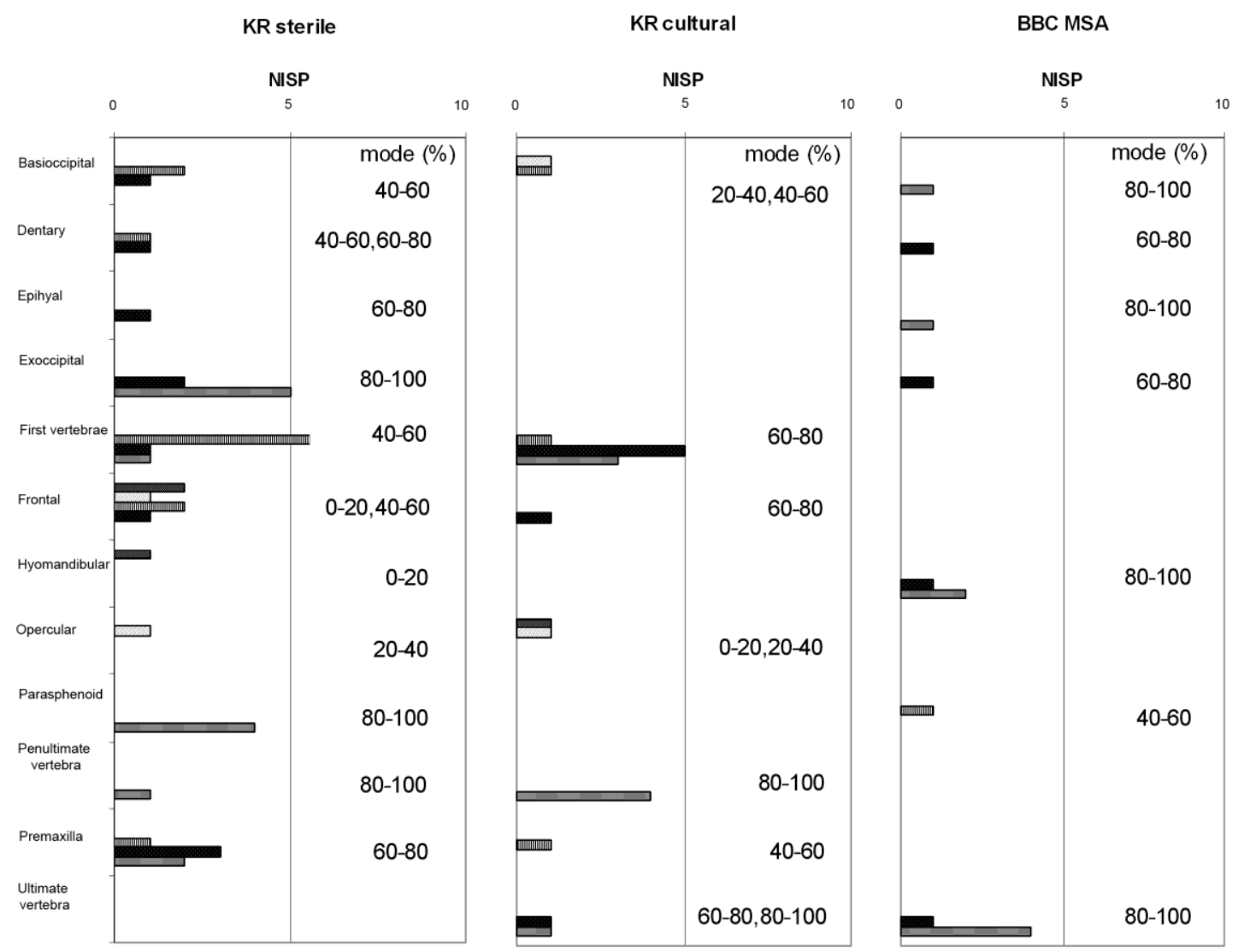

$\square$ 0-20 $\square$ 20-40 $\square$ 40-60 임 60-80 $\square$ 80-100 Element completeness score

Figure 6.22. Distribution of element completeness scores of the most common Liza richardsonii elements from Klasies River main site sterile and cultural units and Blombos Cave Middle Stone Age based on NISP. Stacked bars on the left indicate the completeness scores. Scores are 0-20\%, 20-40\%, 40-60\%, $60-80 \%$ and $80-100 \%$ complete (see legend at bottom of chart). The most frequent score (mode) per element is indicated to the right of that element per assemblage.

Table 6.49. Percentage per completeness score of all elements and species combined from the interior and exterior of the Blombos Cave Middle Stone Age excavations. Calculations are based on NISP of elements for which \% completeness scores were recorded

\begin{tabular}{|l|r|r|r|r|r|r|}
\hline & & \multicolumn{5}{|c|}{ \% COMPLETENESS SCORE } \\
\hline ASSEMBLAGE & \multicolumn{1}{|c|}{ NISP } & $\mathbf{0 - 2 0}$ & $\mathbf{2 0 - 4 0}$ & $\mathbf{4 0 - 6 0}$ & \multicolumn{1}{c|}{$\mathbf{6 0 - 8 0}$} & \multicolumn{1}{c|}{$\mathbf{8 0 - 1 0 0}$} \\
\hline BBC MSA exterior & 37 & 11 & 5 & 24 & 11 & $\mathbf{4 9}$ \\
\hline BBC MSA interior & 193 & 10 & 11 & 10 & 19 & 50 \\
\hline
\end{tabular}

The degree of fragmentation of specimens was compared between the cultural and sterile units per member from KR (Table 6.50). Specimens in the cultural units of MSA I are slightly more complete than those from the sterile units of the MSA I but the difference is not statistically significant $(\mathrm{K}-\mathrm{S}$ statistic $=0.09, \mathrm{p}<0.05)$. In the MSA II, specimens are significantly more complete in the cultural units $(\mathrm{K}-\mathrm{S}$ statistic $=0.25, \mathrm{p}<0.05)$. The degree 
of fragmentation of specimens from the cultural and sterile units of the HP is very similar, with most specimens in the $80-100 \%$ completeness category. Specimens from the cultural and sterile units of the MSA III were the least fragmented, with most in the $80-100 \%$ completeness category, and no specimens represented less than $20 \%$ of an element. The total numbers of specimens per cultural and sterile units of the MSA III were too low to allow significance testing.

Table 6.50. Percentage per completeness score of all elements and species for the cultural and sterile units respectively per member from Klasies River. Calculations are based on NISP of elements for which \% completeness scores were recorded

\begin{tabular}{|l|r|r|r|r|r|r|}
\hline & \multicolumn{6}{|c|}{ \% COMPLETENES SCORE } \\
\hline ASSEMBLAGE & \multicolumn{1}{|c|}{ NISP } & \multicolumn{1}{|c|}{$\mathbf{0 - 2 0}$} & $\mathbf{2 0 - 4 0}$ & $\mathbf{4 0 - 6 0}$ & $\mathbf{6 0 - 8 0}$ & $\mathbf{8 0 - 1 0 0}$ \\
\hline MSA III C & 29 & 0 & 10 & 10 & 28 & 52 \\
\hline MSA III S & 34 & 0 & 3 & 15 & 18 & 65 \\
\hline HP C & 82 & 2 & 9 & 15 & 22 & 52 \\
\hline HP S & 151 & 5 & 7 & 19 & 20 & 50 \\
\hline MSA II C & 163 & 1 & 7 & 12 & 15 & 66 \\
\hline MSA II S & 199 & 5 & 11 & 22 & 21 & 41 \\
\hline MSA I C & 168 & 2 & 11 & 14 & 29 & 44 \\
\hline MSA I S & 171 & 3 & 15 & 19 & 27 & 36 \\
\hline
\end{tabular}

The completeness scores of all elements and species were combined to determine whether there was any general patterning of element fragmentation between assemblages (Table 6.51). With the exception of BBF 6 , where a score of $60-80 \%$ was most common, the most frequent completeness score in all assemblages was 80-100\%. The BBF 6 assemblage is most fragmented, with the highest number of specimens (18\%) in the $0-20 \%$ completeness category.

Table 6.51. Percentage per completeness score of all elements and species combined per assemblage. Calculations are based on NISP of elements for which \% completeness scores were recorded

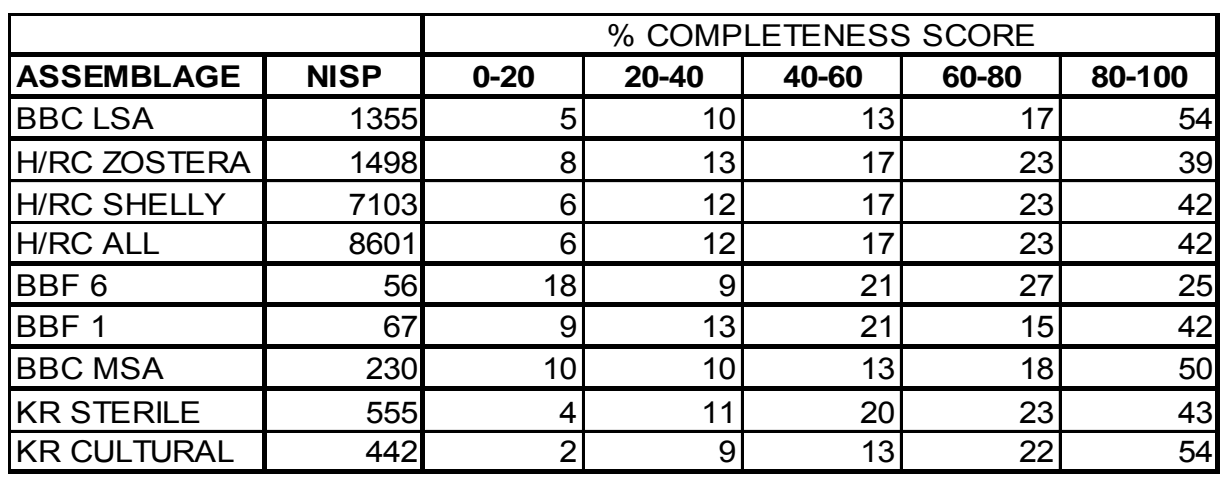

The KR cultural units and the BBC LSA and MSA contained the best preserved elements, with $50 \%$ or more specimens having completeness scores of $80-100 \%$. Element 
preservation is very similar between the Zostera and shelly layers of $\mathrm{H} / \mathrm{RC}$, and the \% completeness scores of the shelly layers are identical to that of the Zostera and shelly layers combined. The BBC LSA elements are significantly less fragmented than those from the combined H/RC layers $(\mathrm{K}-\mathrm{S}$ statistic $=0.13$, p<0.05) and BBF $6(\mathrm{~K}-\mathrm{S}$ statistic $=$ $0.29, \mathrm{p}<0.05)$.

The KR sterile units contain more fragmented elements than the cultural units, and the difference is statistically significant $(\mathrm{K}-\mathrm{S}$ statistic $=0.11, \mathrm{p}<0.05)$. There are no significant differences in element fragmentation between the BBC MSA and KR cultural or sterile units $(\mathrm{K}-\mathrm{S}$ statistic $=0.09, \mathrm{p}<0.05$ and $0.06, \mathrm{p}<0.05$, respectively).

Elements in the KR cultural units are significantly better preserved than those from BBF 1, BBF 6 and H/RC (K-S statistic $=0.20,0.29$ and 0.12, p<0.05, respectively). Element completeness in the KR sterile units is similar to that of $\mathrm{H} / \mathrm{RC}, \mathrm{BBF} 1$ and $\mathrm{BBF} 6$, and differs from BBC LSA in containing more fragmented elements in the $20-80 \%$ score categories $(\mathrm{K}-\mathrm{S}$ statistic $=0.11, \mathrm{p}<0.05)$. Element preservation is very similar between the BBC MSA and LSA assemblages.

\subsection{Skeletal element preservation based on zones}

In addition to assessing element fragmentation based on completeness scores, the zones present on each specimen were recorded to indicate which portion of each element was present. This data was collected to determine whether the same parts of specific species' elements survived between different assemblages. As detailed in Chapter 5, zones lacking the 'MNI feature' were not recorded in order to avoid counting the same element twice, even if more than $50 \%$ of the zone was present. Due to this, some zones of elements may be underrepresented. Fortunately few specimens in the assemblages studied here had $>50 \%$ of a zone present but lacked the 'MNI feature', due to the generally small size of each zone. More zones will be assigned per element in future studies to avoid underrepresenting particular parts of elements when assessing skeletal element preservation based on zones.

Due to the large variation in species and element representation between assemblages, the current data of most species are not sufficient to allow comparison of zone preservation across all assemblages. The zones of elements of Liza richardsonii, a species that did 
occur in all assemblages, were compared to establish whether there was any difference between the parts that survived between sites (Table 6.52).

Table 6.52. Comparison of the zones present of the most common elements of Liza richardsonii between all assemblages. $\mathrm{N}=$ the total number of specimens (NISP) for which zone data was recorded

\begin{tabular}{|c|c|c|c|c|c|c|c|c|c|c|c|c|c|c|c|c|c|}
\hline \multirow{3}{*}{$\begin{array}{l}\text { L. richardsonii } \\
\text { Element } \\
\end{array}$} & \multirow{3}{*}{ ZONE } & \multicolumn{2}{|c|}{\begin{tabular}{l|l} 
BBF 1 \\
\end{tabular}} & \multicolumn{2}{|c|}{$\mathrm{BBF} 6$} & \multicolumn{4}{|c|}{$\overline{B B C}$} & \multicolumn{4}{|c|}{$\mathrm{H} / \mathrm{RC}$} & \multicolumn{4}{|c|}{$\overline{\mathrm{KR}}$} \\
\hline & & & & & & \multicolumn{2}{|c|}{ LSA } & \multicolumn{2}{|c|}{ MSA } & \multicolumn{2}{|c|}{ Zostera } & \multicolumn{2}{|c|}{ Shelly } & \multicolumn{2}{|c|}{ Cultural } & \multicolumn{2}{|c|}{ Sterile } \\
\hline & & $\%$ & $\mathrm{~N}$ & $\%$ & $\mathrm{~N}$ & $\%$ & $\mathrm{~N}$ & $\%$ & $\mathrm{~N}$ & $\%$ & $\mathrm{~N}$ & $\%$ & $\mathrm{~N}$ & $\%$ & $\mathrm{~N}$ & $\%$ & $\mathrm{~N}$ \\
\hline \multirow[b]{3}{*}{ Basioccipital } & $\bar{A}$ & & & 100 & 1 & 100 & 16 & 100 & 1 & 100 & 4 & 100 & 2 & 100 & 2 & 100 & 3 \\
\hline & B & & & 100 & & 94 & & 100 & & 100 & & 100 & & & & & \\
\hline & $\mathrm{C}$ & & & & & 88 & & 100 & & & & & & & & 33 & \\
\hline \multirow[b]{3}{*}{ Ceratohyal } & $\mathrm{A}$ & & & & & 100 & 13 & 100 & 2 & 100 & 4 & 100 & 3 & & & & \\
\hline & $B$ & & & & & 100 & & 100 & & 100 & & 100 & & & & & \\
\hline & $\bar{C}$ & & & & & 100 & & 100 & & 100 & & 100 & & & & & \\
\hline \multirow[b]{4}{*}{ Cleithrum } & $\bar{A}$ & & & & & 72 & 38 & 50 & 2 & 100 & 5 & 100 & 2 & & & & \\
\hline & B & & & & & 75 & & 100 & & 100 & & 100 & & & & & \\
\hline & $\mathrm{C}$ & & & & & 78 & & 50 & & 20 & & 100 & & & & & \\
\hline & D & & & & & 61 & & 50 & & & & 100 & & & & & \\
\hline \multirow[b]{5}{*}{ Dentary } & $\bar{A}$ & & & & & 100 & 7 & 100 & 1 & 100 & 2 & 100 & 2 & & & 100 & 2 \\
\hline & B & & & & & 100 & & 100 & & 100 & & 100 & & & & 100 & \\
\hline & $\mathrm{C}$ & & & & & 100 & & 100 & & 100 & & 100 & & & & 100 & \\
\hline & $\mathrm{D}$ & & & & & 71 & & & & 100 & & 100 & & & & 100 & \\
\hline & $E$ & & & & & 71 & & 100 & & & & & & & . & 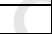 & 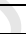 \\
\hline \multirow[b]{3}{*}{ First Vertebra } & $\bar{A}$ & & & & & 100 & 11 & & & 100 & \begin{tabular}{|l|}
3 \\
\end{tabular} & & & 100 & 9 & 100 & 7 \\
\hline & $B$ & & & & & 100 & & & & 100 & & & & 67 & & 86 & \\
\hline & C & & & & & 82 & & & & 67 & & & & 56 & & 100 & \\
\hline \multirow[b]{3}{*}{ Hyomandibular } & $\mathrm{A}$ & & & & & 100 & 13 & 100 & 3 & 100 & \begin{tabular}{|l|}
5 \\
\end{tabular} & 83 & 5 & 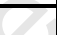 & & 100 & 6 \\
\hline & $B$ & & & & & 77 & & 33 & & 80 & & 67 & - & $\infty$ & & 17 & \\
\hline & $\mathrm{C}$ & & & & & 100 & & 67 & & 60 & & 67 & & & & 50 & \\
\hline \multirow[b]{3}{*}{ Opercular } & $\bar{A}$ & & & & & 71 & 14 & & & & \begin{tabular}{|l|}
2 \\
\end{tabular} & 100 & $\overline{6}$ & 100 & 2 & 100 & 1 \\
\hline & $B$ & & & & & 71 & & & & 100 & & 50 & & & & & \\
\hline & C & & & & & 64 & & & & 50 & & 83 & & & & & \\
\hline \multirow[b]{3}{*}{ Parasphenoid } & $\bar{A}$ & & & & & 89 & 9 & 100 & $\overline{1}$ & 100 & 1 & 50 & 4 & & & & 1 \\
\hline & $B$ & & & & & 78 & & & & & & 75 & & & & & \\
\hline & C & & & & & 56 & & 100 & & 100 & & 25 & & & & 100 & \\
\hline \multirow[b]{2}{*}{ Penultimate vertebra } & $\bar{A}$ & & & & & 100 & 9 & & & 100 & \begin{tabular}{|l|}
6 \\
\end{tabular} & 100 & 20 & 100 & 4 & 100 & $\overline{4}$ \\
\hline & $B$ & & & & & 100 & & & & 100 & & 100 & & 100 & & 100 & \\
\hline \multirow[b]{2}{*}{ Ultimate vertebra } & $\bar{A}$ & 100 & 1 & 100 & 1 & 100 & 15 & 100 & 3 & 100 & 8 & 100 & 5 & 100 & 6 & 100 & 2 \\
\hline & $B$ & 100 & & 100 & & 100 & 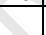 & 100 & & 100 & & 100 & & 100 & & 100 & \\
\hline
\end{tabular}

Where ceratohyals occurred, there was no difference in the zones preserved between the MSA and LSA sites, or between the Zostera and shelly layers of H/RC (Figure A-1d). Only zone A of the opercular was preserved in the KR assemblage, whereas this zone was missing from $\mathrm{H} / \mathrm{RC}$ Zostera, and all zones of this element occured at $\mathrm{H} / \mathrm{RC}$ shelly and BBC LSA (Figure A-1m). Penultimate and ultimate vertebrae were equally well preserved in all assemblages, with both zones $\mathrm{A}$ and $\mathrm{B}$ present. Zone $\mathrm{C}$ of the first vertebra was better preserved in the sterile layers of KR relative to the cultural layers. Zone E of dentaries were present only in the BBC MSA and LSA assemblages (Figure A-1g). All three zones of the hyomandibular bones were present in the assemblages where this element occurred, and zone A was consistently the most frequently occurring zone (Figure A-1j). Parasphenoids (Figure A-1b) in BBC MSA and H/RC Zostera layers lacked zone B, whereas this zone was present in BBC LSA and H/RC shelly. 
Based on this limited data, there does not seem to be any relationship between the Liza richardsonii zones present and site type or age. This exercise was not very satisfactory due to the low number of shared elements between assemblages. Larger sample sizes are needed to make meaningful comparisons of zone preservation between these sites.

As comparisons of the zone data from all assemblages was not feasible, zone preservation of a few of the more frequently occurring species was compared at a smaller scale. The zones present on elements of Galeichthys feliceps at BBC MSA and KR are listed in Table 6.53. At KR, the opercular and vomer have more zones present in the cultural units (Figure A-1m, o). Zone B of the ceratohyal (Figure A-1d) was most common in both the cultural and sterile units. Zone A and E of the dentary (Figure A-1g) was present in KR sterile units, but absent from BBC MSA. Otoliths were represented by both zones in all of the MSA assemblages. With the exception of otoliths, all other elements were present at very low frequencies and the slight differences noted here could relate to the small sample size. There is not sufficient data from the rest of the species and elements to allow further comparison of the cultural and sterile units at KR.

Table 6.53. Comparison of zones present of the most common elements of Galeichthys feliceps between the cultural and sterile layers at Klasies River main site and Blombos Cave MSA. $N=$ the total number of elements (NISP) for which zone data was recorded

\begin{tabular}{|c|c|c|c|c|c|c|c|}
\hline \multirow{4}{*}{ Element } & \multirow{4}{*}{ ZONE } & \multicolumn{6}{|c|}{ G. feliceps } \\
\hline & & \multicolumn{4}{|c|}{ KR } & \multirow{2}{*}{\multicolumn{2}{|c|}{$\begin{array}{l}\text { BBC } \\
\text { MSA }\end{array}$}} \\
\hline & & \multicolumn{2}{|c|}{ cultural } & \multicolumn{2}{|c|}{ sterile } & & \\
\hline & & $\%$ & $\mathrm{~N}$ & $\%$ & $\mathrm{~N}$ & $\%$ & $\mathrm{~N}$ \\
\hline \multirow[b]{3}{*}{ Articular } & $\mathrm{A}$ & 100 & 2 & 100 & 1 & 50 & 6 \\
\hline & $\mathrm{B}$ & 100 & & 100 & & 67 & \\
\hline & $\mathrm{C}$ & 100 & & 100 & $\bar{c}$ & 100 & \\
\hline \multirow[b]{3}{*}{ Ceratohyal } & $\mathrm{A}$ & 50 & 2 & 67 & 3 & & \\
\hline & $\mathrm{B}$ & 100 & & 100 & & & \\
\hline & C & 50 & & 67 & & & \\
\hline \multirow[b]{5}{*}{ Dentary } & A & 0 & 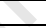 & 100 & 1 & & 1 \\
\hline & $B$ & $\mathrm{~s}_{2}$ & 8 & 100 & & 100 & \\
\hline & $\mathrm{C}$ & 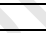 & & 100 & & 100 & \\
\hline & $\mathrm{D}$ & 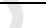 & & & & 100 & \\
\hline & $E$ & 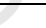 & & 100 & & & \\
\hline \multirow[b]{2}{*}{ Dorsal spine } & $A$ & 100 & 2 & & & 100 & 5 \\
\hline & $B$ & 100 & & & & 60 & \\
\hline \multirow[b]{3}{*}{ Opercular } & $\bar{A}$ & 100 & 2 & 100 & 3 & & \\
\hline & $\mathrm{B}$ & 100 & & & & & \\
\hline & $C$ & & & & & & \\
\hline \multirow[b]{2}{*}{ Otolith } & $\bar{A}$ & 100 & 38 & 100 & 15 & 100 & $\overline{6}$ \\
\hline & $\mathrm{B}$ & 100 & & 100 & & 100 & \\
\hline \multirow[b]{4}{*}{ Vomer } & $\bar{A}$ & 100 & 1 & 100 & 1 & & \\
\hline & $B$ & 100 & & 100 & & & \\
\hline & $\mathrm{C}$ & 100 & & & & & \\
\hline & $\mathrm{D}$ & 100 & & & & & \\
\hline \multirow[b]{2}{*}{ Ultimate vertebra } & $\mathrm{A}$ & 100 & 1 & 100 & 6 & & \\
\hline & $\mathrm{B}$ & 100 & & 67 & & & \\
\hline
\end{tabular}

The zones of the most common elements of Sarpa salpa, Seriola lalandi and Dichistius capensis that occurred in both the Zostera and shelly layers of $\mathrm{H} / \mathrm{RC}$ were compared to determine whether there were any differences in zone preservation between these layers 
(Table 6.54). All zones of $S$. salpa first vertebrae were present in both layers at equal frequencies. The zones of the other common elements of this species occurred at similar frequencies in both the Zostera and shelly layers.

Table 6.54. Comparison of zones present of the most common elements of Sarpa salpa, Seriola lalandi and Dichistius capensis between the Zostera and shelly layers at Hoffman's/Robberg Cave. $N=$ the total number of elements (NISP) for which zone data was recorded

\begin{tabular}{|c|c|c|c|c|c|c|c|c|c|c|c|c|c|}
\hline \multirow{3}{*}{\begin{tabular}{|l} 
Element \\
\end{tabular}} & \multirow{3}{*}{ ZONE } & \multicolumn{4}{|c|}{ S. salpa } & \multicolumn{4}{|c|}{ D. capensis } & \multicolumn{4}{|c|}{ S. lalandi } \\
\hline & & \multicolumn{2}{|c|}{ Zostera } & \multicolumn{2}{|c|}{ Shelly } & \multicolumn{2}{|c|}{ Zostera } & \multicolumn{2}{|c|}{ Shelly } & \multicolumn{2}{|c|}{ Zostera } & \multicolumn{2}{|c|}{ Shelly } \\
\hline & & $\%$ & $\mathrm{~N}$ & $\%$ & $\mathrm{~N}$ & $\%$ & $\mathrm{~N}$ & $\%$ & $\mathrm{~N}$ & $\%$ & $\mathrm{~N}$ & $\%$ & $\mathrm{~N}$ \\
\hline \multirow[b]{3}{*}{ Articular } & $A$ & & & & & 100 & 5 & 100 & 17 & 43 & 7 & 56 & 16 \\
\hline & $\mathrm{B}$ & & & & & 100 & & 100 & & 43 & & 44 & \\
\hline & C & & & & & 100 & & 100 & & 100 & & 88 & \\
\hline \multirow[b]{3}{*}{ Ceratohyal } & $\bar{A}$ & & & & & & & & & 70 & 10 & 100 & 4 \\
\hline & $B$ & & & & & & & & & 80 & & 50 & \\
\hline & $\mathrm{C}$ & & & & & & & & & 20 & & 50 & \\
\hline \multirow[b]{4}{*}{ Cleithrum } & $A$ & & & & & 17 & 6 & 43 & 21 & & 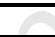 & & \\
\hline & $\mathrm{B}$ & & & & & 17 & & 33 & & & 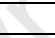 & 8 & \\
\hline & C & & & & & 50 & & 43 & & $\mathrm{c}$ & 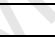 & & \\
\hline & $\mathrm{D}$ & & & & & 33 & & 29 & & 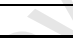 & 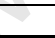 & & \\
\hline \multirow[b]{5}{*}{ Dentary } & $\mathrm{A}$ & 93 & 44 & 92 & 153 & & & & & 57 & 7 & 57 & 21 \\
\hline & $B$ & 98 & & 93 & & & & & 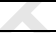 & 57 & & 62 & \\
\hline & $\mathrm{C}$ & 93 & & 97 & & & & & & 71 & & 71 & \\
\hline & $\mathrm{D}$ & 80 & & 78 & & & & & & & & 52 & \\
\hline & $E$ & 66 & & 69 & & & & 8 & & 14 & & 33 & \\
\hline \multirow[b]{3}{*}{ First vertebra } & $A$ & 100 & 26 & 100 & 120 & 100 & 3 & 100 & 3 & & & & \\
\hline & $\mathrm{B}$ & 100 & & 100 & & 100 & & 100 & & & & & \\
\hline & $\mathrm{C}$ & 100 & & 100 & & 100 & 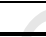 & 100 & & & & & \\
\hline \multirow[b]{3}{*}{ Hyomandibular } & $\mathrm{A}$ & & & & & 100 & 9 & 100 & 9 & & & & \\
\hline & $B$ & & & & & 22 & 10 & 67 & & & & & \\
\hline & $\mathrm{C}$ & & & & & 22 & 2 & 33 & & & & & \\
\hline \multirow[b]{4}{*}{ Maxilla } & $\mathrm{A}$ & 93 & 15 & 99 & 96 & 63 & 8 & 72 & 18 & & & & \\
\hline & $\mathrm{B}$ & 93 & & 99 & & 75 & & 78 & & & & & \\
\hline & $\mathrm{C}$ & 73 & & 80 & & 88 & & 94 & & & & & \\
\hline & $\mathrm{D}$ & 73 & & 76 & & 75 & & 83 & & & & & \\
\hline \multirow[b]{3}{*}{ Palatine } & $A$ & 98 & 46 & 98 & 158 & & & & & & & & \\
\hline & $\mathrm{B}$ & 76 & & 81 & 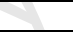 & & & & & & & & \\
\hline & $\mathrm{C}$ & 100 & & 100 & 2 & & & & & & & & \\
\hline & $A$ & & & 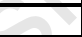 & & & & & & 20 & 5 & 69 & 16 \\
\hline & $\mathrm{B}$ & & 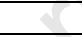 & 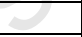 & & & & & & 20 & & 31 & \\
\hline Parasphenoid & C & & 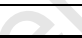 & & & & & & & 80 & & 63 & \\
\hline & $A$ & 85 & 102 & 86 & 231 & 100 & 5 & 46 & 28 & 33 & 9 & 50 & 28 \\
\hline & $\mathrm{B}$ & 95 & & 93 & & 100 & & 79 & & 44 & & 54 & \\
\hline & C & 86 & 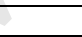 & 94 & & 100 & & 75 & & 56 & & 61 & \\
\hline & D & 68 & & 81 & & 100 & & 57 & & 22 & & 7 & \\
\hline Premaxilla & $E$ & 76 & & 88 & & 100 & & 75 & & 44 & & 43 & \\
\hline & $\mathrm{A}$ & & & & & & & & & 100 & 7 & 100 & 10 \\
\hline Pterygoid & B & & & & & & & & & 86 & & 100 & \\
\hline & $\bar{A}$ & & & & & & & & & 100 & 5 & 56 & 18 \\
\hline & $B$ & & & & & & & & & 60 & & 72 & \\
\hline Quadrate & $\mathrm{C}$ & & & & & & & & & 20 & & 83 & \\
\hline & $A$ & & & & & 100 & 7 & 100 & 7 & & & & \\
\hline Scapula & $B$ & & & & & 57 & & 100 & & & & & \\
\hline
\end{tabular}

Zones B and C of Dichistius capensis hyomandibular bones were better represented in the shelly layers (Table 6.54, Figure A-1j)). All premaxillae zones were present at equal frequencies in the Zostera layers, and there was more variation in zone frequency in the shelly layers. All zones of scapulae were present at the same frequency in the shelly layers, whereas zone B was less common in the Zostera layers (Figure A-1e). Zones A and 
B of cleithra were less frequent in the Zostera layers. Articular (figure A-1f) and first vertebra zones were equally represented in both layers.

Zone A of Seriola lalandi parasphenoid bones was less common in the Zostera layers (Table 6.54, FigureA-1b). Zone D of the dentary was not present in the Zostera layers, whereas more than $50 \%$ of dentaries in the shelly layers contained this zone. This was the only zone of S. lalandi present only in the shelly layers. All other zones of the species listed in (Table 6.54) were present in both the Zostera and shelly layers. Several zones of some elements were less well represented in the Zostera layers of this species but the lower number of zones in the Zostera layers was not consistent as some elements' zones are less common in the shelly layers.

\subsection{Elements used for species identification}

During analysis it was noted which elements were useful or not as species indicators. Some seem too similar to ever identify to more than family, whereas others depend on the level of completeness for identification. Figures 5.1 and 5.2 show the location of the elements referred to in the text. See also the figures in the appendix (Figures A2 - A17) for species specific element illustrations. On the basis of these observations, in addition to the work above, it was possible to narrow down the number of elements that are useful for species identification.

The elements that were most frequently used to identify specific Sparidae are listed in Table 6.55. Frequencies are percentages based on MAU but are not the same as \%MAU, as the frequencies of elements that are not the equivalent of 100\% MAU are not scaled to that number. Elements of the lateral skull and jaw apparatus in particular (articular, dentary, maxilla and premaxilla) were most often used for identification (11 out of 18), and the premaxilla was the most frequently used element for identifying Sparidae (9 out of 18). Many elements of Cymatoceps nasutus are indistinguishable from those of Chrysoblephus cristiceps and/or Chrysoblephus laticeps, unless they fall into a size class bigger than the latter two can attain. The identification of the maxilla, premaxilla, vomer and pterygoid of $C$. nasutus was based on size. The other elements are more reliable for identification of smaller specimens of this species. 
Table 6.55. Frequency (\%) of elements per Sparidae from all sites combined (based on MAU) used for identification. The most frequently identified element per species is shaded. *denotes elements that are only useful for identification if it is from a specimen larger than what morphologically similar species can attain

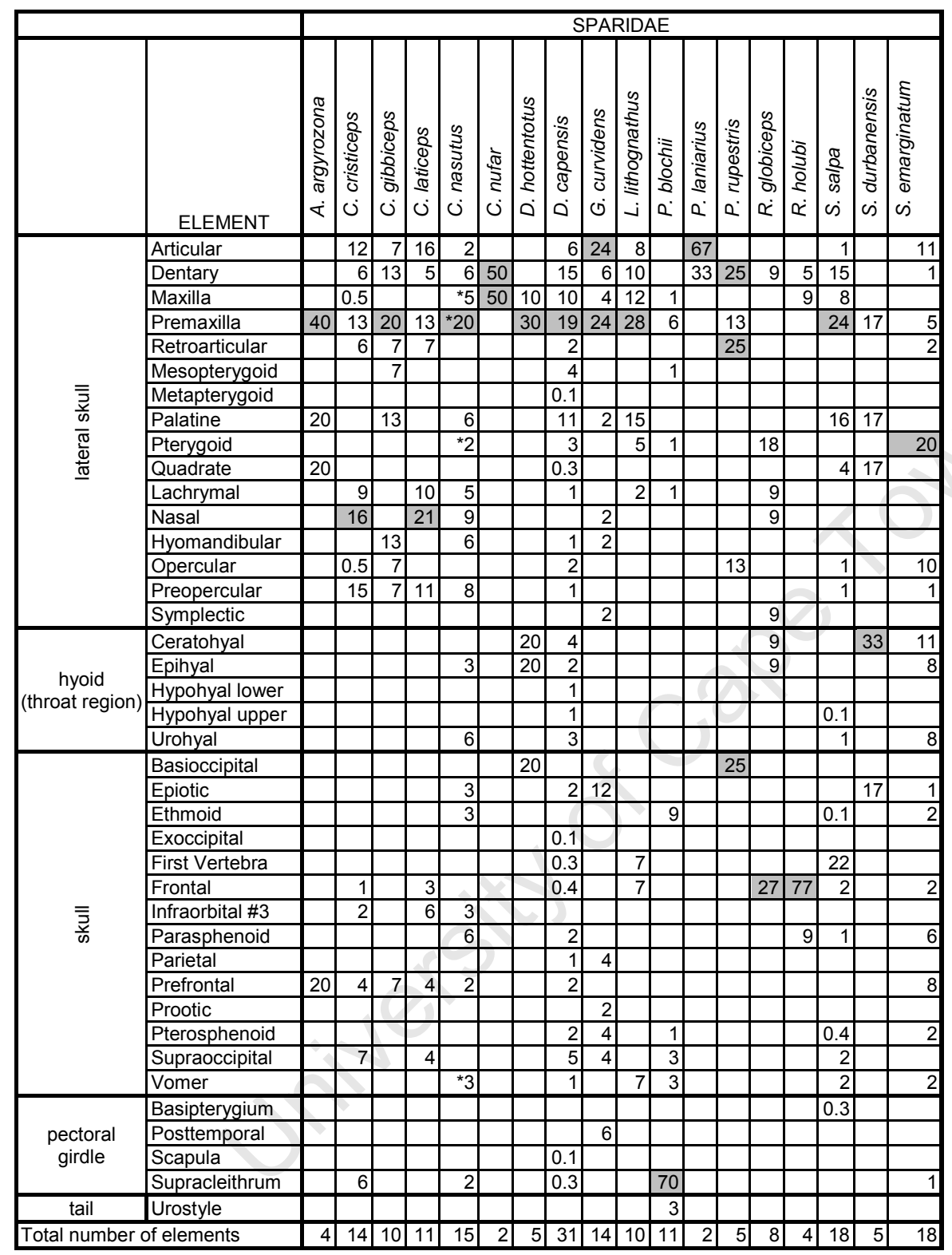

Two species, Rhabdosargus globiceps and Rhabdosargus holubi, were considerably better represented by skull bones, and their numbers would have been considerably lower if counts were based on jaw elements only. Elements from the pectoral region were not good Sparidae indicators except for Pachymetopon blochii, and most Sparidae tail elements could not be identified to species. A total of 41 Sparidae elements provided species identification. 
Table 6.56 lists the elements used for identification of species other than Sparidae. As with Sparidae, jaw elements were often the most frequently used elements. Several species are equally or better represented by bones from the skull. Hyoid region elements provided several identifications but are generally very low in frequency. The urostyle is the most frequent Liza richardsonii element, followed by the basioccipital. Urostyles are not, however, the most common element in all the assemblages, and other elements including basioccipitals were more frequent in some assemblages.

The number of elements that allowed identification of species from families other than Sparidae is much higher (51) (Table 6.56). This is not surprising as several morphologically diverse families are represented. A large number of elements contributed less than $5 \%$ of identifications each. At this stage it would be best to include all elements during identification of species such as Mugil cf. cephalus, Monodactylus falciformis and Oplegnathus conwayi that are represented by only a few elements as other elements not present here might well be useful for identification in assemblages where these species are more common.

Non-Sparid species such as Dichistius capensis, Liza richardsonii, Epinephelus marginatus and Seriola lalandi were identified on a large number of elements, and the frequencies of the most common elements are low $(\leq 10 \%)$. Determining which of the rare elements can be excluded from future analyses needs to be assessed per individual species as in some instances the less common elements are the only ones present in an assemblage. 
Table 6.56. Frequency (\%) of elements per non-Sparid species from all sites combined (based on MAU) used for identification. The most frequently identified element per species is shaded

\begin{tabular}{|c|c|c|c|c|c|c|c|c|c|c|c|c|c|c|c|c|}
\hline & & \multicolumn{15}{|c|}{ SPECIES OTHER THAN SPARIDAE } \\
\hline & ELEMENT & 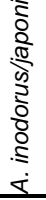 & 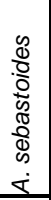 & 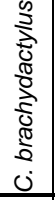 & $\begin{array}{l}\frac{0}{\omega} \\
\frac{1}{8} \\
\frac{2}{8} \\
0 \\
0\end{array}$ & 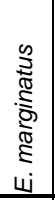 & 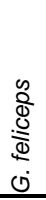 & 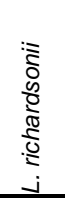 & $\begin{array}{l}0 \\
\frac{0}{0} \\
\frac{d}{d} \\
0 \\
\dot{\Sigma}\end{array}$ & 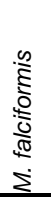 & 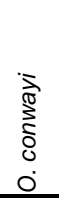 & 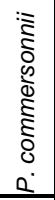 & 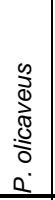 & 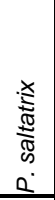 & 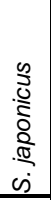 & $\frac{\bar{d}}{\frac{\pi}{\pi}}$ \\
\hline \multirow{18}{*}{ 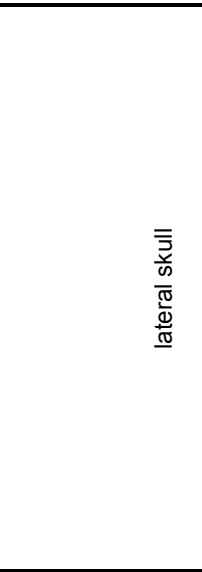 } & Articular & 7 & 10 & & 6 & 2 & 3 & 1 & & & & & & 3 & & 5 \\
\hline & \begin{tabular}{|l|} 
Dentary \\
\end{tabular} & & 7 & 6 & 4 & 3 & 1 & 1 & & & & 50 & & 19 & 20 & 6 \\
\hline & Maxilla & & 5 & & 6 & 3 & & 1 & & 50 & & & & & & 4 \\
\hline & Premaxilla & 2 & 5 & & 8 & 2 & & 1 & & & 100 & & 7 & 10 & 20 & 7 \\
\hline & Retroarticular & & & & 1 & 2 & & & & & & & 4 & & & 2 \\
\hline & Mesopterygoid & & & & 1 & 2 & & & & & & & & & & \\
\hline & Metapterygoid & & & & & 6 & & & & & & & & & & \\
\hline & Palatine & 5 & 2 & & 4 & & & 0.2 & & & & 50 & 4 & 2 & & 3 \\
\hline & Pterygoid & & & & 3 & 3 & & & & & 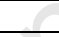 & 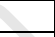 & & 6 & & 3 \\
\hline & Quadrate & 2 & 2 & 2 & 4 & 8 & & 1 & & & $1+$ & 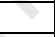 & & 4 & & 6 \\
\hline & \begin{tabular}{|l|} 
Jugal \\
\end{tabular} & & & & & & & & & & + & & & & & 0.3 \\
\hline & Lachrymal & & & & 1 & 2 & & & & + & & & & & & \\
\hline & \begin{tabular}{|l} 
Hyomandibular \\
\end{tabular} & & & & 5 & 3 & 1 & 5 & & +2 & & & 4 & 2 & & 3 \\
\hline & Interopercular & & & & 3 & 2 & & 1 & & 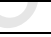 & & & & & & 1 \\
\hline & Opercular & & 2 & & 1 & 5 & 4 & 3 & 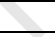 & & & & 4 & & & 0.3 \\
\hline & Preopercular & & & & 3 & 2 & & 1 & & & & & & & & 0.3 \\
\hline & Subopercular & & & & 0.5 & 3 & & 1 & & & & & 4 & & & \\
\hline & Symplectic & & & & & & 1 & 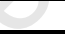 & & & & & & & & \\
\hline \multirow{5}{*}{ 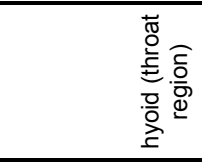 } & \begin{tabular}{|l} 
Ceratohyal \\
\end{tabular} & & 5 & 2 & 4 & & 3 & 4 & & & & & 4 & 2 & & 4 \\
\hline & \begin{tabular}{|l|} 
Epihyal \\
\end{tabular} & 2 & & 2 & 2 & 2 & + & 1 & & & & & 4 & 2 & & 1 \\
\hline & Hypohyal lower & & & & 3 & 2 & & & & & & & & & & 1 \\
\hline & Hypohyal upper & & & & 1 & & & 0.04 & & & & & & 1 & & 1 \\
\hline & \begin{tabular}{|l|} 
Urohyal \\
\end{tabular} & & & & 3 & 6 & & 6 & & & & & 7 & & & \\
\hline \multirow{19}{*}{$\begin{array}{l}\overline{\overline{\bar{y}}} \\
\text { 点 }\end{array}$} & Basioccipital & 32 & 5 & - & 2 & 3 & 1 & 8 & 100 & & & & 14 & 2 & & 5 \\
\hline & Basispenoid & & 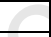 & +2 & & & & & & & & & & & & 3 \\
\hline & Epiotic & & + & 1 & 0.5 & & 1 & 3 & & & & & & & & 1 \\
\hline & Epiotic 2 (L. richardsonii) & 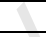 & & & & & & 1 & & & & & & & & \\
\hline & \begin{tabular}{|l|} 
Ethmoid \\
\end{tabular} & 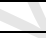 & & & 0.5 & & & 0.2 & & & & & & 2 & & \\
\hline & Exoccipital & 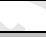 & & & 1 & & & 5 & & & & & & & & 1 \\
\hline & First Vertebra & 36 & 15 & 77 & 4 & 3 & 1 & 5 & & & & & & 11 & 40 & 3 \\
\hline & \begin{tabular}{|l|} 
Frontal \\
\end{tabular} & & 5 & 8 & 1 & & 3 & 3 & & & & & & & & 1 \\
\hline & Opisthotic & & & & & & & 0.1 & & & & & & & & 2 \\
\hline & Otolith & 2 & & & & & 35 & 0.1 & & & & & 7 & & & \\
\hline & Parasphenoid & & 10 & & 2 & 3 & & 3 & & & & & & & & 7 \\
\hline & \begin{tabular}{|l|} 
Parietal \\
\end{tabular} & & & & & 2 & & 0.1 & & & & & 4 & & & 1 \\
\hline & Prefrontal & & & & & 2 & 1 & 0.3 & & & & & & & & \\
\hline & \begin{tabular}{|l|} 
Prootic \\
\end{tabular} & & & & 1 & & & 2 & & & & & & & & 2 \\
\hline & Pterosphenoid & & & & 0.2 & 3 & & 0.04 & & & & & & & & \\
\hline & \begin{tabular}{|l|} 
Pterotic \\
\end{tabular} & & & & 1 & 3 & & 4 & & & & & 4 & 2 & 20 & 3 \\
\hline & Sphenotic & & & & 0.5 & & & 1 & & & & & 7 & & & \\
\hline & Supraoccipital & & & & 4 & & & 3 & & & & & 14 & & & 3 \\
\hline & Vomer & 5 & 20 & & 1 & 3 & 5 & 0.4 & & & & & & 2 & & 4 \\
\hline \multirow{8}{*}{$\begin{array}{l}\frac{0}{\overline{0}} \\
\frac{\overline{0}}{\bar{\sigma}} \\
\overline{0} \\
\overline{0} \\
\frac{0}{0} \\
\varrho\end{array}$} & Basipterygium & & & & 1 & 5 & & 2 & & & & & & & & 1 \\
\hline & Cleithrum & & & & 4 & 5 & 1 & 6 & & 50 & & & 7 & & & 3 \\
\hline & Coracoid & & & & 2 & 2 & & 2 & & & & & & 1 & & 2 \\
\hline & Postcleithrum & & & & 2 & & & 3 & & & & & & & & 0.3 \\
\hline & Posttemporal & & 2 & & 3 & 3 & & 0.3 & & & & & 4 & & & 1 \\
\hline & \begin{tabular}{|l|} 
Scapula \\
\end{tabular} & & & & 4 & 6 & & 2 & & & & & & 2 & & 6 \\
\hline & Supracleithrum & & & & 0.2 & 3 & & 1 & & & & & & & & 2 \\
\hline & Upper postcleithrum & & & & 2 & 2 & & & & & & & & & & 1 \\
\hline \multirow{2}{*}{$\begin{array}{l}\bar{\pi} \\
\underset{\pi}{x}\end{array}$} & Spine & & & & & & 1 & & & & & & & & & \\
\hline & Vertebra & 7 & 3 & 4 & & & 26 & 1 & & & & & & 24 & & \\
\hline \multirow{3}{*}{$\overline{\overline{\mathbb{T}}}$} & \begin{tabular}{|l} 
First anal pterygiophore \\
\end{tabular} & & & & & & 1 & 0.1 & & & & & & & & \\
\hline & Penultimate vertebrae & & & & & & & 6 & & & & & & & & 2 \\
\hline & Urostyle & & & & 2 & & 11 & 10 & & & & & & & & 3 \\
\hline \multicolumn{2}{|c|}{ Total number of elements } & 10 & 15 & 7 & 42 & 33 & 18 & 44 & 1 & 2 & 1 & 2 & 17 & 18 & 4 & 40 \\
\hline
\end{tabular}


From the combined element data, all element frequencies of Dichistius capensis are less than $9 \%$. The only element representing this species in one assemblage (KR MSA III) is the basioccipital, which comprises only $2 \%$ of elements of all sites combined (Tables 6.54, 6.30). If this element was excluded from identification due to its low frequency the species would not have been identified in that assemblage. The same applies to other elements of this species that occur at frequencies below 5\%, such as the lower hypohyal and first vertebra. Exclusion of low frequency elements such as the coracoid of Epinephelus marginatus would also result in this species not being recorded in one of the assemblages (BBC MSA M2) (Table 6.33).

A similar pattern is not seen with Liza richardsonii that is also represented by many elements that all occur at generally low frequencies. Exclusion of the $<5 \%$ frequency elements would not have affected the species composition or counts of this species in any of the assemblages (Table 6.38). The same applies to Seriola lalandi (Table 6.45).

Elements that were not useful for species identification are listed in Table 6.57. Those elements that contributed less than $5 \%$ of species identifications are included in the table. If the elements that could not be identified to Sparidae are excluded, then the number of elements used to identify Sparidae can be reduced to 31. If the ten elements that provided less than $5 \%$ of identifications are ignored, then 21 Sparidae elements are useful for identification. Removing elements that account for less than $5 \%$ of the identifications does not affect the resultant species lists. Nine elements did not provide any identifications of the non-Sparid species present in the assemblages studied here. A total of eight elements could not be used to identify any species and can be excluded from future analysis of assemblages containing similar species (Table 6.57). 
Table 6.57. Elements that were not identifiable to species, and elements that consistently provided less than $5 \%$ of species identifications for Sparidae and non-Sparidae respectively

\begin{tabular}{|c|c|c|c|c|c|}
\hline & Element & \begin{tabular}{|l|} 
Sparidae \\
No identification
\end{tabular} & $\begin{array}{l}\text { Non-Sparidae } \\
\text { No identification }\end{array}$ & $\begin{array}{l}\text { Sparidae } \\
\text { Low identification }\end{array}$ & $\begin{array}{l}\text { Non-Sparidae } \\
\text { Low identification }\end{array}$ \\
\hline \multirow{13}{*}{ 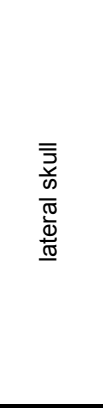 } & Retroarticular & $\square$ & $\square$ & & $x$ \\
\hline & Mesopterygoid & $\square$ & & & $x$ \\
\hline & Metapterygoid & $\square$ & & $x$ & \\
\hline & Jugal & $x$ & & & \\
\hline & Lacrimal & $\square$ & & & $x$ \\
\hline & Nasal & $\square$ & $x$ & & \\
\hline & Temporal & $x$ & $x$ & & \\
\hline & Interopercular & $x$ & $x$ & & $x$ \\
\hline & Pharyngobranchial & $x$ & $x$ & & \\
\hline & Preopercular & $\square$ & & & $x$ \\
\hline & Subopercular & $x$ & & & $x$ \\
\hline & Symplectic & $\square$ & $\square$ & & \\
\hline & Upperpostcleithrum & $x$ & & & $x$ \\
\hline \multirow{3}{*}{ 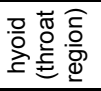 } & Epihyal & $\square$ & & & $x$ \\
\hline & Hypohyal lower & & & $x$ & $x$ \\
\hline & Hypohyal upper & & & $x$ & $x$ \\
\hline \multirow{16}{*}{$\begin{array}{l}\overline{\bar{z}} \\
\frac{\vec{v}}{\omega}\end{array}$} & Basisphenoid & $x$ & $x$ & & $x$ \\
\hline & Circumorbital \#1 & $x$ & $x$ & A & \\
\hline & Circumorbital \#2 & $x$ & $x$ & + & \\
\hline & Epiotic & $\square$ & & +1 & $x$ \\
\hline & Epiotic 2 (L. richardsonii) & $\square$ & & & $x$ \\
\hline & Ethmoid & $\square$ & 4 & 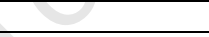 & $x$ \\
\hline & Exoccipital & & & $x$ & \\
\hline & Infraorbital \#4 & $x$ & $x$ & & \\
\hline & Opisthotic & $x$ & $\square$ & & $x$ \\
\hline & Otolith & $x$ & 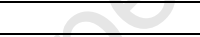 & & \\
\hline & Parietal & $\square$ & & $x$ & $x$ \\
\hline & Prefrontal & $\square$ & 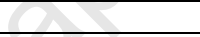 & & $x$ \\
\hline & Prootic & $\square$ & $\mathrm{C}$ & $x$ & $x$ \\
\hline & Pterosphenoid & $\square$ & & $x$ & $x$ \\
\hline & Pterotic & 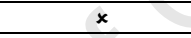 & & & \\
\hline & Sphenotic & $x$ & $\square$ & & \\
\hline \multirow{8}{*}{$\begin{array}{l}\frac{0}{\overline{0}} \\
\frac{0}{\sigma} \\
\frac{\pi}{0} \\
\frac{0}{0} \\
\mathbb{d} \\
\square\end{array}$} & Basipterygium & +2 & & $x$ & \\
\hline & Cleithrum & $x$ & & & \\
\hline & Coracoid & $x$ & & & $x$ \\
\hline & Postcleithrum & $x$ & & & $x$ \\
\hline & Posttemporal & $\square$ & & & $x$ \\
\hline & 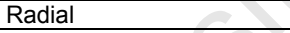 & $x$ & $x$ & & \\
\hline & 00 & & & $x$ & \\
\hline & Supracleithrum & $\square$ & & & $x$ \\
\hline \multirow{2}{*}{ axial } & 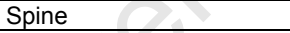 & $x$ & & & $x$ \\
\hline & Vertebra & $x$ & & & \\
\hline \multirow{3}{*}{$\overline{\overline{\mathbb{T}}}$} & First anal pterygiophore & $x$ & & & $x$ \\
\hline & Penultimate vertebrae & $x$ & & & \\
\hline & Urostyle & $\square$ & & $x$ & \\
\hline
\end{tabular}




\section{Chapter 7. Discussion}

In this chapter the results per assemblage from Chapter 6 on species composition, size reconstruction, natural and cultural bone modification and skeletal element representation is used to first determine a) how the fish remains entered the sites; b) what methods were employed to acquire the fish: c) whether methods of capture and habitats exploited changed with time; d) whether the species compositions can give an indication of the season of occupation. Second, the findings will be compared between assemblages studied here as well as with fish data from other archaeological sites, and how the findings relate to the broader picture/our current knowledge of fish remains from South African archaeological sites. Third, and in the final part of this chapter, I summarize the information gleaned from the various data collected on skeletal element preservation.

\subsection{Introduction}

A wide range of fish species were identified in this study and the sites that are geographically situated close to one another have the most species in common. Species diversity is highest in Klasies River main site and Hoffman's/Robberg Cave, both of which are situated further east than the other sites studied. The high species diversity is most likely a reflection of the increased species richness that is typical for this easterly coastline. The species composition of the $\mathrm{KR}$ and $\mathrm{H} / \mathrm{RC}$ assemblages is very similar and they have 22 species in common. The BBC LSA and MSA assemblages have a smaller range of species present, but the species composition is very alike and eight species out of a total of 21 occur in both assemblages. The Blombosfontein 1 and 6 assemblages have very limited numbers of species, all of which also occur in the LSA layers of nearby BBC.

All of the species identified still occur in the area and most species are near-shore inhabitants. A small number of offshore species were found at KR, BBC MSA and H/RC. These are Pterogymnus laniarius (BBC MSA, KR), Argyrozona argyrozona (KR, H/RC) and Triglidae spp. (H/RC). They were presumably acquired as washed up fish as there is no evidence for prehistoric use of seafaring vessels in South Africa. The P. laniarius could have entered the sites as the stomach contents of seals as it is one of their favoured prey items. 


\subsection{Agent of accumulation, method of capture, diachronic change and seasonality}

The primary issue to be addressed when interpreting faunal assemblages is whether the remains were brought into the deposit by humans or other animals. This is particularly significant for the MSA assemblages as fish remains are rare in the MSA and some doubt has been expressed as to whether these remains were accumulated by humans (Deacon 1995; Deacon and Geleijnse 1988; Klein 1995, 1999a, b, 2001, 2008; Klein et al. 2004). This is particularly the case at KR, where fish remains occur in both cultural and sterile units. If humans were the principal accumulators of the fish, then the secondary line of investigation entails determining the means by which the fish were acquired. Thereafter the data can be used to investigate issues such as temporal changes in fishing methods, habitats exploited and season of occupation.

\subsubsection{Hoffman's/Robberg Cave}

\section{Agent of accumulation}

At $\mathrm{H} / \mathrm{RC}$, four of the species present have been recorded in cormorant pellets (Galeichthys feliceps, Acanthistius sebastoides, Spondyliosoma emarginatum and Gobiidae spp.), and one (Mugil cf. cephalus) forms part of the African fish eagle's diet. With the exception of S. emarginatum, the species that form part of cormorant and fish eagles' diets occur only in the shelly layers and in low numbers (total NISP excluding S. emarginatum =11). As neither cormorants nor fish eagles inhabit caves, it is unlikely that these birds contributed to the fish remains at $\mathrm{H} / \mathrm{RC}$. No evidence of gnawing or digestive etching was recorded on any of the specimens.

The high density of fish associated with cultural artefacts, presence of potential fishing gear in the form of sinkers, some evidence for fish processing in the form of cut marks and burning and the complete absence of carnivore gnaw marks or acid etching indicates that humans were the primary accumulators of the fish remains at H/RC.

\section{Method of capture}

Having established that humans were the agents of accumulation of fish at $\mathrm{H} / \mathrm{RC}$, the following step was to determine how they acquired the fish, what technology was employed, and whether this changed over time. Overall, of the 29 species present, 27 can 
be caught with baited hooks; 16 can be caught in tidal traps; ten can be killed by cold water upwelling events, then washed up and scavenged (excluding those where only juveniles are affected as most in the assemblage are not juveniles); twelve species can be speared; two can be caught with nets.

\section{Acquisition through upwelling events}

Dichistius capensis could have been scavenged after an upwelling event but its comparatively higher numbers and presence in all layers, apart from one, suggests that there was a more systematic method of acquiring these fish. Upwellings resulting in fish mortalities are neither a frequent nor a reliable method of acquisition. Sarpa salpa could theoretically also have been acquired through scavenging of washed up specimens as juveniles of this species in particular are very susceptible to sudden changes in water temperature. Adult $S$. salpa move into shallower water during cold water upwellings where they might be easy prey for humans but are seldom killed by the upwelling itself. The majority of $S$. salpa specimens in the assemblage are of adult size (Bennett and Griffiths 1986; Biden 1930; Mann 2000; Smith and Heemstra 1995). Diplodus capensis react to cold water upwelling in the same way as S. salpa. The majority (82\%) of this species fall in the small (151-300mm) category, which can include fish of both juvenile $(<21 \mathrm{~cm})$ and adult size, followed by medium sized fish. It is therefore not known what percentage of the small category comprises adults or juveniles. As with Dichistius capensis, it seems unlikely that fish sheltering in shallow water due to upwellings were the primary source, as both $S$. salpa and Diplodus capensis occur in consistently high frequencies throughout the assemblage. The unidentified Triglidae and single adult Argyrozona argyrozona that only occur offshore were probably collected as washed up specimens, but not necessarily due to cold water upwelling events as it is not known whether they are affected by these events.

\section{Active fishing methods}

Two of the most common species, Dichistius capensis and Diplodus capensis, can be caught by any of the methods listed in Table 3.6 apart from by netting. Sarpa salpa can be caught in tidal traps, by baited hook, or killed by cold water upwelling events. Seriola lalandi is rarely caught in tidal traps, and is more frequently caught on baited hooks or speared. Liza richardsonii is usually only caught in tidal traps or with nets which can result in high catch numbers. Pomatomus saltatrix can be caught with baited hooks and in 
tidal traps, and shoals of these fish shelter in shallow water during cold water upwelling events. Two of the species present, Argyrozona argyrozona (NISP = 3) and Acanthistius sebastoides (NISP $=4$ ), are typically caught only with baited hooks. Adult A. argyrozona are usually only caught offshore, but juveniles occur inshore. Three of the four specimens are juvenile fish, that could have been caught with baited hooks from the shore.

Judging from the large quantity and variety of fish in this assemblage a range of fishing methods must have been employed, and it is unlikely that the inhabitants were dependant on sporadic cold water upwelling events for fish, although they probably exploited these when the opportunity arrived. The low number of offshore species present at $\mathrm{H} / \mathrm{RC}$ suggests that scavenging of washed up specimens probably occurred, but this was not the primary method of acquiring fish. The coastline along the Robberg Peninsula is very steep and unsuitable for stone traps, and none have been recorded in the area. There are some natural rock pools on the Robberg Peninsula where fish could occasionally have been trapped by the receding tide and collected by hand or with nets or baskets. However, as there are large numbers of Liza richardsonii throughout the assemblage, a more systematic method is indicated, and they were most likely caught with nets along the beach. It is not clear why other species that are typically caught with nets are not present.

By excluding stone traps and upwelling events as the primary sources of fish, the constant presence of Pomatomus saltatrix and Sarpa salpa in particular, and to a lesser extent Seriola lalandi, Dichistius capensis and Diplodus capensis, indicates that some kind of hook and line technology was employed. The stone sinkers that have been found in the assemblage could have functioned as net or line sinkers. The large size difference in two of the species most likely caught on hooks ( $S$. salpa and S. lalandi) indicates that the 'hooks', presumably gorges made of wood or thorn as no artefacts that could have functioned as hooks or gorges have been recovered, were made in different sizes. Small species such as S. salpa are not likely to be caught on large hooks intended for fish the size of $S$. lalandi, and vice versa. As these fish are not caught in the same habitats, differences in hook sizes indicate an awareness of the species' ecology, and suggest deliberate targeting of specific sized fish.

The use of spears cannot be excluded as several of the species, including Seriola lalandi, Dichistius capensis, Diplodus capensis and Lithognathus lithognathus, can be caught with 
spears. Bone points are present in the site and these might have been hafted and used for spearing fish, or alternately wooden spears could have been used. As these species can also be caught with baited hooks, there is no clear evidence for the use of spears at H/RC.

The absence of very large Seriola lalandi and Pomatomus saltatrix could relate to the equipment used to catch them, which might not have been strong enough to deal with larger specimens, as both these species are strong fighters and $P$. saltatrix has sharp teeth that can easily bite through light line (Biden 1954; Schoeman and Schoeman 1990).

The very low number of tiny specimens at $\mathrm{H} / \mathrm{RC}$ could be seen as an indication that finemeshed nets were not used. Fine meshed nets are not size discriminatory and can catch fish of all sizes, but a larger mesh size is selective and would exclude tiny fish. Though low in number, the fact that tiny fish are present suggests that the sieve size used $(3 \mathrm{~mm})$ is small enough to retain at least some of these sized specimens. The underrepresentation of tiny fish therefore seems to be a reflection of fishing practices rather than a function of sieve size.

\section{Fish habitats exploited}

With the exception of Liza richardsonii and Pomatomus saltatrix, all the species in the assemblage that frequent estuaries as adults occur only in the shelly layers $(n=6)$. This could be an indication that people were moving further afield towards the Piesangs River, approximately $5 \mathrm{~km}$ from the site, to fish. As these occur in low numbers (NISP between 1 and 9) it could also be a function of the much larger sample size in the shelly layers. The wide range of species present that occupy different habitats indicates that the H/RC inhabitants were adept fishers who exploited all the available habitats in the near vicinity of the cave. This includes sandy beaches, near shore shallows and rock pools, as well as fishing in deeper water from ledges.

\section{Diachronic changes in fish procurement}

The species composition across the Zostera and shelly layers is very similar, and the additional species present in the shelly layers are most likely a function of the larger sample size. As there are also no significant differences in the sizes of the species between the Zostera and shelly layers, the impression is that the methods of capture employed did not change when the site was occupied during these periods. 
The overall higher species diversity in the older layers could be an indication of more intensified exploitation of marine resources prior to 3800 years ago. Alternately, it could simply be a function of the larger sample size in the older shelly layers. Differences in species variety and quantities between specific layers suggests that there were cycles of increased focus on targeted species interspersed with periods of less focused fishing, where a larger range was taken. In the two layers (Ben and Katherine) where large numbers of a limited range of species were recorded, there is an increase in numbers of three of the shoaling species (Seriola lalandi, Sarpa salpa and Pomatomus saltatrix) that occur most frequently in summer. Seriola lalandi and $P$. salatrix are both known to engage in bouts of frenzied feeding close to shore, during which they are often easily caught if a line is thrown into their midst (Biden 1954). In Layer Katherine another shoaling species, Liza richardsonii, occurred at high frequencies. This species occasionally shoals in large numbers close to the shore (Biden 1954), and is a common prey species of S. lalandi and P. saltatrix. The presence of large shoals in the vicinity of $\mathrm{H} / \mathrm{RC}$ could have attracted these predator species. In Layer Ben, however, increased numbers of $S$. lalandi and $P$. saltatrix are not accompanied by increased numbers of $L$. richardsonii. Numbers of Diplodus capensis also increased in Layer Ben. This species is typically caught on baited hooks or speared. It seems therefore that most fishing during this period of occupation was with hook and line rather than nets. Another species that occurred frequently in Layers Ben and Katherine is Dichistius capensis, which is most often captured during winter when the water is more turbulent and visibility is low.

\section{Seasonality}

There is no clear patterning in terms of season of capture as each layer contains species that are more common in either summer or winter. Although summer-caught species are more abundant in the assemblage, it is likely a reflection of fishing conditions in the southern Cape, where a wider range of species can be caught in summer (Bennett and Griffiths 1986; Biden 1930; Smith and Heemstra 1995). The movement of Seriola lalandi and Pomatomus saltatrix close to shore during summer would have provided the H/RC inhabitants with the ideal opportunity to spear or hook these fish. Knowledge of these seasonal abundances would have made summer occupation at the coast attractive. In the southern Cape the turbulent seas that occur frequently during the winter months would have made access to marine resources more difficult than in summer. Winter months 
however can also yield a higher marine harvest as some species of fish, for example Dichistius capensis, develop extra mass in winter. The presence of Dichistius capensis, a winter indicator species, in relatively high numbers in all layers apart from layer Peter, indicates that occupation of the site was not restricted to summer. Overall the data suggest that $\mathrm{H} / \mathrm{RC}$ was occupied throughout the year.

\section{Evidence for processing}

Apart from the clear cut mark on an E. marignatus urohyal (H/RC\#1848) and some potential but currently unverified cut marks on a few other elements, there is very little evidence for fish processing at $\mathrm{H} / \mathrm{RC}$. Some evidence against processing or removal of certain body parts was found in the form of the presence of elements from both the head and tail region of some species. Elements from all areas of the skeletons of Dichistius capensis, Liza richardsonii and Seriola lalandi were present which suggests that these fish were brought into the cave intact. It could indicate that these species were consumed on site, but does not negate the possibility that the fish fillets were removed at the site and taken elsewhere. The lack of tail elements from other species does not imply that these species did not arrive whole at the site, but is rather a reflection of the more readily identifiable tail elements of these particular species.

\subsubsection{Blombosfontein 1}

\section{Agent of accumulation}

The BBF 1 fish was recovered from a context associated with cultural artefacts and no signs of gastric etching or gnawing were recorded. The site's open location makes it an unsuitable habitat for roosting fish-eating birds, and the average size of the fish is larger than that normally found in otter spraints. It is therefore likely that these fish remains were deposited by humans.

\section{Method of capture}

The species present at BBF 1 could have been caught in a variety of ways but the sample size is too small to make any meaningful observations on how they were likely to have been acquired. The presence of Liza richardsonii could indicate the use of nets or tidal traps, but again the sample size is inadequate for making inferences. The lack of specimens of fish less than $15 \mathrm{~mm}$ in length could be due to smaller elements not surviving 
well at exposed locations. This assemblage, however, was heavily brecciated and retrieval methods might have been biased towards larger specimens.

\section{Seasonality}

The presence of Dichistius capensis and absence of any other seasonal indicator species is consistent with a winter occupation, as has been indicated on the basis of oxygen isotope measurements on shells (Henshilwood 1995, 2008).

\section{Comparison with previous analysis}

Two of the species identified in this study were also identified by Poggenpoel, (Henshilwood 1995, 2008), whereas three others he lists could not be found. Two additional species were identified in my analysis. Poggenpoel calculated a total MNI of nine, whereas I have calculated seven. NISP was not given for the BBF assemblages studied by Poggenpoel, and it is therefore not clear whether some of the material might have been accidentally excluded from this study.

\subsubsection{Blombosfontein 6}

\section{Agent of accumulation}

The fish remains from BBF 6 are associated with some cultural artefacts and large amounts of shellfish collected by humans. Some of the specimens are burnt, and some vertebrae show evidence of cut marks. The open site location is unsuitable for roosting birds although seagulls could have contributed fish to the deposit as the site is close to the ocean. No data are available on the fish species taken by gulls, but it is assumed to be variable as they are opportunistic scavengers. The medium and large sized fish in the BBF 6 assemblage are presumably bigger than those gulls can transport over some distance. None of the specimens showed evidence of gastric etching or gnawing. It is therefore assumed that the fish remains were deposited by humans.

\section{Method of capture}

As with the BBF 1 assemblage, the small sample size makes it difficult to reconstruct how the fish were obtained, although the presence of a large Chrysoblephus cristiceps could indicate the use of hook and line technology as this deeper water species is not usually trapped in tidal pools, or netted, speared or affected by cold water upwelling. BBF 6 also has a low number of species present. 


\section{Seasonality}

The only species that could give some indication of season of capture is Chrysoblephus cristiceps, present in Layer HBL, which is most abundant in summer. This contrasts with oxygen isotope values obtained from edge increments of $T$. sarmaticus opercula from this layer which indicated winter occupation (Henshilwood 1995, 2008). Considering that there is only one specimen and that 'most abundant' does not exclude other seasons, this cannot be considered as a strong seasonal indicator.

\section{Comparison with previous analysis}

Only one of the species identified in this study (Diplodus capensis) was also identified by Poggenpoel (Henshilwood 1995, 2008). This discrepancy can in part be attributed to the sample size studied here being smaller as faunal material from three of the six layers could not be located. The low number of identified species from this site and BBF 1 limits the interpretive value of these assemblages relative to the others studied here.

\subsubsection{Blombos Cave Later Stone Age}

\section{Agent of accumulation}

All of the BBC LSA fish remains were recovered from within the cave as, unlike the MSA, the LSA occupation did not extend beyond the dripline. Birds such as cormorants that could deposit fish remains do not inhabit caves and are unlikely to have contributed to the fish assemblage. Species consumed by penguins that sometimes inhabit caves were not found in the assemblage. None of the specimens showed evidence of gastric etching, but the presence of a tooth mark on one specimen indicates that there was some animal activity in the assemblage. The majority of specimens are too large to represent remains from otter spraints. Despite the lack of any obvious fishing gear, the fish remains are associated with cultural material and evidence of cut marks and burning indicates that humans were the primary accumulators.

\section{Method of capture}

The species composition of the older and younger layers is similar, and the additional species in the older layers occur at low frequencies and are thought to be a function of the larger sample size. Of the 13 species present, twelve can be caught with hooks, eight can be speared, seven caught in tidal traps, five can be killed by cold water upwelling, and one species can be caught with nets. 
The presence of Chrysoblephus cristiceps in all layers and Petrus rupestris in a few, one of which is a very large fish $(>100 \mathrm{~cm})$, suggests that the inhabitants possessed some kind of hook and line technology as these species are not usually captured by any of the other methods listed in Table 3.6. Further evidence for the use of hooks is seen in the presence of Spondyliosoma emarginatum in all layers. Although this species can be killed by cold water upwelling events, fish deaths from upwelling events have not been recorded in the vicinity of BBC. This species was therefore most likely caught with hooks (Hanekom et al. 1989). In the absence of any artefacts resembling potential hooks in the assemblage, hooks or gorges may have been made out of wood or thorns that did not survive in the deposit. All of the other species that can be caught on hooks can also be caught with spears. There is at least one bone point in Layer 4 that could have been hafted and used for spearfishing (Henshilwood et al. 2001a), but untipped wooden spears could have also been used. Fish that were trapped in some of the larger rock pools would have made easy spearfishing targets. The absence of $C$. brachydactylidae, a common inhabitant of larger rock pools near BBC, and which is usually only caught by spearing, could indicate that spearfishing was not regularly practiced by the BBC LSA inhabitants.

The relatively high numbers and consistent presence of Liza richardsonii in all layers could indicate the use of stone tidal traps or nets. The area in the direct vicinity of BBC is not suitable for constructing stone walled tidal traps although there are numerous rock pools that could have functioned as ad hoc traps, with or without alteration. I have observed small shoals of $L$. richardsonii trapped in natural rock pools near BBC on numerous occasions. These fish would be easy to collect with nets, and I have observed them being caught in this manner. They could also be 'herded' into a rock corner with bushes, as recorded historically from constructed fish traps in South Africa, and then collected with baskets or by hand. Alternately, nets could have been used to catch shoals from the beach. Although this is the most common species throughout the assemblage, the numbers are not particularly high, particularly in comparison with those from H/RC. No other species that are typically caught with nets occur in the assemblage. The methods used to capture this species at BBC are thus somewhat ambiguous, and there is no clear evidence for the use of nets. 


\section{Diachronic changes in fish procurement}

Despite the lower numbers of species in the younger layers of BBC LSA, there appears to be no difference in the methods used for fishing between the oldest and the youngest layers. The most frequently occurring species are present in both the pre and post $1600 \mathrm{BP}$ layers, and there is no significant difference in the sizes of fish present between these layers. The lower density in the younger layers suggests that fish played a less important role in people's diets around 300 years ago.

\section{Seasonality}

Both species that are more frequently caught during winter or summer occur throughout the sequence. Thus there is no indication that visits to the cave were seasonally scheduled.

\section{Comparison with previous analysis}

The additional data from this study has increased the number of species identified previously by one (Dichistius capensis) (van Niekerk 2005) (Table 3.5). This species is represented by one element only, and its presence is most likely due to the increased sample size. The additional sample has not changed van Niekerk's (2005) previous findings of decreased densities in the younger layers, but has increased the number of species present in Layer 2 from five to seven, and in Layer 4 from nine to ten. The additional sample from this study has, however, contributed significant data in terms of species size, cultural and natural modification of fish bone, skeletal element completeness and element zone data, which was not recorded before.

\subsubsection{Blombos Cave Middle Stone Age}

\section{Agent of accumulation}

Most of the BBC MSA fish comes from within the cave and is associated with cultural material. A smaller amount was retrieved from the excavation beyond the dripline and is associated with cultural material. None of the bones showed signs of digestion or gnawing. No cormorants have been observed roosting directly outside the cave, although this does not rule out the possibility that they did so in the past. Most of the specimens from both the interior and exterior of the cave are from small sized fish which is the size most frequently taken by cormorants, but there are also specimens that are too large to have been brought in by birds. As cormorants do not inhabit caves, it is unlikely that they contributed to the fish assemblage in the interior of BBC. Three of the species that were 
found in the exterior of the cave (Galeichthys feliceps, Acanthistius sebastoides and Clinidae spp.) have been recorded in modern cormorant pellets (von den Driesch 2004). The latter two species are represented by one specimen each. Unlike those from modern cormorant pellets, these specimens showed no evidence of gastric etching. The G. feliceps elements are dominated by vertebrae (75\% in the exterior, $89 \%$ in the interior), unlike those found in cormorant pellets, which are dominated by non-vertebral elements. More than double the number of all specimens from the exterior are vertebrae (NISP $=105$ ), relative to non-vertebral elements (NISP $=51$ ). A wider range of $G$. feliceps elements were present in the exterior $(n=8)$ relative to the interior $(n=5)$ of the cave. Three of the elements from the interior were also found in the exterior, and the difference could relate to the larger sample size in the exterior, as the total NISP of G. feliceps is 84 in the exterior, and 36 in the interior. Burning was recorded on one specimen of this species from the exterior.

The absence of signs of digestion and the lack of haemal and neural spines on several of the vertebrae are consistent with assemblages created by $H$. vocifer, but as this species does not inhabit caves it is an unlikely contributor to the assemblage. Birds that sometimes do inhabit caves, such as S. demersus (jackass penguin) (Simmons and Kemper 2003), prey on species that were not found in the assemblage, with the possible exception of the Gobiidae that could not be identified to species (NISP=7). All the species that occur in the exterior are also present in the interior of the cave, with the exception of the single Acanthistius sebastoides specimen, which is present only in the M3 outside the cave. This might have been deposited by a bird, and is a common component of $P$. carbo's diet (Randall et al. 2002).

Fish that are bigger than those birds could take occur in both the interior and exterior of $\mathrm{BBC}$. The majority of specimens from both areas are mostly intact, but overall the interior specimens are more complete than those from the exterior. This could be a function of the smaller sample from the exterior for which completeness could be recorded (37 specimens from the exterior and 193 from the interior).

In summary, there are no significant differences between the interior and exterior fish in terms of size, all the species that occur in the exterior are also present in the interior, with the exception of one specimen from one species, most elements from the interior and 
exterior are fragmented to the same degree, burnt specimens occur in both the interior and exterior, and no signs of digestion were recorded on any specimens. Based on these similarities between the interior and exterior fish assemblages, it appears that both were deposited by the same agent of accumulation.

Indicators of humans as the primary accumulators of fish bone include the presence of fish too large to have been deposited by birds, with larger fish $(>300 \mathrm{~mm})$ from the interior and exterior combined comprising $31 \%, 58 \%$ and $66 \%$ of the M3, M2 and M1 respectively. In addition, there is an absence of species that are eaten by birds that might inhabit caves, no signs of digestion or gnawing, and burnt bones occur in all phases. Other indications that humans were responsible for bringing fish to the cave include tentative evidence of cooking as seen from burn patterns on the distal ends of $20 \%$ of all spines. Green-stick fractures on some of the Galeichthys feliceps spines indicate that they were broken while fresh, most likely to prevent accidental penetration as they are serrated and covered with toxic mucus which can inflict a painful wound (Poggenpoel, pers. comm.). Similar evidence for deliberate breaking of catfish spines has been recorded by von den Driesch at Karnak-North near Luxor (1983), and disarming of the locking mechanism of catfish spines was recorded at Arenosa Rock Shelter by Jurgens (2008).

\section{Method of capture}

The possible methods employed to acquire the species present are varied as most can be caught by a number of methods. Of the 17 species present, seven are affected by cold water upwelling events, twelve can be caught with hooks, seven can be speared, seven can be caught in tidal traps and one can be caught with nets.

\section{Scavenging of washed up specimens}

Three of the species killed by cold water upwelling occur in the M3, one in M2 and five in M1. In addition, one species (Argyrosomus inodorus/japonicus) which is affected by cold water upwelling events but not usually killed by these events, occurs in the M3. In total, $12 \%$ of the MNI of specimens that were identified to species are from species that are known to be affected by cold water upwelling events. Cold water upwelling events do not occur in the direct vicinity of BBC today, and those that do occur approximately $70 \mathrm{~km}$ eastwards do not cause fish fatalities. Cold water upwelling events affect a wide variety of species, and one would expect to see this variety in an assemblage if scavenging of washed up fish was the primary source of fish, unless the inhabitants were selective in 
their choice of species. As only seven of the nineteen species that are known to be killed or affected by cold water upwelling events occur in the assemblage, and these account for a low percentage of the total assemblage, it appears that upwelling events resulting in fish deaths either did not occur in the vicinity of BBC, or that scavenging of washed up fish was not the primary source of fish.

The two vertebrae of Argyrosomus inodorus/japonicus in the M3 are the only specimens from fish larger than $100 \mathrm{~cm}$ that were found in the assemblage. These were found in the same layer but not square and could belong to the same individual. This large fish might have been collected after washing up on shore, or opportunistically caught after being trapped in a rock pool. The offshore species Pterogymnus laniarius, which occurs in the M2, was probably collected after washing up on the beach, or entered the site as the stomach contents of a seal. Seal remains are present in all three phases of the BBC MSA (Henshilwood et al. 2001).

\section{Active fishing methods}

\section{Hooks}

All of the species from BBC MSA that are affected by cold water upwelling events can also be caught with either baited hooks or speared. Ten of the species present in the M3 can be caught on baited hooks, six in the M2 and nine in the M1. In total, 79\% of the identified species (MNI) can be caught with hooks. The highest number occurs in the M3 (90\% of the total MNI of M3). Three species, Chrysoblephus gibbiceps in the M1 and M2, Chrysoblephus cristiceps in all phases, and Acanthistius sebastoides in the M3, are usually caught with baited hooks, and not by any other methods. These species are not affected by cold water upwelling events.

\section{Spearfishing}

Of the seven species that can be caught by spearing, four occur in the M3, three in the M2 and six in the M1. They account for 54\% of the total MNI. The highest number of fish that could have been speared occurs in the M3 (69\% of the total MNI of M3).

\section{Tidal traps}

Six species that can be caught in tidal traps occur in the M3, four in the M2 and six in the M1. Sixty-nine percent of the total MNI are from species that can be caught in tidal traps. Most occur in the M3, where $83 \%$ of the fish identified can be caught by this method. 


\section{Nets}

One species, Liza richardsonii, can be caught with nets and is present in all phases. The Gobiidae could not be identified to species, and it is unclear whether these were pelagic or rock pool inhabiting species. If the latter, they might have been caught with nets, although these fish can easily be caught with simple technology.

\section{Summary of methods of capture}

The majority of species present in the BBC MSA can be caught by baited hooks, spearing or with tidal traps. The presence of three species that are not typically caught in any other manner apart from with baited hooks suggests that this method formed part of the fishing strategy at BBC during the MSA. Two of these species are seldom hooked from the shore unless deep gullies are present, which do occur in the vicinity of the cave. All of the species that can be caught with spears can also be caught on baited hooks, with the exception of Oplegnathus conwayi, which can either be speared or killed by cold water upwelling events.

Bone points that could have been hafted and used for spearfishing occur in the M1 and M2 phases, but whether these were used for fishing is unclear. Most of the bone points occur in the M2 phase, which contains the lowest number of species that can be caught with spears, which suggests that the bone points served another purpose. Wooden spears, if used, would no longer be preserved, but could have served the same function. As noted earlier under the section on the LSA fish remains from BBC, there are several rock pools in the area that could have functioned as tidal traps with little or no adjustment needed. All of the potentially trapped species, apart from Liza richardsonii, can also be caught on hooks. The latter species could have been caught with nets along the beach, but it is not possible to verify this.

In the absence of any obvious fishing gear, it is not possible to clearly identify one method as the most likely technique used to catch fish based on the current data, although the use of hooks is strongly indicated by three of the species present, and the fact that most of the fish caught by any of the other methods apart from netting can also be caught on hooks. It is probable that a range of techniques were used, with hooks, spears and traps being the most likely. Occasional scavenging of washed up specimens probably occurred, but most of the fish appears to have been acquired through less passive methods. 


\section{Diachronic changes in fish procurement}

The species composition varies across phases but the two most common species (Liza richardsonii and Galeichthys feliceps) occur in all phases. The older M3 phase contains

the highest density of fish in terms of MNI $\left(63 / 10 \mathrm{~m}^{3}\right)$, and contains significantly more fish in the small size category relative to the M2 and M1, which contain more medium sized fish.

The methods by which these fish could have been acquired are varied as most can be caught by several methods, and there are no significant differences in how fish could have been caught between phases. The consistent presence of the two most common fish species suggests that similar methods were employed during all phases. The addition of an extra species that is typically caught with hooks in the M2 and M1 in relatively high numbers could indicate increased use of hooks in the younger phases.

Some of the small sized specimens in the M3 are from the same species that occur in larger sizes in the M2 and particularly the M1. The species are Galeichthys feliceps, Dichistius capensis and Pachymetopon blochii. This could indicate an improvement in technique from the older to the younger phases allowing capture of larger specimens. The number of specimens that were identified to species for which size estimations could be made is however small, and the observed difference could be a function of the small sample size.

\section{Seasonality}

The species composition of all three phases includes species that are common in either winter or summer. As the data has been conflated per phase, it is not possible to infer season of occupation at this scale.

\section{Comparison with previous analysis}

Additional excavation since the initial report on the BBC MSA fish (Henshilwood et al. 2001b) has increased the sample size of the fish from 664 to 860 specimens (NISP) (Table 3.3). The number of species identified has increased from ten to seventeen. The number of species identified has increased from eight to twelve in the M3, five to ten in the M2 and six to twelve in the M1. Nine of the species identified by Poggenpoel were also identified in this study. The one species not identified here, Epinephelus andersoni, which Poggenpoel identified in the exterior M3, is lacking from the comparative collection and is 
probably the same specimen recorded here as unidentified Serranidae in the exterior M3. Some of the specimens from the exterior M3 identified as Dichistius capensis by Poggenpoel are probably the same that were recorded as Dichistiidae spp. here, due to some uncertainty about the exact species. The number of Cymatoceps nasutus identified in this study is considerably lower than that of the preliminary study. This is most likely because Poggenpoel identified several teeth and spines as belonging to this species (pers. comm.), whereas I did not find teeth or most spines apart from those of Galeichthys feliceps to be diagnostic to species.

\subsubsection{Comparison of the MSA and LSA fish assemblages from BBC}

The BBC MSA and LSA fish assemblages are ideal for comparing in terms of differences or similarities in the procurement strategies and abilities of MSA and LSA fishers as the species present and possible methods of capture as both were deposited in the same site, and therefore under similar conditions, barring the age factor, and with the same coastal resources and species available. Based on the shellfish composition of the BBC MSA phases, it is believed that the distance from the cave to the shore was similar to the present during the MSA occupation (Henshilwood et al. 2001b), and it is therefore assumed here that the same species that were available to the LSA inhabitants were also available to the MSA inhabitants. In addition, comparison of the MSA and LSA assemblages can be informative on differences or similarities in the procurement strategies and abilities of MSA and LSA fishers.

\section{Species composition and method of capture}

The species diversity in the BBC MSA is higher than in the LSA, with the MSA containing 17 species, and the LSA 13. There are five more species present in the M3 and M2, and four more in the M1 phase relative to the LSA. Most of the LSA species (9 out of 13) are present in the MSA in total. The more frequently occurring of the eight species that are only present in the MSA phases are Galeichthys feliceps, Chrysoblephus gibbiceps and unidentified Gobiidae and Clinidae. The other species, occurring in low numbers only in the MSA, are Acanthistius sebastoides, Argyrosomus inodorus/japonicus, Pterogymnus laniarius and Oplegnathus conwayi. Species that are present only in the LSA are Diplodus capensis, Gymnocrotaphus curvidens, Petrus rupestris and Sparodon durbanensis. The latter two occur in low numbers (NISP <5). 
The difference in species between the MSA and LSA is not particularly informative in terms of differences as to how they might have been captured. Both the LSA and MSA contain one species each (Petrus rupestris and Chrysoblephus gibbiceps, respectively) that are usually only caught with baited hooks, but the others that occur only in the LSA or MSA can be caught in a number of ways.

The species that occur in both assemblages are most frequently caught with baited hooks, and the impression is that this was the most likely method used to catch fish during both the MSA and the LSA. One species that is consistently present in both the MSA and LSA, Liza richardsonii, is most commonly caught in tidal traps or with nets. The tidal pools directly in front of Blombos Cave would require very little modification to form tidal traps, and these could be the source of the L. richardsonii found in both the MSA and LSA assemblages. Although this is the most frequently occurring species, present in all layers of the LSA and phases of the MSA, it is not very abundant, and, as there are no other species present that are usually caught with nets, there is little evidence to support the use of nets at BBC.

One of the eight species present only in the MSA (Oplegnathus conwayi) is reportedly killed by cold water upwelling events but can also be speared. Another five species that are killed by upwelling, but can also be caught by other means, are present in both the LSA and MSA (Chrysoblephus laticeps, Cymatoceps nasutus, Dichistius capensis, Epinephelus marginatus and Spondyliosoma emarginatum). These species constitute 34\% of the total MNI of fish in the LSA, and only $12 \%$ of the total MNI of fish in the LSA.

\section{Size reconstruction}

In terms of size, the LSA in total contains more medium sized specimens of all species, whereas specimens from the MSA are mostly from smaller fish including tiny specimens that are almost absent from the LSA. The difference is statistically significant (K-S statistic $=0.11, \mathrm{p}<0.05)($ Table 7.1). There is no statistical difference in size classes of all species between the LSA and the M1 and M2. The higher number of small specimens and low amount of large specimens of all species in total in the M3 is statistically different from the LSA (K-S statistic $=0.29, \mathrm{p}<0.05)$. 
Table 7.1. Percentage of NISP and MNI per size class and phase from the Blombos Cave Middle Stone Age and Later Stone Age assemblages. The upper rows include all species, and the lower rows include only those species present in both the MSA and LSA. NISP and MNI are based on elements that could be used for size estimations

\begin{tabular}{|c|c|c|c|c|c|c|c|}
\hline ASSEMBLAGE & & TINY & SMALL & MEDIUM & LARGE & XLARGE & XXLARGE \\
\hline ALL SPECIES & NISP & $0-150 \mathrm{~mm}$ & $151-300 \mathrm{~mm}$ & $301-500 \mathrm{~mm}$ & $501-800 \mathrm{~mm}$ & $801-1000 \mathrm{~mm}$ & $>1000 \mathrm{~mm}$ \\
\hline BBC M1 & 156 & 6 & 27 & 46 & 20 & 0.6 & \\
\hline BBC M2 & 138 & 7 & 36 & 37 & 20 & & \\
\hline BBC M3 & 221 & 8 & 60 & 26 & 5 & & 0.9 \\
\hline BBC MSA ALL & 515 & 8 & 43 & 35 & 13 & 0.2 & 0.4 \\
\hline \multirow[t]{2}{*}{ BBC LSA } & 1322 & 0.7 & 39 & 51 & 10 & & 0.1 \\
\hline & NISP & \multicolumn{6}{|c|}{ SPECIES THAT OCCUR IN BOTH THE MSA AND LSA } \\
\hline BBC M1 & 15 & & 66 & 21 & 14 & & \\
\hline BBC M2 & 17 & 6 & 82 & 6 & 6 & & \\
\hline $\mathrm{BBC} M 3$ & 19 & 5 & 59 & 35 & & & \\
\hline BBC MSA ALL & 50 & 4 & 69 & 21 & 6 & $\Delta$ & \\
\hline \multirow[t]{2}{*}{ BBC LSA } & 400 & 2 & 75 & 15 & 8 & $\sqrt{2}$ & \\
\hline & MNI & \multicolumn{6}{|c|}{ SPECIES THAT OCCUR IN BOTH THE MSA AND LSA } \\
\hline BBC M1 & 11 & & 55 & 27 & 18 & 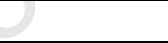 & \\
\hline BBC M2 & 11 & 9 & 73 & 9 & 9 & & \\
\hline $\mathrm{BBC} M 3$ & 16 & 6 & 63 & 31 & $\infty$ & & \\
\hline BBC MSA ALL & 38 & 5 & 63 & 24 & 8 & & \\
\hline BBC LSA & 84 & 5 & 48 & 27 & 20 & & \\
\hline
\end{tabular}

In terms of the species that occur in both the MSA and LSA, there are slightly more specimens in the medium category of the MSA, and more small sized specimens in the LSA, but the difference is not statistically significant $(\mathrm{K}-\mathrm{S}$ statistic $=0.05, \mathrm{p}<0.05)$. Large specimens of the species present in both the LSA and MSA are more common in the M1 than in the LSA.

The interpretation of the data above is based on NISP, which indicates the number of specimens, not the number of fish, and does not take possible interdependence of specimens into account. NISP was used throughout this study to calculate size classes, as it is the only fundamental measure, and allows comparison between assemblages, which was not always possible with MNI as this could not be calculated for some assemblages, and these counts are very low in others. The size classes of the species present in both the LSA and MSA have however been calculated here based on MNI as well, in an attempt to sidestep potential biases in using NISP with regards to interdependence in this particular instance. From the above, based on NISP, it appears that MSA people, particularly those occupying the cave during the M1 and M3 phases, were actually capturing larger fish of the same species that the subsequent LSA inhabitants caught. Using MNI instead of NISP 
for comparing size classes resulted in the LSA containing the highest percentage of large fish, rather than the M1, and correspondingly de-emphasising the number of small sized fishes in the LSA. The proportions of size classes in the LSA and M1 are now very similar, apart from the absence of tiny specimens in the M1. Overall, based on MNI, species that occur in both the LSA and MSA tend to be somewhat smaller in the MSA phases.

\section{Summary}

The main difference between the MSA and LSA assemblages at BBC is in the numbers of species and specimens present. The LSA has more abundant fish of the same size and species that also occur in the MSA, and there is a wider range of species in the MSA than in the LSA. The higher species diversity in the MSA, which includes the presence of small rock pool dwelling species, as well as species that were presumably collected after having washed up on shore, and the presence of one species, Galeichthys feliceps, that is easily caught but absent from the LSA indicates that MSA people exploited a wider range of fish habitats and were less selective about their choice of fish than their LSA counterparts. The number of fish from species that occur in both the MSA and LSA that can be killed by cold water upwelling is considerably higher in the LSA, which suggests that MSA people were not necessarily more reliant on washed up specimens than LSA people, if this was a source of fish.

The higher number of slightly smaller fish of the same species that occur in both the LSA and MSA could indicate that the technology employed by the MSA fishers was either geared towards, or better suited to, catching smaller fish. The similarity in species between the two assemblages suggests most fishing was done with hooks, although other methods were also employed.

As most of the best preserved elements are the same in both the MSA and LSA, it seems that these specific elements possess characteristics that are conducive to better preservation. Most of these elements appear to be fairly robust, which could have contributed to their preservation. Whether these are more robust than the less common elements, is not known. Density values are not available for elements of southern African fish, and previous studies (on cod and salmon bones) have shown that bone survival is not always linked with high densities, that bones with higher densities do not necessarily survive better than those of lower density, and that bone survival is dependant on a 
number of factors, particularly those relating to the shape and size of the bone, as well as cultural practices. Cultural practices such as removal or transport of certain body parts are difficult to establish here as in most instances recognising such practices requires that vertebrae are identified to species, which could not be done here. Whatever the reasons are for their higher frequencies in both the MSA and LSA, these elements can be considered to have better survival potential in older cave assemblages and should be included during identification and quantification.

There is a large deficit of elements in the MSA relative to the LSA, and the most likely reason for this deficit is that age-related taphonomic processes severely reduced the original number of fish that were deposited during the MSA. The similarity in skeletal elements that have survived in both the MSA and LSA reinforces this observation. Based on the comparison of the BBC LSA and MSA fish remains, there is little evidence to support the theory that MSA people were incapable of fishing.

\subsubsection{Klasies River main site}

\section{Agent of accumulation}

Deacon (1995; 2001; Deacon and Geleijnse 1988) has argued that the fish remains at Klasies River main site are the result of regurgitated pellets from cormorants that nested in the cliff above the site, as they do now. They have shown, based on mass, that the majority of fish from the members above the MSA I occur in the non-cultural layers. This observation is supported by the number of specimens counted here (total NISP in cultural units $=5562$, and in sterile units $=7254$ ).

The MSA I member contains more specimens in the cultural units (1886 versus 1408). It is assumed here that the majority of fish remains that occur in the sterile units were accumulated by non-human agents, presumably cormorants but the possibility that other animals were accumulators cannot be dismissed. To investigate whether the fish remains are solely due to cormorants or other non-human accumulators the data from the cultural and sterile layers were compared in terms of species present, species size, cultural or natural modifications, skeletal element representation and completeness.

Significant differences between the fish remains from the cultural and sterile units may be seen as an indication that different agents were responsible for depositing the material. A lack of significant differences could be interpreted to indicate that the same agent was 
responsible for the fish remains in both the cultural and sterile units. Although differences between assemblages can point to different accumulators, similarities do not necessarily indicate that the same agent collected the fish bones. A lack of difference between assemblages could simply be a reflection of the options available to humans and other fish predators in the environment.

\section{Agent of accumulation - comparison of cultural and sterile units}

The species composition of the fish from cultural and sterile units was quite similar, with 20 out of the 31 species present occurring in both. Six species occurred only in the cultural units, and five in only the sterile units. These were present in very low numbers.

In the absence of data on volumes of deposit excavated from KR, it is difficult to assess the significance of differences in numbers between the cultural and sterile units. Considering the microstratigraphic nature of the cultural deposit and fine level of excavation, as well as the effects of the heavy overburden on units, it is difficult to determine how much displacement of the relatively small fish bones could have occurred. A possibility is that non-culturally accumulated fish migrated into cultural units and culturally accumulated fish into sterile units. The MSA III sterile units were considerably thicker than the cultural units, and contained almost double the number of fish specimens. The sterile units of the MSA I were also thicker than the cultural units, but in this member fish were more numerous in the cultural units. This higher number is not due to increased fragmentation of elements leading to higher NISP counts, as the specimens from the cultural units are significantly more complete than those from the sterile units.

In the HP, where numerous cultural units were interspersed with a few thin sterile units, fish remains were more common in the sterile units. The relative volume of cultural versus sterile units could not be assessed for the MSA II, but more fish bones were present in the sterile units. In most of the members the specimens responsible for higher NISP's in the sterile units that could be identified to family or species are from a limited range of fish, namely Clinidae, Gobiidae, shark and, in the MSA III, Galeichthys feliceps. Scutes from unidentified Carangidae accounted for most of the higher NISP in the cultural units of the MSA I. Thus the differences in numbers between the cultural and sterile units relate mostly to an increase in numbers of species that are already present in both units, usually in relatively high quantities. 
Comparison of changes in the relative frequency of species per member indicates some differences between the sterile and cultural units. Most notable are the high frequency $(41 \%)$ of sharks in the HP cultural, relative to a low frequency $(6 \%)$ in the sterile units, and a high frequency (54\%) of Clinidae in the HP sterile, relative to a lower frequency $(21 \%)$ in the cultural units. Changes in the relative frequency of Galeichthys feliceps per member are the same for both the cultural and sterile units, which increase from the oldest to the younger members.

\section{Contribution of fish by cormorants}

Several of the species reportedly preyed on by the white-breasted cormorant, $P$. carbo, were found. These are Clinidae spp., Acanthistius sebastoides, Galeichthys feliceps, Sarpa salpa, Chirodactylus brachydactylus, Spondyliosoma emarginatum and Diplodus capensis. The latter two occurred in very low numbers (total NISP of one and three, respectively). Most of these species were present in both the cultural and sterile units of the members in which they occurred. The few exceptions were the single $S$. emarginatum specimen in the sterile units of MSA I, two C. brachydactylus specimens present only in the sterile units of the MSA III, and seven S. salpa specimens only in the sterile units of the MSA II.

There was some variation in the number of specimens (NISP) from species preyed on by cormorants between the cultural and sterile units per member, but overall there were more specimens in the sterile units of most members. In terms of relative frequency, changes in frequencies of species taken by cormorants are similar in both the cultural and sterile units of most members. The exception is the higher frequency of Clinidae in the sterile units of the HP, whereas sharks, not known to be a cormorant prey species, are the most frequently occurring species in the HP cultural units. Changes in the frequencies per member of other species not taken by cormorants are very similar, particularly in the MSA I and MSA II. In the MSA III, sharks are more frequent in the sterile units, and Galeichthys feliceps in the cultural units.

None of the specimens from either the cultural or sterile units showed any evidence of modification by humans. Very few specimens from the assemblage displayed signs of digestion, and most that did appeared to belong to very fresh specimens, mostly of Acanthistius sebastoides, a known $P$. carbo food prey item. These were excluded from this analysis as they most likely originate from recent cormorant pellets, perhaps 
regurgitated between excavation seasons and accidentally incorporated into the assemblage. Apart from these very fresh looking bones, 19 specimens from the cultural and 15 from the sterile units showed evidence of gastric etching. Ten of the 19 specimens from the cultural units were from species found in cormorant pellets by von den Driesch (2004), and three of the fifteen specimens in the sterile units with evidence of gastric etching were from species identified in cormorant pellets. All gastric etched specimens were from small (151-300mm) sized fish.

Non-vertebral elements were more common than vertebrae in the modern cormorant pellets analysed by von den Driesch (2004). In contrast to this, both the cultural and sterile units of KR are heavily dominated by vertebral elements, with $89 \%$ of the specimens in the cultural units and $93 \%$ in the sterile units being vertebrae.

The majority of specimens in both the cultural and sterile units per member were from small (151-300mm) fish, which is the size class preferred by cormorants. There was some variation between the frequency of size classes between the cultural and sterile units but these differences were not statistically significant. Both the cultural and sterile units contained specimens of larger size classes that could not have been brought in by birds. A few of these larger fish were from species that occurred only in the cultural units (Seriola lalandi and Petrus rupestris - 801-1000mm; Sparodon durbanensis - 501-800mm), whereas species occurring only in the sterile units were generally smaller. Although shark remains were not identified to species, and sizes were not estimated from the many vertebrae present due to a lack of comparative specimens, von den Driesch (2004) identified several shark species that were larger than $80 \mathrm{~cm}$. She did not specify in which units these occurred, but fish of this size could not have been taken by birds.

\section{Contribution of fish by penguins}

Although cormorants are thought to be the most likely contributors of fish remains in the culturally sterile units, the possibility that other non-human animals contributed some or all of the fish remains needs to be considered. Jackass penguins (Spheniscus demersus) could be responsible for the pelagic Clupeidae found in the site (NISP=27), which, along with pelagic Gobiidae, form the main component of penguin diet (Kemper et al. 2007; Maclean 1993). These bones could have entered the site as stomach contents of the birds that either nested there or were brought in as food by humans. The Gobiidae in this assemblage were found in all cultural and sterile layers and could not be identified to 
species so it is unclear whether these are pelagic or near-shore species. The Clupeidae occurred in one cultural and one sterile layer.

\section{Contribution of fish by African fish eagles}

The African fish eagle, Haliaeetus vocifer, is another potential accumulator of fish bone at KR. Its preferred nesting and roosting habitats are in trees close to water, but they sometimes roost on cliffs. Stewart et al. (1999) have shown that a significant number of bones can accumulate below roosts of this species in a relatively short time. Assemblages accumulated by $H$. vocifer are characterised by an average prey size of $35-40 \mathrm{~cm}$, specific prey selection for near shore but less common species, high numbers of spines and cleithra, low frequencies of vertebrae and skull elements, vertebrae lacking processes, an absence of signs of gastric etching and high fragmentation of elements, particularly those from fish larger than $30 \mathrm{~cm}$, presumably due to being swallowed whole.

There are some similarities between these characteristics and the KR assemblage, specifically the very low incidence of gastric etching, the lack of vertebral processes and the high fragmentation of elements in the sterile units. Characteristics that are contrary to those of typical Haliaeetus vocifer assemblages include the fact that the majority of specimens are smaller than that preferred by this species in both the cultural and sterile units, spines and cleithra are underrepresented, and vertebrae are by far the most common elements in the assemblage.

The very low incidence of bones etched by gastric processes is more consistent with an assemblage created by Haliaeetus vocifer than by cormorants, based on our current knowledge of the effects of these birds' digestive processes on fish bone. It is possible that bones that were heavily etched at the time of deposition have not survived due to their increased vulnerability to taphonomic processes, and that those that have survived did so because they were less etched, or not etched at all, and therefore less fragile. Stewart et al.'s (1999) study was based on a relatively 'young' assemblage, accumulated within ten years, yet no signs of digestion were noted on any of the specimens. The assemblage did, however, show a very large deficit in elements, and it might be that this species' digestive processes are efficient enough to render several elements invisible either prior to or shortly after regurgitation. The generally lighter colour of the etched specimens in the KR assemblage makes the exact provenance of these bones uncertain and it was thought best to exclude them from the analysis. 
The lack of haemal or neural processes on the vertebrae from both the cultural and sterile units is similar to that described from assemblages created by Haliaeetus vocifer, but it is possible that other factors are responsible for this condition. Most of the vertebrae from the BBC MSA also lack vertebral processes, but, as BBC is a cave site, it is unlikely that the fish remains were deposited there by $H$. vocifer as it does not inhabit caves.

The specimens from fish smaller than $30 \mathrm{~cm}$ were significantly more complete than those from larger fish in both the cultural $(\mathrm{K}-\mathrm{S}$ statistic $=0.17,<0.05)$ and sterile units $(\mathrm{K}-\mathrm{S}$ statistic $=0.21,<0.05)($ Table 7.2), which conforms with Stewart et al.'s (1999) findings from Haliaeetus vocifer roosts. There were no significant differences between the cultural and sterile units in terms of completeness scores of specimens that were smaller or larger than $30 \mathrm{~cm}$ (K-S statistic $=0.09,<0.05$ and $0.13, \mathrm{p}<0.05$, respectively). The smaller specimens might be more intact as a result of having been swallowed whole by $H$. vocifer, or perhaps cormorants, thereby suffering less damage. It could also reflect differential processing of smaller fish by humans, as recorded by others (Gifford-Gonzalez et al. 1999; Stewart 1991; Zohar and Cooke 1997; Zohar et al. 2001).

Table 7.2. Percentage per completeness score of all elements and species combined of the Klasies River main site cultural and sterile units for specimens from fish smaller and larger than $30 \mathrm{~cm}$ respectively. Calculations are based on NISP of elements for which \% completeness scores as well as size estimations were recorded

\begin{tabular}{|l|r|r|r|r|r|r|}
\hline & \multicolumn{6}{|c|}{ \% COMPLETENESS SCORE } \\
\hline ASSEMBLAGE & \multicolumn{1}{|c|}{ NISP } & $\mathbf{0 - 2 0}$ & $\mathbf{2 0}-\mathbf{4 0}$ & $\mathbf{4 0 - 6 0}$ & $\mathbf{6 0 - 8 0}$ & $\mathbf{8 0 - 1 0 0}$ \\
\hline KR STERILE $<30 \mathrm{~cm}$ & 420 & 3 & 8 & 18 & 23 & $\mathbf{4 8}$ \\
\hline KR STERILE $>30 \mathrm{~cm}$ & 131 & 6 & 21 & 23 & 21 & $\mathbf{2 9}$ \\
\hline KR CULTURAL <30cm & 334 & 1 & 7 & 12 & 23 & 57 \\
\hline KR CULTURAL >30cm & 92 & 2 & 16 & 18 & 23 & $\mathbf{4 0}$ \\
\hline
\end{tabular}

As there is little or no reason to assign a human accumulator to the fish remains in the culturally sterile units of KR, and specimens from smaller fish were found to be more complete in both the sterile and cultural units, the same pattern of element fragmentation might be due to post-depositional processes favouring the preservation of smaller elements at this site. Alternatively, the fish from both the cultural and sterile layers were deposited by Haliaeetus vocifer. The latter seems unlikely when considering the following factors that argue against this bird being a primary accumulator of fish bone at KR.

The majority of fish in the KR assemblage in both the cultural and sterile units are smaller than that reportedly preferred by Haliaeetus vocifer. As the current data of the species taken are from habitats different to that of $\mathrm{KR}$, and this bird is known to have strong 
preferences for particular fish species, depending on its habitat, it is possible that the species preferred in the vicinity of KR might differ in size from those recorded at the other sites, but it is not clear which species are preferred in this region.

Factors arguing against Haliaeetus vocifer being the primary accumulator of fish remains at KR include the information that the most frequently occurring elements found in this species' roosts were spines and cleithra, whereas these occur at very low frequencies in the KR assemblage (31 spines and no cleithra in the cultural units, and 24 spines and seven cleithra in the sterile units). In addition, vertebrae were poorly represented in $H$. vocifer pellets, whereas these constituted the vast majority of elements in the KR assemblage.

\section{Comparison of cultural and sterile units - skeletal element representation}

Some differences in the skeletal element representation of species between the cultural and sterile units were noted. Species known to be preyed on by cormorants are of particular interest here, as some elements that have been through the birds' digestive system might preserve better than others. Four elements of Acanthistius sebastoides occurred in both the cultural and sterile units, whereas there were six other elements present only in the cultural units, and four only in the sterile units. Elements from all regions of the skeleton were present in both the cultural and sterile units. There were twelve Galeichthys feliceps elements in the sterile units, and eight in the cultural units. Six of the elements from the sterile units did not occur in the cultural units, and two elements occurred only in the cultural units. Five elements of Sarpa salpa occurred in the sterile units, and three of the same elements were present in the cultural units. There were five elements of Chirodactylus brachydactylus in the cultural units, and four in the sterile units. Three of the elements occurred in both the cultural and sterile units. There were considerably more elements of Liza richardsonii in the sterile $(n=16)$ than cultural $(n=7)$ units. All the elements in the cultural units also occurred in the sterile units. Although this specific species has not been reported as cormorant prey in the literature, other Mugilidae have been and, as they behave very similarly, might be preyed on by cormorants.

Similar differences in the number of elements preserved in the cultural and sterile units were found in the species not reportedly preyed on by cormorants. In most instances there were more elements preserved in the sterile units, with the exception of Pomatomus saltatrix and Scomber japonicus, which had more elements in the cultural units. Two 
species had the same elements present in both the cultural and sterile units

(Gymnocrotaphus curvidens and Pomadasys olivaceum). In total, of the species that occur in both the cultural and sterile units, a wider range of elements were preserved in the sterile units $(n=75)$ than the cultural units $(n=54)$. This difference does not appear to be a function of the larger overall sample size in the sterile units as vertebrae and specimens that could not be identified to species level were excluded from the skeletal element representation calculations, which resulted in 186 specimens from the sterile units, and 146 from the cultural units being used. Although more elements per species were present in the sterile units, the element completeness data showed that the specimens from the sterile units were significantly more fragmented than those from the cultural units.

\section{Cultural modification indicating human agency}

Most of the burnt bones occurred in the cultural layers of all members, some of which $(5 \%)$ were calcined which indicates direct exposure of defleshed bone to high temperatures (Asmussen 2009). No other signs of cultural modification such as cut marks were found.

\section{Summary of agents of accumulation}

From the above, there appears to be some slight differences between the cultural and sterile units that might indicate different agents of accumulation. The most significant differences are in the wider range of elements preserved in the sterile units, and the higher degree of fragmentation of specimens in these units. The species present are similar in both, and the few that occur only in the cultural or sterile units occur in low numbers. The ranges of fish sizes are similar in both the cultural and sterile units. The majority of fish are of the size that cormorants take, although there are some exceptions of larger fish, some of which occur only in the cultural units, and these tend to be bigger than those that occur only in the sterile units.

Several of the species preyed on by cormorants are present in the assemblage in both the cultural and sterile units, as are many species not reported from these birds' diets. The low number of specimens with signs of gastric etching and the high number of vertebral elements in both the cultural and sterile units is inconsistent with von den Driesch's (2004) findings of the contents of modern regurgitated cormorant pellets. The sample of modern cormorant pellets analysed is small $(n=10)$, and larger samples might produce different results in terms of the number of elements displaying signs of digestion. The low 
incidence of gastric etching is more consistent with pellets cast by $H$. vocifer, but the starkly different element composition argues against this bird being a primary accumulator of fish at KR.

The higher number of calcined bones in the cultural units might indicate that these bones were discarded on the fire after the meat was consumed, which suggests a more direct human agency as birds are unlikely to roost above the site while it was occupied by humans with fires burning below. Unequivocal evidence for cultural modification of fish bone indicating a human agent of accumulation is rare, even in assemblages where fish processing was known to be practiced. The absence of cultural modification is not sufficient to exclude humans as possible accumulators of the fish bone.

The possibility that the specimens showing evidence of etching are from human gastric processes cannot be ruled out, but cannot be definitely established. The presence of species that form part of fish eating birds' diet is not enough evidence for birds being the only accumulators. Humans inhabiting the same landscape are also likely to take the most available species, as does the cormorant $P$. carbo. Although the majority of specimens are from fish of the same size taken by cormorants, there is no reason to assume that all the smaller fishes were brought in by birds. The majority of the same species of fish occurring at $\mathrm{H} / \mathrm{RC}$ are of the same size as those from $\mathrm{KR}$, and there is no doubt that those were accumulated by humans.

The fact that a large quantity of the fish remains at KR occurs in sterile layers unassociated with cultural remains confirms that agents other than humans, most likely cormorants, were responsible for a considerable amount of the fish assemblage. Their role in the accumulation of fish bone in the cultural layers is less clear, and the data from this study does not confirm that they are the only agents of accumulation in the cultural layers. Until further studies of the taphonomic signatures of South African fish predators are conducted, and in the absence of clear digestion or gnaw marks on the KR fish, it is reasonable to assign a human agent of collection to at least some of the fish remains.

\section{Method of capture - species that occur exclusively in either the cultural or sterile units}

The species that are present only in either the cultural or sterile units occur in very low numbers, but their presence indicates slight differences between the cultural and sterile units in terms of how they could have been acquired. Two species present only in the 
cultural units, Petrus rupestris (MSA I and II) and Argyrozona argyrozona (HP) are not affected by cold water upwelling and can only be caught with baited hooks. The $A$. argyrozona specimen is from a juvenile fish, which could have been caught from shore (adults occur offshore). The very large Seriola lalandi in the cultural units of the MSA II could have been caught with baited hooks or, alternatively, speared, perhaps after being trapped in a tidal rock pool. . The large Sparodon durbanensis (from specimens representing either one or two individual fish) in the cultural units of the MSA II could have been caught by the same methods as $S$. lalandi. The medium sized Epinephelus marginatus in the cultural units of the MSA I could have been speared or hooked, or alternately, collected after being killed by a cold water upwelling event. The other species that are present only in either the cultural or sterile units can all be caught in a variety of ways and are not useful indicators of differences between the cultural and sterile units.

\section{Method of capture - all species}

Although it is accepted here that most of the fish in the sterile units derive from fish predators other than humans, allowing for some displacement of fish from the cultural units into the sterile units and vice versa, the potential methods by which the fish were acquired is assessed here for the species from both the cultural and sterile units.

\section{Scavenging of washed up specimens}

Cold water upwelling events resulting in fish being killed or stunned do occur in the vicinity of KR. Ten out of the 18 species that are known to be killed by cold water upwelling events were found. Eight occur in the cultural units overall and eight in the sterile units. In total, there are 31 specimens (NISP) from fish that are killed by cold water in the cultural units, and 50 in the sterile units. In the cultural units, six species occur in the MSA I, and two in each of the MSA II, HP and MSA III members. Seven species killed by upwelling occur in the sterile units of the MSA I, four in the MSA II, two in the HP and one in the MSA III. One of the species affected by cold water upwelling events, Diplodus capensis, is present only in the MSA II, in both the cultural and sterile units. Most of the Diplodus capensis reportedly killed by upwelling are juveniles. The ones found in KR MSA II (cultural and sterile) are in the small size category (155-300mm), which encompasses both juvenile and adult sizes so it is not clear whether these were juveniles that had been washed up and collected. The offshore species Pterogymnus laniarius, present in the sterile units of the MSA I and II, has not been reported to be 
affected by cold water upwelling events but as an offshore species its presence suggests that it was either scavenged after being washed up on shore, or entered the site as the stomach contents of a captured or scavenged seal.

\section{Hooks}

Twenty-three of the species in all units and members combined can be caught with baited hooks from the shore, 19 of which are in the cultural units, and 17 in the sterile units. Thirteen occur in the cultural units of the MSA I, twelve in the MSA II, eight in the HP and six in the MSA III. In the sterile units, 13 species that can be caught on baited hooks occur in the MSA I, twelve in the MSA II, eleven in the HP and five in the MSA III.

\section{Tidal traps and nets}

Fifteen of the species present in the total assemblage can be caught in tidal traps, twelve of which occur in either the cultural or sterile units. Eight occur in the cultural units of the MSA I, ten in the MSA II, seven in the HP and six in the MSA III. Nine species occur in the sterile units of the MSA I, ten in the MSA II and HP and six in the MSA III. One species, Liza richardsonii, which is typically only caught with tidal traps or nets, is present in both the cultural and sterile units of all members. The coastline around KR is steep, but there are some rock pools in the close vicinity of the site which could have functioned as tidal traps, either natural or modified by humans, for capturing species such as L. richardsonii. Gobiidae, excluding the pelagic species of this family, are typically caught in rock pools with small nets today. It is not clear whether the Gobiidae in the assemblage are pelagic or rock pool dwelling species, due to a lack of sufficient comparative specimens.

\section{Spearfishing}

There is a total of twelve species in the assemblage that can be speared. In the cultural units, five occur in the MSA I, nine in the MSA II, five in the HP and three in the MSA III. Six of the species in the sterile units of the MSA I can be speared, seven in the MSA II, eight in the HP and four in the MSA III. One species, Chirodactylus brachydactylus, can only be caught by spearing, and occurs in both the cultural and sterile units of all members apart from the cultural units of the MSA III. 


\section{Sharks}

The shark species that are present in both the cultural and sterile units of all members could have been caught in natural or constructed tidal pools, or caught with baited hooks. It is possible that the Klasies River estuary was larger and perhaps closer to the main site during periods of lower sea levels (von den Driesch 2004). An enlarged estuary would have been a suitable habitat for juvenile sharks that often inhabit inshore waters and estuaries throughout the year, as well as for some species of adult sharks, as they move towards the inner shore and sometimes estuaries during the summer months (Smith and Heemstra 2005; Smale et al. 2007). The high frequency of sharks in the HP cultural layers, at a time when sea levels were lower, could lend support to the hypothesis of a larger estuary. However, only 10 out of the 18 shark species identified by von den Driesch (2004) enter estuaries, and none of the identified species are estuarine dependant. Thus the higher number of shark bones in the HP could relate to more suitable shark habitats due to lowered sea levels, but it does not confirm the presence of a larger estuary in the vicinity. Some of the shark species identified by von den Driesch (2004), such as Rhinobatos annulatus (lesser guitarfish), occur in shallow inshore waters and could have been caught by spearing. Others identified by von den Driesch are more commonly caught with baited hooks.

\section{Summary of methods of capture}

The species that are killed by cold water upwelling events that occur at KR could have been collected by humans. If so, then the higher number of these species in the oldest member could indicate that people were more reliant on washed up fish during this period, and that with time the importance of this resource declined. Alternatively, as cold water upwelling events are summer occurrences, it could indicate a predominantly summer occupation during MSA I, and less seasonal visits during the other time of the other members. These species occur in very low numbers and are not the ones that form the bulk of fish in any of the units or members. Therefore, although it is possible that people took advantage of washed up fish, this was not the primary source of fish for the KR inhabitants.

Many of the species can be caught in a number of ways, but the majority in terms of NISP point to the use of hooks, traps or spears. If the Gobiidae in the assemblage are from rock pool dwelling species, the presence of relatively large numbers of these fish in all 
members could indicate the use of nets, but, as these fish tend to be abundant in rock pools, they could have been collected by hand without the use of any technology.

\section{Diachronic change in fish procurement}

A much wider range of species are present in the MSA I, and the number of species decreases from the oldest to the youngest members. Fish are considerably smaller in the MSA I compared to that of the younger MSA II and HP members. Two species, Galeichthys feliceps and Liza richardsonii, occur most frequently throughout the assemblage, along with unidentified Gobiidae and Clinidae.

Of the species that were present in the cultural units of all members, the relative frequency of Clinidae in particular, and Gobiidae to a lesser extent, decreased from the oldest to the youngest member, whereas the frequency of Galeichthys feliceps increased considerably from the MSA I to the MSA III. The relative frequencies of other species did not change much between members. Clinidae are rock pool dwelling fish that can be easily caught on a small hook, but is also easily collected by hand from the pools. There are several methods by which $G$. feliceps can be caught, including tidal traps, hooks or spears. This significant change in species from Gobiidae and particularly Clinidae to G. feliceps over time indicates either a change in the methods used to procure fish, or a change in the availability of species over time.

Lowered sea levels during the HP would have changed the coastal environment and could have resulted in changes in the available species. Shellfish data indicated that there was an increase in sandy shore species and a decrease in species that live on rocky shores during the HP and MSA III (Thackeray 1988). Increased sandy shores would result in less rocky habitats suitable for Clinidae, but it would still be a suitable habitat for Galeichthys feliceps. The gradual decline of Clinidae from the oldest to the youngest members conforms to the suggestion that sandy shores were dominant and this is confirmed by the shellfish data. The largest change in frequency from Clinidae to G. feliceps at KR however is seen between the HP and the MSA III. There were still considerable quantities of Clinidae in the HP. Thus the high frequency of G. feliceps in the MSA III member relative to others appears to be related to an increased focus on this species. As it can be caught by several methods, it does not necessarily indicate a significant change in technology over time. 


\section{Seasonality}

Pomatomus saltatrix is present in both the cultural and sterile units of each member. It could be an indication of summer occupation but the numbers are low and considering that these data are from conflated units it is not possible to infer seasonality. The large number of shark remains in the KR assemblage, and particularly in the HP cultural layers, could indicate summer occupation as many of these species move to shallow coastal waters and estuaries during the summer. Evidence for summer occupation does not preclude winter occupations. Based on mortality profiles of seal remains at KR, Klein (1994; Klein and Cruz-Uribe 1996) argue that people inhabited KR year round, or alternatively that visits to the coast by MSA people were seasonally unfocussed. Inferring seasonality based on fish species is not ideal, as species identifications are not the best indicators of season. Further work on incremental growth analysis of the KR fish remains has the potential to provide finer resolution on the topic of seasonal occupation.

\section{Comparison with previous analysis}

The total number of specimens (NISP) from the KR assemblage counted here (12816) differs from that counted by von den Driesch (2004) (NISP=13750) as some data had to be excluded from this study due to uncertainty about provenance. The number of species identified from KR main site in this study is considerably lower than those identified by von den Driesch (2004) (Table 3.1). This difference relates mainly to a lack of these species in the comparative collection used in this study (Table 5.1). A total of 15 families and 28 species of bony fish identified by von den Driesch are not in this study's comparative collection. It is not possible to determine what the contribution of the species lacking from the comparative collection was to the overall assemblage as on den Driesch did not specify NISP per species. One of these species, Chorisochismus dentex (Gobiesocidae), did contribute significantly to the assemblage and appears as one of the five most abundant families in several of the squares. Two other species that fell in the most abundant category of some of the squares and members but not present in the comparative collection are Terapon jarbua (Teraponidae, thornfish) and Engraulus japonicus (Engraulidae, anchovies).

Nineteen of the species identified in this study were also recorded by von den Driesch. Six of the species identified by her are present in the comparative collection, but were not 
identified here, and seven of the species identified in this study were not identified by von den Driesch (2004).

As noted in Chapter Four, the KR data in this study is presented separately for the respective cultural and sterile units MSA I, II, HP and MSA III. This differs from von den Driesch's (2004) approach. Her data were combined according to three chronological units, the LBS member (MSA I), the SAS member (upper and lower SAS data presented separately and in some instances combined - MSA II) and the Upper member (HP and MSA III combined).

\subsection{The significance of fish in the diet of Stone Age people}

\section{Later Stone Age fish remains}

\section{Hoffman's/Robberg Cave, Blombos Cave LSA, Blombosfontein 1 and 6}

There are considerably more species present at H/RC than in the BBC LSA, but eleven species occur in both. The majority of specimens from $\mathrm{H} / \mathrm{RC}$ are from small fishes, and at BBC LSA most specimens are from medium sized fish. The size difference is partly due to the presence of small species such as Sarpa salpa at H/RC, but species that occur in both assemblages tend to span a wider range of sizes in H/RC. Most of the species that are present in both assemblages tend to be bigger at BBC. At both BBC (LSA) and H/RC, the presence of both large and small species caught with hooks suggests that people were making and using fishing gear of different sizes, and were aware of specific species' ecology and deliberately targeting certain sized fishes.

At $\mathrm{H} / \mathrm{RC}$ it seems that in some layers people were focused on targeting specific species, whereas in other layers people were taking a wider range of species. This targeting was not noted in the BBC LSA, and this apparent absence could be due to the manner in which the fish data was combined in this study. Fish data from individual units at this site were grouped into five respective layers following Henshilwood (1995; 2008), as the numbers of fish in individual units were small. The very highly resolved nature of the H/RC sequence and large numbers of fish per layer allowed detection of short-term episodes of behaviour that could not be detected at BBC. There were very few fish specimens in the BBF1 and 6 assemblages, so the data from these two sites has not been particularly useful for comparison with other assemblages in terms of species present. 


\section{Hoffman's Cave and Nelson Bay Cave}

The $\mathrm{H} / \mathrm{RC}$ fish assemblage has contributed a large amount of new data that is directly comparable with the fish remains from nearby Nelson Bay Cave. NBC was inhabited by people during the period that $\mathrm{H} / \mathrm{RC}$ was occupied. The renewed exploration of the $\mathrm{H} / \mathrm{RC}$ site showed that artefacts from $\mathrm{H} / \mathrm{RC}$ are typical of the post-Wilton, including informal lithic artefacts made from locally available raw material, whereas at NBC, which is only a few hundred meters away, the transition from Wilton to post-Wilton occurred later, at almost exactly the same time that $\mathrm{H} / \mathrm{RC}$ was no longer occupied. The reason for this difference is being studied.

The species composition at $\mathrm{H} / \mathrm{RC}$ is similar to that at NBC (Inskeep 1987). Species not found at $\mathrm{H} / \mathrm{RC}$ but present at NBC are Lithognathus mormyrus, Petrus rupestris and Argyrosomus inodorus/japonicus. The latter two are present in low numbers at NBC. It is possible that some of the specimens identified as Lithognathus lithognathus at $\mathrm{H} / \mathrm{RC}$ could actually be L. mormyrus if their morphology is similar, as this species is lacking in the comparative collection. Eleven of the species present at $\mathrm{H} / \mathrm{RC}$ were not present at NBC. These are Acanthistius sebastoides, Chrysoblephus laticeps, Cheimerius nufar, Mugil cf. cephalus, Monodactylus falciformis, Pomadasys commersonnii, Pomadasys olivaceum, Spondyliosoma emarginatum, Scomber japonicus, and unidentified Gobiidae and Triglidae. Most occur infrequently and almost all are in the shelly layers. Two of these species occur in both the Zostera and shelly layers (C. nufar and S. emarginatum). The latter is present in several layers. The large numbers of Clinidae/Blenniidae at NBC were not recorded at H/RC. Gobiidae, Clinidae and Blenniidae are very similar in terms of the habitat in which they occur, which is mostly in shallow rock pools, although there are some pelagic Gobiidae. A single element of an unidentified Gobiidae was found in layer TOM.

The reason for the large numbers of species that are present at $\mathrm{H} / \mathrm{RC}$ but absent from NBC is not clear. It cannot relate to sample size as the NBC sample is much larger than that of H/RC, with 14662 fish identified from the Inskeep excavations alone (Inskeep 1987).

There is no consistent patterning in the way that these species can be caught to suggest that people inhabiting $\mathrm{H} / \mathrm{RC}$ were using different methods of capture to those at NBC. Sinkers are present at both sites during $\mathrm{H} / \mathrm{RC}$ 's occupation, but increase significantly at $\mathrm{NBC}$ after $\mathrm{H} / \mathrm{RC}$ was abandoned. The ecologies of these species are also not specific 
enough to infer significant differences in fishing strategies or habitats exploited.

Methodological differences between Poggenpoel's (1996) study of the NBC fish and this

study could account for the lack of some of the species at NBC, as his identifications were based on a limited number of elements, namely premaxillae, dentaries, basioccipitals, first vertebrae and otoliths. Four of the species present only at H/RC, Chrysoblephus laticeps, Monodactylus falciformis, Scomber japonicus and Spondyliosoma emarginatum, were identified on elements other than those used by Poggenpoel.

One of the most striking differences between $\mathrm{H} / \mathrm{RC}$ and $\mathrm{NBC}$ is the complete absence of Clinidae/Blenniidae at $\mathrm{H} / \mathrm{RC}$, whereas these species were common at NBC and contributed a substantial amount in terms of meat weight to the overall fish assemblage during the same time that H/RC was occupied (Inskeep 1987). There is no clear reason why the NBC inhabitants exploited these rock pool dwelling species and the H/RC people did not. It might be that larger samples of H/RC fish would produce some of these species, but that seems an unlikely explanation for their complete absence.

\section{Fish avoidance or taboos}

The absence of Galeichthys feliceps from the BBC LSA, a species that is common in the MSA of both BBC and KR, was noted previously (van Niekerk 2005), and this species is still absent from the BBC LSA despite the increased sample size. As it is a species that is easily caught, I have speculated that it might have been actively avoided, either to avoid injury from its toxic mucous-covered and serrated spines, or perhaps due to a social taboo against eating fish that lack scales. Historical records refer to 'Hottentots' refusing to eat fish without scales (Kolbe 1738, in Thompson 1913). This species occurs in relatively low numbers in some of the LSA assemblages that date to older than $2000 \mathrm{BP}$, but is almost completely absent from younger assemblages. LSA assemblages dating to older than 2000 BP containing G. feliceps are $\mathrm{EBC}(\mathrm{MNI}=15$; NISP=28), TC (MNI=79, NISP=151), SC $(\mathrm{MNI}=2), \mathrm{PKM}(\mathrm{MNI}=1), \mathrm{NBC}(\mathrm{MNI}=3)$ and SRM1 (MNI=1) (Inskeep 1987; Jerardino 1998; Poggenpoel 1996). The only reported G. feliceps remains from assemblages younger than $2000 \mathrm{BP}$ is one specimen from Die Kelders in the oldest LSA occupation layer ( 2000 BP) (Schweitzer 1979). It therefore appears that some people may have been purposefully avoiding this species for one reason or another after about $2000 \mathrm{BP}$. 


\section{Fishing in the Later Stone Age: the contribution of the Blombos Cave LSA and Hoffman's/Robberg Cave studies}

The $\mathrm{H} / \mathrm{RC}$ assemblage contains a very large number of fish relative to the other assemblages studied. From my observation of the faunal remains, the inhabitants' protein sources were almost exclusively marine fauna, mostly fish and shellfish, and very few terrestrial faunal remains are present. This conforms with Inskeep's (1987) findings of increased marine food consumption at NBC after 3300 BP and Sealy's (2006) isotopic studies of human remains which indicated that people living on the Robberg peninsula at this time were consuming more high-trophic-level marine protein than their counterparts at nearby Matjes River Rock Shelter. Seriola lalandi is a carnivorous fish and therefore a higher trophic level source of marine protein than Sarpa salpa, which has a vegetarian diet. Based on average weight reconstructions, the considerably higher contribution of protein from $S$. lalandi relative to $S$. salpa also supports Sealy's (2006) findings.

The BBC LSA inhabitants ate considerably less fish than those at $\mathrm{H} / \mathrm{RC}$, and their diet was more varied and included more terrestrial animals than those of the $\mathrm{H} / \mathrm{RC}$ inhabitants. However the non-fish fauna of $\mathrm{H} / \mathrm{RC}$ has yet to be studied. Fish remains are more common and occur in higher densities in the layers dating to between 2000 and 1800 BP. Thereafter, the numbers of fish decline and it seems that fish played a less significant role in the diet of the inhabitants. Similar low incidences of fish have been noted at many other LSA sites after $2000 \mathrm{BP}$. The reduction in fish numbers from $2000 \mathrm{BP}$ to younger layers is also seen at Nelson Bay Cave, Die Kelders and Byneskranskop. Few sites dating to younger than $1500 \mathrm{BP}$ contain considerable amounts of fish. It appears therefore that the role of fish diminished during the late Holocene, which fits in with the results from stable isotope analysis of human skeletons that have shown reduced dependence on marine foods in the last 2000 years on both the western and southern Cape coasts (Sealy 2006; Sealy and Pfeiffer 2000; Sealy and van der Merwe 1988). This could relate to an increased dependence on domestic stock, as seen at DK, but this is unlikely at BBC, as the inhabitants were not herders, although the site does contain a few sheep bones in the older layers. There are no changes in the mammalian and shellfish record of BBC to suggest that new food items replaced the role of fish in the diet. In fact, the mammalian fauna shows that the range of species exploited reduced significantly after $1600 \mathrm{BP}$. 
Fish bones in LSA assemblages are said to often outnumber mammal bones by an order of magnitude (Avery et al. 2008; Halkett et al. 2003; Klein et al. 2004). This appears to be true for the LSA H/RC assemblage, based on the sample analysed in this study and a visual assessment of the limited mammalian fauna from that sample. The data on mammalian and fish fauna from BBC LSA are not directly comparable as different sample sizes were analysed from different squares, but there does appear to be considerably more fish remains than mammal remains, with approximately 2881 fish specimens (NISP) per $\mathrm{m}^{3}$, and 1681 mammal specimens (NISP) per $\mathrm{m}^{3}$. Several other assemblages where both fish and mammalian fauna were analysed also contained considerably more fish remains, but these were generally limited to sites with larger fish assemblages such as EBC (Klein and Cruz-Uribe 1987; Poggenpoel 1996), TC (Klein and Cruz-Uribe 1987; Poggenpoel 1996), SC (Klein and Cruz-Uribe 1987; Poggenpoel 1996), SBF (Robertshaw 1978), SWB (Marean 1985; Poggenpoel and Robertshaw 1981), GBM (van Noten 1974), DK (Schweitzer 1979), NBC (Inskeep 1987) and PM (marginal difference) (Robertshaw 1977).

A few LSA assemblages contain more mammalian fauna than fish. These are PKM (mammal MNI=55, fish MNI=18) (Jerardino 1998), BNK (mammal MNI=604, fish MNI=265) (Schweitzer and Wilson 1982) and BB (mammal NISP=1117, fish NISP=754) (Maggs and Speed 1967). At BB micromammals are included in the total NISP count, and the difference between fish and mammal remains might not be as great if these were excluded, as they have been for the other assemblages listed above. At most sites with smaller amounts of fish either the fish or the mammalian fauna had not been analysed, and it is not possible to determine whether fish remains were more abundant than mammalian fauna in these assemblages. At BBF1 and BBF6 the mammalian bone was too badly fragmented and weathered to allow identification (Henshilwood 1995, 2008).

\section{Middle Stone Age fish remains}

\section{Comparison of Blombos Cave and Klasies River main site}

There are considerably more species present in KR than in BBC MSA, but twelve species occur in both assemblages. The greater species diversity at KR is probably a reflection of the wider species availability at this location, as mentioned previously. The same two species, Liza richardsonii and Galeichthys feliceps, are the most frequently occurring in both assemblages. The fish remains from BBC MSA and KR differ slightly in terms of 
size, with the majority of KR specimens being from small fish. Small fish are more common in the M3 of BBC, but medium sized fish are more common in the M1 and M2. At both sites fish size increases from the oldest to the youngest phases or members. As the samples are rather small, it is best not to attach significance to this observation. Skeletal element completeness is very similar between $\mathrm{BBC}$ and the cultural units of $\mathrm{KR}$, and elements were more fragmented in the sterile units of KR.

The lack of spinous processes on the majority of vertebrae from BBC MSA and KR contrasts with the condition of vertebrae in the LSA assemblages. The lack of processes appears to relate to age-related taphonomic processes leading to the deterioration of these more fragile extremities over long periods of time.

\section{Middle Stone Age methods of capture}

It is not possible to state with any certainty how MSA people at $\mathrm{BBC}$ or KR were catching their fish, but the data shows that scavenging of washed up fish was not the primary source of fish at either of the sites. Therefore more active methods of fishing are indicated. As there is such a wide variety of species present, it seems that a number of different techniques were employed to catch fish. These could be through the use of hooks, tidal rock traps or spears. Several of the species indicate that some sort of hook was used. It is possible that wood or thorns could have been used as gorges and that these have not survived in archaeological sites. Spears made out of wood would also not survive in the archaeological record, and if natural tidal traps were culturally modified, these modifications would no longer be visible. Natural rock pools can also function as tidal traps under certain circumstances.

\subsection{Skeletal element preservation: a synopsis}

Attributes such as skeletal elements per species, element completeness and element preservation based on zones were recorded on specimens in this study. This data has seldom or never been published from other southern African fish assemblages hence it was not possible to compare these with the findings in this study.

\section{Element survival}

In this study I set out to establish whether there is any correlation in survivorship of specific species' elements through time and under different environmental conditions (open versus cave sites). Some potentially useful information on element survival could be 
gathered for some of the species that were present in both MSA and LSA sites and which were represented by a number of elements. The BBF1 and BBF6 assemblages contained too little fish remains to make a useful contribution.

There are five species that occur in all the larger assemblages (excluding BBF 1 and 6), with considerable variation in numbers of these species between sites. The relative frequency of all elements per species and site were calculated but currently only the data from the more frequently occurring species are useful for comparing skeletal element representation. Larger samples of the less common species are needed to determine whether element preservation patterns are valid or a function of sample size for these species.

\section{Element preservation between MSA and LSA assemblages}

All of the elements of Liza richardsonii that are present in the MSA assemblages are also present in the LSA cave assemblages, and a much wider variety of elements of this species is present in the LSA assemblages. A wider variety of elements of Sarpa salpa, Chrysoblephus cristiceps, Chrysoblephus laticeps, Cymatoceps nasutus, Dichistius capensis, Diplodus capensis and Seriola lalandi also occurred in the LSA assemblages relative to the MSA assemblages.

In terms of skeletal element preservation of the eight species that occur in both the LSA and MSA of BBC, the LSA contained many more elements per species than the MSA for most species, apart from Dichistius capensis, where only one element was present in the LSA and two in the MSA, and Pachymetopon blochii, where there were four elements in the MSA and six in the LSA. There are 125 elements in the LSA and 41 in the MSA of the species that occur in both assemblages. Seven elements from four species only occur in the MSA. These are the coracoid of Epinephelus marginatus, supraoccipital and maxilla of P. blochii, the retroarticular and dentary of Spondyliosoma emarginatum, and the premaxilla and articular of Dichistius capensis. It is notable that the elements that are only present in the MSA are different for each species. The reason for the absence of these elements in the LSA is unclear, but could relate to the LSA assemblage studied here being from a sample. The Dichistius capensis elements present are from such a small sample that it is best to exclude this data until larger samples are available. 
All the other MSA elements $(n=34)$ also occur in the LSA assemblage. Therefore the majority (34 out of 41) of elements in the MSA are also present in the LSA. Fifteen of these belonged to Sparidae, and seventeen to species from other families. The most common Sparidae element preserved in the MSA of species that are also present in the LSA (based on MAU) is the supraoccipital, followed in equal quantities by the dentary, articular, lachrymal, maxilla, nasal, palatine, prefrontal, premaxilla, preopercular, retroarticular and supracleithrum bones. In the LSA nasal and premaxillae are the most frequently occurring elements, followed by the articular and preopercular. The most common elements after these, in descending order of frequency, are the lachrymal, supracleithrum, dentary, prefrontal, retroarticular, supraoccipital, ceratohyal and third infraorbital bones. Thus the eight most commonly preserved Sparidae elements in the LSA are also the best preserved elements in the MSA. The maxilla and palatine, common in the MSA, occur less frequently in the LSA.

The best preserved Liza richardsonii element in the MSA is the urostyle, followed by the hyomandibular, basioccipital, ceratohyal and cleithrum. In the LSA, the urohyal and basioccipital is best preserved, followed by the urostyle, cleithrum and hyomandibular. As with the Sparidae, most of the best preserved elements are the same in both the MSA and LSA. The one element from Epinephelus marginatus (basipterygium) that is present in both the MSA and LSA, is not very common in the LSA, where the metapterygoid was the most frequently occurring element.

There are many more elements preserved per species at $\mathrm{H} / \mathrm{RC}$ than $\mathrm{KR}$, although there are several exceptions where elements are only present at KR. The total number of elements of species that occur in both sites is 257 at $\mathrm{H} / \mathrm{RC}$ and 90 at KR. Thirty-nine of the 90 elements of various species present in KR do not occur in H/RC. These mostly $(n=29)$ occur where the $\mathrm{H} / \mathrm{RC}$ sample sizes are small (less than 6 elements present) and the absence of these elements from $\mathrm{H} / \mathrm{RC}$ probably relates more to sample size than preservation. Therefore most of the elements preserved in the KR MSA site also occur in the H/RC LSA assemblage.

Element preservation: A comparison of open and cave sites

Element preservation between the open (KR, BBF1 and BBF6, BBC MSA exterior) and cave (BBC LSA, BBC MSA interior, H/RC) sites were compared to determine whether 
there is differential preservation between open and cave sites. From the comparison of elements preserved at $\mathrm{H} / \mathrm{RC}$ and $\mathrm{KR}$ above, it was shown that the majority of elements preserved in the open site are also preserved in the cave site, but that the cave site contains considerably higher numbers of elements.

There is considerable variability in element survival between the open and cave MSA sites. Of the eight species that occur in both KR and BBC MSA (interior), different skeletal elements of five of these species only occur in one or the other of the two MSA assemblages. Five elements of Liza richardsonii, a species with several elements present in both $\mathrm{KR}$ and $\mathrm{BBC}$ MSA, are present in both sites. In addition, KR contained ten elements not found at BBC MSA, and BBC MSA contained six different elements that did not occur at KR. Some species' elements present are restricted to different skeletal regions at BBC MSA and KR, but in general elements from all regions of the skeleton occur in both assemblages.

Some variation between the representation of Galeichthys feliceps elements at the interior and exterior of BBC MSA was noted, with more elements present in the exterior. Three out of the five elements present in the interior were also present in the exterior. Two of the elements of this species from the exterior of BBC MSA were not present at KR, but the other six were. In all instances the few elements that were present at the open BBF1 and BBF6 sites also occurred in the LSA cave sites. Some, but not all, of the few elements of species that occurred at BBF1 and BBF6 were also present at KR and BBC MSA. The percentage of vertebrae relative to other elements in cave sites was $44 \%$ at BBC LSA, $32 \%$ at BBC MSA interior and $60 \%$ at $\mathrm{H} / \mathrm{RC}$. At the open sites, $91 \%$ of all elements at KR were vertebrae, $67 \%$ at $\mathrm{BBC}$ exterior, $77 \%$ at $\mathrm{BBF} 6$ and $50 \%$ at $\mathrm{BBF} 1$.

Scales are fairly common at BBC MSA whereas only one was found at KR, which suggests that scales preserve better in MSA caves relative to open sites. Scales were not quantified in the LSA assemblages but it was noted that BBC LSA had considerably more scales present than $\mathrm{H} / \mathrm{RC}$ or BBF 1 and BBF 6. The low number of scales at $\mathrm{H} / \mathrm{RC}$ probably relates to the restricted sample size.

The variation in element survival between species that occur in the MSA in open or cave contexts could be related to differential survival of elements between these contexts. In many instances the sample sizes are small and differences could be due to this. The LSA 
cave sites contain the largest variety of elements, which is not unexpected as these assemblages are relatively young and the cave roof presumably affords some measure of protection that is not present at open sites. The higher frequency of vertebrae in most of the open sites relative to cave sites suggests that vertebrae tend to preserve better than non-vertebral elements.

The difference in elements of Galeichthys feliceps in the interior and exterior of BBC MSA could relate to preferential treatment of this species which has a toxic coating on its spines. Avoidance of injury while handling this species would be easier in the better lit outside area, and the higher number of specimens in the exterior could relate to behavioural factors rather than preservation issues.

\section{Element preservation: comparison of the Zostera and shelly layers at $H / R C$}

The elements per species data from the H/RC assemblage were split between the top Zostera-rich layers and the older layers that contained little Zostera and more shellfish to determine whether the presence of large amounts of vegetable matter had an influence on the preservation of fish remains. Although some differences were noted, it is not clear how much relates to the smaller sample of fish remains in the Zostera layers. The shelly layers consistently contain more elements than the Zostera layers. A few exceptions occur, notably with Epinephelus marginatus which only has a few elements present in the Zostera layers, but these are mostly not present in the shelly layers. This could be an indication of differential preservation of this species' elements under dissimilar conditions.

There is a clear difference between Pomatomus saltatrix element survival in Zostera and shelly layers, with only lateral skull elements preserved in the Zostera layers. These lateral skull elements are also present in the shelly layers. All the $P$. saltatrix elements in the KR MSA layers also occur in the H/RC shelly layers, and none of these are in the Zostera layers. Some of the elements survive in MSA assemblages for tens of millenia and thus it could be argued that the difference in skeletal elements between the Zostera and shelly layers may be due to an additional contributory factor and not just natural attrition. One possibility is that the presence of mats of Zostera grass could have affected the $\mathrm{pH}$ of the surrounding soil and this acidity factor could account for the differential preservation of elements. Alternately, the shellfish in the shelly layers would have contributed calcium 
carbonate that would be beneficial for bone preservation. The latter option seems unlikely as some of the same elements that are also present in the sterile KR MSA layers are not associated with shell but have also survived.

The lack of pectoral elements could be indicative of the processing methods used as these are often missing from assemblages where fish had been processed and parts taken elsewhere (Barrett et al. 1999). The additional lack of skull and hyoid region elements suggests that this is not the case, as these would most likely have remained with the lateral skull elements if processing was involved. The reason for the limited range of elements of this species in the Zostera layers is not clear. There are similar differences between elements of other species from the Zostera and shelly layers, but none are as clearly restricted to one part of the skeleton as is the case with Pomatomus saltatrix.

\section{Discussion: Element survival}

The lower number of elements in the MSA assemblages is due to age related taphonomic processes rather than differences in preservation between open and cave sites. In several instances all the elements that are present in an MSA assemblage are also present in the LSA, which could indicate that these elements have some intrinsic characteristic which allows for better survival over time. These elements are not always those that have been traditionally used for identification or quantification. It is important that these elements that are present in the MSA assemblages are included in future analyses of these particular species.

Based on the current data, it is difficult to establish whether the differences in element preservation between the Zostera and shelly layers of $\mathrm{H} / \mathrm{RC}$ are real, or a function of the larger sample size in the shelly layers. Larger samples are needed to determine whether the patterns observed here are as a result of differential preservation between the Zostera and shelly layers.

\section{Element completeness}

Data on the degree of element completeness allows for comparison of fragmentation across sites. Most of the current data are not sufficient for comparison across sites at a species level, and larger samples are needed. This would be useful for determining whether specific species' elements are more prone to fragmentation, and therefore less likely to survive, and could also inform on differential processing of fish by humans. 
Skeletal element completeness of Liza richardsonii has shown that elements in the sterile units of KR are more fragmented than those from the cultural units of KR and BBC MSA. Likewise, elements of this species are considerably more complete in the BBC LSA than at $\mathrm{H} / \mathrm{RC}$. Recording skeletal element completeness of all species combined in the KR cultural and sterile units has been useful to show that there is a significant difference in fragmentation between these units, whereas other lines of evidence such as size and species composition were less indicative of differences.

The elements in the BBC MSA do not appear to be more fragmented than those from the BBC LSA in total. The degree of fragmentation of elements is very similar, with a slightly higher percentage of very fragmented specimens in the MSA, and slightly more intact specimens in the LSA. The degrees of fragmentation of species that occur in both the MSA and LSA are also very similar, with the majority of specimens in the most complete (80-100\%) category, although the MSA contains slightly more of the most complete specimens, and no specimens in the very fragmented ( $0-20 \%$ complete) category.

The considerable fragmentation of elements at BBF 6 is worth noting as this is an open shell midden, and fish remains are often scarce or underrepresented in shell middens. Excessive fragmentation of fish bones from open sites could render many of the specimens unrecognizable to inexperienced primary sorters.

\section{Zone preservation}

Each element was assigned a number of zones, and these zones were recorded for each specimen. These were recorded to determine which parts of which elements of each species survive, and whether the same parts were preserved at different sites. This data was intended for comparison at a species specific level, but the widely disparate range of elements that were present per species between sites made this exercise less than satisfactory. It has the potential to be useful in the future when more data per element and species are available.

Based on the current data, there seems to be little correlation between the zones per element preserved and site type. Comparison of element zones from open and cave sites did not show consistent differences in preserved zones. Differences in age also did not appear to affect zone preservation. A few differences were noted between the Zostera and shelly layers of $\mathrm{H} / \mathrm{RC}$ in terms of preserved zones of some species' elements. These 
differences are not constant and it is currently not possible to state whether there are any significant differences between the preservation of elements in the Zostera and shelly layers or whether the presence of large amounts of plant material affected element preservation in the upper layers.

Zone data can potentially allow identification of processing activities in the absence of evidence for cut marks if elements are found to consistently lack particular zones in an assemblage. The challenge is to first establish which zones are present or absent in assemblages that have not been culturally modified but have undergone sufficient attrition for some zones to have disappeared. As the amount of attrition as a result of cultural processes in archaeological assemblages is not known, comparison between such assemblages is not feasible. This might be achieved through studying ancient naturally accumulated fish assemblages from episodes such as mass strandings of fish on the shore. I am not aware of any such assemblages from a marine context in southern Africa, but if one should become available, this would be an invaluable resource for future studies into fish processing.

\section{Elements used for identification}

Most previous analyses of southern African fish remains relied on a select number of elements for identification and quantification. These were primarily dentaries, maxillae and premaxillae for Sparidae, and basioccipitals and first vertebrae for Mugilidae. With several species the most frequently occurring elements from this study overall are the same as those used previously for identification and quantification, but on an individual assemblage basis, these elements were not always present, and identifications were often made on other, less common elements. For example, Poggenpoel $(1984 ; 1996)$ found that jaw bones of Liza richardsonii do not survive well and that basioccipitals and first vertebrae provided higher MNI counts for this and other species of Mugilidae. In this study, basioccipitals were present in all assemblages that contained this species, but it was not the most common element in any of the assemblages, and the most frequently occurring element varied between sites.

First vertebrae were the most common elements of Liza richardsonii at KR, but not at other sites, and they were absent from some assemblages. Poggenpoel states that his quantifications were based on the most frequently occurring elements, therefore I assume that these were the most common elements at those particular sites. From the skeletal 
element data collected here, it is clear that many species would be underrepresented and others missed if identification and quantification was based on this limited set of elements. I attempted to identify which elements are useful for species identification in order to eliminate those that are never useful, with the intention of speeding up future identification without compromising data quality. The most common element for identification to species varies between sites, particularly with species other than Sparidae. The elements used for Sparidae identification can confidently be narrowed down to 31, and fairly confidently to 21 . The exclusion of the 10 elements that occur at frequencies below 5\% does not affect the species composition or numbers of the Sparidae in the assemblages studied here.

Narrowing down the number of elements used for identification of species from other families is less straightforward. For some species it is best to analyse all elements, whereas others are not affected if the least frequent elements are excluded from analysis. Based on the variety of elements identified and the lack of consistency in element survival, it would be best to include all elements for identification of these species. As these species tend to be more morphologically distinct than Sparidae, identification of these tend to be less time consuming in any case. Figures A-2 to A17 in the appendix illustrate some of the variations in shape of elements of different species and families.

\subsection{Fish bone survival in MSA assemblages}

Earlier observations (Klein 1974, 1975, 1976; Klein and Cruz-Uribe 1996; Volman 1978) that fish bones were rare relative to mammal bones at Klasies River were based on the material from the Wymer excavations (Singer and Wymer 1982). Fish remains were indeed rare in this assemblage, and only a few vertebrae from fish weighing between 1$4 \mathrm{~kg}$ were reported. The reason for this scarcity is partly due to the large screen sizes used, which would introduce a strong bias against smaller fish bones, but the biggest reason for a lack of fish remains from the Wymer excavations at Klasies River is due to selective retention only of faunal specimens that were considered to be taxonomically identifiable, whereas those that were not considered identifiable were discarded during preliminary sorting in the field (Klein 1976). Fish vertebrae, which comprise the bulk of elements preserved from the Deacon excavation of Klasies River, were almost certainly considered unidentifiable and discarded. The few vertebrae reported (Singer and Wymer 1982) were 
presumably kept due to their larger size. These could not be located for inclusion in this study, but a few (NISP=5) dentaries, maxillae and premaxillae from one medium (30$50 \mathrm{~cm})$, two large $(50-80 \mathrm{~cm})$ and two extra large $(80-100 \mathrm{~cm})$ fish were found. The presence of these elements from larger fish, which were usually considered diagnostic to species (Poggenpoel 1996; Singer and Wymer 1982), confirms that elements were selectively retained and presumably selected for their larger size as well as suitability for identification, as analysis of the fish remains from the Deacon excavation has shown that most of the specimens from Klasies River are from smaller sized fish (von den Driesch 2004; this study). The low incidence of fish remains from the Wymer excavations is therefore directly related to significant sampling bias, and not to a real absence of fish. All material from subsequent excavations at Klasies River by Deacon from 1984 onwards were retained, and it is a sample of the fish remains from these excavations that von den Driesch (2004) reported on, and that was examined for this thesis. The statement that mammalian bone numbers far outweigh fish bone at Klasies River is not entirely accurate, as there are actually more fish bones than mammal bones in the Klasies River MSA assemblage. Van Pletzen (2000) analysed the mammalian fauna from the same squares and units of Klasies River that the fish remains studied here came from. The total NISP of mammalian bone amounted to 5359, whereas there were 12816 specimens of fish recorded in this study. Admittedly, there are less fish bones in the cultural units (NISP=5562, and 7254 in the sterile units), but mammal remains were reported for the combined cultural and sterile units, so the numbers are comparable. There are, however, more mammalian specimens than fish in the Blombos Cave MSA assemblage. It must be noted that, although fish bones outnumber mammal bones at Klasies River, I am not implying that fish were exploited more heavily than mammals, or that fish contributed more to the overall diet than mammals did. As MNI could not be calculated for the assemblage, it is not possible to extrapolate the meat contribution of fish relative to mammals but, as most fish are from small specimens, it is almost certain that mammals contributed significantly more food, as even large bovids such as eland and kudu occur throughout the assemblage (van Pletzen 2000), and the same is true for Blombos Cave.

Apart from the isolated fish bones found at Sibudu (SBC) and the larger numbers of fish from Blombos Cave and Klasies River, there are no other known MSA sites in southern Africa that contain preserved fish in significant quantities. Very few coastal MSA sites 
apart from those listed above contain well preserved bone, and the vertebral fauna from even fewer have been analysed and published. The following section explores whether the absence of marine fish at other coastal MSA sites could be due to poor preservation of organic material and/or sampling methods.

At Die Kelders, where no fish bones were recovered, bone was very fragmented in the upper MSA layers, but large mammalian fauna was well preserved and less fragmented in the lower layers (Avery et al. 1997; Klein and Cruz-Uribe 2000a; Marean et al. 2000a; Marean et al. 2000b). Shellfish preservation was very poor due to acidic groundwater, and so badly decomposed that it could not be recovered intact. The potential effects of acidic environments on fish bones are considered further down in this section.

Nineteen fish bones were recovered from Pinnacle Point 13B (PP13B), which have not yet been studied, but consists mostly of vertebrae, with some spines and two potential cranial specimens (Thompson 2008; pers. comm. 2010). Shellfish was present in small numbers at PP13A and PP13B, but preservation was poor in many instances. The quantity of shellfish could be small because very little was collected by the inhabitants, or else much of the original shellfish had decomposed, as suggested by the friable condition of those that were recovered. The PP assemblages include well preserved large mammal remains, and the good preservation is attributed to calcium carbonate rich waters seeping through the roof and walls of the cave (Marean et al. 2004b). Very few small mammals, tortoises or microfauna such as small rodents, reptiles, amphibians, and bats were recovered. The authors interpreted the low incidences of these smaller species, including tortoises, to indicate that raptors and owls did not play a significant role in the accumulation of the faunal remains. Subsequent analysis has shown that small numbers of microfauna (various species of small rodents) are present in the assemblage, and that the barn owl, Tyto alba, is the most likely accumulator (Matthews et al. 2009). Evidence for carnivore activity in the form of gastric etching and tooth marks was recorded on a very small percentage of the mammalian fauna (Thompson 2008).

The low frequency of tortoises and small mammals from the PP assemblages is unusual compared to other MSA sites. Unlike at PP13A and PP13B, tortoises were an important dietary component at several MSA assemblages such as Blombos Cave, Ysterfontein 1 and Die Kelders, and at these sites humans were the primary accumulators (Halkett et al. 
2003; Henshilwood et al. 2001b; Klein and Cruz-Uribe 2000a). Small mammals were also common food components at other MSA sites. At Blombos Cave dune mole rats (Bathyergus suillus) and rock hyraxes (Procavia capensis) are common in the MSA and were collected by humans (Henshilwood et al. 2001b). Hyraxes at Die Kelders were also most likely accumulated by humans, although the majority of dune mole rats in this assemblage appeared to have been accumulated by eagle owls (Klein and Cruz-Uribe 2000a). Hyraxes were common at Klasies River where both humans and carnivores could have been accumulators, but dune mole rats were rare (van Pletzen 2000). Dune molerats were the most frequently occurring species at Ysterfontein 1, where it was argued that humans were the primary accumulators (Halkett et al. 2003). Hyraxes, hares and dune mole rats dominate the faunal assemblage at Diepkloof Rock Shelter (Texier et al. 2010). Dune mole rats are absent from the PP13A and PP13B assemblages, and hyraxes are absent from PP13A, and scarce at PP13B (NISP=28) (Thompson 2008). Thompson (2008) has considered possible reasons for the lack of dune mole rats and other small mammals and tortoises at PP13B such as a lack of suitable (non-human) predators, unsuitable habitat for these species or their predators, and human choices relating to consumption of these animals at the place of capture, rather than transporting them back to the site as the occupants of Blombos Cave and Die Kelders did. The sample was however too small to determine whether any of these options were likely.

The low number and often poor condition of shellfish, as well as the lack of smaller mammals in the PP assemblages could be due to differential preservation affecting smaller faunal remains to a greater degree than large mammalian fauna. As other MSA sites such as Die Kelders also contain badly preserved shellfish, but small mammal remains and tortoises occur frequently, the low frequency of tortoises and small mammals at PP13B might well be as a result of human processing choices or habitat restrictions on species availability rather than taphonomic processes affecting these animals' remains more severely than that of larger mammals. If that is the case, then the very low incidence of fish bones at PP13B might not be related to differential preservation of smaller animals, although the processes that have lead to the decomposition of some of the shellfish could also have affected survival of the fish bones. The barn owl responsible for the microfauna at PP13B does not prey on fish, but evidence for limited carnivore activity at the site could indicate that agents other than humans accumulated the fish. 
The sites mentioned above are all cave sites located on the south coast alongside the Indian Ocean. Ysterfontein 1 shelter is currently the only MSA site situated on the west coast alongside the Altantic Ocean containing significant amounts of faunal remains that have been analysed and published (Avery et al. 2008; Halkett et al. 2003; Klein et al. 2004). The shelter is currently very exposed, but the remaining roof and walls are formed out of calcrete, and water percolating through this would have contributed calcium carbonate which could have aided bone preservation. Part of the roof collapsed between the deposition of Layers 6 and 7 (Avery et al. 2008), and the material from Layer 6 and above would have been more exposed to the elements than the material below. It is not known whether this exposure of the upper layers affected bone preservation adversely. The few bones from small fish recovered in the disturbed upper layers have been attributed to carnivores or cormorants, and most of the other faunal remains in the upper unit were probably collected by non-human agents (Avery et al. 2008). Shellfish was abundant at Ysterfontein 1 and, although the condition of the shells were not mentioned in the literature, well preserved as maximum lengths could be measured from a large number of the limpet species. Large mammal bone and tortoise carapaces were heavily fragmented, but preserved enough to allow some to be identified to species (Halkett et al. 2003). Microfauna appears to be absent from the assemblage, as none of the three publications on the assemblage make any mention of these small animals. Lack of suitable roosts for raptors in the shelter could explain this absence (Avery et al. 2008; Halkett et al. 2003).

According to the authors (Avery et al. 2008; Halkett et al. 2003; Klein et al. 2004), MSA people at Ysterfontein 1 utilized available food resources in the same way that MSA people at Blombos Cave and Klasies River did, which was less efficiently and more selective than LSA people, and the lack of fish at Blombos Cave and Klasies River was repeatedly emphasized as one of the key similarities between these sites. Other similarities between Ysterfontein 1, Klasies River and Blombos Cave were based on the larger sizes of shellfish and tortoises in the MSA relative to the LSA. The mammal and bird samples from Ysterfontein 1 were too small to allow comparison with the south coast assemblages. As seen from this current and previous studies (Henshilwood et al. 2001b; von den Driesch 2004), fish bones are not absent from Klasies River and Blombos Cave, and many are anthropogenic in origin. Why then, if people were exploiting local resources in the 
same way, are there no fish bones at Ysterfontein 1? Coastal resources were more abundant than terrestrial ones at Ysterfontein 1 as the interior was arid but the Benguela current provided rich nutrients supporting marine life. Harmful algal blooms (red tides), which are most common in late summer on the west coast (Pitcher and Calder 2000), can cause fish mortalities, sometimes in vast numbers, and these fish would have washed up on shore and could have been easily collected. Crayfish (Jasus lalandii) are also affected by these blooms on the west coast, and respond by 'walking out' on land to escape the oxygen depleted water, making them easy prey for humans. Crayfish were also absent from the Ysterfontein 1 assemblage, but did occur in many west coast LSA assemblages, and was another example used to indicate how LSA people exploited marine resources more intensively than MSA people (Avery et al. 2008; Halkett et al. 2003; Klein et al. 2004). It is not clear whether the absence of crayfish at Ysterfontein 1 is because people were more selective about their food resources, or due to poor preservation, as none have been reported from any MSA sites. Washed up fish and stranded crayfish would have provided excellent food gathering opportunities in an area where washups and walkouts occur quite frequently. The source of fish remains from Klasies River and Blombos Cave have in some instances been attributed to just that, humans scavenging washed up fish (Klein 2000; Klein and Cruz-Uribe 2000a).

From this, it seems that there are two possible reasons for the absence of fish at Ysterfontein 1. One is that people at Ysterfontein 1 selected not to fish or scavenge washed up fish, unlike their counterparts at Klasies River and Blombos Cave on the south coast, who did so occasionally. This would suggest a difference in MSA resource exploitation between the west and south coast, which is not supported by the other faunal evidence available to date, from both west and south coast assemblages. The other option is that people at Ysterfontein 1 did catch and/or scavenge fish, as they did at Klasies River and Blombos Cave, but that taphonomic processes at this location were not conducive to fish bone survival. The vertebrate fauna from the few other MSA sites on the west coast are poorly preserved and the samples small, at times biased, and two have not been analysed, yet a few fish bones from two of these indicate that at least some fish were present originally.

At Diepkloof Rock Shelter organic preservation is said to be exceptional, although the mammalian fauna is highly fragmented, and currently under study (Texier et al. 2010, 
Steele pers. comm.). The site is located approximately $18 \mathrm{~km}$ inland from the coast, but some shellfish and seal remains are present, indicating that coastal resources were exploited to a limited extent. The fish remains from the LSA layers at Diepkloof Rock Shelter that date between 390 and 1590 BP were few and very fragmented, which suggests that conditions at this shelter were not conducive to good bone preservation. As the relatively young LSA fish remains were in such a poor state of preservation, it seems unlikely that fish bones would have survived in the much older MSA layers, if they had been present initially.

The MSA shell middens of Sea Harvest and Hoedjies Punt could not be excavated in the conventional manner due to the cemented nature of the deposits, and the assemblages consist of material that had eroded from the base of the middens (Volman 1978). A small amount of bone was recovered from Sea Harvest which includes one fish vertebra. Shellfish was common at Sea Harvest and in good condition but the condition of the few bones recovered were not mentioned. Shells were less well preserved at Hoedjies Punt but no other details of the faunal component were reported. The collected material cannot be considered representative of these two assemblages, and the possibility that more fish remains were present cannot be excluded. Sea Harvest has subsequently been destroyed by road building, but renewed excavation of Hoedjies Punt is planned.

The contents of the MSA shell midden Boegoeberg 2 have not yet been fully analysed or published. I have noticed that there are some fish vertebrae in the unsorted material of this assemblage. Intriguingly, this assemblage contains a higher relative abundance of cormorants than most LSA assemblages (Klein 1999a). It has long been argued that, as with fish, MSA people lacked the ability and technology to catch airborne birds, and that these were the sole domain of LSA people (Avery et al. 2008; Halkett et al. 2003; Klein 1974, 1989a, b, 1992, 1995, 1998, 1999a, 2000, 2001, 2008; Klein et al. 2004; Volman 1984). At all other published MSA assemblages, however, cormorants are less common than penguins.

Only a small amount of material was collected from a rescue excavation of the Herold's Bay shell midden which contained shellfish and a small amount of mammalian fauna (Brink and Deacon 1982). It is not possible to determine whether the absence of fish from this assemblage is real or due to the small sample size. The MSA assemblages from both 
Nelson Bay Cave and Elands Bay Cave do not have any faunal remains preserved, due to waterlogging in the lower deposits of the former (Volman 1981), and leaching at the latter. It is impossible to determine whether these assemblages originally contained fish.

Most actualistic studies on fish bone survival have focused on body part representation and skeletal completeness of specific species after being subjected to different processes such as digestion by humans and other animals, trampling, burial, changing soil $\mathrm{pH}$, burning, cooking and processing methods (Butler and Schroeder 1998; Jones 1984, 1986, 1999; Lubinski 1996; Nicholson 1992b, 1993b, 1995, 1996; Stewart 1991; Stewart and Gifford-Gonzalez 1994; Zohar and Cooke 1997; Zohar et al. 2001), as well as the role of bone density in element survival (Butler and Chatters 1994; Nicholson 1992a). These studies were concerned with which elements had survived, and how the remaining assemblage could help to identify the pre- and post-depositional processes that affected the fish remains. In many instances it was found that the effects of these processes do not affect all species to the same degree. Similar studies have not yet been done on southern African fish species and, as many of these effects appear to be species specific, experimental studies on local species are needed. Some of the results of the abovementioned studies can however help in an attempt to determine why some MSA assemblages lack fish remains.

Nicholson's (1996) experiments on fish bone degradation after burial found considerable differences in element survivorship between soils with similar $\mathrm{pH}$ and drainage after only seven years. The experiments also showed that in one instance, less than $3 \%$ of the original skeletons were preserved in acidic soil (otoliths and vertebrae), and in very poor condition, whereas more elements were preserved, and in better condition, at another location with the same soil profile. The difference is attributed to increased microbial activity at the first location, where burial depth was shallower $(35 \mathrm{~cm})$ than at the other acidic location $(45 \mathrm{~cm})$. This is interesting as the combination of acidic soil and shallow burial would be similar to conditions at Die Kelders, which has an acidic environment, and discarded bones were unlikely to be rapidly covered by deposit exceeding $35 \mathrm{~cm}$ during occupation. The mammalian bones in the lower MSA layers (10 and 11) of Die Kelders showed very little evidence for sub-aerial weathering, indicating that the bones were not exposed for long periods after deposition, which is consistent with burial (Marean et al. 2000a). 
Nicholson (1996) also described the effects of microbial activity on large mammal elements from the acidic location with shallowly buried bones, which showed extensive pitting, channeling, and severe erosion of epiphyseal margins and unfused distal ends of metapodial shafts, particularly on the boiled sheep bones. The described surface condition of mammalian bones from Die Kelders in the lower MSA levels does not resemble that described by Nicholson, although pitting and channeling were not addressed with the Die Kelders fauna. Epiphyseal ends were underrepresented relative to shaft fragments at Die Kelders, which was attributed to carnivores targeting these fat-rich parts, and the underrepresentation of metapodials was attributed to differential transport (Marean et al. 2000a). Superficially, microbial bioerosion can obscure or mimic carnivore tooth marks (Blumenschine et al. 2007; Dominguez-Rodrigo and Barba 2006; Thompson 2005), but Marean et al. (2000a) based their assignment of carnivores on the frequency of tooth marks on mid-shaft fragments, where carnivore tooth marks are more clearly distinguishable. Therefore it is not known to what extent microbial agents affected bone in the Die Kelders MSA assemblage. Assuming that there was a certain amount of microbial activity at Die Kelders, the combination of acidic soil, shallow burial and time could have resulted in the complete loss of fish bones, if they were deposited initially.

Bones in LSA middens tend to be very fragmented and often poorly preserved (e.g. at Duiker Eiland, Blombosfontein 1 \& 6, Still Bay 1, Still Bay 2, Paapkuilfontein 4, 5, 7 \& 11, Gordon's Bay Midden). Unfortunately, many reports on shell middens do no include descriptions of the condition of the faunal remains. Vertebrate remains from shell middens elsewhere such as the Californian coast also tend to be very fragmented (Erlandson et al. 1999). Most of the LSA shell middens with reported fish contain relatively small numbers, although there are exceptions such as Gordon's Bay Midden and Stofbergsfontein. The small quantities of fish in many of the LSA middens could in part relate to economic subsistence choices leading to a preference for or focus on other food sources (Jerardino 1996, 1998; Jerardino and Yates 1996). In some instances the smaller quantities could relate to issues of preservation at these open sites due to exposure to the elements. Even in sites with relatively large numbers of fish like Gordon's Bay Midden, extensive fragmentation of the bone has rendered most specimens unidentifiable, even to element (van Noten 1974). 
At several shell middens, where skeletal elements were reported, vertebrae were the only or most frequently surviving elements (at Blombosfontein $1 \& 6$, Still Bay 1, Still Bay 2, Paapkuilfontein 4 and Stofberfontein). The exception is Duiker Eiland, where bone preservation is poor but cranial elements exceeded vertebrae counts (Poggenpoel 1996). The predominance of fish vertebrae in shell middens have also been recorded elsewhere around the world, such as at Ingane River Mouth in Kwa-Zulu Natal (Schoute-Vanneck and Walsh 1959), Arrawarra I in New South Wales (Gobalet 2005; Vale and Gargett 2002), at a large number of middens on the Central Californian Coast (Gobalet and Jones 1995), at CA-SBA-2057, a midden on the Santa Barbara Coast of California (Rick and Erlandson 2000), Oughtymore in Ulster (Mallory et al. 1984), a few shell middens in Southeast Queensland (Hlinka et al. 2002), in the Arabian Gulf (Beech 2002), to name but a few. In some instances vertebrae were underrepresented in middens, as at the Neolithic site of Konam-Ri in Korea, where the lack of vertebrae might be due to transportation of the fleshy parts including the vertebral column elsewhere (Norton et al. 1999). Thus, barring differential processing and transport of fish, fish vertebrae appear to preserve better in shell middens than other elements. It should be noted that, although vertebrae are more numerous in a fish skeleton than any other individual element, there are many more non-vertebral elements in a fish skeleton (excluding spines and rays) than vertebrae, so the predominance of vertebrae in shell middens is related to preservation and not to skeletal element abundance.

The few open MSA shell midden assemblages in South Africa (Sea Harvest, Hoedjies Punt, Boegoeberg 2 and Herold's Bay) either suffer from sample bias due to inadequate excavation techniques or small sample size or, in the case of Boegoeberg 2 and Hoedjies Punt, have not been analysed. As bones from LSA shell middens are generally in poor condition, and non-vertebral fish elements tend to be poorly represented, it appears that fish remains do not survive well in shell middens, and the most likely fish elements to be encountered in older MSA middens, if they have survived, would be vertebrae. The single vertebra found at Sea Harvest and the few noticed at Boegoeberg 2 is not sufficient evidence to support this observation, although it is notable that the only surviving fish elements in the MSA shell midden assemblages are vertebrae and not cranial elements, suggesting that these elements do preserve better. Vertebrae are also the most common 
elements preserved at Klasies River and the exterior of Blombos Cave MSA, which further supports this observation.

In sum, there are only 13 coastal MSA assemblages in southern Africa which have been excavated by 'modern' methods (i.e., more than just representative samples were kept), that allows some measure of comparison between them (Klasies River, Herold's Bay, PP13A, P13B, Nelson Bay Cave, Blombos Cave, Die Kelders, Ysterfontein 1, Diepkloof Rock Shelter, Elands Bay Cave, Hoedjies Punt, Sea Harvest and Boegoeberg 2). Sibudu Cave has been excluded from this list as its distance from the shore during occupation would have been in excess of $20 \mathrm{~km}$ during most of the MSA occupation (Wadley and Jacobs 2006). Although the same could be said for Diepkloof Rock Shelter, I have included it here as it is in the same geographic location as several of the other sites. Coastal MSA sites that could potentially have contained fish remains, but where only selected material was retained, and therefore not considered here, are Peers Cave (Peers 1929), Trappies Kop (Goodwin 1953), Tunnel Cave (Malan 1955) and Dale Rose Parlour (Goodwin 1953; Schirmer 1975), all located in False Bay near Cape Town.

Two of the MSA assemblages excavated by 'modern' standards have no faunal remains preserved (Nelson Bay Cave and Elands Bay Cave). Two others (Herold's Bay and PP13A) contain very small samples of material, with no fish reported, and these cannot be considered representative of the whole assemblages. The Hoedjies Punt sample is small and the faunal material has not been analysed. Five of the remaining eight assemblages contain fish remains. There are very few fish at Sea Harvest, Boegoeberg 2 and PP13B, and relatively large numbers at Blombos Cave and Klasies River. The Sea Harvest assemblage is small and biased due to the method of excavation employed, and the Boegoeberg 2 assemblage has not yet been studied, but could potentially contain more as yet undiscovered fish remains. The low incidence of fish at PP13B could be due to preservation bias against smaller fauna, although accumulation by carnivores cannot be excluded. The fact that most or all of the surviving elements at Sea Harvest, Boegoeberg 2 and PP13B are vertebrae, which preserve better than other elements, suggests that the original number of fish deposited was probably much higher. The absence or of fish remains from Die Kelders and Diepkloof Rock Shelter could be attributed to excessive fragmentation and poor conditions of bone preservation. In addition, as Diepkloof Rock Shelter is located $18 \mathrm{~km}$ inland, fish remains might never have formed a substantial part of 
the original assemblage. The absence of fish at the remaining MSA assemblage, Ysterfontein 1, could to be due to taphonomic processes resulting in the loss of fish bone and, if the absence of microfauna at this site is real and not an omission from the publications, then these processes also affected other smaller fauna. Data from subsequent excavations (2007 and 2008), that have not yet been published, could confirm or refute this claim.

Thus most of the MSA sites that contain little or no fish bones suffer from one or other disadvantage, as detailed above, which prevents me from asserting that MSA people at sites other than Klasies River and Blombos Cave did not exploit fish, either through scavenging or active fishing. Ysterfontein 1 seems to be the most promising site for determining whether MSA people living on the west coast were taking advantage of fish as the site is well stratified, excavated by modern methods which allows comparison with Klasies River and Blombos Cave, and does not have an acidic environment such as at Die Kelders which compromises shellfish preservation, and potentially that of fish bone. Results from the newer excavations at Ysterfontein 1 are eagerly anticipated. It might seem that much is being made out of very few fish bones here (from Sea Harvest, Boegoeberg 2 and PP13B). It should be kept in mind that if only a single vertebra is found in an assemblage, this represents one fish, and one fish contains many bones. In other words, that one vertebra is all that is left of an original number of more than 200 bones, meaning that a very large number of bones are missing (assuming that the whole skeleton was originally deposited). This study has shown that MSA assemblages of Klasies River and Blombos Cave contain a large deficit of elements, indicating that very few of the originally deposited bones have survived relative to younger LSA assemblages. Thus agerelated taphonomic processes have reduced the original number of bones significantly. These processes would also have affected fish remains from other MSA assemblages, to varying degrees depending on each site's particular conditions, and the few remaining bones serve to indicate that fish were deposited at these sites, but that preservation of fish bones was poor. The original number of fish deposited could have been significantly higher.

In many of these assemblages the bones are reported as heavily fragmented and fragmented fish bones are very difficult to identify with an untrained eye. A potential 
problem with the absence of fish remains in MSA sites is that most primary sorting of excavated material is done by inexperienced sorters, often students, and the possibility that highly fragmented fish bones were missed is high, with the result that reports claiming an absence of fish can be misleading.

In addition to poor preservation of fish remains in the few MSA assemblages that have been excavated, our understanding of coastal resource exploitation during the MSA is further hampered by the fact that an unknown number of MSA sites would have been obliterated by rising sea levels during the Holocene.

The potential reasons for the absence of fish at several coastal MSA sites as discussed above were concerned with physical and taphonomic conditions that could have resulted in fish bone loss over time. The degree of attrition, and therefore the size of the original fish assemblages, is difficult or impossible to determine. The impression is, however, that although people in the MSA were fishing, as seen from this study of Blombos Cave and Klasies River, they were not as focused on procuring fish as is evidenced at some of the LSA sites such as Elands Bay Cave, Nelson Bay Cave and Hoffman's/Robberg Cave, where fish played a significant role in the diet of the inhabitants. Deacon $(1989 ; 2001 \mathrm{a})$ has argued that MSA people at Klasies River did not fish because they ranked other dietary sources, such as shellfish, more highly. Henshilwood and Marean (2003) argued similarly that fish are less common in the MSA as catching fish and flying birds is labourintensive in terms of technology required and the process of capture time-consuming, therefore these were ranked lower in net return than other food sources.

The reason for less fish in the MSA could relate to population sizes. Klein and colleagues (Avery et al. 1997; Avery et al. 2008; Halkett et al. 2003; Klein et al. 2004; Klein and Cruz-Uribe 1983; Steele and Klein 2005; Texier et al. 2010) have argued that human populations were much smaller during the MSA, based on differences in shellfish and tortoise sizes between MSA and LSA assemblages, and that MSA people collected tortoises and shellfish less intensively and more selectively. There are currently no data on the actual demography of southern Africa during the MSA, although population densities are thought to have varied during this period (Behar et al. 2008; Deacon 1989). There are certainly many more LSA than MSA sites in southern Africa, and, even allowing for some preservation bias, LSA population densities appear to have been considerably higher. 
Larger populations would have necessitated increased and intensified exploitation of resources, and are often associated with increased sedentism, whereas smaller populations would have had more mobility, exerted less pressure on available resources, and allowed people to select from a wider subsistence resource base (Broughton 1994; Deacon 1989; Hall 2000; Jerardino 2010; Jerardino et al. 2009; Parkington 2003). Smaller populations exerting less pressure on available resources with a wider range of resources to choose from could account for the lower numbers of fish in MSA assemblages. This could explain why the diversity of fish species found at Klasies River is higher than at Hoffman's/Robberg Cave, and higher in Blombos Cave MSA than LSA. The higher species diversity at Hoffman's/Robberg Cave relative to Blombos Cave MSA is due to environmental differences between these regions, with a wider range of species available in the vicinity of Hoffman's/Robberg Cave. Faunal data from Blombos Cave and Klasies River indicate that a wide range of food resources, including fish and geophytes, were available and exploited (Deacon 1993; Henshilwood et al. 2001b; Klein 1976; van Pletzen 2000). The shellfish composition at Blombos Cave, Klasies River and other MSA sites indicates that people were selectively choosing specific species, probably for their large size, and they were not forced to take whatever they could get (Klein et al. 2004;

Parkington 2003; Steele and Klein 2005).

The sum suggests that MSA populations were smaller, probably more mobile, and people were not compelled to catch fish in order to survive, but that they were aware of this resource, and were able to tap into it when required.

It should be noted that considering the MSA as a single, static, entity is not ideal as there are several periods within this large time-span that show unique characteristics and adaptations, but the current fish samples are too small to allow interpretation on more time-restricted levels.

\subsection{Fish remains and modern human behaviour}

The definition of what constitutes modern human behaviour varies between researchers (Chase and Dibble 1990; Deacon 1998; Foley and M. 1997; Gibson 1996; Henshilwood and Marean 2003, 2006; McBrearty and Brooks 2000; Renfrew 1996). Early models were based on differences in material culture and resource exploitation before and after the transition from the European Middle (MP) to Upper Palaeolithic (UP) about 40k years 
ago, and 'trait' lists were compiled by which modern behaviour could be identified (Ambrose 1998; Ambrose and Lorenz 1990; Bar-Yosef 1998; Chase and Dibble 1987; Clark and Lindly 1989, 1991; Klein 1992, 1995, 1999b, 2000; Mellars 1989; Mellars 1991; Mellars 1996). The differences between the MP-UP were thought to equate with the MSA and LSA transition in Africa around 50-40k years ago, with LSA people exhibiting the same traits as UP people in Europe, whereas MSA people were thought to be 'nonmodern', and behaviourally similar to MP people. The transition from 'non-modern' in the MSA to 'modern' in the LSA was thought to be as abrupt as the MP-UP 'revolution'. The belief that MSA populations were incapable of modern behaviour was based on the absence of the 'traits' that characterised the UP.

These traits, as applied to the African archaeological record and found lacking in the MSA by some researchers, include the following: formal tools and art objects made out of bone, ivory or shell, spatial and temporal variability in lithic raw materials, selection and transport of exotic lithic raw material, changes in diversity and standardization of artefact types, spatial organization of living areas including extensive hearths, evidence for ceremony or ritual, higher population densities, fishing and an increased ability to exploit food resources effectively, including dangerous animals, and recognition of seasonally available resources (Ambrose 1998; Ambrose and Lorenz 1990; Binford 1984; Klein 1989a, 1995).

Some researchers still support a late origin for modern behaviour in Africa around 50k years ago, and argue that a genetic mutation allowed increased cognitive ability (Klein 1989a, b, 1994, 1995, 1999a, 2000, 2008; Klein et al. 2004; Klein and Edgar 2002). Others supported the late origin of modernity at 50k years ago, but did not ascribe it to a genetic mutation (Ambrose 1998; Ambrose and Lorenz 1990; Binford 1984, 1985; Clark 1988; Clark 1989; Tattersall 1995).

A great body of work has been dedicated to illustrating that MSA people in southern Africa were not capable of fully modern human behaviour. The bulk of the argument is based on the absence of fish and evidence for less intensive exploitation of tortoises and shellfish signifying smaller populations during the MSA. Klein and colleagues (Avery et al. 2008; Halkett et al. 2003; Klein 1974, 1975, 1976, 1977a, b, 2008; Klein et al. 2004; Klein and Cruz-Uribe 1996, 2000a; Klein and Steele 2008; Volman 1978, 1981) argue 
that populations were smaller during the MSA because their behaviour was not fully modern as they lacked the cognitive and technological ability to exploit available resources fully, and, somewhat circularly, their inability to do so meant that population size could not increase. Therefore the only way to break this cycle would be through a neurological shift, or genetic mutation of the brain, which they believe occurred around 50k years ago. The supposed absence or rarity of fish at Klasies River (and later, at Blombos Cave) has been used extensively by these authors to argue that MSA people did not exploit coastal resources as effectively as LSA people, and that their inability to catch fish (and flying birds) indicated that they lacked the necessary technological capabilities to do so. Additionally, the lack of fishing gear, particularly bone gorges and sinkers, in MSA sites has been cited repeatedly by the same authors as an indication that MSA people lacked the technology to catch fish routinely. This argument is unsustainable as most LSA assemblages with fish also lack these artefacts, and there is no doubt that these people were actively fishing.

The appropriateness of using eurocentrically derived models and 'trait lists' to indicate the presence or absence of behavioural modernity in the African MSA has been criticized by many authors, as it does not take the vast size and different climatic zones of the African continent compared to Europe into account, which would stimulate diverse responses depending on the context (Chase and Dibble 1990; Deacon 1998, 2001b; Gibson 1996; Henshilwood et al. 2001a; Henshilwood and Marean 2003, 2006; McBrearty and Brooks 2000; Mellars 2005; Renfrew 1996). Despite the unsuitability of expecting some of the traits that characterise the UP, where Homo sapiens were considered to be fully modern, on the African continent, some are still considered useful to identify modernity in MSA populations. Traits thought to indicate modern behaviour by these authors are varied but include qualities such as symbolically mediated behaviour including art and personal ornaments, technological traditions indicating cognitive complexity such as creating composite tools, adhesives, complex hunting technology and advanced technical proficiency, and exploitation of a broad range of food sources.

Proponents for an earlier manifestation of modern human behaviour have based their arguments on a rapidly expanding body of work with evidence for innovation during the MSA from renewed analyses and excavations in southern Africa signifying that MSA people were cognitively more advanced than what was previously thought. (Deacon 1992, 
1993, 2001b; Deacon and Wurz 2002; Henshilwood 2004; Henshilwood and d'Errico 2003; Henshilwood et al. 2002; Henshilwood and Marean 2003, 2006; Lombard and Phillipson 2010; Marean and Assefa 2005; McBrearty 1990; McBrearty and Brooks 2000; Mellars 2005, 2006; Minichillo 2005; Parkington et al. 2005; Stringer 2002, 2003; Texier et al. 2010; Wadley et al. 2009; Wurz 1997, 1999, 2008). These authors argue that the development of modern behaviours in Africa was not sudden, as the MP-UP transition appears to some, but entailed a complex series of events that developed in a mosaic, rather than linear pattern.

Most of these innovations have been recognised from HP and SB assemblages, and include the following: personal ornaments in the form of marine shell beads at Blombos Cave (d'Errico et al. 2005; Henshilwood et al. 2004) and some potential marine shell beads at Sibudu Cave (SBC) (d'Errico et al. 2008), engraved ochre pieces at Blombos Cave (Henshilwood et al. 2009; Henshilwood et al. 2002), Klein Kliphuis (Mackay and Welz 2008), Klasies River (Watts 1998), bone points and awls at Blombos Cave (Henshilwood and Sealy 1997; Henshilwood et al. 2001a) and Sibudu Cave (SBC) (Backwell et al. 2008; Wadley 2006), notched and engraved bone pieces from Klasies River, Blombos Cave (d'Errico and Henshilwood 2007; d'Errico et al. 2001) and SBC (Wadley 2006), evidence for compound adhesive manufacture and composite tool making at SBC, Umhlatuzana (UH) and Rose Cottage Cave (Lombard 2005, 2006a, b, 2007a; Wadley et al. 2009; Wadley et al. 2004), bow and arrow technology at SBC (Backwell et al. 2008; Lombard and Phillipson 2010), the use of traps and snares, which signifies forethought and planning, at SBC (Wadley 2010), intentional pre-heating of lithic raw material prior to knapping at Pinnacle Point 5-6, possibly dating back as far as $164 \mathrm{k}$ years ago at PP13B (Brown et al. 2009), spatial organization of living spaces with discrete sleeping areas and hearths at SBC (Goldberg et al. 2009) and organization around hearths at Klasies River (Deacon 1995; Deacon and Wurz 1996), and variability in food resource exploitation at SBC over time that includes birds and fish (Clark and Plug 2008; Plug 2004, 2006a; Wadley 2006). From the above, it is clear that the once almost universally held idea that throughout the MSA people were incapable of cognitively advanced behaviour is steadily becoming untenable.

Most of the new evidence for increased human cognitive capacity during certain periods of the MSA have either been ignored or considered insignificant in favour of evidence for 
small population sizes in the MSA and an inability to catch fish or flying birds (Avery et al. 2008; Halkett et al. 2003; Klein et al. 2004). The statement that the shellfish and tortoise sizes indicate smaller populations in the MSA is plausible, based on the current data, but whether population size was the factor that inhibited cultural innovations no longer seems credible.

The ability to fish is one of the features on the UP-derived trait list for identifying modern human behaviour. Others have argued that fishing (and fowling) is an economic activity that does not signify cognitive abilities or require intellect, but forms part of subsistence choices related to cost and effort, and the increased effort to catch fish made them less appealing than, for example, easily available shellfish (Deacon 1989, 2001a; Henshilwood and Marean 2003, 2006; Wurz 2000). It follows then that the evidence for fishing at Klasies River and Blombos Cave can either be interpreted, depending on which model is preferred, as evidence that MSA people were cognitively more advanced than previously thought, as they apparently did possess the technological skills to catch fish, or that in certain instances MSA people were prepared to invest in more labour-intensive activities to acquire fish.

At present there is no evidence for marine fishing at other coastal MSA sites in Africa. Whether this absence is due to a lack of suitable excavated sites or an indication that marine fishing was limited to southern Africa during the Middle Stone Age is not yet known. 


\section{Chapter 8. Conclusion}

The methods used in this study to analyse marine fish remains from a number of LSA and MSA assemblages differ from previous methods used on archaeological fish remains in southern Africa. With the exception of vertebrae, scales, teeth, spines and rays, all other fish elements were considered for identification, and a suite of attributes was systematically recorded for each specimen. This has not been done in previous analyses, and has allowed a level of comparison of data between assemblages that was not previously possible.

As a result of this methodological approach, it has been demonstrated that the previously common use of a select few elements to identify fish can result lowered specimen counts on a species level, and, perhaps more importantly, in some species being completely omitted from species lists. Comparison of elements of species occurring in both the MSA and LSA assemblages has identified large deficits of elements in the MSA. The surviving elements in the MSA assemblages were not always the ones traditionally used for identification, and these species would have been missed if all elements were not considered for identification.

The systematic recording of all elements has also allowed recognition of elements that are not suitable for identification to species level, which can be eliminated from future analyses of assemblages containing the same species. Exclusion of these elements will go some way towards speeding up identification, but there are still many elements that should be included during analysis.

The fish from Hoffman's/Robberg Cave has contributed a large, previously unknown of, assemblage to the LSA fish database of the southern Cape. It has also served to substantiate arguments for increased reliance on marine foods on the Robberg Peninsula during the period of occupation.

Detailed examination of the Hoffman's/Robberg Cave and Blombos Cave LSA assemblages have resulted in identification of the first explicit evidence for fish processing during the LSA.

Examination of the MSA assemblages of Klasies River and Blombos Cave has revealed that people were purposely fishing at least some of the time. The confirmation that people 
were capable of fishing in the MSA can be added to the gradually increasing list of traits that were once thought to belong only to the realm of 'modern' LSA people. The evidence for the ability to fish by MSA people, along with all the other new evidence for cognitively advanced abilities in the MSA, does not fit with the model which postulates that a lack of effective resource exploitation and hunting technology, including the ability to catch fish, due to small population sizes, prohibited the development of more complex technologies, thus keeping MSA people in a cycle of 'non-modern' behaviour until they were released from it by an unverifiable genetic mutation 50k years ago. It is better suited to models that espouse a gradual or mosaic development of cognitive abilities during the MSA.

It is telling that most of the studies that have uncovered evidence for increased cognitive abilities during the MSA date to within the last decade, and most are from assemblages excavated by 'modern' standards. This emphasizes the changing nature of archaeological investigations and the shifting paradigms as new evidence comes to light, allowing renewed evaluation of previously held ideas.

\section{Recommendations for future work}

During the course of this investigation, a number of issues that need to be addressed in future studies were identified. One is the issue of the absence of fish remains in the coastal MSA assemblages. As there are so few coastal MSA sites, it is imperative that the material from the unsorted Boegoeberg 2 assemblage and the residues of Hoedjies Punt are examined to establish how many fish bones are present in the former, and whether the latter contains any fish remains at all that might have been missed by primary sorters, in order to recreate a more complete picture of the absence or presence of fish remains in the rather patchy MSA record. Boegoeberg 2 in particular has the potential to add some muchneeded data to the very few MSA sites with preserved fish remains, but unfortunately it has been destroyed by road-building and it will not be possible to enlarge the small sample sizes if it does indeed contain more fish remains.

Subsampling of excavated material from the older, potentially less well sorted MSA assemblages would also help to allay suspicions that very fragmented fish remains were inadvertently overlooked, and to confirm the absence of fish in these assemblages. 
Another future project would be to subject a range of southern African marine fish species to a suite of processes to determine what sequence of events would result in the total destruction of fish remains. The one effect that cannot be replicated experimentally is that of age-mediated destruction of fish bone over several millennia. A potential method of overcoming this would be to look at the elements that have survived in the MSA assemblages studied here, and determine which processes would eliminate these elements that presumably possess characteristics that favour their preservation.

Finally, this study has highlighted the need for more actualistic studies on potential animal agents of accumulation of fish bone in the study area. The pilot study by von den Driesch (2004) on the contents of cormorant pellets needs to be expanded on, both in terms of larger sample sizes, and in terms of more detailed analysis of the element composition. Similar studies need to be done on fish remains from African fish eagle roosts that are located close to the study areas, and therefore contain local species. Both these studies will help to establish what the signatures of these potential fish accumulators are, and will allow recognition of their contribution to archaeological fish assemblages. 


\section{References}

Abe, Y., Marean, C. W., Nilssen, P. J., Assefa, Z. and Stone, E. C. 2002. The analysis of cutmarks on archaeofauna: a review and critique of quantification procedures, and a new image-analysis GIS approach. American antiquity 67 (4): 643-663.

Aldenderfer, M. 1998. Quantitative methods in archaeology: A review of recent trends and developments. Journal of Archaeological Research 6 (2): 91-120.

Allen, M. S., Ladefoged, T. N. and Wall, J. J. 2001. Traditional Rotuman fishing in temporal and regional context. International Journal of Osteoarchaeology 11 (12): 56-71.

Alperson-Afil, N., Sharon, G., Kislev, M., Melamed, Y., Zohar, I., Ashkenazi, S., Rabinovich, R., Biton, R., Werker, E. and Hartman, G. 2009. Spatial Organization of Hominin Activities at Gesher Benot Ya'aqov, Israel. Science 326 (5960): 1677.

Alvarez Arias, B. T. 2000. Ichthyotoxic plants used in Spain. Journal of Ethnopharmacology 73 (3): 505-512.

Ambrose, S. H. 1998. Chronology of the Later Stone Age and Food Production in East Africa. Journal of Archaeological Science 25: 377-392.

Ambrose, S. H. and Lorenz, K. G. 1990. "Social and Ecological Models for the MSA in South Africa," in The Emergence of Modern Humans. Edited by Mellars, P. New York: Cornell University Press.

Andersson, C., Folkvord, A., Geffen, A. and Høie, H. 2008. "Seasonal Temperature Estimates From Late Holocene Barents Sea cod Otoliths: Problems and Potential," in American Geophysical Union, Fall Meeting, Supplementary abstract.

Andrews, A. H., Gobalet, K. W. and Jones, T. L. 2003. Reliability Assessment of Seasonof-Capture Determination from Archaeological Otoliths. Bulletin of the Southern California Academy of Sciences 102.2.

Andrews, P. 1990. Owls, caves, and fossils: predation, preservation, and accumulation of small mammal bones in caves, with an analysis of the Pleistocene cave faunas from Westbury-sub-Mendip, Somerset, UK Chicago: University of Chicago Press.

Andrews, P. 1995. Experiments in Taphonomy. Journal of Archaeological Science 22 (2): 147-153.

Andrews, P. and Cook, J. 1985. Natural modifications to bones in a temperate setting. Man 20 (4): 675-691.

Andrus, C. F. T., Crowe, D. E., Sandweiss, D. H., Reitz, E. J. and Romanek, C. S. 2002. Otolith delta 180 record of mid-Holocene sea surface temperatures in Peru. Science 295 (5559): 1508.

Andrus, C. F. T., Crowe, D. E., Sandweiss, D. H., Reitz, E. J., Romanek, C. S. and Maasch, K. A. 2003. Response to Comment on" Otolith delta 180 Record of MidHolocene Sea Surface Temperatures in Peru". Science 299 (5604): 203.

Ankei, Y. 1989. Folk knowledge of fish among the Songola and the Bwari: comparative ethnoichthyology of the Lualaba River and Lake Tanganyika fishermen. African Study Monographs Supplement 9: 1 - 88.

Arndt, A., van Neer, W., Hellemans, B., Robben, J., Volckaert, F. and Waelkens, M. 2003. Roman trade relationships at Sagalassos (Turkey) elucidated by ancient DNA of fish remains. Journal of Archaeological Science 30 (9): 1095-1105.

Asmussen, B. 2009. Intentional or incidental thermal modification? Analysing site occupation via burned bone. Journal of Archaeological Science 36 (2): 528-536. 
Attwood, C. G. and Bennett, B. A. 1990. A simulation model of the sport-fishery for galjoen coracinus capensis: An evaluation of minimum size limit and closed season. S. Afr. J. mar. Sci. 9: 359-369.

Attwood, C. G. and Bennett, B. A. 1994. Variation in dispersal of galjoen (Coracinus capensis) (Teleostei: Coracinidae) from a marine reserve. Canadian Journal of Fisheries and Aquatic Science 51: 1247-1257.

Attwood, C. G. and Cowley, P. D. 2005. Alternate explanations of the dispersal pattern of galjoen Dichistius capensis. African Journal of Marine Science 27: 141-156.

Avery, D. M. 2001. The Plio-Pleistocene vegetation and climate of Sterkfontein and Swartkrans, South Africa, based on micromammals. Journal of Human Evolution 41 (2): 113-132.

Avery, G. 1975. Discussion on the age and use of tidal fish-traps (visvywers). South African Archaeological Bulletin 30: 105-113.

Avery, G. 1976. A systematic investigation of open station shell middens along the southwestern Cape coast, M.A., University of Cape Town.

Avery, G. and Siegfried, W. R. 1980. Food gatherers along South Africa's seashore. Oceans 13: 32-37.

Avery, G., Halkett, D., Orton, J., Steele, T., Tusenius, M. and Klein, R. G. 2008. The Ysterfontein 1 Middle Stone Age rock shelter and the evolution of coastal foraging. South African Archaeological Society Goodwin Series.

Avery, G., Cruz-Uribe, K., Goldberg, P., Grine, F. E., Lenardi, M. J., Marean, C. W., Rink, J. W., Schwartz, H. P., Thackeray, A. I. and Wilson, M. L. 1997. The 19921993 Excavations at the Die Kelders Middle and Later Stone Age Cave Site, South Africa. Journal of Field Archaeology 24: 263-291.

Backwell, L., D'errico, F. and Wadley, L. 2008. Middle Stone Age bone tools from the Howiesons Poort layers, Sibudu Cave, South Africa. Journal of Archaeological Science 35 (6): 1566-1580.

Bada, J. L. and Deems, L. 1975. Accuracy of dates beyond the 14C dating limit using the aspartic acid racemisation reaction. Nature 255: 218-219.

Bailey, G. 2009. "The Red Sea, coastal landscapes, and hominin dispersals," in The Evolution of Human Populations in Arabia. . Edited by Petraglia, M. and Rose, J., pp. 15-37. Dordrecht: Springer.

Bamford, M. K. and Henderson, Z. L. 2003. A reassessment of the wooden fragment from Florisbad, South Africa. Journal of Archaeological Science 30 (6): 637-650.

Bar-Yosef, O. 1998. "The origin of modern humans.," in The Archaeology of Society in the Holy Land. Edited by Levy, T. E., pp. 111-121. New York: Facts on File.

Barba, R. and Domínguez-Rodrigo, M. 2005. The taphonomic relevance of the analysis of bovid long limb bone shaft features and their application to element identification: study of bone thickness and morphology of the medullary cavity. Journal of Taphonomy 3: 29-42.

Barrett, J. H. 1995. Few know an earl in fishing-clothes, Ph.D, University of Glasgow.

Barrett, J. H. 1997. Fish trade in Norse Orkney and Caithness: a zooarchaeological approach. Antiquity 71 (273): 616-638.

Barrett, J. H. and Beukens, R. P. 2001. Diet and ethnicity during the Viking colonization of northern Scotland: evidence from fish bones and stable carbon isotopes. Antiquity 75 (287): 145-154. 
Barrett, J. H. and Richards, M. P. 2004. Identity, Gender, Religion and Economy: New Isotope and Radiocarbon Evidence for Marine Resource Intensification in Early Historic Orkney, Scotland, UK. European Journal of Archaeology 7 (3): 249-271.

Barrett, J. H., Nicholson, R. A. and Cerón-Carrasco, R. 1999. Archaeo-ichthyological Evidence for Long-term Socioeconomic Trends in Northern Scotland: 3500 BC to AD 1500. Journal of Archaeological Science 26 (4): 353-388.

Barrett, J. H., Locker, A. M. and Roberts, C. M. 2004a. The origins of intensive marine fishing in medieval Europe: the English evidence. Proceedings of the Royal Society of London. Biological sciences 271 (1556): 2417-2421.

Barrett, J. H., Locker, A. M. and Roberts, C. M. 2004b. 'Dark Age Economics' revisited: the English fish bone evidence AD 600-1600. Antiquity 78 (301): 618-636.

Barrett, J. H., Johnstone, C., Harland, J., van Neer, W., Ervynck, A., Makowiecki, D., Heinrich, D., Hufthammer, A. K., Enghoff, I. B. and Amundsen, C. 2008. Detecting the medieval cod trade: a new method and first results. Journal of Archaeological Science 35 (4): 850-861.

Barton, H., Piper, P. J., Rabett, R. and Reeds, I. 2009. Composite hunting technologies from the Terminal Pleistocene and Early Holocene, Niah Cave, Borneo. Journal of Archaeological Science 36: 1708-1714.

Bartosiewicz, L. 2001. Archaeozoology or zooarchaeology?: a problem from the last century. Archaeologia Polona 39: 75-86.

Bartosiewicz, L. 2008. Taphonomy and palaeopathology in archaeozoology. Geobios 41: 69-77.

Bartram, J. L. E., Jr. and Marean, C. W. 1999. Explaining the "Klasies Pattern": Kua Ethnoarchaeology, the Die Kelders Middle Stone Age Archaeofauna, Long BoneFragmentation and Carnivore Ravaging. Journal of Archaeological Science 26: 37-163.

Bazzanella, M., Betti, L. and Wierer, U. 2007. "Mesolithic wetland exploitation at Galgenbühel / Dos de la Forca Italy, Eastern Alps. The fish fauna," in The Role of Fish in Ancient Time ; Proceedings of the 13th Meeting of the ICAZ Fish Remains Working Group in October 4th - 9th, Basel, Augst 2005 Edited by Hüster Plogmann, H., pp. 93-100. Rhaden/Westf: Verlag Marie Leidorf GmbH.

Bearez, P., Devries, T. J. and Ortlieb, L. 2003. Comment on" Otolith delta 180 Record of Mid-Holocene Sea Surface Temperatures in Peru". Science 299 (5604): 203.

Béarez, P. 1998. First archaeological indication of fishing by poison in a sea environment by the Engoroy poulation at Salango (Manabi, Ecuador). Journal of Archaeological Science 25: 943-948.

Beaune, D., Le Bohec, C., Lucas, F., Gauthier-Clerc, M. and Le Maho, Y. 2009. Stomach stones in king penguin chicks. Polar Biology 32 (4): 593-597.

Beech, M. 2002. Fishing in the 'Ubaid: A review of fish-bone assemblages from early prehistoric coastal settlements in the Arabian Gulf. Journal of Oman Studies 12: 25-40.

Beech, M. 2003. "The development of fishing in the UAE: A zooarchaeological perspective." First International Conference on the Archaeology of the U.A.E., 2003 , pp. 289.

Behar, D. M., Villems, R., Soodyall, H., Blue-Smith, J., Pereira, L., Metspalu, E., Scozzari, R., Makkan, H., Tzur, S. and Comas, D. 2008. The dawn of human matrilineal diversity. The American Journal of Human Genetics 82 (5): 1130-1140. 
Behrensmeyer, A. K., Gordon, K. D. and Yanagi, G. T. 1986. Trampling as a cause of bone surface damage and pseudo-cutmarks. Nature 319 (6065): 768-771.

Bennett, B. A. 1991. Conservation in the marine environment: Some problems with the management of shore-angling in the southwestern Cape. Sth. Afr. J. aquat. Sci. 17 $(1 / 2): 12-18$.

Bennett, B. A. and Griffiths, C. L. 1986. Aspects of the biology of Galjoen Coracinus capensis (Cuvier) off the South-Western Cape, South Africa. South African Journal of Marine science 4: 153-162.

Bennett, B. A. and Attwood, C. G. 1991. Evidence for recovery of a surf-zone fish assemblage following the establishment of a marine reserve on the southern coast of South Africa. Marine Ecology Progress series 75: 173-181.

Bertellotti, M. and Yorio, P. 1999. Spatial and Temporal Patterns in the Diet of the Kelp Gull in Patagonia. The Condor 101 (4): 790-798.

Biden, C. L. 1930. Sea-angling fishes of the Cape (South Africa): A Natural History of some of the principal fishes caught by sea anglers and professional fishermen in Cape waters. London: Humphrey Milford.

Biden, C. L. 1954. Sea-angling fishes of the Cape (South Africa): A Natural History of some of the principal fishes caught by sea anglers and professional fishermen in Cape waters, Third edition. London: Humphrey Milford.

Binford, L. R. 1978. Nunamiut ethnoarchaeology. New York: Academic Press.

Binford, L. R. 1984. Faunal remains from Klasies River Mouth. Orlando, Florida: Academic Press.

Binford, L. R. 1985. Human ancestors: changing views of their behavior. Journal of Anthropological Archaeology 4 (4): 292-327.

Binneman, J. N. F. 1996. The symbolic construction of communities during the Holocene Later Stone Age in the south-eastern Cape, PhD thesis, University of the Witwatersrand.

Blumenschine, R. J., Prassack, K. A., Kreger, C. D., Pante, M. C., Dominguez-Rodrigo, M. and Barba, R. 2007. Carnivore tooth-marks, microbial bioerosion, and the invalidation of Domínguez-Rodrigo and Barba's (2006) test of Oldowan hominin scavenging behavior. Authors' reply. Journal of Human Evolution 53 (4): 420-433.

Bobrowsky, P. T. 1982. Olsen and Olsen's Identity Crisis in Faunal Studies. American Antiquity 47 (1): 180-183.

Bökönyi, S. 1970. A new method for the determination of the number of individuals in animal bone material. American Journal of Archaeology 74: 291-292.

Bower, D. and Crawford, R. 1981. A black south-easter. Custos 10 (7): 20-21.

Brackenbury, T. D. and Appleton, C. C. 1997. Acute toxicity evaluation of the plant molluscicide, Apodytes dimidiata (Icacinaceae), to Eisenia fetida (Oligochaeta) and Oreochromis mossambicus (Cichlidae) in South Africa. Acta tropica 63 (1): 114.

Brain, C. K. 1981. The hunters or the hunted? an introduction to African cave taphonomy. Chicago: Chicago University Press.

Branch, G. M. 1985. The impact of predation by Kelp Gulls Larus dominicanus on the sub-Antarctic limpet Nacella delesserti. Polar Biology 4 (3): 171-177.

Branch, M. L. and Beckley, L. E. 1999. Two oceans: a guide to the marine life of southern Africa. Cape Town: Struik.

Breuil, H. 1945. Sea animals amongst the prehistoric rock paintings of Ladybrand. South African Journal of Science XLI: 353-360. 
Brewer, D., J. 1987. Seasonality in the Prehistoric Faiyum Based on the Incremental Growth Structures of the Nile Catfish (Pisces: Clarias). Journal of Archaeological Science 14: 459-472.

Brewer, D. J. 1991. Temperatures in Predynastic Egypt inferred from the remains of the Nile perch. World Archaeology 22 (3): 288-303.

Briggs, D. E. G. 1995. Experimental Taphonomy. PALAIOS 10 (6): 539-550.

Brink, J. S. and Deacon, H. J. 1982. A study of a last interglacial shell midden and bone accumulation at Herolds Bay, Cape Province, South Africa. Palaeoecology of Africa 15: 31-39.

Broadhurst, C. L., Cunnane, S. C. and Crawford, M. A. 1998. Rift Valley lake fish and shellfish provided brain-specific nutrition for early Homo. British Journal of Nutrition 79 (01): 3-21.

Broadhurst, C. L., Wang, Y., Crawford, M. A., Cunnane, S. C., Parkington, J. E. and Schmidt, W. F. 2002. Brain-specific lipids from marine, lacustrine, or terrestrial food resources: potential impact on early African Homo sapiens. Comparative Biochemistry and Physiology 131 (4): 653-673.

Brooks, A., S. and Smith, C. C. 1987. Ishango Revisited: New Age Determinations and Cultural Interpretations. The African Archaeological Review 5: 65-78.

Brooks, A. S., Helgren, D. M., Cramer, J. S., Franklin, A., Hornyak, W., Keating, J. M., Klein, R. G., Rink, W. J., Schwarcz, H., Leith Smith, J. N., Stewart, K., Todd, N. E., Verniers, J. and Yellen, J. E. 1995. Dating and context of three Middle Stone Age sites with bone points in the Upper Semliki Valley, Zaire. Science 268: 548553.

Broughton, J. M. 1994. Late Holocene resource intensification in the Sacramento Valley, California: the vertebrate evidence. Journal of Archaeological Science 21: 501501.

Broughton, J. M., Cannon, V. I., Bogiatto, R., Arnold, S. and Dalton, K. 2006. The taphonomy of owl-deposited fish remains and the origin of the Homestead Cave ichthyofauna. Journal of Taphonomy 4 (2): 69-95.

Brouwer, S. L. and Griffiths, M. H. 2005. Stock separation and life history of Argyrozona argyrozona (Pisces: Sparidae) on the South African east coast. African Journal of Marine Science 27: 585-595.

Brouwer, S. L. and Griffiths, M. H. 2006. Management of Argyrozona argyrozona (Pisces: Sparidae) in South Africa based on per-recruit models. African Journal of Marine Science 28: 89-98.

Brown, K. S., Marean, C. W., Herries, A. I. R., Jacobs, Z., Tribolo, C., Braun, D., Roberts, D. L., Meyer, M. C. and Bernatchez, J. 2009. Fire as an engineering tool of early modern humans. Science 325 (5942): 859.

Bunn, H. T. 1982. Meat-eating and human evolution: studies on the diet and subsistence patterns of Plio-Pleistocene hominids in East Africa, Ph.D, University of California.

Bunn, H. T. 1991. A Taphonomic Perspective on the Archaeology of Human Origins. Annual Review of Anthropology 20: 433-467.

Bunn, H. T. and Kroll, E. M. 1986. Systematic Butchery by Plio/Pleistocene Hominids at Olduvai Gorge, Tanzania [and Comments and Reply]. Current Anthropology: 431452. 
Bunn, H. T., Bartram, L. E. and Kroll, E. M. 1988. Variability in bone assemblage formation from Hadza hunting, scavenging, and carcass processing. Journal of anthropological archaeology(Print) 7 (4): 412-457.

Burchell, W. J. 1824. Travels in the interior of Southern Africa. London: The Batchworth Press.

Butler, V. L. 1993. Natural versus cultural Salmonid remains: origin of the Dalles Roadcut bones, Columbia River, Oregon, USA. Journal of Archaeological Science 20: 1-1.

Butler, V. L. 1996. Tui Chub taphonomy and the importance of marsh resources in the western great basin of North America. American antiquity 61 (4): 699-717.

Butler, V. L. and Chatters, J. C. 1994. The Role of Bone Density in Structuring Prehistoric Salmon Bone Assemblages. Journal of Archaeological Science 21: 413-424.

Butler, V. L. and Bowers, N. J. 1998. Ancient DNA from salmon bone: a preliminary study. Ancient Biomolecules 2: 17-26.

Butler, V. L. and Schroeder, R. A. 1998. Do Digestive Processes Leave Diagnostic Traces on Fish Bones?, Journal of Archaeological Science 25: 957-971.

Butler, V. L. C., J.C. 1994. The role of bone density in structuring prehistoric salmon bone assemblages. Journal of Archaeological Science 21: 413-424.

Butzer, K. W. 1973. Geology of Nelson Bay Cave, Robberg, South Africa. The South African Archaeological Bulletin 28 (111/112): 97-110.

Buxton, C. D. and Smale, M. J. 1984. A preliminary investigation of the marine ichthyofauna in the Tsitsikamma Coastal National Park. Koedoe 27: 13-24.

Cable, C. 1984. Economy and technology in the Late Stone Age of southern Natal. Oxford: British Archaeological Reports International Series 201

Cannon, D. Y. 1987. Marine Fish Osteology - A Manual for Archaeologists. Burnaby: Simon Fraser University Archaeology Press.

Cannon, M. D. 1999. A mathematical model of the effects of screen size on zooarchaeological relative abundance measures. Journal of Archaeological Science 26 (2): 205-214.

Capaldo, S. D. 1997. Experimental determinations of carcass processing by PlioPleistocene hominids and carnivores at FLK 22 (Zinjanthropus), Olduvai Gorge, Tanzania. Journal of Human Evolution 33 (5): 555-597.

Capaldo, S. D. and Blumenschine, J. 1994. A quantitative diagnosis of notches made by hammerstone percussion and carnivore gnawing on bovid long bones. American Antiquity 59: 724-748.

Capriles, J. M., Domic, A. I. and Moore, K. M. 2008. Fish remains from the Formative Period (1000 BC-AD 400) of Lake Titicaca, Bolivia: Zooarchaeology and taphonomy. Quaternary International 180 (1): 115-126.

Carlson, B. A. and Kingston, J. D. 2007a. Docosahexaenoic acid biosynthesis and dietary contingency: Encephalization without aquatic constraint. American Journal of Human Biology 19 (4): 585-588.

Carlson, B. A. and Kingston, J. D. 2007b. Docosahexaenoic acid, the aquatic diet, and hominin encephalization: difficulties in establishing evolutionary links. American Journal of Human Biology 19 (1): 132-141.

Carss, D. N. 1993. Cormorants Phalacrocorax carbo at cage fish farms in Argyll, western Scotland. Seabird 15: 38-44.

Carvajal-Contreras, D. R., Cooke, R. and Jiménez, M. 2008. Taphonomy at two contiguous coastal rockshelters in Panama: Preliminary observations focusing on fishing and curing fish. Quaternary International 180 (1): 90-106. 
Casteel, R. W. 1972. Some archaeological uses of fish remains. American Antiquity 37 (3): 404-419.

Casteel, R. W. 1974. On the remains of fish scales from archaeological sites. American Antiquity 39 (4): 557-581.

Casteel, R. W. 1976. Fish Remains in Archaeology and Paleo-environmental Studies, 1 edition. London: Academic Press.

Casteel, R. W. 1977. Characterization of faunal assemblages and the minimum number of individuals determined from paired elements: continuing problems in archaeology. Journal of Archaeological Science 4 (2): 125-134.

Castley, J. G., Cockcroft, V. G. and Kerley, G. I. H. 1991. A note on the stomach contents of fur seals Arctocephalus pusillus pusillus beached on the south-east coast of South Africa. South African Journal of Marine Science 11 (1): 573-577.

Chase, P. and Dibble, H. L. 1987. Middle Paleolithic symbolism: A review of current evidence and interpretations. Journal of Anthropological Archaeology 6: 263-296.

Chase, P. G. and Dibble, H. L. 1990. On the emergence of modern humans. Current Anthropology 31 (1): 58-66.

Ciesielski, S. and Brzuzan, P. 2003. The genetic analysis of trout skeletal remains: how should the potential of ancient fish DNA be utilized?, Archiwum Rybactwa Polskiego 11 (2): 213-224.

Ciesielski, S., Brzuzan, P. and Luczynski, M. 2002. Recovery and Analysis of Mitochondrial DNA from Ancient Bones of Common Bream, Abramis brama L. Ancient Biomolecules 4 (1): 43-46.

Clark, G. A. and Lindly, J. M. 1989. Modern human origins in the Levant and western Asia: the fossil and archaeological evidence. American Anthropologist 91 (4): 962985.

Clark, G. A. and Lindly, J. M. 1991. On paradigmatic biases and Paleolithic research traditions. Current Anthropology 32 (5): 577-587.

Clark, J. D. 1958. Some stone age woodworking tools in Southern Africa. The South African Archaeological Bulletin: 144-152.

Clark, J. D. 1988. The Middle Stone Age of East Africa and the beginnings of regional identity. Journal of World Prehistory 2: 237-305.

Clark, J. D. 1989. "The origins and spread of Modern Humans: a Broad perspective on the African Evidence," in The Human Revolution. Edited by Mellars, P. and Stringer, C., pp. 566-588. Edinburgh: Edinburgh University Press.

Clark, J. L. and Plug, I. 2008. Animal exploitation strategies during the South African Middle Stone Age: Howiesons Poort and post-Howiesons Poort fauna from Sibudu Cave. Journal of human evolution 54 (6): 886-898.

Clason, A. T. 1986. "Fish and Archaeology," in Fish and Archaeology: Studies in Osteometry, Taphonomy, Seasonality and Fishing Methods, vol. 294. Edited by Clason, A. T. and Brinkhuizen, D. C., pp. 1-8. Oxford: British Archaelogical Reports International Series.

Colley, S. M. 1989. "The Analysis and Interpretation of Archaeological Fish Remains," in Archaeological method and theory, vol. 2, pp. 207-253. Tucson: University of Arizona Press.

Colley, S. M. 1990. "The Analysis and Interpretation of Archaeological Fish Remains," in Archaeological method and theory, vol. 2, pp. 207-253. Tucson: University of Arizona Press.

Cornwall, I. W. 1974. Bones for the archaeologist. London: Dent and Sons. 
Corr, L. T., Sealy, J. C., Horton, M. C. and Evershed, R. P. 2005. A novel marine dietary indicator utilising compound-specific bone collagen amino acid $13 \mathrm{C}$ values of ancient humans. Journal of Archaeological Science 32 (3): 321-330.

Corr, L. T., Richards, M. P., Grier, C., Mackie, A., Beattie, O. and Evershed, R. P. 2009. Probing dietary change of the Kwaday Dan Ts' nchi individual, an ancient glacier body from British Columbia: II. Deconvoluting whole skin and bone collagen d 13 $\mathrm{C}$ values via carbon isotope analysis of individual amino acids. Journal of Archaeological Science 36: 12-18.

Craig, O. E., Forster, M., Andersen, S. H., Koch, E., Crombe, P., Milner, N. J., Stern, B., Bailey, G. N. and Heron, C. P. 2007. Molecular and isotopic demonstration of the processing of aquatic products in norhtern European prehistoric pottery. Archaeometry 49 (1): 135-152.

Crawford, M. A. 2006. Docosahexaenoic acid in neural signaling systems. Nutrition and health (Berkhamsted, Hertfordshire) 18 (3): 263.

Crawford, M. A., Bloom, M., Broadhurst, C. L., Schmidt, W. F., Cunnane, S. C., Galli, C., Gehbremeskel, K., Linseisen, F., Lloyd-Smith, J. and Parkington, J. 1999a. Evidence for the unique function of docosahexaenoic acid during the evolution of the modern hominid brain. Lipids 34: 39-47.

Crawford, R. J. M. 2007. "Trends in numbers of three cormorants Phalacrocorax spp. breeding in South Africa's Western Cape Province," in Final Report of BCLME (Benguela Current Large Marine Ecosystem) Project on Top Predators as Biological Indicators of Ecosystem Change in the BCLME. Edited by Kirkman, S. P., pp. 165-172. Cape Town: Avian Demography Unit.

Crawford, R. J. M., Shannon, L. J. and Whittington, P. A. 1999b. Population dynamics of the African Penguin Spheniscus demersus at Robben Island, South Africa. Marine Ornithology 27: 139-147.

Crawford, R. J. M., Dyer, B. M., Kemper, J., Simmons, R. E. and Upfold, L. 2007. Trends in numbers of Cape Cormorants (Phalacrocorax capensis) over a 50-year period, 1956-57 to 2006-07. Emu 107 (4): 253-261.

Cruz-Uribe, K. and Klein, R. G. 1998. Hyrax and Hare Bones from Modern South African Eagle Roosts and the Detection of Eagle Involvement in Fossil Bone Assemblages. Journal of Archaeological Science 25: 135-147.

Cunnane, S. C. 2007. Docosahexaenoic acid and human brain evolution: missing the forest for the trees? Comments by Cunnane. British Journal of Nutrition 97 (05): 1021-1022.

Cunnane, S. C. and Crawford, M. A. 2003. Survival of the fattest: fat babies were the key to evolution of the large human brain. Comparative Biochemistry and Physiology, Part A 136 (1): 17-26.

Cunnane, S. C., Plourde, M., Stewart, K. and Crawford, M. A. 2007. Docosahexaenoic acid and shore-based diets in hominin encephalization: a rebuttal. American Journal of Human Biology 19 (4): 578-581.

D'errico, F. 2003. The invisible frontier: a multiple species model for the origin of behavioral modernity. Evolutionary Anthropology 12: 188-202.

D'errico, F. and Henshilwood, C. S. 2007. Additional evidence for bone technology in the southern African Middle Stone Age. Journal of Human Evolution 52 (2): 142-163.

D'errico, F., Henshilwood, C. S. and Nilssen, P. 2001. An engraved bone fragment from ca. 75,000-year-old Middle Stone Age levels at Blombos Cave, South Africa: implications for the origin of symbolism and language. Antiquity 75: 309-318. 
D'errico, F., Vanhaeren, M. and Wadley, L. 2008. Possible shell beads from the Middle Stone Age layers of Sibudu Cave, South Africa. Journal of Archaeological Science 35 (10): 2675-2685.

D'errico, F., Henshilwood, C., Vanhaeren, M. and van Niekerk, K. 2005. Nassarius kraussianus shell beads from Blombos Cave: evidence for symbolic behaviour in the Middle Stone Age. Journal of Human Evolution 48: 3-24.

Daly, P. 1969. Approaches to Faunal Analysis in Archaeology. American Antiquity 34 (2): 146-153.

Dart, R. A. 1957. The osteodontokeratic culture of Australopithecus prometheus. Vol. 10: Memoir of the Transvaal Museum.

Darwent, C. M. and Lyman, R. L. 2002. "Detecting the postburial fragmentation of carpals, tarsals, and phalanges," in Advances in forensic taphonomy: Method, theory and archaeological perspectives, pp. 355-377. Boca Raton: CRC Press.

Davis, S. J. M. 1987. The archaeology of animals. London: B. T. Batsford.

Day, J. J. 2002. Phylogenetic relationships of the Sparidae (Teleostei: Percoidei) and implications for convergent trophic evolution. Biological Journal of the Linnean Society 76 (2): 269-301.

De Laguna, F. 1964. Archeology of the Yakutat Bay area, Alaska: US Govt. Print. Off.

De Ruiter, D. J. 2004. "Relative abundance, skeletal part representation and accumulating agents as Swartkrans," in Swartkrans - A cave's chronicle of early Man, 2 edition. Edited by Brain, C. K. Pretoria: Transvaal Museum Monograph 8.

De Ruiter, D. J., Brophy, J. K., Lewis, P. J., Churchill, S. E. and Berger, L. R. 2008. Faunal assemblage composition and paleoenvironment of Plovers Lake, a Middle Stone Age locality in Gauteng Province, South Africa. Journal of Human Evolution 55 (6): 1102-1117.

De Smet, P. 1998. Traditional pharmacology and medicine in Africa Ethnopharmacological themes in sub-Saharan art objects and utensils. Journal of ethnopharmacology 63 (1-2): 1-175.

Deacon, H. J. 1970. Two shell midden occurrences in the Tsitsikamma National Park, Cape Province: A contribution to the study of the ecology of the Strandloopers. Koedoe 13: 37-49.

Deacon, H. J. 1972. A review of the Post-Pleistocene in South Africa. South African Archaeological Bulletin, Goodwin Series 1: 26-45.

Deacon, H. J. 1974. An archaeological study of the Eastern Cape in the Post-Pleistocene period, Ph.D., University of Cape Town.

Deacon, H. J. 1976. Where hunters gathered: a study of Holocene Stone Age people in the eastern Cape. Claremont: South African Archaeological Society.

Deacon, H. J. Editor. 1989. Late Pleistocene palaeoecology and archaeology in the southern Cape, South Africa. The Human Revolution: Behavioural and Biological Perspectives on the Origins of Modern Humans. Edinburgh: Edinburgh University Press.

Deacon, H. J. 1992. "Southern Africa and Modern Human Origins," in The Origin of Modern humans and the impact of chronometric dating. Edited by Aitken, M. J., Stringer, C. B. and Mellars, P. A. New Jersey: Princeton University Press.

Deacon, H. J. 1993. Planting an Idea: An Archaeology of Stone Age Gatherers in South Africa. The South African Archaeological Bulletin 48 (158): 86-93.

Deacon, H. J. 1995. Two Late Pleistocene-Holocene archaeological depositories from the southern Cape, South Africa. South African Archaeological Bulletin 50: 121-131. 
Deacon, H. J. 1998. "Modern Human Emergence: an African archaeological perspective." Dual Congress - The archaeology of modern humans, Sun City, South Africa, 1998.

Deacon, H. J. 2001a. Guide to Klasies River. http://academic.sun.ac.za/archaeology/KRguide2001.PDF

Deacon, H. J. 2001b. "Modern Human Emergence: an African Archaeological Perspective," in Humanity from African Naissance to Coming Millennia Colloquia in Human Biology and Palaeaonthropology. Edited by Tobias, P. V., Raath, M. A., Maggi-Cecchi, J. and Doyle, G. A., pp. 217-226. Florence: Florence University Press.

Deacon, H. J. 2008. The context of the 1967-8 sample of human remains from Cave 1 Klasies River main site. South African Archaeological Society Goodwin Series 10: 143-149.

Deacon, H. J. and Deacon, J. 1963. Scott's Cave: a late Stone Age site in the Gamtoos Valley. Annals of the Cape Provial Museums 3: 96-121.

Deacon, H. J. and Geleijnse, V. B. 1988. The stratigraphy and sedimentology of the main site sequence, Klasies River, South Africa. South African Archaeological Bulletin 43: $5-14$.

Deacon, H. J. and Wurz, S. 1996. "Klasies River main site, cave 2: a Howiesons Poort occurrence," in Aspects of African Archaeology. Edited by Pwiti, G. and Soper, R., pp. 213-218. Harare: University of Zimbabwe.

Deacon, H. J. and Deacon, J. 1999. Human Beginnings in South Africa: Uncovering the secrets of the Stone Age. Cape Town: David Philip.

Deacon, H. J. and Wurz, S. 2002. "Howiesons Poort and the implications for behavior."

Deacon, J. 1978. Changing patterns in the late Pleistocene/Early Holocene prehistory of southern Africa as seen from the Nelson Bay Cave stone artifacts sequence. Quaternary Research 10: 84-111.

Deacon, J. 1984a. The Later Stone Age of southernmost Africa. British Archaeological Reports International Series 213: Only 370-400.

Deacon, J. 1984b. "Later Stone Age people and their descendants in southern Africa," in Southern African prehistory and palaeoenvironments. Edited by Klein, R. G., pp. 221-328. Rotterdam: A.A. Balkema.

Desse, J. 1984. Propositions pour une réalisation collective d'un corpus: fiches d'identification et d'exploitation métrique du squelette des poissons. 2èmes Rencontres d'Archéo-Ichthyologie. Paris: Centre National de la Recherche Scientifique: $67-86$.

Desse, J. and Desse-Berset, N. 1992. "Age et saison de mort des poissons: applications al'archéologie," in Tissus Durs et Age Individuel des Vertébrés. Edited by Bagliniere, J. L., Castanet, J., Conand, F. and Meunier, F. J., pp. 341-353. Paris: ORSTOM.

Desse, J. and Desse-Berset, N. 1996. On the boundaries of osteometry applied to fish. Archaeofauna (5): 171.

Desse, G. and De Buit, M. H. 1971. Diagnostic des pièces rachidiennes des Téléostéens et des Chondrichthyens II: Chondrichthyens. Paris: L'Expansion Scientifique Francaise.

Desse-Berset, N. and Desse, J. 2000. Salsamenta, garum et autres preparations de poissons. Ce qu'en disent les os. Mélanges de l'École Française de Rome, Antiquité 112: 73-97. 
Dobney, K. and Rielly, K. 1988. A method for recording archaeological animal bones: the use of diagnostic zones. Circaea 5 (2): 79-96.

Döckel, W. 1998. Re-investigation of the Matjes River Rock Shelter, M.A., University of Stellenbosch.

Dominguez-Rodrigo, M. and Barba, R. 2006. New estimates of tooth mark and percussion mark frequencies at the FLK Zinj site: the carnivore-hominid-carnivore hypothesis falsified. Journal of Human Evolution 50 (2): 170-194.

Dominguez-Rodrigo, M., Egeland, C. P. and Barba, R. 2007. "The "physical attribute" taphonomic approach," in Deconstructing Olduvai: A Taphonomic Study of the Bed I Sites. Edited by Dominguez-Rodrigo, M., Barba, R. and Egeland, C. P. New York: Springer Verlag.

Dreyer, T. F. 1934. The archaeology of the Matjes River rock shelter. Transactions of the Royal Society of South Africa XXI: 187-209.

Duffy, D. C., Wilson, R. P. and Wilson, M. P. 1987. Spatial and temporal patterns of diet in the Cape Cormorant off southern Africa. Condor 89 (4): 830-834.

Dufour, E., Holmden, C., van Neer, W., Zazzo, A., Patterson, W. P., Degryse, P. and Keppens, E. 2007. Oxygen and strontium isotopes as provenance indicators of fish at archaeological sites: the case study of Sagalassos, SW Turkey. Journal of Archaeological Science 34 (8): 1226-1239.

Dyall, L. K. 2004. "The Aboriginal middens at Birubi," pp. 259. Wallsend.

Eastwood, E. B. 1999. Red lines and arrows: attributes of supernatural potency in San rock art of the Northern Province, South Africa and south-western Zimbabwe. The South African Archaeological Bulletin: 16-27.

Efremov, I. A. 1940. Taphonomy: a new branch of paleontology. Pan-American Geologist 74 (2): 81-93.

Emmerson, W. and Philip, S. 2004. Diets of Cape clawless otters at two South African coastal localities. African Zoology 39 (2): 201-210.

Enviro-Fish-Africa. 2008. Environmental Impact Assessment of the proposed eradication of Invasive Alien Fishes from selected rivers in the Cape Floristic Region. EnviroFish Africa (Pty) Ltd.

Erlandson, J., M., Braje, T. J., Rick, T. C. and Peterson, J. 2005. Beads, Bifaces, and Boats: An Early Maritime Adaptation on the South Coast of San Miguel Island, California. American Anthropologist 107 (4): 677-683.

Erlandson, J. M. 2001. The archaeology of aquatic adaptations: paradigms for a new millennium. Journal of Archaeological Research 9 (4): 287-350.

Erlandson, J. M., Moss, M. L. and Des Lauriers, M. 2008. Life on the edge: early maritime cultures of the Pacific Coast of North America. Quaternary Science Reviews 27 (23-24): 2232-2245.

Erlandson, J. M., Rick, T. C., Vellanoweth, R. L. and J.K., D. 1999. Maritime Subsistence at a 9300 Year Old Shell Midden on Santa Rosa Island, California. Journal of Field Archaeology 26 (3): 255-265.

Erlandson, J. M., Rick, T. C., Collins, P. W. and Guthrie, D. A. 2007. Archaeological implications of a bald eagle nesting site at Ferrelo Point, San Miguel Island, California. Journal of Archaeological Science 34 (2): 255-271.

Fagan, B. M. 1960. The Glentyre Shelter and Oakhurst re-examined. South African Archaeological Bulletin 155: 80-94.

Fairhall, A. W., Young, A. W. and Erickson, J. L. 1976. University of Washington dates IV. Radiocarbon 18 (2): 221-239. 
Faith, J. T., Marean, C. W. and Behrensmeyer, A. K. 2007. Carnivore competition, bone destruction, and bone density. Journal of Archaeological Science 34 (12): 20252034.

Feathers, J. K. 1997. Luminescence dating of sediment samples from White paintings Rockshelter, Botswana. Quaternary Science Reviews 16 (3-5): 321-331.

Feathers, J. K. 2002. Luminescence Dating in Less Than Ideal Conditions: Case Studies from Klasies River Main Site and Duinefontein, South Africa. Journal of Archaeological Science 29 (2): 177-194.

Feathers, J. K. and Bush, D. A. 2000. Luminescence dating of Middle stone age deposits at Die Kelders. Journal of Human Evolution 38 (1): 91-119.

Feathers, J. K. and Migliorini, E. 2001. Luminescence dating at Katanda -- a reassessment. Quaternary Science Reviews 20 (5-9): 961-966.

Fischer, A. 2007. "Computerised bone templates as the basis of a practical procedure to record and analyse graphical zooarchaeological data," in Revista Electrónica de Arqueología PUCP, vol. 2.

Fisher, J. W. 1995. Bone Surface Modifications in Zooarchaeology. Journal of Archaeological Method and Theory 2 (1): 7-68.

Foley, R. and M., L. M. 1997. Mode 3 Technologies and the evolution of Modern Humans. Cambridge Archaeological Journal 7 (1): 3-36.

Froese, R. and Pauly, D. 2009. "FishBase." version (10/2009).

Gabriel, O., Lange, K., Dahm, E. and Wendt, T. Editors. 2005. Von Brandt's Fish catching methods of the world, 4th edition. Oxford: Blackwell Publising.

Gargett, R. H. and Vale, D. 2005. There's something fishy going on around here. Journal of Archaeological Science 32 (4): 647-652.

Gayet, M. and van Neer, W. 1990. Caractères diagnostiques des épines de quelques silures africains. Journal of African Zoology 104 (3): 241-252.

Gibson, K. R. 1996. "The biocultural Human brain, seasonal migrations, and the emergence of the Upper Paleolithic," in Modelling the early human mind. Edited by Mellars, P. and Gibson, K. Oxford: Oxbow books.

Gifford-Gonzalez, D., Stewart, K. M. and Rybczynski, N. 1999. Human activities and site formation at modern lake margin foraging camps in Kenya. Journal of Anthropological Archaeology 18 (4): 397-440.

Gifford, D. P. 1981. Taphonomy and Paleoecology: A Critical Review of Archaeology's Sister Disciplines. Advances in Archaeological Method and Theory 4: 365-438.

Gilbert, A. S. and Singer, B. H. 1982. Reassessing zooarchaeological quantification. World Archaeology 14 (1): 21-40.

Gilmore, H. W. 1953. Hunting Habits of the Early Nevada Paiutes. American Anthropologist 55 (1): 148-153.

Giovas, C. M. 2009. The shell game: analytic problems in archaeological mollusc quantification. Journal of Archaeological Science 36 (7): 1557-1564.

Gobalet, K. W. 2005. Comment on "Size matters: 3-mm sieves do not increase richness in a fishbone assemblage from Arrawarra I, an Aboriginal Australian shell midden on the mid-north coast of New South Wales, Australia" by Vale and Gargett. Journal of Archaeological Science 32 (4): 643-645.

Gobalet, K. W. and Jones, T. L. 1995. Prehistoric Native American fisheries of the central California coast. Transactions of the American Fisheries Society 124 (6): 813-823. Goldberg, P., Miller, C. E., Schiegl, S., Ligouis, B., Berna, F., Conard, N. J. and Wadley, L. 2009. Bedding, hearths, and site maintenance in the Middle Stone age of Sibudu 
cave, KwaZulu-Natal, South Africa. Archaeological and Anthropological Sciences $1(2)$ : 95-122.

Goodwin, A. J. H. 1938. Archaeology of the Oakhurst Shelter. Part 1. Course of the excavation. Transactions of the Royal Society of South Africa XXV: 229-247.

Goodwin, A. J. H. 1946. Prehistoric fishing methods in South Africa. Antiquity 20: 134141.

Goodwin, A. J. H. 1953. Two Caves at Kalk Bay, Cape Peninsula. South African Archaeological Bulletin 8: 59 - 77.

Granadeiro, J. P. and Silva, M. A. 2000. The use of otoliths and vertebrae in the identification and size-estimation of fish in predator-prey studies. Cybium 24 (4): 383-393.

Grayson, D. K. 1973. On the methodology of faunal analysis. American Antiquity: 432439.

Grayson, D. K. 1984. Quantitative zooarchaeology: Topics in the Analysis of Archaeological Faunas. Orlando: Academic Press, Inc.

Grayson, D. K. and Frey, C. J. 2004. Measuring skeletal part representation in archaeological faunas. Journal of Taphonomy 2: 27-42.

Gregory, W. K. 1933. Fish skulls: a study of the evolution of natural mechanisms. Transactions of the American Philosophical Society 23 (2).

Griffiths, M. H. and Heemstra, P. C. 1995. A contribution to the taxonomy of the marine fish genus Argyrosomus (Perciformes: Sciaenidae), with descriptions of two new species from southern Africa. Ichthyological Bulletin 65: 1-40.

Grine, F. E. and Klein, R. G. 1993. Late Pleistocene human remains from the Sea Harvest site, Saldanha Bay, South Africa. South African Journal of Science 89: 145-152.

Grine, F. E. and Henshilwood, C. S. 2002. Additional human remains from Blombos Cave, South Africa:(1999-2000 excavations). Journal of Human Evolution 42 (3): 293-302.

Grine, F. E., Henshilwood, C. S. and Sealy, J. C. 2000. Human remains from Blombos Cave, South Africa: (1997-1998 excavations). Journal of Human Evolution 38: 755-765.

Grobbelaar, C. S. and Goodwin, A. J. H. 1952. Report on the skeletons and implements in association with them from a cave near Bredasdorp, Cape Province. South African Archaelogical Bulletin 7: 95-106.

Groves, C. and Thorne, A. 2000. The affinities of the Klasies River Mouth remains. Perspectives in Human Biology 5: 43-53.

Grün, R., Shackleton, N. J. and Deacon, H. J. 1990. Electron-Spin-Resonance Dating of Tooth Enamel from Klasies River Mouth Cave. Current Anthropology 31 (4): 427432.

Haddad, D. 2003. "Fishing the Khoisan way," in Good Taste, vol. September/October, pp. 102-106.

Halkett, D., Hart, T., Yates, R., Volman, T. P., Parkington, J. E., Orton, J., Klein, R. G., Cruz-Uribe, K. and Avery, G. 2003. First excavation of intact Middle Stone Age layers at Ysterfontein, Western Cape Province, South Africa: implications for Middle Stone Age ecology. Journal of Archaeological Science 30: 955-971.

Hall, S. L. 1990. Hunter-gatherer-fishers of the Fish River Basin: a contribution to the Holocene prehistory of the Eastern Cape, Ph. D., University of Stellenbosch.

Hall, S. L. 1997. Freshwater fish from Mzinyashana Shelter 1, Thukela Basin, KwaZuluNatal. Natal Museum Journal of Humanities 9: 47-55. 
Hall, S. L. 2000. Burial and Sequence in the Later Stone Age of the Eastern Cape Province, South Africa. The South African Archaeological Bulletin 55 (172): 137146.

Hambly, W. D. 1937. "Economic Life \& Hunting Cultures," in Source Book for African Anthropology Part II, vol. 26. Edited by Martin, P. S., pp. 332-333; 602-607. Chicago: Field Museum of Natural History, Chicago.

Hanekom, N., Hutchings, L., Joubert, P. A. and van Der Byl, P. C. N. 1989. Sea temperature variations in the Tsitsikamma coastal National Park, South Africa, with notes on the effect of cold conditions on some fish populations. South African Journal of Marine Science 8: 145-153.

Harland, J. F., Barrett, J. H., Carrott, J., Dobney, K. and Jaques, D. 2003. The York System: An integrated zooarchaeological database for research and teaching. Internet Archaeology 13.

Harper, D. M., Harper, M. M., Virani, M. A., Smart, A., Brooks Childress, R., Adatia, R., Henderson, I. and Chege, B. 2002. Population fluctuations and their causes in the African Fish Eagle,(Haliaeetus vocifer (Daudin)) at Lake Naivasha, Kenya. Hydrobiologia 488 (1): 171-180.

Hbs. http://hbs.bishopmuseum.org/frc/types.html

Heemstra, P. and Heemstra, E. 2004. Coastal Fishes of Southern Africa. Grahamstown: South African Institute for Aquatic Biodiversity and National Inquiry Service Centre.

Heinrich, D. 1983. "Temporal changes in fishery and fish consumption between early medieval Haithabu and its successor, Schleswig," in Animals and Archaeology: Shell middens, fishes and birds. Edited by Grigson, C. and Clutton-Brock, J., pp. 151-156. Oxford: British Archaeological Reports International Series.

Heizer, R. F. 1949. Curved Single-Piece Fishhooks of Shell and Bone in California. American Antiquity 15 (2): 89-97.

Henshilwood, C. S. 1995. Holocene archaeology of the coastal Garcia State Forest, Southern Cape, South Africa, Ph.D, University of Cambridge.

Henshilwood, C. S. 1997. Identifying the collector: evidence for human processing of the Cape dune mole-rat, Bathyergus suillus, from Blombos Cave, southen Cape, South Africa., Journal of Archaeological Science. 24: 659-662.

Henshilwood, C. S. 2004. "The origins of modern human behaviour- exploring the African evidence," in Pre-history in a global perspective, vol. 1210. Edited by Oestigaard, T., Anfinset, N. and Saetersdal, T. Bergen, Norway: BAR International eries.

Henshilwood, C. S. 2005. "Stratigraphic Integrity of the Middle Stone Age Levels at Blombos Cave," in From tools to symbols: from early hominids to modern humans. Edited by D’errico, F. and Backwell, L., pp. 441-458. Johannesburg: Witwatersrand University Press.

Henshilwood, C. S. 2008. Holocene prehistory of the southern Cape, South Africa: excavations at Blombos Cave and the Blombosfontein Nature Reserve. Vol. 75. Cambridge Monographs in African Archaeology. British Archaeological Reports S1860. Oxford: Archaeopress.

Henshilwood, C. S. and Sealy, J. C. 1997. Bone artefacts from the Middle Stone Age at Blombos Cave, southern Cape, South Africa. Current Anthropology 38 (5): 890895.

Henshilwood, C. S., D'errico, F., Marean, C. W., Milo, R. G. and Yates, R. 2001a. An early bone tool industry from the Middle Stone Age at Blombos Cave, South 
Africa: implications for the origins of modern human behaviour, symbolism and language. Journal of Human Evolution 41: 631-678.

Henshilwood, C. S., Sealy, J. C., Yates, R., Cruz-Uribe, K., Goldberg, P., Grine, F. E., Klein, R. G., Poggenpoel, C. A., van Niekerk, K. and Watts, I. 2001b. Blombos Cave, Southern Cape, South Africa: Preliminary Report on the 1992-1999 Excavations of the Middle Stone Age Levels. Journal of Archaeological Science 28: 421-448.

Henshilwood, C. S. and D'errico, F. 2003. "Being modern in the Middle Stone Age: Individuals and innovation," in The individual hominid in context: Archaeological investigations of Lower and Middle Palolithic landscapes, locales and artefacts. Edited by Gamble, C. and Porr, M.: Routledge (Taylor Francis).

Henshilwood, C. S. and Marean, C. W. 2003. The origin of modern human behavior: A review and critique of the models and their test implications. Current anthropology 44 (5): 627-651.

Henshilwood, C. S. and Marean, C. W. 2006. "Remodelling the origins of modern human behaviour - Tracing the lineage of modern man," in The prehistory of Africa. Edited by Soodyall, H., pp. 37-59. Cape Town: Jonathan Ball publishers.

Henshilwood, C. S., D'errico, F. and Watts, I. 2009. Engraved ochres from the Middle Stone Age levels at Blombos Cave, South Africa. Journal of Human Evolution 57: 27-47.

Henshilwood, C. S., D'errico, F., Vanhaeren, M., van Niekerk, K. and Jacobs, Z. 2004. Middle stone age shell beads from South Africa. Science 304 (5669): 404-404.

Henshilwood, C. S., D'errico, F., Yates, R., Jacobs, Z., Tribolo, C., Duller, G. A. T., Mercier, N., Sealy, J. C., Valladas, H. and Watts, I. 2002. Emergence of Modern Human Behavior: Middle Stone Age Engravings from South Africa. Science 295 (5558): 1278.

Herdrich, D. and Armstrong, K. 2008. Historic fishing methods in American Samoa Final Report to PIFSC \& NOAA.

Hesse, B. and Perkins, D., Jr. 1974. Faunal Remains from Karatå̊Ÿ-Semayuk in Southwest Anatolia: An Interim Report. Journal of Field Archaeology 1 (1/2): 149-160.

Higham, T. F. G. and Horn, P. L. 2000. Seasonal Dating Using Fish Otoliths: Results from the Shag River Mouth Site, New Zealand. Journal of Archaeological Science 27: 439-448.

Hine, P. 2008. Stone-walled tidal fish traps: an archaeological and archival investigation, M.Phil., University of Cape Town.

Hine, P., Sealy, J., Halkett, D. and Hart, T. 2010. Antiquity of stone-walled tidal fish traps on the Cape coast, South Africa South African Archaeological Bulletin 65 (191): $35-44$.

Hlinka, V., Ulm, S., Loy, T. and Hall, J. 2002. The genetic speciation of archaeological fish bone: a feasibility study from southeast Queensland. Queensland Archaeological Research 13: 71.

Hoffman, A. C. 1958. New excavations in the Matjes River Rock Shelter. SAMAB 6 (13): 342-348.

Höglund, H. G. 1972. On the Bohuslän Herring During the Great Herring Fishery Period in the Eighteenth Century. Lund: C. Bloms Boktryckeri.

Hornell, J. 1941. 87. Fishing-Poisons. Man 41: 126-128. 
Horton, D. R. 1984. Minimum numbers: a consideration. Journal of Archaeological Science 11: 255-271.

Horwitz, L. K. 1990. The Origin of Partially Digested Bones Recovered from Archaeological Contexts in Israel. Paleorient 16: 97-106.

Howard, H. 1930. A Census of the Pleistocene Birds of Rancho La Brea from the Collections of the Los Angeles Museum. The Condor 32 (2): 81-88.

Howes, F. N. 1930. Fish-poison plants. Bulletin of Miscellaneous Information (Royal Gardens, Kew) 1930 (4): 129-153.

Hu, Y., Shang, H., Tong, H., Nehlich, O., Liu, W., Zhao, C., Yu, J., Wang, C., Trinkaus, E. and Richards, M. P. 2009. Stable isotope dietary analysis of the Tianyuan 1 early modern human. Proceedings of the National Academy of Sciences 106 (27): 10971-10974.

Hufthammer, A. K., Høie, H., Folkvord, A., Geffen, A. J., Andersson, C. and Ninnemann, U. S. 2010. Seasonality of human site occupation based on stable oxygen isotope ratios of cod otoliths. Journal of Archaeological Science 37 (1): 78-83.

Humphreys, A. J. B. 1974. A Preliminary Report on Test Excavations at Dikbosch Shelter I, Herbert District, Northern Cape. The South African Archaeological Bulletin 29 (115/116): 115-119.

Inskeep, R. R. 1972. Nelson's Bay Cave, Robberg Peninsula, Plettenberg Bay. Palaeoecology of Africa \& of the surrounding islands \& Antarctica: 247.

Inskeep, R. R. 1987. Nelson Bay Cave, Cape Province, South Africa: the Holocene levels: BAR.

Inskeep, R. R. and Vogel, J. C. 1985. Radiocarbon Dates from the Holocene Levels at Nelson Bay Cave, and an Interim Report on Their Associations. The South African Archaeological Bulletin 40 (142): 103-105.

Jacobs, Z., Duller, G. A. T., Wintle, A. G. and Henshilwood, C. S. 2006. Extending the chronology of deposits at Blombos Cave, South Africa, back to $140 \mathrm{ka}$ using optical dating of single grains of quartz. Journal of Human Evolution 51: 255 273.

Jacobs, Z., Roberts, R. G., Galbraith, R. F., Deacon, H. J., Grun, R., Mackay, A., Mitchell, P., Vogelsang, R. and Wadley, L. 2008. Ages for the Middle Stone Age of Southern Africa: Implications for Human Behavior and Dispersal. Science 322 (5902): 733-735.

Jerardino, A. 1993. Mid- to Late Holocene sea-level fluctuations: the archaeological evidence at Tortoise Cave, southwestern Cape, South Africa. The Holocene 5 (3): 361-368.

Jerardino, A. 1996. Changing social landscapes of the western Cape coast of southern Africa over the last 4500 years, $\mathrm{PhD}$, University of Cape Town.

Jerardino, A. 1998. Excavations at Pancho's Kitchen Midden, Western Cape Coast, South Africa: Further Observations into the Megamidden Period. The South African Archaeological Bulletin 53 (167): 16-25.

Jerardino, A. 2003. Pre-Colonial Settlement and Subsistence along Sandy Shores South of Elands Bay, West Coast, South Africa. The South African Archaeological Bulletin 58 (178): 53-62.

Jerardino, A. 2010. Large shell middens in Lamberts Bay, South Africa: a case of huntergatherer resource intensification. Journal of Archaeological Science 37 (9): 22912302. 
Jerardino, A. and Yates, R. 1996. Preliminary results from excavations at Steenbokfontein Cave: implications for past and future research. The South African Archaeological Bulletin 51 (163): 7-16.

Jerardino, A. and Yates, R. 1997. Excavations at Mike Taylor's Midden: A Summary Report and Implications for a Re-Characterisation of Megamiddens. The South African Archaeological Bulletin 52 (165): 43-51.

Jerardino, A., Dewar, G. and Navarro, R. 2009. Opportunistic Subsistence Strategies among Late Holocene Coastal Hunter-Gatherers, Elands Bay, South Africa. The Journal of Island and Coastal Archaeology 4 (1): 37-60.

Jones, A. K. G. 1984. "Some effects of the mammalian digestive system on fish bones." 2nd Fish Osteoarchaeology Meeting, Paris, 1984, pp. 61-65 16.

Jones, A. K. G. 1986. "Fish bone survival in the digestive systems of the pig, dog and man: some experiments," in Fish and Archaeology: Studies in Osteometry, Taphonomy, Seasonality and Fishing Methods, vol. 294. Edited by Brinkhuizen, D. C. and Clason, A. T., pp. 53-61. Oxford: British Archaeological Reports International Series.

Jones, A. K. G. 1999. Walking the cod: an investigation into the relative robustness of cod, Gadus morhua, skeletal elements. Internet Archaeology 7.

Joordens, J. C. A., Kuipers, R. S. and Muskiet, F. A. J. 2007. Preformed dietary DHA: The answer to a scientific question may in practice become translated to its opposite. American Journal of Human Biology 19 (4): 582-584.

Joordens, J. C. A., Wesselingh, F. P., De Vos, J., Vonhof, H. B. and Kroon, D. 2009. Relevance of aquatic environments for hominins: a case study from Trinil (Java, Indonesia). Journal of Human Evolution 57 (6): 656-671.

Jousse, H., Obermaier, H., Raimbault, M. and Peters, J. 2008. Late Holocene economic specialisation through aquatic resource exploitation at Kobadi in the Méma, Mali. International Journal of Osteoarchaeology 18 (6): 549-572.

Jurgens, C. J. 2005. Zooarcheology and bone technology from Arenosa Shelter (41 VV99), Lower Pecos region, Texas, Ph.D, The University of Texas at Austin.

Jurgens, C. J. 2008. The fish fauna from Arenosa Shelter (41VV99), Lower Pecos region, Texas. Quaternary International 185 (1): 26-33.

Kemp, L. 2006. Ancient stonewall fish traps on the south coast of South Africa: Documentation, current use, ecological effects and management implications, MSc, University of Cape Town.

Kemp, L. V., Branch, G. M., Attwood, C. A. and Lamberth, S. J. 2009. The'fishery'in South Africa's remaining coastal stonewall fish traps. African Journal of Marine Science 31 (1): 55-62.

Kemper, J., Roux, J. P., Bartlett, P. A., Chesselet, Y. J., Delport, J. A., James, J. A. C., Jones, R., Underhill, L. G., Uhongora, N. N. and Wepener, S. 2007. The African penguin Spheniscus demersus: population estimates, trends, adult survival and age structure from moult and nest counts. Final report of the BCLME (Benguela Current Large Marine Ecosystem) project on top predators as biological indicators of ecosystem change in the BCLME. Cape Town: Avian Demography Unit, University of Cape Town: 69-76.

Kerwath, S. E. 2006. Empirical studies of fish movement behaviour and their application in spatially explicit models for marine conservation, $\mathrm{PhD}$, Rhodes University. 
Kerwath, S. E., Gotz, A., Attwood, C. G., Sauer, W. H. H. and Wilke, C. G. 2007. Area utilisation and activity patterns of roman Chrysoblephus laticeps (Sparidae) in a small marine protected area. African Journal of Marine Science 29: 259-270.

Kirby, J. S., Holmes, J. S. and Sellers, R. M. 1996. Cormorants Phalacrocorax carbo as fish predators: An appraisal of their conservation and management in Great Britain. Biological Conservation 75 (2): 191-199.

Klein, R. G. 1972a. Preliminary report on the July through September 1970 excavations at Nelson Bay Cave, Plettenberg Bay (Cape Province, South Africa). Palaeoecology of Africa and of the Surrounding Islands and Antarctic 6: 177-208.

Klein, R. G. 1972b. The late Quaternary mammalian fauna of Nelson Bay Cave (Cape Province, South Africa): its implications for megafaunal extinctions and environmental and cultural change. Quaternary Research 2: 135-142.

Klein, R. G. 1974. Environment and subsistence of prehistoric man in the southern Cape Province, South Africa. World Archaeology 5 (3): 249-284.

Klein, R. G. 1975. Middle Stone Age man-animal relationships in southern Africa: evidence from Die Kelders and Klasies River Mouth. Science 190: 265-267.

Klein, R. G. 1976. The mammalian fauna of the Klasies River Mouth sites, southern Cape Province, South Africa. South African Archaeological Bulletin 31: 75-98.

Klein, R. G. 1977a. The Ecology of Early Man in Southern Africa. Science 197 (4299): 115-126.

Klein, R. G. 1977b. The mammalian fauna from the Middle and Later Stone Age (Later Pleistocene) levels of Border Cave, Natal Province, South Africa. South African Archaeological Bulletin 32: 14-27.

Klein, R. G. 1983. The Stone Age Prehistory of Southern Africa. Annual Review of Anthropology 12: 25-48.

Klein, R. G. 1986. The prehistory of Stone Age herders in the Cape Province of South Africa. South African Archaeological Society Goodwin Series 5: 5-12.

Klein, R. G. 1988. "Biological and behavioural perspectives on modern human origins in southern Africa," in The human revolution: behavioural and biological perspectives on the origins of modern humans. Edited by Mellars, P. and Stringer, C. B., pp. 529-546. Edinburgh: Edinburgh University Press.

Klein, R. G. 1989a. "Biological and behavioural perpesctives on modern human origins in southern Africa," in The Human Revolution: Behavioural and Biological Perspectives on the Origins of Modern Humans. Edited by Mellars, P. and Stringer, C., pp. 529-546. Edinburgh: Edinburgh University Press.

Klein, R. G. 1989b. The Human Career: Human Biological and Cultural Origins. Chicago: Chicago University Press.

Klein, R. G. 1992. The archaeology of modern human origins. Evolutionary Anthropology 1: 5-14.

Klein, R. G. 1994. "The problems of modern human origins," in Origins of anatomically modern humans. Edited by Nitecki, M. and Nitecki, D., pp. 3-17. New York: Plenum Press.

Klein, R. G. 1995. Anatomy, Behaviour and Modern Human Origins. Journal of World Prehistory 9 (2): 167-197.

Klein, R. G. 1998. "Why anatomically modern people did not disperse from Africa 100 000 years ago," in Neanderthals and Modern Humans in Western Asia. Edited by Akawaza, T., Aoki, K. and Bar-Yosef, O., pp. 509-521. New York: Plenum Press. 
Klein, R. G. 1999a. The human career: human biological and cultural origins, 2 edition. Chicago: University of Chicago Press.

Klein, R. G. 1999b. "The Evolution of Human Behavior."

Klein, R. G. 2000. Archaeology and the Evolution of Human Behaviour. Evolutionary Anthropology 9 (1): 17-36.

Klein, R. G. 2001. Southern Africa and modern human origins. Journal of Anthropological Research 57 (1): 1-16.

Klein, R. G. 2008. Out of Africa and the evolution of human behavior. Evolutionary Anthropology: Issues, News, and Reviews 17 (6): 267-281.

Klein, R. G. and Cruz-Uribe, K. 1983. Stone Age Population Numbers and Average Tortoise Size at Byneskranskop Cave 1 and Die Kelders Cave 1, Southern Cape Province, South Africa. The South African Archaeological Bulletin 38 (137): 2630.

Klein, R. G. and Cruz-Uribe, K. 1984. The Analysis of Animal Bones from Archaeological Sites. Chicago: Chicago University Press.

Klein, R. G. and Cruz-Uribe, K. 1987. "Large mammal and tortoise bones from Eland's Bay Cave and nearby sites, western Cape Province, South Africa," in Papers in the prehistory of the Western Cape, South Africa. Edited by Parkington, J. E. and Hall, M., pp. 132-163.

Klein, R. G. and Cruz-Uribe, K. 1996. Exploitation of large bovids and seals at Middle and Later Stone Age sites in South Africa. Journal of Human Evolution 31: 315334.

Klein, R. G. and Cruz-Uribe, K. 2000a. Middle and Later Stone Age large mammal and tortoise remains from Die Kelders Cave 1, Western Cape Province, South Africa. Journal of Human Evolution 38 (1): 169-195.

Klein, R. G. and Cruz-Uribe, K. 2000b. Middle and Later Stone Age large mammal and tortoise remains from Die Kelders Cave 1, Western Cape Province, South Africa. Journal of Human Evolution 38: 168-195.

Klein, R. G. and Edgar, B. 2002. The dawn of human culture. New York: John Wiley \& Sons.

Klein, R. G. and Steele, T. E. 2008. Gibraltar data are too sparse to inform on Neanderthal exploitation of coastal resources. Proceedings of the National Academy of Sciences 105 (51): E115.

Klein, R. G., Cruz-Uribe, K., Halkett, D., Hart, T. and Parkington, J. E. 1999a. Paleoenvironmental and Human Behavioral Implications of the Boegoeberg 1 Late Pleistocene Hyena Den, Northern Cape Province, South Africa. Quaternary Research 52 (3): 393-403.

Klein, R. G., Cruz-Uribe, K., Halkett, D., Hart, T. and Parkington, J. E. 1999 b. Paleoenvironmental and Human Behavioural Implications of the Boegoeberg 1 Late Pleistocene Hyena Den, Northern Cape Province, South Africa. Quaternary Research 52: 393-403.

Klein, R. G., Avery, G., Cruz-Uribe, K., Halkett, D., Parkington, J. E., Steele, T., Volman, T. P. and Yates, R. 2004. The Ysterfontein 1 Middle Stone Age site, South Africa, and early human exploitation of coastal resources. Proceedings of the National Academy of Sciences of the United States of America (PNAS) 101 (16): 5708-5715.

Knight, C., Power, C. and Watts, I. 1995. The Human Symbolic Revolution: A Darwinian account. Cambridge Archaeological Journal 5 (1): 75-114.

Kolbe, P. 1738. The present state of the Cape of Good-Hope. London: W. Innys. 
Krantz, G. S. 1968. A New Method of Counting Mammal Bones. American Journal of Archaeology 72 (3): 286-288.

Kuang-Ti, L. 2001. Prehistoric Marine Fishing Adaptation in Southern Taiwan. Journal of East Asian Archaeology 3: 47-74.

Kuhn, B. F., Wiesel, I. and Skinner, J. D. 2008. Diet of brown hyaenas (Parahyaena brunnea) on the Namibian coast. Transactions of the Royal Society of South Africa 63: $150-159$.

Kyriacou, K. 2009. The reinvestigation of Hoffman's/Robberg Cave - the artefactual and shellfish assemblages, 2007, M. Phil., University of Cape Town.

Kyriacou, K. and Sealy, J. 2009. The archaeological assemblage from the 1958 excavation of Hoffman's/Robberg Cave and a comparison with Nelson Bay Cave. Navorsinge van die Nasionale Museum, Bloemfontein 25 (2): 49-72.

Lam, Y. M. 1992. Variability in the behaviour of spotted hyaenas as taphonomic agents. Journal of Archaeological Science 19: 389-406.

Lam, Y. M. and Pearson, O. M. 2005. Bone density studies and the interpretation of the faunal record. Evolutionary Anthropology: Issues, News, and Reviews 14 (3).

Lamberth, S. J., Bennett, B. A. and Clark, B. M. 1994. Catch composition of the commercial beach-seine fishery in False Bay, South Africa. South African Journal of Marine Science 14: 69-78.

Lamberth, S. J., Clark, B. M. and Bennett, B. A. 1995. Seasonality of beach-seine catches in False Bay, South Africa, and implications for management. South African Journal of Marine Science 15: 157-167.

Landon, D. B. 2005. Zooarchaeology and Historical Archaeology: Progress and Prospects. Journal of Archaeological Method and Theory 12 (1): 1-36.

Langdon, J. H. 2006. Has an aquatic diet been necessary for hominin brain evolution and functional development?, British Journal of Nutrition 96 (01): 7-17.

Leach, B. F. and Boocock, A. 1995. Estimating live fish catches from archaeological bone fragments of snapper. Pagrus auratus. Tuhinga: Records of the Museum of New Zealand Te papa Tongarewa 3: 1-28.

Leach, B. F., Intoh, M. and Smith, I. W. G. 1984. Fishing, Turtle Hunting, and Mammal Exploitation at Fa'ahia, Huanine, French Polynesia. Journal de la Société des Océanistes 40 (79): 183-197.

Leach, B. F., Davidson, J. M. and Horwood, L. M. 1997. The estimation of live fish size from archaeological cranial bones of the New Zealand blue cod Parapercis colias. International Journal of Osteoarchaeology 7 (5): 481-496.

Leach, F. 1986. A Method for the Analysis of Pacific Island Fishbone Assemblages and an Associated Database Management System. Journal of Archaeological Science 13: $147-159$.

Leach, F. and Davidson, J. 2001. The use of size-frequency diagrams to characterise prehistoric fish catches and to assess human impact on inshore fisheries. International Journal of Osteoarchaeology 11 (1-2): 150-162.

Lechanteur, Y. 2004. Spatial distribution, relative abundance and size distribution of suprabenthic linefish species present over subtidal reef along the Cape Peninsula National Park coastline. Center for Marine Studies, University of Cape Town.

Lee, D. N. and Woodhouse, H. C. 1970. Art on the rocks of Southern Africa.Cape Town Purnell .

Lepiksaar, J. 1983 Osteologia I. Pisces. Privately distributed, Göteborg 
Lepiksaar, J. 1986. Tierreste in einer Romischen Amphore aus Salzburg (Mozartplatz 4)." Bayerische Vorgeschichtsblatter 51: 163-85.

Lepiksaar, J. and Heinrich, D. 1977. Untersuchungen an Fischresten aus der frühmittelalterlichen Siedlung Haithabu. Neumünster: Karl Wachholtz Verlag

Lichtenstein, H. 1930. Travels in Southern Africa in the Years 1803, 1804, 1805, and 1806. 2 vols. Cape Town: van Riebeeck Society.

Linn, I. J. and Campbell, K. L. I. 1992. Interactions Between White-Breasted Cormorants Phalacrocorax carbo (Aves: Phalacrocoracidae) and the Fisheries of Lake Malawi. Journal of Applied Ecology 29 (3): 619-634.

Lombard, M. 2005. Evidence of hunting and hafting during the Middle Stone Age at Sibidu Cave, KwaZulu-Natal, South Africa: a multianalytical approach. Journal of Human Evolution 48 (3): 279-300.

Lombard, M. 2006a. First impressions of the functions and hafting technology of Still Bay pointed artefacts from Sibudu Cave. Southern African Humanities 18 (1): 27-41.

Lombard, M. 2006b. Direct evidence for the use of ochre in the hafting technology of Middle Stone Age tools from Sibudu Cave. Southern African Humanities 18 (1): 57-67.

Lombard, M. 2007a. The gripping nature of ochre: The association of ochre with Howiesons Poort adhesives and Later Stone Age mastics from South Africa. Journal of Human Evolution 53 (4): 406-419.

Lombard, M. 2007b. Evidence for change in Middle Stone Age hunting behaviour at Blombos Cave: results of a macrofracture analysis. South African Archaeological Bulletin 62 (185): 62-67.

Lombard, M. and Phillipson, L. 2010. Indications of bow and stone-tipped arrow use 64 000 years ago in KwaZulu-Natal, South Africa. Antiquity 84: 635-648.

Louw, J. T. 1960. Prehistory of the Matjes River rock shelter. Bloemfontein: Memoirs of the National Museum 1.

Lubinski, P. 1996. Fish heads, fish heads: an experiment on differential bone preservation in a salmonid fish. Journal of Archaeological Science 23 (2): 175-181.

Lucas, C. 1998. A medieval fishery on Whittlesea Mere, Cambridgeshire. Medieval Archaeology 42: 19-40.

Ludwig, B. 2005. A comparison of hunter-gatherer material culture from Matjes River Rock Shelter and Nelson Bay Cave, M.Sc, University of Cape Town.

Luff, R. M. and Bailey, G. N. 2000. Analysis of Size Changes and Incremental Growth Structures in African Catfish Synodontis schall (schall) from Tell el-Amarna, Middle Egypt. Journal of Archaeological Science 27: 821-835.

Lupo, K. D. and O'connell, J. F. 2002. Cut and tooth mark distributions on large animal bones: ethnoarchaeological data from the Hadza and their implications for current ideas about early human carnivory. Journal of Archaeological Science 29 (1): 85109.

Lutjeharms, J. R. E., Cooper, J. and Roberts, M. 2000. Upwelling at the inshore edge of the Agulhas Current. Continental Shelf Research 20 (7): 737-761.

Lyman, R. L. 1982. Nomenclature in Faunal Studies: A Response to Olsen and Olsen. American Antiquity 47 (1): 179-180.

Lyman, R. L. 1986. On the Analysis and Interpretation of Species List Data in Zooarchaeology. Journal of Ethnobiology 6: 67-81.

Lyman, R. L. 1994a. Vertebrate taphonomy. Cambridge: Cambridge University Press. 
Lyman, R. L. 1994b. Quantitative units and terminology in zooarchaeology. American Antiquity: 36-71.

Lyman, R. L. 2006. Identifying bilateral pairs of deer (Odocoileus sp.) bones: how symmetrical is symmetrical enough? , Journal of Archaeological Science 33 (9): 1256-1265.

Lyman, R. L. 2008. Quantitative paleozoology. Cambridge: Cambridge University Press.

Lyman, R. L., Power, E. and Lyman, R. J. 2003. Quantification and sampling of faunal remains in owl pellets. Journal of Taphonomy 1 (1): 3-14.

Mackay, A. and Welz, A. 2008. Engraved ochre from a Middle Stone Age context at Klein Kliphuis in the Western Cape of South Africa. Journal of Archaeological Science 35 (6): 1521-1532.

Maclean, G. L. 1993. Roberts' Birds of Southern Africa, 6 edition. Cape Town: The Trustees of the John Voelcker Bird Book Fund.

Maggs, T. and Speed, E. 1967. Bonteberg Shelter. S.A. Archaelogical Bulletin 22: 90-91.

Malan, B. D. 1955. The Archaeology of Tunnel Cave and Skildergat Kop, Fish Hoek, Cape of Good Hope. South African Archaeology Bulletin 10: 3-9.

Mallory, J. P., Woodman, P. C., Voss, P., van Wijngaarden-Bakker, L., Ross, H. and Monk, M. 1984. Oughtymore: An Early Christian Shell Midden. Ulster Journal of Archaeology 47: 51-62.

Manhire, A. 1993. A report on the excavations of Faraoskop rock- shelter in the Graafwater District of the south-western Cape., Southern African Field Archaeology 2: 2-23.

Manhire, A., Parkington, J. and Yates, R. 1985. Nets and fully recurved bows: Rock painting and hunting methods in the Western Cape, South Africa. World Archaeology 17: 161-173.

Mann, B. Q. Editor. 2000. Southern African marine linefish status reports. Vol. Special Publication No 7. Cape Town: South African Association for Marine Biological Research.

Marean, C. W. 1985. The Faunal Remains from Smitswinkelbaai Cave, Cape Peninsula. The South African Archaeological Bulletin 40 (142): 100-102.

Marean, C. W. 1998. A critique of the evidence for scavenging by Neanderthals and Early Modern Humans: New data from Kobeh Cave (Zagros mountains, Iran) and Die Kelders Cave1 layer 10 (South Africa). Journal of Human Evolution 34: 000-000.

Marean, C. W. and Spencer, L. M. 1991. Impact of carnivore ravaging on zooarchaeological measures of element abundance. American Antiquity 56 (4): 645-658.

Marean, C. W. and Frey, C. J. 1997. Animal bones from caves to cities: Reverse utility curves as methodological artefacts. American Antiquity 62 (4): 698-711.

Marean, C. W. and Kim, S. J. 1998. Mousterian Large-mammal remains from Kobeh cabe - Behavioural implications for Neanderthals and Early Modern Humans. Current Anthropology 39: S79-S113.

Marean, C. W. and Assefa, Z. 1999. Zooarcheological evidence for the faunal exploitation behavior of Neandertals and early modern humans. Evolutionary Anthropology: Issues, News, and Reviews 8 (1): 22-37.

Marean, C. W. and Assefa, Z. 2005. "The Middle and Upper Pleistocene African record for the biological and behavioral origins of modern humans," in African Archaeology: A Critical Introduction Edited by Stahl, A. B., pp. 93-129. Malden (MA): Blackwell 
Marean, C. W., Dominguez-Rodrigo, M. and Pickering, T. R. 2004a. Skeletal element equifinality in zooarchaeology begins with method: the evolution and status of the "shaft critique"'. Journal of Taphonomy 2 (2): 69-98.

Marean, C. W., Abe, Y. A., Frey, C. J. and Randall, R. 2000a. Zooarchaeological and taphonomic analysis of the Die Kelders Cave 1 Layers 10 and 11 Middle Stone Age larger mammal fauna. Journal of Human Evolution 38: 197-233.

Marean, C. W., Abe, Y., Nilssen, P. J. and Stone, E. C. 2001. Estimating the minimum number of skeletal elements (MNE) in zooarchaeology: a review and a new imageanalysis GIS approach. American antiquity: 333-348.

Marean, C. W., Goldberg, P., Avery, G., E, G. F. and Klein, R. G. 2000b. Middle Stone Age stratighraphy and excavations at Die Kelders Cave 1 (Western Cape Province, South Africa): the 1992, 1993, and 1995 field seasons. Journal of Human Evolution 38: 7-42.

Marean, C. W., Nilssen, P. J., Brown, K., Jerardino, A. and Stynder, D. 2004 b. Paleoanthropological investigations of Middle Stone Age sites at Pinnacle Point, Mossel Bay (South Africa): archaeology and hominid remains from the 2000 field season. Paleoanthropology 2: 14-83.

Marean, C. W., Bar-Matthews, M., Bernatchez, J., Fisher, E., Goldberg, P., Herries, A. I. R., Jacobs, Z., Jerardino, A., Karkanas, P. and Minichillo, T. 2007. Early human use of marine resources and pigment in South Africa during the Middle Pleistocene. Nature 449 (7164): 905-908.

Marks, M. M., Brooke, R. K. and Gildenhuys, A. M. 1997. Cape fur seal Arctocephalus pusillus predation on Cape cormorants Phalacrocorax capensis and other birds at Dyer Island, South Africa. Marine Ornithology 25: 9-12.

Marshall, F. and Pilgram, T. 1993. NISP vs. MNI in quantification of body-part representation. American Antiquity 58 (2): 261-269.

Matisoo-Smith, E. 2009. The Commensal Model for Human Settlement of the Pacific 10 Years on - What Can We Say and Where to Now?, The Journal of Island and Coastal Archaeology 4 (2): 151 - 163.

Matthews, T., Marean, C. and Nilssen, P. 2009. Micromammals from the Middle Stone Age (92-167 ka) at Cave PP13B, Pinnacle Point, south coast, South Africa. Palaeontologia Africana 44 (December): 112-120.

Mazel, A.D. 1989. People making history: the last ten thousand years of hunter-gatherer communities in the Thukela Basin. Natal Museum Journal of Humanities 1:1-168.

Mazel, A. and Parkington, J. 1981. Stone tools and resources: a case study from southern Africa. World Archaeology: 16-30.

Mcbrearty, S. 1990. The Origin of Modern Humans. Man [N.S.] 25: 129-43.

Mcbrearty, S. and Brooks, A. 2000. The revolution that wasn't: a new interpretation of the origin of modern human behavior. Journal of Human Evolution 38: 000-000.

Mckechnie, I. 2005. Five thousand years of fishing at a shell midden in the broken group islands, Barkley Sound, British Columbia, Simon Fraser University.

Mellars, P. 1989. Major issues in the emergence of modern humans. Current Anthropology 30 (3): 349-385.

Mellars, P. 1991. Cognitive changes and the emergence of modern humans in Europe. Cambridge Archaeological Journal 1: 63-76.

Mellars, P. 1996. "Symbolism, Language and the Neanderthal mind," in Modelling the Early Human Mind, McDonald Institute Monographs. Edited by Mellars, P. and Gibson, K. London: Oxbow Books. 
Mellars, P. 2005. The Impossible Coincidence: A single species model for the origins of modern human behaviour in Europe. Evolutionary Anthropology 14: 12-27.

Mellars, P. 2006. Why did modern human populations disperse from Africa ca. 60,000 years ago? A new model. PNAS 103 (no 25 June 20, 2006): 9381 - 9386.

Mery, S., Charpentier, V. and Beech, M. 2008. First evidence of shell fish-hook technology in the Gulf. Arabian Archaeology and Epigraphy 19 (1): 15-21.

Miller, M. J., Capriles, J. M. and Hastorf, C. A. 2010. The fish of Lake Titicaca: implications for archaeology and changing ecology through stable isotope analysis. Journal of Archaeological Science 37 (2): 317-327.

Milliken, S. 2007. Neanderthals, Anatomically Modern Humans, and 'Modern Human Behaviour' in Italy. Oxford Journal of Archaeology 26 (4): 331-358.

Milner, N., Craig, O. E., Bailey, G. N., Pedersen, K. and Andersen, S. H. 2004. Something fishy in the Neolithic? A re-evaluation of stable isotope analysis of Mesolithic and Neolithic coastal populations. Antiquity 78 (299): 9-22.

Milo, R. G. 1998. Evidence for Hominid Predation at Klasies River Mouth, South Africa, and its implications for the behaviour of Early Modern Humans. Journal of Archaeological Science 25: 99-133.

Minichillo, T. 2005. Middle Stone Age lithic study, South Africa: an examination of modern human origins, Ph.D, University of Washington.

Morales, A. and Rosenlund, K. 1979. Fish bone measurements: an attempt to standardize the measuring of fish bones from archaeological sites. Copenhagen: Steenstrupia.

Moreno-Garcia, M., Orton, C. and Rackham, J. 1996. A new statistical tool for comparing animal bone assemblages. Journal of archaeological science 23: 437-454.

Morlan, R. E. 1994. Bison Bone Fragmentation and Survivorship: a Comparative Method. Journal of Archaeological Science 21 (6): 797-807.

Morris, E. J. 1938. Primitive Fish-Hooks. The Scientific Monthly 47 (3): 258-261.

Mourre, V., Villa, P. and Henshilwood, C. S. Early Use of Pressure Flaking on Lithic Artifacts at Blombos Cave, South Africa. Science 330 (6004): 659.

Movius, H. L., Jr. 1950. A Wooden Spear of Third Interglacial Age from Lower Saxony. Southwestern Journal of Anthropology 6 (2): 139-142.

Nabo. http://www.nabohome.org/

Nabone. http://www.nabohome.org/products/manuals/fishbone/index.html

Nel, J. A. J. and Somers, M. J. 2007. Distribution and habitat choice of Cape clawless otters, Aonyx capensis, in South Africa. South African Journal of Wildlife Research 37 (1): 61-70.

Neuwinger, H. D. 2004. Plants used for poison fishing in tropical Africa. Toxicon 44: 417430.

Nicholls, A., Matisoo-Smith, E. and Allen, M. S. 2003. A novel application of molecular techniques to pacific archaeofish remains. Archaeometry 45 (1): 133-147.

Nicholson, R. A. 1992a. An assessment of the value of bone density measurements to archaeoichthyological studies. International Journal of Osteoarchaeology 2 (2): 139-154.

Nicholson, R. A. 1992b. Bone survival: The effects of sedimentary abrasion and trampling on fresh and cooked bone. International Journal of Osteoarchaeology 2 (1): 79-90.

Nicholson, R. A. 1993a. A Morphological Investigation of Burnt Animal Bone and an Evaluation of its Utility in Archaeology. Journal of Archaeological Science 20 (4): 411-428. 
Nicholson, R. A. 1993b. An investigation into the effects on fish bone of passage through the human gut: some experiments and comparisons with archaeological material. Circaea 10 (1): 38-51.

Nicholson, R. A. 1995. Out of the frying pan into the fire: what value are burnt fish bones to archaeology? , Archaeofauna (4): 47.

Nicholson, R. A. 1996. Bone degradation, burial medium and species representation: debunking the myths, an experiment-based approach. Journal of Archaeological Science 23 (4): 513-533.

Nicholson, R. A. 1998. Bone degradation in a compost heap. Journal of Archaeological Science 25 (5): 393-403.

Noah, A. C. 1998. Prehistoric Fishing on the San Diego Coast. . Pacific Coast Archaeological Society Quarterly 34 (2): 5-31.

Noble, J. 1875. Descriptive handbook of the Cape Colony: its condition and resources. Cape Town: Juta.

Norton, C. J., Kim, B. and Bae, K. 1999. Differential processing of fish during the Korean Neolithic: Konam-ri. Arctic Anthropology 36 (1/2): 151-165.

O'Connor, T. P. 1996. A critical overview of archaeological animal bone studies. World Archaeology 28 (1): 5-19.

O'Connor, T. P. 2000. The archaeology of animal bones. Texas: Texas A \& M University Press.

O'Connor, T. P. 2001. "Collecting, sieving, and animal bone quantification," in Animals and Man in the Past, vol. 41, pp. 7-16. Groningen: ARC Publicatie.

O'Connor, T. and Barrett, J. 2006. "Animal bones," in Archaeology in Practice: A Student Guide to Archaeological Analyses. Edited by Balme, J. and Paterson, A., pp. 260295. Oxford: Blackwell.

Obomanu, V. G., Ogbalu, O. K., Gabriel, U. U., Fekarurhobo, S. G. K. and Abadi, S. U. 2007. Piscicidal Effects of Lepidagathis alopecuroides on Mudskipper. Research Jourmal of Applied Sciences 2 (4): 382-387.

Olsen, S. J. 1968. Fish, amphibian and reptile remains from archaeological sites: Part 1: Southeastern and Southwestern United States; Appendix: The Osteology of the Wild Turkey. Vol. 56. Cambridge, Massachusetts: Papers of the Peabody Museum of Archaeology and Ethnology.

Olsen, S. J. 1971. Zooarchaeology: Animal Bones in Archaeology and their Interpretation. Reading, Massachusetts: Addison-Wesley Modular Publications.

Olsen, S. L. and Olsen, J. W. 1981. A Comment on Nomenclature in Faunal Studies. American Antiquity 46 (1): 192-194.

Olsen, S. L. and Shipman, P. 1988. Surface modification on bone: Trampling versus butchery. Journal of Archaeological Science 15 (5): 535-553.

Olson, C., Limburg, K. and Söderblom, M. 2008. Stone Age fishhooks-how were they dimensioned? Morphology, strength test, and breakage pattern of Neolithic bone fishhooks from Ajvide, Gotland, Sweden. Journal of Archaeological Science 35 (10): 2813-2823.

Opačak, A., Florijančić, T., Horvat, D., Ozimec, S. and Bodakoš, D. 2004. Diet spectrum of great cormorants (Phalacrocorax carbo sinensis L.) at the Donji Miholjac carp fishponds in eastern Croatia. European Journal of Wildlife Research 50 (4): 173178. 
Orchard, T. J. 2003. An application of the linear regression technique for determining length and weight of six fish taxa: the role of selected fish species in Aleut paleodiet. Vol. 1172: British Archaeological Reports International Series.

Orchard, T. J. 2005. The use of statistical size estimations in minimum number calculations. International Journal of Osteoarchaeology 15 (5).

Orton, J. and Halkett, D. 2007. Excavations at Noetzie Midden: an open site on the Cape south coast. . The Digging Stick 24 (3): 5-7.

Outram, A. K. 2001. A New Approach to Identifying Bone Marrow and Grease Exploitation: Why the "Indeterminate", Fragments should not be ignored. Journal of Archaeological Science 28 (4): 401-410.

Outram, A. K., Knüsel, C. J., Knight, S. and Harding, A. F. 2005. Understanding complex fragmented assemblages of human and animal remains: a fully integrated approach. Journal of Archaeological Science 32 (12): 1699-1710.

Ouzman, S. 1995. The fish, the shaman and the peregrination: San rock paintings of mormyrid fish as religious and social metaphors. Southern African Field Archaeology 4: 3-17.

Pappe, L. 1866. Synopsis of the edible fishes at the Cape of Good Hope, 2d edition. Cape Town: W. Brittain.

Parker, D. M., Burchell, R. K. and Bernard, R. T. F. 2005. The diet of Cape clawless otters at two sites along the Bloukrans River, Eastern Cape Province, South Africa. African Zoology 40 (2): 330.

Parkington, J. E. 1976. Follow the San: an analysis of seasonality in the prehistory of the western Cape, South Africa, Ph.D, University of Cambridge.

Parkington, J. E. 1978. Report on Research in the Olifants River Valley 1977. Nyame Akuma 13: 25-31.

Parkington, J. 1991. Approaches to dietary reconstruction in the western Cape: Are you what you have eaten? Journal of Archaeological Science 18:331-342.

Parkington, J. E. 2001. Presidential Address: Mobility, Seasonality and Southern African Hunter-Gatherers. The South African Archaeological Bulletin 56 (173/174): 1-7.

Parkington, J. E. 2003. Middens and moderns: shellfishing and the middle stone age of the Western Cape, South Africa. South African Journal of Science 99 (5-6): 243-247.

Parkington, J. E. 2006. Shorelines, Strandlopers and shell middens. Cape Town: Creda Communications.

Parkington, J. E. and Poggenpoel, C. 1971. Excavations at De Hangen, 1968. The South African Archaeological Bulletin 26 (101/102): 3-36.

Parkington, J. E. and Poggenpoel, C. 1987. "Diepkloof rock shelter," in Papers in the prehistory of the western Cape, South Africa. Edited by Parkington, J. E. and Hall, M., pp. 269-293.

Parkington, J. E., Poggenpoel, C., Buchanan, B., Robey, T., Manhire, T. and Sealy, J. 1988. Holocene coastal settlement patterns in the western Cape. The archaeology of prehistoric coastlines 22: 41.

Parkington, J. E., Poggenpoel, C., Rigaud, J. P. and Texier, P. J. 2005. From tool to symbol: the behavioural context of intentionally marked ostrich eggshell from Diepkloof, Western Cape. F. d'Errico \& L. Backwell (eds) From tools to symbols: from early hominids to modern humans: 475-492.

Patterson, W. P. 1998. North American continental seasonality during the last millennium: high-resolution analysis of sagittal otoliths. Palaeogeography, Palaeoclimatology, Palaeoecology 138 (1-4): 271-303. 
Patterson, W. P., Smith, G. R. and Lohmann, K. C. 1993. Continental paleothermometry and seasonality using the isotopic composition of aragonitic otoliths of freshwater fishes. Climate change in continental isotopic records 78: 191-202.

Peers, B. 1929. Preliminary report on the archaeology of Fish Hoek - Noord Hoek valley, South African Museum.

Phillipson, D. W. 1970. Notes on the later prehistoric radiocarbon chronology of eastern and southern Africa. Journal of African History 11 (1): 1-15.

Pickering, T. R. and Egeland, C. P. 2006. Experimental patterns of hammerstone percussion damage on bones: implications for inferences of carcass processing by humans. Journal of Archaeological Science 33 (4): 459-469.

Pickering, T. R., Marean, C. W. and Dominguez-Rodrigo, M. 2003. Importance of limb bone shaft fragments in zooarchaeology: a response to "On in situ attrition and vertebrate body part profiles" (2002), by M.C. Stiner. Journal of Archaeological Science 30 (11): 1469-1482.

Pitcher, G. C. and Calder, D. 2000. Harmful algal blooms of the southern Benguela Current: a review and appraisal of monitoring from 1989 to 1997. South African Journal of Marine Science 22 (1): 255 - 271.

Plug, C. and Plug, I. 1990. MNI counts as estimates of species abundance. South African Archaeological Bulletin 45 (151): 53-57.

Plug, I. 1988. Hunters and Herders an Archaeozoological Study of Some Prehistoric Communities in the Kruger National Park, Pretoria.

Plug, I. 1989. Aspects of Life in the Kruger National Park during the Early Iron Age. South African Archaeological Society Goodwin Series 6: 62-68.

Plug, I. 2004. Resource exploitation: animal use during the Middle Stone Age at Sibudu Cave, KwaZulu-Natal. South African journal of science 100 (3-4): 151-158.

Plug, I. 2005. Osteomorphological differences between some skeletal elements of Labeobarbus kimberleyensis, Labeobarbus aeneus and Labeo capensis (Pisces: Cyprinidae). Annals of the Transvaal Museum 42: 5-17.

Plug, I. 2006a. Aquatic animals and their associates from the Middle Stone Age levels at Sibudu. Southern African Humanities 18 (1): 289-299.

Plug, I. 2006b. "The Exploitation of Freshwater Fish during the Later Stone Age of Lesotho: Preliminary Results," in Beyond Affluent Foragers - Rethinking HunterGatherer Complexity, Proceedings of the 9th Conference of the International Council of Archaeozoology, Durham, August 2002. Edited by Grier, C., Jangsuk, K. and Uchiyama, J., pp. 24-33. Oxford: Oxbow Books.

Plug, I. 2008. Fish bone sizes as estimators of standard lengths of three southern African freshwater species with application to archaeological samples: a preliminary investigation. South African Journal of Science 104 (1-2): 71-75.

Plug, I., Mitchell, P. and Bailey, G. 2003. Animal remains from Likoaeng, an open-air river site, and its place in the post-classic Wilton of Lesotho and eastern Free State, South Africa. South African Journal of Science 99: 143-152.

Plug, I., Mitchell, P. and Bailey, G. 2010. Late Holocene fishing strategies in southern Africa as seen from Likoaeng, highland Lesotho. Journal of Archaeological Science In Press, Accepted Manuscript.

Poggenpoel, C. A. 1984. The determination of minimum numbers of individual fish from coastal sites in South Africa. Frontiers: Southern African archaeology today: 36772. 
Poggenpoel, C. A. 1987. "The implications of fish bone assemblages from Eland's Bay Cave, Tortoise cave and Diepkloof rock shelter for changes in the holocene history of the Verlorenvlei."

Poggenpoel, C. A. 1996. The Exploitation of fish during the Holocene in the southwestern Cape, South Africa, MA University of Cape Town.

Poggenpoel, C. A. and Robertshaw, P. T. 1981. The Excavation of Smitswinkelbaai Cave, Cape Peninsula. The South African Archaeological Bulletin 36 (133): 29-35.

Pradervand, P. and Hiseman, R. 2006. An analysis of the recreational shore fishery in the Goukamma Marine Protected Area. African Zoology 41 (2): 275-289.

Pyle, P. 2009. Kolea stones - their features and ethnohistory. Elepaio 69: 25-27.

Quigley, C. 1956. Aboriginal Fish Poisons and the Diffusion Problem. American Anthropologist 58 (3): 508-525.

Radu, V. 2005. Atlas for the identification of bony fish bones from archaeological sites. Vol. Supplementum 1/2005. Asociatia Romana de Arheologie Studii de Preistorie. Bucuresti: Editura Contrast.

Randall, R. M., Tregoning, C., Randall, B. M. and Martin, A. P. 2002. Adaptability of great cormorants Phalacrocorax carbo in a coastal environment demonstrated by their exploitation of introduced prey species and use of artificial breeding sites. South African Journal of Marine Science 24 (1): 317-321.

Raven-Hart, R. 1967. Before van Riebeeck: Callers at South Africa from 1488 to 1652. Cape Town: C. Struik.

Raven-Hart, R. 1971. Cape of Good Hope 1652-1702: The first fifty years of Dutch colonisation as seen by callers. Cape Town: A.A. Balkema.

Reinman, F. M. 1967. "Fishing: An aspect of oceanic economy: An archaeological approach," in Fieldiana: Anthropology, vol. 52, pp. 95-208: Field Museum of Natural History.

Reitz, E. J. and Wing, E. S. 1999. Zooarchaeology Cambridge University Press Cambridge.

Reitz, E. J. and Wing, E. S. 2008. Zooarchaeology, 2nd edition. Cambridge: Cambridge University Press.

Renfrew, C. 1996. "The Sapient Behaviour Paradox: How to test for potential?," in Modelling the Early Human mind. MacDonald Institute Monographs. Edited by Mellars, P. and Gibson, K. Oxford: Oxbow Books.

Richter, J. 1986. Experimental study of heat induced morphological changes in fish bone collagen. Journal of Archaeological Science 13 (5): 477-481.

Rick, T. C. and Erlandson, J. M. 2000. Early Holocene fishing strategies on the California Coast: evidence from CA-SBA-2057. Journal of Archaeological Science 27 (7): 621-633.

Rick, T. C., Jon, M. E. and Vellanoweth, R. L. 2001. Paleocoastal Marine Fishing on the Pacific Coast of the Americas: Perspectives from Daisy Cave, California. American Antiquity 66 (4): 595-613.

Rick, T. C., Erlandson, J. M., Vellanoweth, R. L. and Braje, T. J. 2005. From Pleistocene mariners to complex hunter-gatherers: The archaeology of the California Channel Islands. Journal of World Prehistory 19 (3): 169-228.

Rigaud, J.-P., Texier, P.-J., Parkington, J. and Poggenpoel, C. 2006. Stillbay and Howiesons Poort stone tool techno-complexes. South African Middle Stone Age chronology and its implications., Science Direct Palevol 5: 839 - 849. 
Rightmire, G. P., Deacon, H. J., Schwartz, J. H. and Tattersall, I. 2006. Human foot bones from Klasies River main site, South Africa. Journal of Human Evolution 50 (1): 96-103.

Ringrose, T. J. 1993. Bone counts and statistics: a critique. Journal of Archaeological Science 20: 121-121.

Ritchie, A. 1977. Excavation of Pictish and Viking Age Farmsteads at Buckquoy. Orkney Proceedings of the Society of Antiquaries of Scotland 108: 174-227.

Robbins, L. H., Murphy, M. L., Stewart, K. M., Campbell, A. C. and Brook, G. A. 1994. Barbed Bone Points, Paleoenvironment, and the Antiquity of Fish Exploitation in the Kalahari Desert, Botswana. Journal of Field Archaeology 21 (2): 257-264.

Robbins, L. H., Murphy, M. L., Brook, G. A., Ivester, A. H., Campbell, A. C., Klein, R. G., Milo, R. G., Steward, K. M., Downey, W. S. and Stevens, N. J. 2000. Archaeology, palaeoenvironment, and chronology of the Tsodilo Hills White Paintings Rock Shelter, northwest Kalahari Desert, Botswana. Journal of Archaeological Science 27: 1085-1113.

Robertshaw, P. T. 1977. Excavations at Paternoster, South-Western Cape. The South African Archaeological Bulletin 32 (125): 63-73.

Robertshaw, P. T. 1978. Archaeological investigations at Langebaan Lagoon, Cape Province. Palaeoecology of Africa and the surrounding islands (10): 139-148.

Robertshaw, P. T. 1979. Excavations at Duiker Eiland, Vredenburg District, Cape Province. Annals of the Cape Provincial Museums (Human Sciences) 1 (1): 1-26.

Robey, T. 1984. Burrows and Bedding: Site taphonomy and spatial archaeology at Tortoise Cave, M.A., University of Cape Town.

Robinson, B. S., Jacobson, G. L., Yates, M. G., Spiess, A. E. and Cowie, E. R. 2009. Atlantic salmon, archaeology and climate change in New England. Journal of Archaeological Science 36 (10): 2184-2191.

Rojo, A. L. 1991. Dictionary of Evolutionary Fish Osteology. Florida: CRC Press.

Rowe-Rowe, D. T. 1977. Food Ecology of Otters in Natal, South Africa. Oikos 28 (2/3): 210-219.

Royer, D. F., Lockwood, C. A., Scott, J. E., Grine, F. E. and Feg, L. F. 2009. Size variation in early human mandibles and molars from Klasies River, South Africa: Comparison with other middle and late Pleistocene assemblages and with modern humans. American Journal of Physical Anthropology 140 (2): 312-323.

Rudner, J. and Rudner, I. 1973. A Note on Early Excavations at Robberg. The South African Archaeological Bulletin 28 (111/112): 94-96.

Sadr, K. 2008. Invisible Herders? The Archaeology of Khoekhoe Pastoralists. Southern African Humanities 20 (1): 179-203.

Sadr, K., Smith, A., Plug, I., Orton, J. and Mutti, B. 2003. Herders and foragers on Kasteelberg: interim report of excavations 1999-2002. South African Archaeological Bulletin 58 (177): 27-32.

Sampson, A. 1998. The Neolithic and Mesolithic Occupation of the Cave of Cyclope, Youra, Alonnessos, Greece. The Annual of the British School at Athens, 93: 1-22.

Schapera, I. 1930. The Khoisan peoples of South Africa. Bushmen and Hottentots. London: George Routledge \& Sons.

Schirmer, G. R. 1975. An analysis of lithic material from Dale Rose Parlour, Trappies Kop, Kalk Bay, Cape Peninsula. University of Cape Town Unpublished Archaeology Additional report. 
Schoeman, F. and Schoeman, S. 1990. Strike! The book on Salt water fishing in southern Africa. Cape Town: ABC Press.

Schoute-Vanneck, C. A. and Walsh, R. C. 1959. The Shell Middens at the Ingane River Mouth, Natal South Coast. The South African Archaeological Bulletin 14 (54): 4355.

Schramm, E. 1982. Towards a Rational Nomenclature in Faunal and Ecological Studies. American Antiquity 47 (1): 178-179.

Schrire, C. 1962. Oakhurst: Re-examination and vindication. South African Archaeological Bulletin 17: 181-195.

Schumann, E. H. 1999. Wind-driven mixed layer and coastal upwelling processes off the south coast of South Africa. Journal of Marine Research 57: 671-691.

Schumann, E. H., Perrins, L.-A. and Hunter, I. T. 1982. Upwelling along the South Coast of the Cape Province, South Africa. South African Journal of Science 78: 238-242.

Schwarcz, H. P. and Rink, W. J. 2000. ESR dating of the Die Kelders Cave 1 Site, South Africa. Journal of Human Evolution 38 (1): 121-128.

Schweitzer, F. R. 1970. A Preliminary Report of Excavations of a Cave at Die Kelders. The South African Archaeological Bulletin 25 (99/100): 136-138.

Schweitzer, F. R. 1975. Archaeological evidence for sheep at the Cape. South African Archaeological Bulletin 29: 75-82.

Schweitzer, F. R. 1979. Excavation at Die Kelders, Cape Province, South Africa: the Holocene deposits. Annals of the South African Museum 78: 101-233.

Schweitzer, F. R. and Wilson, M. L. 1978. A Preliminary Report on Excavations at Byneskranskop, Bredasdorp District, Cape. The South African Archaeological Bulletin 33 (128): 134-140.

Schweitzer, F. R. and Wilson, M. L. 1982. Byneskranskop 1, a late Quaternary living site in the southern Cape Province, South Africa. Annals of the South African Museum 88: 1-203.

Sealy, J. C. 1989. The use of chemical techniques for reconstructing prehistoric diets: A case study in the southwestern Cape. South African Archaeological Society Goodwin Series 6: 69-76.

Sealy, J. C. 2006. Diet, Mobility, and Settlement Pattern among Holocene HunterGatherers in Southernmost Africa. Current Anthropology 47 (4): 569-595.

Sealy, J. C. and van der Merwe, N.J. 1985. Isotope assessment of Holocene human diets in the south-western Cape, South Africa. Nature 315:138-140.

Sealy, J. C. and van der Merwe, N.J. 1986. Isotope assessment and the seasonal mobility hypothesis in the south-western Cape, South Africa. Current Anthropology 27:135-150.

Sealy, J. C. and Van Der Merwe, N. J. 1988. Social, spatial and chronological patterning in marine food use as determined by $\delta 13 \mathrm{C}$ measurements of Holocene human skeletons from the south-western Cape, South Africa. World Archaeology 20 (1): 87-102.

Sealy, J. C. and van der Merwe, N.J. 1992. On "Approaches to dietary reconstruction in the western Cape: Are you what you have eaten?" - a reply to Parkington. Journal of Archaeological Science 19:459-466.

Sealy, J. C. and Pfeiffer, S. 2000. Diet, body size, and landscape use among Holocene people in the Southern Cape, South Africa. Current Anthropology 41 (4): 642-654.

Sealy, J. C., Pfeiffer, S., Yates, R., Willmore, K., Manhire, A., Maggs, T., Lanham, J. and Wilmore, K. 2000. Hunter-Gatherer Child Burials from the Pakhuis Mountains, 
Western Cape: Growth, Diet and Burial Practices in the Late Holocene. The South African Archaeological Bulletin 55 (171): 32-43.

Sealy, J. C. and Cox, G. 2003. What can science tell us about slavery: using bone chemistry to identify slaves at the Cape.

Shackleton, N. J. 1973. Oxygen isotope analysis as a means of determining season of occupation of prehistoric midden sites. Archaeometry 15: 133-141.

Shackleton, N. J. 1982. "Stratigraphy and chronology of the Klasies River Mouth deposits: Oxygen isotope evidence," in The Middle Stone Age at Klasies River Mouth in South Africa. Edited by Singer, R. and Wymer, J., pp. 194-199. Chicago: University of Chicago Press.

Shipman, P. 2008. Separating "us" from "them": Neanderthal and modern human behavior. Proceedings of the National Academy of Sciences 105 (38): 1424114242.

Shotwell, J. A. 1955. An Approach to the Paleoecology of Mammals. Ecology 36 (2): 327-337.

Shotwell, J. A. 1958. Inter-Community Relationships in Hemphillian (Mid-Pliocene) Mammals. Ecology 39 (2): 271-282.

Sievers, C. and Wadley, L. 2008. Going underground: experimental carbonization of fruiting structures under hearths. Journal of Archaeological Science 35 (11): 29092917.

Simmons, R. E. and Kemper, J. 2003. Cave breeding by African Penguins near the northern extreme of their range: Sylvia Hill, Namibia. Ostrich-Journal of African Ornithology, 743 (4): 217-221.

Singer, R. and Wymer, J. 1982. The Middle Stone Age at Klasies River Mouth in South Africa. London: University of Chicago Press.

Skelton, P. H. 2000. Heritage for all - The art and culture of fishes in Africa. SAMAB 24 (1): 84-91.

Skelton, P. H. 2001. A complete guide to the freshwater fishes of southern Africa. Halfway House: Southern Book Publishers.

Skinner, J. D. and Chimimba, C. T. 2005. The mammals of the southern African subregion, 3rd edition. Cambridge: Cambridge University Press.

Smale, M. J., Cliff, G., Dicken, M. L. and Booth, A. J. 2007. Spatial and seasonal distribution patterns of juvenile and adult raggedtooth sharks Carcharias taurus tagged off the east coast of South Africa. Marine and Freshwater Research 58 (1): 127-134.

Smith, A. B., Sadr, K., Gribble, J. and Yates, R. 1991. Excavations in the South-Western Cape, South Africa, and the archaeological identity of prehistoric hunter-gatherers within the last 2000 years. South African Archaeological Bulletin 46: 71-91.

Smith, J. L. B. 1953. The sea fishes of southern Africa. Cape Town: The sea fishes of southern Africa book fund.

Smith, M. and Heemstra, P. C. 1995. "Smith's Sea Fishes of Southern Africa," Macmillan, Johannesburg, South Africa.

Smits, L. G. A. 1967. Fishing-Scenes from Botsabelo, Lesotho. The South African Archaeological Bulletin 22 (86): 60-67.

Somers, M. J. 2000. Seasonal variation in the diet of Cape clawless otters (Aonyx capensis) in a marine habitat. African Zoology 35 (2): 261-268.

Soressi, M. 2005. Aux origines de la «modernité» comportementale en Afrique du sud il ya 75000 ans. Annales de la Fondation Fyssen 20: 124-131. 
Speller, C. F. 1999. One fish, two fish, old fish, new fish: Investigating differential distribution of salmon resources in the Pacific Northwest through ancient DNA analysis, M.A., Simon Fraser University.

Speller, C. F., Yang, D. Y. and Hayden, B. 2005. Ancient DNA investigation of prehistoric salmon resource utilization at Keatley Creek, British Columbia, Canada. Journal of Archaeological Science 32 (9): 1378-1389.

Steele, T. E. and Klein, R. G. 2005. Mollusk and tortoise size as proxies for stone age population density in South Africa: Implications for the evolution of human cultural capacity. Munibe. Antropologia-arkeologia (57): 221-237.

Steffen, M. and Mackie, Q. 2005. An Experimental Approach to Understanding Burnt Fish Bone Assemblages within Archaeological Hearth Contexts. Canadian Zooarchaeology 23: 11-38.

Stewart, K. M. 1989. Fishing sites of North and East Africa in the late Pleistocene and Holocene: environmental change and human adaptation. Oxford: BAR International Series.

Stewart, K. M. 1991. Modern fishbone assemblages at Lake Turkana, Kenya: A methodology to aid in recognition of hominid fish utilization. Journal of Archaeological Science 18 (5): 579-603.

Stewart, K. M. 1994. Early hominid utilisation of fish resources and implications for seasonality and behaviour. Journal of Human Evolution 27 (1-3): 229-245.

Stewart, K. M. and Gifford-Gonzalez, D. 1994. An Ethnoarchaeological Contribution to Identifying Hominid Fish Processing Sites. Journal of Archaeological Science 21 (2): 237-248.

Stewart, K. M., Matthiesen, D. P., Leblanc, L. and West, J. 1997. Prey diversity and selectivity of the African fish eagle: data from a roost in northern Kenya. African Journal of Ecology 35 (2): 133-145.

Stewart, K. M., Leblanc, L., Matthiesen, D. P. and West, J. 1999. Microfaunal Remains from a Modern East African Raptor Roost: Patterning and Implications for Fossil Bone Scatters. Paleobiology 25 (4): 483-503.

Steyn, M., Binneman, J. and Loots, M. 2007. The Kouga mummified human remains. South African Archaeological Bulletin 62 (185): 3-8.

Stiner, M. C. 1991. Food procurement and transport by human and non-human predators. Journal of Archaeological Science 18 (4): 455-482.

Stiner, M. C. 1994. Honor among thieves: A zooarchaeological study of Neandertal ecology. Princeton: Princeton University Press.

Stiner, M. C. 2002. On in situ Attrition and Vertebrate Body Part Profiles. Journal of Archaeological Science 29 (9): 979-991.

Stock, C. 1929. A Census of the Pleistocene Mammals of Rancho La Brea, Based on the Collections of the Los Angeles Museum. Journal of Mammalogy 10 (4): 281-289.

Stow, G. W. 1905. The native races of South Africa: a history of the intrusion of the Hottentots and Bantu into the hunting grounds of the Bushmen, the aborigines of the country. London: S. Sonnenschein \& Co., limited.

Straus, L. G. 2001. Africa and Iberia in the Pleistocene. Quaternary International 75 (1): 91-102.

Stringer, C. 2000. Coasting out of Africa. Nature 405: 24-27.

Stringer, C. 2002. Modern human origins: progress and prospects. Philosophical Transactions of the Royal Society of London B 357: 563-579.

Stringer, C. 2003. "The evolution of modern humans: where are we now?," pp. 1-8. 
Stringer, C. B., Finlayson, J. C., Barton, R. N. E., Fernández-Jalvo, Y., Cáceres, I., Sabin, R. C., Rhodes, E. J., Currant, A. P., Rodriguez-Vidal, J., Giles-Pacheco, F. and Riquelme-Cantal, J. A. 2008. Neanderthal exploitation of marine mammals in Gibraltar. Proceedings of the National Academy of Sciences 105 (38): 1431914324.

Stuart, C. and Stuart, T. 2001. Field Guide to Mammals of Southern Africa. Cape Town: Struik.

Sunde, J. and Isaacs, M. 2008. Marine Conservation and Coastal Communities: Who Carries the Costs? , A Study of Marine Protected Areas and Their Impact on Traditional Small-scale Fishing Communities in South Africa. International Collective in Support of Fishworkers. Chennai, India.

Surge, D. and Walker, K. J. 2005. Oxygen isotope composition of modern and archaeological otoliths from the estuarine hardhead catfish (Ariopsis felis) and their potential to record low-latitude climate change. Palaeogeography, Palaeoclimatology, Palaeoecology 228 (1-2): 179-191.

Suter, W. 1995. The Effect of Predation by Wintering Cormorants Phalacrocorax carbo on Grayling Thymallus thymallus and Trout (Salmonidae) Populations: Two Case Studies from Swiss Rivers. Journal of Applied Ecology 32 (1): 29-46.

Swart, V. P. and Largier, J. L. 1987. Thermal structure of Agulhas Bank water. South African Journal of Marine Science 5: 243-252.

Sydow, W. 1973. Contributions to the History and Protohistory of the Topnaar Strandloper Settlement at the Kuiseb River Mouth near Walvis Bay. The South African Archaeological Bulletin 28 (111/112): 73-77.

Tattersall, I. 1995. "The fossil record: How we know what we know about human evolution," New York: Oxford University Press.

Texier, P. J., Porraz, G., Parkington, J., Rigaud, J. P., Poggenpoel, C., Miller, C., Tribolo, C., Cartwright, C., Coudenneau, A. and Klein, R. 2010. A Howiesons Poort tradition of engraving ostrich eggshell containers dated to 60,000 years ago at Diepkloof Rock Shelter, South Africa. Proceedings of the National Academy of Sciences 107 (14): 6180-6185.

Thackeray, A. I. 1992. The Middle Stone Age South of the Limpopo River. Journal of World Prehistory 6: 385-440.

Thackeray, J. F. 1988. Molluscan fauna from Klasies River, South Africa. South African Archaeological Bulletin 43: 27-32.

Thieme, H. 1997. Lower Palaeolithic hunting spears from Germany. Nature 385: 807 810

Thieme, H. 1999. Altpaläolithische Holzgeräte aus Schöningen Lkr. Helmstedt., Germania 77: 451-487.

Thompson, J. 2008. Zooarchaeological tests for modern human behavior at Blombos Cave and Pinnacle Point Cave 13B, southwestern Cape, South Africa. , PhD, Arizona State University.

Thompson, J. C. 2005. The impact of post-depositional processes on bone surface modification frequencies: A corrective strategy and its application to the Loiyangalani site, Serengeti Plain, Tanzania. Journal of Taphonomy 3 (2-3): 6789.

Thompson, W. W. 1913. The Sea Fisheries of the Cape Colony: from van Riebeeck's days to the eve of the union. Cape Town: T. Maskew Miller. 
Todd, L. C. and Rapson, D. J. 1988. Long bone fragmentation and interpretation of faunal assemblages: approaches to comparative analysis. Journal of Archaeological science 15 (3): 307-325.

Tribolo, C. 2003. Apports des méthodes de la luminescence à la chronologie de technofaciès du Middle Stone Age associés aux premiers Hommes modernes d'Afrique du Sud., Ph. D, Université Bordeaux-1.

Tribolo, C., Mercier, N., Selo, M., Valladas, H., Joron, J-L., Reyss, J-L., Henshilwood, C., Sealy, J., Yates, R. 2006. TL dating of burnt lithics from Blombos Cave (South Africa) and the antiquity of modern behaviour. Archaeometry 48 (2): 341-357.

Turner, M. 1970. A Search for the Tsitsikamma Shelters. The South African Archaeological Bulletin 25 (98): 67-70.

Uerpmann, H. P. 1973. Animal bone finds and economic archaeology: a critical study of 'osteo-archaeological' method. World Archaeology: 307-322.

Vale, D. and Gargett, R. H. 2002. Size Matters: 3-mm Sieves Do Not Increase Richness in a Fishbone Assemblage from Arrawarra I, an Aboriginal Australian Shell Midden on the Mid-north Coast of New South Wales, Australia. Journal of Archaeological Science 29 (1): 57-63.

Van Andel, T. H. 1989a. Late Pleistocene sea levels and the human exploitation of the shore and shelf of southern South Africa. Journal of Field Archaeology 16: 133154.

Van Andel, T. H. 1989b. Late Quaternary sea-level changes and archaeology. Antiquity 63: 733-745.

Van Andel, T. H. 1990. Addendum to 'Late Quaternary sea level changes and archaeology'. Antiquity 64: 151-2.

Van Der Elst, R. 1998. A Guide to the Common Sea Fishes of Southern Africa, 3 edition. Cape Town: Struik.

Van Neer, W. 1993. Limits of incremental growth in seasonality studies: The example of the clariid pectoral spines from the Byzantino-Islamic site of Apamea (Syria; sixth to seventh century AD). International Journal of Osteoarchaeology 3 (2): 119127.

Van Neer, W. 1995. "Analysis of the fish remains," in Excavations at Jenne-Jeno, Hambarketolo, and Kaniana (Inland Niger Delta, Mali), the 1981 season. Edited by Mcintosh, S. K. London: University of California Press.

Van Neer, W. and Pieters, M. 1997. Evidence for processing of flatfish at Raversijde, a Late Medieval coastal site in Belgium: Postpalaeolithic Europe I. Anthropozoologica (25-26): 579-586.

Van Neer, W. and Ervynck, A. 1998. "The faunal remains," in Berenike '96. Report of the Excavations at Berenike (Egyptian Red Sea Coast) and the Survey of the Eastern Desert. Special Series, 3. Edited by Sidebotham, S. and Wendrich, W., pp. 349388. Leiden: CNWS Publications.

Van Neer, W. and Ervynck, A. 2004. "Remains of traded fish in archaeological sites: indicators of status or bulk food?," in Behaviour behind Bones. The Zooarchaeology of Ritual, Religion, Status and Identity. Edited by O'day, S. J., Van Neer, W. and Ervynck, A., pp. 203-214. Oxford: Oxbow Books.

Van Neer, W. and Parker, S. T. 2008. First archaeozoological evidence for haimation, the 'invisible'garum. Journal of Archaeological Science 35 (7): 1821-1827. 
Van Neer, W., Loeugas, L. and Rijnsdorp, A. D. 1999. Reconstructing age distribution, season of capture and growth rate of fish from archaeological sites based on otoliths and vertebrae. International Journal of Osteoarchaeology 9 (2): 116-130.

Van Neer, W., Paulissen, E. and Vermeersch, P. 2000. "Chronology, subsistence and environment of the Late Palaeolithic fishing sites of Makhadma-2 and 4," in Palaeolithic living sites in Upper and Middle Egypt. Edited by Vermeersch, P., pp. 271-287. Leuven: Leuven University Press.

Van Neer, W., Ervynck, A., Bolle, L. J. and Millner, R. S. 2004a. Seasonality only works in certain parts of the year: the reconstruction of fishing seasons through otolith analysis. International Journal of Osteoarchaeology 14 (6): 457-474.

Van Neer, W., Hamilton-Dyer, S., Cappers, R., Desender, K. and Ervycnk, A. 2006. The Roman trade in salted Nilotic fish products: some examples from Egypt. Documenta Archaeobiologiae 4: 173-188.

Van Neer, W., Lernau, O., Friedman, R., Mumford, G., Poblome, J. and Waelkens, M. 2004b. Fish remains from archaeological sites as indicators of former trade connections in the Eastern Mediterranean. Paléorient 30 (1): 101-147.

Van Neer, W., Wouters, W., Rutschowscaya, M. H., Delattre, A., Dixneuf, D., Desender, K. and Poblome, J. 2007. "Salted fish products from the Coptic monastery at Bawit, Egypt: evidence from the bones and texts," in The role of fish in ancient time. Edited by Huster Plogmann, H., pp. 147-159. Rahden: Proceedings of the 13th Meeting of the ICAZ Fish Remains Working Group.

Van Niekerk, K. L. 2002. An analysis of the freshwater fish remains from Rose Cottage Cave in the eastern Free State, South Africa, BSc Honours, University of Cape Town.

Van Niekerk, K. L. 2005. The Archaeology of Fishing: Establishing a Comparative Collection and Subsequent Analysis of Marine Fish Bone from Holocene levels at Blombos Cave, South Africa, M.Phil, University of Bergen.

Van Noten, F. L. 1974. Excavations at the Gordon's Bay Shell Midden, South-Western Cape. The South African Archaeological Bulletin 29 (115/116): 122-142.

Van Pletzen, L. 2000. The Large Mammal Fauna from Klasies River, MA, University of Stellenbosch.

Van Wyk, B. E. and Gericke, N. 2000. People's plants: A guide to useful plants of Southern Africa. Pretoria: Briza Publications.

Vellanoweth, R. L., Lambright, M. R., Erlandson, J. M. and Rick, T. C. 2003. Early New World maritime technologies: sea grass cordage, shell beads, and a bone tool from Cave of the Chimneys, San Miguel Island, California, USA. Journal of Archaeological Science 30 (9): 1161-1173.

Verwoerd, D. J. 1987. Observations on the food and status of the Cape clawless otter Aonyx capensis at Betty's Bay, South Africa. South African Journal of Zoology 22 (1): 33-39.

Villa, P., Delagnes, A. and Wadley, L. 2005. A late Middle Stone Age artifact assemblage from Sibudu (KwaZulu-Natal): comparisons with the European Middle Paleolithic. Journal of Archaeological Science 32 (3): 399-422.

Villa, P., Soressi, M., Henshilwood, C. S. and Mourre, V. 2009. The Still Bay points of Blombos Cave (South Africa). Journal of Archaeological Science 36 (2): 441-460.

Villa, P., Soriano, S., Teyssandier, N. and Wurz, S. 2010. The Howiesons Poort and MSA III at Klasies River main site, Cave 1A. Journal of Archaeological Science 37 (3): 630-655. 
Vinnicombe, P. 1960. A Fishing-Scene from the Tsoelike River, South-Eastern Basutoland. The South African Archaeological Bulletin 15 (57): 15-19.

Vinnicombe, P. 1961. A Painting of a Fish-Trap on Bamboo Mountain, Underberg

District, Southern Natal. The South African Archaeological Bulletin 16 (63): 114115.

Vinnicombe, P. 1976. People of the Eland: Rock paintings of the Drakensberg Bushmen as a reflection of their life and thought. Pietermaritzburg: University of Natal Press.

Vogel, J. C. 2001. "Radiometric dates for the Middle Stone Age in South Africa," in Humanity from African Naissance to coming Millennia. Edited by Tobias, P. V., Raath, M. A., Moggi-Cecchi, J. and Doyle, G. A., pp. 261-268. Florence: Florence University Press.

Voigt, E. A. 1982. "The Molluscan Fauna," in The Middle Stone Age at Klasies River Mouth in South Africa. Edited by Singer, R. and Wymer, J. Chicago: University of Chicago Press.

Volman, T. P. 1978. Early Archaeological Evidence for Shellfish collecting. Science 201: 911-913.

Volman, T. P. 1981. The Middle Stone Age of the southern Cape, Ph.D, University of Chicago.

Volman, T. P. 1984. "Early prehistory of southern Africa," in Southern African prehistory and palaeoenvironments. Edited by Klein, R. G., pp. 169-220. Rotterdam: A.A. Balkema.

Von Den Driesch, A. 1983. "Some archaeozoological remarks on fishes in Ancient Egypt," in Animals and Archaeology: Shell middens, fishes and birds, vol. 183. Edited by Grigson, C. and Clutton-Brock, J., pp. 87-111. Oxford: British Archaeological Reports International Series.

Von Den Driesch, A. 2004. The Middle Stone Age Fish Fauna from the Klasies River main site, South Africa. Anthropozoologica 39 (2): 33-59.

Wadley, L. 2001. Preliminary report on excavations at Sibudu Cave, KwaZulu-Natal. South African Humanities 13: 1-17.

Wadley, L. 2006. Partners in grime: results of multi-disciplinary archaeology at Sibudu Cave. Southern African Humanities 18 (1): 315-341.

Wadley, L. 2007. Announcing a Still Bay industry at Sibudu Cave, South Africa. Journal of Human Evolution 52 (6): 681-689.

Wadley, L. 2010. Were snares and traps used in the Middle Stone Age and does it matter? A review and a case study from Sibudu, South Africa. Journal of Human Evolution 58 (2): 179-192.

Wadley, L. and Jacobs, Z. 2006. Sibudu Cave: background to the excavations, stratigraphy and dating. Southern African Humanities 18 (1): 1-26.

Wadley, L., Williamson, B. and Lombard, M. 2004. Ochre in hafting in Middle Stone Age southern Africa: a practical role. Antiquity 78: 661-675.

Wadley, L., Hodgskiss, T. and Grant, M. 2009. Implications for complex cognition from the hafting of tools with compound adhesives in the Middle Stone Age, South Africa. Proceedings of the National Academy of Sciences 106 (24): 9590.

Walker, K. J. and Surge, D. 2006. Developing oxygen isotope proxies from archaeological sources for the study of Late Holocene human-climate interactions in coastal southwest Florida. Quaternary International 150 (1): 3-11. 
Walter, R. C., Buffler, R. T., Brugemann, J. H., Guillaume, M. M. M., Berhe, S. M., Negassi, B., Libsekal, Y., Cheng, H., Edwards, R. L., Von Cosel, R., Neraudeau, D. and Gagnon, M. 2000. Early human occupation of the Red Sea coast of Eritrea during the Last Interglacial. Nature 405: 65-69.

Walters, I. 1988. Fish hooks: Evidence for dual social systems in southeastern Australia. Australian Archaeology 27: 98-114.

Walters, I. 1992. Seasonality of fishing in south-east Queensland. Queensland Archaeological Research 9: 29-34.

Warren, S. H. 1951. The Clactonian flint industry: a new interpretation. Proceedings of the Geological Association 62: 107-135.

Watson, J. P. N. 1979. The estimation of the relative frequencies of mammalian species: Khirokitia 1972. Journal of Archaeological Science 6 (2): 127-137.

Watson, L. H. and Lang, A. J. 2004. Diet of Cape clawless otters in Groenvlei Lake, South Africa: short communications. South African Journal of Wildlife Research 33 (2): 135-137.

Watts, I. 1998. The Origin of Symbolic Culture: The Middle Stone Age of Southern Africa and Khoisan ethnography, Ph.D, University of London.

Wells, M. J. 1965. An analysis of plant remains from Scott's Cave in the Gamtoos Valley. South African Archaeological Bulletin 20: 79-84.

Wheeler, A. and Jones, A. K. G. 1989. Fishes: Cambridge University Press.

White, T. 1987. Cannibals at Klasies: cutmarks on a fragment of human skull joins a growing body of clues to suggest that the Klasies River Caves may hold dark secrets about their early inhabitants., Sagittarius 2 (2): 6-9.

White, T. E. 1952. Observations on the Butchering Technique of Some Aboriginal Peoples: I. American Antiquity 17 (4): 337-338.

White, T. E. 1953. A Method of Calculating the Dietary Percentage of Various Food Animals Utilized by Aboriginal Peoples. American Antiquity 18 (4): 396-398.

White, T. E. 1955. Observations on the Butchering Technics of Some Aboriginal Peoples Numbers 7, 8, and 9. American Antiquity 21 (2): 170-178.

White, T. E. 1956. The Study of Osteological Materials in the Plains. American Antiquity 21 (4): 401-404.

Whitfield, A. K. 2004. Distribution patterns of fishes in a freshwater deprived Eastern Cape estuary, with particular emphasis on the geographical headwater region. Water SA 29 (1): 61.

Whitfield, A. K. and Blaber, S. J. M. 1978. Feeding ecology of piscivorous birds at Lake St. Lucia, Part 1. Diving birds. Ostrich 49: 185-198.

Willcox, A. R. 1963. The Rock art of South Africa. Johannesburg: Nelson.

Willis, L. M., Eren, M. I. and Rick, T. C. 2008. Does butchering fish leave cut marks? , Journal of Archaeological Science 35 (5): 1438-1444.

Wilson, M. L. 1988. Forest Hall Shelter: An Early Excavation on the Southern Cape Coast. The South African Archaeological Bulletin 43 (147): 53-55.

Wilson, R. P. 1985. The jackass penguin (Spheniscus demersus) as a pelagic predator. Marine Ecology Progress Series 25 (3): 219-227.

Wolpoff, M. and Caspari, R. 1996. The modernity mess. Journal of Human Evolution 30 (2): 167-171.

Woodhouse, H. C. 1979. The Bushman art of Southern Africa. London: Purnell. 
Wurster, C. M. and Patterson, W. P. 2001. Late Holocene climate change for the eastern interior United States: evidence from high-resolution [delta] $18 \mathrm{O}$ values of sagittal otoliths. Palaeogeography, Palaeoclimatology, Palaeoecology 170 (1-2): 81-100.

Wurz, S. 1997. The Howiesons Poort at Klasies River: from artefacts to cognition, M.A., University of Stellenbosch.

Wurz, S. 1999. The Howiesons Poort Backed Artefacts from Klasies River: An Argument for Symbolic Behaviour. The South African Archaeological Bulletin 54 (169): 3850 .

Wurz, S. 2000. The Middle Stone Age at Klasies River, South Africa, Ph.D, University of Stellenbosch.

Wurz, S. 2002. Variability in the Middle Stone Age Lithic Sequence, 115,6000-60,000 Years Ago at Klasies River, South Africa. Journal of Archaeological Science 29: 1001-1015.

Wurz, S. 2008. Modern behaviour at Klasies River. South African Archaeological Society Goodwin Series 10 (150-156).

Wymer, J. and Singer, R. 1970. The First Season of Excavations at Clacton-on-Sea, Essex, England: A Brief Report. World Archaeology 2 (1): 12-16.

Yang, D. Y., Cannon, A. and Saunders, S. R. 2004. DNA species identification of archaeological salmon bone from the Pacific Northwest Coast of North America. Journal of Archaeological Science 31 (5): 619-631.

Yellen, J. E. 1996. Behavioural and taphonomic patterning at Katanda 9: A Middle Stone Age site, Kivu Province, Zaire. Journal of Archaeological Science 23: 915-932.

Yellen, J. E. 1998. Barbed bone points: tradition and continuity in Saharan and subSaharan Africa. African Archaeological Review 15: 173-198.

Yellen, J. E., Brooks, A. S., Cornelissen, E., Mehlman, M. J. and Stewart, K. 1995. A Middle Stone Age worked bone industry from Katanda, Upper Semliki Valley, Zaire. Science 268: 553-556.

Zilhão, J., Angelucci, D., Badal-García, E., D’errico, F., Daniel, F., Dayet, L., Douka, K., Higham, T., Martínez-Sánchez, M., Montes-Bernárdez, R., Murcia-Mascarós, S., Pérez-Sirvent, C., Roldán-García, C., Vanhaeren, M., Villaverde, V., Wood, R. and Zapata, J. 2010. Symbolic use of marine shells and mineral pigments by Iberian Neandertals. Proceedings of the National Academy of Sciences 107 (3): 1023-1028.

Zohar, I. and Cooke, R. G. 1997. The impact of salting and drying on fish bones: Preliminary observations on four marine species from Parita Bay, Panama. Archaeofauna (6): 59.

Zohar, I. and Belmaker, M. 2005. Size does matter: methodological comments on sieve size and species richness in fishbone assemblages. Journal of Archaeological Science 32 (4): 635-641.

Zohar, I., Dayan, T. and Spanier, E. 1997. Predicting Grey Triggerfish Body Size from Bones. International Journal of Osteoarchaeology 7: 150-156.

Zohar, I., Dayan, T., Galili, E. and Spanier, E. 2001. Fish Processing during the Early Holocene: A Taphonomic Case Study from Coastal Israel. Journal of Archaeological Science 28: 1041-1053.

Zohar, I., Belmaker, M., Nadel, D., Gafny, S., Goren, M., Hershkovitz, I. and Dayan, T. 2008. The living and the dead: How do taphonomic processes modify relative abundance and skeletal completeness of freshwater fish? , Palaeogeography, Palaeoclimatology, Palaeoecology 258 (4): 292-316. 


\section{Appendix A}

(a)

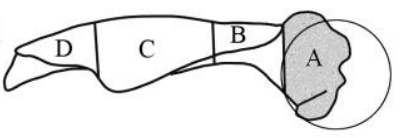

(c)

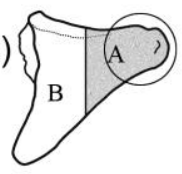

(d)

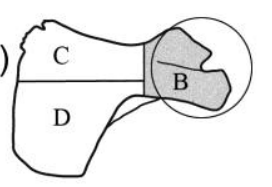

(b)

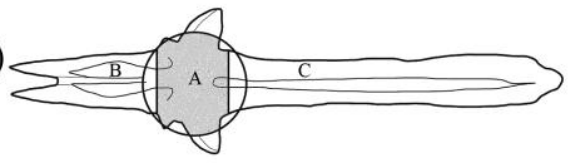

(e)

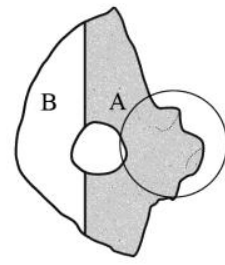

(f)

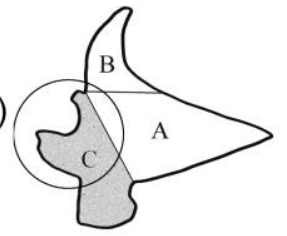

(g)

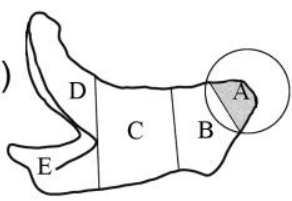

(j)

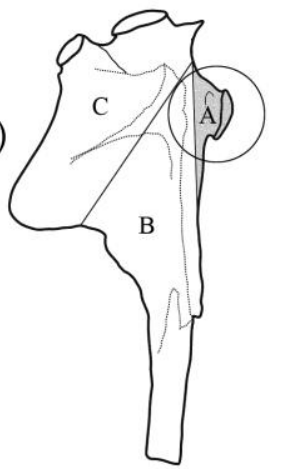

(m)

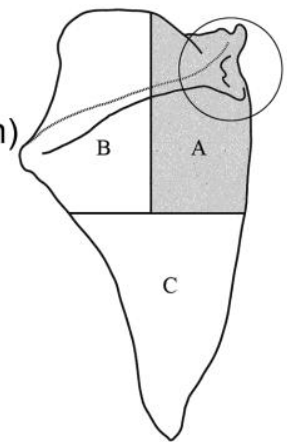

(k)

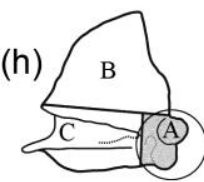

(i)

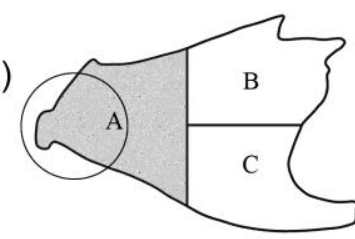

(l)

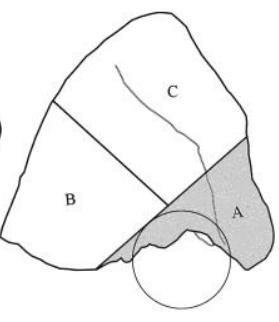

(n)

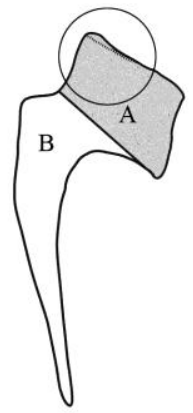

(o)

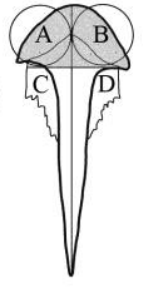

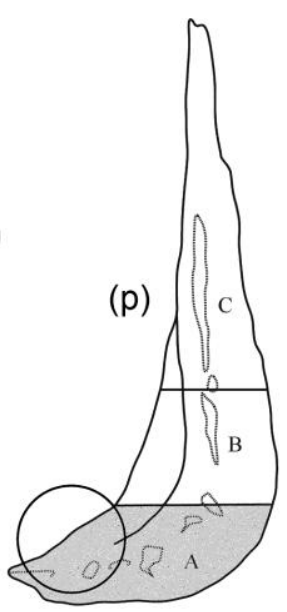

Figure A-1. Illustration of the zones recorded per element and the 'MNI feature' of selected elements. The shaded areas indicate the zone(s) containing the MNI features (circled) that need to be present in order for that bone to be included in MNI calculation. $a=$ maxilla, $b=$ parasphenoid, $c=$ epihyal, $\mathrm{d}=$ ceratohyal, $\mathrm{e}=\mathrm{scapula}, \mathrm{f}=$ articular, $\mathrm{g}=$ dentary, $\mathrm{h}=$ quadrate, $\mathrm{i}=$ urohyal, $\mathrm{j}=$ hyomandibular, $\mathrm{k}=$ palatine, $\mathrm{l}=$ supraoccipital, $\mathrm{m}=$ opercular, $\mathrm{n}=$ coracoid, $\mathrm{o}=$ vomer, $\mathrm{p}=$ preopercular 


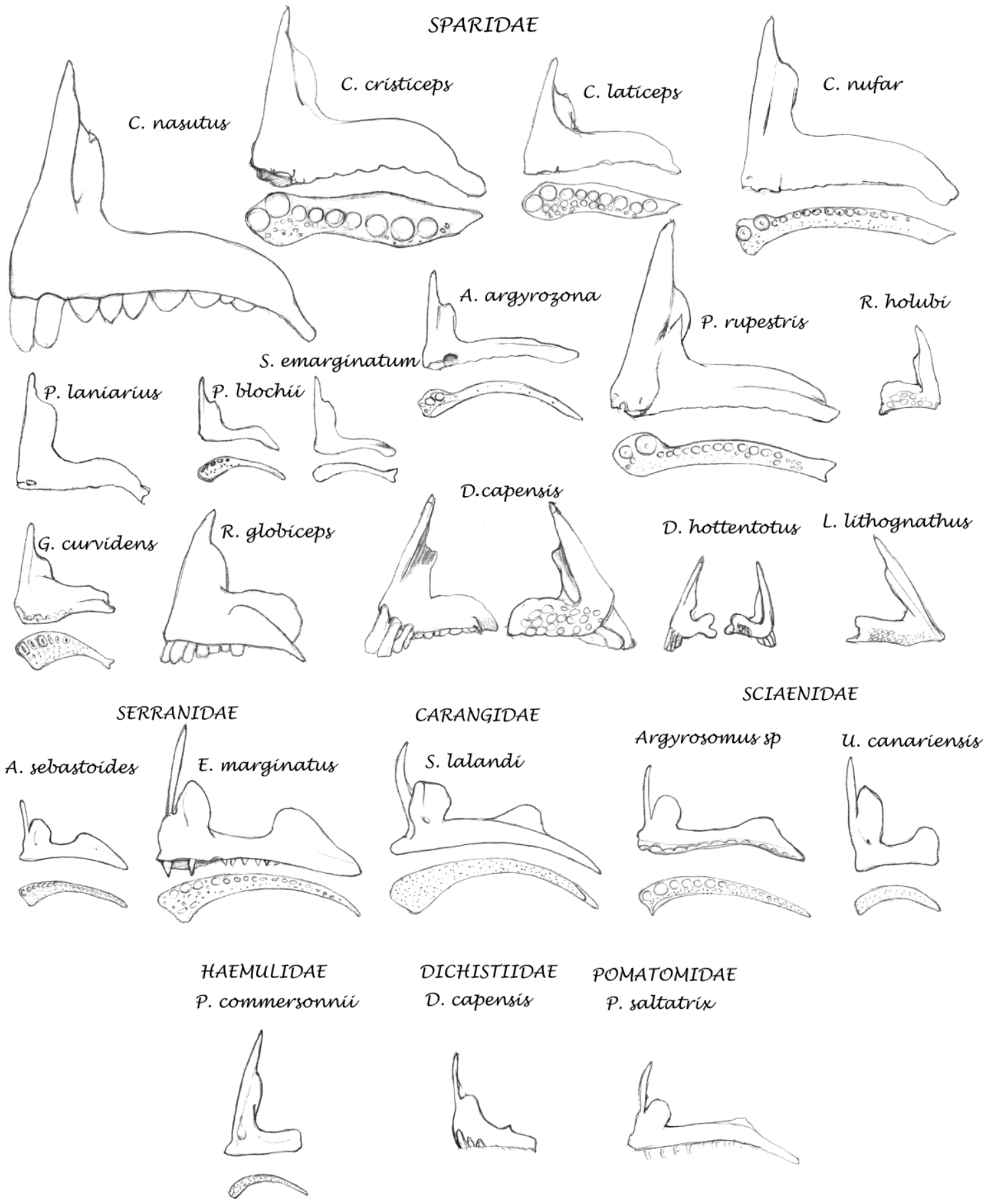

Figure A-2. Illustration of the premaxillae of species from various fish families. Full generic names for figures A2-A17 are: Ariidae: Galeichthys feliceps; Serranidae: Epinephelus marginatus, Acanthistius sebastoides; Pomatomidae: Pomatomys saltatrix; Haemulidae: Pomadasys commersonnii, Pomadasys olivaceum; Sparidae: Argyrozona argyrozona, Cheimerius nufar, Chrysoblephus cristiceps, Chrysoblephus gibbiceps, Chrysoblephus laticeps, Cymatoceps nasutus, Diplodus capensis, Diplodus hottentotus, Gymnocrotaphus curvidens, Lithognathus lithognathus, Pachymetopon blochii, Petrus rupestris, Pterogymnus laniarius, Rhabosargus globiceps, Rhabdosargus holubi, Sarpa salpa, Sparodon durbanensis, Spondyliosoma emarginatum; Dichistiidae: Dichistius capensis; Monodactylidae: Monodactylus falciformis; Sciaenidae: Argyrosomus inodorus/japonicus, Umbrina canariensis; Oplegnathidae: Oplegnathus conwayi; Carangidae: Seriola lalandi, Mugilidae: Liza richardsonii, Mugil cf. cephalus; Scombridae: Scomber japonicus 


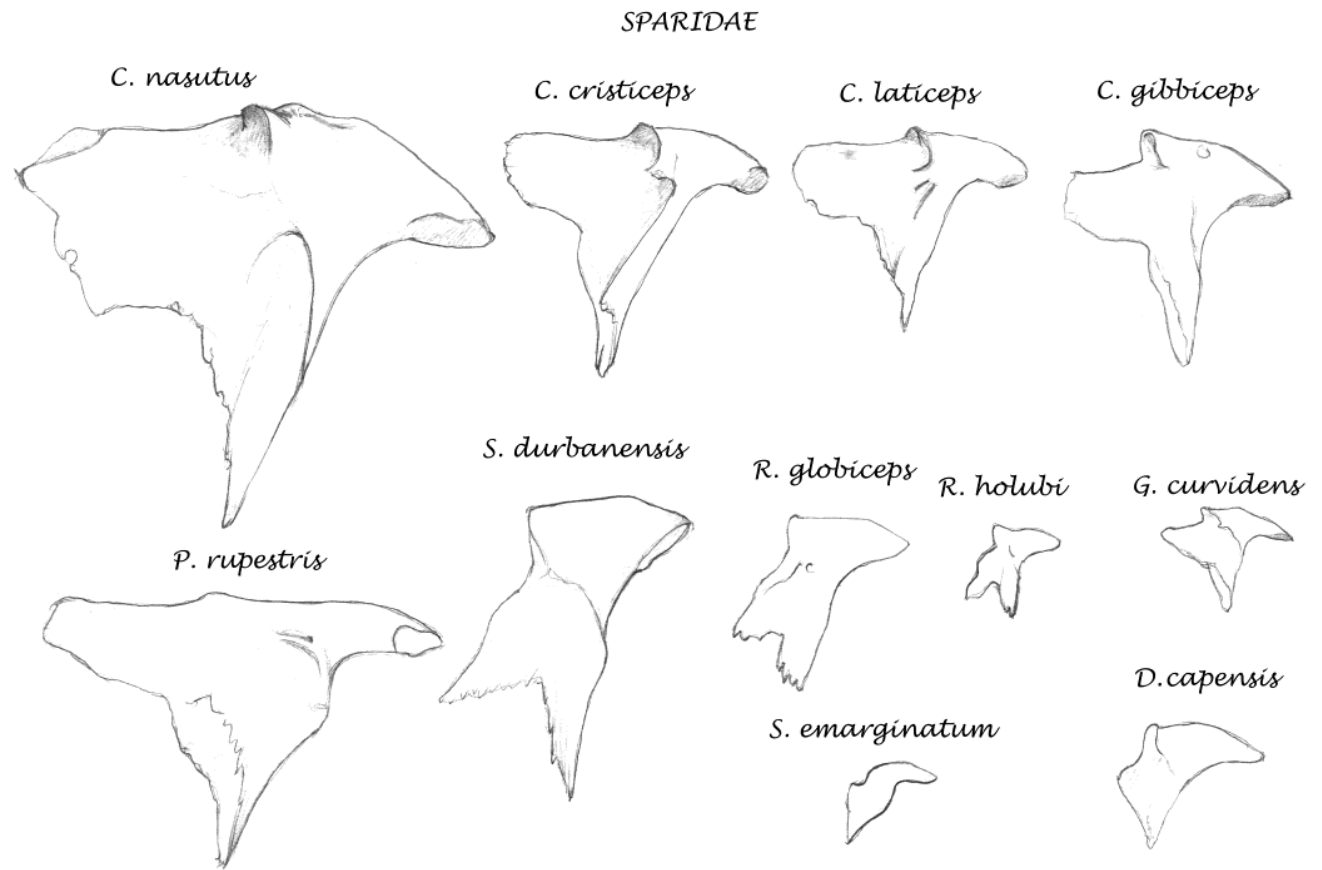

A. argyrozona P.laniarius L. lithognathus S. salpa
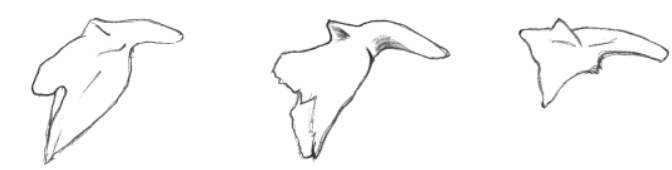

5

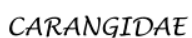

SCIAENIDAE

POMATOMIDAE

SERRANIDAE

S. latandi Argyrosomus sp.

P. saltatrix

E. marginatus

A. sebastoides
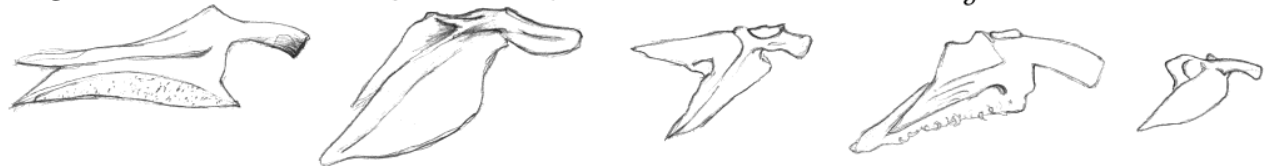

ARIIDAE

DICHISTIIDAE

OPLEGNATHIDAE

SCOMBRIDAE

HAEMULIDAE

G. feliceps

D. capensis

O. conwayí

S. japonicus

P. commersonnii
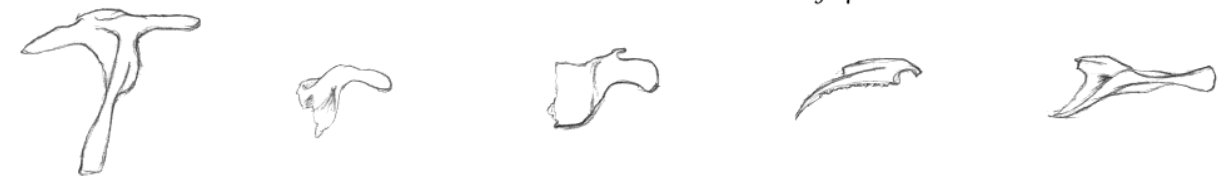

Figure A-3. Illustration of the palatine bone of species from various fish families. 


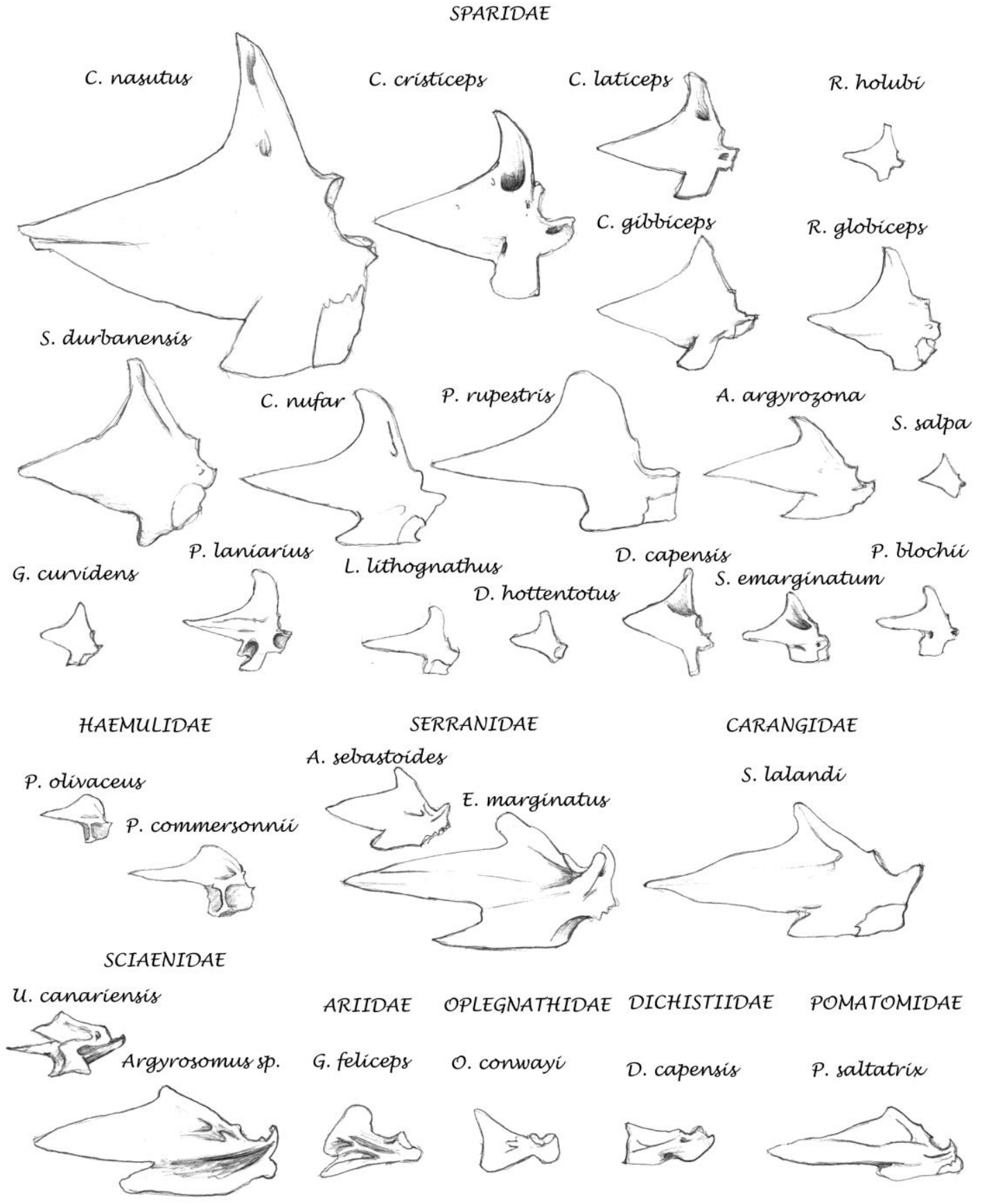

Figure A-4. Illustration of the articular from species from various fish families. 

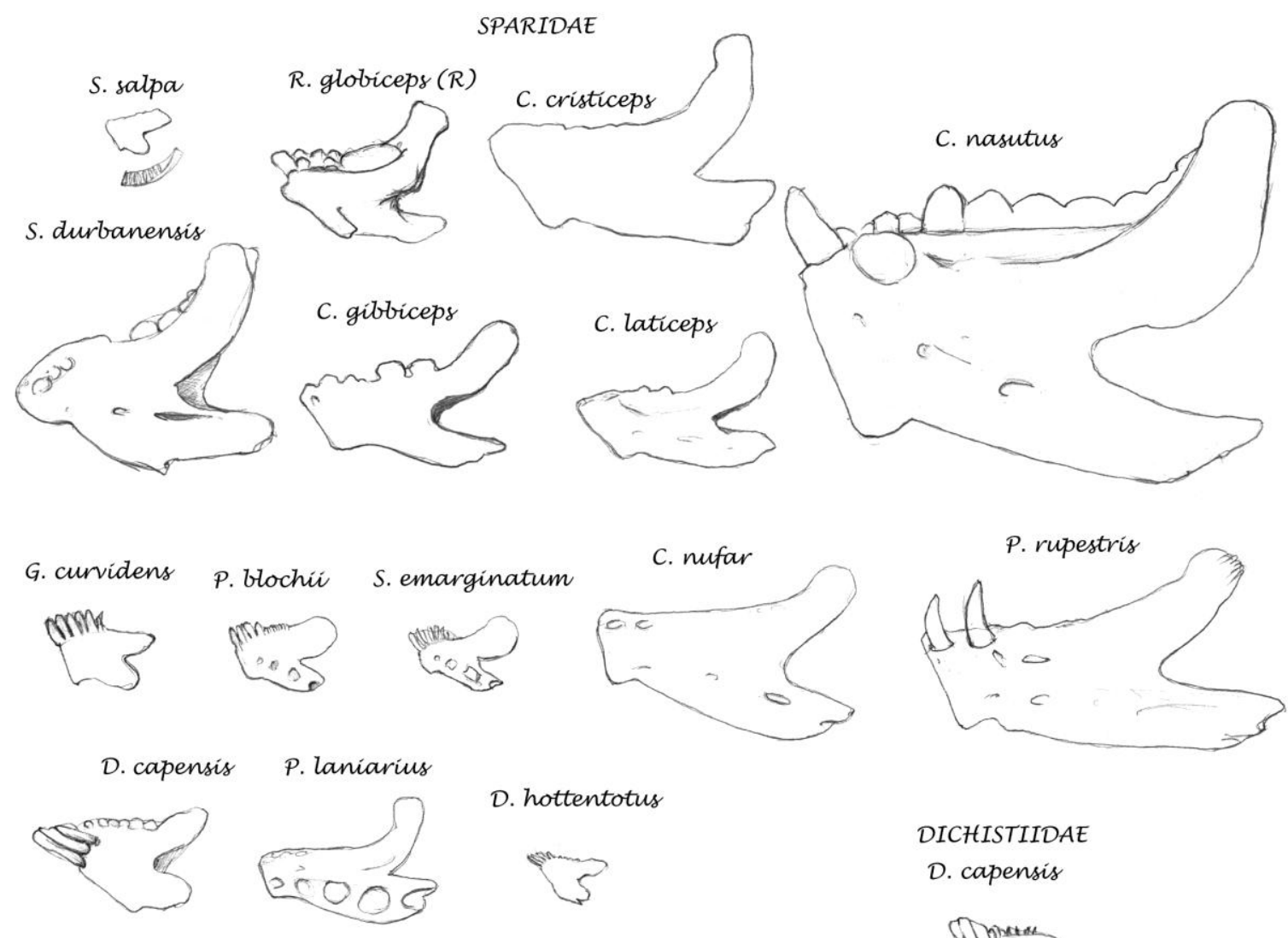

D. hottentotus
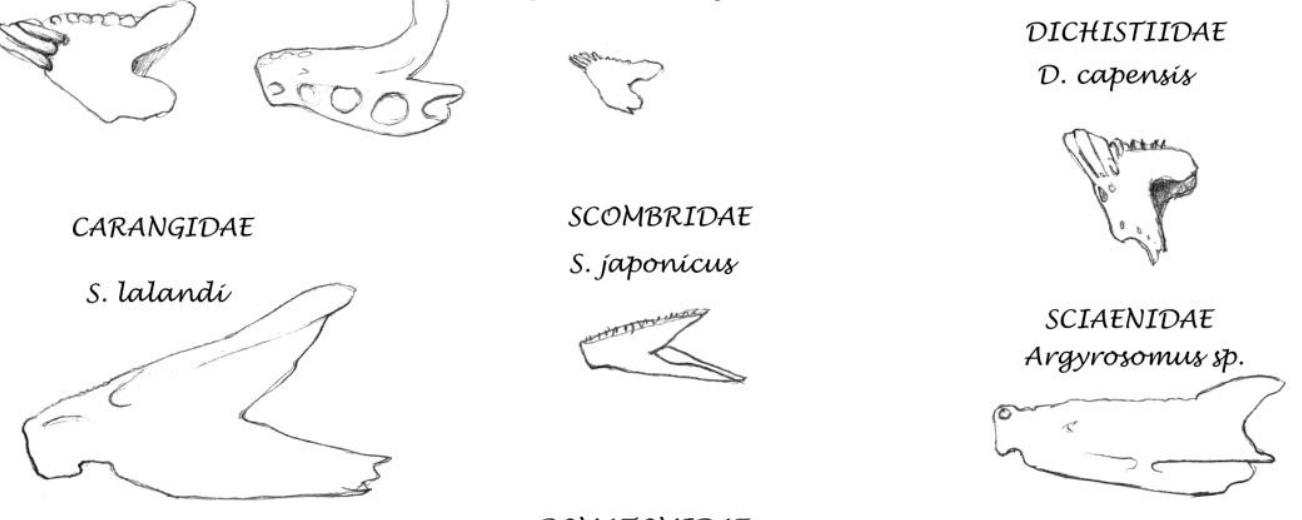

POMATOMIDAE
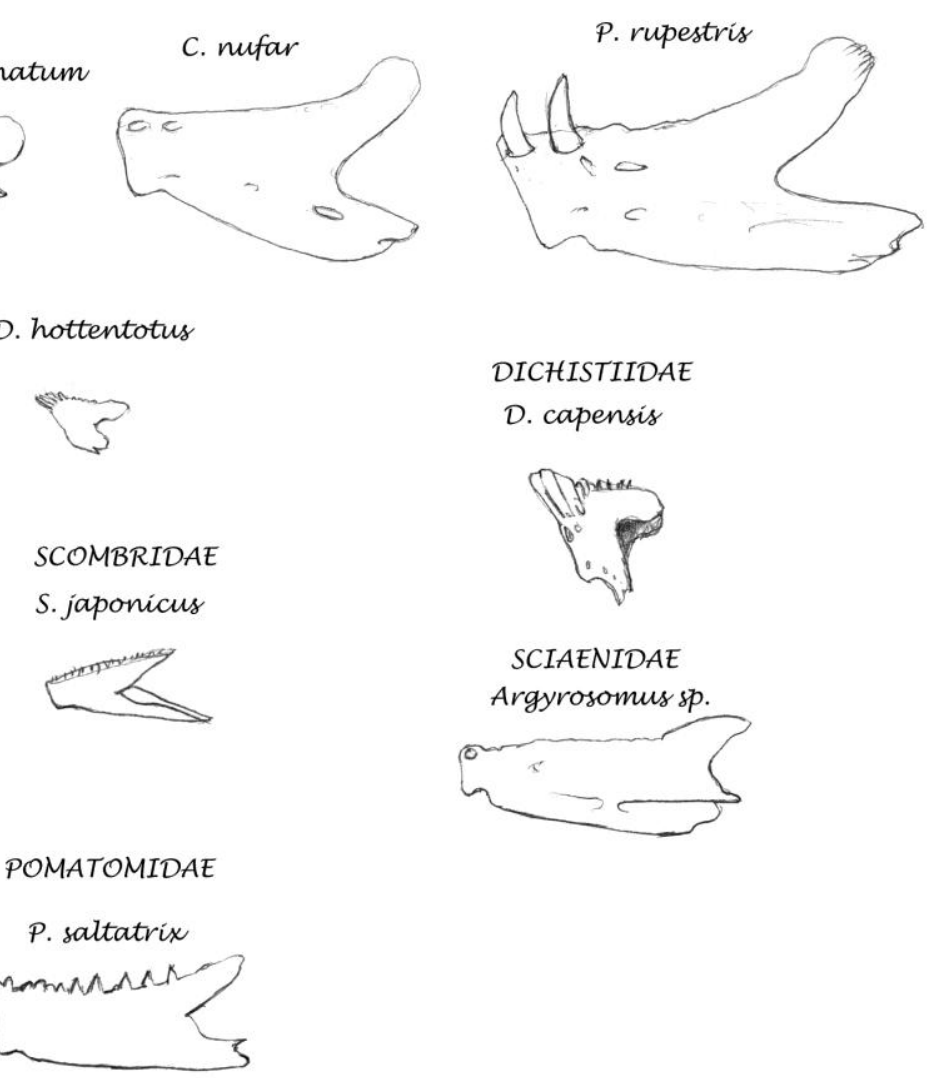

Figure A-5. Illustration of the dentary of species from various families. 


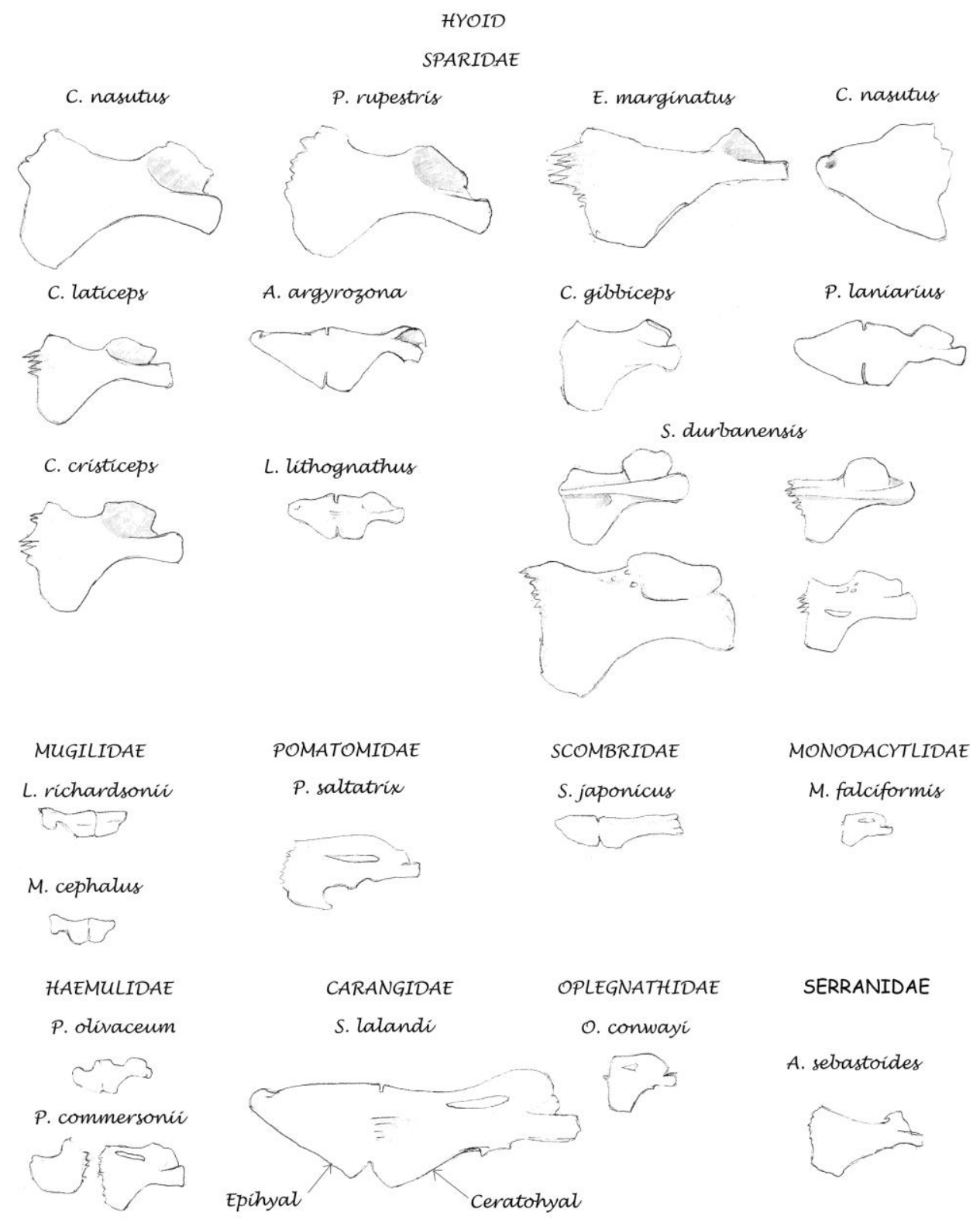

Figure A-6. Illustration of the ceratohyal and epihyal bones from the hyoid region of fish from different families. 


\section{LACHRYMAL}

\section{SPARIDAE}

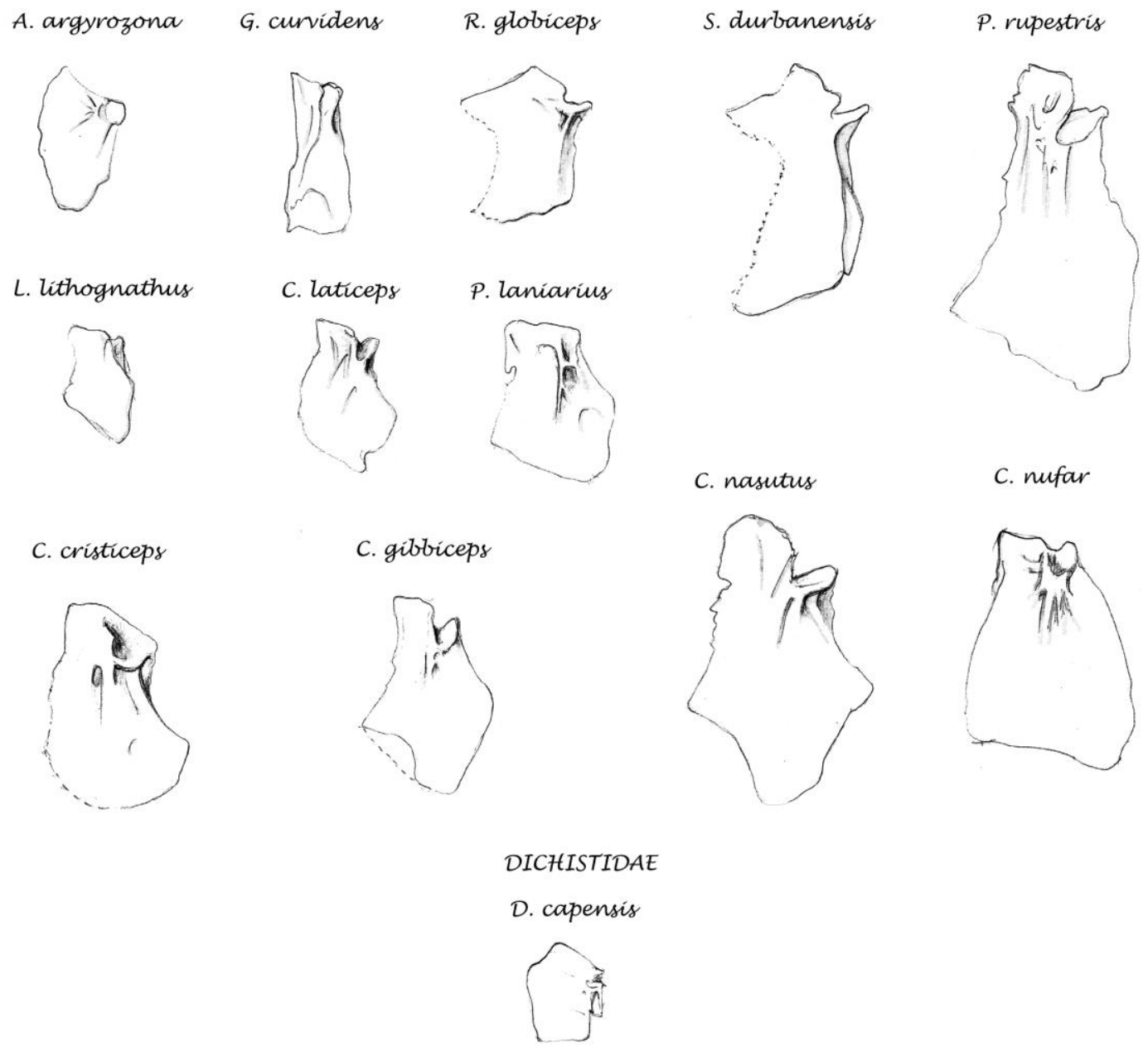

TEMPORAL

JUGAL

SPARIDAE

SPARIDAE

C. cristiceps
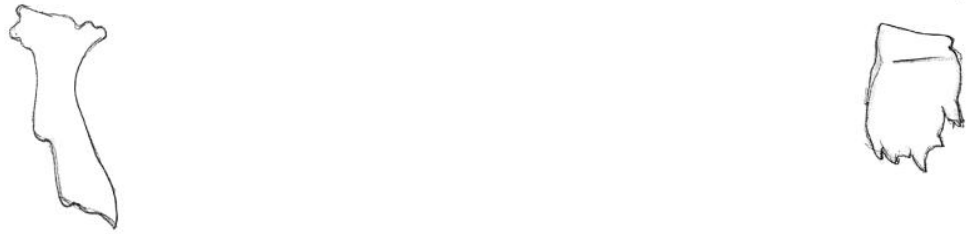

Figure A-7. Illustration of the lachrymal, jugal and and temporal bones of some Sparidae, and the lachrymal of Dichistius capensis. 


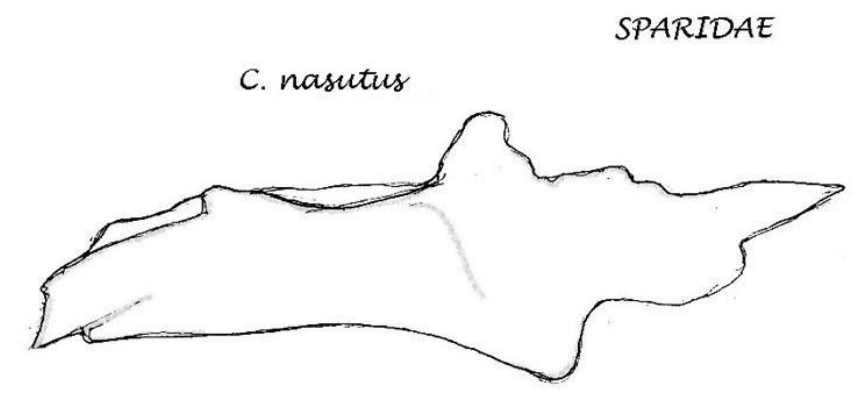

R. hotubi
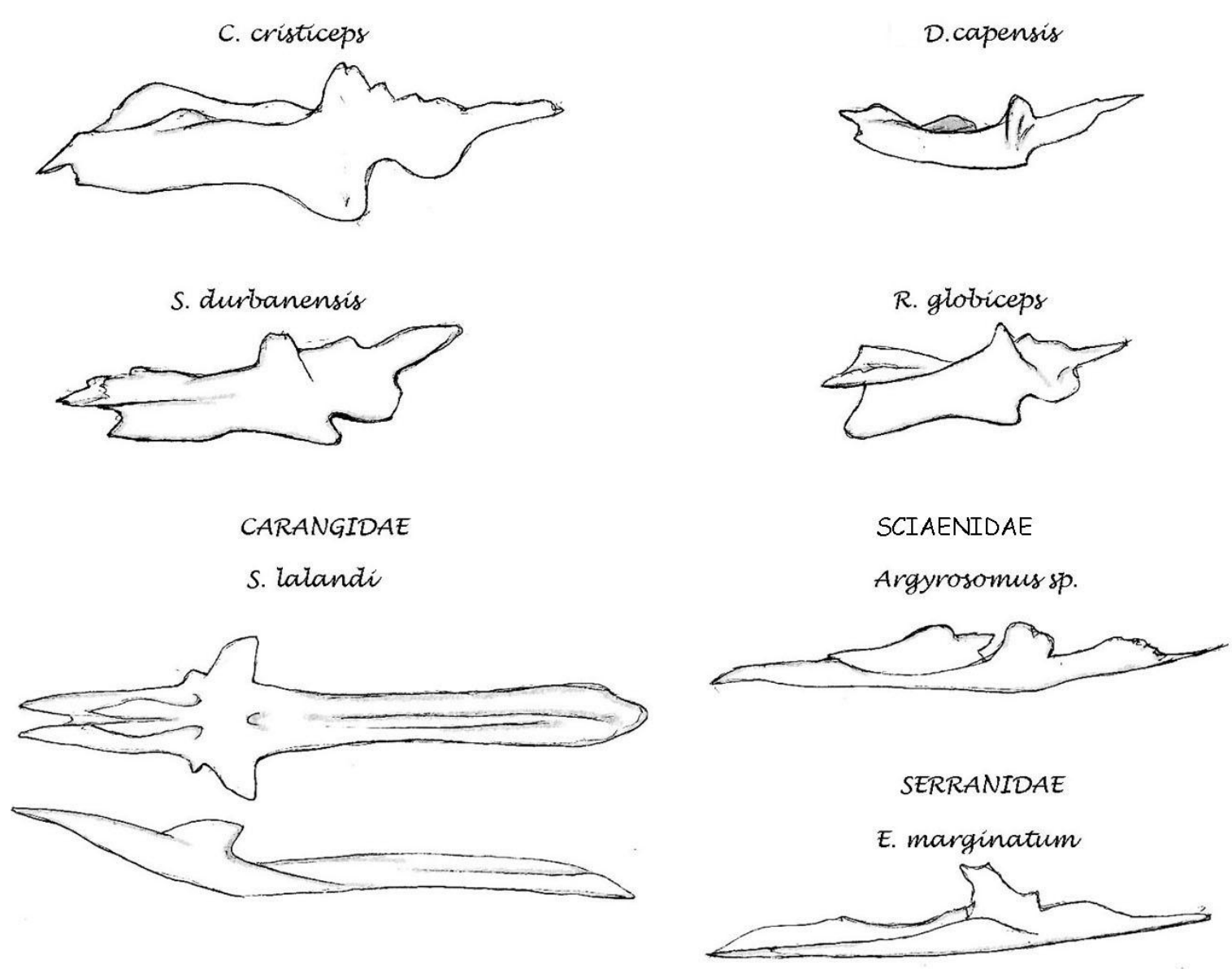

Figure A-8. Illustration of the parasphenoid bone of fish from different families. 
POSTTEMPORAL
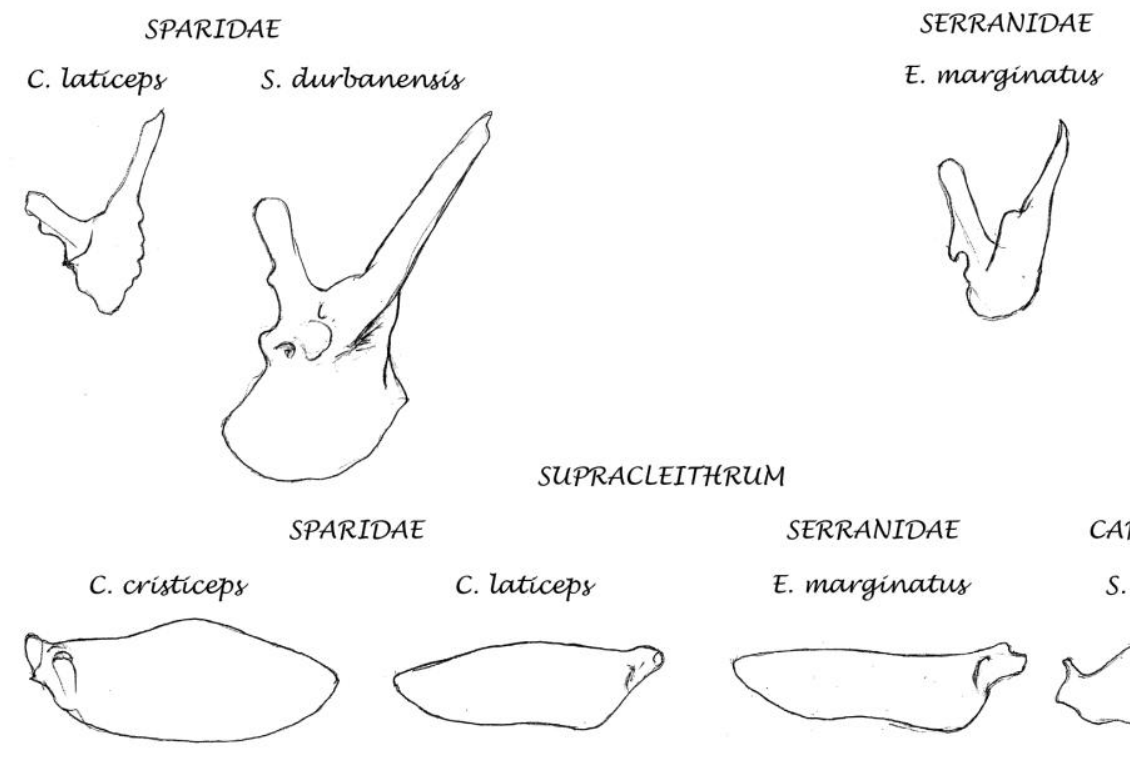

C. laticeps

SERRANIDAE marginatus

CARANGIDAE
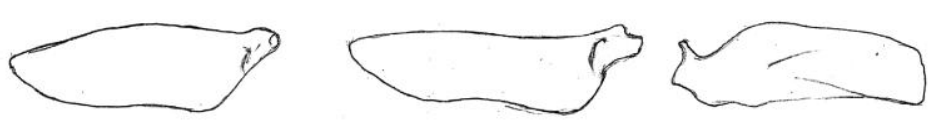

NASAL

SPARIDAE

C. nasutus

C. cristiceps

C. laticeps

S. durbanensis

P. Bochii
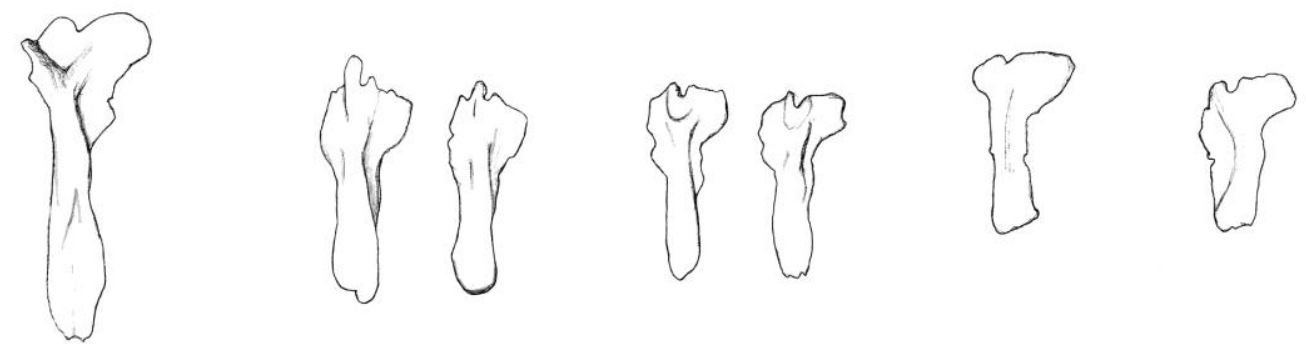

D.capensis

G. curvidens

R. globiceps

C. gibbiceps

P. rupestris

C. nufar
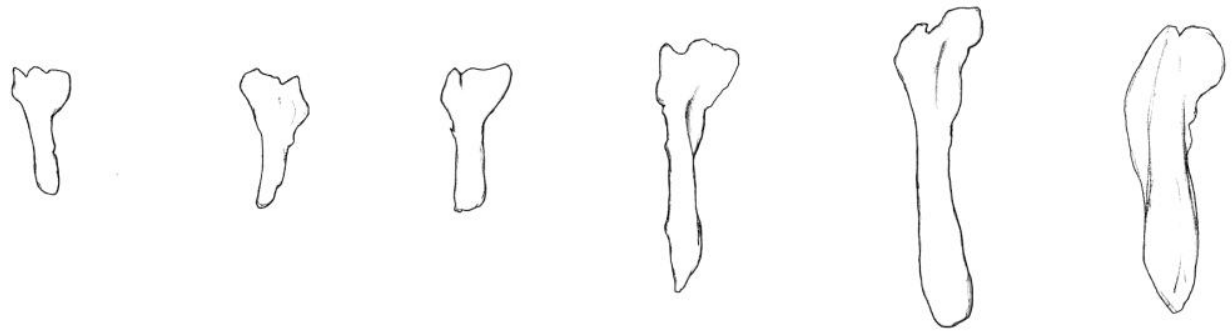

Figure A-9. Illustration of the supracleithrum and posttemporal bones of various fish familes, and the nasal bones of Sparidae. 


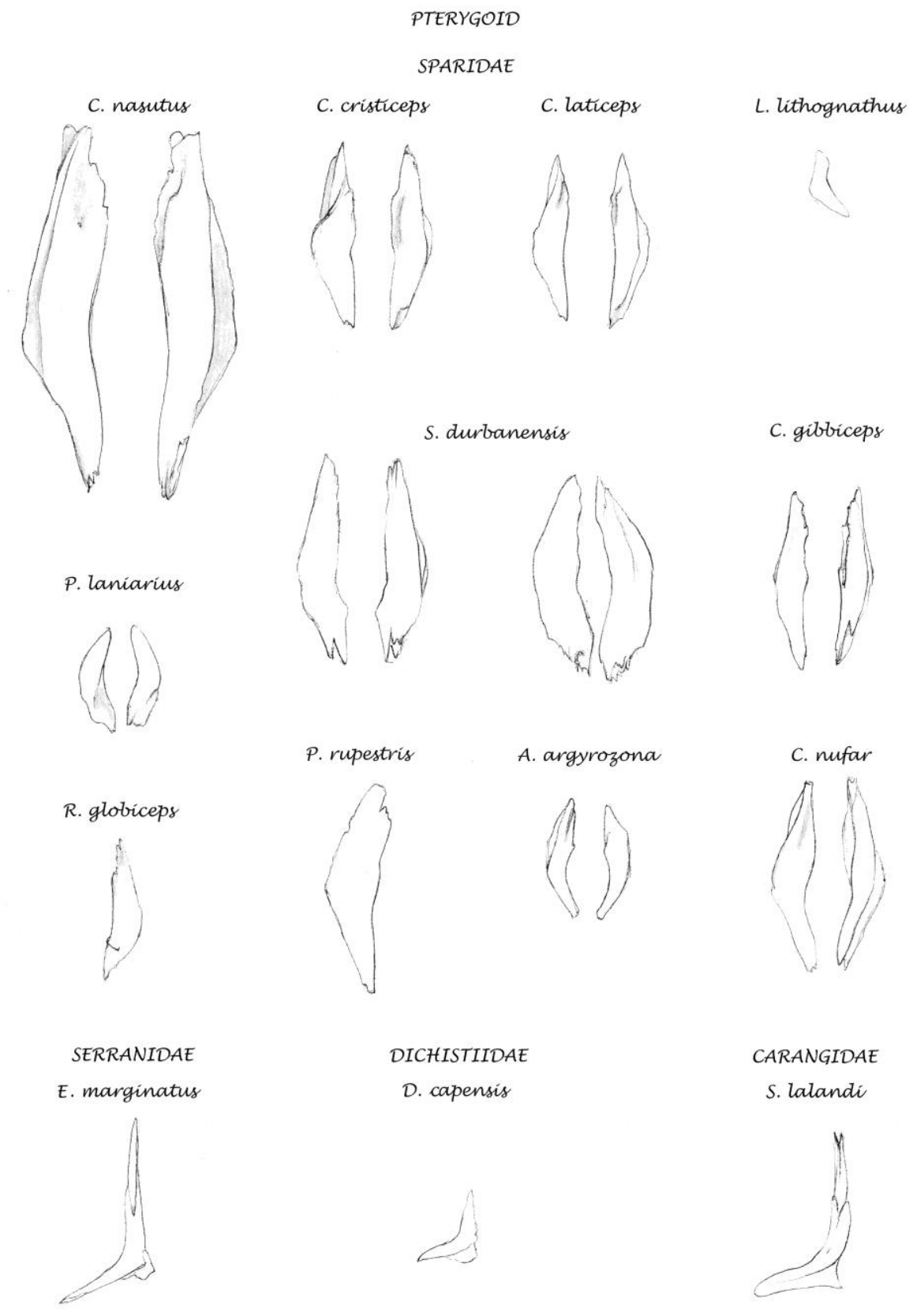

Figure A-10. Illustration of the pterygoid bone of various fish families. 
SCAPULA

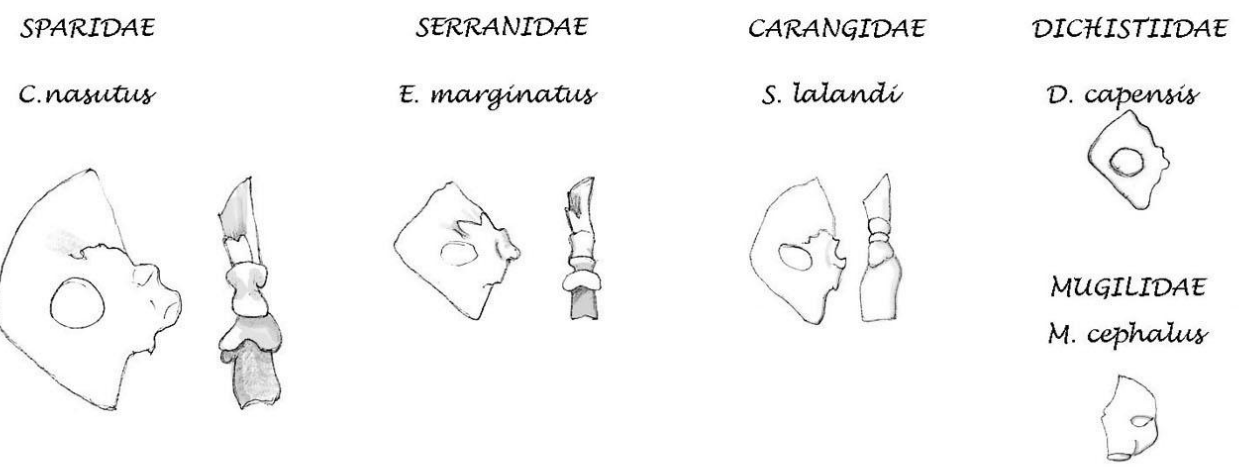

CORACOID

$\begin{array}{lcccc}\text { SPARIDAE } & \text { SERRANIDAE } & \text { SCIAENIDAE } & \text { HAEMULIDAE } & \text { MUGILIDAE } \\ \text { C. laticeps } & \text { E.marginatus } & \text { Argyrosomussp. } & \text { P. commersonnii } & \text { L. richardsonnii }\end{array}$
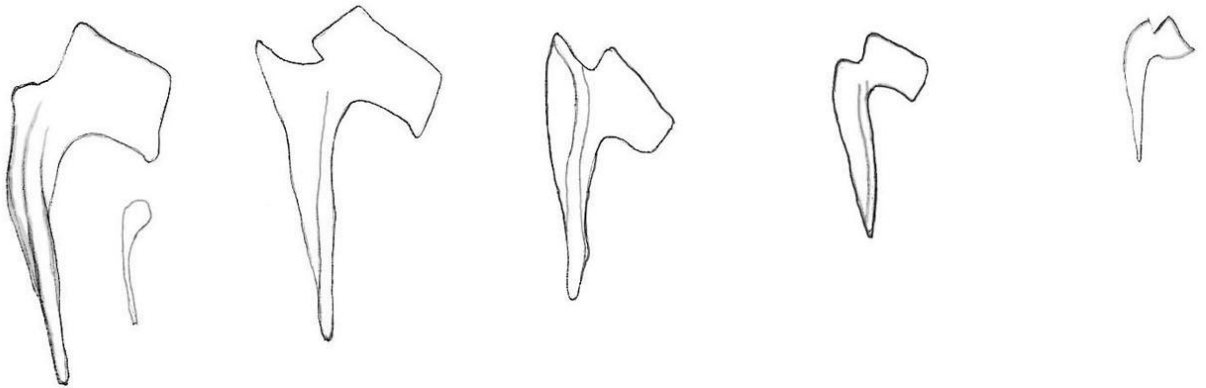

SYMPLECTIC

SPARIDAE

C. cristiceps P. blochiu

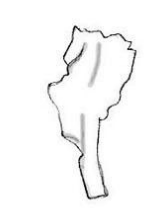

G. curvidens

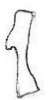

SCIAENIDAE

Argyrosomussp.

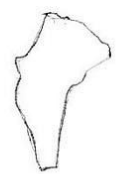

DICHISTIIDAE

D. capensis

G. feliceps
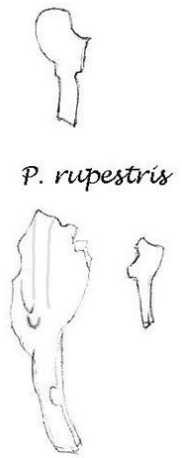
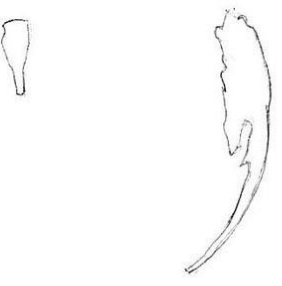

Figure A-11. Illustration of the scapula, coracoid and symplectic bones of various fish families. 
UROHYAL

SPARIDAE

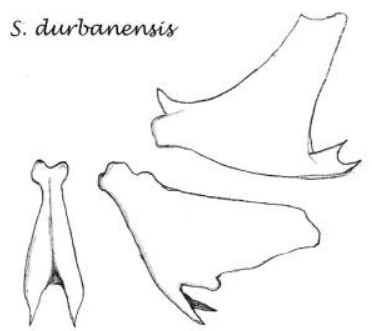

P. rupestris
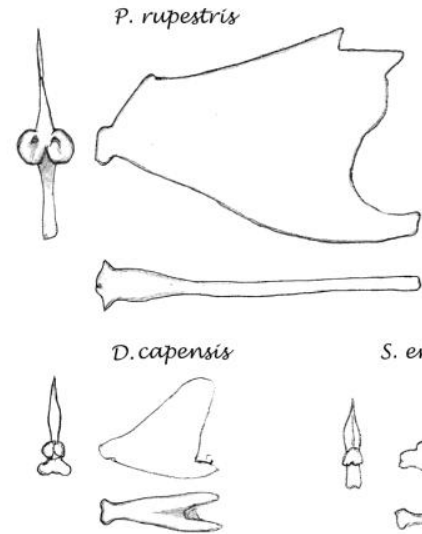

C. gibbiceps

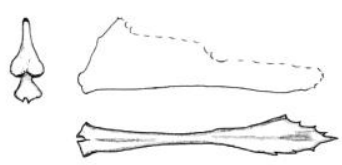

SCIAENIDAE

Argyrosomussp.
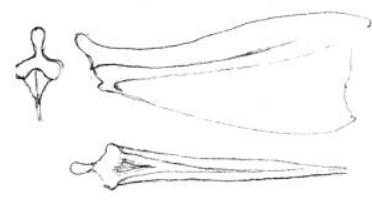

u. canariensis
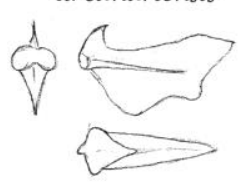

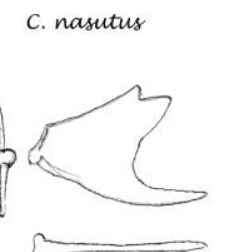

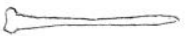

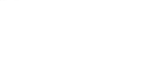

C. cristiceps

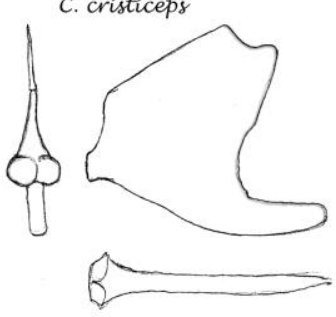

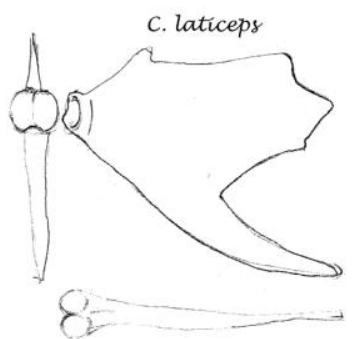

argyrozona

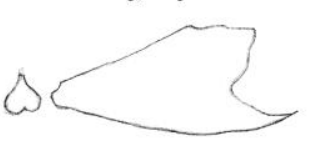

P. laniarius

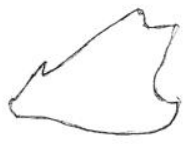

L. lithognathus

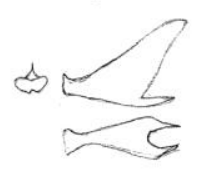

R. holubi

S. salpa

52

R. globiceps

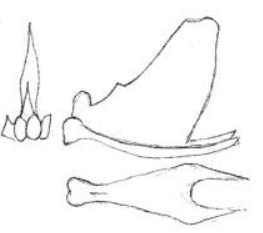

DICFISTIDAE

HAEMULIDAE

P. olivacens

25
$=-3$

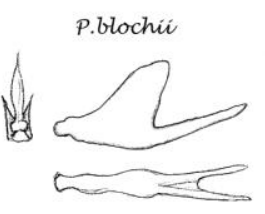

D. capensis

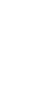

2

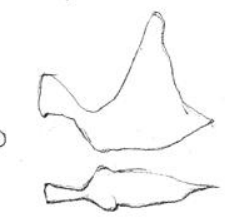

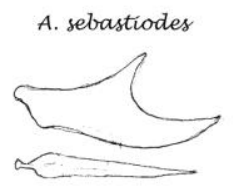

E. marginatus
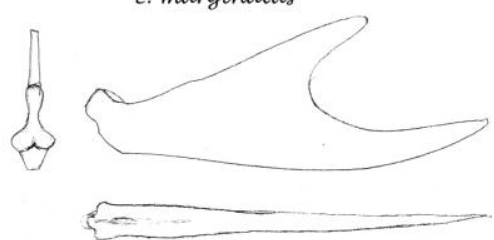

MUGILIDAE

L. richardsonii

इ2

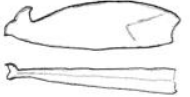

POMATOMIDAE

P. saltatrix

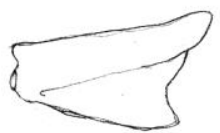

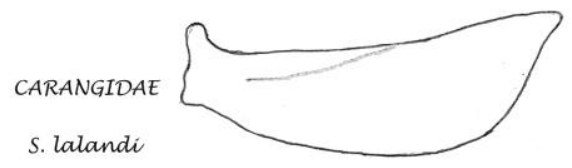

Figure A-12. Illustration of the urohyal bone from various fish families 
VOMER

SPARIDAE
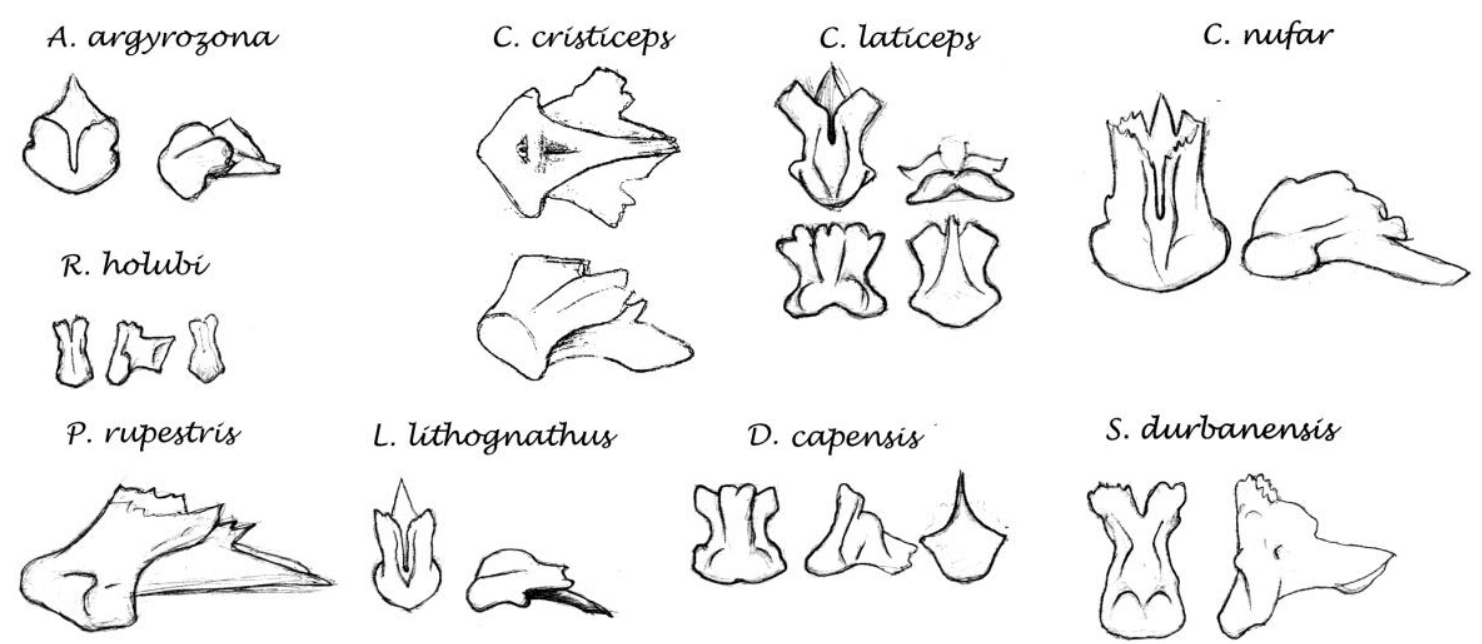

S. durbanensis

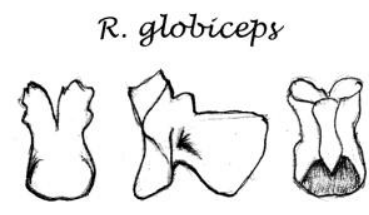

S. emarginatum
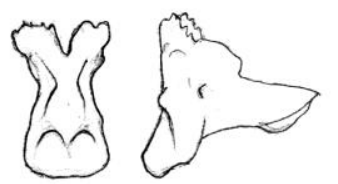
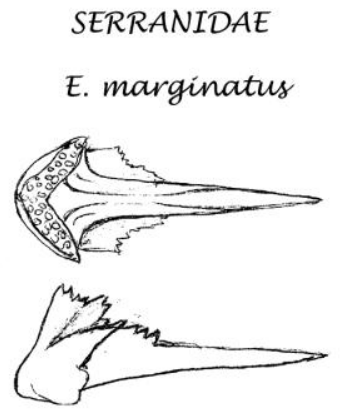

POMATOMIDAE

P. saltatrix

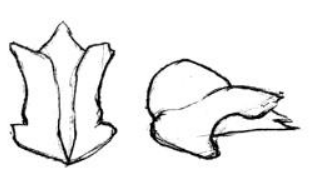

MUGILIDAE

L. richardsonii
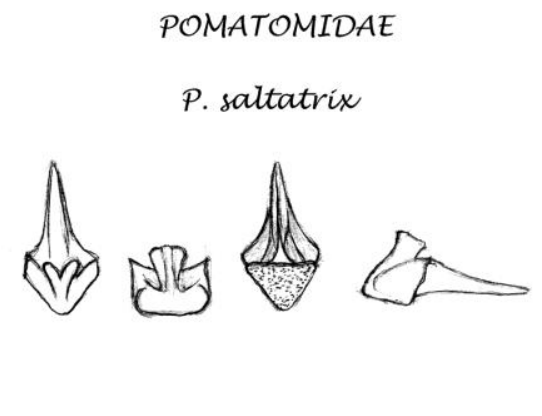

HAEMULIDAE

P. commersonnii
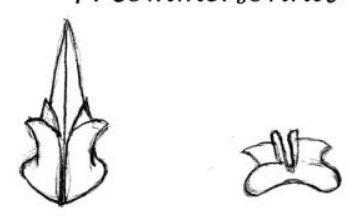

CARANGIDAE

S. latandi

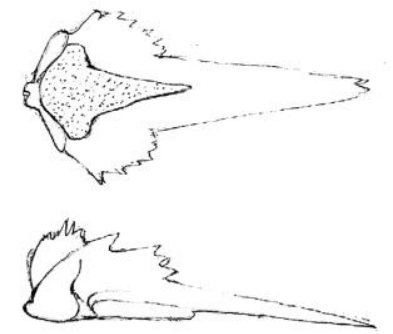

DICHISTIIDAE

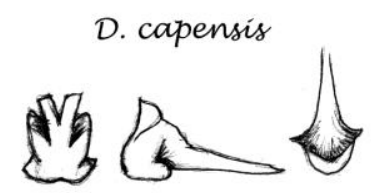

\section{Figure A-13. Illustration of the vomer of species from various fish families}


C. nufar

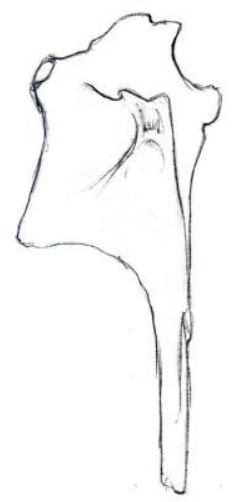

CARANGIDAE

S. latandi

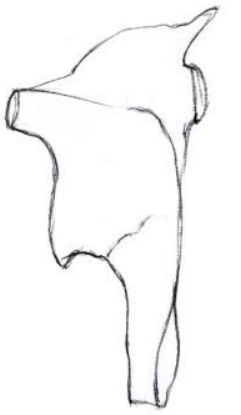

Argyrosomus sp.

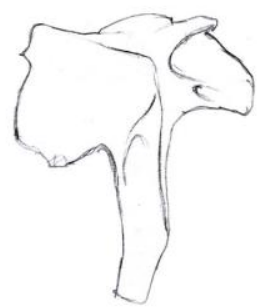

OPLEGNATHIDAE

O. conwayí

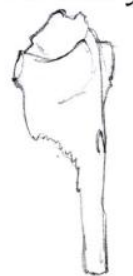

R. globiceps

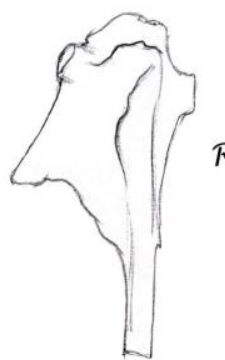

DICHISTIDAE

D. capensis

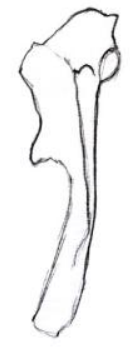

SCIAENIDAE

A. aequidens

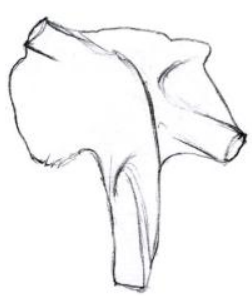

P. saltatrix

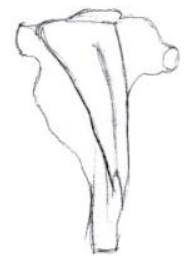

U. canariensis

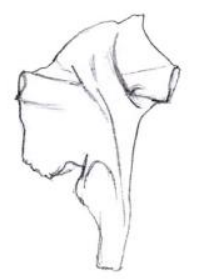

MUGILIDAE

L. richardsonii

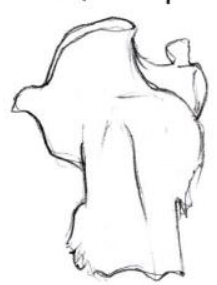

M. cephatus

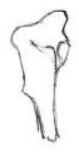

A. argyrozona

S. salpa

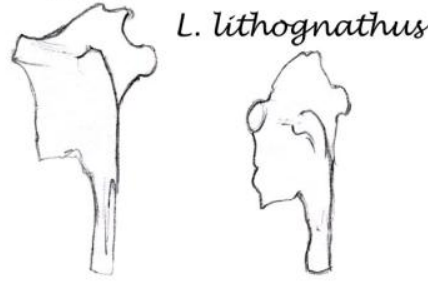

SERRANIDAE

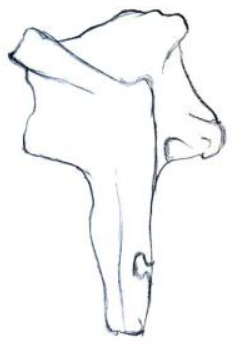

A. sebastoides

HAEMULIDAE

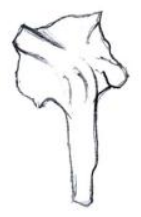

P. commersonniu

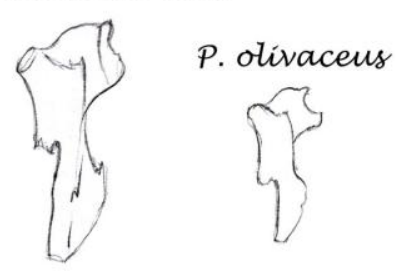

Figure A-14. Illustration of the hymandibular bone of species from various families 


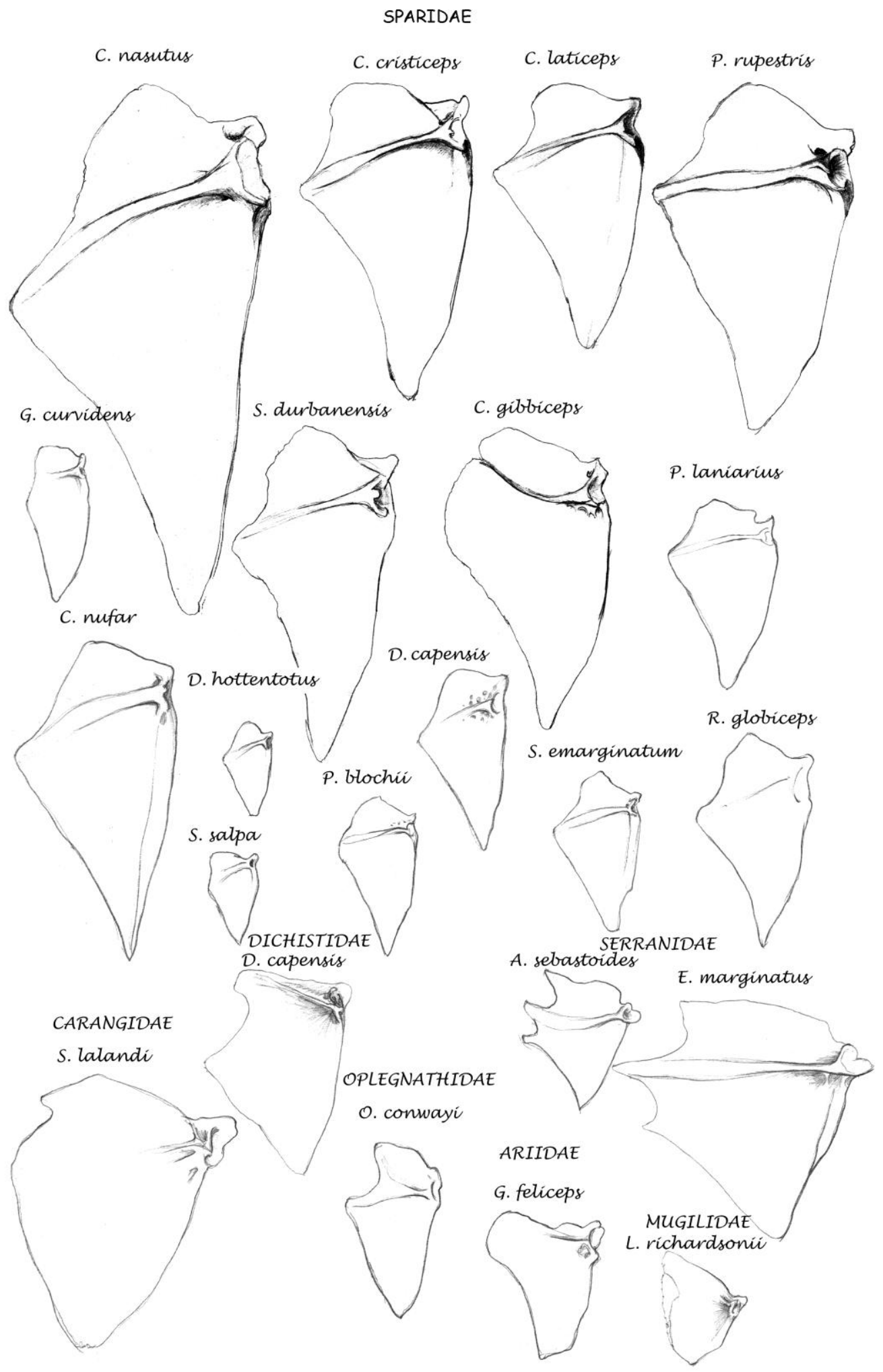

Figure A-15. Illustration of the opercular of species from various fish families 
S. durbanensis

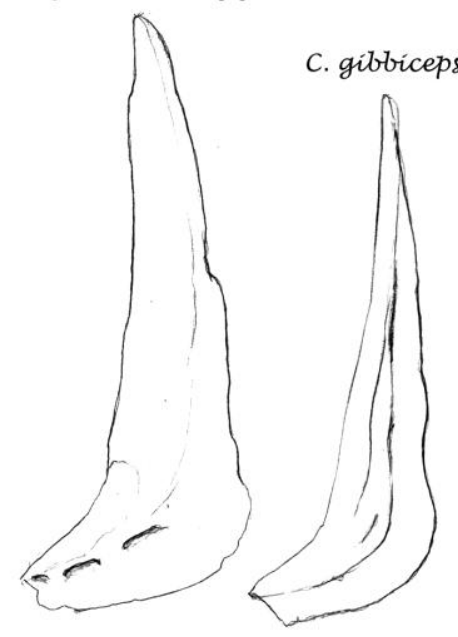

P. blochii
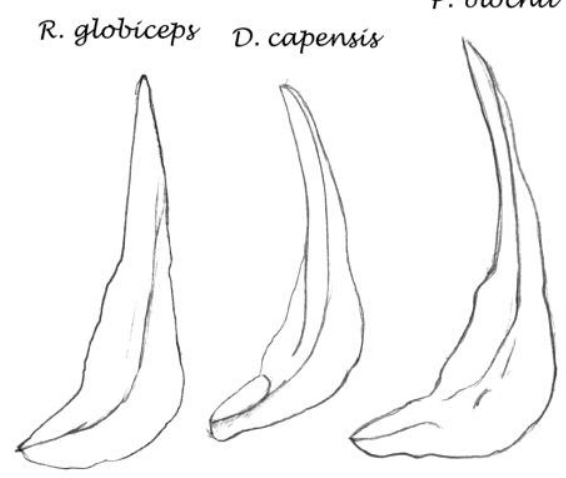

MUGILIDAE

L. richardsoniu

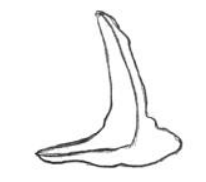

SCOMBRIDAE

S. japonicus

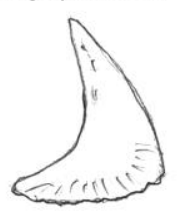

G. curvidens
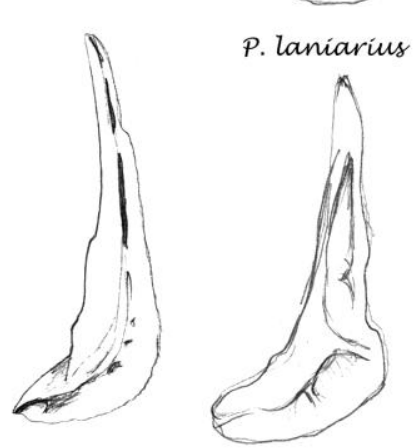

C. laticeps

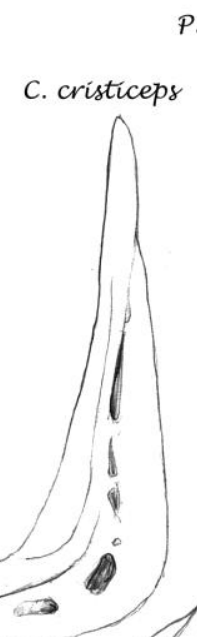

P. rupestris

C. nasutus
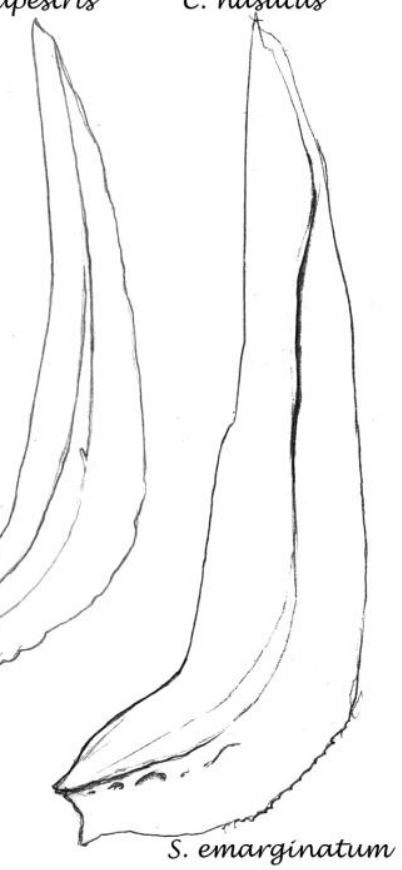

D. hottentotus
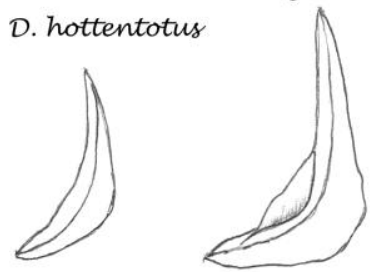

SERRANIDAE

DICHISTIIDAE

D. capensis

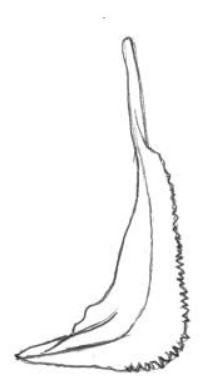

E. marginatus A. sebastoides

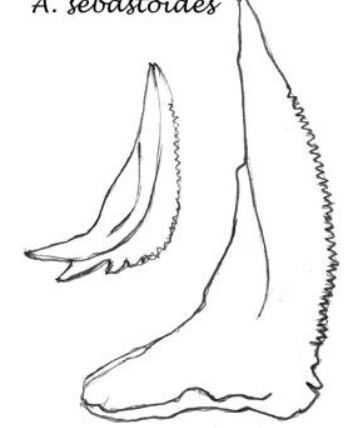

Figure A-16. Illustration of the preopercular of species from various fish families 

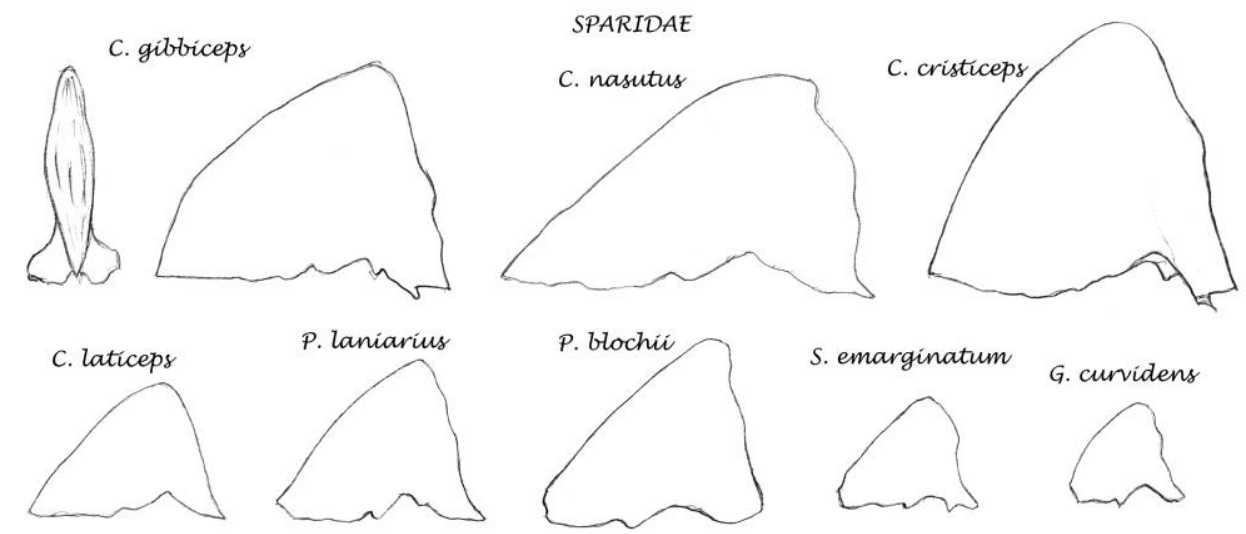

S. emarginatum

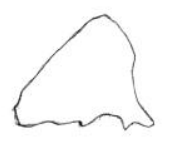

G. curvidens
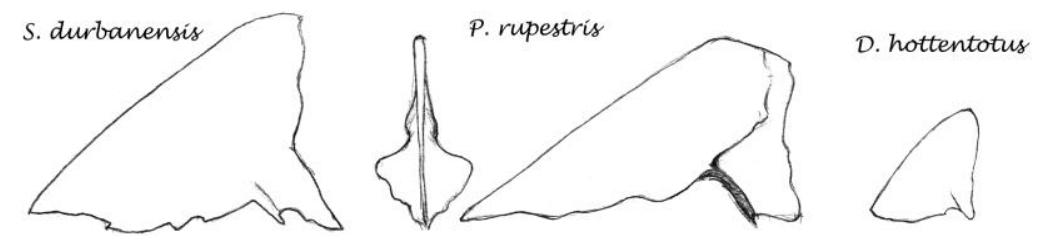

D. capensis
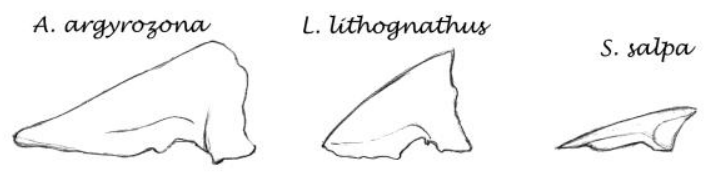

HAEMULIDAE

P. olivaceus
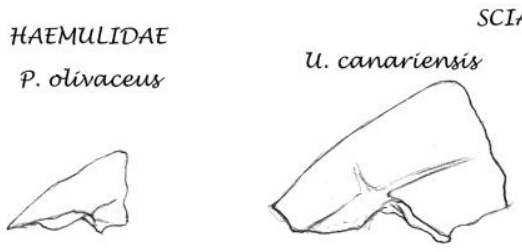

SCIAENIDAE

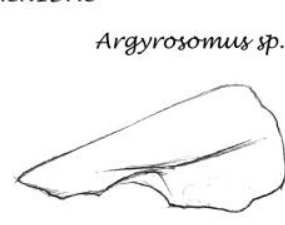

POMATOMIDAE

DICHISTIIDAE OPLEGNATHIDAE
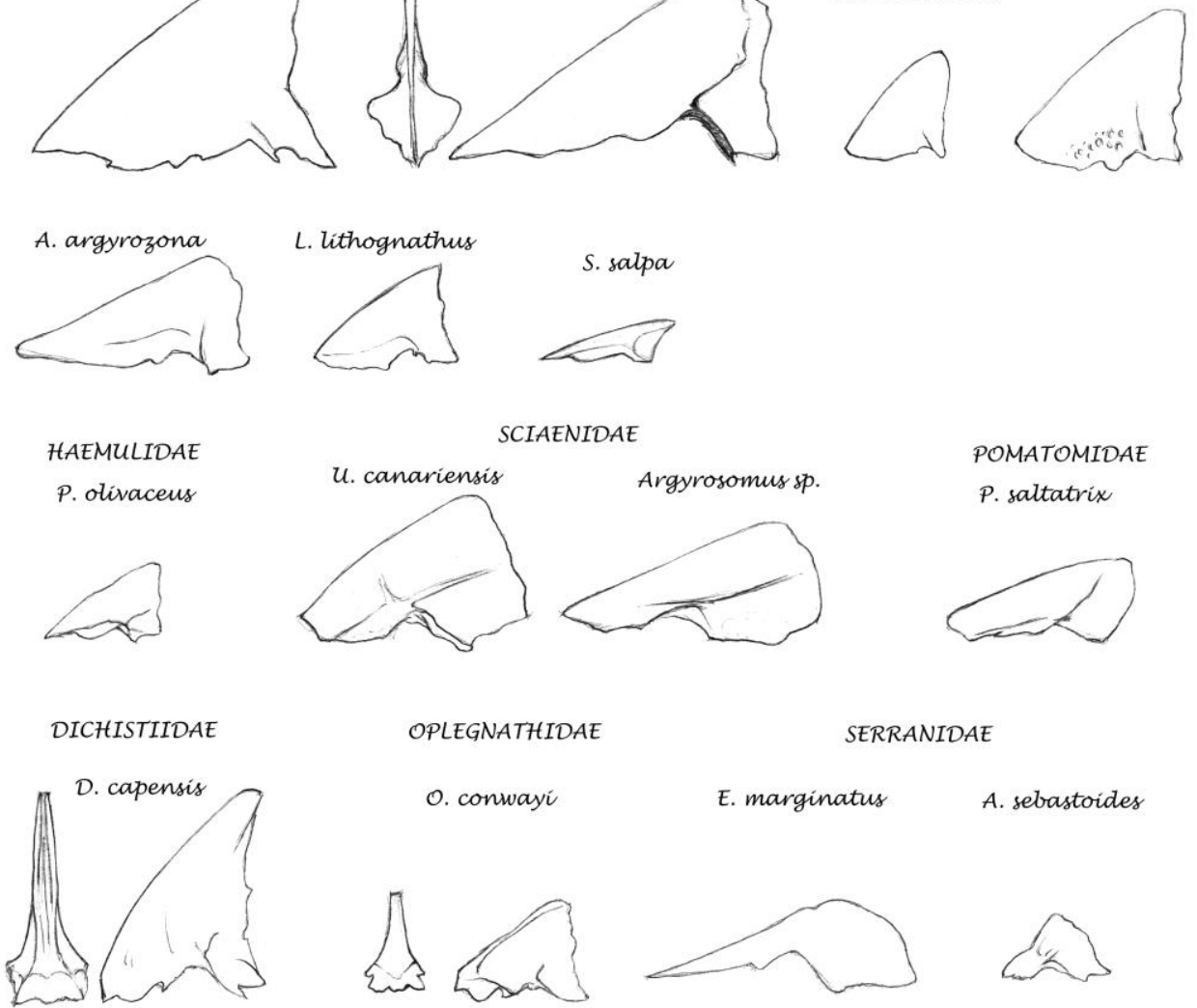

SERRANIDAE

P. saltatrix

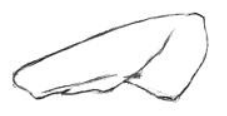

A. sebastoides

Figure A-17. Illustration of the supraoccipital bone of species from various fish families 